\title{
DE-FG02-03ER15448: Modes of Action and Functions of ERECTA-family Receptor-like Kinases in Plant Organ Growth and Development
}

PI: Keiko U. Torii, Ph.D.

Department of Biology

University of Washington

\author{
Final Report
}

\section{Major Research Activities}

Higher plants constitute the central resource for renewable lignocellulose biomass that can supplement for the world's depleting stores of fossil fuels. As such, understanding the molecular and genetic mechanisms of plant organ growth will provide key knowledge and genetic resources that enables manipulation of plant biomass feedstock for better growth and productivity. The goal of this proposal is to understand how cell proliferation and growth are coordinated during aboveground organ morphogenesis, and how cell-cell signaling mediated by a family of receptor kinases coordinates plant organogenesis. The well-established model plant Arabidopsis thaliana is used for our research to facilitate rapid progress. Specifically, we focus on how ERECTA-family leucine-rich repeat receptor kinases (LRR-RLKs) interact in a synergistic manner to promote organogenesis and pattern formation in Arabidopsis.

\section{Specific Aims}

1. Conduct functional analysis of ERECTA-family RLKs: Modes of action and downregulation

2. Link cell-proliferation controlled by ERECTA-family pathways to floral and ovule development

3. Identify downstream targets of ERECTA-family signaling pathways

4. Analyze developmental roles of ERECTA-family RLKs in rice

This project was highly successful, resulted in fourteen publications including nine peerreviewed original research articles. One provisional patent has been filed through this DOE funding (although it was not converted into an actual US Patent). Overall, we identified key developmental programs regulated by the ERECTA-family receptor kinases, flower development including male and female reproductive organ development, stem cell maintenance, and epidermal stomatal patterning. Using stomatal patterning as a model, we identified the downstream target transcription factors that direct differentiation of stomata. In contrast, we were not able to achieve analyzing the developmental role of rice ERECTA-family RLKs. This was mainly due to our inability to grow rice in greenhouse setting in Seattle.

In summary, we have addressed the critical roles for a family of receptor kinases in coordinating proliferation and differentiation of plants, and we successfully elucidated the downstream targets of this signaling pathway in specifying stomatal patterning. On the other hand, our effort to characterize rice mutants was hampered by the lack of facility and expertise to handle rice. Perhaps investigating 'model' cereals would be better option for a laboratory with no expertise in agricultural research. 
Specific Aim 1: During the funding period, we investigated the role of introns in ERECTA expression as well as the role of a juxtamembrane domain for protein stability.

Introns are required for ERECTA expression (Karve et al. 2011 RNA): ERECTA-family RLKs are under complex transcriptional and post-transcriptional regulations. ERECTA coding sequence possesses 26 introns. Interestingly, a cDNA of ERECTA driven by the full-length promoter failed to rescue erecta mutant phenotype, while its genomic region does. We have performed extensive domain-swapping experiments between the genomic and cDNA sequence of ERECTA to address if any specific intron is necessary or sufficient for proper ERECTA mRNA and protein accumulation. We found, surprisingly, that no individual intron is absolutely necessary for ERECTA expression, but rather multiple introns in specific locations increase ERECTA expression in an additive manner. The ability of introns to promote ERECTA expression might be linked to the process of splicing and not to a particular intron sequence.

Regulation of ERECTA protein stability: ERECTA is a membrane-spanning RLK composed of an extracellular ligand-binding leucine-rich repeat (LRR) domain, a membrane-spanning region, and cytoplasmic serine-threonine kinase domain. A juxtamembrane domain is short segment of amino acids between transmembrane domain and kinase domain. We have previously reported that kinase-deleted version of ERECTA $(E R E C T A \triangle K)$ strongly acts as a dominantnegative receptor [1]. Similarly, kinase removal of another ERECTA-family member, ERL1 (ERL1 $1 \Delta \mathrm{K}$ ) confers dominant-negative effects [2]. These kinase-removed receptors accumulates $\sim 100$ times more proteins, indicating that the receptors become stabilized. To gain into the mechanism of receptor stabilization, we have generated a series of deletion constructs. We discovered that the presence of a juxtamembrane domain (=region between the transmembrane domain and the kinase domain) results in instability of ERECTA protein. To understand the potential role of the juxtamembrane domain in regulation of ERECTA activity, we initiated a yeast two-hybrid screen to isolate proteins that may associate with ERECTA cytoplasmic region. We used both versions of cytoplasmic region (with or without the juxtamembrane domain) for screen. We identified several interactors. Dr. Elena Shpak, who was a postdoctoral associate supported by the DOE, took this project with her to her new position as a tenure-track Assistant Professor at the University of Tennessee, Knoxville.

\section{Specific Aim 2:}

During the funding period, we have successfully uncovered specific roles of ERECTA-family RLKs

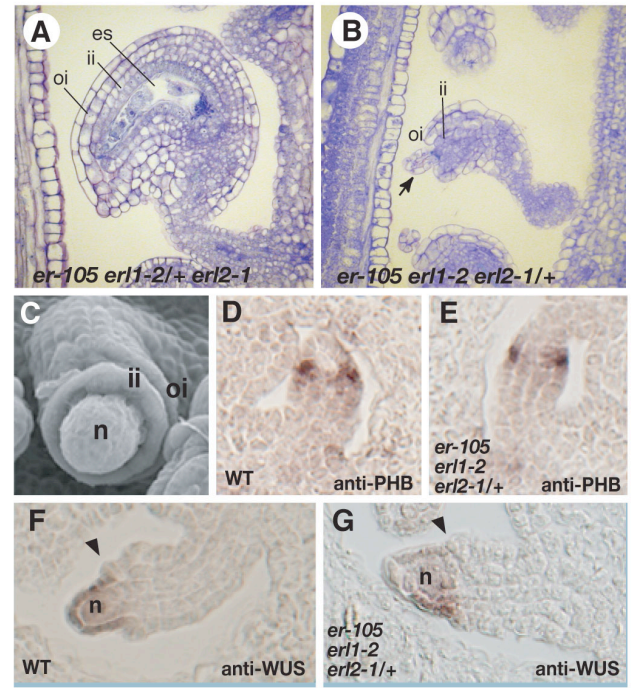

Fig. 1. ERL2 is haploinsufficient for ovule development in the absence of $E R$ and ERL1. (A,B) Thin section through the gametophyte of er-105 erl1-2/+ erl2-1 (A) and er-105 erl1-2 erl2-1/+ (B) ovule at anthesis. In the er-105 erl1-2 erl2-1/+ mutant, the gametophyte has degenerated into a mass of small cells. (C) SEM image of a developing er-105 erl1-2 erl2-1/+ stage 2-I ovule. Both the inner and outer integument initiate as smooth rings at the base of the nucellus, with the outer integument initiating asymmetrically as in wild type. (D-G) Pattern of PHABULOSA (PHB) mRNA accumulation in wild type (D) and er-105 erl1-2 erl2-1/+ (E) and that of WUSCHEL (WUS) in wild type (F) and er-105 erl1-2 erl2-1/+ (G) of approximately stage $2-I$ ovules . No difference in PHB and WUS expression is detected in mutant ovules, indicating normal regional specification. f, funiculus; es, embryo sac; ii, inner integument; oi, outer inteaument: $n$. nucellus 
in controlling development of floral organs, both male and female reproductive organs.

\section{Haploinsufficiency after successive loss of signaling reveals a role for ERECTA-family genes in Arabidopsis ovule development (Pillitteri et al. 2007 Development)}

The Arabidopsis genome contains three ERECTA-family genes, ERECTA (ER), ERL1 and $E R L 2$ that encode leucine-rich repeat receptor-like kinases. This gene family acts synergistically to coordinate cell proliferation and growth during above-ground organogenesis with the major player, ERECTA, masking the loss-of-function phenotypes of the other two members. To uncover the specific developmental consequence and minimum threshold requirement for signaling, $E R$-family gene function was successively eliminated. We report here that ERL2 is haploinsufficient for maintaining female fertility in the absence of ERECTA and ERL1. Ovules of the haploinsufficient er-105 erl1-2 erl2-1/+ mutant exhibit abnormal development with reduced cell proliferation in the integuments and gametophyte abortion (Fig. 1). The early patterning of integuments, on the other hand, appears normal (Fig. 1D-G). Our analysis indicates that progression of integument growth requires ER-family signaling in a dosage-dependent manner and that transcriptional compensation among $E R$-family members occurs to maintain the required signaling threshold. The specific misregulation of cyclin $A$ genes in the er-105 erl1-2 erl2-1/+ mutant suggests that downstream targets of the ERsignaling pathway might include these core cell-cycle regulators. Finally, genetic interaction of the ER family and the WOX-family gene, PFS2, reveals their contribution to integument development through interrelated mechanisms.

\section{Role of ERECTA-family RLKs for flower development-anthers and pollen development} (Hord et al, 2008 MOLECULAR PLANT)

As collaboration with Dr. Hong Ma (Penn State University) and Shuqun Zhang (Univ. Mlssouri), we also investigated the effects of er family mutations on anther development. Mitogenactivated protein kinase (MAPK) and leucine-rich repeat receptor-like kinase (LRR-RLK) signaling pathways have been shown to regulate diverse aspects of plant growth and development. In Arabidopsis, proper anther development relies on intercellular communication to coordinate cell proliferation and differentiation. Two closely related genes encoding MAPKs, MPK3 and MPK6, function redundantly in regulating stomatal patterning. Although the mpk6 mutant has reduced fertility, the function of MPK3 and MPK6 in anther development has not been characterized. Similarly, the ER, ERL1, and ERL2 genes encoding LRR-RLKs function together to direct stomatal cell fate specification and the er-105 erl1-2 erl2-1 triple mutant is sterile. Because the mpk3 mpk6 double null mutant is embryo lethal, anther development

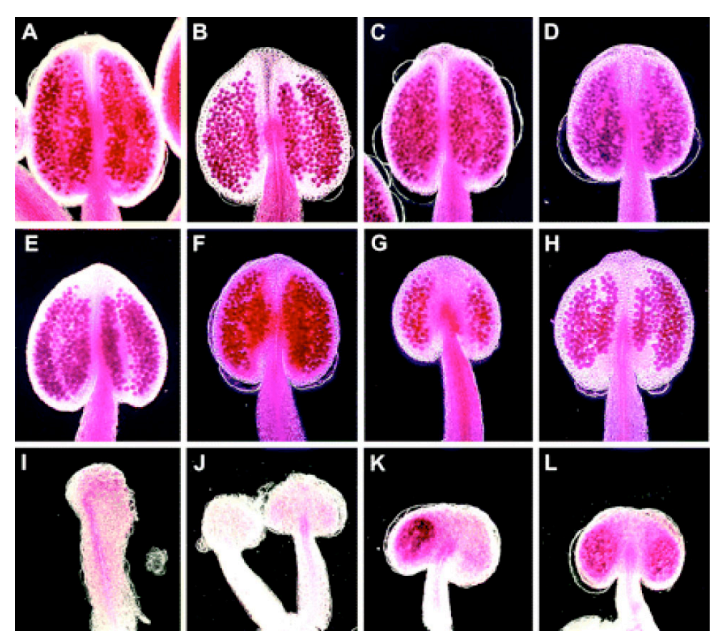

Fig. 2. Pollen phenotype in erecta-family mutant anthers. Pollen viability was determined by the Alxander's staining method (red-purple).(A) Wild-type (Col); (B) er-105; (C) erl1-2; (D) erl2-1; (E) er-105 erl1-2; (F) er-105 erl2-1; (G) erl1-2 erl21; (H) er-105 erl1-2 (+/-) erl2-1; (I) er-105 erl1-2 erl2-1 filament-like stamen with no anther. $(\mathrm{J})$ er105 erl1-2 erl2-1 stamens with underdeveloped anthers.

was characterized in the viable mpk3/+ mpk6/- and er-105 erl1-2 erl2-1 mutants. We found that both mutant anthers usually fail to form one or more of the four anther lobes, with the er105 erl1-2 erl2-1 triple mutant exhibiting more severe phenotypes than those of the $m p k 3 /+$ mpk6/- mutant. The somatic cell layers of the differentiated mutant lobes appeared larger and 
more disorganized than that of wild-type. In addition, the er-105 erl1-2 erl2-1 triple mutant has a reduced number of stamens, the majority of which possess completely undifferentiated or under-differentiated anthers (Fig. 2). Furthermore, sometimes, the mpk3/+ mpk6/- mutant anthers do not dehisce, and the er-105 erl1-2 erl2-1 anthers were not observed to dehisce. Therefore, our results indicate that both ER/ERL1/ERL2 and MPK3/MPK6 play important roles in normal anther lobe formation and anther cell differentiation. The close functional relationship between these genes in other developmental processes and the similarities in anther developmental phenotypes of the two types of mutants reported here further suggest the possibility that these genes might also function in the same pathway to regulate anther cell division and differentiation.

Role for the ERECTA-family genes in specifying the shoot apical meristem (SAM) structure and function.

During the funding period, we also characterized the effects of ERECTA-family genes on development and maintenance of plants' stem cell population, the shoot apical meristem (SAM). In er erl1 erl2 triple mutants, the SAM develops abnormally and precocciously differentiate abnormal floral organs with massive pistils (Fig. 3). Using in situ hybridization analysis, we have characterized the molecular phenotype of the SAM under the complete loss of ERECTA-family functions. We found the ectopic expression of AGAMOUS (AG), a key transcription factor specifying pistil and terminate stem cell fate (not shown). We observed severe disruption of stem cell marker gene (CLAVATA3) expression (Fig. 3C-E), suggesting that ERECTA-family RLKs are critical for proper stem cell function. We have been holding these data. While we discovered interesting effects of ERECTA-family in restricting stem cell markers,

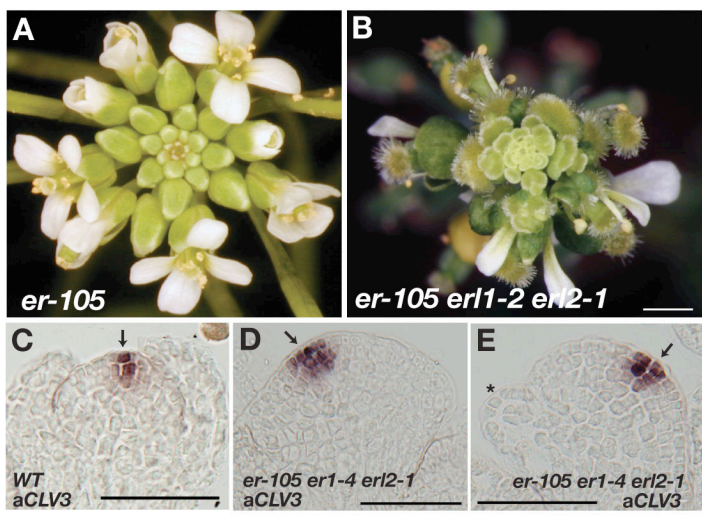

Fig. 3. The complete absence of three ERECTAfamily genes confers severe floral patterning defects. (A, B) In contrast to er-105 (A), flowers of er-105 erl1-2 erl2-1 (B) are severely disorganized with patterning defects. The number of petals and stamens are reduced, while excessive outgrouwth of carpelloid tissues takes place. (C-I) Molecular characterization of er-105 erl1-2 erl2-1 inflorescence and floral meristems by in situ hybridization. (C-E) Stem cell marker CLV3 expression patterns. Unlike wild type (C), the er-105 erl1-2 erl2-1 mutation leads to skewed and off-centered expression of CLV3 in floral meristems (D, E, arrows). The off-centered expression of $C L V 3$ correlates with the asymmetric outgrowth of sepal primordia at the far side (asterisk). we feel that the data are mostly descriptive. As of 2012, we are continuing the project, and we hope to publish strong research article in the near future.

\section{Arabidopsis ERECTA-Family Receptor Kinases Mediate Morphological Alterations Stimulated by Activation of NB-LRR-Type UNI Proteins (Uchida et al. 2011 Plant Cell Physiol)}

Shoot apical meristems (SAMs), which maintain stem cells at the tips of stems, and axillary meristems (AMs), which arise at leaf axils for branch formation, play significant roles in the establishment of plant architecture. Previously, we showed that, in Arabidopsis thaliana, activation of NB-LRR (nucleotide-binding site-leucine-rich repeat)-type UNI proteins affects plant morphology through modulation of the regulation of meristems. However, information about genes involved in the processes was still lacking. Here, we report that ERECTA receptor kinase family members cooperatively mediate the morphological alterations that are stimulated by activation of UNI proteins. uni-1D is a gain-of-function mutation in the UNI gene and uni-1D mutants exhibit early termination of inflorescence stem growth and also formation 
of extra AMs at leaf axils. The former defect involves modulation of the SAM activity and is suppressed by er mutation. Though the AM phenotype is not affected by a single er mutation, it is suppressed by simultaneous mutations of $E R$-family members (Fig. 4). It was previously shown that transzeatin (tZ)-type cytokinins were involved in the morphological phenotypes of uni-1D mutants and that

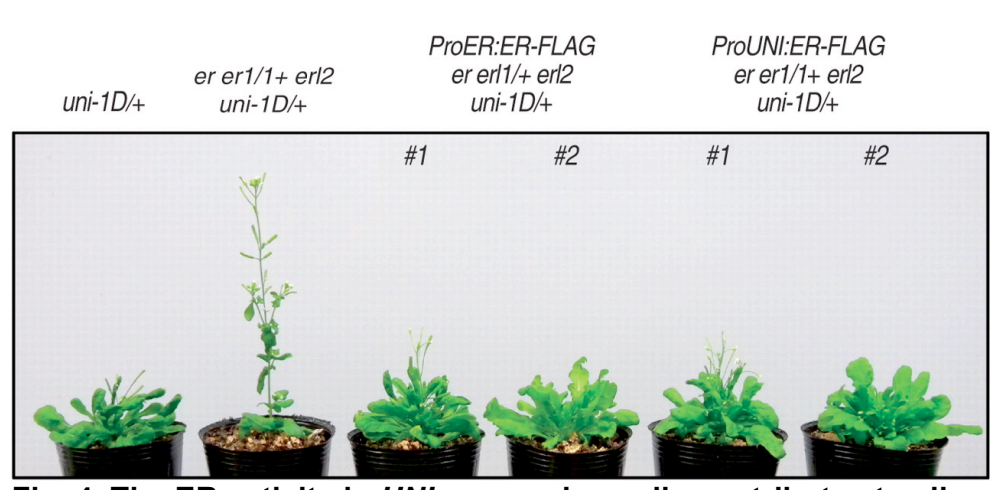

Fig. 4. The ER activity in UNI-expressing cells contributes to all morphological phenotypes of uni-1D/+ mutants. Plants at 48-dayold with the indicated genotypes. Two independent transgenic lines were used to generate ProER:ER-FLAG er erl1/+ erl2 uni-1D/+ and ProUNI:ER-FLAG er erl1/+ erl2 uni-1D/+ plants, respectively. expression of CYP735A2, which is essential for biosynthesis of tZ-type cytokinins, was modulated in uni-1D mutants. We show that this modulation of CYP735A2 expression requires activities of ER-family members. Moreover, the ER activity in UNI-expressing cells contributes to all morphological phenotypes of uni-1D mutants, suggesting that a cross-talk between ER-family-dependent and UNItriggered signaling pathways plays a significant role in the morphological alterations observed in uni-1D mutants.

\section{Aim 3. Identify downstream targets of ERECTA-family signaling pathways}

This aim led to unexpected (and successful) direction through our discovery of a role of ERECTA-family RLKs in stomatal patterning and differentiation. We identified a set of 'master regulatory' transcription factors, SPEECHLESS (SPCH) and MUTE, that sequentially specify cell-state transitions during stomatal development -initiation, proliferation, and differentiation. Genetic analysis suggests that ERECTA-family signaling targets these transcription factors (see below). We further elucidated a role for cell-plate and plasmodesmatal callose synthase in properly restricting stomatal-cell lineages (see brief summaries of accomplishments below).

\section{Termination of Asymmetric Cell Division and Differentiation of Stomata (Pillitteri et al. 2007 Nature)}

During the course of our initial DOE award period, we isolated a novel stomatal mutant, mute, through sensitized screen. This DOE continued to partially support our effort for molecular identification and characterization of MUTE, a 'master switch' gene of stomatal precursor cell differentiation. A technician in the Torii lab, Naomi Bogenschutz, was supported by DOE. We isolated a novel epidermal-patterning mutant mute, in which meristemoids fail to differentiate into guard mother cells. In mute, the meristemoid undergoes excessive rounds of asymmetric division (typically 4-6 rounds) and then arrests. This leaves a rosette like pattern on the epidermis with arrested meristemoid at the center. We performed a map-based cloning and discovered that MUTE encodes a bHLH putative transcription factor. In the absence of MUTE, meristemoids reiterate excessive rounds of asymmetric division and eventually abort instead of differentiating into guard cells. Forced overexpression of MUTE directs the entire epidermis to become stomata. MUTE has two closely-related paralogs: SPEECHLESS and FAMA. FAMA was previously reported by an other group as a regulator of the final step of guard cell differentiation. We show that SPEECHLESS directs the first asymmetric division that initiates the stomatal cell lineage. Together, stomatal differentiation is governed by the sequential actions of three closely-related bHLH proteins at three key transitional states: 
initiation, meristemoid differentiation, and terminal differentiation of guard cells. Our findings highlight the striking parallel between cell-type differentiation in plants and animals (e.g. myogenesis), and further provide implications for related roles played by paralogous genes in the evolution of complex, developmental programs (Fig. 5).

\section{The bHLH protein, MUTE, controls differentiation of stomata and the hydathode pore (Pillitteri et al., 2007. PLANT AND CELL PHYSIOLOGY)}

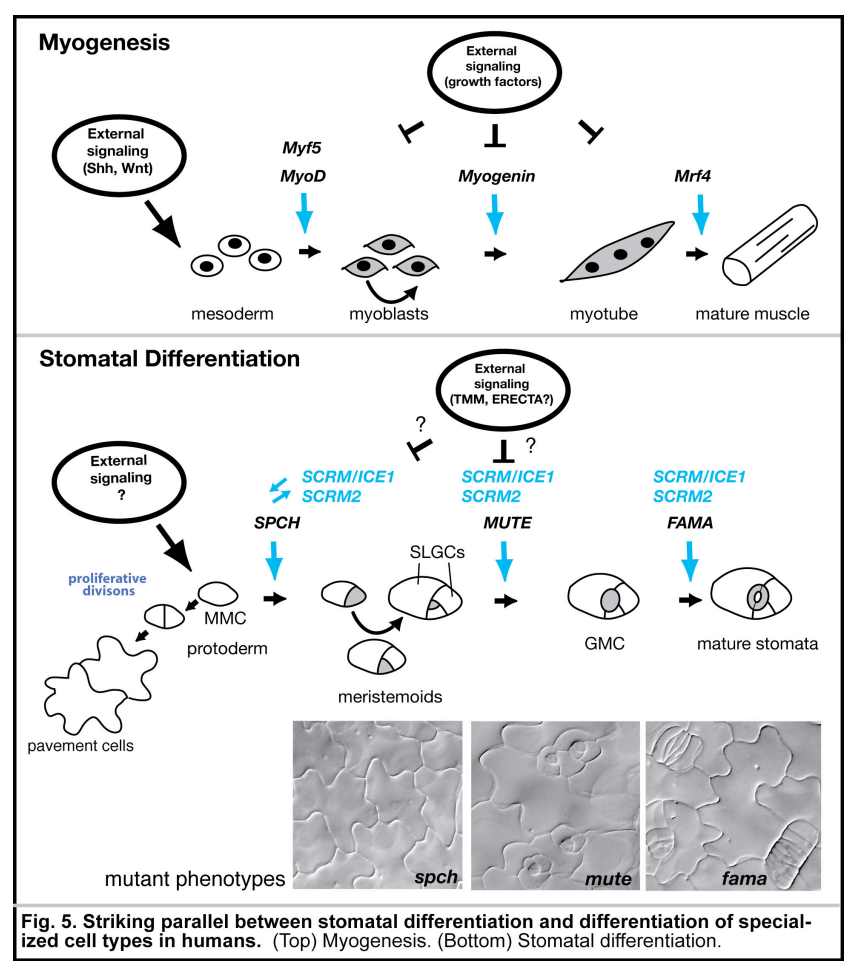

We further analyzed the role of MUTE in differentiation of stomata and hydathode pores in different organs. The genetic control of stomatal spacing across the epidermal surface is variable in different organs. For instance, a distinct suite of genes from those in leaves regulates stomatal patterning in hypocotyls. Here we report that regardless of organ type, MUTE controls downstream events directing stomatal differentiation, specifically the transition from meristemoid to guard mother cell. Ectopic MUTE expression is sufficient to over-ride cell fate specification in cell types that do not normally differentiate stomata. Furthermore, MUTE is required for the production of the structure evolutionarily related to stomata, the hydathode pore. Consistently, MUTE displays expression at the tip of cotyledons and leaves, thus co-localizing with the auxin maxima. However, MUTE itself was not regulated by the auxin, and the absence of hydathode pores in mute did not affect the auxin maxima. Surprisingly, our analysis revealed that the requirement for MUTE could be partially circumvented under conditions of compromised inhibitory signaling. This project was in part supported by the DOE award, which covered a stipend for research technician, Naomi Bogenschutz.

\section{Dysregulation of cell-to-cell connectivity and stomatal patterning by loss-of-function mutation in Arabidopsis CHORUS (Guseman et al. 2010 Development)}

Patterning of stomata, valves on the plant epidermis, requires the orchestrated actions of signaling components and cell-fate determinants. To understand the regulation of stomatal patterning, we performed a genetic screen using a background that partially lacks stomatal signaling receptors. Here, we report the isolation and characterization of chorus (chor), which confers excessive proliferation of stomatal-lineage cells mediated by SPCH. chor breaks redundancy among three ERECTA-family genes and strongly enhances stomatal patterning defects caused by loss-of-function in TOO MANY MOUTHS. chor seedlings also exhibit incomplete cytokinesis and growth defects, including disruptions in root tissue patterning and root hair cell morphogenesis. CHOR encodes a putative callose synthase, GLUCAN SYNTHASE-LIKE 8 (GSL8), that is required for callose deposition at the cell plate, cell wall and plasmodesmata. Consistently, symplastic macromolecular diffusion between epidermal cells is significantly increased in chor, and proteins that do not normally move cell-to-cell, including a fluorescent protein-tagged $\mathrm{SPCH}$, diffuse to neighboring cells. Such a phenotype 
is not a general trait caused by cytokinesis defects. Our findings suggest that the restriction of symplastic movement might be an essential step for the proper segregation of cell-fate determinants during stomatal development.

\section{Aim 4. Analyze developmental roles of ERECTA-family RLKs in rice}

Through the DOE funding period, we have identified transposon insertion lines of rice disrupting OsERECTA. We tried our best effort to grow rice for phenotypic characterization. However, green house in Seattle with very little availability and intensity of sunlight, we were not able to grow and propagate rice. We admit that this Aim 4 was perhaps overly ambitious, especially given the size of the lab (3-4 full time personnel). As we successfully increase the laboratory size, or alternative, as collaboration with rice researchers we would like to pursue the aim in the future.

\section{Publications supported by DOE and acknowledging the sponsor}

1. Pillitteri, L.J., Sloan, D.B., Bogenschutz, N.L, and Torii, K.U. (2007) Termination of asymmetric cell division and differentiation of stomata. Nature 445: 501-505 (Article)

2. Bemis, S.M. and Torii, K.U. (2007) Autonomy of cell proliferation and developmental programs during Arabidopsis aboveground organ morphogenesis. Developmental Biology 304: 367-381

3. Torii, K.U., Kanaoka, M.M., Pillitteri, L.J., and Bogenschutz, NL. (2007) Stomatal development: Three steps for cell-type differentiation. Plant Signaling \& Behavior 2: 5

4.Pillitteri, L.J., Bemis, S.M., Shpak, E.D., and Torii, K.U. (2007) Haploinsufficiency after successive loss of signaling reveals a role for ERECTA-family genes in ovule development. Development 134: 3099-3109.

5. Pillitteri, L.J. and Torii, K.U. (2007) Breaking the silence: three bHLH genes direct cell-fate decisions during stomatal development. BioEssays 29: 861-870

6. Hord, C.L.H., Yu-Jin Sun, Y.-J., Pillitteri, L.J., Torii, K.U., Wang, H., Zhang, S., and Ma, H. (2008) Regulation of Arabidopsis Early Anther Development by the Mitogen-Activated Protein Kinases, MPK3 and MPK6, and the ERECTA and Related Receptor-Like Kinases. Molecular Plant 4: 645-658.

7. Pillitteri, L.J., Bogenschutz, N.L., Torii, K.U. (2008) The bHLH protein, MUTE, controls differentiation of stomata and the hydathode pore in Arabidopsis. Plant and Cell Physiology 49: 934-943

8. Torii, K.U. (2007) Stomatal patterning and guard cell differentiation. In Cell Division Controls in Plants, Eds. By DPS Verma and Z Hong, Springer-Verlag, Heidelberg, pp. 343-359.

9. Torii, K.U. (2008) Transmembrane receptors in plants: receptor kinases and their ligands. In: Intracellular Signaling in Plants. Annual Plant Reviews 33, Ed. by Z. Yang. Blackwell Publishing, pp1-29.

10. van Zanten $M^{*}$, Basten Snoek L, van Eck-Stouten E, Proveniers MC, Torii K.U., Voesenek LA,Peeters AJ, Millenaar FF. (2009). Ethylene-induced hyponastic growth in Arabidopsis thaliana is controlled by ERECTA. Plant Journal 61: 82-95

11. Guseman, J.M., Lee, J.S., Bogenschutz, N.L, Peterson, K.M., Virata, R.E., Xie, B., Kanaoka, M.M., Hong, Z., and Torii, K.U. (2010) Dysregulation of cell-to-cell connectivity and stomatal patterning by loss-of-function mutation in Arabidopsis CHORUS/GLUCAN SYNTHASE-LIKE8. Development 137: 1731-1741 
12. Peterson, K.M., Rychel, A.L., and Torii, K.U.* (2010) Out of the mouths of plants: the molecular basis of the evolution and diversity of stomatal development. Plant Cell 22: 296-306

13. Uchida, N., Igari, K., Bogenschutz, N.L., and Torii, K.U, and Tasaka, M. (2011) Arabidopsis ERECTA-family Receptor Kinases Mediate Morphological Alternations Stimulated by Activation of NB-LRR-type UNI proteins. Plant Cell Physiology 52:804814

14. Karve, R., Liu, W. Willet, S.G., Torii, K.U., Shpak, E.D. (2011) The presence of multiple introns is essential for ERECTA expression in Arabidopsis. RNA 17:1901-1920

\section{Patent/Invention dsclosure from this DOE award}

\section{Methods for modulating plant growth}

Inventors

Keiko U Torii, Elena D Shpak

Publication date

2004/12/30

Patent office

US

Application number

11027304

Description

The invention provides methods for modulating plant height and organ shape, comprising the step of expressing a transgene in a plant, wherein the transgene encodes an ERECTAlike protein lacking an active kinase domain and wherein expression of the transgene modulates plant height or organ shape. The invention also provides methods for for enhancing the yield of a crop plant, transgenic plants comprising a gene encoding an ERECTA-like protein, vectors encoding ERECTA-like proteins and host cells and/or cell cultures comprising these vectors, and isolated nucleic acid sequences.

${ }^{* *}$ This provisional patent was not converted into an actual patent*

\section{Referencee}

1. Shpak, E.D., M.B. Lakeman, and K.U. Torii (2003) Dominant-negative receptor uncovers redundancy in the Arabidopsis ERECTA leucin-rich repeat receptor-like kinase signaling pathway that regulates organ shape. Plant Cell 15: 1095-1110.

2. Lee, J.S., T. Kuroha, M. Hnilova, D. Khatayevich, M.M. Kanaoka, J.M. McAbee, M. Sarikaya, C. Tamerler, and K.U. Torii (2012) Direct interaction of ligand-receptor pairs specifying stomatal patterning. Genes Dev 26: 126-36. 


\title{
ARTICLES
}

\section{Termination of asymmetric cell division and differentiation of stomata}

\author{
Lynn Jo Pillitteri ${ }^{1}$, Daniel B. Sloan ${ }^{1}$, Naomi L. Bogenschutz ${ }^{1}$ \& Keiko U. Torii ${ }^{1,2,3}$
}

Stomata consist of a pair of guard cells that mediate gas and water-vapour exchange between plants and the atmosphere. Stomatal precursor cells-meristemoids-possess a transient stem-cell-like property and undergo several rounds of asymmetric divisions before further differentiation. Here we report that the Arabidopsis thaliana basic helix-loop-helix (bHLH) protein MUTE is a key switch for meristemoid fate transition. In the absence of MUTE, meristemoids abort after excessive asymmetric divisions and fail to differentiate stomata. Constitutive overexpression of MUTE directs the entire epidermis to adopt guard cell identity. MUTE has two paralogues: FAMA, a regulator of guard cell morphogenesis, and SPEECHLESS (SPCH). We show that SPCH directs the first asymmetric division that initiates stomatal lineage. Together, SPCH, MUTE and FAMA bHLH proteins control stomatal development at three consecutive steps: initiation, meristemoid differentiation and guard cell morphogenesis. Our findings highlight the roles of closely related bHLHs in cell type differentiation in plants and animals.

A key developmental innovation of land plants was the evolution of specialized epidermal structures called stomata. In Arabidopsis, a stomatal lineage arises from an undifferentiated protodermal cell called a meristemoid mother cell (MMC), which divides asymmetrically to give rise to two daughter cells with distinct identities: a pavement cell and a meristemoid. The meristemoid undergoes several rounds of asymmetric division before differentiating into a round, guard mother cell (GMC). The GMC divides symmetrically to generate a pair of guard cells that surround a microscopic pore ${ }^{1}$. Density and distribution of stomata are influenced by environmental factors as well as developmental programmes ${ }^{2,3}$. Recent studies have revealed that stomatal patterning is controlled by positional cues. The current model suggests that the subtilisin-like protease STOMATAL DENSITY AND DISTRIBUTION 1 (SDD1) generates a cell-cell signal that is interpreted by the transmembrane receptor TOO MANY MOUTHS (TMM) and three ERECTA family receptor-like kinases ERECTA, ERECTA-LIKE 1 (ERL1) and ERL2. This signalling pathway is proposed to be mediated via mitogen-activated protein kinase (MAP kinase) cascades $^{4-8}$. Loss-of-function mutations in these loci disrupt epidermal patterning with stomata produced adjacent to each other or in clusters. In addition, two putative transcription factors FAMA and FOUR LIPS (FLP) regulate the final differentiation of guard cells: their loss-of-function mutations result in reiterative symmetric divisions of $\mathrm{GMCs}^{7,9}$.

Although our understanding of genes regulating stomatal development has greatly advanced, an intrinsic factor that specifies differentiation of a meristemoid had not yet been discovered. If such a gene exists, then the loss-of-function mutation may extend the lifespan of meristemoids, such that mutant plants should exhibit excessive rounds of asymmetric division but no GMC differentiation. On the basis of this hypothesis, we conducted a sensitized genetic screen and identified MUTE.

\section{Key switch for meristemoid transition}

The loss-of-function mute mutation resulted in the complete absence of stomata. mute plants are small, pale and sterile, presumably owing to severe defects in gas exchange and transpiration (Fig. 1a). Wildtype meristemoids undergo $1-3$ rounds (mean $=2.23 \pm 0.13$ ) of asymmetric division before differentiating into a GMC (Fig. 1b, d) ${ }^{1}$. In contrast, mute meristemoids undergo $3-6$ rounds $($ mean $=4.46 \pm$ 0.20 ) of asymmetric division, suggesting that mute meristemoids have an extended period of replication (Fig. 1c, d). The inward-spiral nature of the reiterative asymmetric divisions created a rosette pattern in the mute epidermis with an arrested, small triangular meristemoid at the centre (Fig. 1).

The molecular character of arrested meristemoids was investigated using stomatal cell-type-specific markers (Fig. 1e-1). TMM and ERL1 mark stomatal lineage cells with the highest expression in meristemoids (Fig. 1e, $\mathrm{f})^{6,8}$. The arrested mute meristemoids expressed high levels of TMM::TMM-GFP, a translational fusion of TMM with a green fluorescent protein, and ERL1::GUS, a reporter $\beta$-glucuronidase driven by the ERL1 promoter ${ }^{6,8}$ (Fig. $1 \mathrm{i}$ and j, respectively). In addition, the rosette of cells surrounding the arrested meristemoid exhibited faint expression of these reporters (Fig. 1e, f, i, j), suggesting their identity as stomatal lineage ground cells (SLGCs)-larger daughter cells derived from the reiterative asymmetric divisions of a single meristemoid $^{8}$.

To investigate whether arrested meristemoids progress into GMCs, we further examined the expression of FAMA::FAMA-GFP, a translational fusion of GFP and FAMA (Supplementary Methods). In the wild-type leaf epidermis, FAMA-GFP was specifically detected in the nuclei of GMCs and early, immature guard cells, but not in meristemoids (Fig. 1g). In mute, no FAMA-GFP expression was detected (Fig. 1k). Likewise, the arrested meristemoids in mute did not express the mature-guard-cell-specific GFP marker E994 (Fig. 1h, $1)^{10}$, suggesting that these cells failed to establish either GMC or guard cell identity ${ }^{7,9}$. On the basis of these findings, we conclude that MUTE controls differentiation of meristemoids to GMCs.

\section{Genetic interactions}

To place MUTE within the context of genetic pathways for stomatal patterning and differentiation, we next investigated genetic 

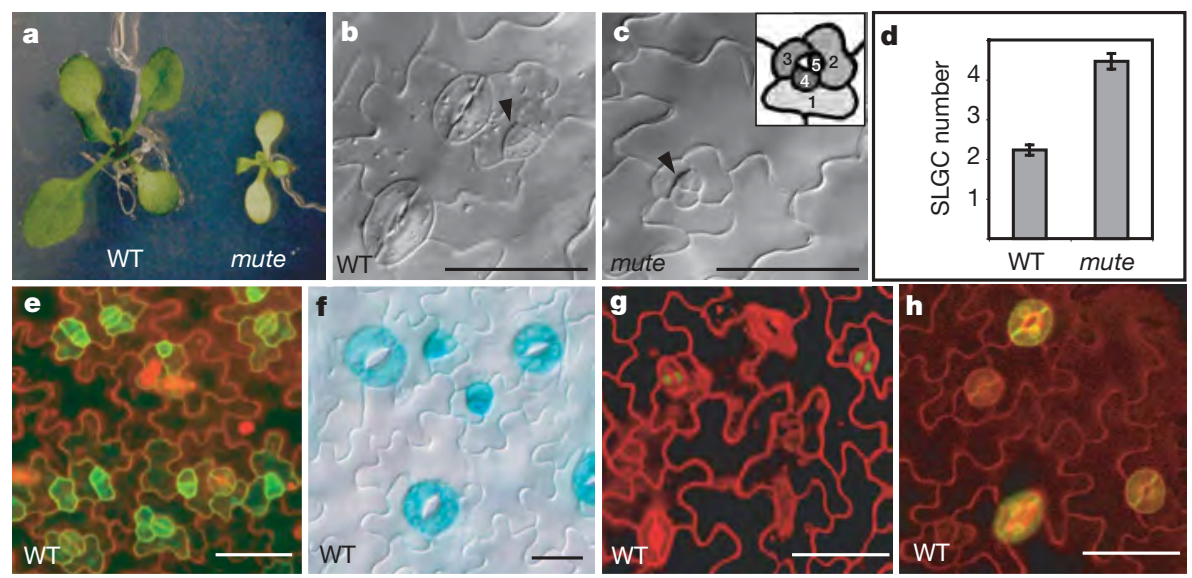

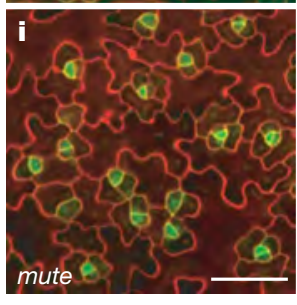

TMM::TMM-GFP

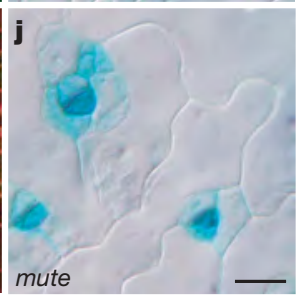

ERL $1:: G U S$

Figure $1 \mid$ MUTE is required for meristemoid differentiation. a, mute phenotype. Unlike the wild type (WT), the mute plant is pale and stunted. b, c, Abaxial leaf epidermis of WT (b) and mute (c), with meristemoids indicated by arrowheads. Inset, tracing of a mute meristemoid with successive numbering of SLGCs. d, Mean numbers of SLGCs surrounding an immature stoma (WT) or an arrested meristemoid (mute). Mean is \pm s.e.m., $n=25$ ( $t$-test, $P<0.00001)$. e-l, Expression of molecular markers in WT

interactions of MUTE with known regulators of stomatal development. First, double and quadruple mutants were produced between mute and cell-cell signalling mutants tmm, sdd 1 and erecta; erl1; erl2 (Fig. 2a-f). The leaf epidermis of tmm or erecta; erl1; erl2 mutants exhibits clusters of adjacent stomata (Fig. 2a, e), whereas that of $s d d 1$ shows increased overall stomatal density with a few
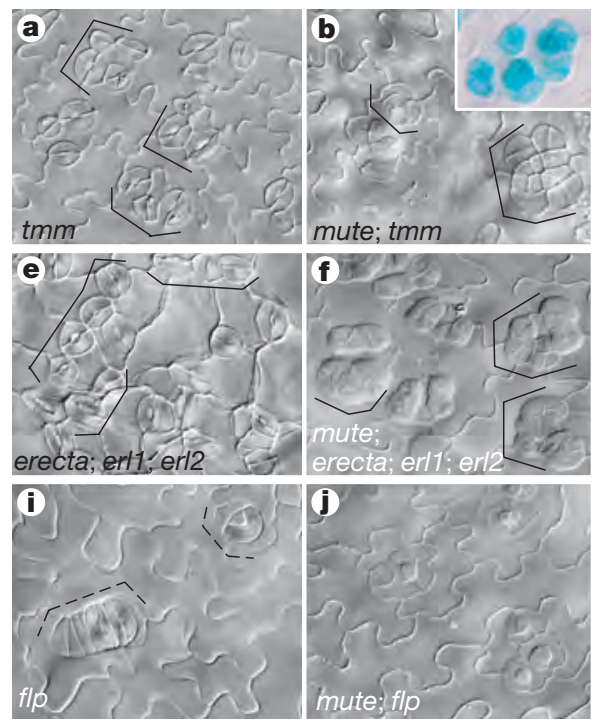

Figure 2 | Genetic interactions between MUTE and known regulators of stomatal patterning and differentiation. a-I, Abaxial leaf epidermis of $t \mathrm{tmm}$ (a), mute; tmm (b), sdd1 (c), mute; sdd1 (d), erecta; erl1; erl2 (e), mute; erecta; erl1; erl2 (f), erecta; erl2 (g), mute; erecta; erl2 (h), flp (i), mute; flp (j), fama (k) and mute; fama (I). Solid brackets indicate clusters of stomata (a, c, e) or meristemoids (b, d, f). Dashed brackets indicate stacks of symmetrically

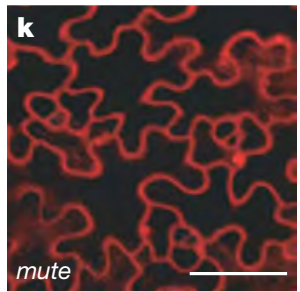

FAMA::FAMA-GFP

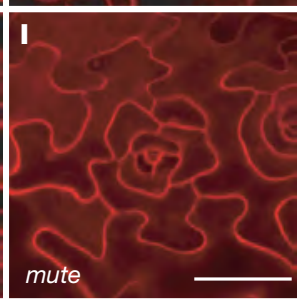

E994

(e-h) and mute (i-I). Arrested meristemoids in mute express high levels of stomatal lineage markers TMM::TMM-GFP (green; i) and ERL1::GUS (blue; j), but does not express GMC/immature guard cell marker

$F A M A:: F A M A-G F P(\mathbf{k})$ and mature guard cell marker E994 (I). For $\mathbf{e}, \mathbf{g}-\mathbf{i}, \mathbf{k}$, $\mathrm{I}$, the epidermal cell periphery is highlighted by propidium iodide (red). Scale bars, $20 \mu \mathrm{m}$.

stomatal clusters (Fig. 2c). 'Bubble-like' clusters of small cells were observed in the epidermis of mute; tmm and mute; erecta; erl1; erl2 (Fig. 2b, f). These small cells expressed TMM::GUS and ERL1::GUS at high levels, indicating that they are stomatal lineages, most probably meristemoids and SLGCs (Fig. 2b inset; Supplementary Fig. 1). The cells in these clusters are oriented in random angles, indicating the
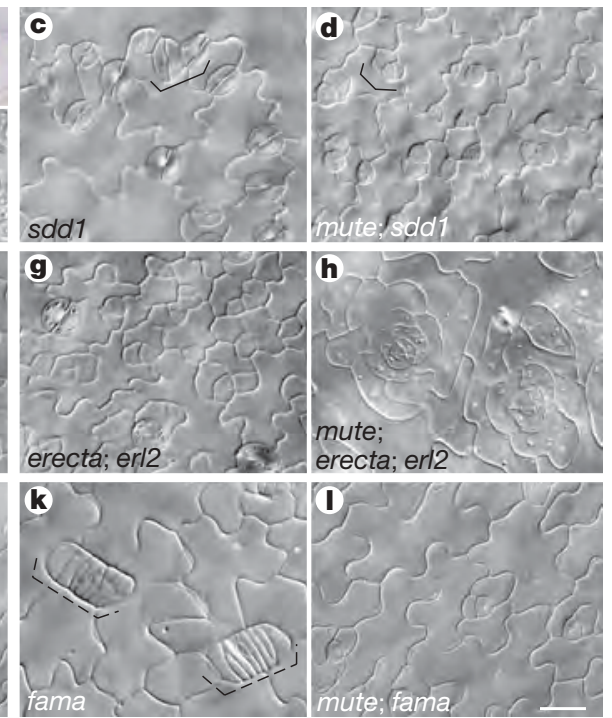

divided GMC or guard cells (i, k). The bubble-like clusters of cells in mute; tmm exhibit high expression of ERL1::GUS (b, inset). For the phenotypes of wild type and mute, see Fig. 1b, c. For expression of ERL1::GUS and TMM::GUS in wild type, mute and tmm; mute, see Supplementary Fig. 1. Images are taken under the same magnification. Scale bar, $20 \mu \mathrm{m}$. 
occurrence of randomized asymmetric division. This is consistent with the roles of TMM and the three ERECTA-family genes in regulating the frequency and orientation of asymmetric division in stomatal lineages ${ }^{8,11}$. The epidermis of mute; sdd1 has an increased density of arrested meristemoids with two meristemoids adjacent to each other observed occasionally (Fig. 2d). Overall, these results suggest that MUTE and the cell-cell signalling genes interact additively, whereas MUTE is epistatic with regard to guard cell differentiation.

erecta; erl 2 double mutants exhibit an increased frequency of asymmetric divisions with occasional patches of SLGCs that do not include stomata (Fig. 2g). The phenotype is due to inhibitory effects of ERL1 in the binary fate decision of a meristemoid to be a GMC rather than an $\mathrm{SLGC}^{8}$. Interestingly, the erecta; erl 2 double mutation a

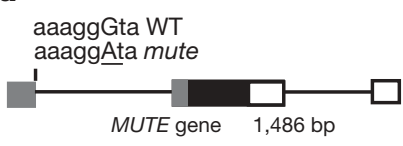

b

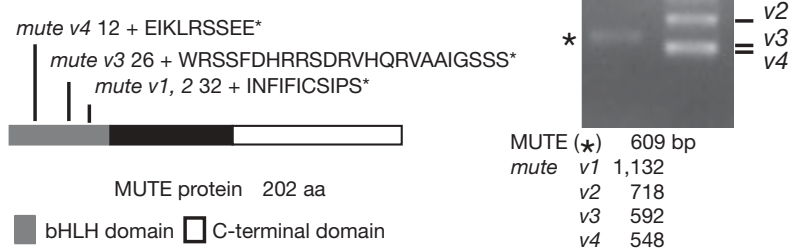

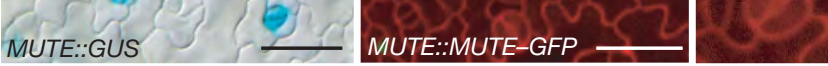
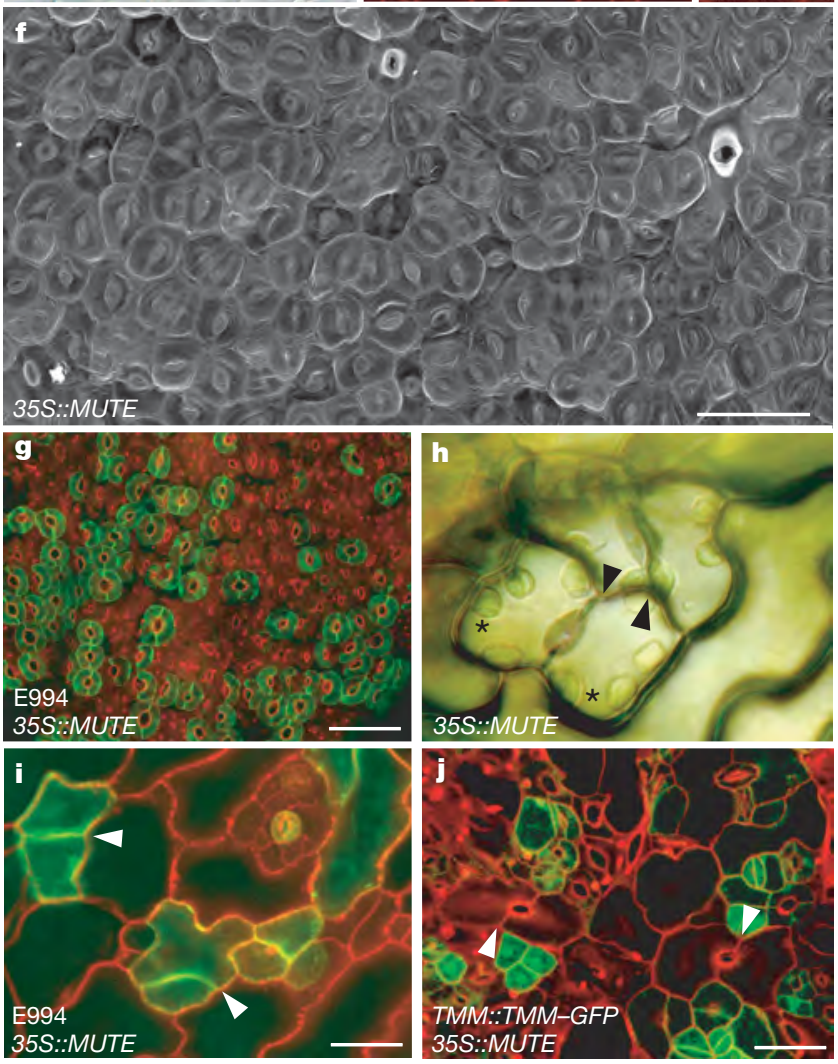

increased the number of SLGCs surrounding the single, arrested mute meristemoid, resulting in a striking, 'large rosette' phenotype (Fig. 2h). Therefore, ERL1 may enhance the longevity of meristemoids in the mute background.

In $f l p$ and fama, differentiation of a meristemoid to a GMC occurs normally. However, subsequent guard cell differentiation is abnormal; the GMC reiterates symmetric divisions and forms stacks of cell clusters (Fig. 2i, k) ${ }^{7,9}$. The phenotypes of mute; flp and mute; fama double mutants were indistinguishable from that of mute (Fig. 2i-1). The epistasis of MUTE over FLP and FAMA is consistent with MUTE acting at an earlier step of stomatal differentiation.

\section{MUTE triggers stomatal differentiation}

By map-based cloning, we identified the MUTE gene as At3g06120, which encodes the putative bHLH transcription factor bHLH45 (Supplementary Methods; Supplementary Fig. 2) ${ }^{12}$. mute possesses a single $\mathrm{G} \rightarrow \mathrm{A}$ nucleotide substitution at the splice donor site of the first intron (Fig. 3a). This abolishes the proper splicing of transcripts and results in splicing variants, which confer premature truncations at various positions within the bHLH domain (Fig. 3b, c). The bHLH domain is required for both DNA binding and dimerization ${ }^{13}$, and therefore mute is most probably a null allele. Introduction of the bHLH45 open reading frame with its native promoter into mute plants fully restored the wild-type phenotype, confirming that bHLH45 is MUTE (Supplementary Fig. 3).

To examine promoter activity and subcellular localization, we used the native MUTE promoter to drive expression of the GUS reporter (MUTE::GUS) and the translational fusion of a full-length MUTE protein and GFP (MUTE::MUTE-GFP). The latter construct complemented the mute phenotype, indicating that the MUTE-GFP fusion protein is functional (Supplementary Fig. 3). MUTE::GUS expression was highest in a subset of meristemoids that have undergone a few rounds of asymmetric division, with residual activity in GMC and immature guard cells (Fig. 3d). The MUTE-GFP fusion protein was localized in the nuclei of a subset of meristemoids that have undergone a few rounds of asymmetric division and in recently transitioned GMCs (Fig. 3e). No GFP signal was detected in other epidermal cells, including newly formed meristemoids and both immature and mature guard cells (Fig. 3e). These findings support the hypothesis that MUTE acts as an intrinsic transcription factor that creates changes in meristemoids to terminate their stem-cell-like properties and trigger GMC differentiation.

We next ectopically overexpressed MUTE. CaMV35S::MUTE plants exhibited a striking phenotype, converting all epidermal cells into stomata (Fig. 3f). Many of these ectopic stomata expressed the mature guard cell marker E994 (Fig. 3g). In less severe lines, a subset of epidermal pavement cells adopted partial guard cell identity. These

Figure 3 | MUTE bHLH protein triggers stomatal differentiation. a, MUTE gene structure. In mute, the $\mathrm{G} \rightarrow \mathrm{A}$ substitution occurred at the first intron splice donor site (underlined). b, MUTE protein structure. Four mute splicing variants (see c) result in a truncation within the bHLH domain. Position of frameshifts and the out-of-frame amino acid (aa) sequence up to the stop codon (asterisk) are indicated. For wild-type MUTE protein sequence, see Supplementary Fig. 4. c, PCR with reverse transcription (RT-PCR) analysis of splicing variants. Size of each transcript indicated. d, MUTE::GUS marks a subset of meristemoids and GMCs with residual activity in guard cells $(+)$. Young meristemoids (arrowhead) do not express GUS activity. e, A functional, translational fusion protein MUTE-GFP is detected in the nuclei of meristemoids that have undergone asymmetric divisions, but not in newly formed meristemoids (arrowhead), immature guard cells (asterisk) or mature guard cells. Insets: enlargements. $\mathbf{f}, \mathbf{g}$, Ectopic overexpression of MUTE converts the entire epidermis to guard cells. f, Scanning electron microscope image. g, Mature guard cell marker E994. $\mathbf{h}-\mathbf{j}$, Weak overexpression lines. Epidermal cells with mixed pavement-guard-cell identity possess chloroplasts (asterisks, h), express E994 (i), and form a symmetric division plane with a 'faux pore' (h-j, arrowheads). Scale bars, $20 \mu \mathrm{m}$. 
pavement cells contain chloroplasts, express the mature guard cell marker E994, and produce a symmetric division plane with a 'faux pore' (Fig. $3 \mathrm{~h}-\mathrm{j}$ ). Together, these results demonstrate that MUTE is capable of directing all protoderm cells to differentiate into guard cells and, depending on the strength of MUTE action, these cells may express characteristics of both pavement and guard cells.
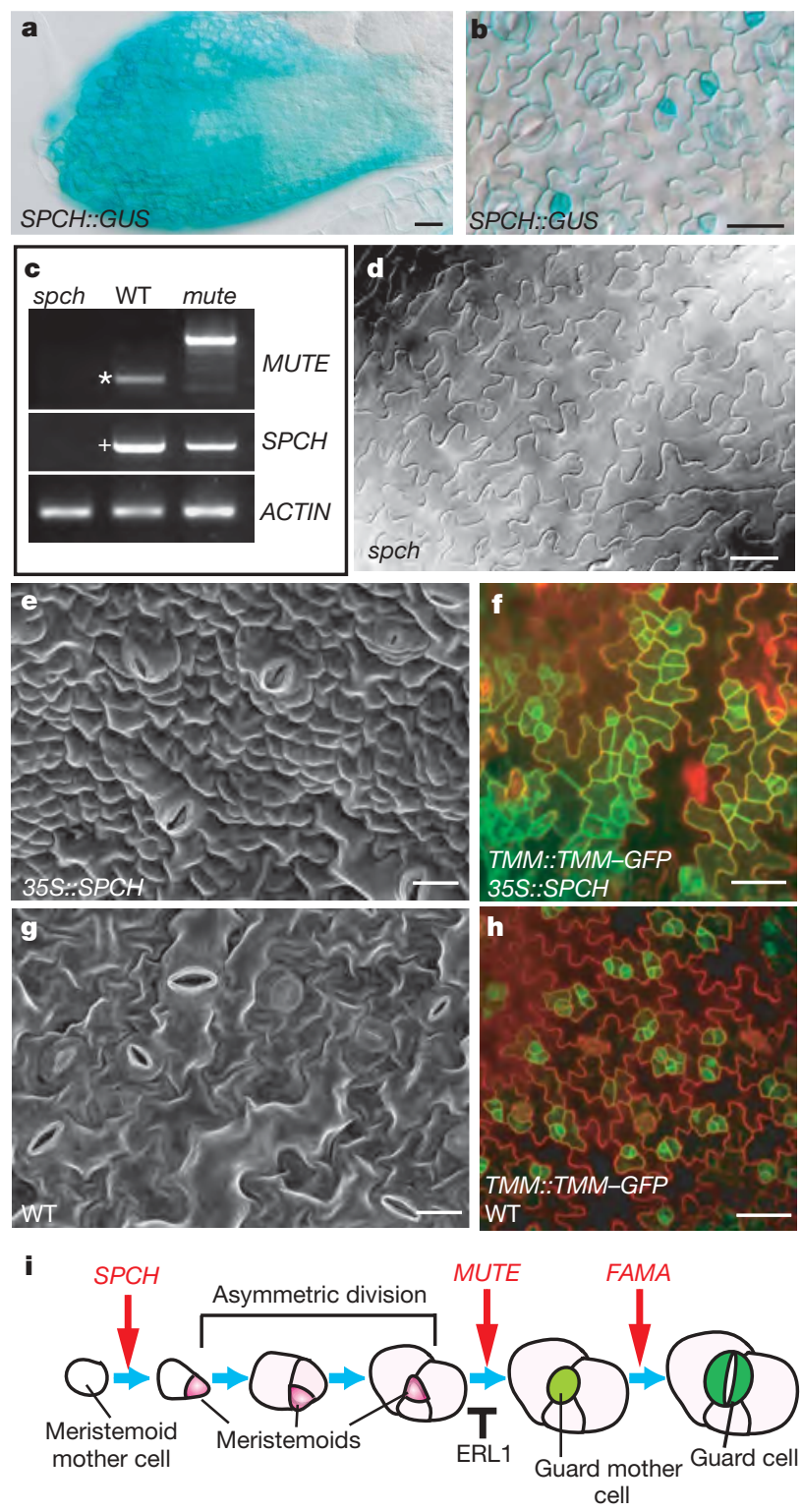

Figure 4 | SPCH, a paralogue of MUTE, initiates stomatal cell lineages. a, b, SPCH::GUS expression in the first leaf primordia of a 4-day-old seedling (a) and in the abaxial leaf epidermis of a 3-week-old seedling (b). c, RT-PCR analysis. The T-DNA insertion spch mutant does not accumulate $S P C H(+)$ or MUTE (asterisk) transcripts. Note that mute produces aberrant splice variants. ACTIN serves as a positive control. d, spch develops an epidermis solely made of pavement cells. e-h, Ectopic overexpression of SPCH confers a highly divided epidermis with small cells. e, Scanning electron microscope image. f, These small cells express high levels of TMM::TMM-GFP.

$\mathbf{g}, \mathbf{h}$, Control WT epidermis. Scale bars, $20 \mu \mathrm{m}$. i, A model for consecutive actions of three $b H L H$ genes. SPCH drives the first asymmetric division that initiates stomatal development. MUTE controls termination of asymmetric division and differentiation of meristemoids into GMCs. FAMA regulates differentiation of mature guard cells from GMCs. ERL1 affects the binary decision of meristemoid versus GMC identity and hence inhibits GMC differentiation. Red arrow, positive regulation; black T-bar, negative regulation.

\section{Consecutive actions of three bHLH proteins}

The genome-wide molecular phylogenetic analysis of Arabidopsis bHLH family showed that MUTE belongs to subfamily III with two closely related paralogues, SPEECHLESS (SPCH)/bHLH98 (At5g53210) and FAMA/bHLH97 (At3g24140) ${ }^{12,14-16}$ (Supplementary Fig. 4). They share high sequence similarity in the bHLH domain and carboxy-terminal region. However, the MUTE protein lacks the amino-terminal extension domain (Supplementary Fig. 4). FAMA is expressed in GMCs and immature guard cells (Supplementary Fig. 5; Fig. $1 \mathrm{~g}$ ), consistent with its known role in controlling the transition from GMC to guard cells ${ }^{7}$.

To unravel the developmental function of $S P C H$, the closest paralogue of MUTE, we next investigated its expression pattern as well as loss-of-function and gain-of-function phenotypes. SPCH promoter activity (SPCH::GUS) was observed broadly in the protoderm of leaf primordia and localized in stomatal lineage cells later in development (Fig. 4a, b; Supplementary Fig. 5). The T-DNA insertion spch loss-offunction mutant exhibited an intriguing epidermal phenotype consisting solely of jigsaw-puzzle-shaped pavement cells, thus lacking any stomatal lineage cells (Fig. 4c, d). In contrast, ectopic overexpression of SPCH by the CaMV35S promoter produced a highly divided epidermis with numerous small cells (Fig. 4e, g). These cells strongly express TMM::TMM-GFP, indicating that they probably have the identity of stomatal lineage cells (Fig. 4f, h). On the basis of these results, we conclude that $S P C H$ directs the first asymmetric division that initiates the stomatal lineage. The spch mutant does not express MUTE transcripts, consistent with SPCH acting before MUTE at the initial step of stomatal development (Fig. 4c).

\section{Discussion}

We have shown that MUTE is a key switch gene for meristemoids to acquire GMC identity and that ectopic MUTE expression triggers stomatal differentiation. Our findings highlight both similarities and differences in the control of asymmetric division and cell type differentiation between plants and animals. During Drosophila melanogaster central nervous system development, a neuroblast divides asymmetrically to produce another neuroblast and a differentiated ganglion mother cell ${ }^{17}$. A transcription factor, Prospero, is unequally allocated during asymmetric division to specify cell fate ${ }^{18}$. In contrast, MUTE protein is first detected in the nuclei of meristemoids just before GMC differentiation. This suggests that plants are able to monitor the age of meristemoids, possibly by tracking the rounds of asymmetric division, and subsequently induce MUTE (Fig. 3e). The longevity of meristemoids in mute; erecta; erl 2 triple mutants (Fig. 2h) implies that positional signals mediated by ERL1 might intersect with this fate decision (Fig. 4i).

We and others ${ }^{19}$ discovered that three closely related bHLH proteins control three critical steps of stomatal development: initiation by $\mathrm{SPCH}$, meristemoid differentiation by MUTE, and final guard cell differentiation by FAMA (Fig. 4i; ref. 19). SPCH triggers asymmetric division whereas MUTE terminates asymmetrically dividing meristemoids. Therefore, these bHLH proteins may act in an opposing manner in cell division control, but cooperate in stomatal differentiation. The basal lineages of extant land plants, such as Selaginella sp., seem to differentiate GMCs without reiterative asymmetric divisions of a precursor cell ${ }^{20,21}$. Diversification of this subfamily of bHLH proteins, possibly by gene duplication, may have allowed plants to acquire complex stomatal patterning as they conquered terrestrial environments.

Our discoveries emphasize the roles of bHLH proteins in cell type determination and differentiation in plants and animals. During myogenesis and neurogenesis in animals, a series of closely related bHLH proteins, such as the four mammalian myogenic regulatory proteins, MyoD, Myf-5, myogenin and MRF4, as well as the Drosophila achaete-scute complex, regulate sequential events in a hierarchical fashion ${ }^{22-24}$. Muscle fibres and neurons are specialized cell types, numbers of which are tightly regulated by extrinsic cell-cell signals $^{25}$. Similarly, proper spacing and density of stomata, which is 
critical for plant physiology and function, are controlled by positional signals mediated by receptor kinases and MAP kinase cascades. Understanding the interplay between cell-cell signals and $\mathrm{SPCH}$, MUTE and FAMA function, as well as tracing evolutionary origins of the three paralogous bHLH proteins, may elucidate the conservation and innovation of asymmetric division and cell type differentiation adopted by land plants.

\section{METHODS}

Plant materials. The Arabidopsis thaliana Columbia (Col) accession was used as a wild type. All mutants are in the Col background, except for $f l p-7$, which is in the Landsberg erecta background. $s d d 1$ was originally in C24 and was outcrossed into Col twice. The mute mutant was backcrossed into Col three times before analysis. T-DNA insertion alleles of FAMA (Salk_100073) and SPCH (SAIL_36_B06) were obtained from SIGnAL (Salk Institute) and ABRC (Ohio State University). For details, see Supplementary Methods.

Genetic screen and map-based cloning. mute was identified as a novel, arrestedmeristemoid phenotype from an ethyl-methanesulphonate-treated $\mathrm{M}_{1}$ population of erecta-105; erl2. A subsequent test cross revealed that mute segregates as a single recessive trait: 14 of $32 \mathrm{~F}_{1}$ plants $(43.8 \%)$ carried the mute gene on the basis of the segregation in the next generation $\left(\chi^{2}=0.5, P=0.48\right)$, and 207 of $851 \mathrm{~F}_{2}$ plants $(24.3 \%)$ showed the mute phenotype $\left(\chi^{2}=0.21, P=0.65\right) . \mathrm{F}_{2}$ mapping populations were generated from a single cross of $\mathrm{M}_{3}$ mutel+; erecta-105; erl $\times$ Ler. For details, see Supplementary Methods. A list of primer sequences is available as Supplementary Tables 1 and 2.

Plasmids and transgenic plants. The following constructs were generated for this study: SPCH::GUS (pLJP149), MUTE::GUS (pLJP138), FAMA::GUS (pLJP146), MUTE::MUTE-GFP (pLJP155), FAMA::FAMA-GFP (pLJP156), MUTE: MUTE-HA (pLJP158), CaMV35S::SPCH (pLJP152), and CaMV35S::MUTE (pLJP151). See Supplementary Table 3 for a list of primer sequences used for plasmid construction. For details, see Supplementary Methods.

Quantitative analysis of SLGCs. The number of asymmetric divisions per stomatal lineage between mute and wild-type plants was quantified using a TMM::GUS reporter line $e^{8}$. Cells surrounding a meristemoid (mute) or an immature stoma (wild type) expressing $\beta$-glucuronidase were considered SLGCs and counted for the analysis.

Microscopy. Detailed protocols, sample preparations, microscopy, and histochemical staining for $\beta$-glucuronidase activity are available in Supplementary Methods.

\section{Received 17 October; accepted 21 November 2006.}

Published online 20 December 2006.

1. Nadeau, J. A. \& Sack, F. D. Stomatal development in Arabidopsis. In The Arabidopsis Book (eds Somerville, C. \& Meyerowitz, E. M.) doi:10.1199/tab.0066 (American Society of Plant Biologists, 2002).

2. Hetherington, A. M. \& Woodward, F. I. The role of stomata in sensing and driving environmental change. Nature 424, 901-908 (2003)

3. Gray, J. E. et al. The HIC signalling pathway links $\mathrm{CO}_{2}$ perception to stomatal development. Nature 408, 713-716 (2000).

4. Berger, D. \& Altmann, T. A subtilisin-like serine protease involved in the regulation of stomatal density and distribution in Arabidopsis thaliana. Genes Dev. 14, 1119-1131 (2000).

5. von Groll, U., Berger, D. \& Altmann, T. The subtilisin-like serine protease SDD1 mediates cell-to-cell signaling during Arabidopsis stomatal development. Plant Cell 14, 1527-1539 (2002)

6. Nadeau, J. A. \& Sack, F. D. Control of stomatal distribution on the Arabidopsis leaf surface. Science 296, 1697-1700 (2002).

7. Bergmann, D. C., Lukowitz, W. \& Somerville, C. R. Stomatal development and pattern controlled by a MAPKK kinase. Science 304, 1494-1497 (2004).
8. Shpak, E. D., McAbee, J. M., Pillitteri, L. J. \& Torii, K. U. Stomatal patterning and differentiation by synergistic interactions of receptor kinases. Science 309, 290-293 (2005)

9. Lai, L. B. et al. The Arabidopsis R2R3 MYB proteins FOUR LIPS and MYB88 restrict divisions late in the stomatal cell lineage. Plant Cell 17, 2754-2767 (2005).

10. Poethig, R. S. EnhancerTraps 〈http://enhancertraps.bio.upenn.edu/〉 (2006).

11. Geisler, M. J., Deppong, D. O., Nadeau, J. A. \& Sack, F. D. Stomatal neighbor cell polarity and division in Arabidopsis. Planta 216, 571-579 (2003).

12. Toledo-Ortiz, G., Huq, E. \& Quail, P. H. The Arabidopsis basic/helix-loop-helix transcription factor family. Plant Cell 15, 1749-1770 (2003).

13. Murre, C., McCaw, P. S. \& Baltimore, D. A new DNA binding and dimerization motif in immunoglobulin enhancer binding, daughterless, MyoD, and myc proteins. Cell 56, 777-783 (1989).

14. Bailey, P. C. et al. Update on the basic helix-loop-helix transcription factor gene family in Arabidopsis thaliana. Plant Cell 15, 2497-2502 (2003).

15. Buck, M. J. \& Atchley, W. R. Phylogenetic analysis of plant basic helix-loop-helix proteins. J. Mol. Evol. 56, 742-750 (2003).

16. Heim, M. A. et al. The basic helix-loop-helix transcription factor family in plants: a genome-wide study of protein structure and functional diversity. Mol. Biol. Evol. 20, 735-747 (2003)

17. Doe, C. Q. \& Bowerman, B. Asymmetric cell division: fly neuroblast meets worm zygote. Curr. Opin. Cell Biol. 13, 68-75 (2001)

18. Ikeshima-Kataoka, H., Skeath, J. B., Nabeshima, Y., Doe, C. Q. \& Matsuzaki, F. Miranda directs Prospero to a daughter cell during Drosophila asymmetric divisions. Nature 390, 625-629 (1997).

19. MacAlister, C. A., Ohashi-Ito, K. \& Bergmann, D. C. Transcription factor control of asymmetric cell divisions that establish the stomatal lineage. Nature doi:10.1038/ nature05491 (this issues)

20. Dengler, N. The developmental basis of anisophylly in Selaginella martensii. II. Histogenesis. Am. J. Bot. 70, 193-206 (1983)

21. Brown, R. \& Lemmon, B. Development of stomata in Selaginella: Division polarity and plastid movements. Am. J. Bot. 72, 1914-1925 (1985).

22. Weintraub, $H$. The MyoD family and myogenesis: redundancy, networks, and thresholds. Cell 75, 1241-1244 (1993).

23. Olson, E. N. MyoD family: a paradigm for development? Genes Dev. 4, 1454-1461 (1990).

24. Campuzano, S. \& Modolell, J. Patterning of the Drosophila nervous system: the achaete-scute gene complex. Trends Genet. 8, 202-208 (1992).

25. Olson, E. N. Interplay between proliferation and differentiation within the myogenic lineage. Dev. Biol. 154, 261-272 (1992).

Supplementary Information is linked to the online version of the paper at www.nature.com/nature.

Acknowledgements We thank C. Doe, B. Wakimoto, J. McAbee, and T. Kakimoto for commenting on the manuscript; F. Sack and T. Altmann for a gift of flp, sdd1 and TMM::TMM-GFP; S. Poethig and D. Bergmann for E994; T. Nakagawa and ABRC for cloning vectors; SIGnAL and ABRC for T-DNA insertion lines; and P. Chan for confocal microscopy expertise. Our thanks to D. Bergmann for sharing unpublished results and discussion about stomatal development. This work was supported in part by the grants from DOE and NSF to K.U.T. L.J.P. was supported by the NRI USDA/CSREES fellowship, and K.U.T. is a CREST JST investigator.

Author Contributions K.U.T. supervised the entire project. K.U.T. and L.J.P. conceived and designed the experiments, and wrote the manuscript with comments from co-authors. L.J.P. isolated the mute mutant and performed characterization of mutants and transgenic plants with N.L.B. D.B.S. identified the MUTE gene by map-based cloning.

Author Information The NCBI/GenBank accession numbers for the genes described in this manuscript are: DQ863645 (MUTE mRNA), DQ864972 (MUTE genomic) and DQ868373 (SPCH mRNA). Reprints and permissions information is available at www.nature.com/reprints. The authors declare no competing financial interests. Correspondence and requests for materials should be addressed to K.U.T. (ktorii@u.washington.edu). 


\title{
Haploinsufficiency after successive loss of signaling reveals a role for ERECTA-family genes in Arabidopsis ovule development
}

\author{
Lynn Jo Pillitteri, Shannon M. Bemis, Elena D. Shpak* and Keiko U. Torii ${ }^{\dagger}$
}

The Arabidopsis genome contains three ERECTA-family genes, ERECTA (ER), ERECTA-LIKE 1 (ERL1) and ERL2 that encode leucinerich repeat receptor-like kinases. This gene family acts synergistically to coordinate cell proliferation and growth during aboveground organogenesis with the major player, $E R$, masking the loss-of-function phenotypes of the other two members. To uncover the specific developmental consequence and minimum threshold requirement for signaling, $E R$-family gene function was successively eliminated. We report here that $E R L 2$ is haploinsufficient for maintaining female fertility in the absence of $E R$ and $E R L 1$. Ovules of the haploinsufficient er-105 er/1-2 er/2-1/+ mutant exhibit abnormal development with reduced cell proliferation in the integuments and gametophyte abortion. Our analysis indicates that progression of integument growth requires ER-family signaling in a dosage-dependent manner and that transcriptional compensation among ER-family members occurs to maintain the required signaling threshold. The specific misregulation of cyclin A genes in the er-105 erl1-2 erl2-1/+ mutant suggests that downstream targets of the ER-signaling pathway might include these core cell-cycle regulators. Finally, genetic interaction of the $E R$ family and the WOX-family gene, PFS2, reveals their contribution to integument development through interrelated mechanisms.

KEY WORDS: Arabidopsis, Integument growth, Ovule, Receptor-like kinase, Haploinsufficient, Cell proliferation

\section{INTRODUCTION}

The final size and shape of plant organs are determined by developmental programs that coordinate cell proliferation, cell expansion and cell-type differentiation (Mizukami, 2001; Potter and $\mathrm{Xu}, 2001)$. The Arabidopsis ovule has a relatively simple structure and a specific, well-defined differentiation pattern, making it a useful model for understanding the regulation of growth and organogenesis. The ovule originates from the placenta as an elongate protrusion with a defined proximal/distal axis that can be separated into three regions. The distal portion, called the nucellus, is the site of megasporogenesis and embryo sac development. The proximal region differentiates into the funiculus or stalk, which attaches the ovule to the carpel wall. Two integuments initiate at the central chalazal region and eventually envelop the nucellus and form the seed coat. Similar to the process of leaf laminar expansion, initiation and expansion of the integument requires the juxtaposition of abaxial and adaxial factors such as those encoded by PHABULOSA (PHB), KANADI1 (KAN1), KAN2 and KAN3, ABERRANT TESTA SHAPE (also known as KAN4 - TAIR), and the YABBY-family member INNER NO OUTER (Eshed et al., 2001; Eshed et al., 2004; McAbee et al., 2006; Sieber et al., 2004; Villanueva et al., 1999). In addition, transcription factors such as AINTEGUMENTA and NOZZLE (also known as SPOROCYTELESS), and the mitochondria ribosomal protein HUELLENLOS, have been identified as necessary for integument initiation (Elliott et al., 1996; Schneitz et al., 1998; Skinner et al., 2004; Villanueva et al., 1999).

Department of Biology, University of Washington, Seattle WA 98195 USA.

*Present address: Department of Biochemistry and Cellular and Molecular Biology, University of Tennessee, Knoxville, TN 37996, USA

${ }^{\dagger}$ Author for correspondence (e-mail: ktorii@u.washington.edu)

Accepted 20 June 2007
A second group of loci affect the progression of integument growth after initiation. Several genes, including PRETTY FEW SEEDS 2 (PFS2), SHORT INTEGUMENTS 1 and 2 (SIN1 and 2) and TSO1, affect cell proliferation or expansion of the integuments, and their loss-of-function mutations result in reduction or loss of fertility (Hauser et al., 2000; Park et al., 2004; Park et al., 2005; Schneitz et al., 1997). Embryo sac failure is a secondary consequence of the absence of integuments; the gametophyte fails to develop in cases where the nucellus is not enclosed (Gasser et al., 1998). The cause of gametophyte abortion is not clear, but is likely to lie in the requirement for tight coordination of cell division and expansion within the ovule and communication between gametophytic and sporophytic tissue (Gasser et al., 1998). Relatively little is known about the genes involved in the coordination of growth or cell-cell communication within the ovule. This might be due to redundancy among the genes involved in this process, or to pleiotropic effects resulting from the loss of these genes. For instance, TSO1, TOUSLED (TSL) and SIN1 have multiple, sometimes detrimental, effects on vegetative and floral development in addition to ovule defects (Ehsan et al., 2004; Lang et al., 1994).

ERECTA $(E R)$ and its two paralogs, ERECTA-LIKE 1 (ERLI) and $E R L 2$, regulate organ shape and inflorescence architecture and are members of the leucine-rich repeat receptor-like kinase (LRR-RLK) gene family in Arabidopsis (Shiu and Bleecker, 2001; Torii, 2004; Torii et al., 1996). Based on phylogeny, it was suggested that ERL1 and $E R L 2$ evolved by recent gene duplication and are functionally related to $E R$, while maintaining overlapping but unique transcript expression patterns (Shpak et al., 2004). We report here that in the absence of functional ER and ERL1, Arabidopsis plants heterozygous at the ERL2 locus exhibit specific defects in integument development. Based on data from genetic and gene expression analysis, the ER family plays a key role in ovule development and fertility by regulating cell proliferation in the integuments. Our study highlights the unequal redundancy and dosage compensation among $E R$-family genes, and further reveals 
a potential molecular consequence of their reduced dosage for cell division control of integument development and fertility. Finally, genetic interactions of the ER family with PFS2, a WUSCHEL (WUS)-type homeodomain gene, revealed an unexpected intersection of two pathways required for proper integument growth and embryo sac development.

\section{MATERIALS AND METHODS}

\section{Plant material and growth conditions}

The Arabidopsis thaliana ecotype Columbia ( $\mathrm{Col}$ ) was used as wild type. All mutants are in the Col background unless otherwise indicated. The er105, erl1-2 and erl2-1 mutations were described previously (Shpak et al., 2004). The erl1-1 allele (YJ133) was identified as an enhancer-trapped line by Dr John Bowman (University of California, Davis, CA) and kindly provided as a gift. The line was originally in the Landsberg erecta (Ler) background and outcrossed into $\mathrm{Col}$ three times. The erl1-4 allele was isolated from ethyl methanesulfonate-mutagenized Arabidopsis er-105 erl2 seeds and outcrossed to Col. The $p f s 2-1$ allele was a gift from Dr Bernard Hauser (University of Florida, Gainesville, FL) and was in the Ler background and outcrossed to Col. The $p f s 2-1$ mutant was crossed with the er-105 erl1-2 (+/-) erl2-1 mutant for further analysis. All plants were grown under long-day conditions as described previously (Shpak et al., 2003).

\section{Genotyping}

PCR-based genotyping for er-105, erl1-2 and erl2-1 mutations was described previously (Shpak et al., 2004). Detection of the erl1-1 T-DNA insertion was performed with PCR primers: ERLK-185.rc, 5' CGTAGGTCTCCAATACGTGGA-3' and GUS R, 5'-CAGTTGCAACCACCTGTTGAT-3'. The erl1-4 mutation was detected by derived Cleaved Amplified Polymorphic sequences (dCAPs) using primers: erl14dcaps1151, 5'-GACGATGTTCACAACAGTGACTTGTGTTCTAG-3' and erl1-4dcaps1447.rc, 5'-CATCGAAATCAACAGAGAAAGAAAGGG$3^{\prime}$, and subsequent digestion with $\mathrm{XbaI}$ for 2 hours at $37^{\circ} \mathrm{C}$, which cuts the mutant erl1-4 allele sequence.

\section{In situ hybridization}

Tissue preparation and in situ hybridization were performed as described previously (McAbee et al., 2006). Probe template construction was performed as follows. For the ERL1 probe template, the ERL1 sense and antisense probes were produced by linearizing plasmid pLJP562 (contains ERL1 kinase domain) with BamHI or XhoI and transcribing in vitro with T3 or T7 RNA polymerase, respectively. For the $E R$ probe template, the $E R$ sense and antisense probes were synthesized by linearizing pLJP131 (contains the $E R$ kinase domain and partial 3'UTR) with XhoI or BamHI and transcribing in vitro with T3 or T7 RNA polymerase, respectively. The WUS sense and antisense probes were produced by linearizing pSMB106 (nt 627-816 of the $W U S$ cDNA) with EcoRI and $X b a \mathrm{I}$ and transcribing in vitro with T3 or T7 RNA polymerase, respectively. PFS2 probe template construction and hybridization conditions were according to Park et al. (Park et al., 2005). Sense and antisense probes for $P H B$ were produced by linearizing plasmid pPHB (a gift from Dr Kiyotaka Okada, Kyoto University, Kyoto, Japan) with SacI or KpnI and transcribing in vitro with T7 or T3 RNA polymerase, respectively.

\section{GUS histochemical analysis}

proERL1::GUS and proERL2::GUS constructs were described previously (Shpak et al., 2004). Histochemical staining for $\beta$-glucuronidase (GUS) activity was performed as described previously (Sessions et al., 1999).

\section{RNA extraction and quantitative real-time RT-PCR}

Total RNA was isolated from Arabidopsis stage 12 gynoecia (Schneitz et al., 1995) or from ovules isolated from stage 12 gynoecia using the RNeasy Plant Mini Kit (Qiagen, Valencia, CA) and treated with DNase I (Amp Grade, Invitrogen). First-strand cDNAs were generated by ThermoScript Reverse Transcriptase (Invitrogen) and random hexamers using $1.0 \mu \mathrm{g}$ of RNA according to manufacturer's instructions. Real-time PCR was performed with a Chromo4 thermocycler (BioRad, Hercules, CA) and analyzed using Opticon Monitor 3 software (BioRad). Triplicate reactions were performed in $25 \mu \mathrm{l}$ total volume containing iTaq SYBR Green mix
Table 1. Primers for RT-PCR and probe synthesis

Primer Sequence ( $5^{\prime}$ to $3^{\prime}$ )

\begin{tabular}{lll}
\hline \multicolumn{2}{l}{ Real-time RT-PCR primers } \\
\hline ERECTA & sbr ER F & \\
& sbr ER R & GACTGGACAACTTCACCTTCTTCG \\
ERL1 & sbr ERL1 F & GAACTGCTTCACTCTTGTCAATCTG \\
& sbr ERL1 R & CATACCTGGCTGGAGCAAAACG \\
ERL2 & ERL2 3085 & CTGTCTGGCAACAATTTCTCA \\
& sbr ERL2 R & AACACGTACAGATTGGCAAGAC \\
ACTIN & sbr actin F & ATGTCGCCATCCAAGCTGTTCTC \\
& sbr actin r & CTCCTTGATGTCTCTTACAATTTCC \\
PFS2 & pfs2 F & ATGGGCTACATCTCCAACAA \\
CYCLIN A2;2 & sbr PFS2 R & CTTCTAGCGTCGTGATCTGC \\
& sbr CYCA22 F & GAGCTAAAAAAGCCATGGGAA \\
CYCLIN A2;3 & Sbr CYCA22 R & CACCTGATCCATCTTCTTGTTGC \\
& sbr CYCA23 21 R & GTCTCGGCCATTCACTCG \\
& & TTTGGCTCAAGTATACCTG
\end{tabular}

RT-PCR primers

CYCLIN A2;1 CYCA21 153 GAAGCGTGTTGCTAGACCG

CYCA21 347.rC CTACCAAACCATCTTCTTC

CYCLIN A2:2 CYCA22 597 GCTAAAAAAGCCATGGGAAGA

CYCLIN A2;3

CYCA22 1103.rC TGCTCAGTAATTTCTTCATCTT

CYCA23 21 GTCTCGGCCATTCACTCG

CYCA23 359.RC CCTGCTGTATTTGATGCAAC

CYCA24 (-26) TTGGATCGTGTCAAAGGG

CYCA24 518.rC ACTTCTCTCAACTGTCCC

cdkb11610 CAATGGCCGGGTGTTTCC

cdkb11+69.rc TGTCGGAAAGAAAGATAG

cdkb12 615 CAATGGCCGGGTGTAATG

cdkb12 +68.rc GAGCAATAAACTTGACGG

cdkb21 (-40) AGAGAGAGAGAGCGCCG

cdkb21 352.rc GAATGTTCTTGCCAGTGCT

cdkb22 (-32) GAGAGAAGAGTTGTCTTG

cdkb22 358.rc GAATGTTCTGTCCAGCTTG

ER 2248

ER3016.rC

ERL1 2846

$\begin{array}{ll} & \text { ERL1 4411.rC } \\ \text { ERL2 } & \text { ERL2 } 3085\end{array}$

ERL2 4254.rC

ACTIN 2 act2-1

PFS2 $\quad$ pfs2 F

AAGAAGTCATTCAAAGATGTGA

AGAATTTCCAGGTTTGGAATCTGT

TATCCCACCGATACTTGGCA

CCGGAGAGATTGTTGAAGGA

CTGTCTGGCAACAATTTCTCA

AGCCATGTCCATGTGAAGAA

GCCATCCAAGCTGTTCTCTC

GCTCGTAGTCAACAGCAACAA

pfs2 $\mathrm{F}$ ATGGGCTACATCTCCAACAA

pfs2 R TCAGTTCTTCAGAGGCATGA

In situ hybridization probe primers

ERECTA ER 7040 GTAAAGATCTCGGTGTGG

ER 7467.rc GAAGACACATATTCACACC

(BioRad) and $1.0 \mu \mathrm{l}$ of the first-strand reaction. ACTIN 2 was used as an internal control in parallel reactions for each run. The relative level of expression of each target gene was calculated with respect to ACTIN 2 expression for each genotype. Real-time reactions were repeated in two biological replicates and the average fold-difference relative to wild type was calculated. Oligomers and DNA sequences used for PCR are shown in Table 1

\section{Microscopy}

Pictures of plants were taken with an Olympus SZX12 dissecting microscope attached to an Olympus C-2020Z digital camera (Center Valley, PA). Tissue fixation, plastic embedding, sectioning, light- and scanning electron microscopy were performed as described previously (Shpak et al., 2003). To examine ovules using differential interference contrast (DIC) optics, carpels were dissected from flowers and incubated in 9:1 (v:v) ethanol:acetic acid overnight, rinsed with water and placed in choral hydrate (1:8:1 v:v glycerol:chloral hydrate:water). Cleared ovules and in situ hybridization sections were viewed under DIC optics using an Olympus BX51 microscope equipped with a DP70 digital camera. 


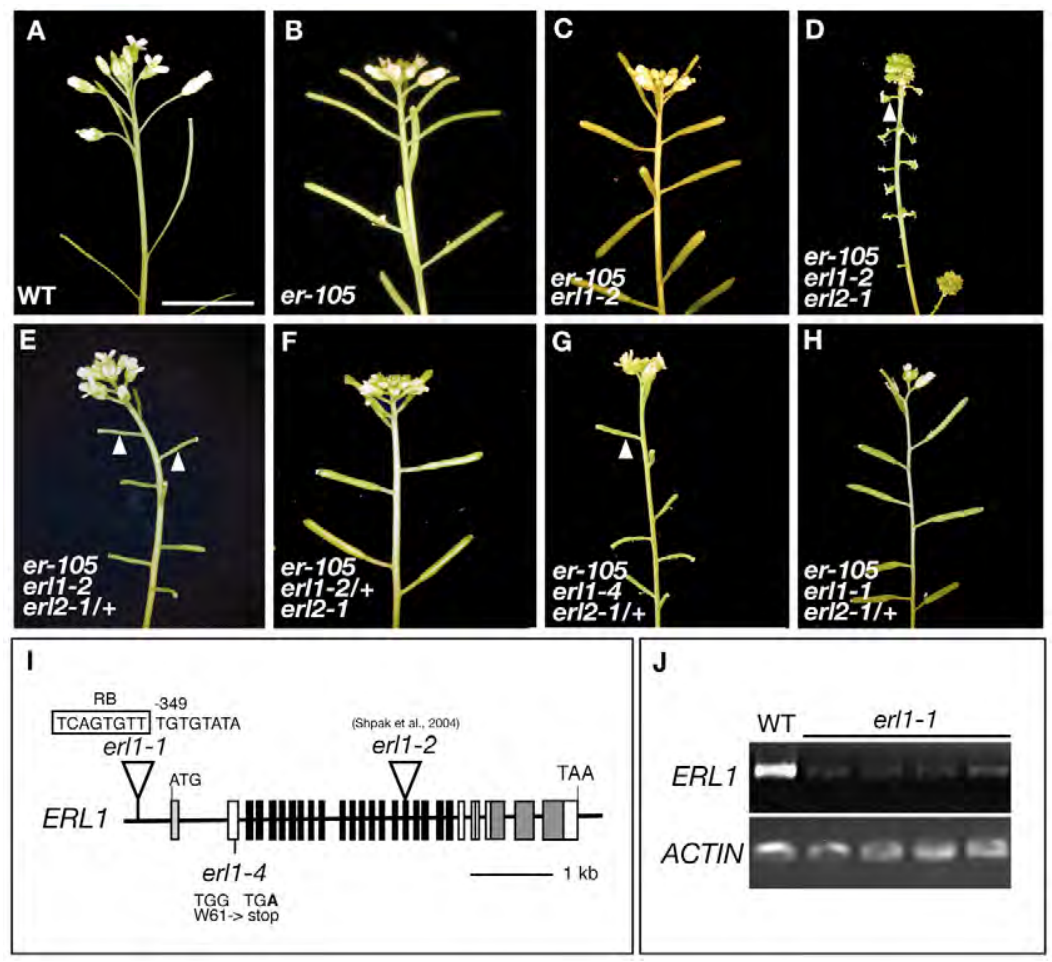

Fig. 1. Growth phenotype of wild-type and erfamily mutant Arabidopsis. (A-H) Wild-type and erfamily mutant inflorescence stems. (A) Wild type (WT); (B) er-105; (C) er-105 erl1-2; (D) er-105 erl1-2 erl2-1; (E) er-105 erl1-2 erl2-1/+; (F) er-105 erl1-2/+ erl2-1; (G) er105 er/1-4 erl2-1/+; and (H) er-105 er/1-1 er/2-1/+. Wild type and all combination mutants (A-C,E-F) except er105 erl1-2 erl2-1 triple mutant (D) maintain proper floral organ patterning and elongation. Among the combinations, er-105 erl1-2 erl2-1/+ (E), er-105 erl1-4 erl2-1/+ $(H)$ and the triple mutant $(D)$, are female sterile. Scale bar: $1 \mathrm{~cm}$. (I) Schematic of the ERL 1 gene with location of T-DNA insertions for er/1-1 and erl2-1, and the point mutation for erl1-4. Exons are indicated as vertical bars, introns as lines. The T-DNA right border (RB) is boxed. (J) RT-PCR analysis of four separate erl1-1 plants with reduced levels of ERL1 transcript compared with wild type. ACTIN 2 serves as a positive control.
Cell size and number measurements

Cell size was measured from images of plastic-embedded sections of wild-type and er erl1-2 erl2-1/+ carpels. The length and width of the first ten cells (nucellar to chalazal end) of the outer layer of the outer integument were measured using pixel distance in Photoshop Pro (Adobe Systems, San Jose, CA). Images used for these measurements corresponded to similar cross-sections through the nucellus of mature wild-type and er erl1-2 erl2-1/+ ovules. Cell numbers were counted from cleared ovules at stages 1-II, 2-IV, 3-II and 3-VI (Schneitz et al., 1995). At stages 2-IV, 3-II and 3-VI, counting started at the tip of the outer integument (nucellar end) and stopped where cells of the funiculus appeared obvious. At stage 1-II, all cells in the L1 layer of the ovule primordia were counted.

\section{RESULTS}

\section{$E R L 2$ is haploinsufficient for female fertility in the} absence of ER and ERL1

As reported previously, er, er erll and er erl2 null mutants developed compact but otherwise normal inflorescences, whereas the loss of all ER-family genes led to extremely dwarf and sterile plants with aberrant flowers disrupted in organ patterning and differentiation (Shpak et al., 2004) (Fig. 1A-D). To unravel the specific functions of $E R L 1$ and $E R L 2$ during inflorescence and floral organ growth, we investigated the phenotypes conferred by reduced dosages of ERL1 and ERL2 in the absence of ER. For this purpose, 100 self-fertilized progenies of er-105 erll-2/+ erl2-1/+, preselected for the presence of the erll-2 T-DNA insertion, were grown to maturity. This population segregated seven extremely dwarf and sterile plants, consistent with the expected $8.3 \%$ ratio for the er-105 erl1-2 erl $2-1$ triple mutant (dwarf plants/total $=7 / 100 ; \chi^{2}=0.221$, $P=0.637$ ). Seventeen additional plants from this population were completely sterile and produced no seeds. However, unlike the triple er-family mutants, they did not exhibit severe dwarfism, disrupted phyllotaxis or floral organ patterning defects. A reciprocal crosspollination with wild type revealed that these plants were female sterile (data not shown).
To determine the genetic basis for this novel phenotype, we next analyzed the genotypes of all 100 plants in this population. All seventeen female-sterile, non-dwarf plants were homozygous for $\mathrm{er}$ 105 and erl1-2 and heterozygous for erl2-1 (i.e. er-105 erl1-2 erl2$1 /+)$ (Fig. 1E). This was highly consistent with the expected ratio of $16.7 \%$ for this population $\left(\chi^{2}=0.006, P=0.936\right)$. Unlike $\mathrm{er}-105 \mathrm{erl} 1$ 2 erl $2-1 /+$, the $e r-105$ erll-2/+ erl2-1 plants were fully fertile (Fig. $1 \mathrm{~F})$. Reintroduction of functional copies of $E R, E R L 1$ and $E R L 2$ into respective loss-of-function mutants confirmed these data (see Table $\mathrm{S} 1$ in the supplementary material). The results indicate that, in the absence of $E R$ and $E R L 1, E R L 2$ is haploinsufficient for female fertility, whereas $E R L 1$ is haplosufficient in the absence of $E R$ and $E R L 2$. By contrast, the lack of defects in floral morphology (see Fig. $\mathrm{S} 1$ in the supplementary material) and phyllotaxis indicate that a single copy of ERL2 is sufficient for inflorescence elongation and floral patterning.

\section{Slight increase in ERL1 activity is sufficient to rescue ERL2 haploinsufficiency}

To further investigate the importance of ERLI function in female fertility, we generated mutant combinations with two additional erll alleles. The erll-1 allele carries a T-DNA insertion 349 bases upstream of the initiation codon and represents a weak allele (Fig. 1I,J). The erll-4 allele has a G-to-A substitution at nucleotide position +750 . This creates a stop codon at amino acid 61, leading to a premature truncation of the protein prior to the LRR domain (Fig. 1I). Therefore, erll-4 is a null allele.

The er-105 erl1-1 erl2-1, er-105 erll-2 erl2-1 and er-105 erl1-4 erl2-1 triple-mutant plants were almost indistinguishable from each other (data not shown). Similar to er-105 erl1-2 er $-1 /+$ plants (Fig. 1E), er-105 erll-4 erl2-1/+ plants were completely female sterile (Fig. 1G). By contrast, er-105 erl1-1 er2-1/+ plants were fertile and produced viable seed (Fig. $1 \mathrm{H})$. These results demonstrate that a slight increase in ERLI activity is sufficient to rescue the haploinsufficiency of ERL2. However, because erl1-1 failed to 

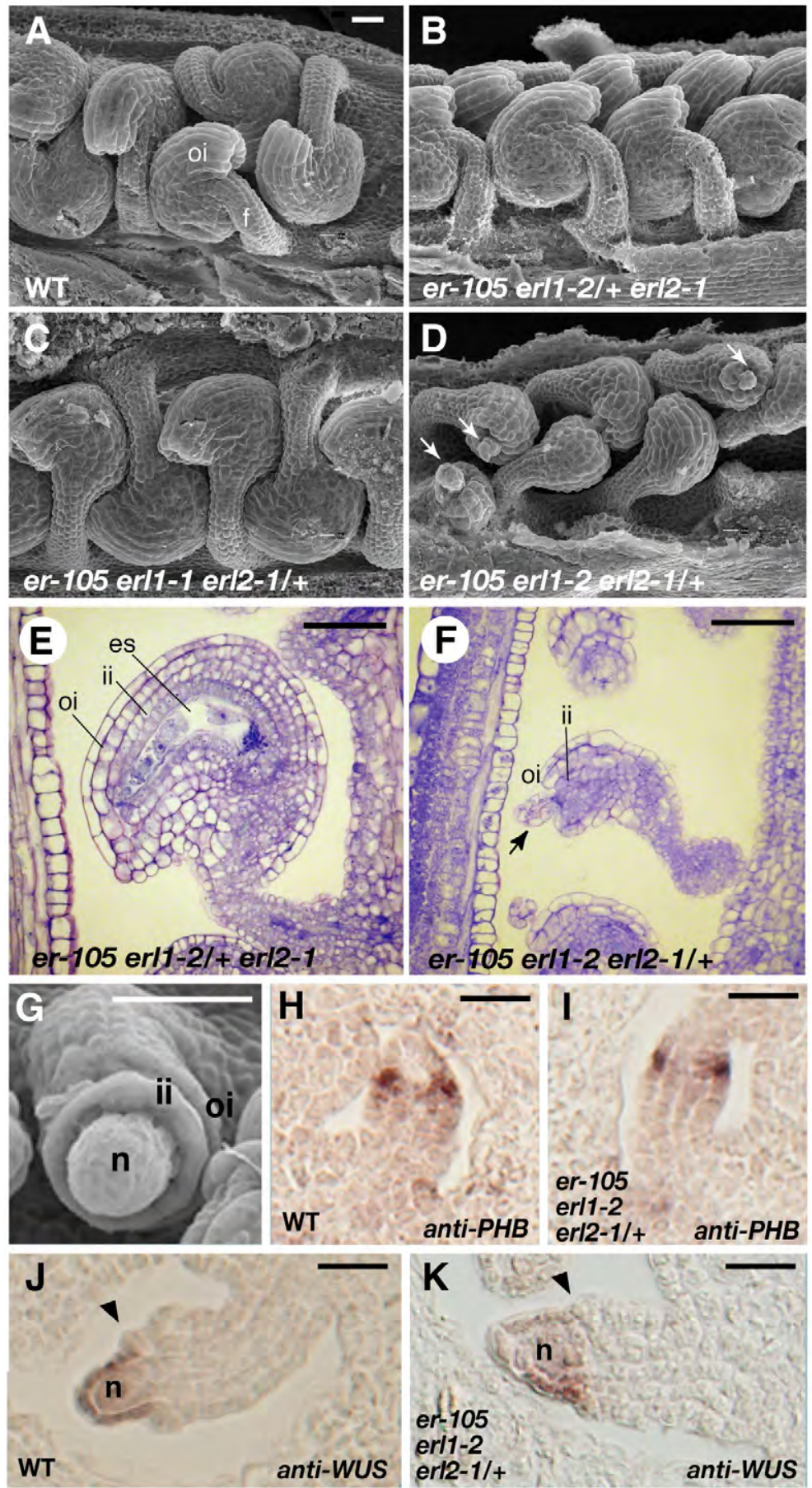

Fig. 2. Phenotypic effects of loss of er-family function on ovule development. (A-D) Scanning electron micrographs (SEMs) of Arabidopsis ovules at anthesis. (A) Wild type; (B) er-105 erl1-2/+ erl2-1; (C) er-105 erl1-1 erl2-1/+; (D) er-105 er/1-2 er/2-1/+. In er-105 er/1-2 er/2-1/+, both inner and outer integuments are shorter leaving the nucellus exposed (arrows). $(\mathbf{E}, \mathbf{F})$ Sagittal sections through the gametophyte of a er-105 er/1-2/+ erl2-1 (E) and er-105 erl1-2 erl2-1/+ (F) ovule at anthesis. In the er-105 erl1-2 er/2-1/+ mutant, the gametophyte has degenerated into a mass of small cells. (G) SEM of a developing er-105 er/1-2 erl2-1/+ stage 2-I ovule. Both the inner and outer integument initiate as smooth rings at the base of the nucellus, with the outer integument initiating asymmetrically as in wild type. (H-K) In situ hybridization for $(\mathrm{H}, \mathrm{I}) \mathrm{PHB}$ and $(\mathrm{J}, \mathrm{K})$ WUS expression. No difference in PHB or WUS expression is detected in mutant ovules, indicating normal regional specification. No signal was detected with PHB or WUS sense probe. f, funiculus; es, embryo sac; ii, inner integument; oi, outer integument; n, nucellus. Scale bars: $20 \mu \mathrm{m}$. promote organ growth, proper floral patterning or fertility in the absence of $E R$ and $E R L 2$, the results highlight a strict ER-family signaling threshold required to promote female fertility.

\section{Haploinsufficiency of ERL2 confers aberrant ovule growth and abortion of embryo sac development}

To gain insight into the developmental basis of er-105 erll-2 erl2-1/+ female sterility, we examined the morphology of mature ovules (stage 13 flowers, anthesis) (Smyth et al., 1990) (Fig. 2). Similar to wild type, er-105 erl1-2/+ erl2-1 exhibited typical amphitropic ovule morphology (Fig. 2A,B,E). Ovules of er-105 erl1-1 erl2$1 /+$ were fertile, but had slightly shorter integuments than wild type, resulting in the micropyle resting slightly away from the funiculus (Fig. 2C). By contrast, the mature ovules of er-105 erll-2 erl2-1/+ displayed aberrant morphology. Growth of both inner and outer integuments as well as the funiculus was dramatically compromised, leading to small, stunted ovules that often had an exposed nucellus (Fig. 2D,F). The embryo sac of mature er-105 erl1-2 erl2-1/+ ovules was absent and replaced by a disorganized mass of small cells (Fig. 2F).

Initiation and polarity of integuments was not disrupted in er-105 erll-2 erl2-1/+ (Fig. 2G). To determine whether integument initiation was defined normally, we used WUS and $P H B$ as a nucellar regional marker and adaxial marker, respectively (Fig. 2H-K). WUS is expressed exclusively in the nucellus of wild-type ovules and promotes integument initiation in the chalazal region proximal to its expression domain (Groß-Hardt et al., 2002), whereas PHB expression marks the inner integument (Sieber et al., 2004). No alteration in $W U S$ or $P H B$ expression was detected between wild type and er-105 erll-2 erl2-1/+. These data suggest that loss of ER-family signaling does not disrupt regional domain specification or radial patterning.

\section{Integument outgrowth is compromised in er-105 er/1-2 er/2-1/+ mutants}

To determine the onset of developmental defects, wildtype and er-105 erll-2 erl2-1/+ ovules were examined at sequential developmental stages (Fig. 3) (Schneitz et al., 1995). Early ovule initiation and patterning were normal in er-105 erll-2 erl2-1/+, consistent with the normal $P H B$ and $W U S$ expression patterns (Fig. 2I,K). Similar to wild type, ovule primordia initiated and elongated from the placental wall (stage 1-II; Fig. $3 \mathrm{~A}, \mathrm{E}, \mathrm{I})$. At stage 2-II to 2-V, outer and inner integument primordia arose from the chalaza and grew toward the nucellus (Fig. 3B,F,J). However, starting at stage 3-I, the cells of er-105 erll-2 erl2-1/+ outer integuments had a more disorganized appearance than wild type (Fig. $3 \mathrm{C}, \mathrm{G}, \mathrm{K})$. Enlargement of the megaspore mother cell (MMC) and tetrad formation proceeded as in wild type within the nucellus of er-105 erl1-2 erl2-1/+ ovules (Fig. 3I,M). However, mutant ovules arrested at a twonucleate embryo sac (Fig. 3N). We never observed er105 erl1-2 erl2-1/+ embryo sacs beyond this twonucleate stage. Based on our observations, we conclude that progression of integument growth in the absence of 

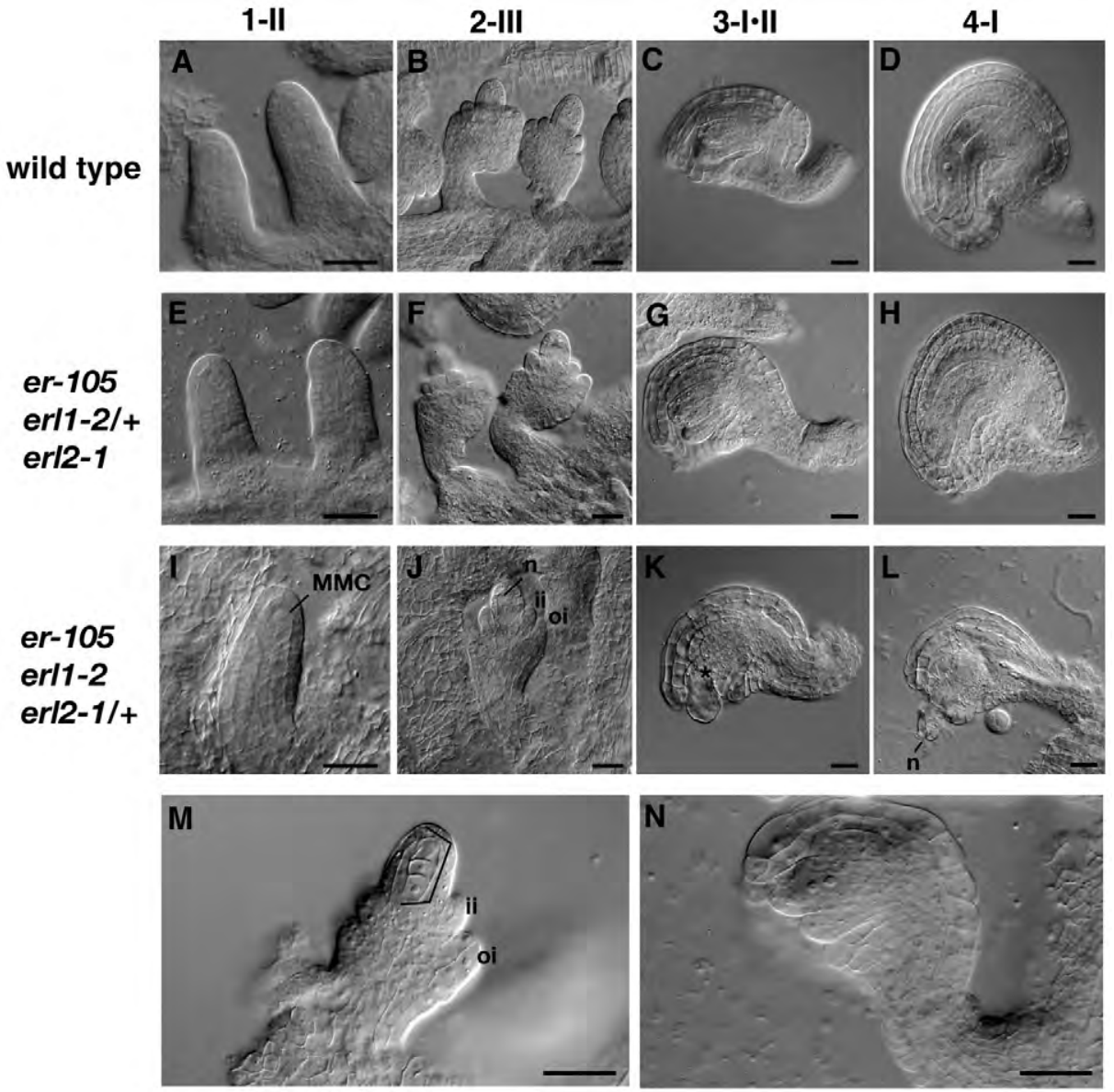

Fig. 3. Developmental series of ovule development in wild-type, er-105 erl12/+ erl2-1 and er-105 erl1-2 erl2-1/+

Arabidopsis. (A-L) DIC images of ovules at stages: 1-II $(A, E, I) ; 2-I I I(B, F, J) ; 3-I I I(C, G, K)$ and $4-I(D, H, L)$. Differences in development are seen at stage 3-I-II (C, G,K). Asymmetric growth of the outer integument occurs in all genotypes; however, er-105 erl1-2 erl2$1 /+$ integuments appear less organized and gametophyte development has ceased (K). By anthesis $(D, H, L)$, the integuments of er105 er/1-2 er/2-1/+ are short resulting in the nucellus protruding from the integuments (L). By contrast, the er-105 erl1-2/+ er/2-1 ovule has nearly normal appearance, with a very subtle phenotype (H). (M,N) er-105 erl1-2 erl2-1/+ ovule at stage 2-V with tetrad ( $M$, bracket) and stage 3-II (N) showing two-nuclear gametophyte. MMC, megaspore mother cell; n, nucleus; ii, inner integument; oi, outer integument. Scale bars: $20 \mu \mathrm{m}$.
$E R$ is dependent on ERL1 and 2 in a dosage-dependent manner. At a minimum, ERL1 in a heterozygous state or ERL2 in a homozygous state is required.

\section{Reduced cell numbers in er-105 erl1-2 erl2-1/+ mutants lead to reduced integument size}

Change in organ size reflects an alteration in cell size, cell number, or both. To understand the mechanism underlying the phenotype of er-105 erl1-2 erl2-1/+ ovules, we examined the size and number of cells in developing ovule integuments and compared them with wild type. In wild type, integument cells at the micropylar end were much more elongated than those at the chalazal end (Fig. 2E). The er erll erl2/+ ovules appeared to lack this characteristic polar elongation of integument cells (Fig. 2F). This made the outer integument cells of er-105 erll-2 erl2-1/+ ovules less organized and slightly more uniform in length than wild type. However, no significant difference in outer integument cell size area was found between wild-type and mutant ovules (Fig. 4A-C).

We next assessed the possibility of endoreduplication in mutant integument cells by observing the nuclear size using 4',6-diamidino2-phenylindole (DAPI). Endoreduplication is the repetitive duplication of chromosomal DNA without mitosis or cytokinesis, resulting in an increase in cell ploidy level (Kondorosi et al., 2000). In plants, a positive correlation between ploidy level and cell size has been observed (Kondorosi et al., 2000; Melaragno et al., 1993). No qualitative difference in the size (area) of nuclei between wild-type and er-105 erl1-2 erl2-1/+ outer integument cells was observed (see Fig. S2 in the supplementary material), consistent with the absence of a cell size difference between wild-type and mutant integuments.
To investigate whether $\mathrm{er}-105 \mathrm{erll}-2 \mathrm{erl}-1 / \mathrm{+}$ ovule integument defects were due to a decrease in cell division, we quantified the number of cells contributing to the epidermis at stage 1-II and to the outer integument at three subsequent stages (see Materials and methods). At stages 1-II and 2-II, no difference in cell number was observed between $e r$-family combination mutants and wild type (Fig. 4D). However, er-105 erll-2 erl2-1/+ outer integuments had significantly fewer cells per outer integument than wild type from stage 3 -III (31\% reduction) to maturity (57\% reduction). A similar trend was observed for er -105 erll-2/+ erl $2-1$ (15\% reduction) and er erl1-1 erl2-1/+ (30\% reduction) ovules, which had an intermediate number of integument cells between that of wild type and er-105 erl $1-2$ erl2-1/+ at maturity (Fig. 4D). Together, these data provide evidence that a reduction in cell division is responsible for the abnormal integument morphology of er-105 erl1-2 erl2-1/+ ovules, and that the number of cell divisions is sensitive to the dosage of ERL1.

\section{Specific misregulation of cell-cycle regulators is associated with the arrested ovule development in er-105 erl1-2 erl2-1/+ mutants}

Because cell divisions were reduced in er-105 erl1-2 erl2-1/+ ovules, we examined the expression of cell-cycle-regulatory genes to determine if their transcript accumulation is altered in er-105 erll2 erl2-1/+ ovules. The cell cycle is regulated at multiple points by cyclin-dependent kinases (CDKs) that form complexes with appropriate cyclins (De Veylder et al., 2003; Dewitte and Murray, 2003). Plants have a large number of core cell-cycle genes including plant specific B-type CDKs that are regulated at the transcriptional 

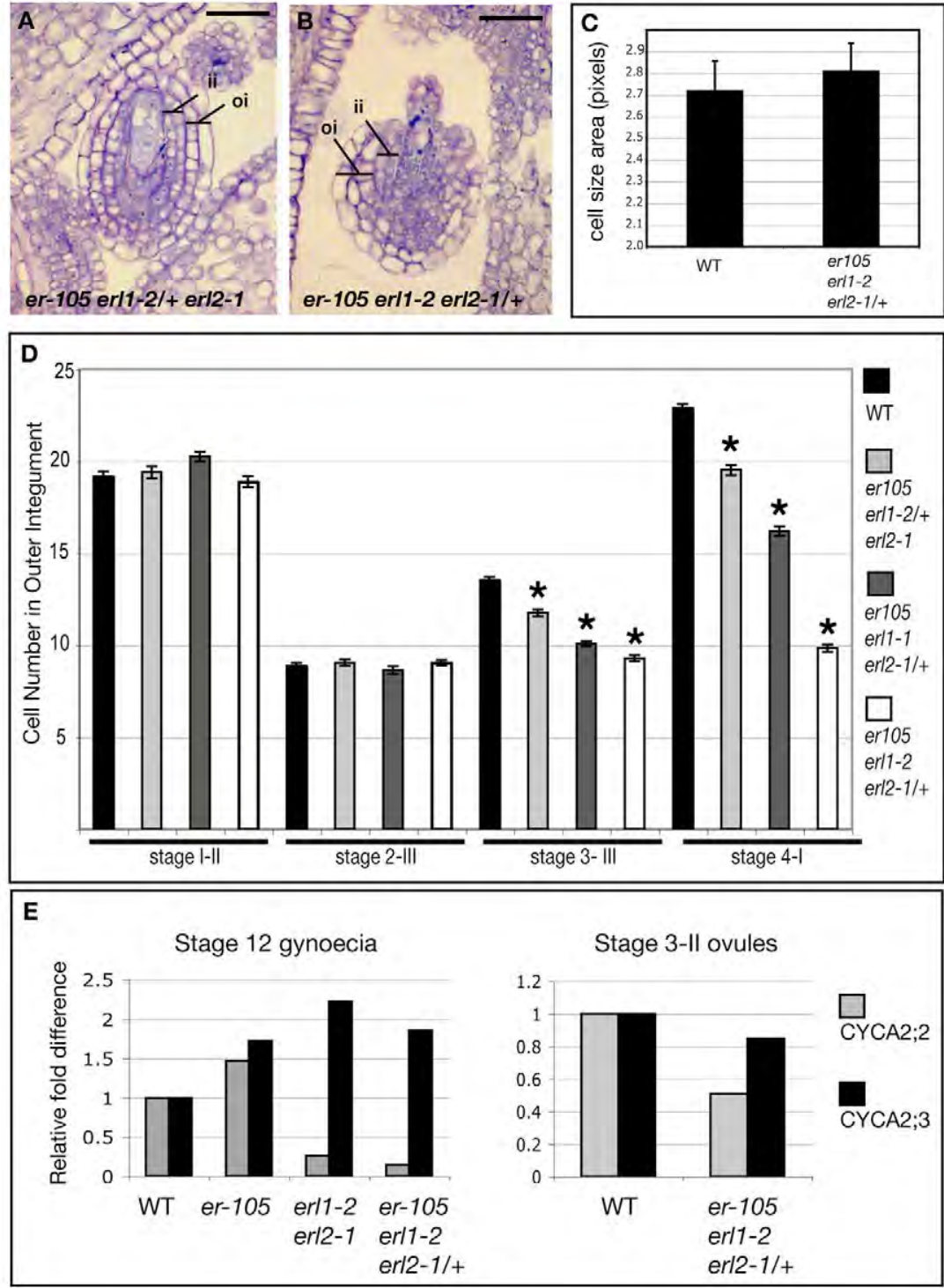

Fig. 4. ERL2 is haploinsufficient to maintain integument growth. (A,B) Transverse (horizontal) sections of Arabidopsis ovules at anthesis. (A) er-105 erl1-2/+erl2-1 has a fully encased and developed embryo sac. (B) er-105 erl1-2 erl2-1/+ ovule has shorter integuments, protruding nucellus with no observable embryo sac. (C) Mean cell area ( \pm s.e.m.) of the outer integument cells of wild type and er-105 er/1-2 er/2-1/+ ( $n=25$, Student's t-test, $P=0.331)$. (D) Cell number ( \pm s.e.m.) in epidermis of ovules (stage 1-II) and the outer layer of the outer integument (stage 2-III, 3-III and 4-I) of wild-type and er-family mutant ovules at the indicated stages $(n=25)$. Asterisks indicate a significant difference from the respective wild type within each stage using Student's $t$-test $(P=0.01)$. At stage 3 -III, a decrease in the number of cells in the outer integument is detected between wild type and er-family mutants. By stage 4-II, the number of cells in the outer integument is dramatically reduced compared with wild type. (E) Real-time RT-PCR analysis of selected cyclins in stage 12 carpels (left) and stage 3 -II ovules (right). Loss of ER-family function results in a disruption of cyclin gene expression. Scale bars: $20 \mu \mathrm{m}$. level (De Veylder et al., 2003). Plant cyclin genes can be divided into four groups (A, B, D and $\mathrm{H})$. The evolution of three A-type subclasses (A1, A2 and A3), compared with only one in animals, has led to the idea that A-type cyclins might fulfil plant-specific roles (Caubet-Gigot, 2000; Dewitte and Murray, 2003).

To detect alterations in expression levels of cell-cycle regulatory genes, we performed RT-PCR analysis on RNA from stage 12 carpels (see Fig. S3 in the supplementary material). According to Schneitz et al. (Schneitz et al., 1995), this stage of carpel corresponds to the point in ovule development when we observed noticeable changes in integument morphology between $\mathrm{er}$ - $105 \mathrm{erll}$ 2 erl2-1/+ and wild type. Most of the B-type CDKs and cyclin A, B and $\mathrm{D}$ genes did not show a dramatic change in transcript abundance in ER-family loss-of-function mutants. However, CYCA2;2 showed a pronounced decrease in expression in er-105 erll-2 erl2-1/+ (see Fig. S3 in the supplementary material; data not shown). We next quantified the relative decrease in $C Y C A 2 ; 2$ transcripts using real-time PCR. We found that $C Y C A 2 ; 2$ consistently shows a significant decrease in expression after loss of $E R$-family function in both carpels and individual ovules (Fig. 4E). Conversely, $C Y C A 2 ; 3$ had the opposite trend, showing increased transcript accumulation after $E R$-family loss-of-function, perhaps owing to compensation (Fig. 4E). The results suggest that reduced levels of $E R$-family gene function may impact cell division through disruption of specific cell-cycle-regulatory gene expression.

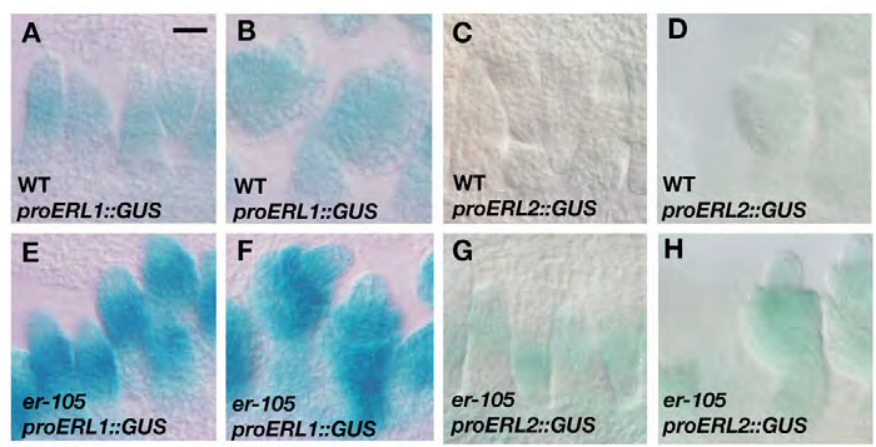

Fig. 5. The ER family of LRR-RLKs are expressed in ovules throughout development. (A,B) proERL $1::$ GUS expression in wildtype Arabidopsis. (C,D) proERL2::GUS expression in wild type. $(\mathbf{E}, \mathbf{F})$ proERL 1::GUS expression in er-105. $(\mathbf{G}, \mathbf{H})$ proERL2::GUS expression in er-105. ERL1 and ERL2 promoter activity is higher in the er-105 background than in wild type. Scale bar: $20 \mu \mathrm{m}$. 

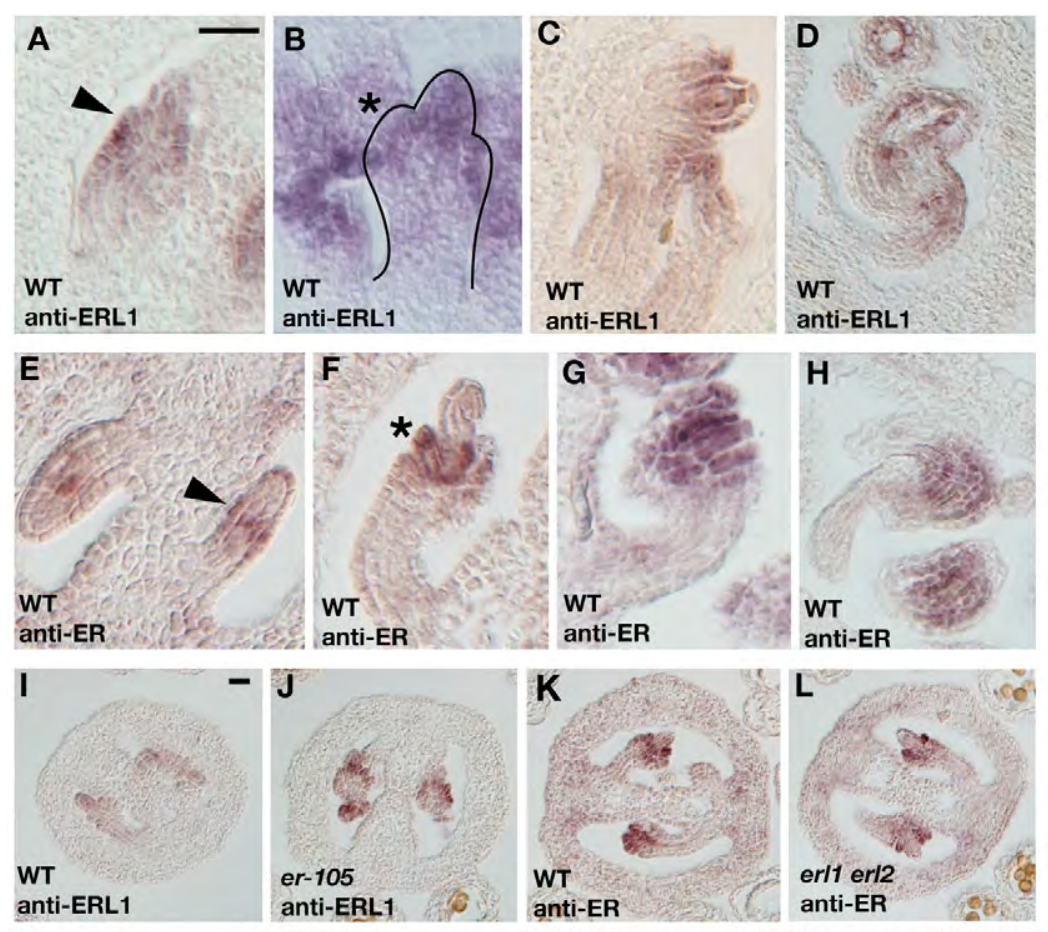

M
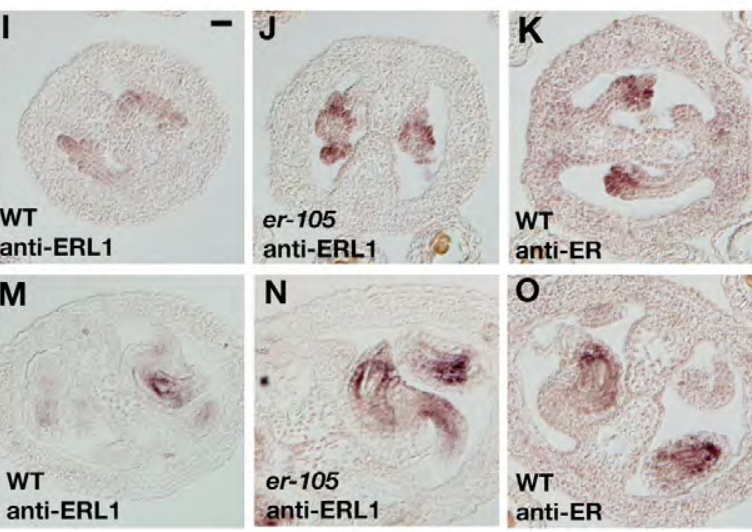

N

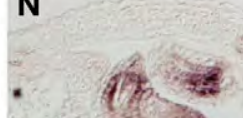

O

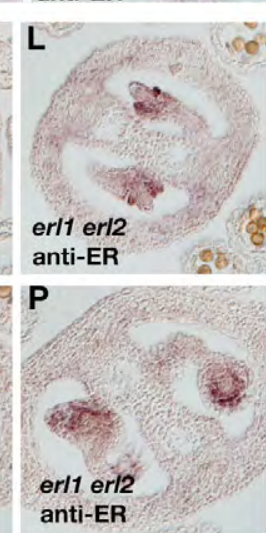

Fig. 6. ERL1 and $E R$ expression patterns. (A-H) In situ hybridization in wild-type Arabidopsis using an ERL1 $(\mathrm{A}-\mathrm{D})$ or $E R(\mathrm{E}-\mathrm{H})$ antisense probe. Both show broad expression in ovules with increased signal intensity in the chalazal region ( $A, E$, arrowheads) and developing integuments (B,F, asterisks). Ovule is outlined in B.

$(\mathrm{D}, \mathrm{H})$ Signal is still visible at maturity. (I-P) Comparison of $E R L 1(\mathrm{I}, \mathrm{J}, \mathrm{M}, \mathrm{N})$ or $E R(\mathrm{~K}, \mathrm{~L}, \mathrm{O}, \mathrm{P}) \mathrm{mRNA}$ accumulation in wild type and ER-family mutants examined by in situ hybridization. (Q) Real-time RT-PCR analysis of ER, ERL1 and $E R L 2$ in stage 12 carpels of wild type and $E R$-family mutants. Transcript abundance of ER,ERL1 and ERL2 are higher in the absence of the other family members. Scale bars: $20 \mu \mathrm{m}$.

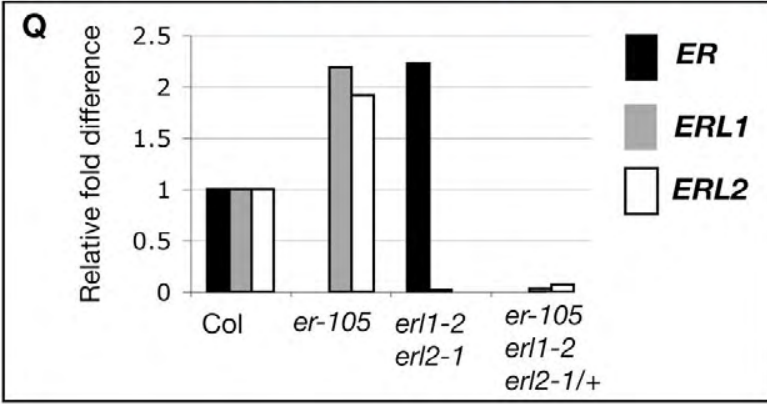

Alternatively, altered levels of these cyclin genes could be the consequence of reduced cell division produced by a yet unknown mechanism.

\section{ERL1 and ERL2 are expressed in ovule primordia and developing integuments}

To determine the expression pattern of the $E R$ family during ovule development, we first observed their promoter activity using reporter $\beta$-glucuronidase (GUS) fusion constructs (Fig. 5) (Shpak et al., 2004). ERL1 and ERL2 promoter activity was first detected in the placental wall prior to ovule primordia initiation (data not shown). They became broadly active in ovules with a slight increase in activity in the central chalazal region (Fig. 5A-D). In mature ovules, faint GUS expression was restricted to the funiculus (data not shown). Interestingly, proERL1::GUS was notably stronger in the er-105 background than in wild type (Fig. 5E,F).

Because integument growth was sensitive to the dosage of ERLI and because the promoter activity of ERL1 and ERL2 are similar, we focused our analysis of expression overlap on $E R$ and $E R L 1$. We analyzed the mRNA expression patterns of $E R$ and $E R L 1$ by in situ hybridization (Fig. 6). In reproductive structures, ERL1 transcripts were detected in floral meristems and primordia (see Fig. S4 in the supplementary material). During ovule development, ERL1 transcripts were detected throughout ovule primordia from initiation to stage 3-II, with increased hybridization intensity in the chalazal region and developing integuments (Fig. 6A-C). At maturity, ERL1 hybridization signal was still detectable in the integuments and funiculus (Fig. 6D).

Expression of $E R$ was observed in floral meristems as well as the carpel wall and developing petals (see Fig. S4 in the supplementary material). Similar to $E R L 1, E R$ transcripts were detected broadly in the developing ovule, with more intense signal in the chalazal region and developing integuments (Fig. 6E-G). ER expression lessened later in development, but was still detectable in the carpel and ovule integuments at maturity (Fig. $6 \mathrm{H}$ ). As the probes for $E R$ and $E R L 1$ did not cross-hybridize with each other (see Fig. S4 in the supplementary material), our results suggest that overlapping expression patterns of $E R$ and $E R L 1$ account for their redundant roles in ovule development. 

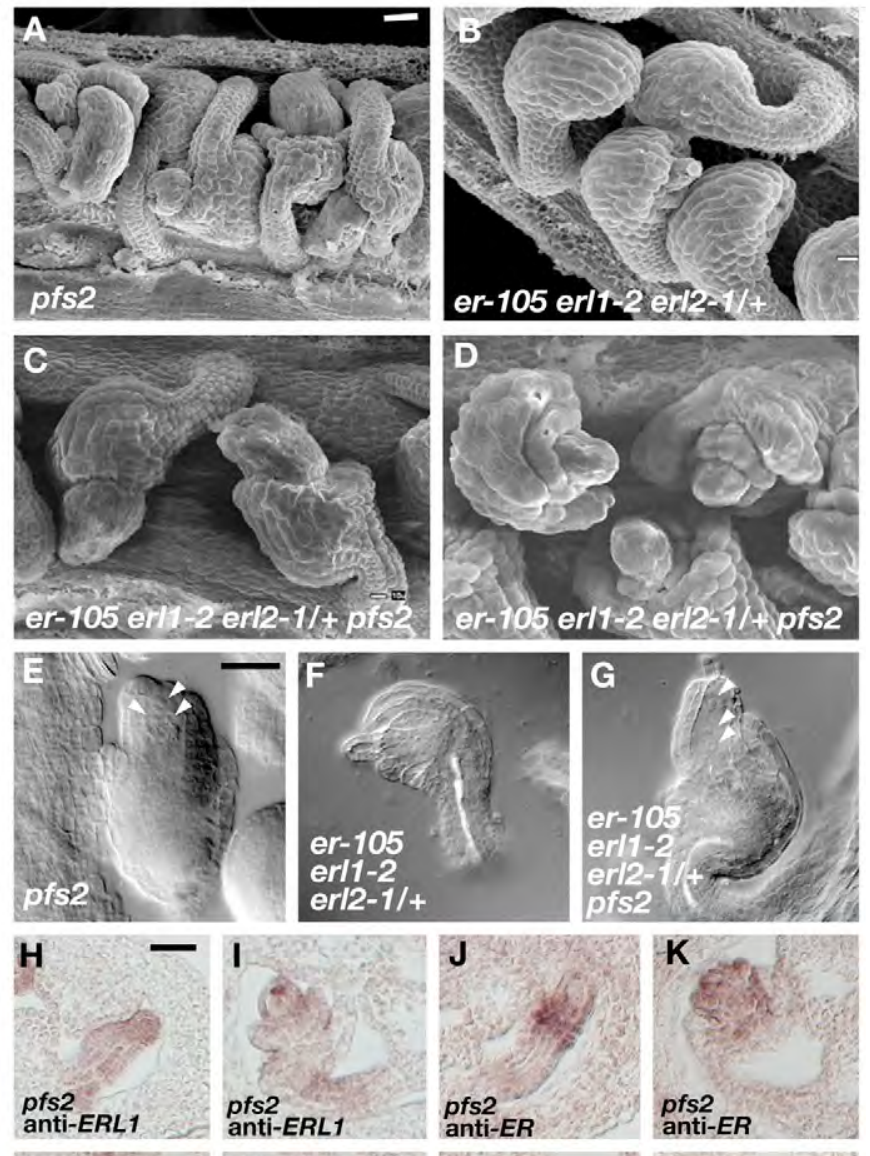

$\mathbf{L}$
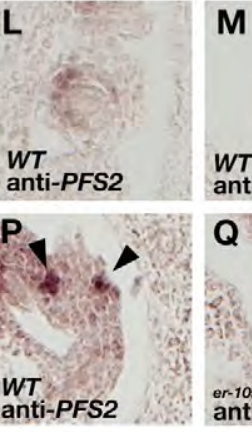

M
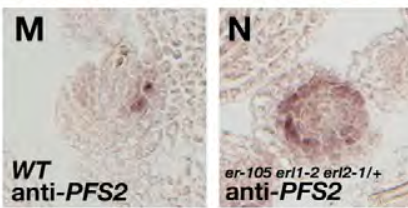

o

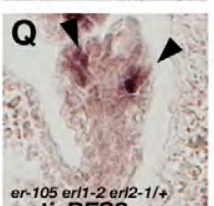
er-105 erl1-2 erl2-1/+
anti-PFS2

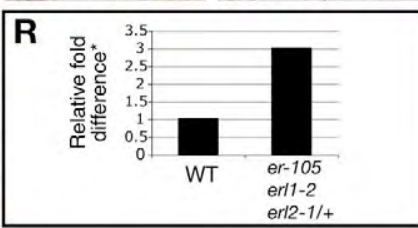

\section{Unequal dosage compensation among ER-family genes}

Given the overlapping expression patterns of $E R$-family genes in the ovule and their ability to functionally substitute for one another (Shpak et al., 2004), we tested whether loss of specific $E R$-family gene function would lead to changes in expression of the other family members. To assess changes in transcript abundance, quantitative real-time RT-PCR analysis was performed on RNA from stage 12 carpels (Fig. 6Q). An increase in both ERL1 and ERL2 transcripts was observed in the er-105 background as compared with wild type and, reciprocally, the accumulation of $E R$ transcripts was higher in the erll erl2 mutant background. In situ hybridization determined that the spatial expression patterns of $E R$ and ERLI were not disrupted in the er-105 and erll erl2 mutant backgrounds (Fig. 6I-P), respectively.

Taken together, the results suggest that transcriptional upregulation occurs in the absence of $E R$-family members, allowing each family member to functionally compensate for each other. This
Fig. 7. Genetic interactions of ER-family genes with PFS2.

(A-D) SEMs of mature Arabidopsis ovules. (A) pfs2-1 ovules have aberrant integument development. (B) er-105 er/1-2 er/2-1/+ ovules. (C,D) er-105 erl1-2 erl2-1/+ pfs2-1 mutant ovules have variability in integument development with reduced growth of the outer integument and some bifurcation of developing integuments. (E-G) DIC images of mature ovules. (E) pfs2-1 ovules have some partially developed embryo sacs. (F) er-105 er/1-2 er/2-1/+ has a penetrant phenotype of a small mass of cells in place of the embryo sac. (G) er-105 erl1-2 erl2-1/+ pfs2-1 mutant ovules display variability in embryo sac development similar to the pfs2-1 single mutant. Embryo sac nuclei are indicated by white arrowheads. (H-K) In situ hybridization in the pfs2-1 mutant using $E R L 1(\mathrm{H}, \mathrm{I})$ and $E R(\mathrm{~J}, \mathrm{~K})$ antisense probes. $(\mathbf{L}, \mathbf{M})$ In situ hybridization in wild type using a PFS2 antisense probe. $(\mathbf{N}, \mathbf{0})$ In situ hybridization in er-105 erl1-2 er/2-1/+ using a PFS2 antisense probe. $(\mathbf{P}, \mathbf{Q})$ Extended exposure of PFS2 antisense probe in wild type and er-105 er/1-2 er/2-1/+. Intense signal was observed in the inner integuments (arrowheads). No signal was detected with sense probes for ERL1, ER or PFS2. Scale bars: $20 \mu \mathrm{m}$. (R) Real-time RT-PCR analysis of PFS2 expression in stage 3-II ovules, given as fold-difference relative to wild type. ${ }^{*}$, Raw data collected as PFS2 transcript abundance relative to ACTIN 2 (wild type versus er-105 er/1-2 er/2-1/+ Experiment 1: $0.205 \pm 0.04$ versus $0.351 \pm 0.02$; Student's $t$-test $P=0.03$; 1.72 fold increase in er-105 erl1-2 erl2-1/+. Experiment 2: $0.141 \pm 0.003$ versus $0.603 \pm 0.04$; Student's $t$-test $P=0.001 ; 4.2$ fold increase in er105 erl1-2 erl2-1/+).

is consistent with the findings of Shpak et al. (Shpak et al., 2004) that ER-family members are able to substitute for each other if their temporal and spatial expression patterns overlap. Fertility in the null er single mutant or erll erl 2 double mutant might require dosage compensation by the remaining family members to maintain signaling above a required threshold. This threshold is not met in the er-105 erll erl2/+ mutant, resulting in the arrest of integument growth.

\section{Interaction of pfs2 with er er/1-2 er/2-1/+}

$P F S 2$, a WUS-related homeobox gene also known as WOX6, affects ovule patterning and regulates integument cell proliferation and gametophyte differentiation (Park et al., 2004; Park et al., 2005). It has been well-documented that the competing actions of the transcription factor WUS, and the CLAVATA (CLV) LRR-RLK signaling pathway, maintain meristem size in Arabidopsis (Brand et al., 2000). Numerous WOX genes display region-specific expression, but relatively little is known about their regulation (Haecker et al., 2004). To investigate whether a mechanism including PFS2 and ER signaling directs integument size during ovule development, we produced the combination mutant er-105 erl1-2 erl2-1/+ pfs $2-1$ and characterized the phenotype.

The $p f s$-1-null mutation causes the production of ovules with similar characteristics to $\mathrm{er}-105 \mathrm{erl1}-2 \mathrm{erl2}-1 /+$. pfs $2-1$ ovules have aberrant integument growth after initiation and embryo sac arrest (Park et al., 2004) (Fig. 7A). However, growth defects are variable in pfs2-1 mutants: some ovules complete various stages of embryo sac development and a low percentage of viable seed are produced (Park et al., 2005). Similar to $p f s 2-1$ alone, we observed a variable degree of integument development in the er-105 erl1-2 erl2-1/+ pfs 2-1 mutant (Fig. 7C,D). The phenotype ranged from an outer integument that covered the inner integument to a near absence of the outer integument. In addition, subtle changes in integument 
development were observed including lobing of the outer integument during development (Fig. 7C,D). Such variability in integument growth was not observed in er-105 erll-2 erl2-1/+, in which the phenotype showed little variation (Fig. 7B). Surprisingly, some ovules of er-105 erll-2 erl2-1/+pfs $2-1$ developed beyond the two-celled nuclei stage typical of er-105 erll-2 erl2-1/+ ovules, although no viable seed could be collected from these plants (Fig. $7 \mathrm{E}-\mathrm{G})$. Therefore, the loss of PFS 2 function partially rescues the $\mathrm{er}$ 105 erll -2 erl2 $-1 /+$ ovule defects.

Because pfs $2-1$ ovules show aberrant integument growth, we performed in situ hybridization using ER and ERL1 probes in the pfs 2-1 ovules to determine whether loss of ER-family signaling contributed to the abnormal integument phenotype of $p f s 2-1$ ovules (Fig. 7H-K). Loss or change in expression domain of ER or ERL1 was not detected in the $p f s 2-1$ mutant, suggesting that the required threshold of ER-signaling is met in the pfs $2-1$ mutant.

The phenotypic rescue of er-105 erll-2 erl2-1/+ ovules by additional $p f s 2$ mutation implies that it is advantageous not to have PFS2 under the sub-threshold level of ER-family signaling. To test whether the er-105 erll-2 erl2-1/+ phenotype was due in part to misexpression of $P F S 2$, we performed in situ hybridization using a PFS2 probe (Fig. 7L-O). PFS2 expression was detected broadly in developing carpels and initiating ovule primordia (data not shown) with strong expression in the inner integument during initiation and growth (Fig. 7L-O). The expression domain of PFS2 was not altered in the er-105 erl1-2 erl2-1/+ ovules (Fig. 7P,Q), indicating that the gametophytic defects in er-105 erl1-2 erl2-1/+ are not due to PFS2 misexpression. However, quantitative real-time RT-PCR detected an increase in PFS2 transcript abundance in er-105 erll-2 erl2-1/+ stage 3-II ovules compared with wild type, indicating a potential inhibitory role for the ER family in PFS2 expression. Phenotypic rescue of $\mathrm{er}-105 \mathrm{erl} 1-2 \mathrm{erl}-1 / \mathrm{t}$ by the addition of the $p f s$ mutation suggests that ovule development requires a proper balance of both $E R$ family and PFS2 function.

\section{DISCUSSION Unequal functional redundancy among the ER- family LRR-RLKs}

Gene duplication is a common occurrence and has led to the production of numerous gene families in Arabidopsis, one of the largest being the RLK-coding gene family, which has over 600 members (Shiu and Bleecker, 2001; Vision et al., 2000). Following a gene duplication event, decreased selection pressure on one of the gene copies can produce proteins with novel function or expression resulting in partial redundancy among gene family members (Hardtke et al., 2004). If the shared function of duplicated genes becomes unequally distributed between gene copies, unequal redundancy among the gene family members may result, as described for the ER family, where both erll and erl 2 confer no growth phenotype but enhance the er growth defects in a unique manner (Kempin et al., 1995; Shpak et al., 2004). In many cases, unequal redundancy is produced by differences in the expression level or expression patterns between gene copies, where the duplicated gene(s) may have redundant function with the ancestral gene, but the expression might be lower or restricted. A good example is the three Arabidopsis brassinosteroid receptor genes. The major receptor, $B R I 1$, is broadly expressed at high levels, whereas expression of BRL2 and BRL3 is restricted to vasculature (CanoDelgado et al., 2004). Successive loss of family members enhances the phenotype of the ancestral gene and a true null phenotype is observed only in the absence of the entire family as has been shown for ER, ERL1 and ERL2 (Shpak et al., 2004).
Our study uncovered unequal contributions and dosage compensation among gene family members with overlapping expression patterns. The most significant difference between $\mathrm{er}-105$ erll-2/+ erl2-1 and er-105 erl1-2 erl2-1/+ plants is the loss of female fertility owing to reduced cell division and outgrowth of the integuments in er-105 erll-2 erl2-1/+. The expression pattern for all three family members overlaps in the distal, central and proximal regions, consistent with all three regions being reduced when successive family members are removed. An erl1-2 erl2-1 double or er single mutant shows no obvious defects in integument development, indicating that the upregulation of $E R$ or ERL1 and 2 in these backgrounds can maintain expression above the threshold necessary to direct integument development (Fig. 6Q). However, as successive family members are lost, low levels of ERL2 are insufficient to maintain signaling, and integument growth is compromised. Consistent with this dosage-dependence model, the ovules of er-105 erl1-2 erl2-1/+ are significantly larger and more developed than those of the $E R$-family triple mutant (see Fig. S5 in the supplementary material).

\section{ERL2 haploinsufficiency specifically affects cell division of the ovule integuments}

The ER-family LRR-RLKs probably maintain cell-cell communication, a reduction of which results in the irregular and reduced cell division during integument outgrowth in er-105 erll-2 erl2-1/+ ovules. They do not regulate ovule primordia patterning or integument initiation, as these aspects are not affected even by the complete loss of ER-family function (Fig. 2 and see Fig. S5 in the supplementary material).

Our results suggest that the ER-family RLKs promote cell division after integument initiation through potential regulation of core cellcycle genes, specifically $C Y C A 2 ; 2$ (Fig. 4). Among over 30 cyclins present in Arabidopsis, ten belong to the A-type (Dewitte and Murray, 2003; Vandepoele et al., 2002). Recent studies suggest that Arabidopsis cyclin A2 families act as negative regulators of endoreduplication (Burssens et al., 2000; Dewitte and Murray, 2003; Imai et al., 2006; Yu et al., 2003). For example, loss-of-function mutations in CYCA2;3 led to elevated ploidy levels in mature organs (Imai et al., 2006). Consistently, the dominant, activation-tagged mutation in INCREASED LEVEL OF POLYPLOIDY1, a conserved repressor of $C Y C A 2$ transcription, caused an increase in endoreduplication (Yoshizumi et al., 2006). However, cyclin A2 families may also function to regulate mitosis. In Medicago, CYCA2;2 $(\mathrm{MedsaCYCA2;2)}$ is required for meristem formation or activity but dispensable for endoreduplication (Roudier et al., 2000). Similar to Medsa;CYCA2;2, all four Arabidopsis cyclin A2 genes show high promoter activity in meristems and young organ primordia (Burssens et al., 2000; Imai et al., 2006). Therefore, they might redundantly control cell division in actively proliferating tissues, where the ERfamily signaling pathway is required. Consistent with this hypothesis, flow-cytometric analysis of DNA content in er-105 suggested possible cell-cycle arrest (Shpak et al., 2003).

All members of the ER family are expressed in overlapping domains in additional areas of the plant including shoot and inflorescence meristems and floral primordia (Shpak et al., 2004). The lack of any visual er-105 erll-2 erl2-1/+ phenotype in these tissues could be due to redundancy by other signaling factors that are not expressed in the ovule. Alternatively, ovule integuments might be particularly susceptible to cell-cell signaling disruption. Unlike meristems and developing leaves and floral organs, integuments are solely derived from the L1 layer (Schneitz et al., 1997). Therefore, cell proliferation in integuments is likely to 
occur with limited inter cell-layer communication. Such a scenario was suggested for loss-of-function mutants of the RLK gene ARABIDOPSIS CRINKLY4, which display localized defects in integument development, despite its expression in a wider variety of tissues (Gifford et al., 2003).

\section{Genetic interaction of ER-family genes and PFS2}

PFS2 is thought to control ovule primordial patterning by regulating the timing of cellular differentiation (Park et al., 2005). In $p f s 2-1$, premature differentiation of the cells contributing to the gametophyte or integument primordia could account for decreased integument length and defects in the embryo sac (Park et al., 2004). The addition of $p f s-1$ to er-105 erl1-2 erl2-1/+ mutations led to increased variability in both integument and gametophyte development, similar to $p f s 2-1$ alone, indicating that the $p f s$ 2- 1 mutation is epistatic to the $e r$-family mutations. One explanation for these results is that the ER-family RLKs and PFS2 both contribute to integument outgrowth through distinct but interrelated mechanisms. The ER family are components of a signaling pathway that sustain organized cell divisions, potentially through the regulation of core cell-cycle genes such as $C Y C A 2 ; 2$. Without activation of this signaling pathway, integument cells may differentiate before undergoing sufficient cell divisions. PFS2 might be responsible for maintaining integument cells in a state that allows them to respond to factors required for coordinated cell division. In this scenario, the loss of PFS2 function would be epistatic to the loss of the ER family.

WOX genes generally have a spatially restricted expression pattern (Matsumoto and Okada, 2001). Our in situ hybridization shows that PFS2 expression is spatially restricted to the inner integument (Fig. 7). Therefore, $P F S 2$ might be required to properly coordinate the developmental states of the sporophytic integument tissues and gametophytic embryo sac. If so, the loss of PFS2 uncouples embryo sac development from abrogated integument growth under the subthreshold level of ER-family signaling, therefore partially rescuing the embryo sac defects in erecta-105 erl1-2 erl2-1/+ ovules.

In the well-established model of $C L V / W U S$ interaction in the shoot meristem, CLV3 limits the expression of WUS, while WUS in turn promotes the expression of $C L V 3$, causing a feedback loop that maintains a stem cell population (Brand et al., 2000; Clark, 2001; Laux et al., 1996). Our results indicate that the ER family does not spatially restrict PFS2 expression and that factors yet to be identified are likely to be responsible for the limiting PFS2 expression. However, the ER family might restrict the expression levels of PFS2, and elevated expression of PFS2 might account for the abortion of gametophytes in er-105 erl1-2 erl2-1/+ ovules. Consistently, embryo sacs of er-105 erll -2 erl2-1/+ are filled with a small group of cells, which resembles the phenotype of PFS2 overexpression described by Park et al. (Park et al., 2005).

Haploinsufficiency refers to a phenotype associated with the inactivation of a single allele leading to a half-normal amount of gene product, which is insufficient to maintain the wild-type phenotype. In this report, we have shown a novel phenotype for successive ER-family loss-of-function; ERL2 is haploinsufficient in the absence of $E R$ and $E R L 1$ to direct integument growth, but sufficient to drive floral organ growth, elongation and patterning. We further uncovered evidence of dosage compensation within the ER family and their potential regulatory relationships with the WOX-family transcription factor. Our work has identified the specific timing of ERL1 action during integument cell proliferation, which might provide a biological system to further elucidate the signaling components.
We thank Dr John Bowman for the erl1-1 enhancer-trap line, Dr Bernard Hauser for pfs2-1 seeds, Drs Kiyotaka Okada, Mitsuhiro Aida and Masao Tasaka for PHB probes, Dr Caroline Josefsson for assistance in RT-PCR analysis of erl1-1, Dr Kay Schneitz for advice on the design of the genetic screen and Dr Jessica Messmer-McAbee for commenting on the manuscript. This work was supported by the USDA CREES postdoctoral fellowship to L.J.P. and by the DOE grant (DE-FG02-03ER15448) to K.U.T. The DIC microscopy was supported by the CREST award from Japan Science and Technology Agency to K.U.T

\section{Supplementary material}

Supplementary material for this article is available at http://dev.biologists.org/cgi/content/full/134/17/3099/DC1

\section{References}

Brand, U., Fletcher, J. C., Hobe, M., Meyerowitz, E. M. and Simon, R. (2000). Dependence of stem cell fate in Arabidopsis on a feedback loop regulated by CLV3 activity. Science 289, 617-619.

Burssens, S., Engler, J. A., Beeckman, T., Richard, C., Shaul, O., Ferreira, P., Van Montagu, M. and Inzé, D. (2000). Developmental expression of the Arabidopsis thaliana CycA2;1 gene. Planta 211, 623-631.

Cano-Delgado, A., Yin, Y., Yu, C., Vafeados, D., Mora-Garcia, S., Cheng, J., Nam, K. H., Li, J. and Chory, J. (2004). BRL1 and BRL3 are novel brassinosteroid receptors that function in vascular differentiation in Arabidopsis. Development 131, 5341-5351.

Caubet-Gigot, N. (2000). Plant A-type cyclins. Plant Mol. Biol. 43, 659-675.

Clark, S. E. (2001). Cell signaling at the shoot apical meristem. Nat. Rev. Mol. Cell Biol. 2, 276-284.

De Veylder, L., Joubes, L. and Inzé, D. (2003). Plant cell cycle transitions. Curr. Opin. Plant Biol. 6, 536-543.

Dewitte, W. and Murray, J. A. H. (2003). The plant cell cycle. Annu. Rev. Plant Biol. 54, 235-264.

Ehsan, H., Reichheld, J. P., Durfee, T. and Roe, J. L. (2004). TOUSLED kinase activity oscillates during the cell cycle and interacts with chromatin regulators. Plant Physiol. 134, 1488-1499.

Elliott, R. C., Betzner, A. S., Huttner, E., Oakes, M. P., Tucker, W. Q. J., Gerentes, D., Perez, P. and Smyth, D. R. (1996). AINTEGUMENTA, an APETALA2-like gene of Arabidopsis with pleiotropic roles in ovule development and floral organ growth. Plant Cell 8, 155-168

Eshed, Y., Baum, S. R., Perea, J. V. and Bowman, J. L. (2001). Establishment of polarity in lateral organ of plants. Curr. Biol. 11, 1251-1260.

Eshed, Y., Izhaki, A., Baum, S. R., Floyd, S. K. and Bowman, J. L. (2004) Asymmetric leaf development and blade expansion in Arabidopsis are mediated by KANADI and YABBY activities. Development 127, 725-734

Gasser, C. S., Broadhvest, J. and Hauser, B. A. (1998). Genetic analysis of ovule development. Annu. Rev. Plant Physiol. Plant Mol. Biol. 49, 1-24.

Gifford, M. L., Dean, S. and Ingram, G. C. (2003). The Arabidopsis ACR4 gene plays a role in cell layer organisation during ovule integument and sepal margin development. Development 130, 4249-4258.

Groß-Hardt, R., Lenhard, M. and Laux, T. (2002). WUSCHEL signaling functions in interregional communicaton during Arabidopsis ovule development. Genes Dev. 16, 1129-1138.

Haecker, A., Groß-Hardt, R., Geiges, B., Sarkar, A., Breuninger, H., Herrmann, M. and Laux, T. (2004). Expression dynamics of WOX genes mark cell fate decisions during early embryonic patterning in Arabidopsis thaliana. Development 131, 657-668

Hardtke, C. S., Ckurshumova, W., Vidaurre, D. P., Singh, S. A., Stamatiou, G., Tiwari, S. B., Hagenn, G., Guilfoyle, T. J. and Berleth, T. (2004). Overlapping and non-redundant functions of the Arabidopsis auxin response factors MONOPTEROS and NONPHOTOTROPIC HYPOCOTYL 4. Development 131, 10891100

Hauser, B. A., He, J. Q., Park, S. O. and Gasser, C. S. (2000). TSO1 is a novel protein that modulates cytokinesis and cell expansion in Arabidopsis. Development 127, 2219-2226

Imai, K. K., Ohashi, Y., Tsuge, T., Yoshizumi, T., Matsui, M., Oka, A. and Aoyama, T. (2006). The A-type cyclin CYCA2;3 is a key regulator of ploidy levels in Arabidopsis Endoreduplication. Plant Cell 18, 382-396.

Kempin, S. A., Savidge, B. and Yanofsky, M. F. (1995). Molecular basis of the cauliflower phenotype in Arabidopsis. Science 267, 522-525.

Kondorosi, E., Roudier, F. and Gendreau, E. (2000). Plant cell size control: growing by ploidy? Curr. Opin. Plant Biol. 3, 488-492.

Lang, J. D., Ray, S. and Ray, A. (1994). sin1, a mutation affecting female fertility in Arabidopsis, interacts with mod1, its recessive modifier. Genetics 137, 1101-1110. Laux, T., Mayer, K. F., Berger, J. and Jurgens, G. (1996). The WUSCHEL gene is required for shoot and floral meristem integrity in Arabidopsis. Development 122, 87-96.

Matsumoto, N. and Okada, K. (2001). A homeobox gene, PRESSED FLOWER, regulates lateral axis-dependent development of Arabidopsis flowers. Genes Dev. $15,3355-3364$. 
McAbee, J. M., Hill, T. A., Skinner, D. J., Izhaki, A., Hauser, B. A., Meister, R. J., Reddy, G. V., Meyerowitz, E. M., Bowman, J. L. and Gasser, C. S. (2006). ABERRANT TESTA SHAPE encodes a KANADI family member, linking polarity determination to separation and growth of Arabidopsis integuments. Plant J. 46 522-531.

Melaragno, J. E., Mehrotra, B. and Coleman, A. W. (1993). Relationship between endopolyploidy and cell size in epidermal tissue of Arabidopsis. Plant Cell 5, 16611668

Mizukami, Y. (2001). A matter of size: developmental control of organ size in plants. Curr. Opin. Plant Biol. 4, 533-539.

Park, S. O., Hwang, S. and Hauser, B. A. (2004). The phenotype of Arabidopsis ovule mutants mimics the morphology of primitive seed plants. Proc. R. Soc. Lond. B Biol. Sci. 271, 311-316.

Park, S. O., Zheng, Z., Oppenheimer, D. G. and Hauser, B. A. (2005). The PRETTY FEW SEEDS2 gene encodes an Arabidopsis homeodomain protein that regulates ovule development. Development 132, 841-849.

Potter, C. J. and Xu, T. (2001). Mechanisms of size control. Curr. Opin. Genet. Dev. 11, 279-286

Roudier, F., Fedoova, E., Gyorgyey, J., Feher, A., Brown, S., Kondorosi, A. and Kondorosi, E. (2000). Cell cycle function of a Medicago sativa A2-type cyclin interating with PSTAIRE-type cylin-dependent kinase and retinoblastoma protein. Plant J. 23, 73-83.

Schneitz, K., Hülskamp, M. and Pruitt, R. E. (1995). Wild-type development in Arabidopsis thaliana: a light microscope study of cleared whole-mount tissue. Plant J. 7, 731-749.

Schneitz, K., Hülskamp, M., Kopczak, S. and Pruitt, R. E. (1997). Dissection of sexual organ ontogenesis: a genetic analysis of ovule development in Arabidopsis thaliana. Development 124, 1367-1376.

Schneitz, K., Baker, S. C., Gasser, C. S. and Redweik, A. (1998). Pattern formation and growth during floral organogenesis: HUELLENLOS and AINTEGUMENTA are required for the formation of the proximal region of the ovule primordium in Arabidopsis thaliana. Development 125, 2555-2563.

Sessions, A., Weigel, D. and Yanofsky, M. F. (1999). The Arabidopsis thaliana MERISTEM LAYER 1 promoter specifies epidermal expression in meristems and young primordia. Plant J. 20, 259-263.

Shiu, S. H. and Bleecker, A. B. (2001). Receptor-like kinases from Arabidopsis form a monophyletic gene family related to animal receptor kinases. Proc. Natl. Acad. Sci. USA 98, 10763-10768.

Shpak, E. D., Lakeman, M. B. and Torii, K. U. (2003). Dominant-negative receptor uncovers redundancy in the Arabidopsis ERECTA leucine-rich repeat receptor-like kinase signaling pathway that regulates organ shape. Plant Cell 15, 1095-1110.

Shpak, E. D., Berthiaume, C. T., Hill, E. J. and Torii, K. U. (2004). Synergistic interaction of three ERECTA-family receptor-like kinases controls Arabidopsis organ growth and flower development by promoting cell proliferation. Development 131, 1491-1501.

Sieber, P., Gheyselinck, J., Gross-Hardt, R., Laux, T., Gossniklaus, U. and Schneitz, K. (2004). Pattern formation during early ovule development in Arabidopsis thaliana. Dev. Biol. 273, 321-334.

Skinner, D. J., Hill, T. A. and Gasser, C. S. (2004). Regulation of ovule development. Plant Cell 16, S32-S45.

Smyth, D. R., Bowman, J. L. and Meyerowitz, E. M. (1990). Early flower development in Arabidopsis. Plant Cell 2, 755-767.

Torii, K. U. (2004). Leucine-rich repeat receptor kinases in plants: structure, function and signal transduction. Int. Rev. Cytol. 234, 1-46.

Torii, K. U., Mitsukawa, N., Oosumi, T., Matsuura, Y., Yokoyama, R., Whittier, R. F. and Komeda, Y. (1996). The Arabidopsis ERECTA gene encodes a putative receptor protein kinase with extracellular leucine-rich repeats. Plant Cell 8, 735-746.

Vandepoele, K., Raes, J., De Veylder, L., Rouze, P., Rombauts, S. and Inze, D. (2002). Genome-wide analysis of core cell cycle genes in Arabidopsis. Plant Cell 14, 903-913.

Villanueva, J. M., Broadhvest, J., Hauser, B. A., Meister, R. J., Schneitz, K. and Gasser, C. S. (1999). INNER NO OUTER regulates abaxial-adaxial patterning in Arabidopsis ovules. Genes Dev. 13, 3160-3169.

Vision, T. J., Rown, D. G. and Tanksley, S. D. (2000). The origins of genomic duplications in Arabidopsis. Science 290, 2114-2117.

Yoshizumi, T., Tsumoto, Y., Takigushi, T., Nagata, N., Yamamoto, Y. Y., Kawashima, M., Ichikawa, T., Nakaawa, M., Yamamoto, N. and Matsui, M. (2006). INCREASED LEVEL OF POLYPLOIDY1, a conserved repressor of CYCLINA2 transcription, controls endoreduplication in Arabidopsis. Plant Cell 18, 2452-2468.

Yu, Y., Steinmetz, A., Peng, C.-Y., Yee, A. S. and Piwnica-Worms, H. (2003). The tobacco A-type Nicta;CYCA3;2, at the nexus of cell division and differentiation. Plant Cell 15, 2763-2777. 


\title{
Regulation of Arabidopsis Early Anther Development by the Mitogen-Activated Protein Kinases, MPK3 and MPK6, and the ERECTA and Related Receptor-Like Kinases
}

\author{
Carey L.H. Hord ${ }^{a, b, c, 2}$, Yu-Jin Sun ${ }^{a, d, 2}$, Lynn J. Pillitterie, Keiko U. Toriie, Huachun Wang ${ }^{\mathrm{f}, \mathrm{g}}$, Shuqun Zhang $^{\mathrm{g}}$ \\ and Hong $\mathrm{Ma}^{\mathrm{a}, \mathrm{b}, 1}$ \\ a Department of Biology and the Huck Institutes of the Life Sciences, Pennsylvania State University, University Park, PA 16802, USA \\ b The Intercollege Graduate Program in Plant Biology, Pennsylvania State University, University Park, PA 16802 USA \\ c Present address: 3015 Quinby Dr., Columbus, OH 43232, USA \\ d Present address: 318 Coker Hall (CB\# 3280), Department of Biology, University of North Carolina at Chapel Hill, Chapel Hill, NC 27599-3280, USA \\ e Department of Biology, University of Washington, Seattle, WA 98195, USA \\ f Present address: Monsanto Company, 700 Chesterfield Pkwy North, Mailzone GG4G, Chesterfield, MO 63017, USA \\ g Department of Biochemistry and Bond Life Sciences Center, University of Missouri, Columbia, MO 65211, USA
}

ABSTRACT Mitogen-activated protein kinase (MAPK) and leucine-rich repeat receptor-like kinase (LRR-RLK) signaling pathways have been shown to regulate diverse aspects of plant growth and development. In Arabidopsis, proper anther development relies on intercellular communication to coordinate cell proliferation and differentiation. Two closely related genes encoding MAPKs, MPK3 and MPK6, function redundantly in regulating stomatal patterning. Although the mpk6 mutant has reduced fertility, the function of MPK3 and MPK6 in anther development has not been characterized. Similarly, the ERECTA (ER), ERECTA-LIKE1 (ERL1) and ERL2 genes encoding LRR-RLKs function together to direct stomatal cell fate specification and the er-105 erl1-2 er/2-1 triple mutant is sterile. Because the mpk3 mpk6 double null mutant is embryo lethal, anther development was characterized in the viable mpk3/+ mpk6/- and er-105 erl1-2 erl2-1 mutants. We found that both mutant anthers usually fail to form one or more of the four anther lobes, with the er-105 erl1-2 erl2-1 triple mutant exhibiting more severe phenotypes than those of the $m p k 3 /+m p k 6 /-$ mutant. The somatic cell layers of the differentiated mutant lobes appeared larger and more disorganized than that of wild-type. In addition, the er-105 erl1-2 erl2-1 triple mutant has a reduced number of stamens, the majority of which possess completely undifferentiated or under-differentiated anthers. Furthermore, sometimes, the mpk3/+ mpk6/- mutant anthers do not dehisce, and the er-105 er/1-2 er/2-1 anthers were not observed to dehisce. Therefore, our results indicate that both ER/ERL1/ERL2 and MPK3/MPK6 play important roles in normal anther lobe formation and anther cell differentiation. The close functional relationship between these genes in other developmental processes and the similarities in anther developmental phenotypes of the two types of mutants reported here further suggest the possibility that these genes might also function in the same pathway to regulate anther cell division and differentiation.

\section{INTRODUCTION}

The coordination of cell proliferation and differentiation is essential for normal plant growth and fertility. The male reproductive organ, the stamen, is composed of a filament and an anther. The filament provides nutrients and structural support to the anther and elongates prior to and during dehiscence (pollen release). The anther has four lobes where pollen grains are produced. Anther development in Arabidopsis is divided into 14 stages based on anther histogenesis (Sanders et al., 1999). An epidermis encases the entire anther, and, within the locules, adjacent rings of cells are formed surrounding the sporogenous cells. Stage 5 is marked by the establishment

\footnotetext{
${ }^{1}$ To whom correspondence should be addressed. E-mail hxm16@psu.edu, fax 814-863-1357.

2 These authors contributed equally to this paper.

( $)$ The Author 2008. Published by the Molecular Plant Shanghai Editorial Office in association with Oxford University Press on behalf of CSPP and IPPE, SIBS, CAS. doi: $10.1093 / \mathrm{mp} / \mathrm{ssn} 029$
} 
of the five cell layers present within each lobe. From outer to inner, they are: epidermis, endothecium, middle layer, tapetum, and pollen mother cells (PMCs). Subsequent to stage 5, PMCs undergo meiosis to produce haploid microspores followed by two mitotic divisions to form pollen. In addition, the tapetum and middle layer degenerate and eventually undergo dehiscence to allow the release of mature pollen grains.

Stamen identity is conferred by the combined action of APETELA3 (AP3), PISTILLATA (PI), and AGAMOUS (AG), which regulate a subset of genes that promote anther development (Bowman et al., 1989, 1991; Ito et al., 2004). The SPOROCYTELESS/ NOZZLE (SPL/NZZ) gene encodes a putative transcription factor that acts very early in anther development and promotes microsporogenesis in the anther under the control of $A G$ (Schiefthaler et al., 1999; Yang et al., 1999; Ito et al., 2004). In addition, two other genes encoding CLAVATA1 (CLV1)related LRR-RLKs, BAM1 and BAM2, act redundantly in anther development to promote the somatic cell types (DeYoung et al., 2006; Hord et al., 2006). Several other genes were found to promote the differentiation of the tapetal cell type, including EXCESS MICROSPOROCYTES1IEXTRA SPOROGENOUS CELLS (EMS1/EXS), SOMATIC EMBRYOGENESIS 1 (SERK1), SERK2, and TAPETUM DETERMINANT1 (TPD1) (Canales et al., 2002; Zhao et al., 2002; Yang et al., 2003; Albrecht et al., 2005; Colcombet et al., 2005; Ma, 2005; Yang et al., 2005).

Normal tapetum development and degeneration are essential for proper pollen development (Mariani et al., 1990, 1991; Denis et al., 1993; Owen and Makaroff, 1995; Wu et al., 1997; Bih et al., 1999; Kapoor et al., 2002; Zheng et al., 2003). Some of the genes known to be important for tapetum development and function in Arabidopsis are MYB33 and MYB65, DYSFUNCTIONAL TAPETUM1 (DYT1), ABORTED MIRCROSPORES (AMS), ARABIDOPSIS THALIANA GLYCEROL-3-PHOSPHATE ACYLTRANSFERASE 1 (AtGPAT1), and MALE STERILE1 (MS1). The myb33 myb65 double mutant and the single mutants of the other genes are aberrant in tapetum development at different stages, but all result in partial or complete male sterility, underscoring the importance of the tapetum in pollen development.

Normal plant growth is dependant upon signal transduction pathways. Signaling pathways have been shown to involve mitogen-activated protein kinases (MAPKs) and/or leucine-rich repeat receptor-like kinases (LRR-RLKs). For example, the MAPKs MPK3 and MPK6 and the LRR-RLKs are involved in pathogen response and are essential for normal guard cell patterning and formation (Yang et al., 2001; Zhang and Klessig, 2001; Asai et al., 2002; Shpak et al., 2005; Wang et al., 2007). Interestingly, several of these signaling components have been shown to play roles in multiple developmental processes. The LRR-RLK SERK1, for example, was originally isolated for its ability to promote somatic embryogenesis (Schmidt et al., 1997; Hecht et al., 2001). In addition to a role in anther development, $B A M 1$ and $B A M 2$ work with $B A M 3$ to maintain meristem size and to promote proper development of leaf vasculature (DeYoung et al., 2006). The ERECTA (ER) gene has long been known to be important for plant height and was recently found to be involved in resistance to bacterial and fungal pathogens (Godiard et al., 2003; Llorente et al., 2005). ER has also been shown to function with ERECTA-LIKE1 (ERL1) and ERL2 to promote cell proliferation, control guard cell differentiation, and direct ovule development (Rédei, 1992; Torii et al., 1996; Shpak et al., 2004, 2005; Pillitteri et al., 2007). MPK3 and MPK6 have also been shown recently to regulate ovule development (Wang et al., 2008). In addition, it has recently been reported that MPK6 and the ER-family LRR-RLKs are involved in stamen development (Shpak et al., 2003, 2004; Bush and Krysan, 2007), although details are not clear.

In this study, we show that MPK3, MPK6, and the ER-family genes are important for cell differentiation during anther development in Arabidopsis. The majority of mpk3/+ mpk6/mutants and er-105 erl1-2 erl2-1 triple mutant stamens have anthers that do not form several normal cell types. When mutant anthers do form somatic and sporogenous cell types, the cells are larger and more disorganized than wild-type cells. Also, the tapetum and middle-layer cells may be delayed in their development, and the mutant anthers often fail to dehisce. Interestingly, the er-105 erl1-2 erl2-1 triple mutant appears to have a more severe phenotype than the $m p k 3 /+$ mpk6/- mutant. Together, these results suggest that MPK3, $M P K 6$, and the $E R$-family genes are key regulators of cell proliferation and differentiation during anther development.

\section{RESULTS}

\section{Anther Size and Male Fertility are Reduced in the mpk6-1 and $m p k 3 /+m p k 6 /-$ Mutants}

MPK3 and MPK6 are closely related homologs that act redundantly in several biological processes (Yang et al., 2001; Asai et al., 2002; Wang et al., 2007). Together, they are essential for plant development, as the mpk3 mpk6 double homozygous mutant is embryo lethal (Wang et al., 2007). The mpk6 mutant was previously reported to have reduced fertility and smaller anthers compared to wild-type, but a detailed histological analysis has not been completed (Bush and Krysan, 2007). In order to better understand the roles of MPK3 and MPK6 in male fertility, we examined plant growth and anther development in plants carrying a mutation in one or both of these genes (Figure 1). The mpk3 single mutant produced viable offspring and appeared similar to wild-type in overall plant growth and silique length (Figure 1A and 1B and data not shown). In line with previous results, the mpk6-1 mutant displayed both long wild-type-like siliques and shorter siliques and set many seeds (Figure $1 \mathrm{C}$ and data not shown) (Bush and Krysan, 2007). The mpk3/+ mpk6-1/plants did not produce wild-type-like long siliques, indicating that fertility was greatly reduced (Figure 1D). It was found that female fertility was severely impaired (Wang et al., 2008). Furthermore, where pollen grains were clearly visible on the anthers of wild-type, $m p k 3$, and $m p k 6-1$ open flowers, much less or no pollen could be seen on the anthers of $m p k 3 /+$ mpk6-1/- flowers (Figure 1E-1H) when plants were grown under long-day conditions (16 h light/8 h dark) and were about 


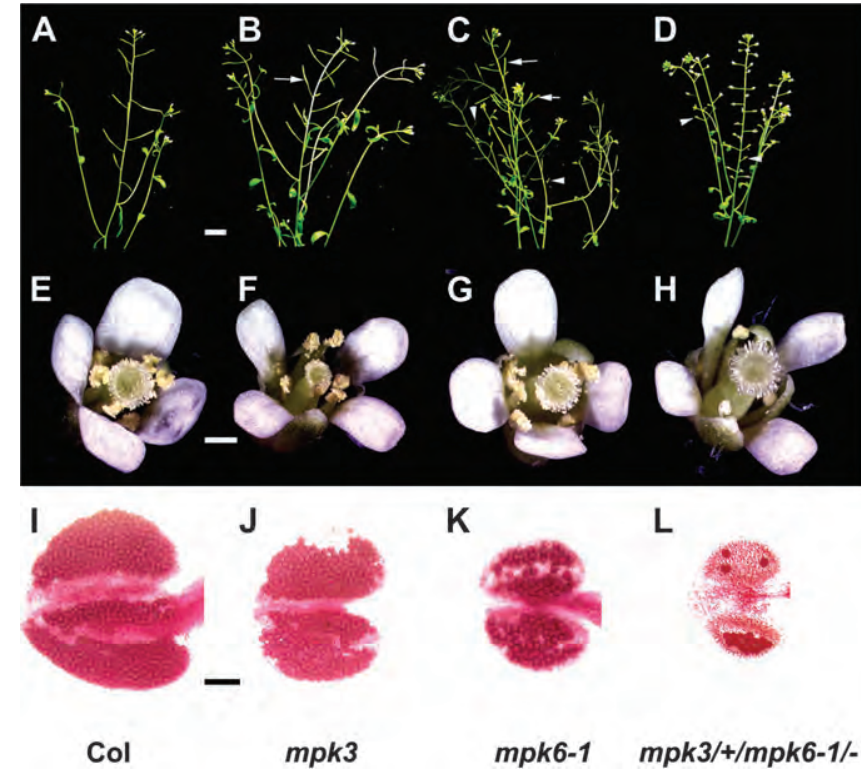

Figure 1. Phenotypes of Wild-Type (Col), $m p k 3, m p k 6-1$, and $m p k 3 /$ + mpk6-1/- mutants.

(A) A Col plant.

(B) An mpk3 plant with normal siliques (arrows).

(C) An mpk6-1 plant with many normal siliques (arrows) and some small siliques (arrowheads).

(D) An $m p k 3 /+m p k 6-1 /-$ plant with very small siliques (arrowheads).

(E) A Col flower.

(F) An mpk3 flower.

(G) An mpk6-1 flower.

(H) An mpk3/+ mpk6-1/- flower.

(I) A Col anther with pollen grains.

(J) An mpk3 anther with pollen grains.

(K) An mpk6-1 anther with fewer pollen grains.

(L) An $m p k 3 /+m p k 6-1 /-$ anther with only a few pollen grains.

Scale bars: $10 \mathrm{~mm}$ in (A)-(D); $500 \mu \mathrm{m}$ in (E)-(H); $20 \mu \mathrm{m}$ in (I)-(K).

half the size of wild-type. When $m p k 3 /+m p k 6-1 /-$ were grown under less stressful conditions, such as moderate light (80-100 $\mathrm{m}^{-2} \mathrm{~s}^{-1} ; 12 \mathrm{~h}$ light/12 $\mathrm{h}$ dark), they were about the same size as $m p k 6-1 /$ - plants and slightly smaller than wild-type (Wang et al., 2008) and produced less than a quarter of the normal amount of pollen (data not shown).

To determine whether the mpk3/+ mpk6-1/- and single mutants produce viable pollen, Alexander's staining was performed. While the mpk3 and mpk6-1 mutants produced viable pollen grains, the $m p k 3$ anthers were reduced in size, in both height and width, compared to wild-type (Figure $1 \mathrm{l}$ and $1 \mathrm{~J}$ ). The mpk6-1 anther produced less pollen and was smaller in size than both the mpk3 mutant and wild-type anthers (Figure $1 \mathrm{I}-1 \mathrm{~K}$ ). Furthermore, the mpk3/+ mpk6-1/- mutant anther was even smaller than either single mutant and produced very few pollen grains (Figure 1J-1L). No dead pollen was observed in any of the mutants examined, suggesting that the numbers of meiotic cells were reduced, but either no nonviable pollen was produced or they degenerated relatively quickly. The reduced anther size was further confirmed by scanning electron microscopy (SEM) (Figure 2). Both the $m p k 3 /+m p k 6-1 /-$ and $m p k 3 /+m p k 6-2 /-$ mutants produced anthers that were smaller than wild-type (Figure 2). SEM analysis also showed that the entire $m p k 3 /+m p k 6-1 /-$ flower was smaller overall than wild-type and the pistil was shorter and wider than wild-type.

\section{The $m p k 3 /+m p k 6-1 /-$ Mutant Anthers are Defective in Cell Differentiation}

In order to better understand the developmental defects of mpk3/+ mpk6-1/- anthers, semi-thin transverse sections of wild-type and mutant flowers were prepared and analyzed (Figure 3). At anther stage 2, wild-type anthers are roughly oval-shaped and the clusters of cells at the four corners begin to establish cell layers parallel to the epidermis (Figure $3 \mathrm{~A}$ ). During anther stages 2 and early 3, no significant difference was detected between wild-type and $m p k 3 /+m p k 6-1 /-$ mutant anthers (Figure $3 \mathrm{~A}$ and $3 \mathrm{E}$ and data not shown). At late anther stage 3 to stage 4, wild-type anthers form four distinct lobes and rings of cells became visible in each lobe (Figure $3 \mathrm{~B}$ ). At these stages, the $m p k 3 /+m p k 6-1 /-$ mutant displayed a range of anther phenotypes (Figure 3F). A few mutant anthers produced the four lobes but they appeared smaller than wild-type anthers, while the majority of mutant anthers remain relatively oval-shaped and appear highly vacuolated. Thus, the $m p k 3 /+m p k 6-1 /-$ anther developmental defect could be seen as early as anther stage 4 . At anther stages 5 and 6 , a transverse section of a wild-type anther is butterfly-shaped and each of the four lobes has four distinct, concentric layers of cells surrounding the pollen mother cells (Figure 3C and 3D). In contrast, a range of defects was observed at these stages in $m p k 3 /+m p k 6-1 /-$ anthers (Figure $3 \mathrm{G}$ and $3 \mathrm{H}$ ). Occasionally, an $m p k 3 /+m p k 6-1 /-$ anther formed all four lobes; however, usually, the $m p k 3 /+m p k 6-1 /-$ anthers appeared misshapen, with one or more lobes failing to form. In general, abaxial lobes appeared to develop more frequently than adaxial lobes. Although cell layers could sometimes be recognized in $\mathrm{mpk} 3 /+$ mpk6-1/- anthers, often, such layers were not well organized or were otherwise abnormal (Figure $3 \mathrm{~L}$ ). For example, the middle layer was often absent. In addition, presumptive tapetal cells and meiocytes/microspores were sometimes abnormally enlarged and irregularly shaped.

During stages 7 and 8 in wild-type anthers, microspores are released from tetrads, the tapetum becomes a thin ring of darkly staining cells, endothecium cells are vacuolated, and the middle layer is degenerated (Figure $3 \mathrm{I}$ and $3 \mathrm{~J}$ ). At these stages, $m p k 3 /+m p k 6-1 /-$ anther locules occasionally displayed similar cell patterning and structure to wild-type (Figure $3 \mathrm{~L}$ ). However, most of the cell types in mutant anthers appeared abnormal at stages 7 and 8 (Figure $3 \mathrm{~L}$ and $3 \mathrm{M}$ ). The $m p k 3 /+$ mpk6-1/- mutant tapetum did not form a thin and darkly stained ring around the microspores, but remained thick and disorganized (Figure $3 \mathrm{M}$ ). Interestingly, unlike $\mathrm{mpk3/+}$ $m p k 6-1 /-, m p k 3 /-m p k 6-1 /+$ anthers appeared to develop normal cell layers, similar to wild-type (Figure $3 \mathrm{~N}$ ). 

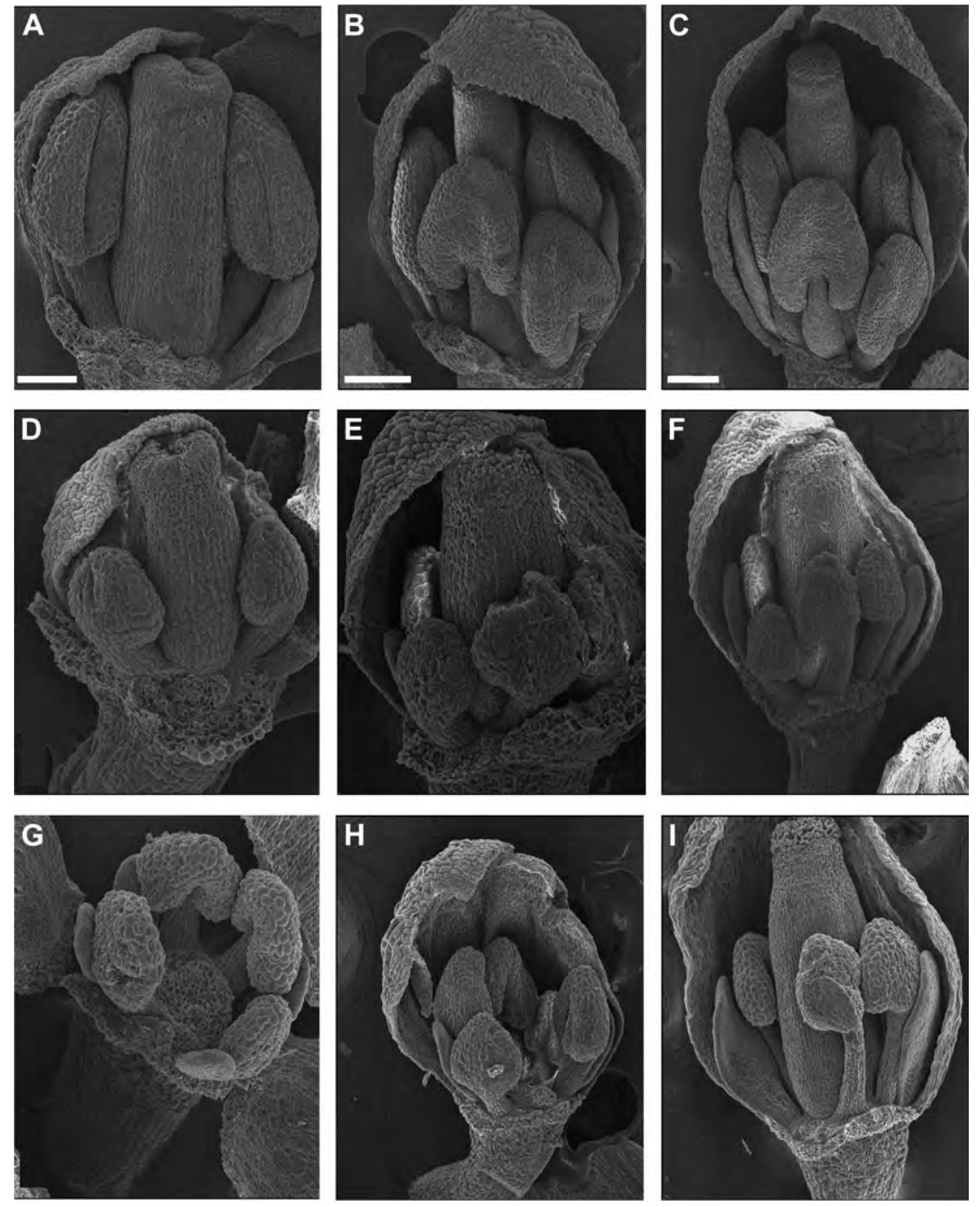

Figure 2. Scanning Electron Microscopy Images of Wild-Type (Col), mpk3/+ mpk6-1/-, and mpk3/+ mpk6-2/- floral buds.

(A-C) Wild-type (Col) floral buds.

(D-F) $m p k 3 /+m p k 6-1 /-$ floral buds.

(G-I) $m p k 3 /+m p k 6-2 /-$ floral buds.

$(A),(D)$, and $(G)$ : stage 9 floral buds with same magnification. (B), (E), and (H): stage 10 floral buds with same magnification. (C), (F), and (I): stage 11 floral buds with same magnification. Scale bars: $50 \mu \mathrm{m}$ in (A); $100 \mu \mathrm{m}$ in (B)-(C). Flower stages taken from Smyth et al. (1990).

\section{The $m p k 3 /+m p k 6-1 /-$ Mutant Inflorescence Displays Reduced Expression of Several Anther Genes}

In order to gain insights into the relationship between MPK3/ MPK6 and other genes important for anther development, we examined the expression of several genes (Figure 4). In our previous microarray analysis (Wijeratne et al., 2007), the expression levels of MPK3 and MPK6 were not significantly different in the $s p /$ or ems1 mutants compared to wild-type, and MPK6 was expressed at a slightly higher level than $M P K 3$ in anthers (Figure $4 A$ ). In addition, real-time PCR performed with RNA from wild-type and mutant inflorescences revealed that the expression of several genes important for anther development did not appear altered in mpk3/+ mpk6-1/- flowers (Figure 4B). No significant difference was observed between mutant and wild-type expression of TPD1, EMS1, and MYB33 (Figure 4B). However, the expression of $S P L$ and $A M S$ were significantly reduced in the $m p k 3 /+m p k 6-1 /-$ mutant flowers by about 50 and $75 \%$ that of wild-type, respectively (Figure 4B). Interestingly, the mpk6-1 mutant showed an increased expression of TPD1 and MYB33-two genes involved in tapetum development.

The er-105 erl1-2 erl2-1 Triple Mutant Displays a Range of Anther Defects and Reduced Number of Stamens

As previously described, the er-105 er/1-2 erl2-1 triple mutant forms stamen with anthers that do not differentiate normally 

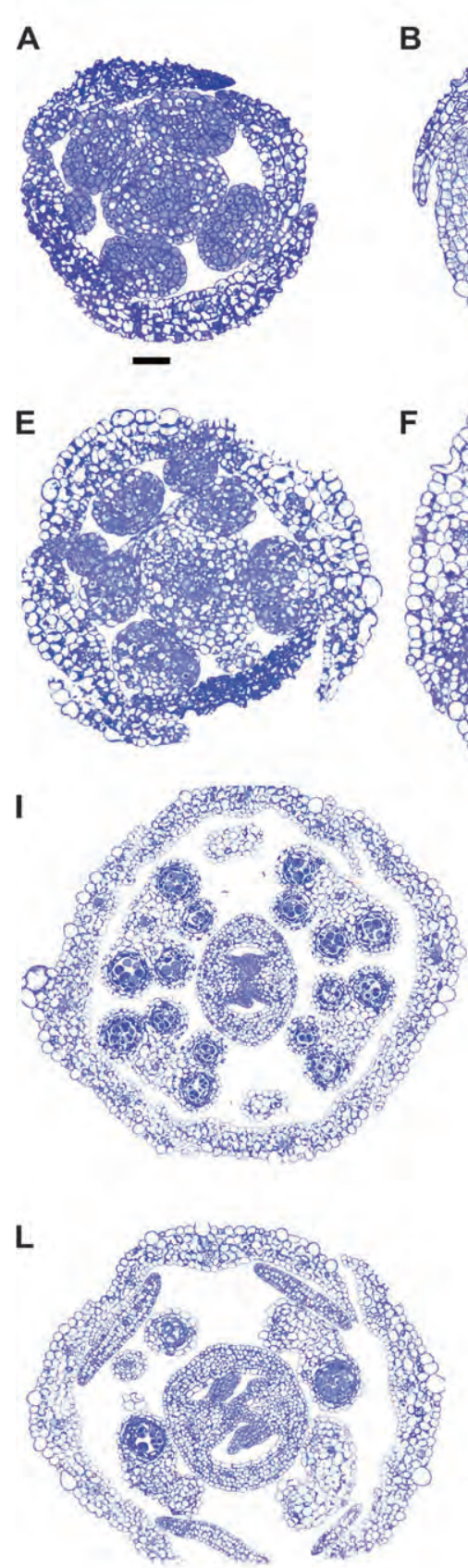
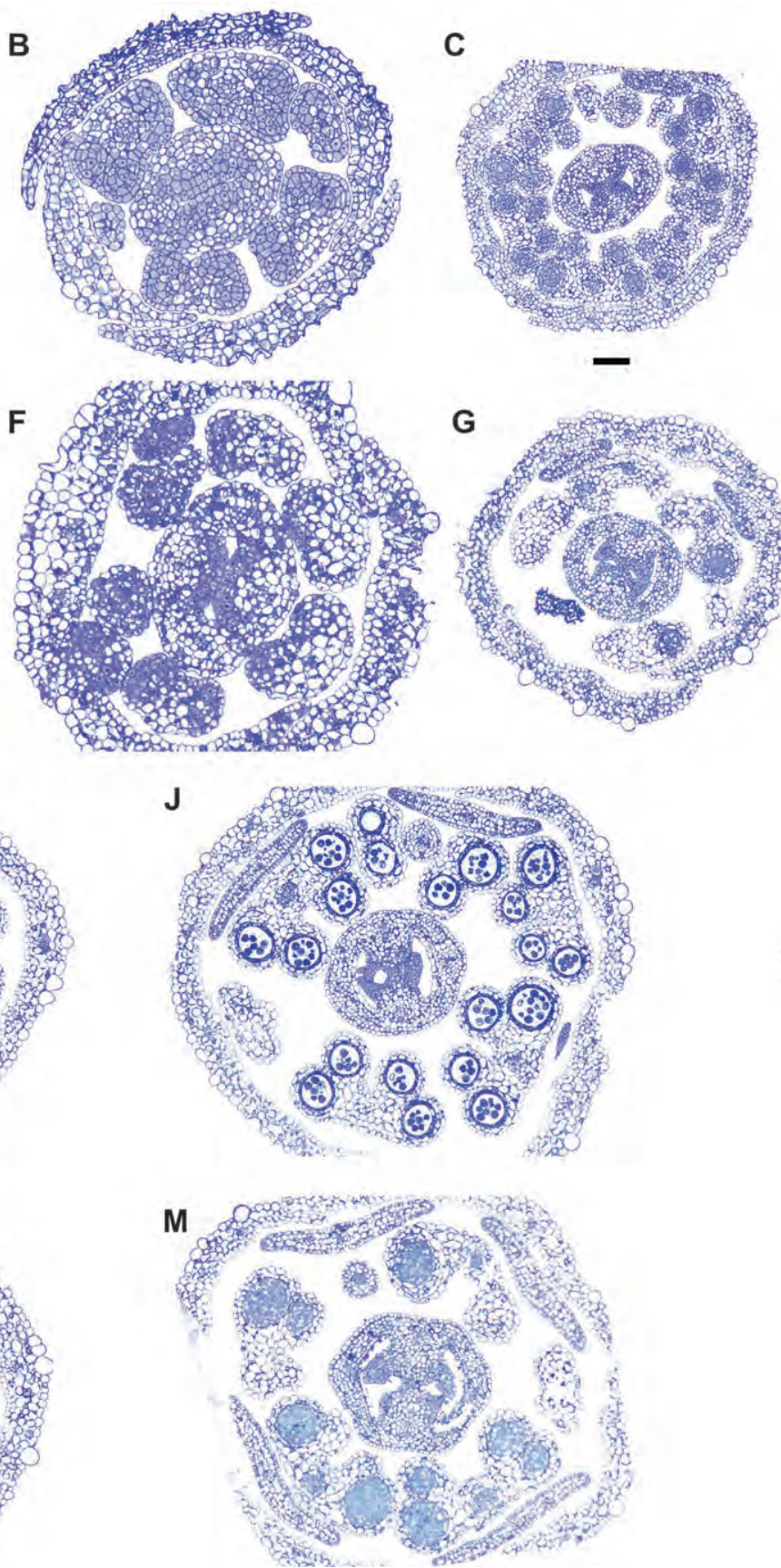
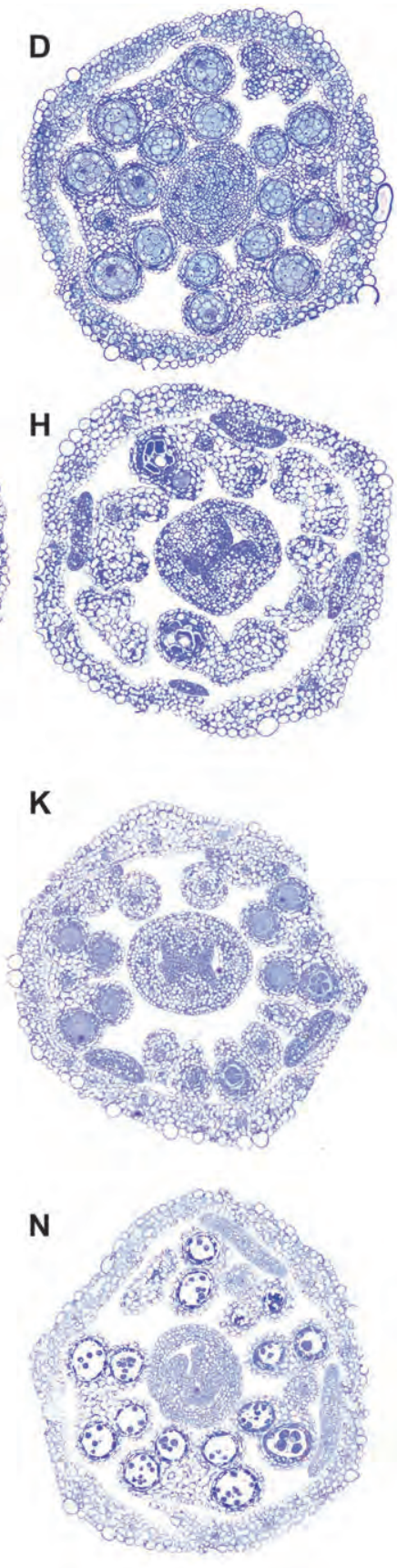

Figure 3. Anther Development from Stage 2 to Stage 8 in the Wild-Type (Col) and mpk3/+ mpk6-1/- mutant.

(A-D) and (I-J) Wild-type anther cross-sections.

(E-H) and (L-M) mpk3/+ mpk6-1/- anther cross-sections.

(K) A mpk6-1 anther cross-section.

(N) A $m p k 3 /-m p k 6-1 /+$ anther cross-section.

(A) and (E): most anthers were at stage 2. (B) and (F): most anthers were at late stage 3 to stage 4. (C), (G), and (K) are at anther stage 5. (D) and $(\mathrm{H})$ are at anther stage 6. (I), (L), and $(\mathrm{N})$ are at anther stage $7 .(\mathrm{J})$ and $(\mathrm{M})$ are at anther stage 8 . Scale bars: $20 \mu \mathrm{m}$ in $(\mathrm{A}) ; 40 \mu \mathrm{m}$ in $(\mathrm{C})$. (A), $(B),(E)$, and $(F)$ are at the same magnification. (C), (D), and (G)-(N) are at the same magnification.

(Shpak et al., 2004). To better understand the defect in anther and pollen development, Alexander's stain was used to determine pollen viability in wild-type and mutants (Figure 5). The er-105, erl1-2, erl2-1 single mutants, er-105 erl1-2, er-105 erl2-
1, erl1-2 erl2-1 double mutants, and er-105 erl1-2 (+/-) erl2-1 mutant had anthers that were comparable in size to wild-type and produced round, uniform pollen grains that were viable, similar to wild-type (Figure $5 \mathrm{~A}-5 \mathrm{H}$ ). 

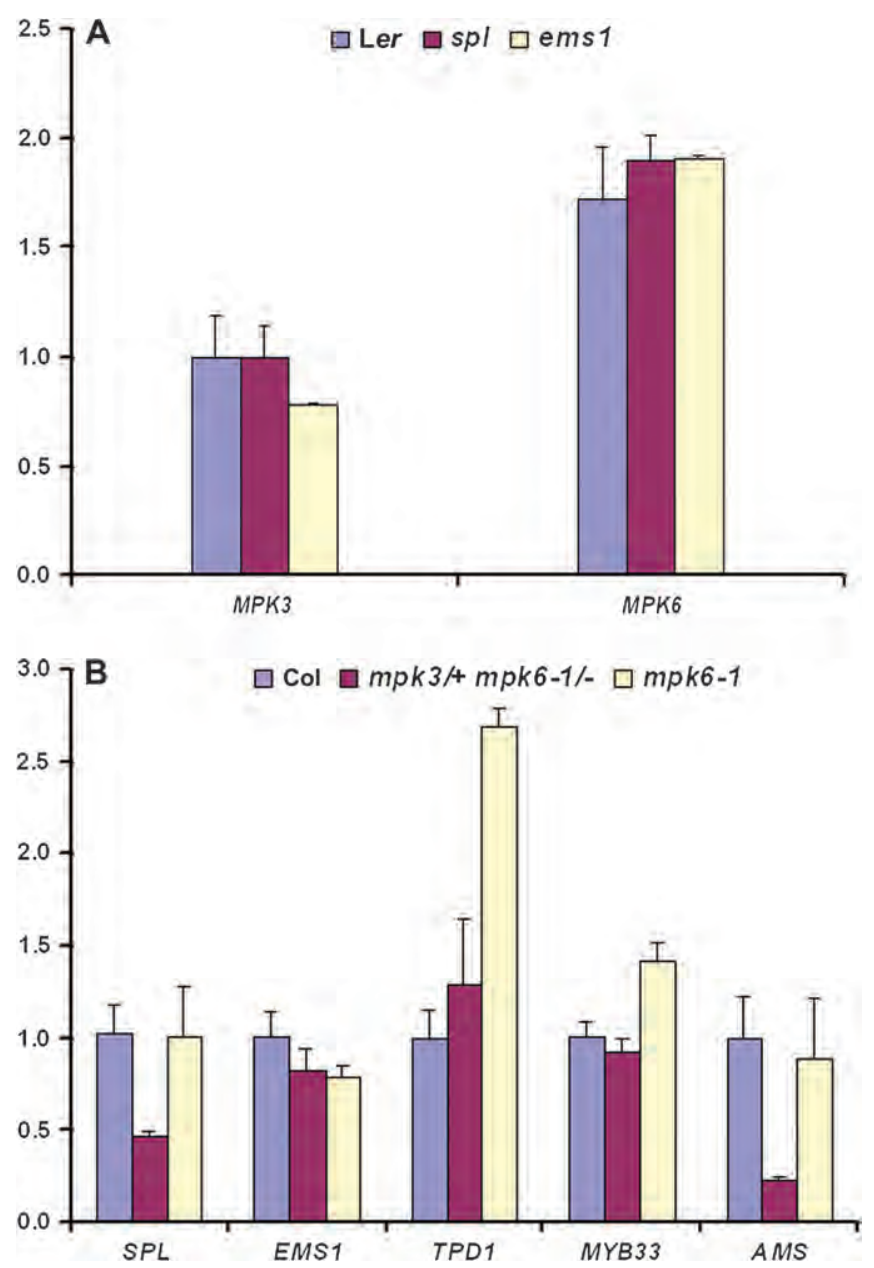

Figure 4. Comparison of Gene Expression.

(A) Expression levels of MPK3 and MPK6 in wild-type (Ler), spl, and ems1 anthers at stages 4-6.

(B) Real-time PCR results of selected anther genes in young inflorescences of wild-type (Col), mpk3/+ mpk6-1/- and mpk6-1.

Many of the er-105 erl1-2 erl2-1 flowers did not produce stamens (Shpak et al., 2004 and data not shown). In er-105 erl1-2 erl2-1 flowers that did produce stamens, the anther developmental defect varied in severity. The most severe defect observed was filament-like stamens that appeared to completely lack an anther (Figure $5 \mathrm{I}$ ). Some stamens had apparently undifferentiated anthers of variable sizes (Figure 5J). A few stamens produced viable pollen in one or two of their anther locules (Figure 5K and $5 \mathrm{~L}$ ). These anthers were larger than those that did not differentiate, but were still drastically reduced in size, mainly in height, but also in width, compared to wild-type.

Interestingly, the average number of anthers in the er-105 erl1-2 erl2-1 triple mutant was reduced and varied more compared to wild-type (3.06 \pm 1.18 vs $5.77 \pm 0.56$, respectively) (Figure 6A). Almost three-fourths of the stamens observed (69/95) had under-developed anthers, about one-tenth were filament-like (10/95), and less than one-fifth (16/95) produced pollen (Figure 6B). No dead pollen was ever observed in er-105 erl1-2 erl2-1 triple mutant anthers. Interestingly, pollen grains in the triple mutant appeared slightly smaller than wild-type (not shown). In addition, although they produced viable pollen, these anthers did not dehisce and pollen grains adhered to the anther wall when anthers were manually broken, indicating additional later defects.

\section{The er-105 erl1-2 erl2-1 Triple Mutant is Defective in Anther Formation and Differentiation}

To gain further insight into the development of anther cell layers, semi-thin transverse sections of wild-type and the ER-family mutants were analyzed (Figure 7 and Supplemental Figure 1). At anther stage 5, each wild-type locule had the five normal cell layers (Figure 7A). Similar to wild-type, the single, double and er-105 er/1-2 (+/-) erl2-1 mutants produced the five anther cell layers (Supplemental Figure 1). In general, these cell layers were similar in appearance to wild-type cell layers.

Consistent with the er-105 erl1-2 erl2-1 stamen phenotypes observed using the Alexander's stain, cross-sections of the triple mutant flowers showed stamens that were defective in anther development to varying degrees (Figure 7C-7F). Filament-like stamens appeared fairly round with a central vascular bundle (Figure 7E and 7F). Stamens that produced underdeveloped anthers varied in size and shape, but were overall oval or kidney-shaped (Figure 7C-7F). These anthers generally had a vascular bundle near the center surrounded by large, disorganized cells that were similar in appearance to connective tissue. When anther lobes with the sporophytic cell types were formed, they occupied only the abaxial positions; therefore, no more than two of the four locules were ever observed to form.

In general, when a locule of an er-105 erl1-2 erl2-1 anther was formed, the five cell layers could be seen, on the basis of position and overall appearance. The cells of the epidermis and endothecium appeared slightly larger in the triple mutant than in wild-type, but were otherwise fairly normal. The middle-layer cells appear adjacent to each other in wild-type (Figure $7 \mathrm{~A}$ and $7 \mathrm{~B}$ ). In contrast, the middle-layer cells appear to overlap with one another in the triple mutant (Figure $7 C$ and 7D). In addition, sometimes, two periclinally adjacent middle-layer cells were observed, indicating an additional cell division, whereas, other times, the middle-layer cells did not appear to completely surround the tapetum (Figure 7D). Furthermore, mutant middle-layer cells were also larger at stage 6 than in wild-type, suggesting that they persisted longer than normal (Figure 7F and 7G). The most striking cellular defect was seen in tapetal cells. In wild-type, the tapetum forms a single layer of rectangular cells surrounding the PMCs at stage 5 (Figure 7A), which become separated from each other and from meiocytes by a callose layer during stage 6 (Figure 7B and 7E). In the er-105 erl1-2 er/2-1 mutant, tapetal cells did not form a single layer, but appeared as a largely disorganized mass of cells that were greater in number compared to wild- 

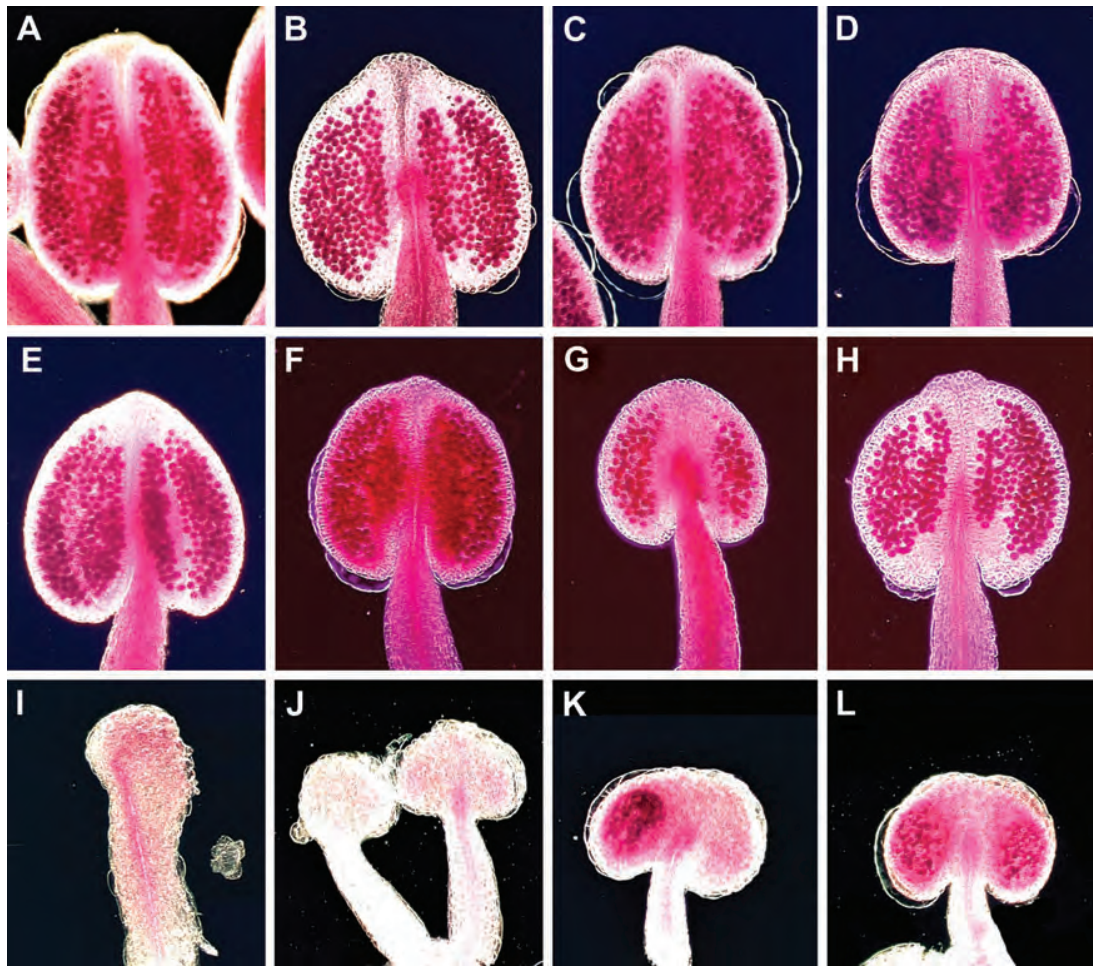

Figure 5. Alexander's Staining for Viable Pollen.

(A) Wild-type (Col).

(B) er-105.

(C) erl1-2.

(D) erl2-1.

(E) er-105 erl1-2.

(F) er-105 erl2-1.

(G) erl1-2 erl2-1.

(H) er-105 erl1-2 (+/-) erl2-1.

(I) er-105 er/1-2 erl2-1 filament-like stamen with no anther.

(J) er-105 erl1-2 erl2-1 stamens with underdeveloped anthers.

(K) er-105 er/1-2 er/2-1 anther showing one locule with viable pollen.

(L) er-105 erl1-2 erl2-1 anther possessing two locules with viable pollen. Images were taken at the same magnification.

type (Figure 7D, 7F, and 7G). In addition, the tapetum did not always completely surround the meiocytes, possibly due to the degradation of some of tapetal cells. Furthermore, the tapetal cells were greatly enlarged and abnormally shaped. They possessed large vacuoles and did not appear to separate from each other during stage 6. When PMCs had developed into microspores (stage 8), tapetal cells remained predominately attached to each other and to other somatic cells surrounding them and their degeneration appeared delayed (not shown).

In summary, the $E R$-family single, double, and er-105 erl1-2 (+/-) erl2-1 mutants produced viable pollen and formed five normal cell layers similar to wild-type. The er-105 erl1-2 erl2-1 triple mutant produced fewer stamens and was defective in anther development, with phenotypes varying from antherless stamens to anthers with no more than the two abaxial lobes with differentiated cell layers. In addition, cells of the triple mutant anthers appeared larger and more disorganized than wild-type. Tapetum development appeared partic- ularly affected, and tapetum and middle-layer cells may have been delayed in their development. Finally, although the triple mutant anthers sometimes produced viable pollen, they did not dehisce.

\section{DISCUSSION}

\section{ER/ERL1/ERL2 and MPK3/MPK6 Are Important for Anther Formation and Development}

Previous studies indicate that the ER/ERL1/ERL2 and MPK6 genes are involved in stamen development (Shpak et al., 2003, 2004; Bush and Krysan, 2007). Our results demonstrate that the ERIERL1/ERL2 and MPK3/MPK6 genes are important for normal anther development. Specifically, the ER/ERL1/ $E R L 2$ genes function together to regulate both anther lobe formation and anther cell differentiation. The overlapping expression of $E R, E R L 1$, and ERL2 during anther development (Supplemental Figure 2) and normal anther development in 

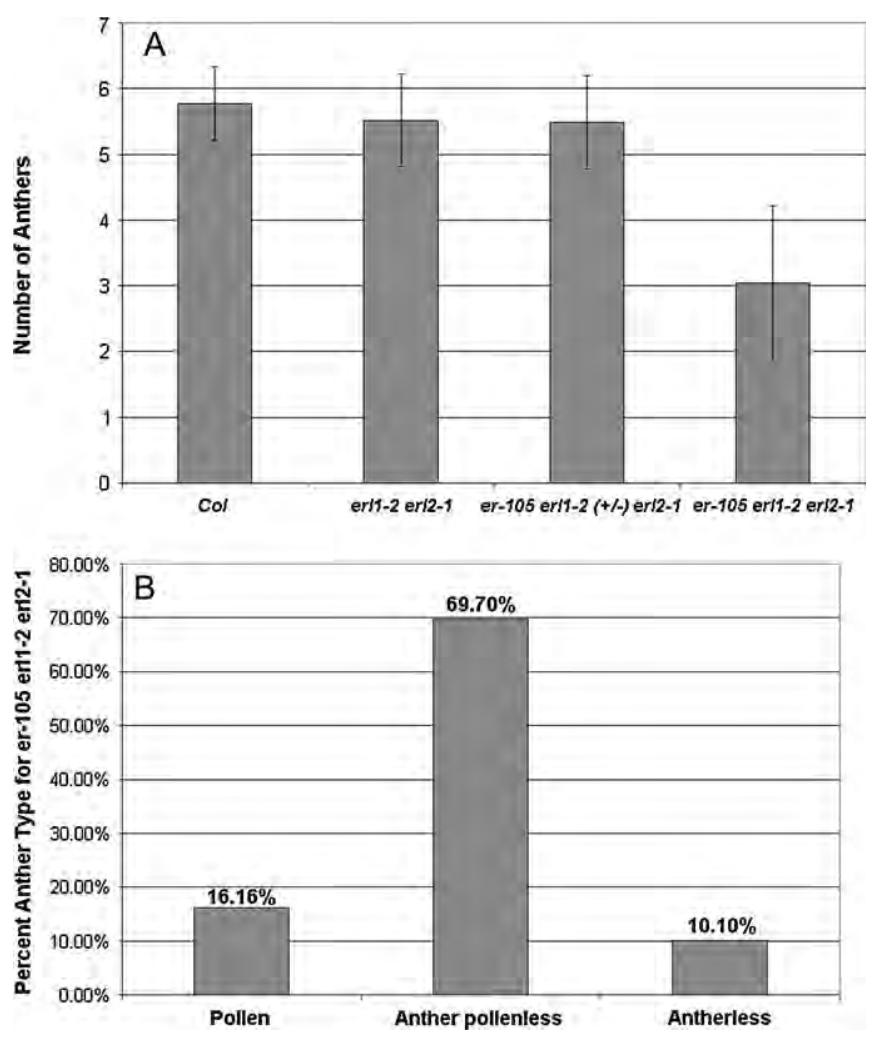

Figure 6. Average Number of Stamens and Anther Type.

(A) The average number of stamens \pm standard deviation in wildtype (Col) ( $n=78)$, erl1-2 erl2-1 ( $n=26)$, er-105 erl1-2 (+l-) erl2-1 ( $n=54)$, and er-105 erl1-2 erl2-1 mutants $(n=95)$.

(B) The percentage of anther type in the er-105 erl1-2 erl2-1 mutant.

the single, double and er-105 erl1-2 (+/-) erl2-1 mutants indicate that these genes act redundantly and that a single functional copy of ERL1 is sufficient for anther development. Interestingly, ERL2 was recently shown to be haploinsufficient for promoting normal ovule development in the absence of $E R$ and ERL1 (Pillitteri et al., 2007). Further study will be required to determine whether ERL2 is also haploinsufficient in directing anther development.

Similarly, our results of the $m p k 3 /+m p k 6-1 /-$ mutant indicate that these two genes together play crucial roles in anther lobe formation and anther cell differentiation. The ability of the $m p k 3 /-m p k 6-1 /+$ mutant to develop anther cell layers, similar to those in wild-type, suggests that one copy of MPK6 is sufficient to direct anther cell differentiation in the absence of MPK3. However, the mpk6-1 mutant sometimes failed to develop the normal cell layers and most of the mpk3/+ mpk6-1/anthers had an aberrant phenotype (Bush and Krysan, 2007, and this study). Therefore, MPK3 is not sufficient to consistently direct normal cell differentiation in the absence of MPK6. Furthermore, loss of one function copy of MPK3 in the mpk6-1 mutant greatly enhances the mutant phenotype. These observations indicate that the MPK3 and MPK6 genes have partially redundant functions, with MPK6 playing a more prominent role. MPK6 is expressed at a higher level than that of $M P K 3$, providing a possible explanation for the functional difference between these two genes. Because the mpk3 mpk6 double homozygous mutant is embryo lethal, it was not possible to determine the anther phenotypes of the double mutant; nevertheless, it is reasonable to speculate that the double mutant might have more severe anther development phenotypes than the $m p k 3 /+m p k 6-1 /-$ mutant. Because ER/ERL1/ $E R L 2$ and MPK3/6 are members of gene families that include more distant members, it is possible other family members may also have some overlapping functions.

\section{The ER/ERL1/ERL2 and MPK3/6 Functions in Anther Development Are Analogous to their Roles in Other Developmental Processes}

The ER/ERL1/ERL2 genes promote cell proliferation and control differentiation during plant development (Torii et al., 1996; Shpak et al., 2003, 2004, 2005). For example, the cortex of the er-105 er/1-2 erl2-1 mutant pedicel had somewhat larger, irregularly shaped cells that appeared disorganized and had gaps between them (Shpak et al., 2004). The triple mutant was severely reduced in longitudinal cell number, but not affected in the number of cell files (Shpak et al., 2004). In contrast, the number of guard cells seen in the er-105 erl1-2 erl2-1 mutant epidermis was dramatically increased, indicating a possible synergistic function of these genes in negatively regulating asymmetric cell division and guard mother cell differentiation (Shpak et al., 2005). Thus, the ER-family genes promote cell proliferation and regulate differentiation, linking the two to cell patterning and organ growth (Shpak et al., 2003, 2004, 2005).

Analogous roles can be seen for ER/ERL1/ERL2 in anther development. At very early stages of anther formation and development, $E R, E R L 1$, and $E R L 2$ redundantly promote the propagation of cells necessary for normal anther formation. The enlarged and disorganized cells observed in some of the er-105 erl1-2 erl2-1 triple mutant anthers are similar to the phenotype observed in the pedicel cortex. While disruption of the cell cycle can lead to increased cell size, this does not account for the aberrant cell patterning or increased number of tapetum cells (Urbani et al., 1995; Wang et al., 2000; De Veylder et al., 2001). The ER/ERL1/ERL2 genes may communicate signals necessary for normal tapetum cell division and differentiation, perhaps analogous to their role in stomatal cell specification. In anther locule formation, asymmetric cell divisions occur periclinally, while symmetric cell divisions occur longitudinally and anticlinally. Hence, anther cell differentiation is distinct from epidermal cell patterning in that symmetric cell divisions do not typically occur in the same plane as the asymmetric cell division events (Sanders et al., 1999; Scheres and Benfey, 1999; Geisler et al., 2000; Scott et al., 2004). Therefore, in the er-105 erl1-2 er/2-1 mutant, the lack of ERIERL1/ERL2 function leads to aberrant cell patterning and increased numbers of 
A

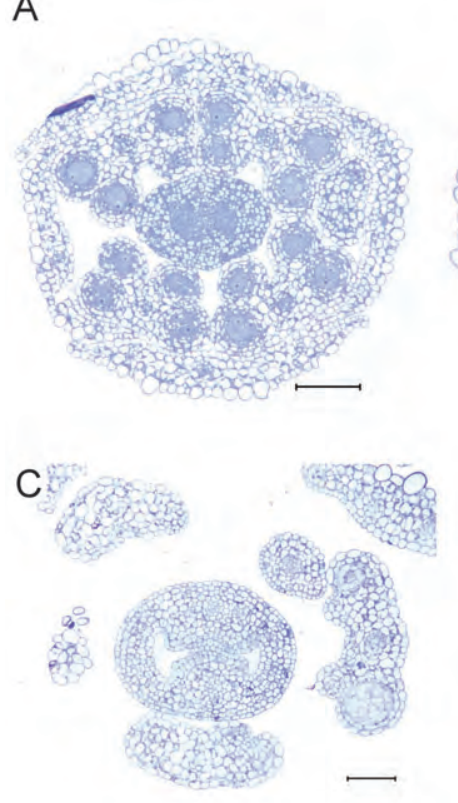

E

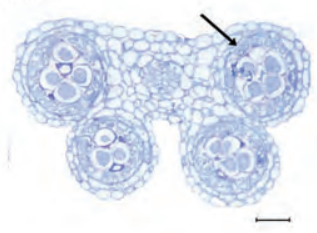

$\mathrm{F}$
B
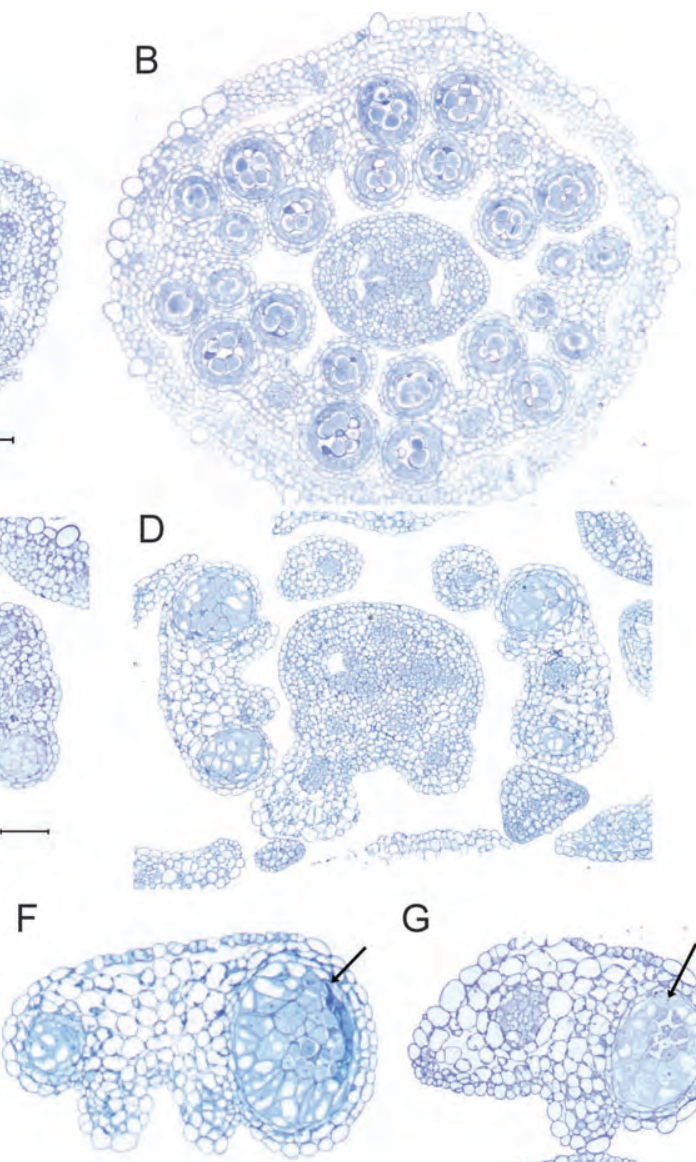

G

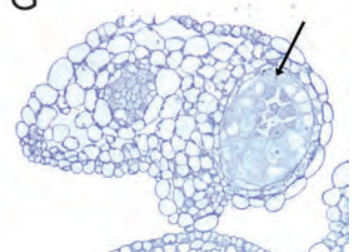

Figure 7. Semi-Thin Cross-sections of Flowers and Anthers.

(A) A wild-type (Col) bud at anther stage 5.

(B) A wild-type (Col) bud at anther stage 6.

(C) An er-105 erl1-2 erl2-1 bud with an anther at stage 5 .

(D) An er-105 erl1-2 erl2-1 bud with anthers at stage 6 .

(E) A wild-type anther at stage 6 .

(F) An er-105 er/1-2 erl2-1 anther at stage 6 with the two abaxial locules that have differentiated. The tapetum cells are highly vacuolated, enlarged and disorganized compared to wild-type in (E), and some of the tapetal cells may have been degraded. The other cell layers are larger than wild-type in (E).

(G) An er-105 er/1-2 erl2-1 anther at stage 6 with one differentiated abaxial locule. The tapetum cell layer is similar to (F), and the middle layer appears somewhat discontinuous. Arrows in (E)-(G) point to tapetal cells. Panels (A) and (B) are at the same magnification, (C) and (D) are at the same magnification, and $(E)-(G)$ are at the same magnification. Bar in $(A)$ and $(C)=50 \mu \mathrm{m}$. Bar in $(E)=20 \mu \mathrm{m}$.

tapetum cells and sometimes the middle-layer cells, suggesting a function of these genes in repressing periclinal cell division events and promoting longitudinal cell divisions in anther development, as well as contributing to normal cell patterning.

The phenotypes of the $m p k 3 /+m p k 6 /-$ mutant anthers suggest that the MPK3/6 genes are important for cell division in the very early stages of anther development to promote the formation of anther lobes. In addition, the later mutant defects in the differentiation of anther cell layers, particularly the tapetum, with enlarged cells suggest that the signal transduction process mediated by the MPK3/6 kinases is also impor- tant for the regulation of anther cell fates. Both ER/ERL and MPK3/MPK6 are required for proper anther development, and their loss of function led to similar anther phenotypes. The $E R / E R L$ and MPK3/6 genes have also been shown to have similar functions in other developmental processes (Bush and Krysan, 2007; Shpak et al., 2004, 2005; Pillitteri et al., 2007; Wang et al., 2007, 2008). Both the ER/ERL and MPK3/6 genes are key regulators of guard cell patterning (Shpak et al., 2005; Wang et al., 2007). Furthermore, the ER/ERL and MPK3/6 genes are important for ovule development in a haplosufficient manner (Pillitteri et al., 2007; Wang et al., 2008). Our study of anther development further extends the overlapping roles 
of ER/ERL and MPK3/MPK6 in a broad range of developmental processes.

\section{Possible Relationship between ER/ERL1/ERL2 and MPK3/6 and with Other Anther Genes}

ER/ERL1/ERL2 are LRR-RLKs that are thought to mediate signaling events at the cell surface (Torii et al., 1996; Shiu and Bleecker, 2003; Shpak et al., 2003, 2004, 2005; Pillitteri et al., 2007), whereas MPK3/6 are MAP kinases that are components of a signal transduction protein kinase cascade that include MAPKK (MAPK kinase) and MAPKKK (MAPKK kinase) as upstream regulators (Yang et al., 2001). As discussed above, ER/ERL1/ERL2 together are critical for several developmental processes, including cell proliferation, guard cell differentiation and female fertility (Torii et al., 1996; Shiu and Bleecker, 2003; Shpak et al., 2003, 2004, 2005; Pillitteri et al., 2007). In addition, MPK3/MPK6 have also been shown to regulate stomatal and ovule development, and function downstream of MKK4/MKK5, two functionally redundant MAPKKs, and YODA (a MAPKKK) (Bergmann et al., 2004; Wang et al., 2007, 2008). Both the er erl1 erl2 triple mutant and the mpk3/+ mpk6/mutant show somewhat similar abnormal anther phenotypes, including the failure to form one or more anther lobes and abnormal anther cell differentiation, suggesting that these two sets of genes regulate related aspects of anther development. Therefore, a reasonable hypothesis is that they act in the same pathway that is important for normal anther development.

The observation that both the er er/1 erl2 triple mutant and the $m p k 3 /+m p k 6 /-$ anthers often fail to form one or more anther lobes suggests these genes are important at very early stages of anther development. Detailed analysis of wild-type anther development indicates that archesporial cells are formed in the L2 layer of the anther primordia and they divide to form the four lobes (Sanders et al., 1999). Therefore, the failure to form an anther lobe could be due to either the absence or abnormal function of archesporial cells at one or more locations for future anther lobes, suggesting that the ERIERL1/ERL2 and MPK3/MPK6 genes either play a role in specifying the archesporial cell identity or in promoting the function of archesporial cells to generate the proper progeny cells. Previous studies indicate that the SPL and BAM1/2 genes are important for controlling the cell differentiation of archesporial cell progeny (Yang et al., 1999; Hord et al., 2006). In the spl and bam 1 bam 2 anthers, cell differentiation is dramatically altered, although a four-lobed structure can still be formed. In particular, the $s p /$ mutant fails to form L2 derived somatic cell types and sporogenous cells. We found that the SPL expression was approximately half of the normal level in the $m p k 3+/-$ mpk6/- mutant, whereas the MPK3 and MPK6 expression did not seem affected in the sp/ mutant. It is possible that $E R / E R L$ and MPK3/6 modulate the expression of SPL, although these genes are not required for SPL expression, consistent with the formation of some lobes in er erl1 erl2 and mpk3/6 mutants.
Among the anther cell differentiation defects we observed in this study, tapetal cells were disorganized with abnormally large vacuoles. Recently, it has been shown that the ems1/exs, serk1 serk2, and tpd1 mutants fail to produce the tapetum layer and form excess PMCs instead, indicating that these genes likely function in the same pathway to specify tapetum formation (Canales et al., 2002; Zhao et al., 2002; Yang et al., 2003; Albrecht et al., 2005; Colcombet et al., 2005). As the er105 erl1-2 erl2-1 and mpk3/+ mpk6/- anthers can still form the tapetum, the $E R / E R L$ and MPK3/6 genes probably act in a pathway distinct from that involving the EMS1/EXS, SERK1, SERK2, and TPD1 genes. This is further supported by the observation that MPK3/6 were expressed at near normal levels in the ems1 mutant and EMS1 and TPD1 expression was not reduced in the mpk3/+ mpk6/- mutant. In the myb33 myb65 and dyt1 mutants, the tapetum becomes highly vacuolated around anther stage 5 (Millar and Gubler, 2005; Zhang et al., 2006). Subsequently, the tapetal cells enlarge inward, leaving little space inside the locule, while the PMCs degenerate (Millar and Gubler, 2005; Zhang et al., 2006). However, neither of these phenotypes was observed in the er-105 erl1-2 erl2-1 mutant. Furthermore, none of the known loss-of-function Arabidopsis tapetum mutants resembles the er-105 erl1-2 erl2-1 mutant in abnormal tapetum patterning (Wilson et al., 2001; Canales et al., 2002; Ito and Shinozaki, 2002; Sorensen et al., 2002; Zhao et al., 2002; Steiner-Lange et al., 2003; Yang et al., 2003; Zheng et al., 2003; Albrecht et al., 2005; Colcombet et al., 2005; Millar and Gubler, 2005). Therefore, the ER/ERL1/ERL2 and $M P K 3 / 6$ genes define a new function in the regulation of anther development.

\section{Implication of the Variable Phenotypes in er erl1 erl2 and mpk3/+ mpk6/- mutants}

One striking feature shared by both the er erl1 erl 2 and $m p k 3 /+$ mpk6/- mutants is that the anther phenotypes are quite variable, ranging from anthers lacking any lobes to anthers with two fully formed lobes containing several cell types, unlike previously described mutants, such as spl, bam1 bam2, or ems1, which produce anthers that have rather consistent morphologies. As discussed above, the lack of some anther lobes is likely due to a defect in the formation or function of archesporial cells. However, the fact that some lobes are formed indicates that these genes are not essential for archesporial cell formation or function. It is possible that mutants still had some residual function due to the existence of one functional copy (MPK3) or even potential functionally overlapping paralogs in these families; such partial function might be near the threshold of the needed activity, and small fluctuations of gene function might be just enough for archesporial formation or function sometimes, but not other times.

Alternatively, the function(s) provided by the ER/ERL and MPK3/6 genes might not be essential but serve to modulate the activity of other essential genes, such as SPL. In this scenario, the ER/ERL-mediated signaling event and MPK3/6dependent signal transduction promote archesporial 
formation or function by enhancing the expression of SPL and/ or other key anther developmental genes. In particular, because the adaxial lobes normally develop slightly later than the abaxial ones and, in the mutant anthers, the adaxial lobes were more likely to be absent, perhaps ER/ERL and MPK mediate cell-cell communications that assist the formation of the adaxial lobes following the initiation of the abaxial lobes. In other words, the ER/ERL and MPK3/6 genes may increase the robustness of the regulatory machinery of early anther development, thereby ensuring proper cell division and differentiation. In the absence of the ER/ERL and MPK3/6 gene functions, the genes that directly control anther development, such as $S P L$, are vulnerable to factors that disrupt the normal activity of these genes. The fact that the ER/ERL and MPK genes likely mediate cell-cell communication suggests that proper coordination between cells is important for robust anther development. Such a mechanism of coordination might extend to other developmental processes, given that the ER/ERL and $M P K 3 / 6$ genes are also required for stomatal patterning and ovule development (Bush and Krysan, 2007; Shpak et al., 2004, 2005; Pillitteri et al., 2007; Wang et al., 2007, 2008).

A third possibility is that these genes are important for promoting anther development under variable environments, since ER and MPK3/6 are known to be environmentally responsive (Zhang and Klessig, 2001; Godiard et al., 2003; Llorente et al., 2005; Wang et al., 2007). It is possible that slightly different growth conditions can impact normal anther development; in wild-type, genes such as ER/ERL and MPK3/6 act to modulate the intrinsic anther developmental program to allow proper development in response to environmental changes. When these genes are mutated, response to environmental changes is hampered, resulting in developmental defects. Depending on the environment, the defects might be severe or mild. This type of speculation is supported by the observation that the $m p k 3 /+m p k 6 /-$ mutant can have milder phenotypes under some growth conditions, such as reduced light intensity and/or duration (data not shown). Because plants naturally experience variable growth conditions, genes that promote normal development under environmental stresses likely play important roles in ensuring developmental successes. The variable cellular defects in anther development of er/erl and mpk3/mpk6 mutants revealed by this study provide potentially valuable tools to unravel the RLK-MAP kinase signal transduction pathways that might be crucial for plant development in response to environmental changes.

It is known that the number of pollen-producing sacs (anther lobes in Arabidopsis) in a stamen can vary among related species. For example, species of Chloranthus (Chloranthaceae) have evolved to have twice as many pollen-producing sacs as species of Sarcandra, the closest relative of Chloranthus (Kong et al., 2002). In addition, when microsporogenesis was induced in petal-like organs of the Arabidopsis agamous mutant by AGAMOUS-independent SPL expression (Ito et al., 2004), often, two locules are formed, suggesting that two locules might be a more basal state. Because the er erl1 erl2 triple mutant and $m p k 3+/-m p k 6-/-$ anthers produced reduced number of locules, it is tempting to speculate that these genes and their counterparts in other plants might have also contributed to the evolution of the number of pollen sacs during angiosperm history.

\section{METHODS}

\section{Plant Material and Growth Conditions}

The mpk6-1 (CS31099), mpk6-2 (Salk_073907), and mpk3 (SALK_151594) T-DNA insertion mutants have been previously described (Liu and Zhang, 2004; Wang et al., 2007). The mpk6-1 mutant was originally in the $W s-O$ background and obtained from the Wisconsin Arabidopsis Knockout Facility, and the mpk6-2 mutant, Col background, was obtained from the Arabidopsis Biological Resource Center (ABRC). The mutant alleles used for ERECTA (At2g26330), ERECTA-LIKE1 (ERL1) (At5g62230), and ERL2 (At5g07180) were described previously (Torii et al., 1996; Shpak et al., 2004). Briefly, the er-105 allele was generated in the Columbia (Col) ecotype in the glabrous1 (g/1) mutant background by fast-neutron-irradiated seeds and contains a T-DNA insertion from an unknown source in the ERECTA promoter region (Lehle Seeds, Round Rock, TX, USA) (Torii et al., 1996). The erl1-2 and erl2-1 mutants carry T-DNA insertions in the LRR domain and were generated by the ABRC (Shpak et al., 2004). Col plants were used as the wild-type control and g/1 mutant as a background control for the er-105 mutant. Arabidopsis thaliana seeds were planted directly, or transplanted after germinating on MS plates, on potting mixture and were grown with a $16 \mathrm{~h}$ light $/ 8 \mathrm{~h}$ dark cycle at $18-23^{\circ} \mathrm{C}$.

\section{Expression of ERECTA, ERL1 and ERL2 in Arabidopsis Anthers}

The GUS expressing lines used were described previously (Shpak et al., 2004). GUS staining of floral tissue was performed essentially as described previously (http://www.its. caltech.edu/ plantlab), placing the tissue directly into $100 \%$ ethanol after staining instead of going through a graded ethanol series. Anthers and other floral organs were dissected and images were taken using a Nikon dissecting microscope (Nikon Corp., Tokyo, Japan) and an Optronics Digital camera (Optronics Inc., Goleta, CA, USA).

\section{Characterization of the Mutant Phenotype}

Pollen viability was determined by fixing flowers in Carnoy's fixative $(100 \%$ ethanol:Chloroform:Acetic Acid $=6: 3: 1)$ for at least $1 \mathrm{~h}$ and staining with Alexander's stain for at least $7 \mathrm{~h}$ at $55-73^{\circ} \mathrm{C}$ (Alexander, 1969). Flowers were then briefly washed with $10 \%$ glycerol and anthers were dissected from them. Images were taken using either a Nikon Eclipse E400 or E800 microscope (Nikon Corp.) with an Optronics Digital camera (Optronics Inc). Average anther number, standard deviations and percent anther type were calculated using 
Microsoft Excel (Seattle, WA, USA). Flower buds and inflorescences were prepared for sectioning by embedding in Spurr's resin as previously described (Owen and Makaroff, 1995; Zhao et al., 2002). Semi-thin $(0.5 \mu \mathrm{m})$ sections were made using an Ultracut UCT ultramicrotome (Leica Microsystems, Wetzlar, Germany) and were stained with either 0.05 or $0.1 \%$ of Toluidine Blue in 0.05 or $0.1 \% \mathrm{Na}_{2} \mathrm{~B}_{4} \mathrm{O}_{7}$ (respectively) for up to $1 \mathrm{~min}$. Images were photographed using an Olympus BX51 microscope (Tokyo, Japan) and a SPOT II RT Slider digital camera with SPOT software version 3.5.8 for Windows (Diagnostic Instruments, Inc., Sterling Heights, MI, USA). The SEM experiment was performed as described previously ( $\mathrm{Hu}$ and $\mathrm{Ma}$, 2006). Images were edited using PHOTOSHOP 7.0 (Adobe system Inc., San Jose, CA, USA).

\section{Expression Analysis}

A set of genes essential for anther development were selected for expression analysis by real-time PCR. The primers for realtime PCR are listed in Supplemental Table 1. Inflorescences at approximately floral stages 1 through 10 were collected and immediately frozen in liquid nitrogen. The total RNA extraction, cDNA synthesis, real-time PCR, and data analysis were performed as described previously (Ni et al., 2004; Zhang et al., 2006).

\section{SUPPLEMENTARY DATA}

Supplementary Data are available at www.mplant. oxfordjournals.org.

\section{FUNDING}

This work was supported by grants from the Department of Energy (DE-FG02-02ER15332) to H.M., from the Department of Energy (DE-FG02-03ER15448) to K.U.T., and from the National Science Foundation (IBN-0133220) to S.Z.

\section{ACKNOWLEDGMENTS}

We would like to thank Danielle Asquino for assistance in quantifying the mutant phenotype. We also express appreciation to Bridget Leyland, Gavilange Nestor, Danielle Asquino, and Jiong Wang for help with plant care, and Yi Hu for help with photography of GUS-stained flowers.

No conflict of interest declared.

\section{REFERENCES}

Albrecht, C., Russinova, E., Hechtm, V., Baaijens, E., and de Vries, S. (2005). The Arabidopsis thaliana SOMATIC EMBRYOGENESIS RECEPTOR-LIKE KINASES1 and 2 control male sporogenesis. Plant Cell. 17, 3337-3349.

Alexander, M.P. (1969). Differential staining of aborted and nonaborted pollen. Stain Technol. 44, 117-122.
Asai, T., Tena, G., Plotnikova, J., Willmann, M.R., Chiu, W.L., GomezGomez, L., Boller, T., Ausubel, F.M., and Sheen, J. (2002). MAP kinase signalling cascade in Arabidopsis innate immunity. Nature. 415, 977-983.

Bergmann, D.C., Lukowitz, W., and Somerville, C.R. (2004). Stomatal development and pattern controlled by a MAPKK kinase. Science. 304, 1494-1497.

Bih, F.Y., Wu, S.S., Ratnayake, C., Walling, L.L., Nothnagel, E.A., and Huang, A.H. (1999). The predominant protein on the surface of maize pollen is an endoxylanase synthesized by a tapetum mRNA with a long 5' leader. J. Biol. Chem. 274, 22884-22894.

Bowman, J.L., Smyth, D.R., and Meyerowitz, E.M. (1989). Genes directing flower development in Arabidopsis. Plant Cell. 1, 37-52.

Bowman, J.L., Smyth, D.R., and Meyerowitz, E.M. (1991). Genetic interactions among floral homeotic genes of Arabidopsis. Development. 112, 1-20.

Bush, S.M., and Krysan, P.J. (2007). Mutational evidence that the Arabidopsis MAP kinase MPK6 is involved in anther, inflorescence, and embryo development. J. Exp. Bot. 58, 2181-2191.

Canales, C., Bhatt, A.M., Scott, R., and Dickinson, H. (2002). EXS, a putative LRR receptor kinase, regulates male germline cell number and tapetal identity and promotes seed development in Arabidopsis. Curr. Biol. 12, 1718-1727.

Colcombet, J., Boisson-Dernier, A., Ros-Palau, R., Vera, C.E., and Schroeder, J.I. (2005). Arabidopsis SOMATIC EMBRYOGENESIS RECEPTOR KINASES1 and 2 are essential for tapetum development and microspore maturation. Plant Cell. 17, 3350-3361.

De Veylder, L., Beeckman, T., Beemster, G.T., Krols, L., Terras, F., Landrieu, I., van der Schueren, E., Maes, S., Naudts, M., and Inze, D. (2001). Functional analysis of cyclin-dependent kinase inhibitors of Arabidopsis. Plant Cell. 13, 1653-1668.

Denis, M., Delourme, R., Gourret, J.P., Mariani, C., and Renard, M. (1993). Expression of engineered nuclear male sterility in Brassica napus. Plant Physiol. 101, 1295-1304.

DeYoung, B.J., Bickle, K.L., Schrage, K.J., Muskett, P., Patel, K., and Clark, S.E. (2006). The CLAVATA1-related BAM1, BAM2 and BAM3 receptor kinase-like proteins are required for meristem function in Arabidopsis. Plant J. 45, 1-16.

Geisler, M., Nadeau, J., and Sack, F.D. (2000). Oriented asymmetric divisions that generate the stomatal spacing pattern in Arabidopsis are disrupted by the too many mouths mutation. Plant Cell. 12, 2075-2086.

Godiard, L., Sauviac, L., Torii, K.U., Grenon, O., Mangin, B., Grimsley, N.H., and Marco, Y. (2003). ERECTA, an LRR receptorlike kinase protein controlling development pleiotropically affects resistance to bacterial wilt. Plant J. 36, 353-365.

Hecht, V., Vielle-Calzada, J.P., Hartog, M.V., Schmidt, E.D., Boutilier, K., Grossniklaus, U., and de Vries, S.C. (2001). The Arabidopsis SOMATIC EMBRYOGENESIS RECEPTOR KINASE 1 gene is expressed in developing ovules and embryos and enhances embryogenic competence in culture. Plant Physiol. 127, 803-816.

Hord, C.L.H., Chen, C., DeYoung, B.J., Clark, S.E., and Ma, H. (2006). The BAM1/BAM2 receptor-like kinases are important regulators of Arabidopsis early anther development. Plant Cell. 18, 1667-1680. 
Hu, W., and Ma, H. (2006). Characterization of a novel putative zincfinger gene MIF1: involvement in multiple hormonal regulation of Arabidopsis development. Plant J. 45, 399-422.

Ito, T., and Shinozaki, K. (2002). The MALE STERILITY1 gene of Arabidopsis, encoding a nuclear protein with a PHD-finger motif, is expressed in tapetal cells and is required for pollen maturation. Plant Cell Physiol. 43, 1285-1292.

Ito, T., Wellmer, F., Yu, H., Das, P., Ito, N., Alves-Ferreira, M., Riechmann, J.L., and Meyerowitz, E.M. (2004). The homeotic protein AGAMOUS controls microsporogenesis by regulation of SPOROCYTELESS. Nature. 430, 356-360.

Kapoor, S., Kobayashi, A., and Takatsuji, H. (2002). Silencing of the tapetum-specific zinc finger gene TAZ1 causes premature degeneration of tapetum and pollen abortion in petunia. Plant Cell. 14, 2353-2367.

Kong, H.-Z., Lu, A.-M., and Endress, P.K. (2002). Floral organogenesis of Chloranthus sessilifolius, with special emphasis on the morphological nature of the androecium of Chloranthus (Chloranthaceae). Plant Syst. Evol. 232, 181-188.

Liu, Y., and Zhang, S. (2004). Phosphorylation of 1-Aminocyclopropane-1-Carboxylic Acid Synthase by MPK6, a stress-responsive mitogen-activated protein kinase, induces ethylene biosynthesis in Arabidopsis. Plant Cell. 16, 3386-3399.

Llorente, F., Alonso-Blanco, C., Sánchez-Rodriguez, C., Jorda, L., and Molina, A. (2005). ERECTA receptor-like kinase and heterotrimeric $\mathrm{G}$ protein from Arabidopsis are required for resistance to the necrotrophic fungus Plectosphaerella cucumerina. Plant J. 43, 165-180.

Ma, H. (2005). Molecular genetic analyses of microsporogenesis and microgametogenesis in flowering plants. Annu. Rev. Plant Biol. 56, 393-434.

Mariani, C., De Beuckeleer, M., Truettner, J., Leemans, J., and Goldberg, R.B. (1990). Induction of male sterility in plants by a chimaeric ribonuclease gene. Nature. 347, 737-741.

Mariani, C., Goldberg, R.B., and Leemans, J. (1991). Engineered male sterility in plants. Symp. Soc. Exp. Biol. 45, 271-279.

Millar, A.A., and Gubler, F. (2005). The Arabidopsis GAMYB-like genes, MYB33 and MYB65, are microRNA-regulated genes that redundantly facilitate anther development. Plant Cell. 17, 705-721.

Ni, W., Xie, D., Hobbie, L., Feng, B., Zhao, D., Akkara, J., and Ma, H. (2004). Regulation of flower development in Arabidopsis by SCF complexes. Plant Physiol. 134, 1574-1585.

Owen, H.A., and Makaroff, C.A. (1995). Ultrastructure of microsporogenesis and microgametogenesis in Arabidopsis thaliana (L.) Heynh. ecotype Wassilewskija (Brassicaciae). Protoplasma. 185, 7-21.

Pillitteri, L.J., Bemis, S.M., Shpak, E.D., and Torii, K.U. (2007). Haploinsufficiency after successive loss of signaling reveals a role for ERECTA-family genes in Arabidopsis ovule development. Development. 134, 3099-3109.

Rédei, J.P. (1992). A note on Columbia wild type and Landsberg erecta. In Methods in Arabidopsis Research, C. Konca, Chua, N.-H. and J. Schell, eds (Singapore: World Scientific), p. 3.

Sanders, P.M., Bui, A.Q., Weterings, K., Mclntire, K.N., Hsu, Y., Lee, P.Y., Truong, M.T., Beals, T.P., and Goldberg, R.B. (1999). An- ther development defects in Arabidopsis thaliana male-sterile mutants. Sex. Plant Reprod. 11, 297-322.

Scheres, B., and Benfey, P.N. (1999). Asymmetric cell division in plants. Annu. Rev. Plant Physiol. Plant Mol. Biol. 50, 505-537.

Schiefthaler, U., Balasubramanian, S., Sieber, P., Chevalier, D., Wisman, E., and Schneitz, K. (1999). Molecular analysis of NOZZLE, a gene involved in pattern formation and early sporogenesis during sex organ development in Arabidopsis thaliana. Proc. Natl Acad. Sci. U S A. 96, 11664-11669.

Schmidt, E.D., Guzzo, F., Toonen, M.A., and de Vries, S.C. (1997). A leucine-rich repeat containing receptor-like kinase marks somatic plant cells competent to form embryos. Development. 124, 2049-2062.

Scott, R.J., Spielman, M., and Dickinson, H.G. (2004). Stamen structure and function. Plant Cell. 16 Suppl, S46-S60.

Shiu, S.H., and Bleecker, A.B. (2003). Expansion of the receptor-like kinase/Pelle gene family and receptor-like proteins in Arabidopsis. Plant Physiol. 132, 530-543.

Shpak, E.D., Berthiaume, C.T., Hill, E.J., and Torii, K.U. (2004). Synergistic interaction of three ERECTA-family receptor-like kinases controls Arabidopsis organ growth and flower development by promoting cell proliferation. Development. 131, 1491-1501.

Shpak, E.D., Lakeman, M.B., and Torii, K.U. (2003). Dominantnegative receptor uncovers redundancy in the Arabidopsis ERECTA leucine-rich repeat receptor-like kinase signaling pathway that regulates organ shape. Plant Cell. 15, 10951100.

Shpak, E.D., McAbee, J.M., Pillitteri, L.J., and Torii, K.U. (2005). Stomatal patterning and differentiation by synergistic interactions of receptor kinases. Science. 309, 290-293.

Smyth, D.R., Bowman, J.L., and Meyerowitz, E.M. (1990). Early flower development in Arabidopsis. Plant Cell. 2, 755-767.

Sorensen, A., Guerineau, F., Canales-Holzeis, C., Dickinson, H.G., and Scott, R.J. (2002). A novel extinction screen in Arabidopsis thaliana identifies mutant plants defective in early microsporangial development. Plant J. 29, 581-594.

Steiner-Lange, S., Unte, U.S., Eckstein, L., Yang, C., Wilson, Z.A., Schmelzer, E., Dekker, K., and Saedler, H. (2003). Disruption of Arabidopsis thaliana MYB26 results in male sterility due to non-dehiscent anthers. Plant J. 34, 519-528.

Torii, K.U., Mitsukawa, N., Oosumi, T., Matsuura, Y., Yokoyama, R., Whittier, R.F., and Komeda, Y. (1996). The Arabidopsis ERECTA gene encodes a putative receptor protein kinase with extracellular leucine-rich repeats. Plant Cell. 8, 735-746.

Urbani, L., Sherwood, S.W., and Schimke, R.T. (1995). Dissociation of nuclear and cytoplasmic cell cycle progression by drugs employed in cell synchronization. Exp. Cell Res. 219, 159-168.

Wang, H., Liu, Y., Bruffett, K., Lee, J., Hause, G., Walker, J.C., and Zhang, S. (2008). Haplo-insufficiency of MPK3 in MPK6 mutant background uncovers a novel function of these two MAPKs in Arabidopsis ovule development. Plant Cell, 20, 602-613.

Wang, H., Ngwenyama, N., Liu, Y., Walker, J.C., and Zhang, S. (2007). Stomatal development and patterning are regulated by environmentally responsive mitogen-activated protein kinases in Arabidopsis. Plant Cell. 19, 63-73. 
Wang, H., Zhou, Y., Gilmer, S., Whitwill, S., and Fowke, L.C. (2000). Expression of the plant cyclin-dependent kinase inhibitor ICK1 affects cell division, plant growth and morphology. Plant J. 24, 613-623.

Wijeratne, A.J., Zhang, W., Sun, Y., Liu, W., Albert, R., Zheng, Z., Oppenheimer, D.G., Zhao, D., and Ma, H. (2007). Differential gene expression in Arabidopsis wild-type and mutant anthers: insights into anther cell differentiation and regulatory networks. Plant J. 52, 14-29.

Wilson, Z.A., Morroll, S.M., Dawson, J., Swarup, R., and Tighe, P.J. (2001). The Arabidopsis MALE STERILITY1 (MS1) gene is a transcriptional regulator of male gametogenesis, with homology to the PHD-finger family of transcription factors. Plant J. 28, 27-39.

Wu, S.S.H., Platt, K.A., Ratnayake, C., Wang, T.W., Ting, J.T., and Huang, A.H. (1997). Isolation and characterization of neutrallipid-containing organelles and globuli-filled plastids from Brassica napus tapetum. Proc. Natl Acad. Sci. U S A. 94, 12711-12716.

Yang, K.Y., Liu, Y., and Zhang, S. (2001). Activation of a mitogenactivated protein kinase pathway is involved in disease resistance in tobacco. Proc. Natl Acad. Sci. U S A. 98, 741-746.

Yang, S.L., Jiang, L.X., Puah, C.S., Xie, L.F., Zhang, X.Q., Chen, L.Q., Yang, W.C., and Ye, D. (2005). Overexpression of TAPETUM DETERMINANT1 alters the cell fates in the Arabidopsis carpel and tapetum via genetic interaction with EXCESS MICROSPOROCYTES1IEXTRA SPOROGENOUS CELLS. Plant Physiol. 139, 186-191.
Yang, S.L., Xie, L.F., Mao, H.Z., Puah, C.S., Yang, W.C., Jiang, L., Sundaresan, V., and Ye, D. (2003). TAPETUM DETERMINANT1 is required for cell specialization in the Arabidopsis anther. Plant Cell. 15, 2792-2804.

Yang, W.C., Ye, D., Xu, J., and Sundaresan, V. (1999). The SPOROCYTELESS gene of Arabidopsis is required for initiation of sporogenesis and encodes a novel nuclear protein. Genes Dev. 13, 2108-2117.

Zhang, S., and Klessig, D.F. (2001). MAPK cascades in plant defense signaling. Trends Plant Sci. 6, 520-527.

Zhang, W., Sun, Y., Timofejeva, L., Chen, C., Grossniklaus, U., and Ma, H. (2006). Regulation of Arabidopsis tapetum development and function by DYSFUNCTIONAL TAPETUM1 (DYT1) encoding a putative bHLH transcription factor. Development. 133, 3085-3095.

Zhao, D.Z., Wang, G.F., Speal, B., and Ma, H. (2002). The EXCESS MICROSPOROCYTES1 gene encodes a putative leucine-rich repeat receptor protein kinase that controls somatic and reproductive cell fates in the Arabidopsis anther. Genes Dev. 16, 2021-2031.

Zheng, Z., Xia, Q., Dauk, M., Shen, W., Selvaraj, G., and Zou, J. (2003). Arabidopsis AtGPAT1, a member of the membranebound glycerol-3-phosphate acyltransferase gene family, is essential for tapetum differentiation and male fertility. Plant Cell. 15, 1872-1887. 


\title{
Haploinsufficiency after successive loss of signaling reveals a role for ERECTA-family genes in Arabidopsis ovule development
}

\author{
Lynn Jo Pillitteri, Shannon M. Bemis, Elena D. Shpak* and Keiko U. Torii ${ }^{\dagger}$
}

The Arabidopsis genome contains three ERECTA-family genes, ERECTA (ER), ERECTA-LIKE 1 (ERL1) and ERL2 that encode leucinerich repeat receptor-like kinases. This gene family acts synergistically to coordinate cell proliferation and growth during aboveground organogenesis with the major player, $E R$, masking the loss-of-function phenotypes of the other two members. To uncover the specific developmental consequence and minimum threshold requirement for signaling, $E R$-family gene function was successively eliminated. We report here that $E R L 2$ is haploinsufficient for maintaining female fertility in the absence of $E R$ and $E R L 1$. Ovules of the haploinsufficient er-105 er/1-2 er/2-1/+ mutant exhibit abnormal development with reduced cell proliferation in the integuments and gametophyte abortion. Our analysis indicates that progression of integument growth requires ER-family signaling in a dosage-dependent manner and that transcriptional compensation among ER-family members occurs to maintain the required signaling threshold. The specific misregulation of cyclin A genes in the er-105 er/1-2 er/2-1/+ mutant suggests that downstream targets of the ER-signaling pathway might include these core cell-cycle regulators. Finally, genetic interaction of the $E R$ family and the WOX-family gene, PFS2, reveals their contribution to integument development through interrelated mechanisms.

KEY WORDS: Arabidopsis, Integument growth, Ovule, Receptor-like kinase, Haploinsufficient, Cell proliferation

\section{INTRODUCTION}

The final size and shape of plant organs are determined by developmental programs that coordinate cell proliferation, cell expansion and cell-type differentiation (Mizukami, 2001; Potter and $\mathrm{Xu}, 2001)$. The Arabidopsis ovule has a relatively simple structure and a specific, well-defined differentiation pattern, making it a useful model for understanding the regulation of growth and organogenesis. The ovule originates from the placenta as an elongate protrusion with a defined proximal/distal axis that can be separated into three regions. The distal portion, called the nucellus, is the site of megasporogenesis and embryo sac development. The proximal region differentiates into the funiculus or stalk, which attaches the ovule to the carpel wall. Two integuments initiate at the central chalazal region and eventually envelop the nucellus and form the seed coat. Similar to the process of leaf laminar expansion, initiation and expansion of the integument requires the juxtaposition of abaxial and adaxial factors such as those encoded by PHABULOSA (PHB), KANADI1 (KAN1), KAN2 and KAN3, ABERRANT TESTA SHAPE (also known as KAN4 - TAIR), and the YABBY-family member INNER NO OUTER (Eshed et al., 2001; Eshed et al., 2004; McAbee et al., 2006; Sieber et al., 2004; Villanueva et al., 1999). In addition, transcription factors such as AINTEGUMENTA and NOZZLE (also known as SPOROCYTELESS), and the mitochondria ribosomal protein HUELLENLOS, have been identified as necessary for integument initiation (Elliott et al., 1996; Schneitz et al., 1998; Skinner et al., 2004; Villanueva et al., 1999).

Department of Biology, University of Washington, Seattle WA 98195 USA.

*Present address: Department of Biochemistry and Cellular and Molecular Biology, University of Tennessee, Knoxville, TN 37996, USA

${ }^{\dagger}$ Author for correspondence (e-mail: ktorii@u.washington.edu)

Accepted 20 June 2007
A second group of loci affect the progression of integument growth after initiation. Several genes, including PRETTY FEW SEEDS 2 (PFS2), SHORT INTEGUMENTS 1 and 2 (SIN1 and 2) and TSO1, affect cell proliferation or expansion of the integuments, and their loss-of-function mutations result in reduction or loss of fertility (Hauser et al., 2000; Park et al., 2004; Park et al., 2005; Schneitz et al., 1997). Embryo sac failure is a secondary consequence of the absence of integuments; the gametophyte fails to develop in cases where the nucellus is not enclosed (Gasser et al., 1998). The cause of gametophyte abortion is not clear, but is likely to lie in the requirement for tight coordination of cell division and expansion within the ovule and communication between gametophytic and sporophytic tissue (Gasser et al., 1998). Relatively little is known about the genes involved in the coordination of growth or cell-cell communication within the ovule. This might be due to redundancy among the genes involved in this process, or to pleiotropic effects resulting from the loss of these genes. For instance, TSO1, TOUSLED (TSL) and SIN1 have multiple, sometimes detrimental, effects on vegetative and floral development in addition to ovule defects (Ehsan et al., 2004; Lang et al., 1994).

ERECTA $(E R)$ and its two paralogs, ERECTA-LIKE 1 (ERLI) and $E R L 2$, regulate organ shape and inflorescence architecture and are members of the leucine-rich repeat receptor-like kinase (LRR-RLK) gene family in Arabidopsis (Shiu and Bleecker, 2001; Torii, 2004; Torii et al., 1996). Based on phylogeny, it was suggested that ERL1 and $E R L 2$ evolved by recent gene duplication and are functionally related to $E R$, while maintaining overlapping but unique transcript expression patterns (Shpak et al., 2004). We report here that in the absence of functional ER and ERL1, Arabidopsis plants heterozygous at the ERL2 locus exhibit specific defects in integument development. Based on data from genetic and gene expression analysis, the ER family plays a key role in ovule development and fertility by regulating cell proliferation in the integuments. Our study highlights the unequal redundancy and dosage compensation among $E R$-family genes, and further reveals 
a potential molecular consequence of their reduced dosage for cell division control of integument development and fertility. Finally, genetic interactions of the ER family with PFS2, a WUSCHEL (WUS)-type homeodomain gene, revealed an unexpected intersection of two pathways required for proper integument growth and embryo sac development.

\section{MATERIALS AND METHODS}

\section{Plant material and growth conditions}

The Arabidopsis thaliana ecotype Columbia ( $\mathrm{Col}$ ) was used as wild type. All mutants are in the Col background unless otherwise indicated. The er105, erl1-2 and erl2-1 mutations were described previously (Shpak et al., 2004). The erl1-1 allele (YJ133) was identified as an enhancer-trapped line by Dr John Bowman (University of California, Davis, CA) and kindly provided as a gift. The line was originally in the Landsberg erecta (Ler) background and outcrossed into $\mathrm{Col}$ three times. The erl1-4 allele was isolated from ethyl methanesulfonate-mutagenized Arabidopsis er-105 erl2 seeds and outcrossed to Col. The $p f s 2-1$ allele was a gift from Dr Bernard Hauser (University of Florida, Gainesville, FL) and was in the Ler background and outcrossed to Col. The $p f s 2-1$ mutant was crossed with the er-105 erl1-2 (+/-) erl2-1 mutant for further analysis. All plants were grown under long-day conditions as described previously (Shpak et al., 2003).

\section{Genotyping}

PCR-based genotyping for er-105, erl1-2 and erl2-1 mutations was described previously (Shpak et al., 2004). Detection of the erl1-1 T-DNA insertion was performed with PCR primers: ERLK-185.rc, 5' CGTAGGTCTCCAATACGTGGA-3' and GUS R, 5'-CAGTTGCAACCACCTGTTGAT-3'. The erl1-4 mutation was detected by derived Cleaved Amplified Polymorphic sequences (dCAPs) using primers: erl14dcaps1151, 5'-GACGATGTTCACAACAGTGACTTGTGTTCTAG-3' and erl1-4dcaps1447.rc, 5'-CATCGAAATCAACAGAGAAAGAAAGGG$3^{\prime}$, and subsequent digestion with $\mathrm{XbaI}$ for 2 hours at $37^{\circ} \mathrm{C}$, which cuts the mutant erl1-4 allele sequence.

\section{In situ hybridization}

Tissue preparation and in situ hybridization were performed as described previously (McAbee et al., 2006). Probe template construction was performed as follows. For the ERL1 probe template, the ERL1 sense and antisense probes were produced by linearizing plasmid pLJP562 (contains ERL1 kinase domain) with BamHI or XhoI and transcribing in vitro with T3 or T7 RNA polymerase, respectively. For the $E R$ probe template, the $E R$ sense and antisense probes were synthesized by linearizing pLJP131 (contains the $E R$ kinase domain and partial 3'UTR) with XhoI or BamHI and transcribing in vitro with T3 or T7 RNA polymerase, respectively. The WUS sense and antisense probes were produced by linearizing pSMB106 (nt 627-816 of the $W U S$ cDNA) with EcoRI and $X b a \mathrm{I}$ and transcribing in vitro with T3 or T7 RNA polymerase, respectively. PFS2 probe template construction and hybridization conditions were according to Park et al. (Park et al., 2005). Sense and antisense probes for $P H B$ were produced by linearizing plasmid pPHB (a gift from Dr Kiyotaka Okada, Kyoto University, Kyoto, Japan) with SacI or KpnI and transcribing in vitro with T7 or T3 RNA polymerase, respectively.

\section{GUS histochemical analysis}

proERL1::GUS and proERL2::GUS constructs were described previously (Shpak et al., 2004). Histochemical staining for $\beta$-glucuronidase (GUS) activity was performed as described previously (Sessions et al., 1999).

\section{RNA extraction and quantitative real-time RT-PCR}

Total RNA was isolated from Arabidopsis stage 12 gynoecia (Schneitz et al., 1995) or from ovules isolated from stage 12 gynoecia using the RNeasy Plant Mini Kit (Qiagen, Valencia, CA) and treated with DNase I (Amp Grade, Invitrogen). First-strand cDNAs were generated by ThermoScript Reverse Transcriptase (Invitrogen) and random hexamers using $1.0 \mu \mathrm{g}$ of RNA according to manufacturer's instructions. Real-time PCR was performed with a Chromo4 thermocycler (BioRad, Hercules, CA) and analyzed using Opticon Monitor 3 software (BioRad). Triplicate reactions were performed in $25 \mu \mathrm{l}$ total volume containing iTaq SYBR Green mix
Table 1. Primers for RT-PCR and probe synthesis

Primer Sequence ( $5^{\prime}$ to $3^{\prime}$ )

\begin{tabular}{lll}
\hline \multicolumn{2}{l}{ Real-time RT-PCR primers } \\
\hline ERECTA & sbr ER F & \\
& sbr ER R & GACTGGACAACTTCACCTTCTTCG \\
ERL1 & sbr ERL1 F & GAACTGCTTCACTCTTGTCAATCTG \\
& sbr ERL1 R & CATACCTGGCTGGAGCAAAACG \\
ERL2 & ERL2 3085 & CTGTCTGGCAACAATTTCTCA \\
& sbr ERL2 R & AACACGTACAGATTGGCAAGAC \\
ACTIN & sbr actin F & ATGTCGCCATCCAAGCTGTTCTC \\
& sbr actin r & CTCCTTGATGTCTCTTACAATTTCC \\
PFS2 & pfs2 F & ATGGGCTACATCTCCAACAA \\
CYCLIN A2;2 & sbr PFS2 R & CTTCTAGCGTCGTGATCTGC \\
& sbr CYCA22 F & GAGCTAAAAAAGCCATGGGAA \\
CYCLIN A2;3 & Sbr CYCA22 R & CACCTGATCCATCTTCTTGTTGC \\
& sbr CYCA23 21 R & GTCTCGGCCATTCACTCG \\
& & TTTGGCTCAAGTATACCTG
\end{tabular}

RT-PCR primers

CYCLIN A2;1 CYCA21 153 GAAGCGTGTTGCTAGACCG

CYCA21 347.rC CTACCAAACCATCTTCTTC

CYCLIN A2:2 CYCA22 597 GCTAAAAAAGCCATGGGAAGA

CYCLIN A2;3

CYCA22 1103.rC TGCTCAGTAATTTCTTCATCTT

CYCA23 21 GTCTCGGCCATTCACTCG

CYCA23 359.RC CCTGCTGTATTTGATGCAAC

CYCA24 (-26) TTGGATCGTGTCAAAGGG

CYCA24 518.rC ACTTCTCTCAACTGTCCC

cdkb11610 CAATGGCCGGGTGTTTCC

cdkb11+69.rc TGTCGGAAAGAAAGATAG

cdkb12 615 CAATGGCCGGGTGTAATG

cdkb12 +68.rc GAGCAATAAACTTGACGG

cdkb21 (-40) AGAGAGAGAGAGCGCCG

cdkb21 352.rc GAATGTTCTTGCCAGTGCT

cdkb22 (-32) GAGAGAAGAGTTGTCTTG

cdkb22 358.rc GAATGTTCTGTCCAGCTTG

ER 2248

ER3016.rC

ERL1 2846

$\begin{array}{ll} & \text { ERL1 4411.rC } \\ \text { ERL2 } & \text { ERL2 } 3085\end{array}$

ERL2 4254.rC

ACTIN 2 act2-1

PFS2 $\quad$ pfs2 F

AAGAAGTCATTCAAAGATGTGA

AGAATTTCCAGGTTTGGAATCTGT

TATCCCACCGATACTTGGCA

CCGGAGAGATTGTTGAAGGA

CTGTCTGGCAACAATTTCTCA

AGCCATGTCCATGTGAAGAA

GCCATCCAAGCTGTTCTCTC

GCTCGTAGTCAACAGCAACAA

pfs2 $\mathrm{F}$ ATGGGCTACATCTCCAACAA

pfs2 R TCAGTTCTTCAGAGGCATGA

In situ hybridization probe primers

ERECTA ER 7040 GTAAAGATCTCGGTGTGG

ER 7467.rc GAAGACACATATTCACACC

(BioRad) and $1.0 \mu \mathrm{l}$ of the first-strand reaction. ACTIN 2 was used as an internal control in parallel reactions for each run. The relative level of expression of each target gene was calculated with respect to ACTIN 2 expression for each genotype. Real-time reactions were repeated in two biological replicates and the average fold-difference relative to wild type was calculated. Oligomers and DNA sequences used for PCR are shown in Table 1

\section{Microscopy}

Pictures of plants were taken with an Olympus SZX12 dissecting microscope attached to an Olympus C-2020Z digital camera (Center Valley, PA). Tissue fixation, plastic embedding, sectioning, light- and scanning electron microscopy were performed as described previously (Shpak et al., 2003). To examine ovules using differential interference contrast (DIC) optics, carpels were dissected from flowers and incubated in 9:1 (v:v) ethanol:acetic acid overnight, rinsed with water and placed in choral hydrate (1:8:1 v:v glycerol:chloral hydrate:water). Cleared ovules and in situ hybridization sections were viewed under DIC optics using an Olympus BX51 microscope equipped with a DP70 digital camera. 


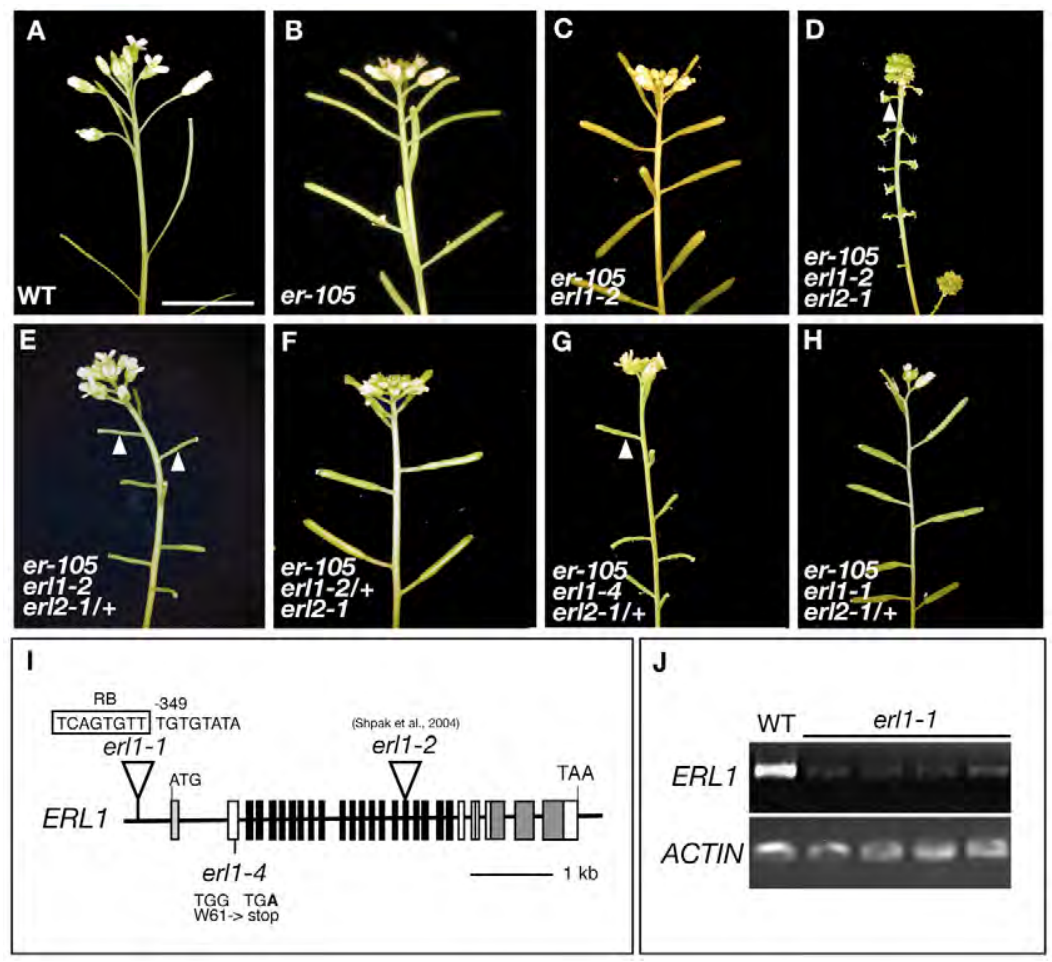

Fig. 1. Growth phenotype of wild-type and erfamily mutant Arabidopsis. (A-H) Wild-type and erfamily mutant inflorescence stems. (A) Wild type (WT); (B) er-105; (C) er-105 erl1-2; (D) er-105 erl1-2 erl2-1; (E) er-105 erl1-2 erl2-1/+; (F) er-105 erl1-2/+ erl2-1; (G) er105 er/1-4 erl2-1/+; and (H) er-105 er/1-1 er/2-1/+. Wild type and all combination mutants (A-C,E-F) except er105 erl1-2 erl2-1 triple mutant (D) maintain proper floral organ patterning and elongation. Among the combinations, er-105 erl1-2 erl2-1/+ (E), er-105 erl1-4 erl2-1/+ $(H)$ and the triple mutant $(D)$, are female sterile. Scale bar: $1 \mathrm{~cm}$. (I) Schematic of the ERL 1 gene with location of T-DNA insertions for er/1-1 and erl2-1, and the point mutation for erl1-4. Exons are indicated as vertical bars, introns as lines. The T-DNA right border (RB) is boxed. (J) RT-PCR analysis of four separate erl1-1 plants with reduced levels of ERL1 transcript compared with wild type. ACTIN 2 serves as a positive control.
Cell size and number measurements

Cell size was measured from images of plastic-embedded sections of wild-type and er erl1-2 erl2-1/+ carpels. The length and width of the first ten cells (nucellar to chalazal end) of the outer layer of the outer integument were measured using pixel distance in Photoshop Pro (Adobe Systems, San Jose, CA). Images used for these measurements corresponded to similar cross-sections through the nucellus of mature wild-type and er erl1-2 erl2-1/+ ovules. Cell numbers were counted from cleared ovules at stages 1-II, 2-IV, 3-II and 3-VI (Schneitz et al., 1995). At stages 2-IV, 3-II and 3-VI, counting started at the tip of the outer integument (nucellar end) and stopped where cells of the funiculus appeared obvious. At stage 1-II, all cells in the L1 layer of the ovule primordia were counted.

\section{RESULTS}

\section{$E R L 2$ is haploinsufficient for female fertility in the} absence of ER and ERL1

As reported previously, er, er erll and er erl2 null mutants developed compact but otherwise normal inflorescences, whereas the loss of all ER-family genes led to extremely dwarf and sterile plants with aberrant flowers disrupted in organ patterning and differentiation (Shpak et al., 2004) (Fig. 1A-D). To unravel the specific functions of $E R L 1$ and $E R L 2$ during inflorescence and floral organ growth, we investigated the phenotypes conferred by reduced dosages of ERL1 and ERL2 in the absence of ER. For this purpose, 100 self-fertilized progenies of er-105 erll-2/+ erl2-1/+, preselected for the presence of the erll-2 T-DNA insertion, were grown to maturity. This population segregated seven extremely dwarf and sterile plants, consistent with the expected $8.3 \%$ ratio for the er-105 erl1-2 erl $2-1$ triple mutant (dwarf plants/total $=7 / 100 ; \chi^{2}=0.221$, $P=0.637$ ). Seventeen additional plants from this population were completely sterile and produced no seeds. However, unlike the triple er-family mutants, they did not exhibit severe dwarfism, disrupted phyllotaxis or floral organ patterning defects. A reciprocal crosspollination with wild type revealed that these plants were female sterile (data not shown).
To determine the genetic basis for this novel phenotype, we next analyzed the genotypes of all 100 plants in this population. All seventeen female-sterile, non-dwarf plants were homozygous for $\mathrm{er}$ 105 and erl1-2 and heterozygous for erl2-1 (i.e. er-105 erl1-2 erl2$1 /+)$ (Fig. 1E). This was highly consistent with the expected ratio of $16.7 \%$ for this population $\left(\chi^{2}=0.006, P=0.936\right)$. Unlike $\mathrm{er}-105 \mathrm{erl} 1$ 2 erl $2-1 /+$, the $e r-105$ erll-2/+ erl2-1 plants were fully fertile (Fig. $1 \mathrm{~F})$. Reintroduction of functional copies of $E R, E R L 1$ and $E R L 2$ into respective loss-of-function mutants confirmed these data (see Table $\mathrm{S} 1$ in the supplementary material). The results indicate that, in the absence of $E R$ and $E R L 1, E R L 2$ is haploinsufficient for female fertility, whereas $E R L 1$ is haplosufficient in the absence of $E R$ and $E R L 2$. By contrast, the lack of defects in floral morphology (see Fig. $\mathrm{S} 1$ in the supplementary material) and phyllotaxis indicate that a single copy of ERL2 is sufficient for inflorescence elongation and floral patterning.

\section{Slight increase in ERL1 activity is sufficient to rescue ERL2 haploinsufficiency}

To further investigate the importance of ERLI function in female fertility, we generated mutant combinations with two additional erll alleles. The erll-1 allele carries a T-DNA insertion 349 bases upstream of the initiation codon and represents a weak allele (Fig. 1I,J). The erll-4 allele has a G-to-A substitution at nucleotide position +750 . This creates a stop codon at amino acid 61, leading to a premature truncation of the protein prior to the LRR domain (Fig. 1I). Therefore, erll-4 is a null allele.

The er-105 erl1-1 erl2-1, er-105 erll-2 erl2-1 and er-105 erl1-4 erl2-1 triple-mutant plants were almost indistinguishable from each other (data not shown). Similar to er-105 erl1-2 er $-1 /+$ plants (Fig. 1E), er-105 erll-4 erl2-1/+ plants were completely female sterile (Fig. 1G). By contrast, er-105 erl1-1 er2-1/+ plants were fertile and produced viable seed (Fig. $1 \mathrm{H})$. These results demonstrate that a slight increase in ERLI activity is sufficient to rescue the haploinsufficiency of ERL2. However, because erl1-1 failed to 

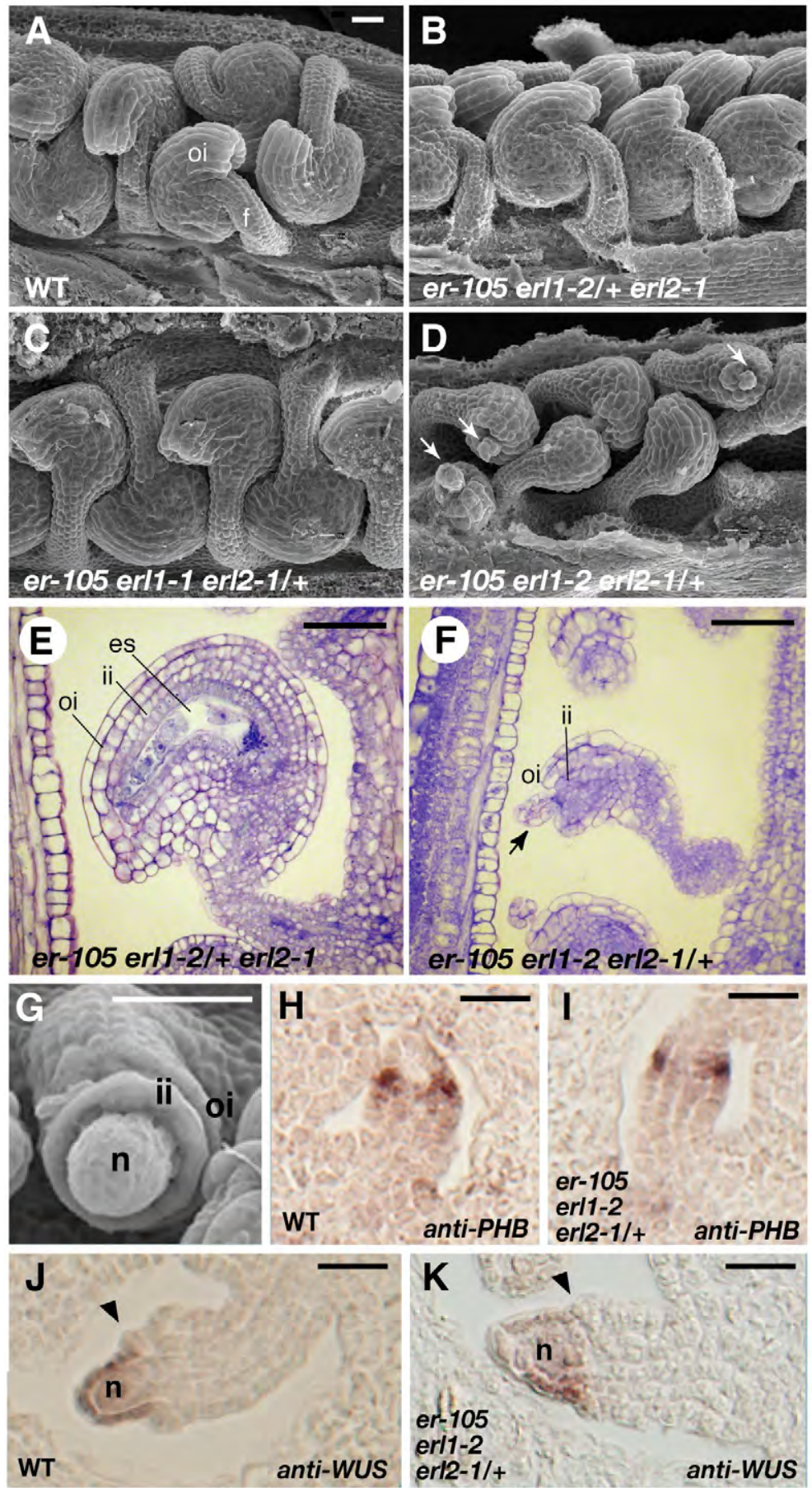

Fig. 2. Phenotypic effects of loss of er-family function on ovule development. (A-D) Scanning electron micrographs (SEMs) of Arabidopsis ovules at anthesis. (A) Wild type; (B) er-105 erl1-2/+ erl2-1; (C) er-105 erl1-1 erl2-1/+; (D) er-105 er/1-2 er/2-1/+. In er-105 er/1-2 er/2-1/+, both inner and outer integuments are shorter leaving the nucellus exposed (arrows). $(\mathbf{E}, \mathbf{F})$ Sagittal sections through the gametophyte of a er-105 er/1-2/+ erl2-1 (E) and er-105 erl1-2 erl2-1/+ (F) ovule at anthesis. In the er-105 erl1-2 er/2-1/+ mutant, the gametophyte has degenerated into a mass of small cells. (G) SEM of a developing er-105 er/1-2 erl2-1/+ stage 2-I ovule. Both the inner and outer integument initiate as smooth rings at the base of the nucellus, with the outer integument initiating asymmetrically as in wild type. (H-K) In situ hybridization for $(\mathrm{H}, \mathrm{I}) \mathrm{PHB}$ and $(\mathrm{J}, \mathrm{K})$ WUS expression. No difference in PHB or WUS expression is detected in mutant ovules, indicating normal regional specification. No signal was detected with PHB or WUS sense probe. f, funiculus; es, embryo sac; ii, inner integument; oi, outer integument; n, nucellus. Scale bars: $20 \mu \mathrm{m}$. promote organ growth, proper floral patterning or fertility in the absence of $E R$ and $E R L 2$, the results highlight a strict ER-family signaling threshold required to promote female fertility.

\section{Haploinsufficiency of ERL2 confers aberrant ovule growth and abortion of embryo sac development}

To gain insight into the developmental basis of er-105 erll-2 erl2-1/+ female sterility, we examined the morphology of mature ovules (stage 13 flowers, anthesis) (Smyth et al., 1990) (Fig. 2). Similar to wild type, er-105 erl1-2/+ erl2-1 exhibited typical amphitropic ovule morphology (Fig. 2A,B,E). Ovules of er-105 erl1-1 erl2$1 /+$ were fertile, but had slightly shorter integuments than wild type, resulting in the micropyle resting slightly away from the funiculus (Fig. 2C). By contrast, the mature ovules of er-105 erll-2 erl2-1/+ displayed aberrant morphology. Growth of both inner and outer integuments as well as the funiculus was dramatically compromised, leading to small, stunted ovules that often had an exposed nucellus (Fig. 2D,F). The embryo sac of mature er-105 erl1-2 erl2-1/+ ovules was absent and replaced by a disorganized mass of small cells (Fig. 2F).

Initiation and polarity of integuments was not disrupted in er-105 erll-2 erl2-1/+ (Fig. 2G). To determine whether integument initiation was defined normally, we used WUS and $P H B$ as a nucellar regional marker and adaxial marker, respectively (Fig. 2H-K). WUS is expressed exclusively in the nucellus of wild-type ovules and promotes integument initiation in the chalazal region proximal to its expression domain (Groß-Hardt et al., 2002), whereas PHB expression marks the inner integument (Sieber et al., 2004). No alteration in $W U S$ or $P H B$ expression was detected between wild type and er-105 erll-2 erl2-1/+. These data suggest that loss of ER-family signaling does not disrupt regional domain specification or radial patterning.

\section{Integument outgrowth is compromised in er-105 er/1-2 er/2-1/+ mutants}

To determine the onset of developmental defects, wildtype and er-105 erll-2 erl2-1/+ ovules were examined at sequential developmental stages (Fig. 3) (Schneitz et al., 1995). Early ovule initiation and patterning were normal in er-105 erll-2 erl2-1/+, consistent with the normal $P H B$ and $W U S$ expression patterns (Fig. 2I,K). Similar to wild type, ovule primordia initiated and elongated from the placental wall (stage 1-II; Fig. $3 \mathrm{~A}, \mathrm{E}, \mathrm{I})$. At stage 2-II to 2-V, outer and inner integument primordia arose from the chalaza and grew toward the nucellus (Fig. 3B,F,J). However, starting at stage 3-I, the cells of er-105 erll-2 erl2-1/+ outer integuments had a more disorganized appearance than wild type (Fig. $3 \mathrm{C}, \mathrm{G}, \mathrm{K})$. Enlargement of the megaspore mother cell (MMC) and tetrad formation proceeded as in wild type within the nucellus of er-105 erl1-2 erl2-1/+ ovules (Fig. 3I,M). However, mutant ovules arrested at a twonucleate embryo sac (Fig. 3N). We never observed er105 erl1-2 erl2-1/+ embryo sacs beyond this twonucleate stage. Based on our observations, we conclude that progression of integument growth in the absence of 

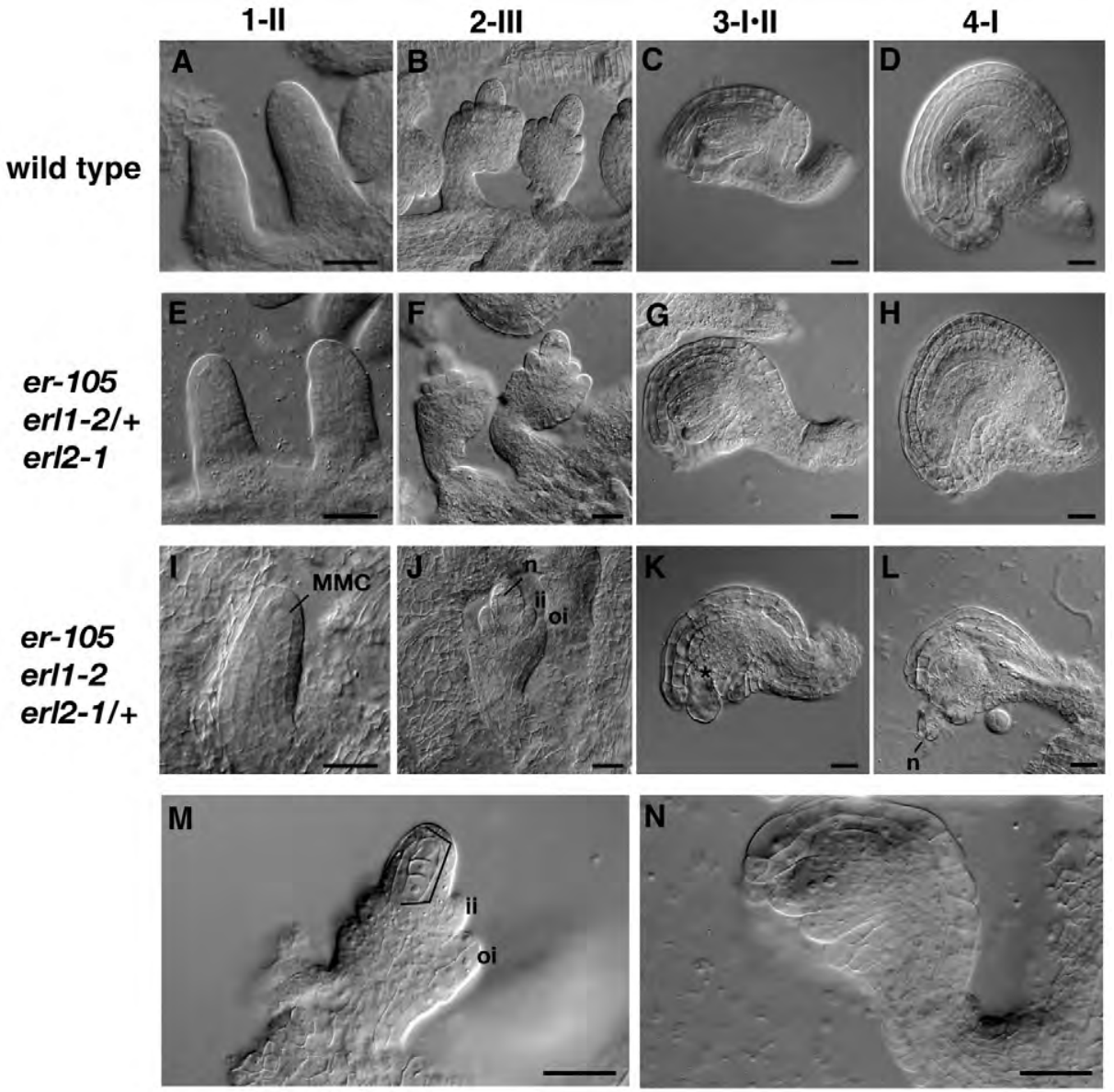

Fig. 3. Developmental series of ovule development in wild-type, er-105 erl12/+ erl2-1 and er-105 erl1-2 erl2-1/+

Arabidopsis. (A-L) DIC images of ovules at stages: 1-II $(A, E, I) ; 2-I I I(B, F, J) ; 3-I I I(C, G, K)$ and $4-I(D, H, L)$. Differences in development are seen at stage 3-I-II (C, G,K). Asymmetric growth of the outer integument occurs in all genotypes; however, er-105 erl1-2 erl2$1 /+$ integuments appear less organized and gametophyte development has ceased (K). By anthesis $(D, H, L)$, the integuments of er105 er/1-2 er/2-1/+ are short resulting in the nucellus protruding from the integuments (L). By contrast, the er-105 erl1-2/+ er/2-1 ovule has nearly normal appearance, with a very subtle phenotype (H). (M,N) er-105 erl1-2 erl2-1/+ ovule at stage 2-V with tetrad ( $M$, bracket) and stage 3-II (N) showing two-nuclear gametophyte. MMC, megaspore mother cell; n, nucleus; ii, inner integument; oi, outer integument. Scale bars: $20 \mu \mathrm{m}$.
$E R$ is dependent on ERL1 and 2 in a dosage-dependent manner. At a minimum, ERL1 in a heterozygous state or ERL2 in a homozygous state is required.

\section{Reduced cell numbers in er-105 erl1-2 erl2-1/+ mutants lead to reduced integument size}

Change in organ size reflects an alteration in cell size, cell number, or both. To understand the mechanism underlying the phenotype of er-105 erl1-2 erl2-1/+ ovules, we examined the size and number of cells in developing ovule integuments and compared them with wild type. In wild type, integument cells at the micropylar end were much more elongated than those at the chalazal end (Fig. 2E). The er erll erl2/+ ovules appeared to lack this characteristic polar elongation of integument cells (Fig. 2F). This made the outer integument cells of er-105 erll-2 erl2-1/+ ovules less organized and slightly more uniform in length than wild type. However, no significant difference in outer integument cell size area was found between wild-type and mutant ovules (Fig. 4A-C).

We next assessed the possibility of endoreduplication in mutant integument cells by observing the nuclear size using 4',6-diamidino2-phenylindole (DAPI). Endoreduplication is the repetitive duplication of chromosomal DNA without mitosis or cytokinesis, resulting in an increase in cell ploidy level (Kondorosi et al., 2000). In plants, a positive correlation between ploidy level and cell size has been observed (Kondorosi et al., 2000; Melaragno et al., 1993). No qualitative difference in the size (area) of nuclei between wild-type and er-105 erl1-2 erl2-1/+ outer integument cells was observed (see Fig. S2 in the supplementary material), consistent with the absence of a cell size difference between wild-type and mutant integuments.
To investigate whether $\mathrm{er}-105 \mathrm{erll}-2 \mathrm{erl}-1 / \mathrm{+}$ ovule integument defects were due to a decrease in cell division, we quantified the number of cells contributing to the epidermis at stage 1-II and to the outer integument at three subsequent stages (see Materials and methods). At stages 1-II and 2-II, no difference in cell number was observed between $e r$-family combination mutants and wild type (Fig. 4D). However, er-105 erll-2 erl2-1/+ outer integuments had significantly fewer cells per outer integument than wild type from stage 3 -III (31\% reduction) to maturity (57\% reduction). A similar trend was observed for er -105 erll-2/+ erl $2-1$ (15\% reduction) and er erl1-1 erl2-1/+ (30\% reduction) ovules, which had an intermediate number of integument cells between that of wild type and er-105 erl $1-2$ erl2-1/+ at maturity (Fig. 4D). Together, these data provide evidence that a reduction in cell division is responsible for the abnormal integument morphology of er-105 erl1-2 erl2-1/+ ovules, and that the number of cell divisions is sensitive to the dosage of ERL1.

\section{Specific misregulation of cell-cycle regulators is associated with the arrested ovule development in er-105 erl1-2 erl2-1/+ mutants}

Because cell divisions were reduced in er-105 erl1-2 erl2-1/+ ovules, we examined the expression of cell-cycle-regulatory genes to determine if their transcript accumulation is altered in er-105 erll2 erl2-1/+ ovules. The cell cycle is regulated at multiple points by cyclin-dependent kinases (CDKs) that form complexes with appropriate cyclins (De Veylder et al., 2003; Dewitte and Murray, 2003). Plants have a large number of core cell-cycle genes including plant specific B-type CDKs that are regulated at the transcriptional 

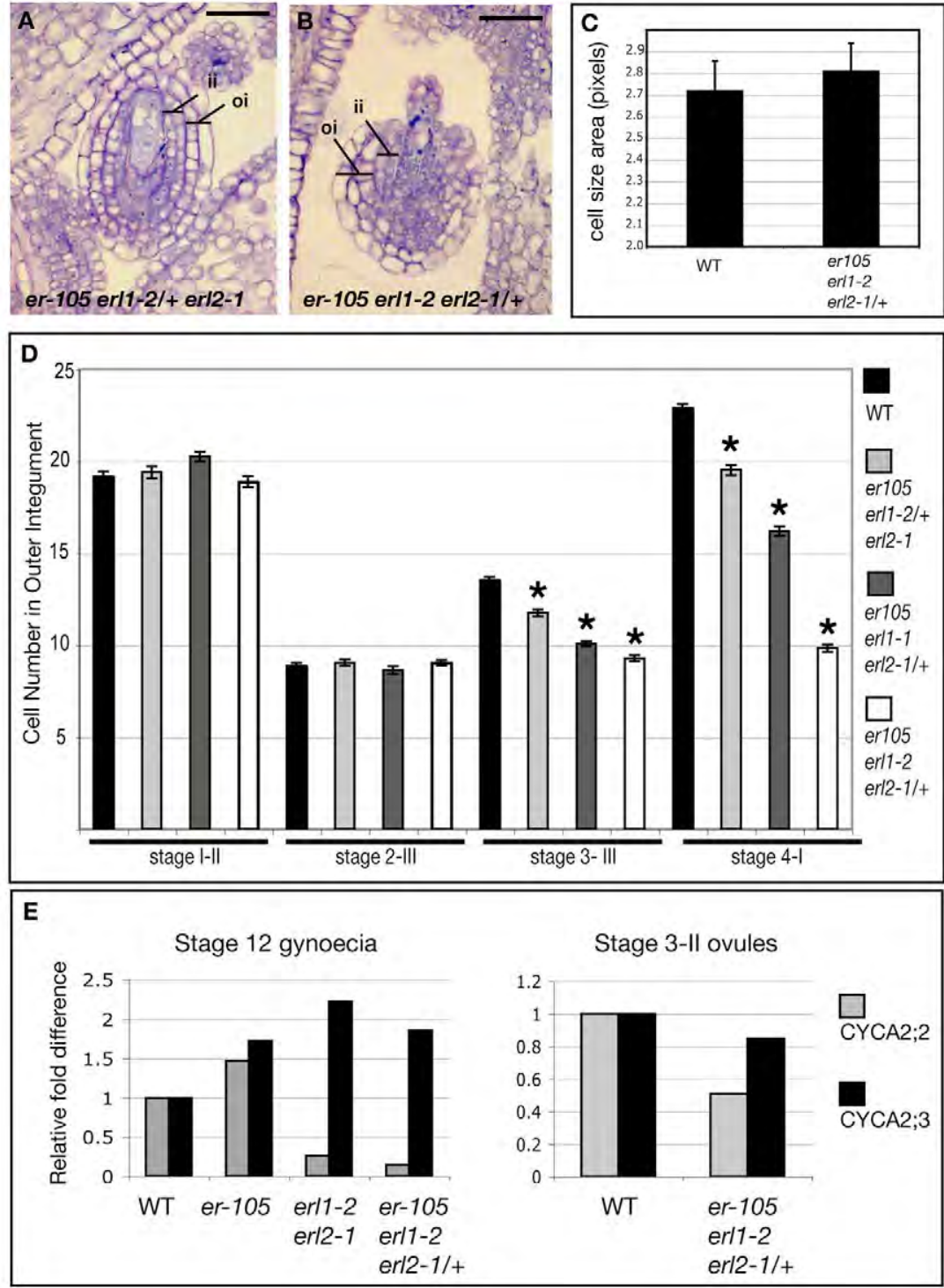

Fig. 4. ERL2 is haploinsufficient to maintain integument growth. (A,B) Transverse (horizontal) sections of Arabidopsis ovules at anthesis. (A) er-105 erl1-2/+erl2-1 has a fully encased and developed embryo sac. (B) er-105 erl1-2 erl2-1/+ ovule has shorter integuments, protruding nucellus with no observable embryo sac. (C) Mean cell area ( \pm s.e.m.) of the outer integument cells of wild type and er-105 er/1-2 er/2-1/+ ( $n=25$, Student's t-test, $P=0.331)$. (D) Cell number ( \pm s.e.m.) in epidermis of ovules (stage 1-II) and the outer layer of the outer integument (stage 2-III, 3-III and 4-I) of wild-type and er-family mutant ovules at the indicated stages $(n=25)$. Asterisks indicate a significant difference from the respective wild type within each stage using Student's $t$-test $(P=0.01)$. At stage 3 -III, a decrease in the number of cells in the outer integument is detected between wild type and er-family mutants. By stage 4-II, the number of cells in the outer integument is dramatically reduced compared with wild type. (E) Real-time RT-PCR analysis of selected cyclins in stage 12 carpels (left) and stage 3 -II ovules (right). Loss of ER-family function results in a disruption of cyclin gene expression. Scale bars: $20 \mu \mathrm{m}$. level (De Veylder et al., 2003). Plant cyclin genes can be divided into four groups (A, B, D and $\mathrm{H})$. The evolution of three A-type subclasses (A1, A2 and A3), compared with only one in animals, has led to the idea that A-type cyclins might fulfil plant-specific roles (Caubet-Gigot, 2000; Dewitte and Murray, 2003).

To detect alterations in expression levels of cell-cycle regulatory genes, we performed RT-PCR analysis on RNA from stage 12 carpels (see Fig. S3 in the supplementary material). According to Schneitz et al. (Schneitz et al., 1995), this stage of carpel corresponds to the point in ovule development when we observed noticeable changes in integument morphology between $\mathrm{er}$ - $105 \mathrm{erll}$ 2 erl2-1/+ and wild type. Most of the B-type CDKs and cyclin A, B and $\mathrm{D}$ genes did not show a dramatic change in transcript abundance in ER-family loss-of-function mutants. However, CYCA2;2 showed a pronounced decrease in expression in er-105 erll-2 erl2-1/+ (see Fig. S3 in the supplementary material; data not shown). We next quantified the relative decrease in $C Y C A 2 ; 2$ transcripts using real-time PCR. We found that $C Y C A 2 ; 2$ consistently shows a significant decrease in expression after loss of $E R$-family function in both carpels and individual ovules (Fig. 4E). Conversely, $C Y C A 2 ; 3$ had the opposite trend, showing increased transcript accumulation after $E R$-family loss-of-function, perhaps owing to compensation (Fig. 4E). The results suggest that reduced levels of $E R$-family gene function may impact cell division through disruption of specific cell-cycle-regulatory gene expression.

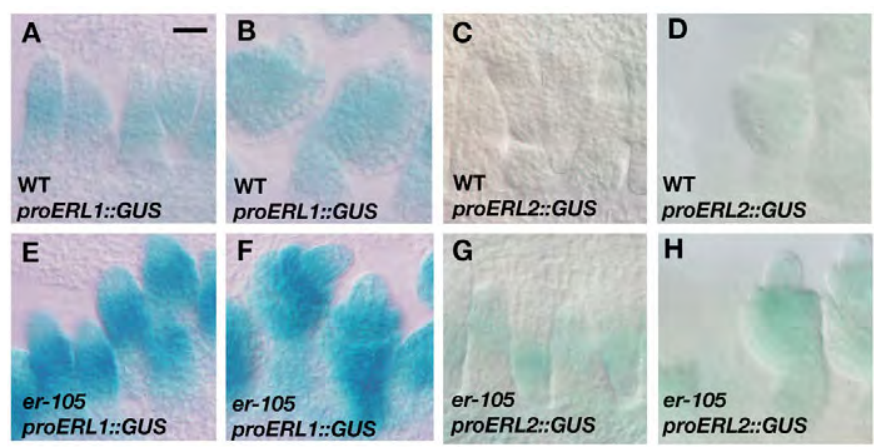

Fig. 5. The ER family of LRR-RLKs are expressed in ovules throughout development. (A,B) proERL $1::$ GUS expression in wildtype Arabidopsis. (C,D) proERL2::GUS expression in wild type. $(\mathbf{E}, \mathbf{F})$ proERL 1::GUS expression in er-105. $(\mathbf{G}, \mathbf{H})$ proERL2::GUS expression in er-105. ERL1 and ERL2 promoter activity is higher in the er-105 background than in wild type. Scale bar: $20 \mu \mathrm{m}$. 

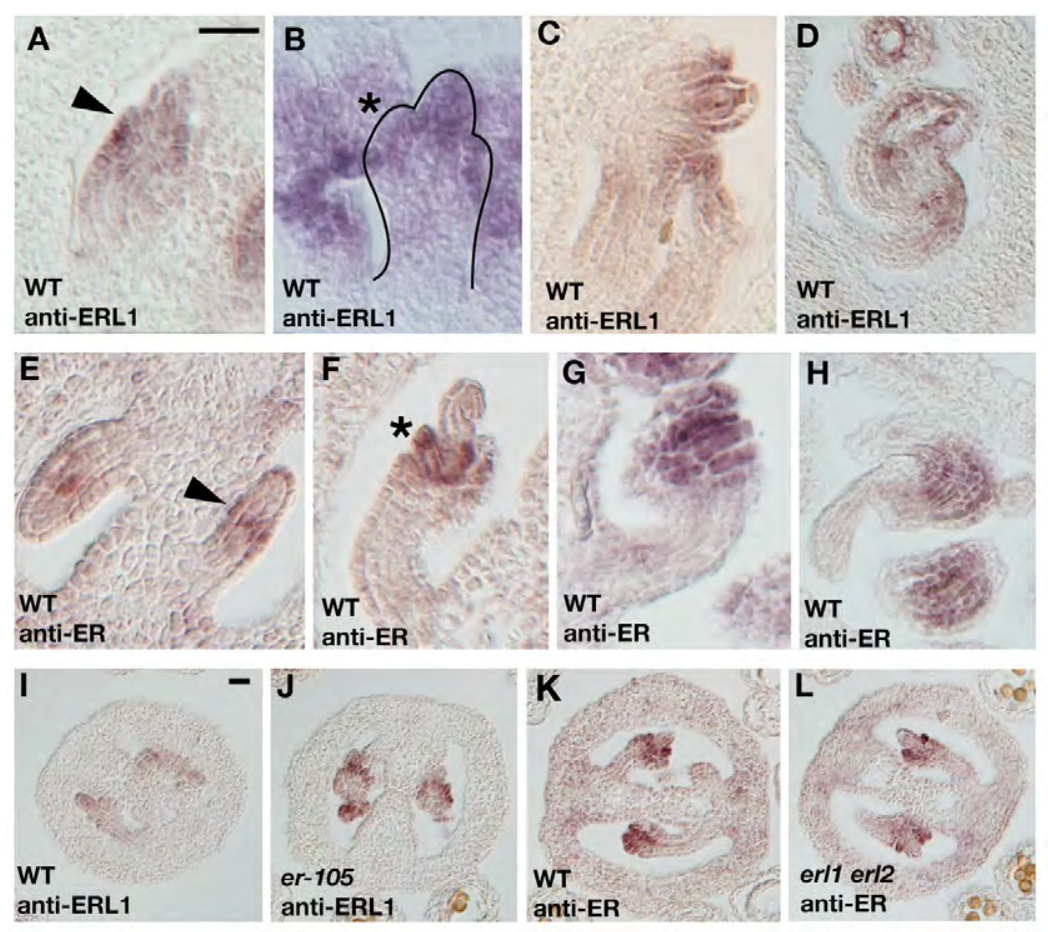

M
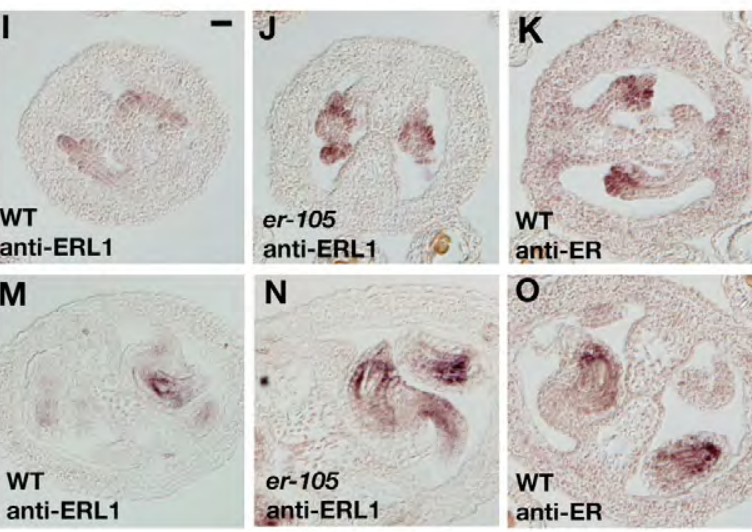

N

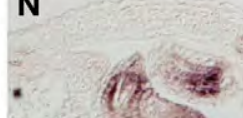

O

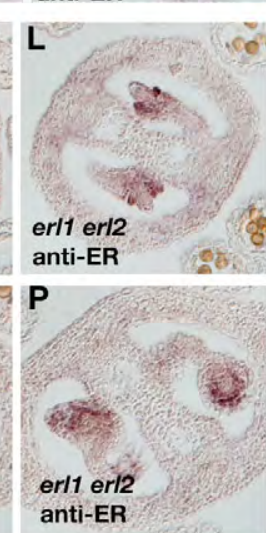

Fig. 6. ERL1 and $E R$ expression patterns. (A-H) In situ hybridization in wild-type Arabidopsis using an ERL1 $(\mathrm{A}-\mathrm{D})$ or $E R(\mathrm{E}-\mathrm{H})$ antisense probe. Both show broad expression in ovules with increased signal intensity in the chalazal region ( $A, E$, arrowheads) and developing integuments (B,F, asterisks). Ovule is outlined in B.

$(\mathrm{D}, \mathrm{H})$ Signal is still visible at maturity. (I-P) Comparison of $E R L 1(\mathrm{I}, \mathrm{J}, \mathrm{M}, \mathrm{N})$ or $E R(\mathrm{~K}, \mathrm{~L}, \mathrm{O}, \mathrm{P}) \mathrm{mRNA}$ accumulation in wild type and ER-family mutants examined by in situ hybridization. (Q) Real-time RT-PCR analysis of ER, ERL1 and $E R L 2$ in stage 12 carpels of wild type and $E R$-family mutants. Transcript abundance of ER,ERL1 and ERL2 are higher in the absence of the other family members. Scale bars: $20 \mu \mathrm{m}$.

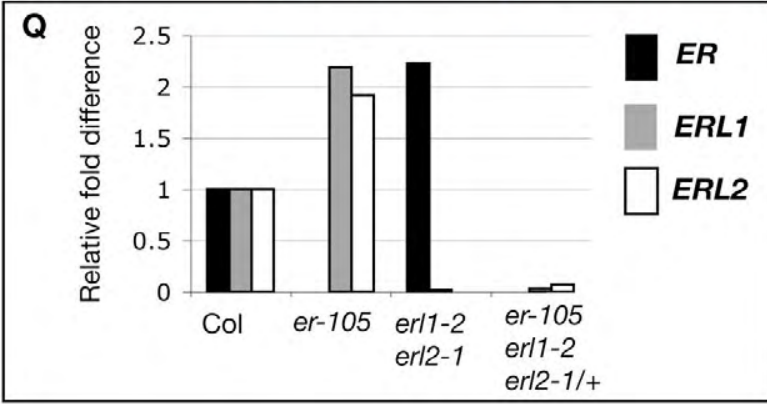

Alternatively, altered levels of these cyclin genes could be the consequence of reduced cell division produced by a yet unknown mechanism.

\section{ERL1 and ERL2 are expressed in ovule primordia and developing integuments}

To determine the expression pattern of the $E R$ family during ovule development, we first observed their promoter activity using reporter $\beta$-glucuronidase (GUS) fusion constructs (Fig. 5) (Shpak et al., 2004). ERL1 and ERL2 promoter activity was first detected in the placental wall prior to ovule primordia initiation (data not shown). They became broadly active in ovules with a slight increase in activity in the central chalazal region (Fig. 5A-D). In mature ovules, faint GUS expression was restricted to the funiculus (data not shown). Interestingly, proERL1::GUS was notably stronger in the er-105 background than in wild type (Fig. 5E,F).

Because integument growth was sensitive to the dosage of ERLI and because the promoter activity of ERL1 and ERL2 are similar, we focused our analysis of expression overlap on $E R$ and $E R L 1$. We analyzed the mRNA expression patterns of $E R$ and $E R L 1$ by in situ hybridization (Fig. 6). In reproductive structures, ERL1 transcripts were detected in floral meristems and primordia (see Fig. S4 in the supplementary material). During ovule development, ERL1 transcripts were detected throughout ovule primordia from initiation to stage 3-II, with increased hybridization intensity in the chalazal region and developing integuments (Fig. 6A-C). At maturity, ERL1 hybridization signal was still detectable in the integuments and funiculus (Fig. 6D).

Expression of $E R$ was observed in floral meristems as well as the carpel wall and developing petals (see Fig. S4 in the supplementary material). Similar to $E R L 1, E R$ transcripts were detected broadly in the developing ovule, with more intense signal in the chalazal region and developing integuments (Fig. 6E-G). ER expression lessened later in development, but was still detectable in the carpel and ovule integuments at maturity (Fig. $6 \mathrm{H}$ ). As the probes for $E R$ and $E R L 1$ did not cross-hybridize with each other (see Fig. S4 in the supplementary material), our results suggest that overlapping expression patterns of $E R$ and $E R L 1$ account for their redundant roles in ovule development. 

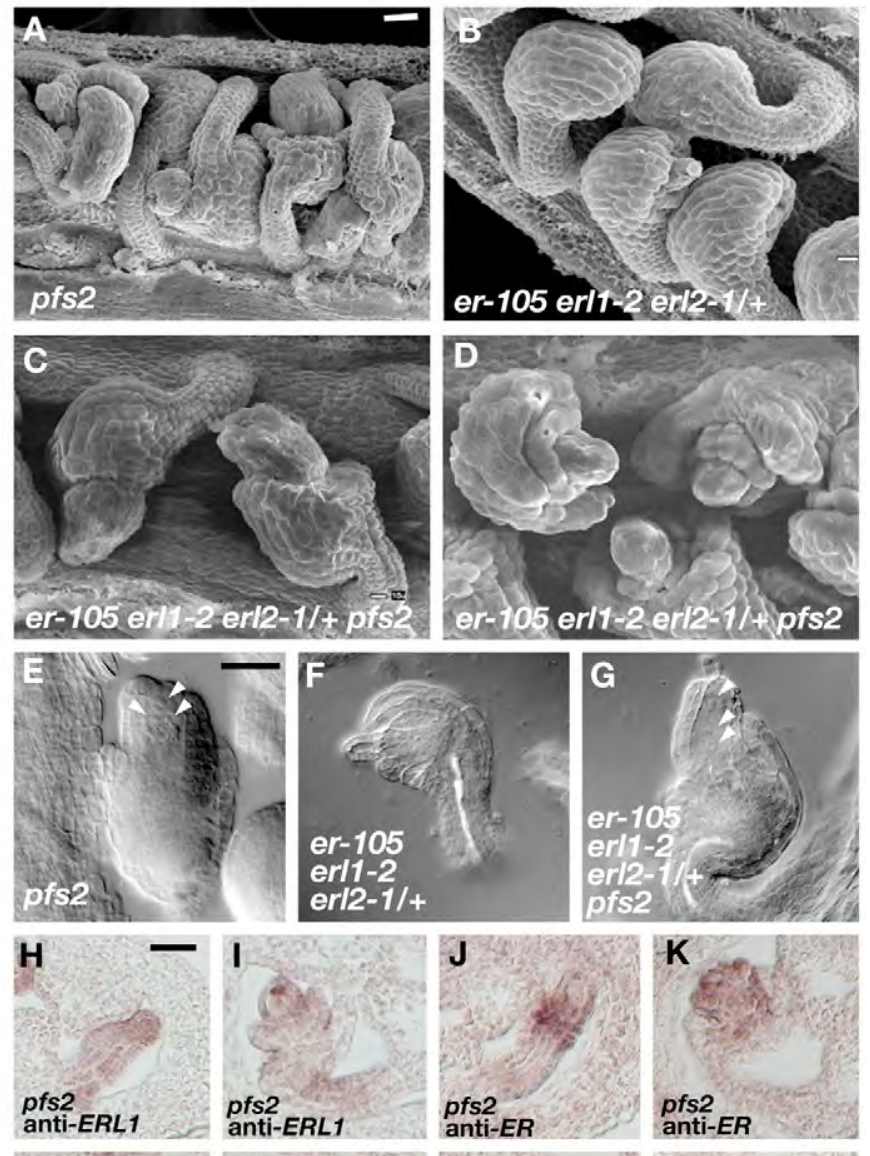

$\mathbf{L}$
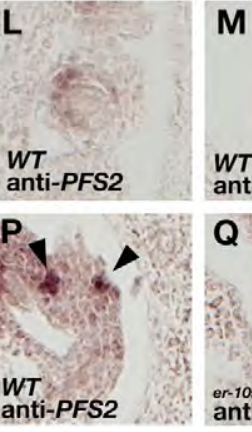

M
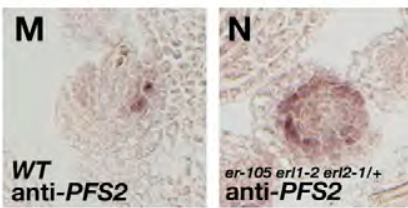

o

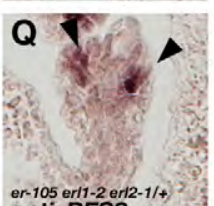
er-105 erl1-2 erl2-1/+
anti-PFS2

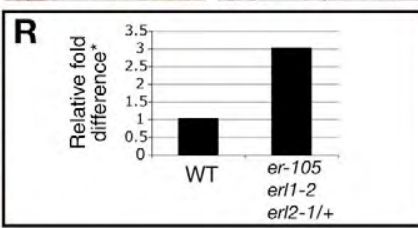

\section{Unequal dosage compensation among ER-family genes}

Given the overlapping expression patterns of $E R$-family genes in the ovule and their ability to functionally substitute for one another (Shpak et al., 2004), we tested whether loss of specific $E R$-family gene function would lead to changes in expression of the other family members. To assess changes in transcript abundance, quantitative real-time RT-PCR analysis was performed on RNA from stage 12 carpels (Fig. 6Q). An increase in both ERL1 and ERL2 transcripts was observed in the er-105 background as compared with wild type and, reciprocally, the accumulation of $E R$ transcripts was higher in the erll erl2 mutant background. In situ hybridization determined that the spatial expression patterns of $E R$ and ERLI were not disrupted in the er-105 and erll erl2 mutant backgrounds (Fig. 6I-P), respectively.

Taken together, the results suggest that transcriptional upregulation occurs in the absence of $E R$-family members, allowing each family member to functionally compensate for each other. This
Fig. 7. Genetic interactions of ER-family genes with PFS2.

(A-D) SEMs of mature Arabidopsis ovules. (A) pfs2-1 ovules have aberrant integument development. (B) er-105 er/1-2 er/2-1/+ ovules. (C,D) er-105 erl1-2 erl2-1/+ pfs2-1 mutant ovules have variability in integument development with reduced growth of the outer integument and some bifurcation of developing integuments. (E-G) DIC images of mature ovules. (E) pfs2-1 ovules have some partially developed embryo sacs. (F) er-105 er/1-2 er/2-1/+ has a penetrant phenotype of a small mass of cells in place of the embryo sac. (G) er-105 erl1-2 erl2-1/+ pfs2-1 mutant ovules display variability in embryo sac development similar to the pfs2-1 single mutant. Embryo sac nuclei are indicated by white arrowheads. (H-K) In situ hybridization in the pfs2-1 mutant using $E R L 1(\mathrm{H}, \mathrm{I})$ and $E R(\mathrm{~J}, \mathrm{~K})$ antisense probes. $(\mathbf{L}, \mathbf{M})$ In situ hybridization in wild type using a PFS2 antisense probe. $(\mathbf{N}, \mathbf{0})$ In situ hybridization in er-105 erl1-2 er/2-1/+ using a PFS2 antisense probe. $(\mathbf{P}, \mathbf{Q})$ Extended exposure of PFS2 antisense probe in wild type and er-105 er/1-2 er/2-1/+. Intense signal was observed in the inner integuments (arrowheads). No signal was detected with sense probes for ERL1, ER or PFS2. Scale bars: $20 \mu \mathrm{m}$. (R) Real-time RT-PCR analysis of PFS2 expression in stage 3-II ovules, given as fold-difference relative to wild type. ${ }^{*}$, Raw data collected as PFS2 transcript abundance relative to ACTIN 2 (wild type versus er-105 er/1-2 er/2-1/+ Experiment 1: $0.205 \pm 0.04$ versus $0.351 \pm 0.02$; Student's $t$-test $P=0.03$; 1.72 fold increase in er-105 erl1-2 erl2-1/+. Experiment 2: $0.141 \pm 0.003$ versus $0.603 \pm 0.04$; Student's $t$-test $P=0.001 ; 4.2$ fold increase in er105 erl1-2 erl2-1/+).

is consistent with the findings of Shpak et al. (Shpak et al., 2004) that ER-family members are able to substitute for each other if their temporal and spatial expression patterns overlap. Fertility in the null er single mutant or erll erl 2 double mutant might require dosage compensation by the remaining family members to maintain signaling above a required threshold. This threshold is not met in the er-105 erll erl2/+ mutant, resulting in the arrest of integument growth.

\section{Interaction of pfs2 with er er/1-2 er/2-1/+}

$P F S 2$, a WUS-related homeobox gene also known as WOX6, affects ovule patterning and regulates integument cell proliferation and gametophyte differentiation (Park et al., 2004; Park et al., 2005). It has been well-documented that the competing actions of the transcription factor WUS, and the CLAVATA (CLV) LRR-RLK signaling pathway, maintain meristem size in Arabidopsis (Brand et al., 2000). Numerous WOX genes display region-specific expression, but relatively little is known about their regulation (Haecker et al., 2004). To investigate whether a mechanism including PFS2 and ER signaling directs integument size during ovule development, we produced the combination mutant er-105 erl1-2 erl2-1/+ pfs $2-1$ and characterized the phenotype.

The $p f s$-1-null mutation causes the production of ovules with similar characteristics to $\mathrm{er}-105 \mathrm{erl1}-2 \mathrm{erl2}-1 /+$. pfs $2-1$ ovules have aberrant integument growth after initiation and embryo sac arrest (Park et al., 2004) (Fig. 7A). However, growth defects are variable in pfs2-1 mutants: some ovules complete various stages of embryo sac development and a low percentage of viable seed are produced (Park et al., 2005). Similar to $p f s 2-1$ alone, we observed a variable degree of integument development in the er-105 erl1-2 erl2-1/+ pfs 2-1 mutant (Fig. 7C,D). The phenotype ranged from an outer integument that covered the inner integument to a near absence of the outer integument. In addition, subtle changes in integument 
development were observed including lobing of the outer integument during development (Fig. 7C,D). Such variability in integument growth was not observed in er-105 erll-2 erl2-1/+, in which the phenotype showed little variation (Fig. 7B). Surprisingly, some ovules of er-105 erll-2 erl2-1/+pfs $2-1$ developed beyond the two-celled nuclei stage typical of er-105 erll-2 erl2-1/+ ovules, although no viable seed could be collected from these plants (Fig. $7 \mathrm{E}-\mathrm{G})$. Therefore, the loss of PFS 2 function partially rescues the $\mathrm{er}$ 105 erll -2 erl2 $-1 /+$ ovule defects.

Because pfs $2-1$ ovules show aberrant integument growth, we performed in situ hybridization using ER and ERL1 probes in the pfs 2-1 ovules to determine whether loss of ER-family signaling contributed to the abnormal integument phenotype of $p f s 2-1$ ovules (Fig. 7H-K). Loss or change in expression domain of ER or ERL1 was not detected in the $p f s 2-1$ mutant, suggesting that the required threshold of ER-signaling is met in the pfs $2-1$ mutant.

The phenotypic rescue of er-105 erll-2 erl2-1/+ ovules by additional $p f s 2$ mutation implies that it is advantageous not to have PFS2 under the sub-threshold level of ER-family signaling. To test whether the er-105 erll-2 erl2-1/+ phenotype was due in part to misexpression of $P F S 2$, we performed in situ hybridization using a PFS2 probe (Fig. 7L-O). PFS2 expression was detected broadly in developing carpels and initiating ovule primordia (data not shown) with strong expression in the inner integument during initiation and growth (Fig. 7L-O). The expression domain of PFS2 was not altered in the er-105 erl1-2 erl2-1/+ ovules (Fig. 7P,Q), indicating that the gametophytic defects in er-105 erl1-2 erl2-1/+ are not due to PFS2 misexpression. However, quantitative real-time RT-PCR detected an increase in PFS2 transcript abundance in er-105 erll-2 erl2-1/+ stage 3-II ovules compared with wild type, indicating a potential inhibitory role for the ER family in PFS2 expression. Phenotypic rescue of $\mathrm{er}-105 \mathrm{erl} 1-2 \mathrm{erl}-1 / \mathrm{t}$ by the addition of the $p f s$ mutation suggests that ovule development requires a proper balance of both $E R$ family and PFS2 function.

\section{DISCUSSION Unequal functional redundancy among the ER- family LRR-RLKs}

Gene duplication is a common occurrence and has led to the production of numerous gene families in Arabidopsis, one of the largest being the RLK-coding gene family, which has over 600 members (Shiu and Bleecker, 2001; Vision et al., 2000). Following a gene duplication event, decreased selection pressure on one of the gene copies can produce proteins with novel function or expression resulting in partial redundancy among gene family members (Hardtke et al., 2004). If the shared function of duplicated genes becomes unequally distributed between gene copies, unequal redundancy among the gene family members may result, as described for the ER family, where both erll and erl 2 confer no growth phenotype but enhance the er growth defects in a unique manner (Kempin et al., 1995; Shpak et al., 2004). In many cases, unequal redundancy is produced by differences in the expression level or expression patterns between gene copies, where the duplicated gene(s) may have redundant function with the ancestral gene, but the expression might be lower or restricted. A good example is the three Arabidopsis brassinosteroid receptor genes. The major receptor, $B R I 1$, is broadly expressed at high levels, whereas expression of BRL2 and BRL3 is restricted to vasculature (CanoDelgado et al., 2004). Successive loss of family members enhances the phenotype of the ancestral gene and a true null phenotype is observed only in the absence of the entire family as has been shown for ER, ERL1 and ERL2 (Shpak et al., 2004).
Our study uncovered unequal contributions and dosage compensation among gene family members with overlapping expression patterns. The most significant difference between $\mathrm{er}-105$ erll-2/+ erl2-1 and er-105 erl1-2 erl2-1/+ plants is the loss of female fertility owing to reduced cell division and outgrowth of the integuments in er-105 erll-2 erl2-1/+. The expression pattern for all three family members overlaps in the distal, central and proximal regions, consistent with all three regions being reduced when successive family members are removed. An erl1-2 erl2-1 double or er single mutant shows no obvious defects in integument development, indicating that the upregulation of $E R$ or ERL1 and 2 in these backgrounds can maintain expression above the threshold necessary to direct integument development (Fig. 6Q). However, as successive family members are lost, low levels of ERL2 are insufficient to maintain signaling, and integument growth is compromised. Consistent with this dosage-dependence model, the ovules of er-105 erl1-2 erl2-1/+ are significantly larger and more developed than those of the $E R$-family triple mutant (see Fig. S5 in the supplementary material).

\section{ERL2 haploinsufficiency specifically affects cell division of the ovule integuments}

The ER-family LRR-RLKs probably maintain cell-cell communication, a reduction of which results in the irregular and reduced cell division during integument outgrowth in er-105 erll-2 erl2-1/+ ovules. They do not regulate ovule primordia patterning or integument initiation, as these aspects are not affected even by the complete loss of ER-family function (Fig. 2 and see Fig. S5 in the supplementary material).

Our results suggest that the ER-family RLKs promote cell division after integument initiation through potential regulation of core cellcycle genes, specifically $C Y C A 2 ; 2$ (Fig. 4). Among over 30 cyclins present in Arabidopsis, ten belong to the A-type (Dewitte and Murray, 2003; Vandepoele et al., 2002). Recent studies suggest that Arabidopsis cyclin A2 families act as negative regulators of endoreduplication (Burssens et al., 2000; Dewitte and Murray, 2003; Imai et al., 2006; Yu et al., 2003). For example, loss-of-function mutations in CYCA2;3 led to elevated ploidy levels in mature organs (Imai et al., 2006). Consistently, the dominant, activation-tagged mutation in INCREASED LEVEL OF POLYPLOIDY1, a conserved repressor of $C Y C A 2$ transcription, caused an increase in endoreduplication (Yoshizumi et al., 2006). However, cyclin A2 families may also function to regulate mitosis. In Medicago, CYCA2;2 $(\mathrm{MedsaCYCA2;2)}$ is required for meristem formation or activity but dispensable for endoreduplication (Roudier et al., 2000). Similar to Medsa;CYCA2;2, all four Arabidopsis cyclin A2 genes show high promoter activity in meristems and young organ primordia (Burssens et al., 2000; Imai et al., 2006). Therefore, they might redundantly control cell division in actively proliferating tissues, where the ERfamily signaling pathway is required. Consistent with this hypothesis, flow-cytometric analysis of DNA content in er-105 suggested possible cell-cycle arrest (Shpak et al., 2003).

All members of the ER family are expressed in overlapping domains in additional areas of the plant including shoot and inflorescence meristems and floral primordia (Shpak et al., 2004). The lack of any visual er-105 erll-2 erl2-1/+ phenotype in these tissues could be due to redundancy by other signaling factors that are not expressed in the ovule. Alternatively, ovule integuments might be particularly susceptible to cell-cell signaling disruption. Unlike meristems and developing leaves and floral organs, integuments are solely derived from the L1 layer (Schneitz et al., 1997). Therefore, cell proliferation in integuments is likely to 
occur with limited inter cell-layer communication. Such a scenario was suggested for loss-of-function mutants of the RLK gene ARABIDOPSIS CRINKLY4, which display localized defects in integument development, despite its expression in a wider variety of tissues (Gifford et al., 2003).

\section{Genetic interaction of ER-family genes and PFS2}

PFS2 is thought to control ovule primordial patterning by regulating the timing of cellular differentiation (Park et al., 2005). In $p f s 2-1$, premature differentiation of the cells contributing to the gametophyte or integument primordia could account for decreased integument length and defects in the embryo sac (Park et al., 2004). The addition of $p f s-1$ to er-105 erl1-2 erl2-1/+ mutations led to increased variability in both integument and gametophyte development, similar to $p f s 2-1$ alone, indicating that the $p f s$ 2- 1 mutation is epistatic to the $e r$-family mutations. One explanation for these results is that the ER-family RLKs and PFS2 both contribute to integument outgrowth through distinct but interrelated mechanisms. The ER family are components of a signaling pathway that sustain organized cell divisions, potentially through the regulation of core cell-cycle genes such as $C Y C A 2 ; 2$. Without activation of this signaling pathway, integument cells may differentiate before undergoing sufficient cell divisions. PFS2 might be responsible for maintaining integument cells in a state that allows them to respond to factors required for coordinated cell division. In this scenario, the loss of PFS2 function would be epistatic to the loss of the ER family.

WOX genes generally have a spatially restricted expression pattern (Matsumoto and Okada, 2001). Our in situ hybridization shows that PFS2 expression is spatially restricted to the inner integument (Fig. 7). Therefore, $P F S 2$ might be required to properly coordinate the developmental states of the sporophytic integument tissues and gametophytic embryo sac. If so, the loss of PFS2 uncouples embryo sac development from abrogated integument growth under the subthreshold level of ER-family signaling, therefore partially rescuing the embryo sac defects in erecta-105 erl1-2 erl2-1/+ ovules.

In the well-established model of $C L V / W U S$ interaction in the shoot meristem, CLV3 limits the expression of WUS, while WUS in turn promotes the expression of $C L V 3$, causing a feedback loop that maintains a stem cell population (Brand et al., 2000; Clark, 2001; Laux et al., 1996). Our results indicate that the ER family does not spatially restrict PFS2 expression and that factors yet to be identified are likely to be responsible for the limiting PFS2 expression. However, the ER family might restrict the expression levels of PFS2, and elevated expression of PFS2 might account for the abortion of gametophytes in er-105 erl1-2 erl2-1/+ ovules. Consistently, embryo sacs of er-105 erll -2 erl2-1/+ are filled with a small group of cells, which resembles the phenotype of PFS2 overexpression described by Park et al. (Park et al., 2005).

Haploinsufficiency refers to a phenotype associated with the inactivation of a single allele leading to a half-normal amount of gene product, which is insufficient to maintain the wild-type phenotype. In this report, we have shown a novel phenotype for successive ER-family loss-of-function; ERL2 is haploinsufficient in the absence of $E R$ and $E R L 1$ to direct integument growth, but sufficient to drive floral organ growth, elongation and patterning. We further uncovered evidence of dosage compensation within the ER family and their potential regulatory relationships with the WOX-family transcription factor. Our work has identified the specific timing of ERL1 action during integument cell proliferation, which might provide a biological system to further elucidate the signaling components.
We thank Dr John Bowman for the erl1-1 enhancer-trap line, Dr Bernard Hauser for pfs2-1 seeds, Drs Kiyotaka Okada, Mitsuhiro Aida and Masao Tasaka for PHB probes, Dr Caroline Josefsson for assistance in RT-PCR analysis of erl1-1, Dr Kay Schneitz for advice on the design of the genetic screen and Dr Jessica Messmer-McAbee for commenting on the manuscript. This work was supported by the USDA CREES postdoctoral fellowship to L.J.P. and by the DOE grant (DE-FG02-03ER15448) to K.U.T. The DIC microscopy was supported by the CREST award from Japan Science and Technology Agency to K.U.T

\section{Supplementary material}

Supplementary material for this article is available at http://dev.biologists.org/cgi/content/full/134/17/3099/DC1

\section{References}

Brand, U., Fletcher, J. C., Hobe, M., Meyerowitz, E. M. and Simon, R. (2000). Dependence of stem cell fate in Arabidopsis on a feedback loop regulated by CLV3 activity. Science 289, 617-619.

Burssens, S., Engler, J. A., Beeckman, T., Richard, C., Shaul, O., Ferreira, P., Van Montagu, M. and Inzé, D. (2000). Developmental expression of the Arabidopsis thaliana CycA2;1 gene. Planta 211, 623-631.

Cano-Delgado, A., Yin, Y., Yu, C., Vafeados, D., Mora-Garcia, S., Cheng, J., Nam, K. H., Li, J. and Chory, J. (2004). BRL1 and BRL3 are novel brassinosteroid receptors that function in vascular differentiation in Arabidopsis. Development 131, 5341-5351.

Caubet-Gigot, N. (2000). Plant A-type cyclins. Plant Mol. Biol. 43, 659-675.

Clark, S. E. (2001). Cell signaling at the shoot apical meristem. Nat. Rev. Mol. Cell Biol. 2, 276-284.

De Veylder, L., Joubes, L. and Inzé, D. (2003). Plant cell cycle transitions. Curr. Opin. Plant Biol. 6, 536-543.

Dewitte, W. and Murray, J. A. H. (2003). The plant cell cycle. Annu. Rev. Plant Biol. 54, 235-264.

Ehsan, H., Reichheld, J. P., Durfee, T. and Roe, J. L. (2004). TOUSLED kinase activity oscillates during the cell cycle and interacts with chromatin regulators. Plant Physiol. 134, 1488-1499.

Elliott, R. C., Betzner, A. S., Huttner, E., Oakes, M. P., Tucker, W. Q. J., Gerentes, D., Perez, P. and Smyth, D. R. (1996). AINTEGUMENTA, an APETALA2-like gene of Arabidopsis with pleiotropic roles in ovule development and floral organ growth. Plant Cell 8, 155-168

Eshed, Y., Baum, S. R., Perea, J. V. and Bowman, J. L. (2001). Establishment of polarity in lateral organ of plants. Curr. Biol. 11, 1251-1260.

Eshed, Y., Izhaki, A., Baum, S. R., Floyd, S. K. and Bowman, J. L. (2004) Asymmetric leaf development and blade expansion in Arabidopsis are mediated by KANADI and YABBY activities. Development 127, 725-734

Gasser, C. S., Broadhvest, J. and Hauser, B. A. (1998). Genetic analysis of ovule development. Annu. Rev. Plant Physiol. Plant Mol. Biol. 49, 1-24.

Gifford, M. L., Dean, S. and Ingram, G. C. (2003). The Arabidopsis ACR4 gene plays a role in cell layer organisation during ovule integument and sepal margin development. Development 130, 4249-4258.

Groß-Hardt, R., Lenhard, M. and Laux, T. (2002). WUSCHEL signaling functions in interregional communicaton during Arabidopsis ovule development. Genes Dev. 16, 1129-1138.

Haecker, A., Groß-Hardt, R., Geiges, B., Sarkar, A., Breuninger, H., Herrmann, M. and Laux, T. (2004). Expression dynamics of WOX genes mark cell fate decisions during early embryonic patterning in Arabidopsis thaliana. Development 131, 657-668

Hardtke, C. S., Ckurshumova, W., Vidaurre, D. P., Singh, S. A., Stamatiou, G., Tiwari, S. B., Hagenn, G., Guilfoyle, T. J. and Berleth, T. (2004). Overlapping and non-redundant functions of the Arabidopsis auxin response factors MONOPTEROS and NONPHOTOTROPIC HYPOCOTYL 4. Development 131, 10891100

Hauser, B. A., He, J. Q., Park, S. O. and Gasser, C. S. (2000). TSO1 is a novel protein that modulates cytokinesis and cell expansion in Arabidopsis. Development 127, 2219-2226

Imai, K. K., Ohashi, Y., Tsuge, T., Yoshizumi, T., Matsui, M., Oka, A. and Aoyama, T. (2006). The A-type cyclin CYCA2;3 is a key regulator of ploidy levels in Arabidopsis Endoreduplication. Plant Cell 18, 382-396.

Kempin, S. A., Savidge, B. and Yanofsky, M. F. (1995). Molecular basis of the cauliflower phenotype in Arabidopsis. Science 267, 522-525.

Kondorosi, E., Roudier, F. and Gendreau, E. (2000). Plant cell size control: growing by ploidy? Curr. Opin. Plant Biol. 3, 488-492.

Lang, J. D., Ray, S. and Ray, A. (1994). sin1, a mutation affecting female fertility in Arabidopsis, interacts with mod1, its recessive modifier. Genetics 137, 1101-1110. Laux, T., Mayer, K. F., Berger, J. and Jurgens, G. (1996). The WUSCHEL gene is required for shoot and floral meristem integrity in Arabidopsis. Development 122, 87-96.

Matsumoto, N. and Okada, K. (2001). A homeobox gene, PRESSED FLOWER, regulates lateral axis-dependent development of Arabidopsis flowers. Genes Dev. $15,3355-3364$. 
McAbee, J. M., Hill, T. A., Skinner, D. J., Izhaki, A., Hauser, B. A., Meister, R. J., Reddy, G. V., Meyerowitz, E. M., Bowman, J. L. and Gasser, C. S. (2006). ABERRANT TESTA SHAPE encodes a KANADI family member, linking polarity determination to separation and growth of Arabidopsis integuments. Plant J. 46 522-531.

Melaragno, J. E., Mehrotra, B. and Coleman, A. W. (1993). Relationship between endopolyploidy and cell size in epidermal tissue of Arabidopsis. Plant Cell 5, 16611668

Mizukami, Y. (2001). A matter of size: developmental control of organ size in plants. Curr. Opin. Plant Biol. 4, 533-539.

Park, S. O., Hwang, S. and Hauser, B. A. (2004). The phenotype of Arabidopsis ovule mutants mimics the morphology of primitive seed plants. Proc. R. Soc. Lond. B Biol. Sci. 271, 311-316.

Park, S. O., Zheng, Z., Oppenheimer, D. G. and Hauser, B. A. (2005). The PRETTY FEW SEEDS2 gene encodes an Arabidopsis homeodomain protein that regulates ovule development. Development 132, 841-849.

Potter, C. J. and Xu, T. (2001). Mechanisms of size control. Curr. Opin. Genet. Dev. 11, 279-286

Roudier, F., Fedoova, E., Gyorgyey, J., Feher, A., Brown, S., Kondorosi, A. and Kondorosi, E. (2000). Cell cycle function of a Medicago sativa A2-type cyclin interating with PSTAIRE-type cylin-dependent kinase and retinoblastoma protein. Plant J. 23, 73-83.

Schneitz, K., Hülskamp, M. and Pruitt, R. E. (1995). Wild-type development in Arabidopsis thaliana: a light microscope study of cleared whole-mount tissue. Plant J. 7, 731-749.

Schneitz, K., Hülskamp, M., Kopczak, S. and Pruitt, R. E. (1997). Dissection of sexual organ ontogenesis: a genetic analysis of ovule development in Arabidopsis thaliana. Development 124, 1367-1376.

Schneitz, K., Baker, S. C., Gasser, C. S. and Redweik, A. (1998). Pattern formation and growth during floral organogenesis: HUELLENLOS and AINTEGUMENTA are required for the formation of the proximal region of the ovule primordium in Arabidopsis thaliana. Development 125, 2555-2563.

Sessions, A., Weigel, D. and Yanofsky, M. F. (1999). The Arabidopsis thaliana MERISTEM LAYER 1 promoter specifies epidermal expression in meristems and young primordia. Plant J. 20, 259-263.

Shiu, S. H. and Bleecker, A. B. (2001). Receptor-like kinases from Arabidopsis form a monophyletic gene family related to animal receptor kinases. Proc. Natl. Acad. Sci. USA 98, 10763-10768.

Shpak, E. D., Lakeman, M. B. and Torii, K. U. (2003). Dominant-negative receptor uncovers redundancy in the Arabidopsis ERECTA leucine-rich repeat receptor-like kinase signaling pathway that regulates organ shape. Plant Cell 15, 1095-1110.

Shpak, E. D., Berthiaume, C. T., Hill, E. J. and Torii, K. U. (2004). Synergistic interaction of three ERECTA-family receptor-like kinases controls Arabidopsis organ growth and flower development by promoting cell proliferation. Development 131, 1491-1501.

Sieber, P., Gheyselinck, J., Gross-Hardt, R., Laux, T., Gossniklaus, U. and Schneitz, K. (2004). Pattern formation during early ovule development in Arabidopsis thaliana. Dev. Biol. 273, 321-334.

Skinner, D. J., Hill, T. A. and Gasser, C. S. (2004). Regulation of ovule development. Plant Cell 16, S32-S45.

Smyth, D. R., Bowman, J. L. and Meyerowitz, E. M. (1990). Early flower development in Arabidopsis. Plant Cell 2, 755-767.

Torii, K. U. (2004). Leucine-rich repeat receptor kinases in plants: structure, function and signal transduction. Int. Rev. Cytol. 234, 1-46.

Torii, K. U., Mitsukawa, N., Oosumi, T., Matsuura, Y., Yokoyama, R., Whittier, R. F. and Komeda, Y. (1996). The Arabidopsis ERECTA gene encodes a putative receptor protein kinase with extracellular leucine-rich repeats. Plant Cell 8, 735-746.

Vandepoele, K., Raes, J., De Veylder, L., Rouze, P., Rombauts, S. and Inze, D. (2002). Genome-wide analysis of core cell cycle genes in Arabidopsis. Plant Cell 14, 903-913.

Villanueva, J. M., Broadhvest, J., Hauser, B. A., Meister, R. J., Schneitz, K. and Gasser, C. S. (1999). INNER NO OUTER regulates abaxial-adaxial patterning in Arabidopsis ovules. Genes Dev. 13, 3160-3169.

Vision, T. J., Rown, D. G. and Tanksley, S. D. (2000). The origins of genomic duplications in Arabidopsis. Science 290, 2114-2117.

Yoshizumi, T., Tsumoto, Y., Takigushi, T., Nagata, N., Yamamoto, Y. Y., Kawashima, M., Ichikawa, T., Nakaawa, M., Yamamoto, N. and Matsui, M. (2006). INCREASED LEVEL OF POLYPLOIDY1, a conserved repressor of CYCLINA2 transcription, controls endoreduplication in Arabidopsis. Plant Cell 18, 2452-2468.

Yu, Y., Steinmetz, A., Peng, C.-Y., Yee, A. S. and Piwnica-Worms, H. (2003). The tobacco A-type Nicta;CYCA3;2, at the nexus of cell division and differentiation. Plant Cell 15, 2763-2777. 


\title{
The bHLH Protein, MUTE, Controls Differentiation of Stomata and the Hydathode Pore in Arabidopsis
}

\author{
Lynn Jo Pillitteri ${ }^{1}$, Naomi L. Bogenschutz ${ }^{1}$ and Keiko U. Torii ${ }^{1,2, *}$ \\ ${ }^{1}$ Department of Biology, University of Washington, Seattle, WA 98195 USA \\ ${ }^{2}$ Institute for Stem Cell and Regenerative Medicine, University of Washington, Seattle, WA 98195, USA
}

Stomata are turgor-driven epidermal valves on the surface of plants that allow for efficient gas and water exchange between the plant and its environment. The Arabidopsis thaliana basic helix-loop-helix (bHLH) protein, MUTE, is a master regulator of stomatal differentiation where it is required for progression through the stomatal lineage and the differentiation of stomata. The genetic control of stomatal spacing across the epidermal surface is variable in different organs. For instance, a distinct suite of genes from those in leaves regulates stomatal patterning in hypocotyls. Here we report that regardless of organ type, MUTE controls downstream events directing stomatal differentiation, specifically the transition from meristemoid to guard mother cell. Ectopic MUTE expression is sufficient to over-ride cell fate specification in cell types that do not normally differentiate stomata. Furthermore, MUTE is required for the production of the structure evolutionarily related to stomata, the hydathode pore. Consistently, MUTE displays expression at the tip of cotyledons and leaves, thus co-localizing with the auxin maxima. However, MUTE itself was not regulated by the auxin, and the absence of hydathode pores in mute did not affect the auxin maxima. Surprisingly, our analysis revealed that the requirement for $M U T E$ could be partially circumvented under conditions of compromised inhibitory signaling.

Keywords: Arabidopsis thaliana - bHLH protein - Cellcell signaling - Cell type differentiation - Hydathode Stomata.

Abbreviations: bHLH, basic helix-loop-helix; CaMV, cauliflower mosaic virus; DIC, differential interference contrast; GFP, green fluorescent protein; GMC, guard mother cell; GUS, $\beta$-glucuronidase; MS, Murashige and Skoog; NPA, naphthylphthalamic acid; SLGC, stomatal lineage ground cell.

\section{Introduction}

Stomata are plant epidermal structures composed of two specialized guard cells surrounding a pore. Guard cells respond to numerous environmental signals to control the aperture of the pore, which regulates the amount of carbon dioxide and water vapor exchange between the plant and the atmosphere. Stomata originate from a specialized epidermal cell lineage and are vital for plants to optimize carbon assimilation and water use (Nadeau and Sack 2003). In Arabidopsis, the basic helix-loop-helix (bHLH) protein SPEECHLESS (SPCH) is required for the initial asymmetric division for entry into the stomatal lineage, which produces a small stomatal precursor cell called a meristemoid (MacAlister et al. 2007, Pillitteri et al. 2007). The meristemoid has transient stem cell-like properties and can reiterate itself over several rounds of divisions. After each division, a meristemoid and its sister cell, called a stomatal lineage ground cell (SLGC) are produced. The bHLH protein, MUTE, is required for the transition from meristemoid to guard mother cell (GMC) (Pillitteri et al. 2007). The GMC is the final precursor cell and divides once symmetrically to produce two terminally differentiated guard cells of the mature stoma. Several genes including the bHLH protein, FAMA, and the R2R3 MYB proteins, FOUR LIPS (FLP) and MYB88, are involved in the final division and differentiation step from GMC to guard cell (Lai et al. 2005, Ohashi-Ito and Bergmann 2006). Unlike flp plants that produce parallel rows of stomata, fama plants fail to express mature guard cell markers, indicating that FAMA is required for guard cell differentiation (Ohashi-Ito and Bergmann 2006). Among the three known stomatal differentiation bHLH proteins, MUTE is capable of directing the differentiation of complete mature stomata when constitutively overexpressed, whereas the overexpression of SPCH or FAMA produces increased asymmetric divisions and single guard cells, respectively (Ohashi-Ito and Bergmann 2006, MacAlister et al. 2007, Pillitteri et al. 2007). This makes $M U T E$ an excellent tool for investigating the mechanisms of stomatal formation.

Although stomatal density and number can vary, stomatal spacing is stable and results in stomata being separated from one another by at least one intervening cell (one-celled spacing rule) (Sachs 1991, Nadeau and Sack 2002b). This spacing pattern is controlled by positiondependent intercellular communication, which directs the position and orientation of divisions across the epidermal surface (Nadeau and Sack 2003, Bergmann 2006). Numerous signaling components have been implicated in this process. These include a small secreted protein

*Corresponding author: E-mail, ktorii@u.washington.edu; Fax, +1-206-685-1728. 
EPIDERMAL PATTERNING FACTOR1 (EPF1), a subtilisin-like protease STOMATAL DENSITY AND DISTRIBUTION (SDD1), a transmembrane receptor-like protein TOO MANY MOUTHS (TMM), a family of receptor-like kinases ERECTA (ER), ERECTA-LIKE1 (ERL1) and ERL2, and several genes of a mitogenactivated kinase cascade, including YODA, MPK3/MPK6 and MKK5/MKK4 (Berger and Altmann 2000, Geisler et al. 2000, von Groll et al. 2002, Bergmann et al. 2004, Shpak et al. 2005, Hara et al. 2007, Wang et al. 2007). Loss of function of many of these components leads to an increase in and misorientation of epidermal divisions, which ultimately results in a breakdown of the one-cell spacing rule and the production of adjacent stomata. Conversely, overexpression or gain of function in EPF1, YODA and mitogen-activated protein (MAP) kinases reduce stomatal production, indicating that these signaling components inhibit the entry into the stomatal lineage (Bergmann et al. 2004, Hara et al. 2007, Wang et al. 2007).

It has been well established that many genes involved in stomatal spacing follow organ-specific rules. For instance, while loss-of-function tmm plants produce stomatal clusters in organs such as leaves, stomata are absent in hypocotyls and stems (Yang and Sack 1995, Geisler et al. 1998, Geisler et al. 2000). The er-family and yoda mutants also have organ-specific effects with varied stomatal cluster size in different organs (Shpak et al. 2005, Bergmann 2006). Detailed combination mutant analysis between tmm and the er-family has revealed intricate and complex stomatal development phenotypes which vary in different organs (Shpak et al. 2005). Additional organ-specific rules for patterning have been identified in the embryonic stem or hypocotyl (Berger et al. 1998). Numerous genes including TRANSPARENT TESTA GLABRA (TTG) and GLABRA2 (GL2), which encode a WD-40 and HD-ZIP protein, respectively, have been shown to be required for patterning stomata in specific non-protruding cell files of the hypocotyl, but do not impact stomatal patterning in nonembryonic organs (Berger et al. 1998, Hung et al. 1998, Schiefelbein 2003). Therefore, the mechanisms by which plants pattern and distribute stomata across the epidermal surface are complex and are not consistent throughout the life cycle of the plant or among different organs.

To date, there has not been a detailed examination of the organ-specific or genotype-specific regulation of the stomatal cell specification genes ( $S P C H, M U T E$ and $F A M A$ ). In this study, we investigated (i) whether the master regulator of GMC differentiation, MUTE, has a consistent role in stomatal differentiation independent of organ type or genetic background; (ii) whether ectopic MUTE activity is sufficient to over-ride cell fate specification in cell types that do not normally differentiate stomata; and (iii) whether MUTE function is conserved in the production of the structure evolutionarily related to the stoma, the hydathode pore. We show that MUTE functions as a master regulator of GMC/stomata differentiation in all aerial organs and related stomatal structures. However, we reveal that the requirement for MUTE could be circumvented under conditions of compromised inhibitory signaling.

\section{Results}

MUTE is required for stomata differentiation in all organs

To determine whether MUTE is absolutely required for stomatal differentiation regardless of the organ type, we examined the effect of MUTE loss of function (mute-1) in all organs that produce stomata. As described previously in Pillitteri et al. (2007), the original mute allele (mute-1) produces aberrant transcripts, all of which produce a premature stop codon within the bHLH domain. The phenotype of the T-DNA insertion allele, mute-2, which produces no detectable transcript, is indistinguishable from that of mute-1 (data not shown). This indicates that both alleles are complete loss of function. We observed the production of aborted meristemoids surrounded by excessive SLGCs in embryonic organs, cotyledons and hypocotyls, as well as all post-embryonic organs that normally produce stomata, including leaves, stems, pedicels and floral organs (Fig. 1). Normal stomatal cell lineage patterning was maintained in the hypocotyls of mute plants, where we observed aborted meristemoids only in non-protruding cell files (Fig. 1D). There was no detectable mute phenotype in roots or petals, which do not normally produce stomata (data not shown). These data suggest that MUTE has an elemental role in stomatal cell lineage determination (the transition from meristemoid to GMC) regardless of organ identity.

MUTE is transiently expressed in all organs that produce stomata

To examine organ-specific effects on the spatial and temporal expression pattern of MUTE, we utilized a construct consisting of $1.9 \mathrm{~kb}$ of the MUTE promoter region driving the expression of $\beta$-glucuronidase (MUTEpro::GUS) or of a translational fusion of the fulllength MUTE protein and green fluorescent protein (GFP; MUTEPro::MUTE-GFP). The MUTEpro::MUTE-GFP construct is nuclear localized and has been demonstrated to rescue the mute phenotype (Pillitteri et al. 2007). In both vegetative and floral organs, MUTEpro:GUS and MUTEPro::MUTE-GFP were similarly expressed in a subset of meristemoids (Fig. 2). While expression of MUTEpro:GUS was observed in meristemoids, GMCs and mature stomata (Fig. 2A-G), the MUTEpro::MUTE$G F P$ protein product was transient and disappeared quickly 

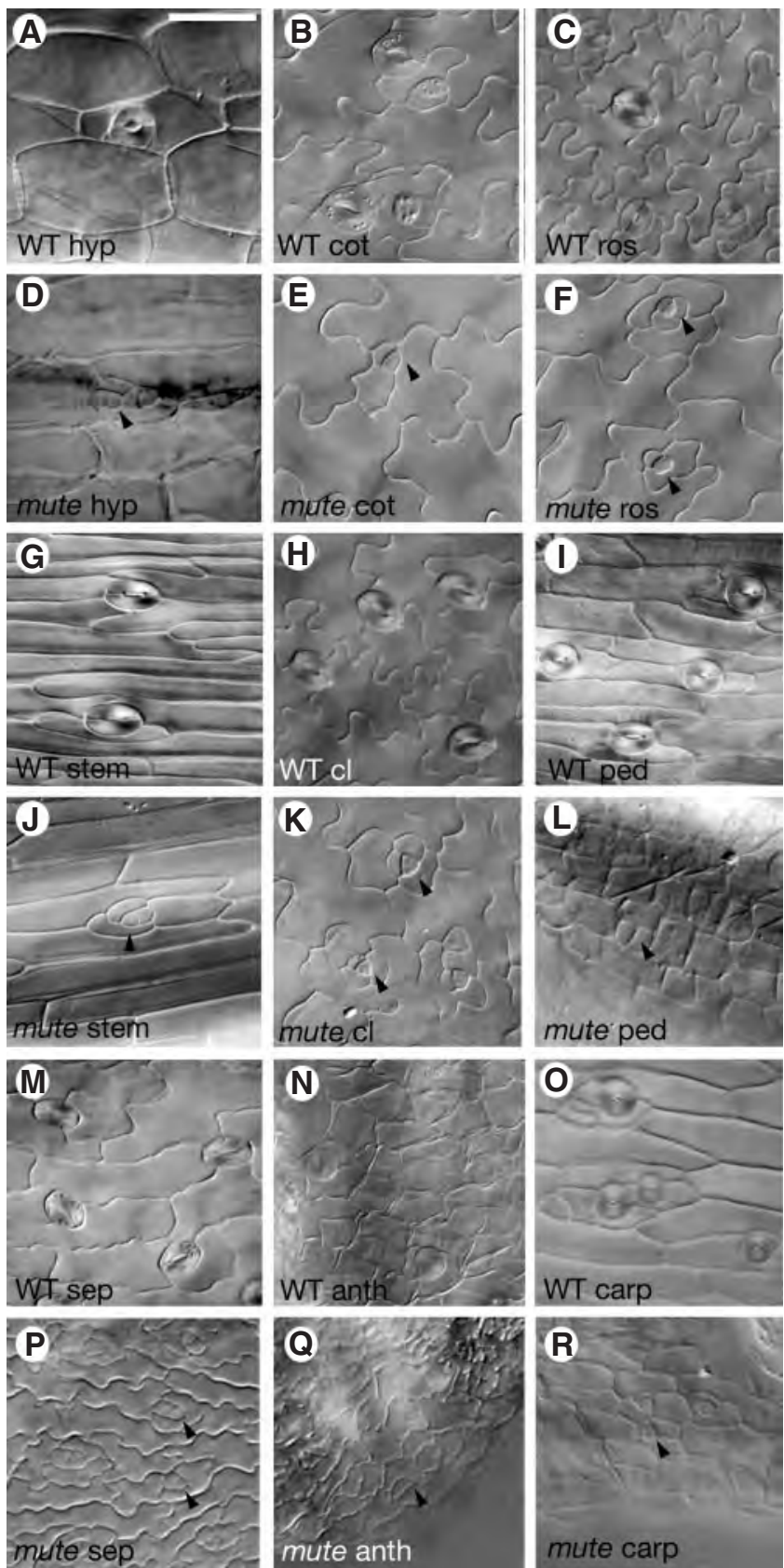

Fig. 1 Loss-of-function mute phenotype in diverse aerial organs. Comparison of wild type (WT; A-C, G-I, M-O) and mute (D-F, J-L, $\mathrm{P}-\mathrm{R})$ in hypocotyls, hyp $(\mathrm{A}, \mathrm{D})$, cotyledons, $\cot (\mathrm{B}, \mathrm{E})$, rosette leaf, ros $(C, F)$, stem $(G, J)$, cauline leaf, $c l(H, K)$, pedicle, ped $(I, L)$, sepal, sep $(M, P)$ anther, anth $(N, Q)$ and carpel, carp $(\mathrm{O}, \mathrm{R})$. No stomata are produced in the mute mutant. Arrowheads indicate examples of aborted meristemoids. Scale bar, $20 \mu \mathrm{m}$.

from GMCs, and was never observed in guard cells (Fig. 2I-L). No MUTE expression was detected in other actively dividing areas such as the root tip (Fig. $2 \mathrm{H}$ ) or in internal tissue layers (data not shown). The spatial and temporal expression of MUTE suggests that it serves as a trigger for GMC differentiation in all stomata-producing organs.

\section{MUTE expression is not mediated by autoregulation}

Positive autoregulation is a common mechanism by which many transcription factors regulate and stabilize their own expression. To establish if MUTE is required to regulate its own expression, we introduced MUTEpro::GUS into mute plants (Fig. 3). Most meristemoids in mute expressed the MUTEpro::GUS reporter (Fig. 3B), but no expression was observed in any other epidermal cell or internal tissue. No qualitative decrease or increase of MUTE promoter activity in the mute background was observed in comparison with the wild type. This suggests that autoregulation is not a factor in the activation or maintenance of MUTE expression during meristemoid to GMC transition.

Ectopic expression of MUTE can over-ride cell fate in nonstomatal producing organs

To investigate whether MUTE is sufficient to induce stomatal fate in organs or cell types that do not normally undergo stomatal development, we overexpressed MUTE using the cauliflower mosaic virus (CaMV) 35S promoter (35S::MUTE). Although many plants that overexpress MUTE are severely compromised and do not bolt, some plants do enter reproductive development and produce flowers. From this group of plants, we analyzed the epidermal phenotype of the petal (Fig. 4A, B). In wildtype plants, both surfaces of the petal lack stomatal lineage cells and stomata (Bowman 1993). Petal development in $35 S: \because M U T E$ plants was disrupted; petals were significantly shorter than those of the wild type, leaving the carpel exposed prior to anthesis (data not shown). Microscopic examination revealed that $35 S: \because M U T E$ petals produced significant numbers of stomata, which were often clustered (Fig. 4B). We did not observe any obvious asymmetric divisions, and some of the ectopic stomata retained the lobed shape of a petal cell, suggesting that ectopic MUTE expression in the petal probably converted those cells directly into stomata without initiation of a stomatal lineage. Ectopic stomata were not found in the root epidermis and internal tissues in either strong or weak $35 S:: M U T E$ lines (data not shown). This implies that only the aerial epidermis is competent to respond to ectopic MUTE activity to trigger stomatal differentiation.

MUTE is downstream of hypocotyl patterning genes and is sufficient to over-ride hypocotyl-specific stomatal patterning

Stomatal patterning in the hypocotyl is controlled in part by a suite of genes, including GL2 and TTG (Berger et al. 1998, Hung et al. 1998, Schiefelbein 2003). Lossof-function $g l 2$ or $\operatorname{tg} 1$ plants produce stomata in both 

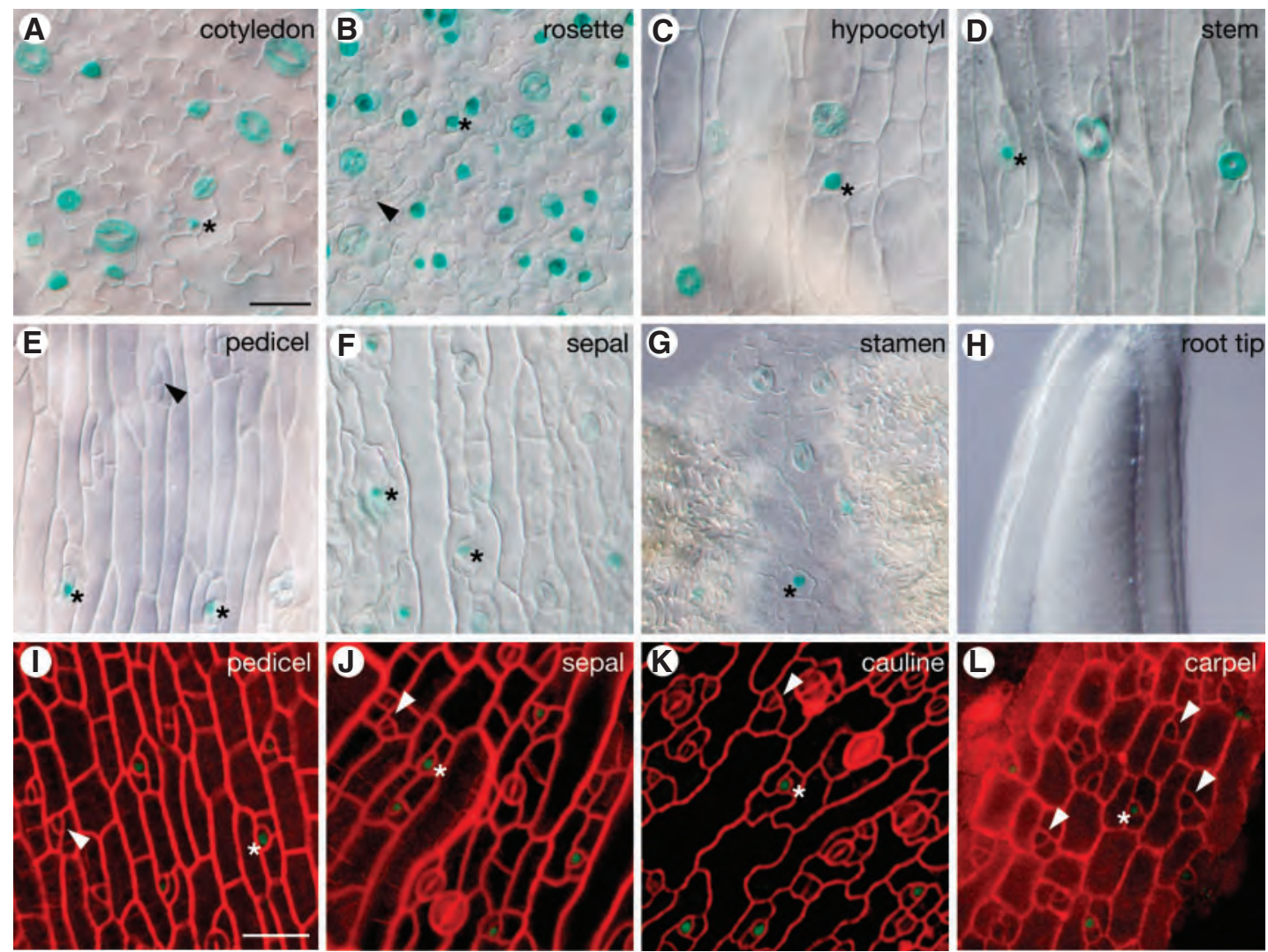

Fig. 2 MUTE gene expression. (A-H) MUTEpro::GUS reporter expression in cotyledons (A), rosette leaf (B), hypocotyls (C), stem (D), pedicel (E), sepal (F), stamen (G) and root tip (H). No MUTE expression is detected in roots. (I-L) Confocal images of MUTEpro::MUTE-GFP protein fusion in pedicel $(\mathrm{I})$, sepal $(\mathrm{J})$, cauline leaf $(\mathrm{K})$ and carpel $(\mathrm{L})$. Tissue for confocal microscopy is stained with propidium iodide to highlight cell borders. Asterisks indicate examples of meristemoids expressing MUTE. Arrowheads indicate examples of meristemoids not expressing MUTE. Scale bar, $20 \mu \mathrm{m}$.
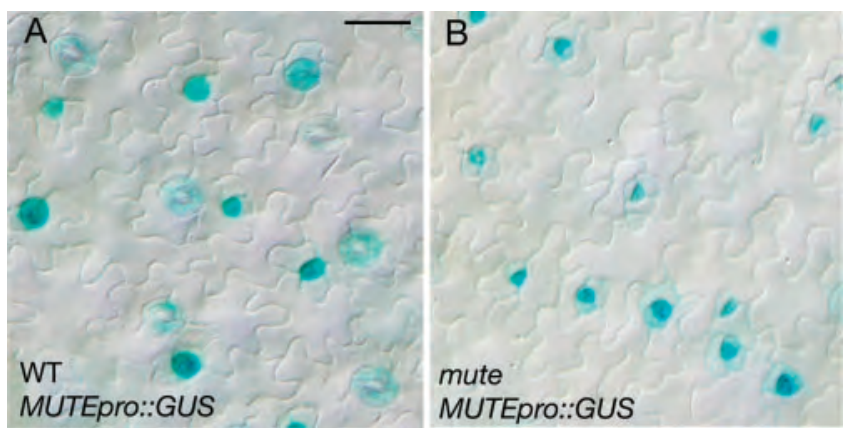

Fig. 3 MUTE is not under autoregulatory control. (A, B) MUTEpro::GUS reporter expression in the leaf abaxial epidermis of 14-day-old seedlings of wild type (A) and mute (B). Scale bar, $20 \mu \mathrm{m}$.

protruding and non-protruding cell files of hypocotyls, unlike the wild type, which restricts stomatal development to only non-protruding cell files (Hung et al. 1998). To determine if MUTE activity is necessary for stomatal production in both hypocotyl cell files, we introduced the mute mutation into either the $g l 2$ or $\operatorname{ttg} 1$ mutant background and examined the phenotype of the hypocotyl. Wild-type plants produced stomata only in non-protruding cell files (Fig. 4C). In contrast, ttgl or gl2 mutant plants initiate stomata in both protruding and non-protruding cell files (Fig. 4D, E). Both ttg1; mute and gl2; mute double mutants produced a mute phenotype consisting of aborted meristemoids in both cell files of the hypocotyl (Fig. 4F, G). This indicated that MUTE acts downstream of the organspecific patterning genes in hypocotyls. We never observed the production of stomata in hypocotyls of double mutant plants, indicating that mute and hypocotyl patterning genes interact additively and that MUTE is absolutely necessary for stomatal differentiation in the hypocotyls.

To determine if MUTE activity is sufficient to over-ride the position-dependent epidermal cell patterning of the hypocotyl, we tested whether ectopic MUTE expression $(35 S: \because M U T E)$ triggers stomatal differentiation in both hypocotyl cell files. The $35 S: \because M U T E$ construct caused the complete conversion of all cells in both files into stomata (Fig. 4J). The conversion of stomata in protruding cell files 

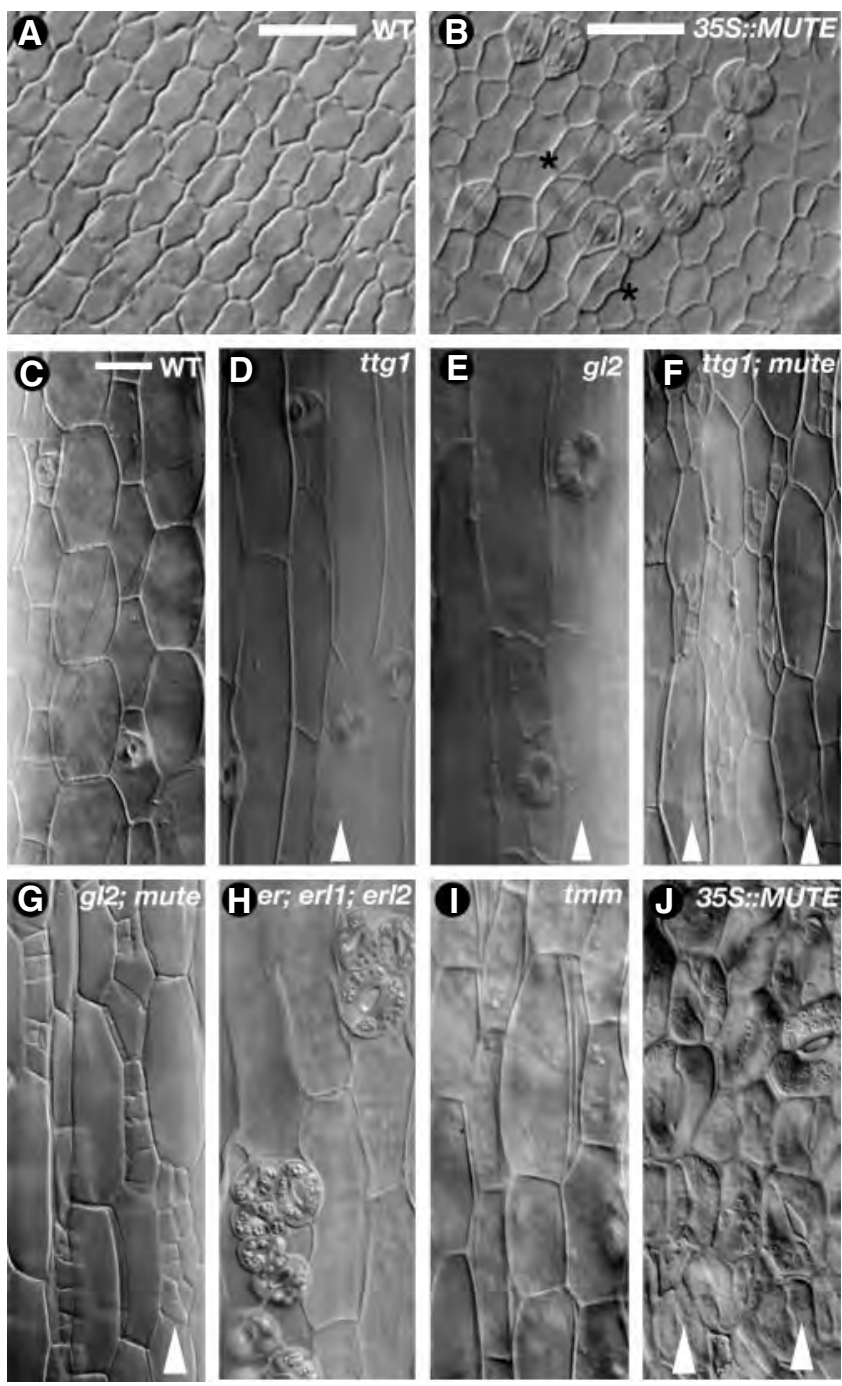

Fig. 4 Ectopic overexpression of MUTE over-rides organ- and cell file-specific rules. (A, B) DIC image of wild type (A) and 35S::MUTE (B) petal epidermis. The asterisk indicates aberrant shaped stomata. (C-J) Hypocotyl epidermis of WT (C), ttg (D), g/2 (E), ttg; mute (F), gl2; mute (G), er; erl1; erl2 (H), tmm (I), 35S::MUTE (J). Arrowheads indicate protruding cell files with ectopic stomata or meristemoids. Scale bar, $20 \mu \mathrm{m}$.

was specific to MUTE overexpression and was not a general characteristic of plants that produce excessive stomata. For instance, er; erl1; erl2 mutant plants did not violate cell patterning specification in hypocotyls (Fig. 4H). The stomata of 35S:MUTE plants were much larger than those of the wild type, suggesting that similar to petals, ectopic expression of MUTE converted cells directly into stomata without initiation of asymmetric division (Fig. 4J). Taken together, our analysis indicates that MUTE functions downstream of hypocotyl patterning genes and is sufficient to trigger stomatal differentiation without initiation of a stomatal lineage.
Release of inhibitory patterning signals can circumvent the requirement for MUTE

Both the expression patterns and loss-of-function phenotype of MUTE indicate that MUTE acts downstream of the negative regulators $T M M, E R$ family and $Y D A$. Consistently, there is no disruption of entry or spacing divisions in the mute mutant (Supplementary Fig. S1). To understand better how inhibitory signals may integrate into the stomatal differentiation pathway, we conducted a detailed phenotypic analysis of combination mutants tmm; mute and er; erl1; erl2; mute. While tmm and er; erl1; erl2 plants produce clustered stomata in leaves (Fig. 5A, C), both tmm; mute and er; erll; erl2; mute plants produced clusters of meristemoid-like cells in leaves and most other aerial organs, indicating an additive interaction (Pillitteri et al. 2007) (Fig. 5D, E and data not shown). As previously described, er; erl1; erl2 plants produce clustered stomata in stems, whereas tmm mutants produce no stomata in stems (Geisler et al. 1998, Shpak et al. 2005) (Fig. 5F, H). No stomata or meristemoids were observed in tmm; mute stems, whereas clusters of meristemoid-like cells were observed in er; er 1l; erl2; mute stems (Fig. 5I, J). Surprisingly, unlike the mute single mutant, which never produces stomata (Fig. 5B, G), we observed that a small number of meristemoids in tmm; mute and er; er 1l; erl2; mute can differentiate into a stoma (Fig. $5 \mathrm{~K}, \mathrm{~L}$ ). This was also true for yda; mute (data not shown). These stomata expressed the mature stomata GFP marker E994 (Fig. 5M-O). Although some rescue of the mute growth phenotype was observed in tmm; mute plants, these plants were still pale in color, stunted and did not produce seed. We quantified the number of stomata and arrested meristemoid-like cells per cotyledon in mutant backgrounds, and observed that the number of stomata produced in tmm; mute or er; erll; erl2; mute was between 0.3 and $1.3 \%$ of the number of meristemoids (Table 1 ). In contrast, no stomata are produced in the mute mutant alone (Table 1). We did not observe any obvious relationship between a converted meristemoid and its location within a meristemoid cluster (i.e. edge or center) in tmm; mute or er; erll; erl2; mute. These data suggest that when the signaling pathway directing stomatal patterning is compromised, the absolute requirement for MUTE can be circumvented.

\section{MUTE acts as a key regulator of hydathode pore development}

Hydathode pores are oversized stomatal structures that are incapable of regulating pore aperture and therefore remain permanently open to release water and dissolved solutes from the xylem (Esau 1977). To understand the role of MUTE in the production of these modified stomata, we examined MUTE expression in hydathodes. Using the MUTEpro::GUS reporter, promoter activity was first observed at the early torpedo stage at the very tips of 

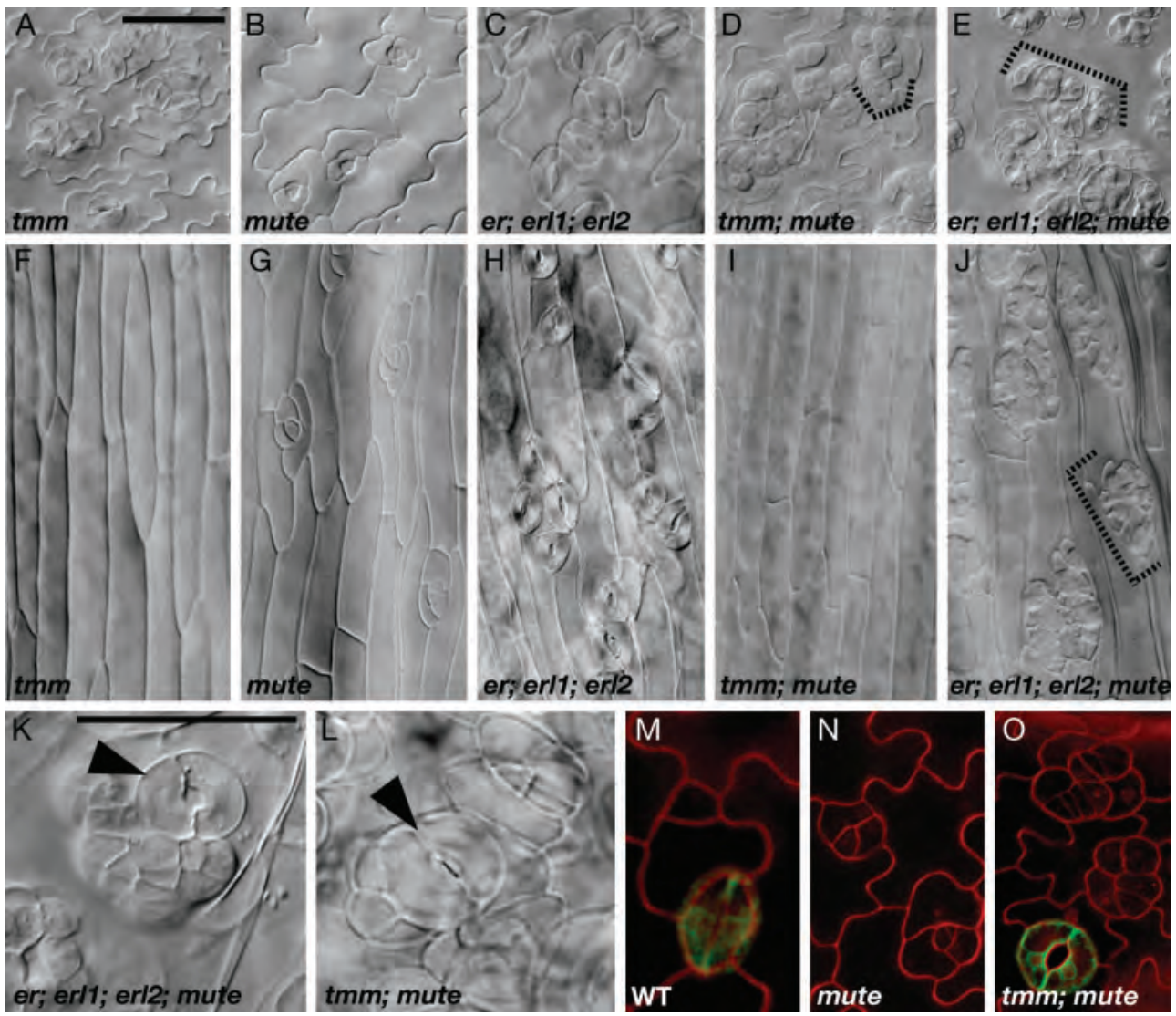

Fig. 5 Negative regulation of stomatal differentiation by stomatal spacing genes. (A-E) DIC images of abaxial epidermis of rosette leaves of tmm (A), mute (B), er; erl1; erl2 (C), tmm; mute (D) and er; erl1; erl2; mute (E). (F-J) DIC images of stem of tmm (F), mute (G), er; erl1; erl2 $(\mathrm{H}), \mathrm{tmm}$; mute (I) and er; erl1; erl2; mute (J). Dashed brackets highlight meristemoid-like cell clusters. DIC image of a stoma on the abaxial rosette leaf epidermis (arrowhead) in er; erl1; erl2; mute (K) and tmm; mute (L). (M-O) Confocal image of a stoma on the abaxial rosette leaf expressing the mature stomata GFP marker E994 in the wild type (M), mute (N) and tmm; mute (O). Scale bar, $20 \mu \mathrm{m}$.

Table 1 Estimated total number of stomata and meristemoids on the abaxial surface of cotyledons of stomatal mutants

\begin{tabular}{lccrr}
\hline \multirow{2}{*}{ Genotype } & \multicolumn{2}{c}{ Stomata/cotyledon } & \multicolumn{2}{c}{ Meristemoid/cotyledon } \\
\cline { 2 - 4 } & Cotyledon 1 & Cotyledon 2 & Cotyledon 1 & Cotyledon 2 \\
\hline Wild type & 890.5 & 722.9 & 26.2 & 11.8 \\
tmm & $4,000.6$ & $3,946.9$ & 247.1 & 332.7 \\
er; erl1; erl2 & $2,358.7$ & $2,673.2$ & 317.1 & 282.1 \\
mute & 0 & 0 & 733.9 & 463.7 \\
tmm; mute & $1^{a}$ & $6^{a}$ & $1,869.7$ & $2,357.7$ \\
er; erl1; erl2; mute & $2^{a}$ & $13^{a}$ & $9,133.8$ & $4,517.8$
\end{tabular}

The numbers of stomata and meristemoids were estimated from the abaxial surface of 14-day-old cotyledons. The numbers of stomata or meristmoids were counted in three separate regions of each cotyledon. Total numbers from each region were averaged and extrapolated to estimate the total number/cotyledon.

${ }^{a}$ The numbers are not estimates, but the actual number of stomata observed on the entire cotyledon.

the cotyledon, where the hydathode originates (Fig. 6A). In emerging leaves, the same expression pattern was observed, with MUTE expression first appearing at the hydathode origin at the very tip of the leaf (Fig. 6B). Water and solutes exiting the leaf from the xylem empty through densely packed mesophyll cells called the epithem to the hydathode pore in a process called guttation (Esau 1977). Wild-type plants differentiated hydathode pores which were much larger than typical stomata (Fig. 6C; Supplementary Fig. S2); however, mute plants did not differentiate 


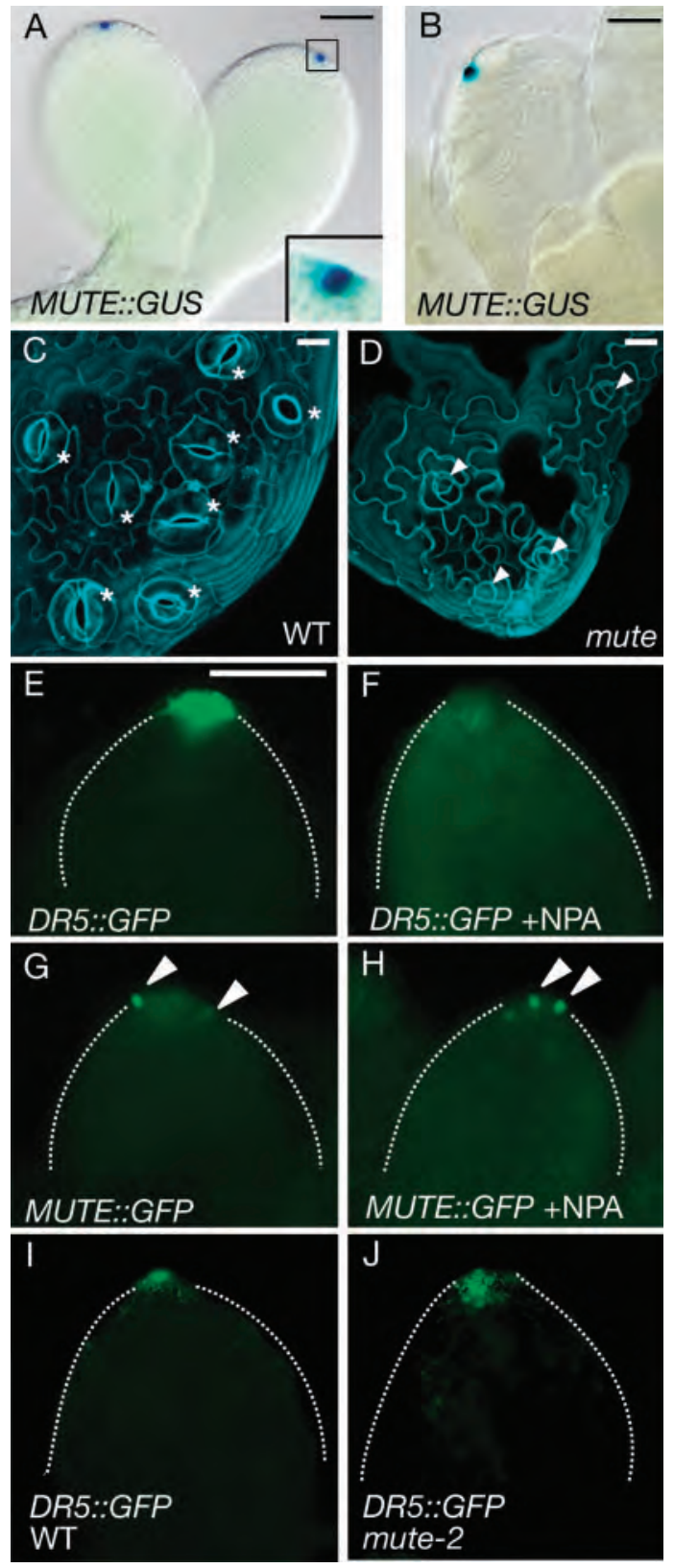

Fig. 6 Effects of mute on hydathode development. (A, B) MUTEpro::GUS reporter expression in a wild-type torpedo-stage embryo (A) and developing rosette leaf (B). Promoter activity is detected at the tip where hydathodes initiate. The inset in A is an optical magnification of the region outlined, showing MUTE expression in a single cell. (C, D) Confocal image of a rosette leaf of wild type (C) and mute (D) hydathode pores. Asterisks indicate hydathode pores; arrowheads indicate undifferentiated hydathode pores. (E-H) Image of a developing wild-type leaf expressing the DR5pro::GFP reporter without $(\mathrm{E})$ and with $(\mathrm{F}) \mathrm{NPA}$ treatment or the MUTEpro::GFP reporter without $(\mathrm{G})$ or with $(\mathrm{H})$ NPA treatment. Arrowheads indicate early MUTE expression. MUTE promoter activity is not affected by disruption of auxin transport. (I, J). Image of a developing wild-type leaf (I) and mute-2 leaf (J) expressing DR5pro::GFP. Leaves are outlined with white dashed lines for clarity. Scale bar, $100 \mu \mathrm{m}(\mathrm{A}, \mathrm{B}, \mathrm{E}-\mathrm{J})$ and $20 \mu \mathrm{m}$ in all other panels. hydathode pores. Instead, the characteristic mute phenotype of an aborted meristemoid surrounded by SLGCs was observed (Pillitteri et al. 2007) in place of hydathode pores (Fig. 6C, D). mute plants did differentiate normal epithem at the end of xylem strands similar to wild-type plants (Supplementary Fig. S2). Based on our observations, hydathode pore formation did not occur prior to germination, suggesting that MUTE acts as a determination marker for cells that will eventually differentiate into the hydathode pore.

Aloni (2001) proposed that auxin synthesis sites are the main factors regulating vascular differentiation during development and that hydathodes are the main regions of auxin synthesis during leaf morphogenesis. Initial auxin maxima coincide with the onset of MUTE expression (prior to maturation of the hydathode pore). To determine if MUTE expression is affected by disruption of auxin maxima, we examined the effects of the auxin transport inhibitor, naphthylphthalamic acid (NPA) on MUTE expression. The NPA treatment caused mislocalization of auxin-responsive DR5pro::GFP expression but no change in the expression of MUTEpro::GFP (Fig. 6E-H). Therefore, while early MUTE expression is coincidental with early auxin maxima, the MUTE promoter is not inherently regulated by auxin. We next tested whether the absence of the hydathode pores in mute leaves affects auxin maxima at the tip. As shown in Fig. 6I and J, DR5pro::GFP expression patterns were not altered in the mute-2 background, indicating that the hydathode pore is not required for initiation or maintenance of auxin maxima in emerging leaves. Consistent with this, the mute mutant shows no obvious vascular defects (data not shown).

\section{Discussion}

MUTE is both necessary and sufficient to direct stomatal differentiation in the epidermis of all aerial organs

Stomatal number and distribution vary among different organs and can also vary within an organ (Yang and Sack 1995, Nadeau and Sack 2002a). Despite this variation, a consistent feature of stomatal development in both embryonic and non-embryonic organs is the requirement for $M U T E$ in the transition from meristemoid to GMC (Fig. 1). MUTE expression is observed in a subset of meristemoids that are probably 'final-stage', and is downregulated in GMCs (Fig. 2), indicative of MUTE being a marker for cell fate transition and not a meristemoid marker per se (Pillitteri et al. 2007). mute mutants produce meristemoids surrounded by an excessive number of SLGCs, implicating that without a trigger for transition, meristemoids remain in a self-renewal cycle until spatial restriction inhibits further division. The mechanistic 
similarities of the stomatal bHLHs (SPCH, MUTE and FAMA) to the myogenic bHLHs (MyoD, myogenin, MRF4 and MYF5) was described previously (MacAlister et al. 2007, Pillitteri et al. 2007). Autoregulation during myogenesis contributes to myogenic commitment and inhibits transdifferentiation during muscle development (Thayer et al. 1989, Shimokawa et al. 1998). Unlike myogenesis, maintenance of MUTE expression may not be likely to include an autoactivation mechanism as has been described for myogenic bHLH proteins (Thayer et al. 1989). Similar to our findings, Ohashi-Ito and Bergmann (2006) described that the stomatal bHLH, FAMA, does not regulate its own expression. Both MUTE and FAMA expression are transient (Ohashi-Ito and Bergmann 2006, Pillitteri et al. 2007). The transition from meristemoid to GMC (MUTE) or from GMC to guard cell (FAMA) can occur in as little as $24 \mathrm{~h}$ (Geisler et al. 2000). Therefore, stabilization of the MUTE or FAMA signal may not be required. In fact, due to the transient nature of cell states within the stomatal lineage, quick turnover may be critical for proper development of stomata.

In this study, we used the ubiquitous CaMV 35S promoter to determine if MUTE expression was sufficient to convert cells directly into stomata and over-ride cell fate specification in organs that do not normally differentiate stomata. From our observations, the cells of both the petal and the protruding files of the hypocotyl are capable of responding to the action of MUTE even though they have not undergone any obvious asymmetric divisions typical of the stomatal lineage (Fig. 4). These data imply that either (i) MUTE alone can actively turn on stomatal differentiation genes either directly or indirectly; or (ii) partners of MUTE are expressed in these cell types and can activate differentiation only in the presence of MUTE.

Interestingly, while examining the requirement for MUTE in the hypocotyl, we observed that unlike $g / 2$ or ttgl alone, hypocotyl cells in gl2; mute and ttgl; mute become highly divided (Fig. 4), similar to what has been observed by the constitutive expression of CYCD4;2 in hypocotyls (Kono et al. 2007). CYCD4;2 has been shown to be involved in the proliferation of stomatal precursors in hypocotyls, but not in leaves or other aerial organs (Kono et al. 2007). It is intriguing to speculate that hypocotyl patterning genes such as GL2 and TTG inhibit the continued action of $C Y C D 4 ; 2$ after stomatal differentiation. However, in gl2; mute or ttg1; mute double mutants, stomatal differentiation is inhibited, resulting in the continued expression of $C Y C D 4 ; 2$ and proliferation of cell divisions. Further experiments to test the link between hypocotyl epidermal patterning genes and the cell cycle regulators are necessary to clarify this hypothesis.

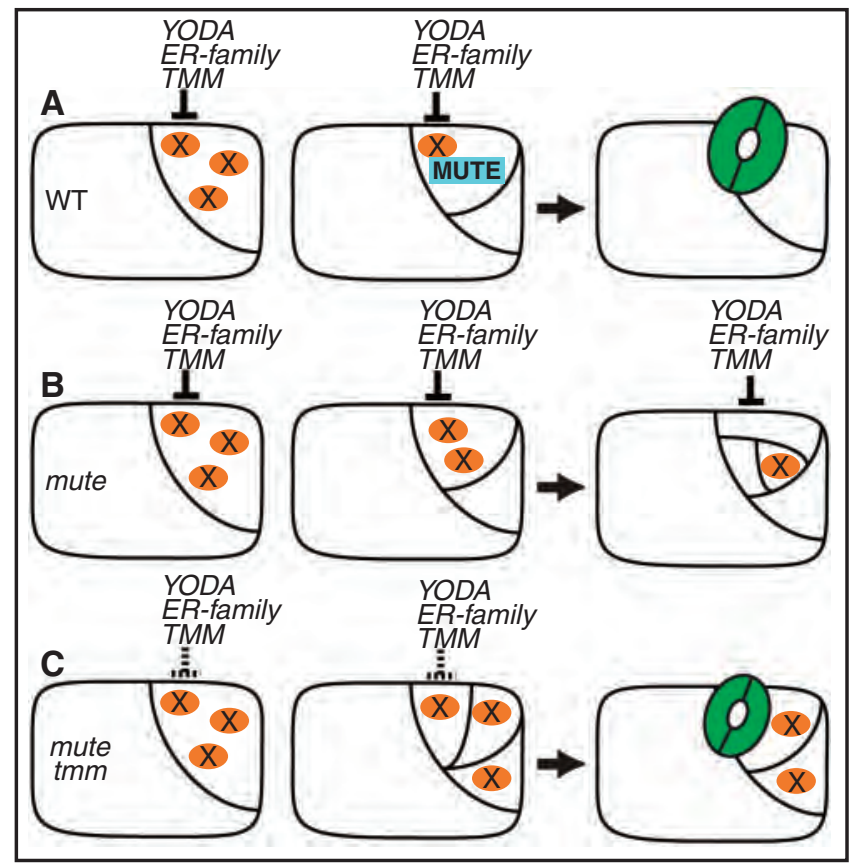

Fig. 7 Proposed model for inhibitory regulation of stomatal differentiation. (A) The signal transduction cascade through YODA, the ER-family and TMM inhibits the action of a meristemoid-specific factor ' $X$ ', which requires MUTE to direct stomatal differentiation. In mute (B), the signal transduction cascade exerts inhibition on factor ' $X$ ' and, with no MUTE protein present, stomatal differentiation does not take place. Under conditions of reduced inhibitory signaling and MUTE loss of function $(C)$, the inhibition on factor ' $X$ ' is lifted and, even in the absence of MUTE, can restore a minimal amount of stomatal differentiation.

Integrating inhibitory signals into the stomatal differentiation pathway

The number of cells entering the stomatal lineage is controlled by inhibitory cell-cell signals. This network controls the number of stomata produced by regulating the number of entry divisions, amplifying divisions and the orientation of spacing divisions (Yang and Sack 1995, Bergmann et al. 2004, Shpak et al. 2005). Under wild-type conditions, MUTE is absolutely required to produce stomata. However, we uncovered the surprising finding that under conditions of compromised cell-cell signaling, such as in tmm, er; erll; erl2 or $y d a$, the MUTE requirement can be partially circumvented. The loss of MUTE function in combination with tmm or er; erll; erl2 mutants resulted in the randomization of cellular divisions and clustered meristemoid-like cells, with the rare production of single stomata (Fig. 5). Relief of inhibitory regulation on a downstream target of MUTE could cause this phenotype. Alternatively, reduced cell-cell signaling could alleviate inhibitory regulation on a protein that works in parallel with MUTE, but requires MUTE activity to function efficiently (Fig. 7). In the latter scenario, the loss of 
inhibitory signaling would increase the activity or amount of the parallel protein and therefore restore some stomatal differentiation programs, albeit a small amount, even in the absence of MUTE. The identification of additional proteins regulating stomatal fate transitions is necessary to understand and determine fully the mechanism underlying the circumvention of MUTE.

Early MUTE expression is indicative of hydathode pore initiation

During the early stages of leaf development, hydathodes are a center for free auxin production (Aloni et al. 2003). It has been demonstrated that mature guard cells and hydathodes can share similar gene expression patterns and are both producers of free auxin (Jacobs and Roe 2005). We demonstrated here that hydathode pores and stomata also share a similar developmental pathway, with MUTE being required for their differentiation. Previous work has indicated that auxin accumulation at the leaf tip during morphogenesis is responsible for the differentiation of the midvein as proposed by the leaf venation hypothesis (Aloni 2001). Initial auxin accumulation is coincidental with the onset of MUTE expression. In the wild type, free auxin production from the mature hydathode lasts for a substantial time (Aloni et al. 2003) and is thought to maintain production of new leaf veins. Our data indicate that MUTE activity is required for the production of the hydathode pore; however, the production of the pore structure itself is not required for auxin accumulation at leaf tips.

\section{Materials and Methods}

\section{Plant material}

The Arabidopsis thaliana accession Columbia $(\mathrm{Col})$ was used as the wild type. All mutants are in the Col background except ttg-1 [CS89, from the Arabidopsis Biological Resource Center (ABRC)], which is in the Landsberg erecta background. The gl2-1 seeds were obtained from ABRC. The mutants er, erll and erl2 refer to the knockout er-105, erl1-2 and erl2-1 alleles, respectively. Unless stated otherwise, the mute-1 allele was used for all phenotypes and expression in this study, and was described peviously (Pillitteri et al. 2007). The mute-2 allele is a T-DNA insertion mutant (FLAG 225D03) obtained from the Institut National de la Recherche Agronomique (INRA, France). All mutant combinations were produced by crossing with mute/+ plants. The DR5pro::GFP seed were a generous gift from Dr. Jiri Friml (Ghent, Belgium). Plants were grown under long day conditions $(18 \mathrm{~h} / 6 \mathrm{~h}$, day $/$ night $)$ at $21^{\circ} \mathrm{C}$.

\section{Histochemical staining and microscopy}

Histochemical staining for GUS activity was performed as described previously (Sessions et al. 1999). To examine tissue using differential interference contrast (DIC) optics, tissue was incubated in $9: 1(\mathrm{v} / \mathrm{v})$ ethanol: acetic acid overnight, rinsed with water and placed in choral hydrate $(1: 8: 1$ by vol. glycerol: chloral hydrate: water). GUS-stained samples were taken through an ethanol series to remove chlorophyll and placed in chloral hydrate for $\sim 1 \mathrm{~h}$. All samples were viewed under the DIC optics using an Olympus (Center Valley, PA, USA) BX51 microscope equipped with a DP70 digital camera. Confocal images were taken using the Zeiss (Thornwood, NY, USA) META 510 confocal microscope. Cell borders were visualized using propidium iodide (Molecular Probes, Carlsbad, CA, USA). Tissue for confocal imaging was incubated in $20 \%(\mathrm{v} / \mathrm{v})$ propidium iodide solution for $10 \mathrm{~min}$ prior to visualization.

\section{Construction of plasmids and transgenic plants}

The MUTEpro::GUS and MUTEPro::MUTE-GFP constructs (pLJP38 and pLJP155, respectively) were described previously (Pillitteri et al. 2007). For 35S::MUTE-4xMYC (pLJP209), pLJP135 containing the MUTE promoter and an open reading frame lacking the stop codon in TOPO-D/pENTR (Invitrogen, Carlsbad, CA, USA) was combined with GWB14 (Nakagawa et al. 2007) in an LR Clonase reaction to produce the vector containing the $\mathrm{C}$-terminal $4 \times$ MYC-tagged MUTE under control of the $35 \mathrm{~S}$ CaMV promoter. For MUTEpro::GFP (pLJP220), pLJP219 containing the MUTE promoter in TOPO$\mathrm{D} / \mathrm{pENTR}$ (Invitrogen) was combined with GWB4 (Nakagawa et al. 2007) in an LR Clonase reaction to produce the vector containing the MUTE promoter driving expression of full-length GFP. All plasmids were transformed into Agrobacterium strain GV3101 by electroporation. Transformation of Arabidopsis by vacuum infiltration and selection of transgenic plants were performed as described by Shpak et al. (2003).

\section{Auxin inhibitor assay}

NPA (Chem Services, Westchester, PA, USA) was added to standard Murashige and Skoog (MS) media at a final concentration of $20 \mu \mathrm{M}$. DR5pro::GFP and MUTEpro::GFP plants were germinated on non-selective media for $7 \mathrm{~d}$ and transferred to NPAsupplemented media for $48 \mathrm{~h}$. Control plants were maintained on non-selective MS media.

\section{Supplementary material}

Supplementary material mentioned in the article is available to online subscribers at the journal website www.pcp. oxfordjournals.org.

\section{Funding}

The National Science Foundation (IOB0520548 to K.U.T.); the Department of Energy (DG-FG02-03ER15448 to K.U.T.); United States Department of Agriculture Cooperative State Research, Education, and Extension Service postdoctoral fellowship (to L.J.P.).

\section{Acknowledgments}

We thank Dr. Jiri Friml and ABRC (Ohio State University) for proDR5::GFP and $g l 2-1$ seeds, and Dr. Tsuyoshi Nakagawa for transformation vectors.

\section{References}

Aloni, R. (2001) Foliar and axial aspects of vascular differentiation: hypotheses and evidence. J. Plant Growth Regul. 20: 22-34. 
Aloni, R., Schwalm, K., Langhans, M. and Ullrich, C.I. (2003) Gradual shifts in sites of free-auxin production during leaf primordium development and their role in vascular differentiation and leaf morphogenesis. Planta 216: 841-853.

Berger, D. and Altmann, T. (2000) A subtilisin-like serine protease involved in the regulation of stomatal density and distribution in Arabidopsis thaliana. Genes Dev. 14: 1119-1131.

Berger, F., Linstead, P., Dolan, L. and Haseloff, J. (1998) Stomata patterning on the hypocotyl of Arabidopsis thaliana is controlled by genes involved in the control of root epidermal patterning. Dev. Biol. 194: 226-234.

Bergmann, D.C. (2006) Stomatal development: from neighborly to global communication. Curr. Opin. Plant Biol. 9: 1-6.

Bergmann, D.C., Lukowitz, W. and Somerville, C.R. (2004) Stomatal development and pattern controlled by a MAPKK kinase. Science 304: 1494-1497.

Bowman, J.L. (1993) Arabidopsis: An Atlas of Morphology and Development. Springer-Verlag, New York.

Esau, K. (1977) Hydathodes. In Anatomy of Seed Plants. pp. 205-206. John Wiley \& Sons, New York.

Geisler, M., Nadeau, J. and Sack, F.D. (2000) Oriented asymmetric divisions that generate the stomatal spacing pattern in Arabidopsis are disrupted by the too many mouths mutation. Plant Cell 12: 2075-2086.

Geisler, M., Yang, M. and Sack, F.D. (1998) Divergent regulation of stomatal initiation and patterning in organ and suborgan regions of the Arabidopsis mutants too many mouths and four lips. Planta 205: 522-530.

Hara, K., Kajita, R., Torii, K.U., Bergmann, D.C. and Kakimoto, T. (2007) The secretory peptide gene EPF1 enforces the stomatal one-cell spacing rule. Genes Dev. 21: 1720-1725.

Hung, C.-Y., Lin, Y., Xhang, M., Pollick, S., Marks, M.D. and Schiefelbein, J. (1998) A common position-dependent mechanism controls cell-type patterning and GLABRA2 regulation in the root and hypocotyl epidermis of Arabidopsis. Plant Physiol. 117: 73-84.

Jacobs, J. and Roe, J.L. (2005) SKS6, a multicopper oxidase-like gene, participates in cotyledon vascular patterning during Arabidopsis thaliana development. Planta 222: 652-666.

Kono, A., Umeda-Hata, C., Adachi, S., Nagata, N., Konomi, M., Nakagawa, T., Uchimiya, H. and Umeda, M. (2007) The Arabidopsis D-type cyclin CYCD4 controls cell division in the stomatal lineage of the hypocotyl epidermis. Plant Cell 19: 1265-1277.

Lai, L.B., Nadeau, J.A., Lucas, J., Lee, E.K., Nakagawa, T., Zhao, L., Geisler, M. and Sack, F.D. (2005) The Arabidopsis R2R3 MYB proteins FOUR LIPS and MYB88 restrict divisions late in the stomatal cell lineage. Plant Cell 17: 2754-2767.

MacAlister, C.A., Ohashi-Ito, K. and Bergmann, D.C. (2007) Transcription factor control of asymmetric divisions that establish the stomatal lineage. Nature 445: 537-540.
Nadeau, J.A. and Sack, F.D. (2002a) Control of stomatal distribution on the Arabidopsis leaf surface. Science 296: 1697-1700.

Nadeau, J.A. and Sack, F.D. (2002b) Stomatal Development in Arabidopsis. In The Arabidopsis Book, Edited by Sommerville, C.R. and Meyerowitz, E.M. American Society of Plant Biologists, Rockville, MD

Nadeau, J.A. and Sack, F.D. (2003) Stomatal development: cross talk puts mouths in place. Trends Plant Sci. 8: 294-299.

Nakagawa, T., Kurose, T., Hino, T., Kawamukai, M., Niwa, N., Toyooka, K., Matsuoka, K., Jinbo, T. and Kimura, T. (2007) Development of series of gateway binary vectors, pGWBs, for realizing efficient construction of fusion genes for plant transformation. J. Biosci. Bioeng. 104: 33-41.

Ohashi-Ito, K. and Bergmann, D.C. (2006) Arabidopsis FAMA controls the final proliferation/differentiation switch during stomatal development. Plant Cell 18: 2493-2505.

Pillitteri, L.J., Sloan, D.B., Bogenschutz, N. and Torii, K.U. (2007) Termination of asymmetric cell division and differentiation of stomata. Nature 445: 501-505.

Sachs, T. (1991) In Pattern Formation in Plant Tissues pp. 205-206. Cambridge University Press, Cambridge.

Schiefelbein, J. (2003) Cell-fate specification in the epidermis: a common patterning mechanism in the root and shoot. Curr. Opin. Plant Biol. 6: 74-78.

Sessions, A., Weigel, D. and Yanofsky, M.F. (1999) The Arabidopsis thaliana MERISTEM LAYER 1 promoter specifies epidermal expression in meristems and young primordia. Plant J. 20: 259-263.

Shimokawa, T., Kato, M., Ezaki, O. and Hashimoto, S. (1998) Transcriptional regulation of muscle-specific genes during myoblast differentiation. Biochem. Biophys. Res. Commun. 246: 287-292.

Shpak, E.D., McAbee, J.M., Pillitteri, L.J. and Torii, K.U. (2005) Stomatal patterning and differentiation by synergistic interactions of receptor kinases. Science 309: 290-293.

Thayer, M.J., Tapscott, S.J., Davis, R.L., Wright, W.E., Lassar, A.B. and Weintraub, H. (1989) Positive autoregulation of myogenic determination gene MyoD. Cell 58: 241-248.

von Groll, U., Berger, D. and Altmann, T. (2002) The subtilisin-like serine protease SDD1 mediates cell-to-cell signaling during Arabidopsis stomatal development. Plant Cell 14: 1527-1539.

Wang, H., Ngwenyama, N., Liu, Y., Walker, J.C. and Zhang, S. (2007) Stomatal development and patterning are regulated by environmentally responsive mitogen-activated protein kinases in Arabidopsis. Plant Cell 19: 63-73.

Yang, M. and Sack, F.D. (1995) The too many mouths and four lips mutations affect stomatal production in Arabidopsis. Plant Cell 7: $2227-2239$.

(Received March 17, 2008; Accepted April 19, 2008) 


\title{
Breaking the silence: three bHLH proteins direct cell-fate decisions during stomatal development
}

\author{
Lynn Jo Pillitteri and Keiko U. Torii*
}

\begin{abstract}
Summary
Stomata are microscopic pores on the surface of land plants used for gas and water vapor exchange. A pair of highly specialized guard cells surround the pore and adjust pore size. Studies in Arabidopsis have revealed that cell-cell communication is essential to coordinate the asymmetric cell divisions required for proper stomatal patterning. Initial research in this area identified signaling molecules that negatively regulate stomatal differentiation. However, genes promoting cell-fate transition leading to mature guard cells remained elusive. Now, three closely related basic helix-loop-helix (bHLH) proteins, SPEECHLESS, MUTE and FAMA have been identified as positive regulators that direct three consecutive cell-fate decisions during stomatal development. The identification of these genes opens a new direction to investigate the evolution of stomatal development and the conserved functions of bHLH proteins in cell type differentiation adopted by plants and animals. BioEssays 29:861-870, 2007. (c) 2007 Wiley Periodicals, Inc.
\end{abstract}

\section{Introduction}

Stomata are plant epidermal structures that consist of two specialized epidermal cells called guard cells, which surround a microscopic pore. The size of the pore opening dictates the amount of gas and water vapor exchange that can take place between the plant and the atmosphere. Stomatal development requires a series of asymmetric divisions followed by a single symmetric division to produce the paired guard cells ${ }^{(1)}$ (Fig. 1). During stomatal development in Arabidopsis thaliana, the stomatal lineage is initiated by a subset of protodermal cells called meristemoid mother cells (MMC). The MMC undergoes an asymmetric "entry" division that produces a small triangular cell called a meristemoid, and a larger sister cell called a

Department of Biology, University of Washington, Seattle, WA.

Funding agencies: Our research on stomatal development has been supported by the grant from NSF (IOB-0520548), DOE (DE-FG0203ER15448), and the CREST award from Japan Science and Technology Agency to K.U.T. L.J.P was supported by a CSREES USDA postdoctoral fellowship.

${ }^{*}$ Correspondence to: Keiko U. Torii, Department of Biology, University of Washington, Seattle, WA 98195. E-mail: ktorii@u.washington.edu DOI 10.1002/bies.20625

Published online in Wiley InterScience (www.interscience.wiley.com). stomatal-lineage ground cell (SLGC), which often becomes a cuticularized epidermal pavement cell. The meristemoid is self-renewing and can repeat multiple asymmetric amplifying divisions that regenerate the meristemoid as well as additional SLGCs. Meristemoids ultimately lose their self-renewing character and differentiate into a round guard mother cell (GMC). New meristemoids are always oriented away from an existing GMC or stomata, which ensures stomata develop at least one-cell apart from one another. The GMC undergoes a final symmetric division to produce two highly specialized guard cells, which work in concert to control the size of the pore. The mature guard cells are terminally differentiated and do not divide further.

This process has been studied in Arabidopsis over the past decade and factors that inhibit stomatal development have been identified $^{(2-5)}$ (Table 1). These genes are involved in the regulation of proper spacing and density of stomata, and their loss of function leads to the formation of aggregated stomata. More recent studies have identified three master switch genes that positively direct three steps of cell-fate transition through the stomatal lineage, from protoderm to differentiated guard cells $^{(6-8)}$ (Table 1). We discuss the genetic and molecular functions of the master switch genes SPEECHLESS (SPCH), MUTE and FAMA in Arabidopsis stomatal development and their utility as tools to investigate stomatal patterning diversity, stomatal evolution and the conservation of developmental programs between plants and animals.

\section{Stomatal patterning requires \\ cell-cell communication}

Stomata (from the Greek work 'mouth') are separated from one another by at least one non-stomatal cell because proper opening and closing of stomata requires water and ions supplied from surrounding cells. ${ }^{(9)}$ In addition to genetic control, placement of stomata across the epidermal surface is also affected by numerous extrinsic factors through both local and long-distance signaling. ${ }^{(10)}$ Conserved cell-surface receptors and intracellular signaling molecules have been implicated in mediating the perception and response to these signals. Mutations in these signaling molecules result in increased stomatal density and mis-orientation of asymmetric divisions that result in adjacent stomata (clustering). The 

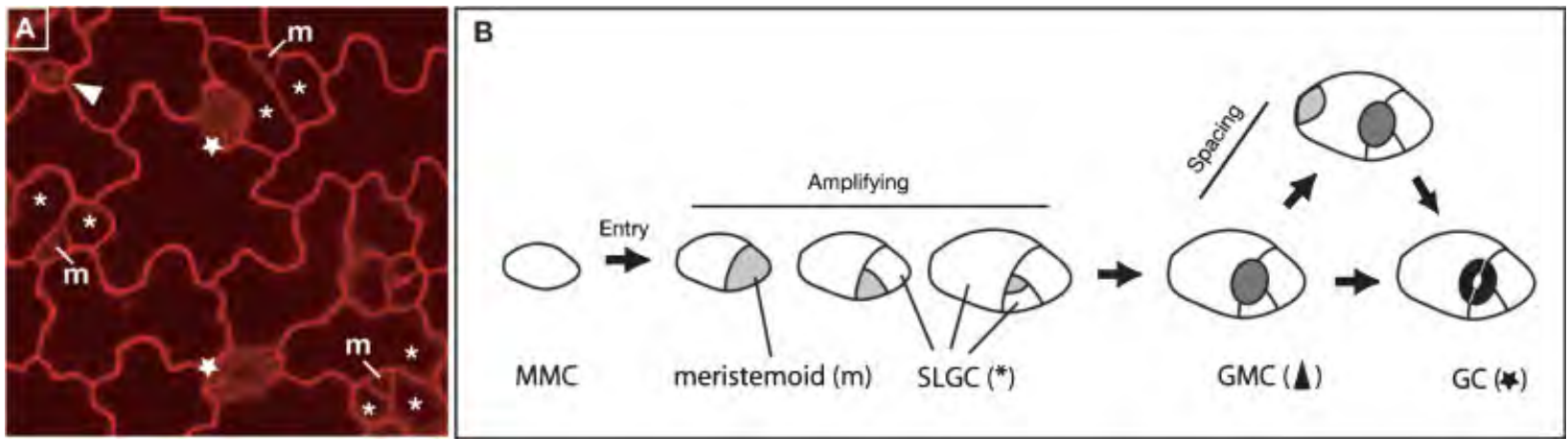

Figure 1. Cell types of the stomatal lineage. A: Confocal image of the abaxial epidermis of a wild-type cotyledon. Cell walls are stained with propidium iodide. Cell types are labeled as described in panel B. B: Stages of divisions during stomatal development. A protodermal cell (not shown) is converted into a meristemoid mother cell (MMC) through an undefined mechanism. The MMC undergoes an asymmetric entry division to create a meristemoid (light grey). Meristemoids are self-renewing and can reiterate one to three rounds of amplifying divisions before differentiating into a guard mother cell (GMC, dark grey). The stomatal lineage ground cells (SLGCs) produced from amplifying divisions can initiate an entry division that is always oriented away from an existing GMC or stomata (spacing division). GMCs divide symmetrically to produce two guard cells (GC, black), which eventually form the mature stomata.

current model suggests that STOMATAL DENSITY AND DISTRIBUTION 1 (SDD1), a subtilisin-like serine protease, acts to process a yet unidentified ligand. ${ }^{(5)}$ The SDDprocessed ligand is perceived by TOO MANY MOUTHS through potential interaction with the three ERECTA family proteins (ERECTA, ERECTA-LIKE 1 and ERL2). TMM and the ERECTA family are a plasma-membrane localized leucine-rich repeat receptor-like protein (LRR-RLP) and

Table 1. Genes involved in Arabidopsis stomatal development in rosette leaves*

\begin{tabular}{|c|c|c|c|c|}
\hline Gene name & Symbol & Molecular homology & Mutant Phenotype & $\begin{array}{l}\text { Overexpression/Constitutively } \\
\text { Active Phenotype }\end{array}$ \\
\hline \multicolumn{5}{|l|}{ Patterning genes } \\
\hline $\begin{array}{l}\text { STOMATAL DENSITY } \\
\text { AND DISTRIBUTION } 1\end{array}$ & $S D D 1$ & Subtilisin-like protease & Increased SI, small clusters & $\begin{array}{l}\text { Represses stomatal divisions } \\
\text { Arrested meristemoids and GMCs }\end{array}$ \\
\hline TOO MANY MOUTHS & $T M M$ & $\begin{array}{l}\text { Leucine-rich repeat receptor-like } \\
\text { protein }\end{array}$ & Increased SI, clusters & ND \\
\hline ERECTA-family & ER.ERL1, ERL2 & $\begin{array}{l}\text { Leucine-rich repeat receptor-like } \\
\text { kinase }\end{array}$ & $\begin{array}{l}\text { Greatly increased SI, large } \\
\text { clusters }\end{array}$ & $\begin{array}{l}\text { ERECTA - no phenotype } \\
\text { ERL1 and ERL2-ND }\end{array}$ \\
\hline$Y O D A$ & $Y D A$ & $\begin{array}{l}\text { Mitogen-activated protein kinase } \\
\text { kinase kinase }\end{array}$ & $\begin{array}{l}\text { Greatly increased SI, large } \\
\text { clusters }\end{array}$ & No stomata, pavement cell only \\
\hline MAPKK4/MAPKK5 & MKK4/MKK5 & $\begin{array}{l}\text { Mitogen-activated protein kinase } \\
\text { kinase }\end{array}$ & $\begin{array}{l}\text { Entire epidermis converted to } \\
\text { stomata }\end{array}$ & No stomata, pavement cell only \\
\hline MAPKЗ/MAPK6 & MPKЗ/MPK6 & Mitogen-activated protein kinase & $\begin{array}{l}\text { Entire epidermis converted to } \\
\text { stomata }\end{array}$ & No stomata, pavement cell only \\
\hline \multicolumn{5}{|l|}{ Differentiation genes } \\
\hline SPEECHLESS & $\mathrm{SPCH}$ & bHLH protein & $\begin{array}{l}\text { No initiation of asymmetric cell } \\
\text { division in the epidermis-no } \\
\text { stomata }\end{array}$ & $\begin{array}{l}\text { Excessive epidermal divisions, } \\
\text { no extra stomata }\end{array}$ \\
\hline MUTE & MUTE & $\mathrm{bHLH}$ protein & $\begin{array}{l}\text { Initiation and reiteration of } \\
\text { asymmetric cell division in the } \\
\text { epidermis-no stomata }\end{array}$ & $\begin{array}{l}\text { Entire epidermis converted to } \\
\text { stomata }\end{array}$ \\
\hline FAMA & FAMA & $\mathrm{bHLH}$ protein & $\begin{array}{l}\text { Reiterative divisions of the } \\
\text { GMC—no stomata }\end{array}$ & $\begin{array}{l}\text { Entire epidermis converted to } \\
\text { single guard cells }\end{array}$ \\
\hline FOUR LIPS & FLP & R2R3 MYB protein & $\begin{array}{l}\text { Reiterative divisions of the GMC, } \\
\text { small clusters }\end{array}$ & ND \\
\hline MYB88 & MYB88 & R2R3 MYB protein & None, enhances flp phenotype & ND \\
\hline
\end{tabular}

${ }^{*}$ TMM, ER-family and YODA have varying phenotypes in different organs. SI, stomatal index (number of stomata/total cell number); ${ }^{+}$, E.D. Shpak and K.U. Torii, unpublished; ND, no data.

\section{BioEssays 29.9}


LRR-receptor-like kinases (RLK), respectively. ${ }^{(3,11,12)}$ The identification of several mutants has implicated a mitogenactivated protein kinase cascade in transduction of the receptor signal to the ultimate gene targets. Mutations in the MAP kinase kinase kinase (MAPKKK) gene, YODA (YDA), cause the formation of large stomatal clusters. ${ }^{(13)}$ Recently, two MAP kinase kinase genes and two MAP kinase genes, $M K K 4 / M K K 5$ and MPK3/MPK6, respectively, were shown to act downstream of YDA in this signaling pathway. ${ }^{(14)}$ The culmination of these studies revealed that cell-cell signals were required for proper orientation of divisions, density of stomata, and control of entry and amplifying divisions (Fig. 2). However, they are not intrinsically required for differentiating cell types within the stomatal lineage. Now, three genes have been identified that lift the "black box" in our understanding of how stomata are produced.

\section{Stomatal differentiation requires the consecutive action of three bHLH proteins}

SPEECHLESS (SPCH), MUTE and FAMA encode closely related bHLH proteins required for stomatal development. A mutation in any one gene results in aerial organs that lack stomata. The gene names reflect their phenotypes: SPCHand
MUTE have 'no-mouths' and FAMA is the goddess of rumor with 'fake mouths'. Expression analysis and loss- and gain-offunction phenotypes indicate that these genes make up a three-step transcriptional cascade where each gene has a distinct role in key transitional states of the stomatal lineage: ${ }^{(6-8)}$

(1) the transition from MMC to meristemoid by $\mathrm{SPCH}$, (2) meristemoid to GMC by MUTE, and (3) GMC to guard cell by FAMA (Fig. 3A).

$S P C H$ is the first gene from this trio to be expressed. $S P C H$ was identified through a genetic screen to isolate genes necessary to promote stomatal fate. ${ }^{(6)}$ The strong spch-1 mutant is completely devoid of stomatal cell lineages, meaning the epidermis comprises jig-saw-puzzle-shaped pavement cells with no stomata (Fig. $3 \mathrm{C}$ ). Therefore, $\mathrm{SPCH}$ is required for the first transition to initiate the asymmetric entry division of the stomatal lineage. The weak spch-2 allele has a reduced number of entry divisions and stomata compared to wild type. Close examination of spch-2 revealed that fewer SLGCs were associated with mature stomata suggesting that $\mathrm{SPCH}$ may have an additional role in promoting amplifying divisions. Consistent with this, SPCH promoter activity is detected early, broadly in the protoderm but also later in a stomatal-lineagespecific pattern. ${ }^{(6,8)} \mathrm{SPCH}$ is not only necessary but also

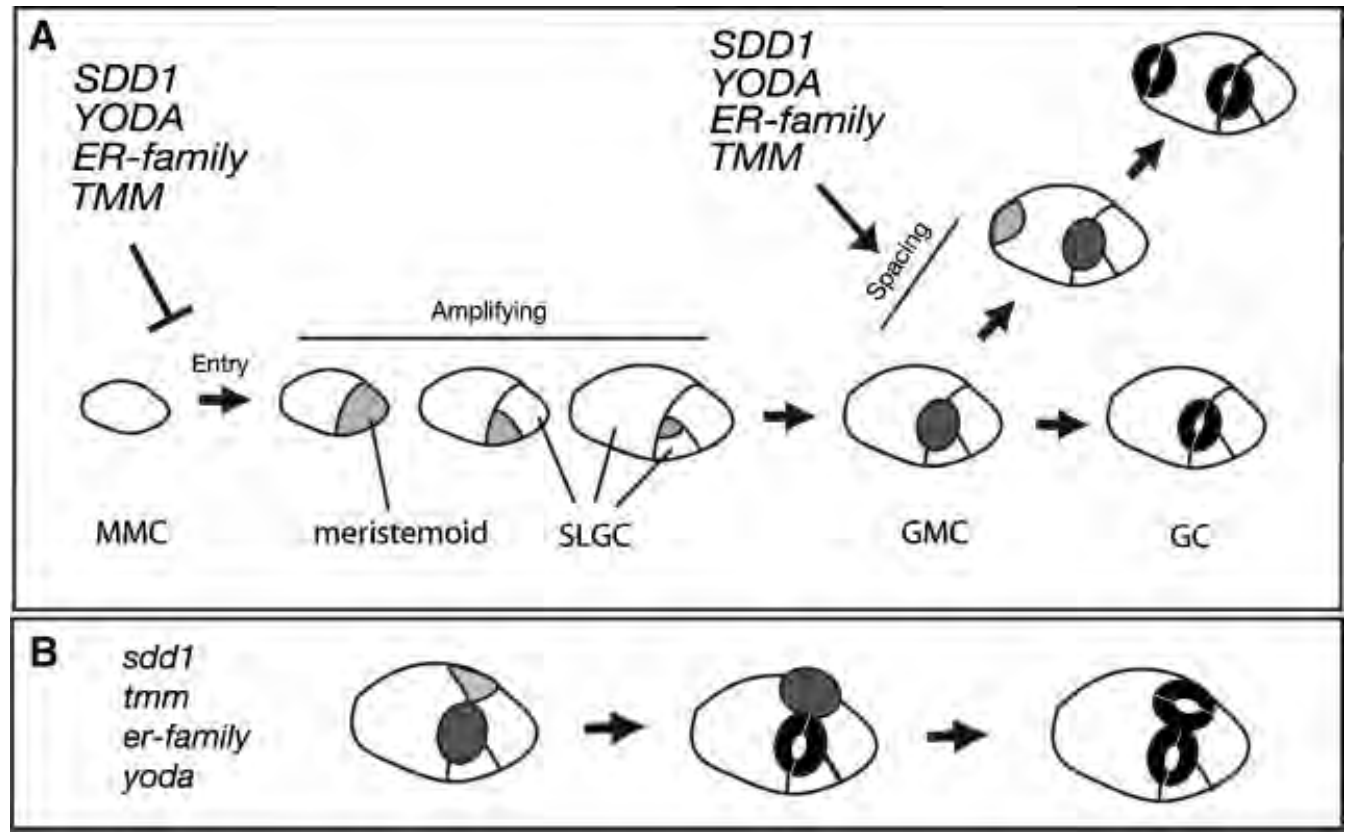

Figure 2. Genetic control of stomatal lineage cell divisions. A: Diagram of the stages of divisions during stomatal development and the points of action of several signaling components implicated in mediating the perception and response to external signals to inhibit stomata production via control of cell lineage divisions. SDD1 (subtilisin protease), YODA (MAPKKK), TMM (LRR-RLP) and the ER family (LRRLRKs) act as negative regulators of stomatal development through limiting the number entry divisions. They also act to inhibit stomatal cluster formation through orienting spacing divisions. B: Example of signaling component mutants showing misorientation of spacing divisions and the production of a stomatal cluster. The role of TMM in entry division inhibition is organ specific. MMC, meristemoid mother cell; SLGC, stomatal lineage ground cell; GMC, guard mother cell; GC, guard cell. 
A
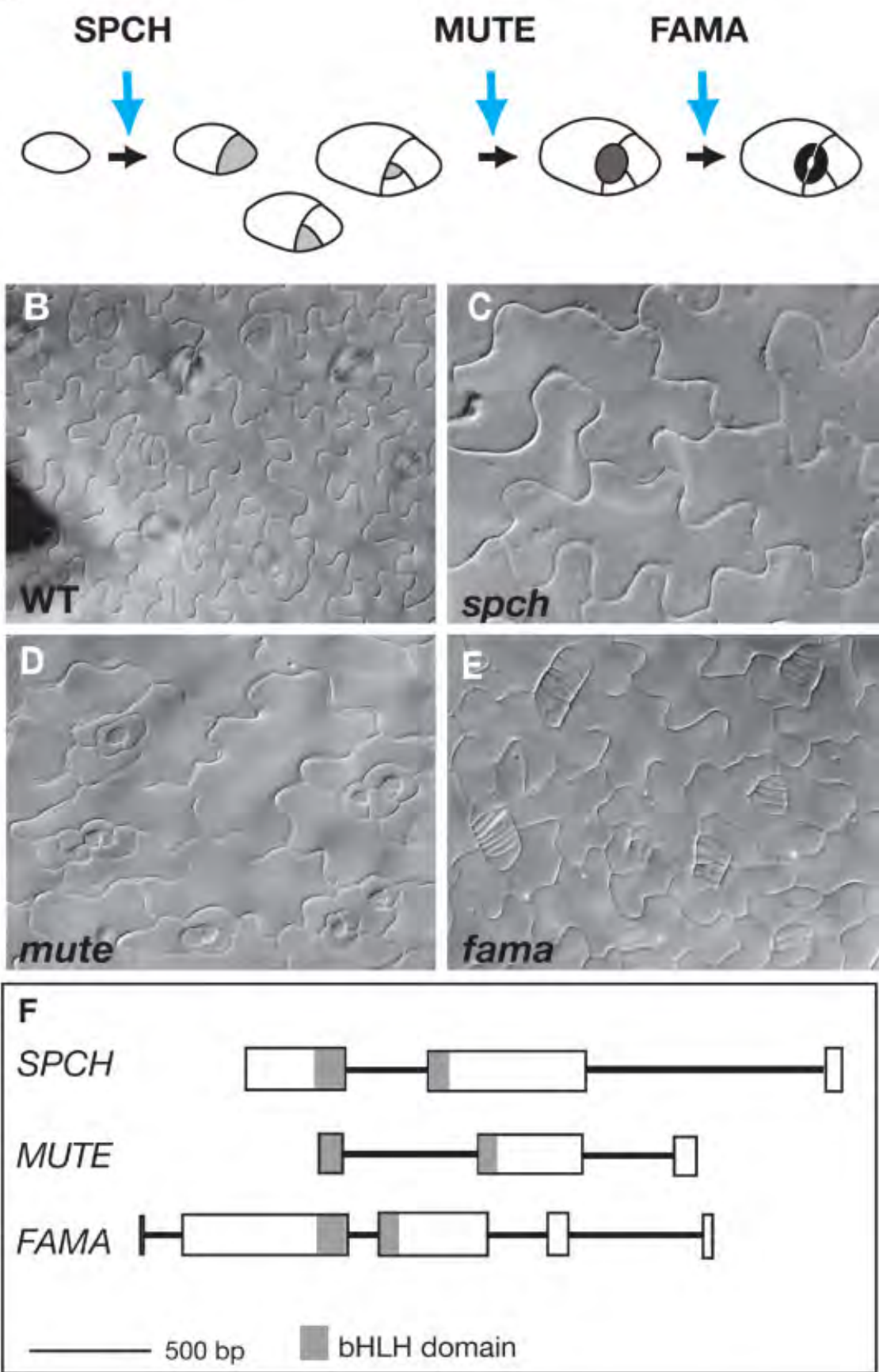

Figure 3. $b H L H$ protein mutant phenotypes. A: Site of stomatal bHLH protein action: the three bHLH proteins work in a three-step transcriptional cascade starting at the transition from MMC to meristemoid by SPCH, meristemoid to GMC by MUTE, and GMC to guard cell by FAMA Blue arrows indicate points of gene action. B-E: Abaxial leaf epidermal surface of wild type (B), spch (C), mute (D), and fama (E). F: Schematic diagram of SPCH, MUTE and FAMA. Exons are indicated as boxes, introns as lines. The bHLH domain of each gene is indicated as a grey box. 
sufficient to create the stomatal lineage, as ectopic inducible expression of $S P C H$ produced excess cell divisions in cells, which do not normally divide (i.e. differentiated pavement cells). ${ }^{(6,8)}$ In addition, these highly divided cells expressed the stomatal lineage markers TMM::TMM-GFP and $E R L 1:: G U S .^{(8)} \mathrm{SPCH}$ activity is required for the expression of both MUTE and FAMA, and SPCH function may be specific to stomatal development, as asymmetric divisions in other organ types, such as the root or embryo, were not affected by SPCH loss-of-function. ${ }^{(6)}$

MUTE activity drives the second transition: the transition from meristemoid to GMC. MUTE was identified through a sensitized genetic screen for genes required for stomatal development. ${ }^{(8)}$ The epidermis of mute plants produces no stomata, but unlike spch, mute plants are able to initiate the first entry division in the stomatal lineage and produce a meristemoid. Typically, this meristemoid completes an excessive number of amplifying divisions, but fails to transition to a GMC. Therefore, the mute epidermis is characterized by inwardly spiraled SLGCs with a triangular, aborted meristemoid at the center (Fig. 3D). MUTE promoter activity and MUTE protein is only observed in a subset of meristemoids, presumably those that have undergone a sufficient number of amplifying asymmetric divisions and are 'ready' to transition. The fact that mute meristemoids are surrounded by an excess number of SLGCs suggests that MUTE is also involved in limiting the number of amplifying divisions, most likely as a secondary consequence of the failure of the self-renewing meristemoid to differentiate. The mechanism through which a plant monitors the number of cell divisions and triggers MUTE is not understood, however, the genetic study by Pillitteri et al. ${ }^{(8)}$ suggested that the cell signaling receptor ERL1 may be involved in regulating the number of amplifying asymmetric division. In any event, the timing of MUTE expression is critical for proper stomatal spacing and function. This is strikingly demonstrated by the ectopic overexpression of MUTE in the epidermis, which converts the entire epidermis to stomata. ${ }^{(6,8)}$ The weaker ectopic overexpression of MUTE conferred hybrid pavement/guard cells that possess chloroplasts and form a symmetric division with a faux pore. ${ }^{(8)}$ This peculiar phenotype indicates that a moderate level of forced MUTE expression can cause epidermal cells to adopt both pavement- and guard cell developmental programs.

FAMA acts at the third transition and was identified through a genome-wide search for genes that are expressed at higher levels in yda mutant plants compared to wild type. ${ }^{(13)}$ The strong fama-1 mutant produces abnormal 'caterpillar-like' rows of GMCs (tumors) on the epidermal surface (Fig. 3E) and its expression is restricted to GMCs and newly divided stomata. Therefore, FAMA regulates the critical differentiation event between GMC and guard cell and, in addition, halts cell division at the final stage of the stomatal lineage to ensure a single symmetric division. ${ }^{(7)}$ Its role in guard cell differentiation was demonstrated by overexpression analysis, which led to the formation of unpaired guard cells even in non-epidermal tissues, indicating that FAMA is sufficient to specify guard cell fate. In addition, fama tumors do not express the mature guard cell marker KAT1::GUS, suggesting that they do not progress past GMC, further supporting a role for FAMA in final differentiation. Negative regulation of the cyclin-dependent kinase, CDKB1;1, by FAMA was suggested as a possible mechanism for halting cell divisions in guard cells. ${ }^{(7)}$

SPCH, MUTE and FAMA are the three most-closely related bHLHs of Arabidopsis subfamily 3. ${ }^{(15)}$ This subgroup shares high amino-acid conservation in addition to similar intron distribution and position (Fig. 3F). For bHLH proteins, the basic region is involved in DNA binding and the $\mathrm{HLH}$ region functions in dimerization. Dimer formation is required for DNA binding and subsequent transcriptional activation. Although they are closely related, the function of each gene is specific as complementation experiments indicated that they can not functionally substitute for one another. ${ }^{(6)}$ Compared to either SPCH or FAMA, a notable structural feature of MUTE is that the $\mathrm{N}$-terminal region is missing. For MUTE, the presumed translational start site begins three amino acids into the basic region. Interestingly, induced expression of FAMA variants containing only the $\mathrm{HLH}$ and C-terminal domain or a mutated DNA-binding domain produced a phenotype similar to that described for MUTE overexpression (i.e. all stomata). These variants were not affected in dimerization properties. OhashiIto and Bergmann ${ }^{(7)}$ postulated that the FAMA variants may act in a dominant negative manner by sequestering other bHLHs in non-functional heterodimers or, alternatively, acquired neomorphic activity within the stomatal differentiation pathway. To test whether the neomorphic phenotype of the FAMA variants is an artifact of overexpression or mimicry of MUTE requires further investigation into the DNA-binding properties of the stomatal bHLHs.

The strong fama-1 phenotype is similar to that described for a double mutant knockout of FOUR LIPS (FLP) and MYB88 (R2R3 MYB-type transcription factors). ${ }^{(4,16)}$ In addition, weak fama-2 mutants are similar to flp-1 single mutants that produce 3- to 4-celled stomata but no tumors. In root epidermal patterning, bHLH and R2R3 MYB proteins interact to produce a complex that controls cell-fate specification. ${ }^{(17)}$ Genetic interaction studies did not suggest that FAMA and MYB88/FLP form heterodimers. In addition, FAMA failed to interact with FLP or MYB88 in yeast or in plants. Together, Ohashi-lto and Bergmann $^{(7)}$ concluded that FAMA and FLP/MYB88 do not form an active complex similar to that in root epidermal cellfate specification.

Possible FAMA dimerization partners may include the bHLH071 and bHLH093, which were identified from a yeast two-hybrid screen. ${ }^{(7)}$ Both bHLH071 and bHLH093 belong to the same subgroup as SPCH, MUTE and FAMA. ${ }^{(15)}$ While loss-of-function mutations in bHLH071 and bHLH093 did not 
produce a noticeable phenotype, overexpression of either gene produced a mild fama phenotype. ${ }^{(7)}$ It is possible that these genes have redundant roles in suppressing FAMA function. Whether they enhance the phenotype of fama or other stomatal bHLH mutations remains to be addressed.

\section{Drawing parallels between plants and animals}

The last common ancestor of plants and animals was likely unicellular and existed around 1.6 billion years ago. ${ }^{(18)}$
Therefore, plants and animals independently developed mechanisms for cell communication and pattern formation even though the molecules that carry out these processes are often unrelated. ${ }^{(19)}$ In contrast, the discovery that three closely related bHLH proteins control stomatal lineage cell-fate decisions is strikingly parallel to the consecutive use of closely related bHLH proteins during myogenesis (Fig. 4). The myogenic bHLH proteins, including MyoD, Myf5, myogenin and MRF4 ${ }^{(20-23)}$ act in a consecutive manner to direct cell-fate

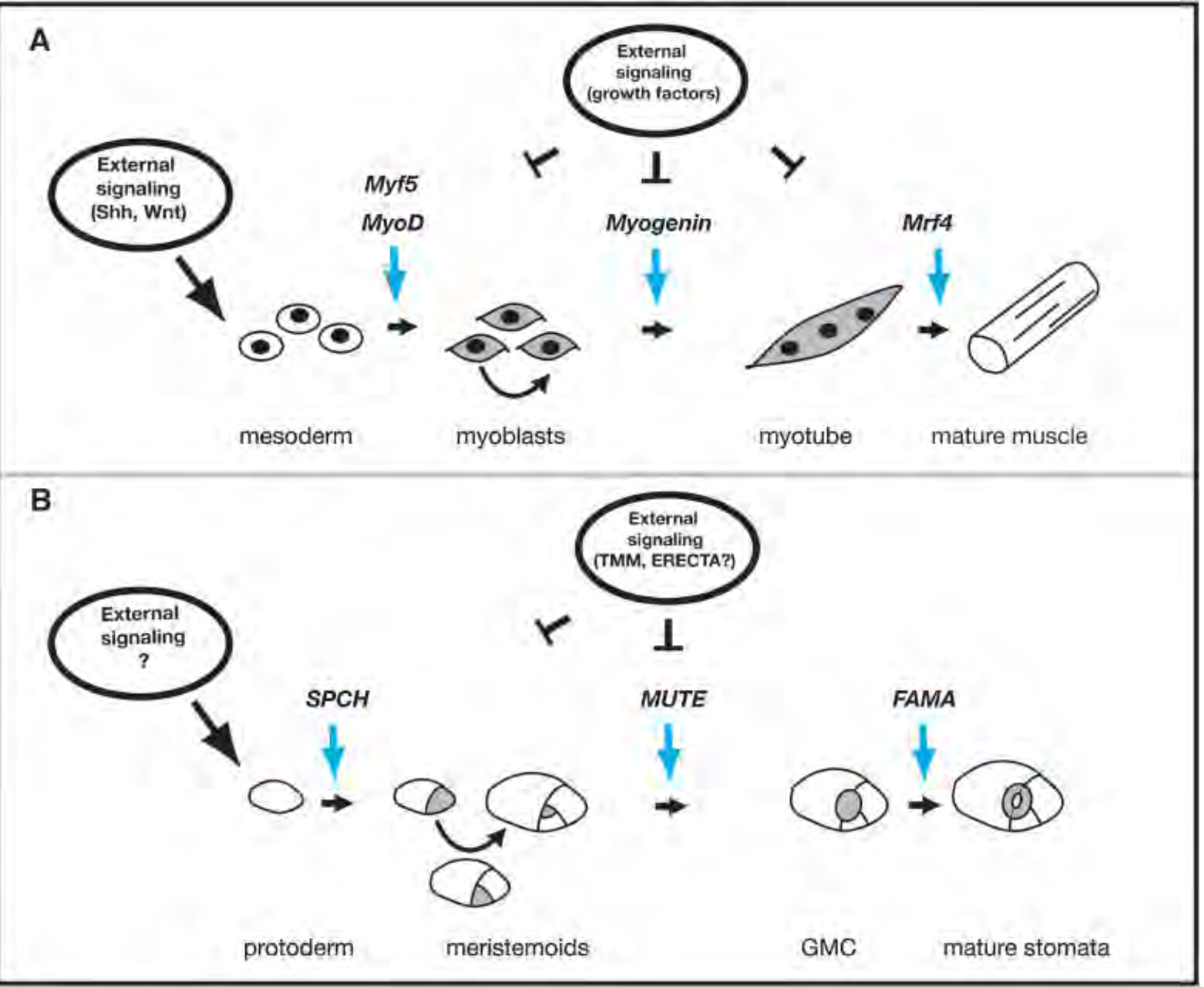

Figure 4. Comparison of the distinct waves of bHLH gene expression during muscle and stomatal development. A: Muscle development in animals (based on Weintraub H 1993 Cell 75:1241-1244). The action of MyoD or Myf5 are required for myoblast determination from mesoderm cells. Myogenin acts in the terminal differentiation of myoblasts. Mrf4 acts downstream of Myogenin during muscle differentiation. B: Stomata development in plants. SPCH is required for the determination of the stomatal lineage: initiation of the entry division. MUTE is required for differentiation of the meristemoid to GMC. FAMA is necessary for terminal differentiation of the GMC to GC. External signaling from Sonic hedgehog (Shh) and Wnt (animals) and potential unidentified ligands (plants) is required for the initiation of the lineages. Additional external signals from growth factors (animals) and from the signal transduction components, TMM, ER family and YDA (plants), act as negative regulators of the differentiation process. Negative regulation is indicated by T-bars. Blue arrows indicate points of gene action.

\section{BioEssays 29.9}


decisions during successive steps in myoblast differentiation: MyoD and Myf5 specifying myoblast precursor state, and myogenin and MRF4 directing terminal differentiation of muscles. ${ }^{(24)}$ The MyoD family proteins are considered 'master regulators' of skeletal myogenesis, as their forced expression is capable of converting nearly all cell types into skeletal muscle fibers. ${ }^{(25,26)}$ Similarly, overexpression of SPCH, MUTE and FAMA induces ectopic stomatal-lineage cells. Both stomatal and MyoD family bHLHs are expressed in a spatially restricted manner.

The myogenic bHLH proteins induce muscle-specific transcription via binding of their basic domain to the canonical DNA E-box consensus (CANNTG) of downstream target genes and regulating their expression. Comparatively, SPCH, MUTE and FAMA all contain the conserved $\mathrm{H}-\mathrm{E}-\mathrm{R}$ amino acid residues in the basic domain characteristic of proteins that bind the E-box. ${ }^{(27)}$ Therefore, SPCH, MUTE and FAMA may act as transcription factors that bind directly to the regulatory elements (promoters) of target genes to direct cell-fate transition. Mutating these critical residues in the FAMA basic domain resulted in a protein that could not rescue the fama-1 mutant. ${ }^{(7)}$

For the MyoD protein, homodimers are transcriptionally inactive, however, heterodimerization with ubiquitously expressed bHLHs (E proteins) results in a complex with high DNA-binding affinity, which is pivotal for controlling downstream targets. ${ }^{(28,29)}$ Therefore, in addition to conserved transcriptional molecules, stomatal development may also share similar regulatory mechanisms with myogenesis. This idea stems from the identification of the two ubiquitously expressed bHLHs that specifically bind to FAMA. ${ }^{(7)}$ Multiple mutant analyses with these two bHLHs will provide some insight as to whether this type of regulatory conservation exists between these two developmental programs.

In animals, non-DNA-binding HLH proteins lacking the basic region, such as human Id protein, act as transcriptional inhibitors. ${ }^{(30)}$ They negatively regulate bHLH transcription factors via direct protein-protein interaction that results in non-functional heterodimers. The Arabidopsis genome contains at least 27 proteins that are categorized as non-DNAbinding HLHs. ${ }^{(15)}$ Among them, KIDARI inhibits photomorphogenesis by direct association with the bHLH protein HFR1, a known downstream component of light signal transduction. ${ }^{(31)}$ It will be fascinating to address whether the activity of $\mathrm{SPCH}$, MUTE and FAMA may also be regulated by such non-DNAbinding HLHs.

The activity of bHLH transcription factors has also been shown in several systems to be regulated by phosphorylation/ dephosphorylation circuits, which can influence the choice and affinity for dimerization partners. Specifically during myogenesis, the MAP kinase p38 phospohorylates the MyoD-binding partner, E47. ${ }^{(32)}$ This phosphorylation event is required for the formation of a functional MyoD/E47 hetero- dimer. Furthermore, DNA-binding affinity of bHLHs is also regulated by phosphorylation. Growth factors, such as FGF (fibroblast growth factor), promote cell proliferation and inhibit terminal differentiation of muscles by inactivating the myogenic bHLHs. ${ }^{(33)}$ This inhibition is mediated by protein kinase $\mathrm{C}$, which phosphorylates the conserved site in the DNAbinding domain of myogenic bHLH and prevents its association to target DNA sequence. ${ }^{(34)}$ During stomatal development, a MAP kinase cascade, including YDA, MKK4/MKK5 and MPK3/MPK6, negatively regulates asymmetric division and stomatal formation, and their loss-of-function mutations resulting in large clusters of stomata. ${ }^{(13,14)}$ Conversely, constitutively active forms of YDA and MPKs result in a phenotype resembling spch-1, consisting of an epidermis of pavement cells only. ${ }^{(13,14)}$ YDA and the ERECTA family of receptor-like kinases promote cell proliferation and organ growth while inhibiting entry divisions to limit stomatal formation. ${ }^{(2,3)}$ Therefore, the parallel between myogenesis and stomatal development may extend to how these master regulatory bHLHs are regulated by extrinsic cell-cell signals (Fig. 4). It remains to be seen whether the phosphorylation circuit works directly or indirectly to regulate stomatal bHLH activity.

Although not directly paralleled in myogenesis, regulation of the stomatal bHLHs may involve a C-terminal regulatory region. MUTE, SPCH and FAMA contain a C-terminal domain weakly similar to an ACT domain, which serves as a regulatory module in a diverse group of proteins. The ACT domain has been most studied in amino acid metabolic enzymes where it is involved in allosteric regulation through the binding of specific small molecule ligands. ${ }^{(35,36)}$ This domain is involved in protein-protein interactions and recently shown to be important for dimerization of several plant bHLH proteins. ${ }^{(37)}$ Dimerization and function of MUTE, SPCH or FAMA with specific partners may require the ACT domain. Evidence for this comes from the loss-of-function spch-1 mutant, which results in a 7-amino acid C-terminal truncation of the wild-type protein, yet is indistinguishable from the complete T-DNA knock-out allele. ${ }^{(6)}$ The truncation removes part of the ACT domain, highlighting the importance of this domain in $\mathrm{SPCH}$ function.

\section{Epidermal diversity: SPCH, MUTE and FAMA action}

The paralogous relationship among SPCH, MUTE and FAMA strongly suggests that they are derived from a genomic duplication event. It appears that both expression and functional changes have occurred within the stomatal bHLHs given that their expression patterns do not significantly overlap and that they cannot functionally substitute for one another. ${ }^{(6)}$ One can hypothesize that the variation of stomatal patterning among land plants could in part be a result of the diversification of the stomatal bHLHs through the course of evolution. 
Numerous models relating to SPCH, MUTE or FAMA action can be postulated to explain stomatal pattern variation. For instance, the simplest model predicts that expression of MUTE immediately after the initial entry division without additional amplifying divisions would produce a modified pattern to that described for Arabidopsis. Indeed, many monocots, including the Liliacea, have linear rows of elongated epidermal cells. Stomata develop at the basal end of these cells after a single asymmetric division with no subsidiary cells present. ${ }^{(38)}$ For Liliacea, spacing of stomata could be ensured by a mechanism that allowed the meristemoid to be produced only at the basal end of cells leaving each stomata at least one cell away from the next (Fig. 5). Additional models could be postulated to explain stomatal complexes with subsidiary cells totaling $>4$, as is seen in the Araceae. ${ }^{(38)}$ In these cases, a delay in MUTE activity could predict this phenotype as is observed in the mute mutant itself. Previous work has determined that stomatal patterning and the division series required for a functional stomatal complex in the model monocot Zea mays varies from Arabidopsis. ${ }^{(39)}$ The large amount of genetic and molecular tools available for maize will allow for testing of some of these models.

Finally, beyond examining the evolutionary diversity of stomatal patterning, the key stomatal regulators are useful tools to investigate how stomata initially developed in early land plants. The earliest land plants include the liverworts, hornworts and mosses, collectively called the Bryophytes. The gametophyte of these plants do not produce stomata; however, the sporophyte of some mosses and hornworts do produce responsive stomata. Further, the fern Selaginella appears to produce stomata without extensive asymmetric division of a precursor cell. ${ }^{(40)}$ Perhaps, prototypical function of stomatal bHLHs may be to trigger initial entry asymmetric divisions and then immediately specify GMC differentiation. Similar to the extensive work done piecing together the evolution of flowering using floral master regulatory genes such as $L E A F Y$ ( $L F Y$ ), these 'primitive' plants provide an excellent comparative system to investigate the stomatal bHLHs and their importance in land plant evolution. The true challenge may lie in identifying true homologous stomatal bHLH genes in other plant systems. Unlike $L F Y$, which is generally found as a unique single copy transcription factor, the bHLH gene family has undergone a large radiation in plants, ${ }^{(15)}$ containing hundreds of members. However, based on homology, convincing stomatal bHLH homologues have been identified in rice (K.U.T., unpublished data) and as more basal plant genomes are sequenced, homology searches will likely reveal others. Identifying whether the stomatal bHLHs have alternate roles in astomatous gametophyte plants in the Bryophytes, as was discovered for $L F Y$ in the non-flowering moss Physcomitrella patens, ${ }^{(41)}$ is an area of exciting future research.

\section{Conclusions}

Stomatal development shares a common theme with other developmental programs in which cells must communicate and coordinate to adopt different cell fates. Many aspects of the emerging model for stomatal development remain to be tested. These include how the cell-cell signaling pathway specifying patterning integrates with the cell differentiation
A

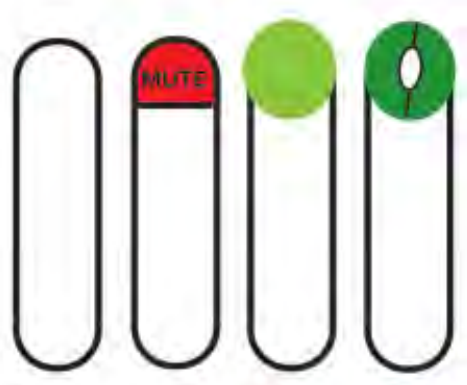

C

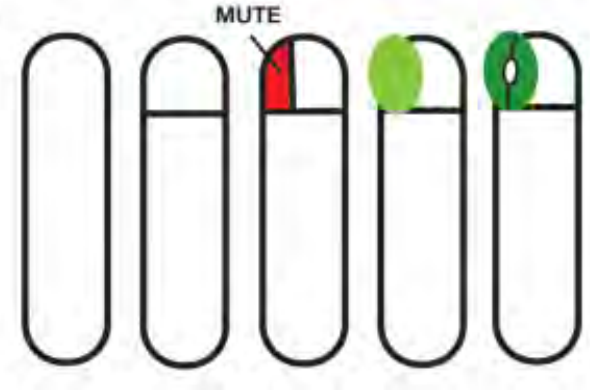

B

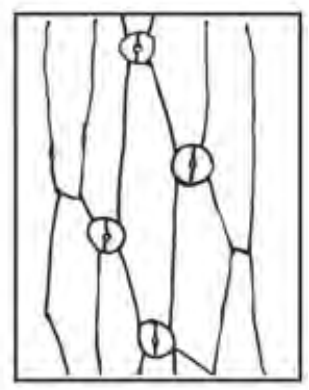

D

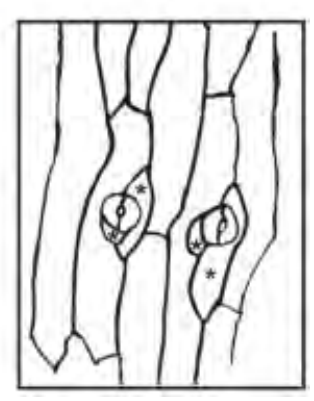

Figure 5. Simple model for stomatal pattern diversity. Alteration in the temporal expression pattern of the bHLH proteins can explain stomatal arrangement diversity observed among land plants. A: Schematic of MUTE expression immediately after the entry division. This scenario would produce stomata with no SLGCs as is observed for many monocots such as Liliaceae. B: Tracing of the epidermis of Liliaceae, redrawn from Stebbins GL and Khush GS 1961 Am J Bot 48:5159. C: Schematic of MUTE expression after one round of amplifying divisions as is observed in Arabidopsis and other dicot species. D: Tracing of Arabidopsis silique epidermis with stomata and additional SLGCs surrounding stomata (asterisks). SLGC, stomatal lineage ground cell. 
pathway. What is the mechanism by which a subset of cells are "chosen" from a seemingly identical pool of protodermal cells that uniformly express SPCH? Similarly, what mechanism determines if MUTE is activated in meristemoids after one division or three? How or when were the stomatal bHLHs recruited for stomata production? The identification of additional players, including up and downstream factors and potential binding partners, and the molecular examination of basal land plants will aid in our understanding. Because the second and third transitions of the stomatal lineage can be interpreted as cell-cycle exit, one potential gene group to investigate as downstream targets are core cell-cycle genes as was suggested by the negative regulation of CDKB1;1 by FAMA. Though many details remain to be addressed, numerous parallels have emerged between the molecules and regulatory mechanisms used by plant and animal developmental programs, even though each group independently recruited bHLHs for these processes through evolution. This makes the isolation of the three stomatal bHLHs a valuable tool for plant and animal comparative analysis.

\section{Acknowledgments}

We thank Dr. Masa Kanaoka for the confocal image used in Fig. 1.

\section{References}

1. Geisler M, Nadeau J, Sack FD. 2000. Oriented asymmetric divisions that generate the stomatal spacing pattern in Arabidopsis are disrupted by the too many mouths mutation. Plant Cell 12:2075-2086.

2. Lukowitz W, Roeder A, Parmenter D, Somerville C. 2004. A MAPKK kinase gene regulates extra-embryonic cell fate in Arabidopsis. Cell 116 109-119.

3. Shpak ED, McAbee JM, Pillitteri LJ, Torii KU. 2005. Stomatal patterning and differentiation by synergistic interactions of receptor kinases. Science 309:290-293.

4. Yang M, Sack FD. 1995. The too many mouths and four lips mutations affect stomatal production in Arabidopsis. Plant Cell 7:2227-2239.

5. Berger D, Altmann T. 2000. A subtilisin-like serine protease involved in the regulation of stomatal density and distribution in Arabidopsis thaliana. Genes Dev 14:1119-1131.

6. MacAlister CA, Ohashi-Ito K, Bergmann DC. 2007. Transcription factor control of asymmetric divisions that establish the stomatal lineage. Nature 445:537-540

7. Ohashi-Ito K, Bergmann DC. 2006. Araidopsis FAMA controls the final proliferation/differentiation switch during stomatal development. Plant Cell 18 2493-2505.

8. Pillitteri LJ, Sloan DB, Bogenschutz N, Torii KU. 2007. Termination of asymmetric cell division and differentiation of stomata. Nature 445:501505

9. Schroeder JI, Allen G, Hugouvieux V, Kwak JM, Wagner D. 2001. Guard cell signal transduction. Annu Rev Plant Physiol Plant Mol Biol 52:627658

10. Coupe SA, Palmer BG, Lake JA, Overy SA, Oxborough K, Woodward FI Gray JE, Quick WP. 2006. Systemic signaling of environmental cues in Arabidopsis leaves. J Exp Bot 57:329-341.

11. Nadeau JA, Sack FD. 2002. Control of stomatal distribution on the Arabidopsis leaf surface. Science 296:1697-1700.

12. Torii KU, Mitsukawa N, Oosumi T, Matsuura Y, Yokoyama R, et al. 1996 The Arabidopsis ERECTA gene encodes a putative receptor protein kinase with extracellular leucine-rich repeats. Plant Cell 8:735-746.

13. Bergmann DC, Lukowitz W, Somerville CR. 2004. Stomatal development and pattern controlled by a MAPKK kinase. Science 304:1494-1497.
14. Wang H, Ngwenyama N, Liu Y, Walker JC, Zhang S. 2007. Stomatal development and patterning are regulated by environmentally responsive mitogen-activated protein kinases in Arabidopsis. Plant Cell 19: 63-73.

15. Toledo-Ortiz G, Huq E, Quail PH. 2003. The Arabidopsis basic/helixloop-helix transcription factor family. Plant Cell 15:1749-1770.

16. Lai LB, Nadeau JA, Lucas J, Lee EK, Nakagawa T, et al. 2005. The Arabidopsis R2R3 MYB proteins FOUR LIPS and MYB88 restrict divisions late in the stomatal cell lineage. Plant Cell 17:2754-2767.

17. Lee MM, Schiefelbein JW. 1999. WEREWOLF, a MYB-related protein in Arabidopsis, is a position-dependent regulator of epidermal cell patterning. Cell 99:473-483.

18. Wang DYC, Kudar S, Hedges SB. 1999. Divergence time estimates for the early history of animal phyla and the origin of plants, animals and fungi. Proc R Soc Lond B 266:163-171.

19. Meyerowitz EM. 2002. Plants compared to animals: The broadest comparative study of development. Science 295:1482-1485

20. Braun T, Buschhausen-Deker G, Bober E, Tannich E, Arnold HH. 1989. A novel human muscle factor related to but distinct from MyoD1 induces myogenic conversion in 10T1/2 fibroblasts. EMBO J 8:701-709.

21. Davis RL, Weintraub $H$, Lassar AB. 1987. Expression of a single transfected cDNA converts fibroblasts to myoblasts. Cell 51:9871000

22. Edmonson DG, Olson EN. 1989. A gene with homology to the myc similarity region of MyoD1 is expressed during myogenesis and is sufficient to activate the muscle differentiation program. Genes Dev 3: 628-640.

23. Rhodes SJ, Konieczny SF. 1989. Identification of MR F4:A new member of the muscle regulatory factor gene family. Genes Dev 3:20502061

24. Weintraub H. 1993. The MyoD family and myogenesis: redundancy, networks, and thresholds. Cell 75:1241-1244.

25. Olson EN. 1990. MyoD family: a paradigm for development? Genes Dev 4:1454-1461

26. Weintraub H, Davis RL, Tapscott SJ, Thayer M, Krause M, Benezra R, Blackwell TK, Turner D, Rupp R, Hollenberg S. 1991. The myoD gene family: nodal point during specification of muscle cell lineage. Science 251:761-766

27. Shimizu T, Toumoto A, Ihara K, Shimizu M, Kyogoku Y, et al. 1997 Crystal structure of $\mathrm{PHO} 4$ bHLH domain-DNA complex: flanking base recognition. EMBO J 16:4689-4697.

28. Murre C, McCaw PS, Baltimore D. 1989. A new DNA binding and dimerization motif in immunoglobulin enhancer binding, daughterless, MyoD, and myc proteins. Cell 56:777-783.

29. Blackwell TK, Weintraub H. 1990. Differences and similarities in DNAbinding preferences of MyoD and E2A proteins complexes revealed by binding site selection. Science 250:1104-1110.

30. Jan YN, Jan LY. 1993. HLH proteins, fly neurogenesis, and vertebrate myogenesis. Cell 75:827-830.

31. Hyun Y, Lee I. 2006. KIDARI, encoding a non-DNA binding bHLH protein, represses light signal transduction in Arabidopsis thaliana. Plant Mol Biol 61:283-286.

32. Lluis T, Ballestar E, Suelves M, Esteller M, Munoz-Canoves P. 2005. E47 phosphorylation by p38 MAPK promotes MyoD/E47 association and muscle-specific gene transcription. EMBO J 24:974-984.

33. Olson EN. 1992. Interplay between proliferation and differentiation within the myogenic lineage. Dev Biol 154:261-272.

34. Li L, Zhou J, James G, Heller-Harrison R, Czech MP, Olsen EN. 1992. FGF inactivates myogenic helix-loop-helix proteins through phosphorylation of a conserved protein kinase $\mathrm{C}$ site in their DNA-binding domains. Cell 71:1181-1194.

35. Aravind L, Koonin EV. 1999. Gleaning non-trivial structural, functional and evolutionary information about proteins by iterative database searches. J Mol Biol 287:1023-1040.

36. Liberles JS, Thorolfsson M, Martinez A. 2005. Allosteric mechanisms in ACT domain containing enzymes involved in amino acid metabolism. Amino Acids 28:1-12.

37. Feller A, Hernandez JM, Grotewold E. 2006. An ACT-domain participates in the dimerization of several plant basic-helix-loop-helix transcription factors. J Biol Chem 281:28964-28974. 
Review articles

38. Stebbins GL, Khush GS. 1961. Variation in the organization of the stomatal complex in the leaf epidermis of monocotyledons and its bearing on their phylogeny. Am J Bot 48:51-59.

39. Gallagher K, Smith GL. 2000. Roles for polarity and nuclear determinants in specifying daughter cell fates following an asymmetric division in the maize leaf. Curr Biol 10:1229-1232.
40. Dengler N. 1983. The developmental basis of anisophylly in Selaginella martensii. II. Histogenesis. Am J Bot 70:193-206.

41. Tanahashi T, Sumikawa N, Kato M, Hasebe M. 2005. Diversification of gene function: homologs of the floral regulator FLO/LFY control the first zygotic cell division in the moss Physcomitrella patens. Development 132:1727-1736

\section{BioEssays 29.9}




\title{
Stomatal Patterning and Guard Cell Differentiation
}

\author{
Keiko U. Torii \\ Department of Biology, University of Washington, Seattle, WA 98195, USA \\ ktorii@u.washington.edu
}

\begin{abstract}
Gas exchange between plants and the atmosphere takes place through stomata (singular, stoma), which are microscopic valves on the plant epidermis composed of paired guard cells. Stomatal differentiation involves a series of asymmetric divisions of precursor cells followed by a single symmetric cell division that produces terminally differentiated guard cell pairs. Stomatal development emerged as a model system to study how environmental- and cell-cell signals translate into site/orientation of asymmetric cell division and cell-type differentiation. This chapter focuses on cell-state transition events leading to guard cell differentiation in the model plant Arabidopsis, and cell-cell signaling mechanisms controlling stomatal patterning. Understanding how cell-cycle regulators influence stomatal patterning and differentiation will advance our knowledge of cell division control in plant development.
\end{abstract}

1

Introduction

The evolution of land plants relied on the acquisition of mechanisms that protected themselves from the dry atmosphere and harmful UV rays, while allowing gas exchange for photosynthesis; and transpiration for stimulating water movement from the soil to aboveground tissues. The innovation of two distinct cell types on the plant epidermis was critical for solving this challenge. Epidermal pavement cells are tightly-sealed interlocking cells with thick cuticle layers. Stomata act as turgor-driven valves that allow gas exchange and transpiration. It is therefore not surprising that evolutionary biologists believe that the emergence of stomata predates the evolution of leaves, flowers, or even vasculature (Edwards et al. 1998). A stoma consists of a microscopic pore surrounded by a pair of guard cells, which open and close upon sensing environmental signals, such as drought, light, and $\mathrm{CO}_{2}$ concentrations. Given the importance of stomatal function for plant growth and survival, significant research has been done on physiological and molecular bases of stomatal opening/closure as well as their eco-physiological and environmental consequences (Assmann and Shimazaki 1999; Schroeder et al. 2001, 2001; Hetherington and Woodward 2003).

For developmental biology, stomata serve as a superb system to understand cell-cell signaling, cell division, stem cell differentiation, cell polarity, and cellular morphogenesis in plants. The steps leading to the differentiation 
of guard cells are uniquely coupled with specific types of cell divisions, the reiterative asymmetric division of precursor stem cells and a single symmetric division that generates a pair of guard cells. The simplicity and tractable nature of the leaf epidermis makes the study of stomatal development technically amenable (Nadeau and Sack 2002; Bergmann et al. 2004). Recent advances in model plant molecular genetics have begun to unravel how genetic and environmental signals act in controlling stomatal patterning. In this chapter, I will introduce the cellular processes of stomatal development with emphasis on the model plant Arabidopsis, and provide the latest updates on emerging cell-cell signaling mechanisms specifying the correct spacing and differentiation of stomata. Potential interactions of cell-cell signaling with intrinsic developmental regulators as well as environmental cues will be explored. Finally, future prospects on integrating cell cycle regulators in the context of stomatal patterning will be presented.

\section{2}

\section{Stomatal Development in Arabidopsis}

Arabidopsis stomata are typically found in complexes with three subsidiary cells, one being distinctly smaller than the others, surrounding a pair of guard cells (Esau 1977; Zhao and Sack 1999; Serna and Fenoll 2000; Nadeau and Sack 2002). These are characteristic "anisocytic" stomatal complexes and are generated through stereotypical cell division patterns (Esau 1977). Stomatal development initiates post-embryonically when populations of protodermal cells, termed meristemoid mother cells (MMC), enter into asymmetric division (Nadeau and Sack 2002). This initial asymmetric division generates two daughter cells with distinct fates. The larger daughter cell differentiates into an epidermal pavement cell. In contrast, the smaller daughter cell, termed a meristemoid, possesses stem-cell like characteristics, as it continues to divide asymmetrically to renew itself over several rounds of divisions (Nadeau and Sack 2002). Typically, meristemoids reiterate 3 rounds of asymmetric division. The repeated asymmetric division of meristemoids will be hereafter referred to as amplifying asymmetric division, as each division increases the number of cells, which we termed stomatallineage ground cells (SLGC), larger daughter cells that function as subsidiary cells (Shpak et al. 2005). SLGCs are also referred to as "subsidiary cells" or "pavement cells" in literature. The meristemoid then differentiates into a round guard mother cell (GMC), which divides symmetrically once to generate a pair of guard cells (Nadeau and Sack 2002). This results in an anisocytic complex with three clonally-related subsidiary cells (Berger and Altmann 2000; Serna et al. 2002). However, the number of asymmetric divisions as well as the clonal relationship among cells constituting the stomatal complex is plastic and variable. For example, a detailed clonal analysis 
of stomatal complexes in the adaxial epidermis of Arabidopsis Ler accession by Serna et al. (2002) revealed that, while the vast majority (87\%) of anisocytic stomatal complexes derived from single precursor cells, the rest were of polyclonal origins. Geisler et al. (2000) reported that the number of asymmetric divisions in the Columbia accession varies from zero to three. This plastic nature of stomatal ontogeny reflects a dynamic intrinsic developmental program that integrates external cues for adaptation and survival. In fact, environmental factors, such as humidity and $\mathrm{CO}_{2}$ concentrations, are known to affect stomatal density and patterning (Gray et al. 2000; Lake et al. 2002).

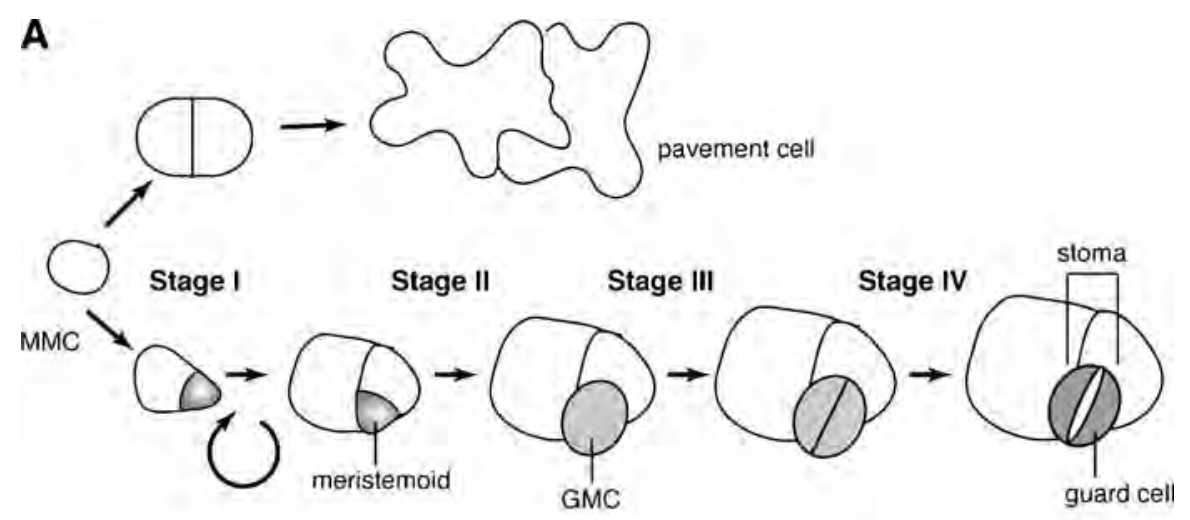

B

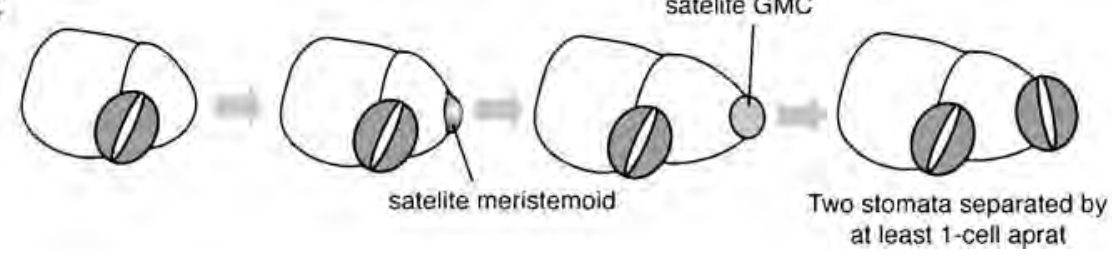

Fig. 1 Stomatal development in Arabidopsis. A Cartoon showing the key steps of stomatal differentiation. Undifferentiated cells in the protoderm can undergo either proliferative division to form pavement cells or asymmetric division to initiate stomatal development. Stage I: a subset of protodermal cells, a meristemoid mother cell (MMC) divides asymmetrically and forms a self-renewing meristemoid that reiterate a few rounds of asymmetric division. Stage II: the meristemoid then differentiates into a round, guard mother cell (GMC). Stage III: the GMC undergoes a single symmetric division. Stage IV: a pair of immature guard cells achieves final morphogenesis to form a functional stoma. The amplifying asymmetric division of meristemoids generates surrounding stomatallineage ground cells (SLGCs) that provide water and ions for stomatal opening and closure. B A polarity of asymmetric division during satellite meristemoid formation. The secondary asymmetric division occurs away from the existing stoma, thereby assuring that two stomata are separated by at least one cell apart (1-cell spacing rule). Modified from Torii (2006) 
The entire process of stomatal patterning and differentiation in Arabidopsis can be divided into the following four critical stages (Fig. 1A). Stage I initiates the entry into the stomatal-lineage via emergence of MMCs (Stage I-a) and commitment to reiterative asymmetric division (Stage I-b). Stage II represents the differentiation of meristemoids into GMCs associated with the loss of potential for asymmetric division. Stage III includes the acquisition of symmetric division potential in GMCs, and finally Stage IV, or guard cell morphogenesis, concludes the process of stomatal development (Fig. 1A).

Occasionally, SLGCs initiate asymmetric division and produce satellite meristemoids (Fig. 1B). This secondary asymmetric division occurs in a nonrandom fashon away from the existing stoma (Yang and Sack 1995; Geisler et al. 2000, 2003; Nadeau and Sack 2002). As a consequence, stomata are sep-
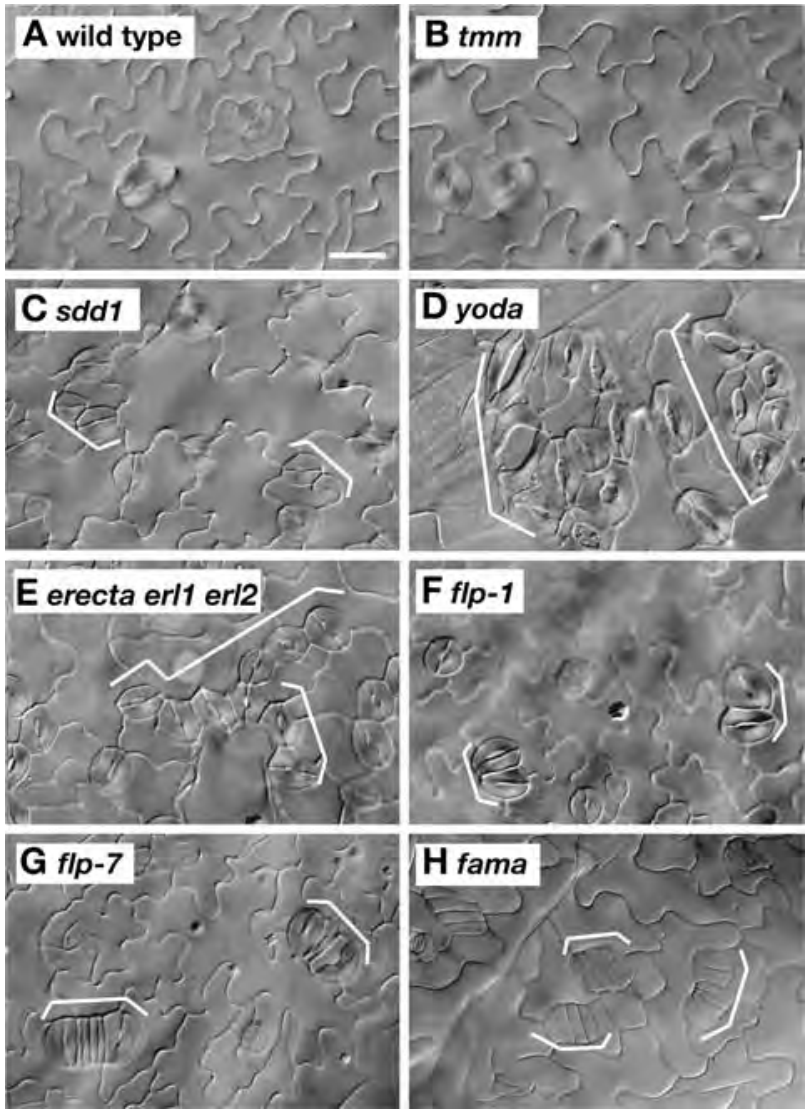

Fig. 2 Stomatal patterning mutants. Shown are the DIC (differential interference contrast) microscopy images of the abaxial rosette leaf epidermis of: A wild type; $\mathbf{B} t m m$; $\mathbf{C} s d d 1$; D yoda; $\mathbf{E}$ erecta erl1 erl2; $\mathbf{F} f l p-1$; G $f l p-7$; and $\mathbf{H}$ fama. Images are taken under the same magnification. A scale bar $=20 \mu \mathrm{m}$ 
arated by at least one cell (known as the "one-cell spacing rule") (Fig. 1B, Fig. 2) (Nadeau and Sack 2002). Proper spacing is critical for physiological functions of stomata, because guard cells must exchange water and ions (e.g. $\mathrm{K}^{+}$and $\mathrm{Cl}^{-}$) with surrounding subsidiary cells in order to open and close (Assmann and Shimazaki 1999; Schroeder et al. 2001, 2001; Hetherington and Woodward 2003). The observed, "one-cell spacing rule" indicates that the newly forming meristemoid "knows" the location of pre-existing stoma and avoids stomatal cluster formation by orienting the site of secondary asymmetric division. This suggests the presence of cell-cell communication.

In the following sections, I will describe the cytological events during stomatal differentiation and emerging roles of key regulatory genes of stomatal patterning.

\section{3}

\section{Stage I-a: Entry Into Asymmetric Division}

\section{1}

\section{Regulation of Orientation and Frequency of Asymmetric Division}

Thus far, no molecular markers have been reported for MMC identity. The earliest cytological event that clearly distinguishes the MMC is the polarization of the cytoskeleton, which predicts the site of asymmetric division (Lucas et al. 2005).

The orientation and frequency of the initial asymmetric divisions and cell-cell interaction among the daughter cells determine the proper density and spacing of stomata. Genes implicated in signal transduction play important roles in stomatal patterning. They include TOO MANY MOUTHS (TMM), STOMATAL DENSITY AND DISTRIBUTION1 (SDD1), YODA (YDA), and three ERECTA-family genes, ERECTA (ER), ERECTA-LIKE1 (ERL1), and ERL2 (Table 1) (Berger and Altmann 2000; Nadeau and Sack 2002; Bergmann et al. 2004; Shpak et al. 2005). Loss-of-function mutations in these genes confer clustered stomata, thus violating the "1-cell spacing rule" (Fig. 2). However, phenotypes of these mutants are not identical, suggesting that their relationships are not simply linear.

$T M M$ encodes a receptor-like protein with an extracellular leucine-rich repeat (LRR-RLP), which likely acts as a receptor for a positional cue that specifies the site of asymmetric division (Nadeau and Sack 2002). The phenotypes of $t m m$ mutant plants are organ-dependent and complex: the cotyledons and leaves produce clustered stomata (Fig. 2B); the stems produce no stomata; and pedicels exhibit a gradient of no stomata to stomatal clusters (Yang and Sack 1995; Geisler et al. 1998). This complex phenotype implies that TMM triggers contrasting developmental events in a dosage-dependent manner and that each organ requires a different dosage of TMM. Perhaps, TMM potentiates 
Table 1 Arabidopsis genes regulating stomatal development

Gene name

AGI Gene Product (putative) Refs.

number

1: Genes regulating asymmetric division and stomatal patterning

$\operatorname{ERECTA}(E R)^{\mathrm{a}, \mathrm{b}}$

At2g26330 LRR-receptor-like kinase (Shpak et al. 2005)

ERECTA-LIKE1 (ERL1) ${ }^{\mathrm{a}, \mathrm{b}}$ At5g62230 LRR-receptor-like kinase (Shpak et al. 2005)

ERECTA-LIKE2 (ERL2) ${ }^{\text {a }}$ At5g07180 LRR-receptor-like kinase (Shpak et al. 2005)

STOMATAL DENSITY

AND DISTRIBUTION1

At1g04110

subtilicin-like

(Berger \& Altmann 2000;

$(S D D 1)^{\mathrm{a}}$

proteinase

von Groll et al. 2002)

TOO MANY MOUTHS

At1g80080 LRR-receptor protein

(Yang and Sack 1995;

$(T M M)^{\mathrm{a}}$

$Y O D A(Y D A)^{\mathrm{a}}$

At1g63700 MAPkinase kinase

Nadeau and Sack 2002)

kinase

(Bergmann et al. 2004)

2: Genes regulating guard cell differentiation

\begin{tabular}{|c|c|c|c|}
\hline$F A M A^{\mathrm{a}}$ & A3g24140 & bHLH protein & (Bergmann et al. 2004) \\
\hline FOUR LIPS $(F L P)^{\mathrm{a}}$ & At1g14350 & R2R3 Myb protein & (Lei et al. 2005) \\
\hline$M Y B 88^{\mathrm{b}}$ & At2g02820 & R2R3 Myb protein & (Lei et al. 2005) \\
\hline \multicolumn{4}{|c|}{ 3: Genes regulating guard cell cytokinesis/morphogenesis } \\
\hline $\begin{array}{l}\text { CYCLIN-DEPENDENT } \\
\text { KINASE B1;1 (CDKB1;1) }\end{array}$ & At3g54180 & cyclin-dependent kinase & (Boudolf et al. 2004) \\
\hline $\begin{array}{l}\text { CYTOKINESIS } \\
\text { DEFECTIVE1 (CYD1) }\end{array}$ & NA & NA (not cloned) & (Yang et al. 1999) \\
\hline$K E U L E^{\mathrm{c}}$ & At1g12360 & Secl protein & (Sollner et al. 2002) \\
\hline $\begin{array}{l}\text { STOMATAL } \\
\text { CYTOKINESIS } \\
\text { DEFECTIVE1 }(\text { SCD1 })^{\mathrm{c}}\end{array}$ & At1g49040 & DENN-WD40 protein & (Fabel et al. 2003) \\
\hline \multicolumn{4}{|c|}{ 4: Genes mediating environmental control of stomatal density } \\
\hline $\begin{array}{l}\text { HIGH CARBON } \\
\text { DIOXIDE }(H I C)\end{array}$ & At2g46720 & $\begin{array}{l}\text { Long-chain fatty acid } \\
\text { biosynthesis }\end{array}$ & (Gray et al. 2000) \\
\hline
\end{tabular}

Notes: Genes are in alphabetical orders

a Photographic images provided in Fig. 2

b Phenotypes largely redundant. Combination of double (or triple) mutations among closely related paralogs revealed synergistic interactions

c Weak- or temperature sensitive alleles show guard cell cytokinesis defects. The defects in severe alleles are pleiotropic

the entry into the stomatal pathway at lower concentrations (e.g. in MMC), but at high concentration (e.g. in meristemoids) TMM ensures guard cell differentiation, while inhibiting its neighboring cells from further asymmetric division.

SDD1 encodes a subtilicin-like putative extracytoplasmic protease (Berger and Altmann 2000). In animals, this protease family is known to process pep- 
tidic ligands to a mature form (Cui et al. 1998). The $s d d 1$ mutant exhibits high stomatal density but with few stomatal clusters (Fig. 2C). This implies that SDD1 may primarily regulate the frequency of initial asymmetric division with lesser effects on specifying the orientation in relation to existing stoma.

YDA encodes a putative mitogen activated protein kinase kinase kinase (MAPKKK), a cytoplasmic protein kinase acting at the entry point of MAPK cascades (Bergmann et al. 2004). In plants, animal, and fungi, MAPK cascades integrate and amplify signals transmitted from the upstream cell-surface receptors and activate downstream gene expression in the nucleus to regulate cellular processes. (Serger and Krebs 1995). Unlike tmm and $s d d 1$, the $y d a$ mutation is highly pleiotropic. $y d a$ was first reported as a mutation defective in the initial asymmetric division of zygote with disrupted embryo patterning (Lukowitz et al. 2004). In addition, $y d a$ plants show severe dwarfism, disrupted floral patterning, and male and female sterility (Lukowitz et al. 2004). The epidermis of $y d a$ leaves produces high-density stomatal clusters (Bergmann et al. 2004). This phenotype is much more severe than in tmm and $s d d 1$ (Fig. 2D). YDA may act as an on-off switch to repress initial entry into stomatal development when a cell receives a signal from its neighbors, while TMM and SDD1 translate the signal gradient. Consistently, the overlyactive form of YDA (YDA $\triangle N B$ ) severely inhibits the onset of initial asymmetric divisions, resulting in the epidermis consisting solely of pavement cells (Bergmann et al. 2004).

Unexpectedly, the ERECTA-family genes were recently shown to play a role in stomatal patterning. ERECTA is a well-known gene regulating plant architecture, in which loss-of-function mutation confers a characteristic compact inflorescence with short pedicels and blunt fruits (Torii et al. 1996). ERECTA encodes an LRR receptor-like kinase (LRR-RLK), a prevalent family of RLKs that play important roles in developmental, steroid-hormone signal transduction, and defense against pathogens (Torii et al. 1996, 2004; Becraft 2002). ERECTA and its two paralogous genes, ERL1 and ERL2, interact in a synergistic manner in regulating stomatal patterning (Shpak et al. 2005). The erecta erl1 erl2 triple loss-of-function mutations confer severe dwarfism, disrupted floral patterning, male- and female sterility, and a high-density stomatal clustering phenotype (Fig. 2E) (Shpak et al. 2004, 2005). Overall, these phenotypes highly resemble those of the $y d a$ single mutant, suggesting that the YDA MAPK cascade may function downstream of ERECTA-family RLKs in multiple developmental processes.

\section{2}

\section{Genetic Interactions and Hierarchy of Signal Transduction}

Studies of genetic interactions are now illuminating possible cell-cell signal transduction mechanisms regulating stomatal patterning. The overexpres- 
sion of SDD1 inhibited the initial asymmetric division and reduced stomatal density (von Groll et al. 2002). However, the effect was reversed by the tmm mutation, thus placing TMM downstream of SDD1 (von Groll et al. 2002). The simplest interpretation of these results is that the excessive production of mature ligands by overexpression of SDD1 overly inhibited entry into stomatal development via the TMM receptor.

$Y D A$ most likely acts downstream of the SDD1-TMM pathway. A single copy of $Y D A \triangle N B$, which by itself does not completely inhibit the entry into stomatal development, was able to suppress the stomatal cluster phenotype of $s d d 1$ and $t m m$ (Bergmann et al. 2004). This indicates that a slight increase in YDA activity was able to resume a normal level of signal transduction in the absence of SDD1 or TMM. Perhaps, signals mediated via TMM are transmitted to the YDA-MAPK cascades to suppress neighboring cells to enter the stomatal-lineage.

TMM does not possess any cytoplasmic stretch (Nadeau and Sack 2002). In fact, the TMM protein molecule ends with a 23 amino-acid-long membranespanning region, suggesting that the C-terminal end of TMM does not extend from the plasma membrane to the cytoplasm. In addition, the C-terminus of TMM possesses a GPI (glycosylphosphatidylinositol)-anchor motif, suggesting that TMM may be anchored to the membrane surface (Nadeau and Sack 2002). Such structural features make TMM unlikely to transmit signals by itself, and it is reasonable to speculate that TMM forms a receptor complex with a partner molecule that possesses a cytoplasmic effector domain. Scientists predicted that the partner of TMM would be an LRR-RLK, in light of other systems such as CLAVATA (CLV) in shoot apical meristem development, whereby CLV1 LRR-RLK is thought to form a receptor dimer with CLV2 LRR-RLP (Clark et al. 1997; Jeong et al. 1999).

The three ERECTA-family LRR-RLKs are attractive candidates of the TMM partner. The genetic interaction of TMM and ERECTA-family by Shpak et al. (2005) suggest that TMM may restrict the inhibitory action of ERECTAfamily RLKs on the initial entry into asymmetric division. This was evident from their interactions in the stems. The tmm stems give rise to no stomata, while the erecta erl1 erl2 stems form high-density stomatal clusters (Nadeau and Sack 2002; Shpak et al. 2005). The epistatic relationship of TMM and ERECTA-family genes in the stem epidermis exhibited stoiochiometric dynamics: While TMM is epistatic to each one of the ERECTA-family genes, three ERECTA-family genes together are epistatic to TMM. Removing TMM and two out of three ERECTA-family genes resumed nearly wild-type stomatal phenotype (Shpak et al. 2005). It is exciting to speculate that TMM modulates the activity of ERECTA-family RLKs via direct association. However, in cotyledons and leaves, both tmm and erecta-family triple mutants display stomatal clusters. Do they act cooperatively in these organs while acting antagonistically in the stems? Establishing the molecular bases of receptor interactions and identifying their ligands is critical for elucidating the 
complex action of TMM and ERECTA-family receptors. By analogy to known LRR-RLPs and LRR-RLKs, the ligands for TMM/ERECTA-family receptors are most likely small peptides.

\section{4 \\ Stage I-b and II: Amplifying Asymmetric Division and Differentiation of Meristemoids}

The amplifying asymmetric division of a meristemoid occurs in an inward spiral. Serna et al. (2002) documented that the division angle of meristemoids in the Ler adaxial leaf epidermis is exactly 60 degrees with little deviation. Therefore, a newly formed, triangular-shaped meristemoid likely re-establishes its polarity away from the polar end of the previous asymmetric division. This implies the presence of a chemical gradient and a cellular system to translate gradient to determine the site of cytokinesis. Lucas et al. (2006) reported that it is common to have two adjacent meristemoids in wild-type epidermis, given that the initial entry asymmetric division occurs randomly. However, two adjacent meristemoids always divide away from each other to avoid further contact. Such polarity is disrupted in the tmm mutant, indicating that $T M M$ functions in perceiving the positional cues during amplifying asymmetric division.

Flexible numbers of amplifying asymmetric division allows developmental plasticity to adjust stomatal density in response to environmental changes. Furthermore, it provides a way to "correct" erroneous division to avoid clustered stomata. The number of amplifying asymmetric divisions may be regulated by ERECTA-LIKE (ERL) genes. The pedicel epidermis of erl1 erl2 double mutants gave rise to stomatal complexes with reduced number of SLGC (stomatal lineage ground cells), implying that the meristemoids completed reduced rounds of asymmetric division and precociously differentiated into GMCs.

ERECTA-family regulates the binary decision of daughter cells of an asymmetric division to adopt stomata vs. SLGC fates. Consistently, the epidermis of erecta single mutant produced occasional patches of 2-3 cells that appear to have undergone asymmetric division but failed to differentiate into guard cells. Expression of stomatal-lineage markers, TMM::GUS and ERL1::GUS in these groups of cells supports the hypothesis that both daughter cells became SLGCs. The tmm mutation greatly enhanced this "patches of cells with no stomata" phenotype. (Shpak et al. 2005). Indeed, the tmm erecta double mutations completely eliminated stomata from cauline-leaf and carpel epidermis, leaving numerous small cells that likely underwent asymmetric division and then became SLGC without accompanying guard cells. (Shpak et al. 2005). In both cases, termination of stomatal fate is likely due to misregulation of ERL1, which becomes overly inhibitory in repressing GMC 
differentiation. Consistent with this hypothesis, the additional erl1 mutation reversed the no-stomata phenotype of tmm erecta. Whether TMM regulates ERL1 via direct association awaits further biochemical analysis.

A positive regulator of differentiation of a meristemoid has not yet been identified. If such a gene exists, then the loss-of-function mutation may extend the lifespan of meristemoids' stem cell-like activity, and consequently mutant plants should exhibit excessive rounds of amplifying asymmetric division. If a default pathway of a meristemoid is to adopt pavement cell fate, then the mutant plants lacking the positive regulator may form two pavement cells after entry into asymmetric division, just like those observed in the tmm erecta double mutant background (Shpak et al. 2005). Further isolation of such mutants and molecular cloning of causal genes will help us elucidate the molecular mechanisms of stem cell differentiation in the plant epidermis.

\section{5}

\section{Stage VI: GMC Division - Intrinsic Regulation by Transcriptional Factors}

Once the meristemoid commits to becoming a GMC, it loses its potential for asymmetric division. GMC differentiation is evident from its changes in cellular morphology. The triangular meristemoid cell expands and become oval in shape with characteristic, end wall thickening (Lucas et al. 2006). The GMC achieves, precisely, a single symmetric division. This reflects the fundamental importance of having a pair of guard cells for proper stomatal function. Therefore, mechanisms must be present to ensure exactly one symmetric division occurs. Two genes, FOUR LIPS (FLP) and FAMA, prevent excessive (more than one) symmetric division of the GMC (Bergmann et al. 2004; Lai et al. 2005)

The $f l p-1$ mutant was initially isolated from the phenotype of paired stomata, which gives four aligned guard cells (Fig. 2F) (Lai et al. 2005). While adjacent stomata are arranged randomly in stomatal patterning mutants $(\mathrm{tmm}$, $s d d 1$, erecta-family, and yoda), paired stomata in flp are in parallel due to their origin from a single GMC. In the severe allele $f l p-7$, the GMC undergoes reiterative symmetric divisions, which result in formation of a row of "caterpillar-like" guard cell clusters (Fig. 2G). FLP encodes an atypical R2R3type Myb protein, which most likely functions as DNA-binding transcription factor (Lai et al. 2005). Therefore, an attractive hypothesis is that FLP suppresses expression of positive regulators of cell cycle progression promoting GMC division. Interestingly, both weak- $(f l p-1, f l p-2)$ and severe alleles $(f l p-7)$ are predicted to produce truncated proteins with incomplete Myb domains, with weaker alleles producing shorter fragments than the severe one (Lai et al. 2005). This apparent discrepancy between the severity of phenotypes and impacts on protein structure implies a dominant-negative activity of $f l p-7$ gene products, which may interfere with redundant components. Consistent with 
this idea, the T-DNA inserted knockout alleles of $f l p$ display weak phenotypes (Lai et al. 2005).

The paralogous Myb gene, MYB88, is most likely the redundant factor. MYB88 shares high sequence identity ( $91 \%$ amino-acid identity in the MYB domain, $71 \%$ overall) and exhibits similar expression pattern with FLP (Lai et al. 2005). Interestingly, while complete loss-of-function mutations in MYB88 failed to confer any visible phenotype, they dramatically enhanced the size of "caterpillar-like" guard cell stacks in the flp mutant (Lai et al. 2005). Moreover, introduction of an extra genomic copy of MYB88 with its own promoter rescued the $f l p$ phenotype. The combined dosage of FLP/MYB88 may be critical for guard cell differentiation.

FAMA was identified from transcriptional profiling as a gene upregulated in yoda (in which the epidermis is predominantly stomata) compared to $Y D A \triangle N B$ (in which the epidermis is predominantly pavement cells) (Bergmann et al. 2004). The loss-of-function fama phenotype highly resembles that of $f l p$, suggesting that FAMA and FLP function in the same step of GMC differentiation. However, unlike $f l p$, the abnormal "caterpillar-like" clusters in fama never form mature guard cells, suggesting that FAMA suppresses GMC division but in addition promotes the guard cell differentiation program (Fig. 2H). FAMA encodes a bHLH (basic-Helix Loop Helix) protein and most likely acts as a DNA- binding transcription factor.

The molecular identity of FLP and FAMA as Myb and bHLH transcription factors highlights an intriguing link to underlying mechanisms of epidermal cell-type differentiation in leaves and roots. Differentiation of trichomes and root hairs requires orchestrated actions of Myb transcriptional activators, which associate with bHLH proteins (Schiefelbein 2000, 2003). It would be therefore of special interest to address whether FLP and FAMA physically associate with each other and constitute a transcriptional regulatory complex.

\section{6}

\section{Stage IV: Guard Cell Morphogenesis}

The final stage of stomatal differentiation involves guard cell morphogenesis, a step leading to the formation of paired guard cells. After a symmetric division of the GMC, the new cell wall forms along the side of division, which develops a pore. The guard cells adopt a characteristic microtubule and microfibril organization (Hepler and Palevitz 1974). Defective cytokinesis leads to abnormal guard cell morphology. The cytokinesis defective1 (cyd1) mutant forms abnormal guard cells with various degrees of cytokinesis defects: $\sim 20 \%$ form a single, large round cell lacking any ventral wall or pore, $\sim 10 \%$ have incomplete wall with pore, and $\sim 5 \%$ form a single round guard cell with partial cell wall protrusions (Yang et al. 1999). These abnormal cells are either single- or bi-nucleated, each correlating with the extent of cytoki- 
netic defects (Yang et al. 1999). The molecular identity of CYD1 is not known. The temperature-sensitive stomatal cytokinesis-defective1-1 (scd1-1) mutant exhibits abnormal guard cells similar to the cyd1 mutant (Falbel et al. 2003). $S C D 1$ encodes a protein with two domains (DENN domain and WD-40 repeats), and these structural features imply a possible role for SCD1 in vesicle trafficking during cell-plate formation. Consistently, the null alleles of $s c d 1$ exhibit pleiotropic defects in cytokinesis and polar cell expansion. Therefore, abnormal guard cell morphology may be a sensitive indicator of general cytokinesis defects, which can be exploited to recover weak alleles of key regulatory genes for cytokinesis. Consistently, the weak alleles of KNOLLE and $K E U L E$, two genes initially isolated as regulators of embryogenesis, display abnormal stomatal morphology (Sollner et al. 2002). KNOLLE and KEULE encode syntaxin and Sec1, respectively, two physically-interacting proteins required for vesicle fusions at the nascent cell plate (Lukowitz et al. 1996; Waizenegger et al. 2000). In addition to cytokinesis, the cell cycle defects may confer abnormal guard cells (see below).

\section{7}

\section{Cell Cycle Regulation in Stomatal Patterning}

Stomatal patterning and differentiation is tightly coupled with specific types of cell division: the initial asymmetric division of MMCs, the amplifying asymmetric division of meristemoids, and a single symmetric division of GMCs. In addition, genome replication is strictly controlled during stomatal development, as guard cells remain at 2C (diploid) unlike the rest of epidermal cells that undergo endoreduplication (Melaragno et al. 1993). The obvious questions are whether specific cell cycle regulators control distinct cell division types during stomatal development and, if so, whether forcing cell cycle switches can invoke/suppress stomatal development. Studies suggest that cell cycle regulators may influence stomatal patterning, but they do not impinge on stomatal differentiation.

The promoter of the Arabidopsis CTD1 gene, which encodes a subunit of the DNA-replication licensing complex together with AtCDC6, is highly active in stomatal-lineage cells (Castellano Mdel et al. 2004). The AtCTD1::GUS promoter activity resembles that of TMM and ERL1, with highest activity in meristemoids and GMCs, and moderate activity in SLGCs (Castellano Mdel et al. 2004). Overexpression of AtCDT1 and AtCDC6 slightly increased the numbers of stomata, but it did not lead to formation of adjacent stomata. These results suggest that the DNA-replication licensing complex may promote stomatal asymmetric division and that forcing G1-to-S phase transition may slightly increase the MMC specification. However, this is not sufficient to overcome negative regulation by cell-cell signaling components encoded by $S D D 1, T M M, Y O D A$, and ERECTA-family genes. 
Arabidopsis B-type cyclin-dependent kinase gene $C D K B 1,1$ is also expressed in stomatal-lineage cells with high expression in meristemoids, GMCs, and in guard cells (Boudolf et al. 2004). Overexpression of a dominantnegative form of $C D K B 1,1$ led to a significant reduction in SLGCs due to reduced amplifying asymmetric division. Intriguingly, the mature stomata in the dominant-negative transgenic plants exhibited aberrant morphology, with unicellular round or kidney-shaped single guard cells without a pore (Boudolf et al. 2004). These unicellular stomata have a nuclear content of 4C, indicating that they are arrested in the G2 phase. Therefore, inhibition of $C D K B 1,1$ prevents division of both meristemoids and GMCs without interfering with the guard cell differentiation program.

How stomatal developmental regulatory genes influence cell cycle machinery is an open question. At least four members of stomatal cell-cell signal transduction, YDA and three ERECTA-family RLKs, are required for cell proliferation during normal plant growth, as both $y d a$ and erecta erl1 erl2 triple mutant plants are severely dwarfed with reduced cell numbers (Lukowitz et al. 2004; Shpak et al. 2004). Conversely, the overly-active $Y D A \Delta N B$ plants show excessive stem elongation due to increased cell numbers (McAbee and Torii, unpublished). How do YDA and three ERECTA-family RLKs promote cell proliferation while suppressing entry into the stomatal lineage? RT-PCR analysis of erecta erl1 erl2 triple mutant plants by Shpak et al. (2004) did not reveal any increase in mRNA levels of G1-cyclins that are known to promote auxinmediated organ growth (Mizukami and Fischer 2000; Hu et al. 2003). It is possible that cell proliferation is modulated by a mechanism other than G1cyclin expression. Better understanding of the exact cell cycle defects in these mutants may link cell cycle regulation and stomatal patterning.

\section{8}

\section{Environmental Control of Stomatal Patterning}

Plants sense environmental changes and adjust stomatal density accordingly. Numerous environmental factors, including light, humidity, drought, ozone, and atmospheric $\mathrm{CO}_{2}$ concentrations affect stomatal density and/or stomatal index (Holroyd et al. 2002). Among these factors, $\mathrm{CO}_{2}$ concentrations and stomatal density show an inverse correlation in a wide variety of plant species (Holroyd et al. 2002). How do plants integrate environmental signals to modulate intrinsic stomatal developmental programs? Identification of the HIGH CARBON DIOXIDE (HIC) gene by Gray et al. (2000) brought new insight into this important question. The Arabidopsis hic mutant has no apparent phenotype in ambient conditions. However, the hic mutant is greatly increased in stomatal density (approx. $40 \%$ increase) under the elevated $\mathrm{CO}_{2}$ concentration (Gray et al. 2000). HIC encodes a putative 3-keto acyl Co-A synthase, an enzyme regulating synthesis of very-long-chain fatty acids (VLCFA), which 
constitute epicuticular wax. HIC is expressed specifically in developing guard cells (but not in the meristemoid or GMC).

Consistent with the role of HIC in epicuticular wax biosynthesis, mutations in two additional epicuticular wax biosynthesis genes, CER 1 and CER6, confer significant increases in stomatal density even in the ambient $\mathrm{CO}_{2}$ levels (Gray et al. 2000; Holroyd et al. 2002). Unlike HIC, CER1 and CER6 affect wax composition in the entire epidermis, including pavement cells (Aarts et al. 1995; Fiebig et al. 2000). One scenario is that the altered composition in the guard cell extracellular matrix changes the concentration gradient of a diffusible inhibitor of stomatal development to neighboring cells. Under high $\mathrm{CO}_{2}$ concentrations, altering wax composition only in the guard cells (but not the entire epidermis) is sufficient to trigger excess stomatal formation. Obviously, identifying the elusive diffusible signal is the key for understanding environmental control of stomatal patterning.

\section{9}

\section{Future Perspectives}

Recent years have seen a dramatic advancement in our understanding of molecular mechanisms of stomatal patterning and differentiation. The identification of SDD1, TMM,YODA, and ERECTA-family genes now allows us to investigate the biochemical basis of stomatal cell-cell signaling. Establishing molecular interactions among these signaling molecules is the obvious next step. It would be particularly interesting to see whether TMM and ERECTAfamily RLKs form receptor heterodimers. However, several key regulatory molecules are still missing. For example, we do not know the identity of ligands or downstream MAPK components for stomatal patterning. Likewise, nothing is known about the positive regulators, which specify meristemoid identity as well as differentiation of meristemoids to GMCs. In animals, control of asymmetric division and cell-type differentiation is controlled by the orchestrated actions of cell-cell signaling, cell division programs, and transcription factors that drive the fate decision. Some key transcription factors for stomatal differentiation may have yet to be discovered. An integrated approach, taking advantage of modern "omics" as well as classical forward- and reverse genetics, may lead to a breakthrough in filling the gap in our knowledge of the molecular bases of stomatal development.

Updates: Since the original book chapter was submitted, four significant publications have appeared. Wang et al. (2007) identified two MAPKs (AtMPK3 and AtMPK6) and two upstream MAPKKs (AtMKK4/AtMKK5) as redundant negative regulators of stomatal differentiation. Both biochemical and genetic data indicate that these kinases act downstream of YODA. Interestingly, AtMPK3/6 and AtMKK4/5 are known to regulate environmental- 
and biotic (pathogen) stress. Findings by Wang et al. (2007) provide tantalizing evidence that both developmental and stress-induced signaling pathways converge at the downstream MAPK cascades.

Second, a trio of genes directing three key steps of stomatal differentiation was identified (MacAlister et al. 2007; Ohashi-Ito and Bergmann, 2006; Pillitteri et al. 2007). Loss-of-function mutations in the gene SPEECHLESS $(S P C H)$ confer an epidermis solely made of pavement cells, thus lacking any stomatal lineage cells. Loss-of-function mutations in MUTE lead to excessive asymmetric division of the meristemoids that fail to differentiate into GMC. Both SPCH and MUTE encode basic helix-loop-helix (bHLH) proteins closely-related with each other as well as with FAMA. Therefore, stomatal differentiation is directed by sequential actions of the three "key switch" bHLH genes: $S P C H$ at initiation (from MMCs to meristemoids), MUTE at precursor differentiation (from meristemoids to GMCs), and FAMA at terminal differentiation of guard cells (from GMCs to guard cells). The findings highlight an intriguing parallel between stomatal cell-type differentiation and muscle- and neuron cell-type differentiation in animals.

Acknowledgements I thank members of my laboratory, especially Lynn Pillitteri, Jessica McAbee, and Naomi Bogenschutz for providing photographic images and helpful comments. My research programs are supported by the US-Department of Energy (DEFG02-03ER15448), Japan Science \& Technology Agency (CREST award), and by the US-National Science Foundation (IOB-0520548).

\section{References}

Aarts M, Keijzer C, Stiekema W, Pereira A (1995) Molecular characterization of the CER1 gene of Arabidopsis involved in epicuticular wax biosynthesis and pollen fertility. Plant Cell 7:2115-2127

Assmann S, Shimazaki K (1999) The multisensory guard cell. Stomatal responses to blue light and abscisic acid. Plant Physiol 119:809-816

Becraft PW (2002) Receptor kinase signaling in plant development. Annu Rev Cell Develop Biol 18:163-192

Berger D, Altmann T (2000) A subtilisin-like serine protease involved in the regulation of stomatal density and distribution in Arabidopsis thaliana. Genes Dev 14:1119-1131

Bergmann DC, Lukowitz W, Somerville CR (2004) Stomatal development and pattern controlled by a MAPKK kinase. Science 304:1494-1497

Castellano Mdel M, Boniotti MB, Caro E, Schnittger A, Gutierrez C (2004) DNA replication licensing affects cell proliferation or endoreplication in a cell type-specific manner. Plant Cell 16:2380-2393

Clark SE, Williams RW, Meyerowitz EM (1997) The CLAVATA1 gene encodes a putative receptor kinase that controls shoot and floral meristem size in Arabidopsis. Cell 89:575-585

Cui Y, Jean F, Thomas G, Christian JL (1998) BMP-4 is proteolytically activated by furin and /or PC6 during vertebrate embryonic development. EMBO J 17:4735-4743 
Edwards D, Kerp H, Haas H (1998) J Exp Bot 49:255-278

Esau K (1977) Stomata. In: Anatomy of seed plants. Wiley, New York, pp 88-99

Falbel TG, Koch LM, Nadeau JA, Segui-Simarro JM, Sack FD, Bednarek SY (2003) SCD1 is required for cytokinesis and polarized cell expansion in Arabidopsis thaliana [corrected]. Development 130:4011-4024

Fiebig A, Mayfield J, Miley N, Chau S, Fischer R, Preuss D (2000) Alteration in CER6, a gene identical to CUT1, differentially affect long-chain lipid contects on the surface of pollen and stems. Plant Cell 12:2001-2008

Geisler M, Nadeau J, Sack FD (2000) Oriented asymmetric divisions that generate the stomatal spacing pattern in Arabidopsis are disrupted by the too many mouths mutation. Plant Cell 12:2075-2086

Geisler M, Yang M, Sack FD (1998) Divergent regulation of stomatal initiation and patterning in organ and suborgan regions of the Arabidopsis mutants too many mouths and four lips. Planta 205:522-530

Geisler MJ, Deppong DO, Nadeau JA, Sack FD (2003) Stomatal neighbor cell polarity and division in Arabidopsis. Planta 216:571-579

Gray JE, Holroyd GH, van der Lee FM, Bahrami AR, Sijmons PC, Woodward FI, Schuch W, Hetherington AM (2000) The HIC signalling pathway links $\mathrm{CO}_{2}$ perception to stomatal development. Nature 408:713-716

Hepler P, Palevitz B (1974) Microtibules and microfilaments. Annu Rev Plant Physiol 25:309-362

Hetherington AM, Woodward FI (2003) The role of stomata in sensing and driving environmental change. Nature 424:901-908

Holroyd GH, Hetherington AM, Gray JE (2002) A role for the cuticular waxes in the environmental control of stomatal development. New Phytol 153:433-439

$\mathrm{Hu}$ Y, Xie Q, Chua NH (2003) The Arabidopsis auxin-inducible gene ARGOS controls lateral organ size. Plant Cell 15:1951-1961

Jeong S, Trotochaud AE, Clark SE (1999) The Arabidopsis CLAVATA2 gene encodes a receptor-like protein required for the stability of the CLAVATA1 receptor-like kinase. Plant Cell 11:1925-1933

Lai LB, Nadeau JA, Lucas J, Lee EK, Nakagawa T, Zhao L, Geisler M, Sack FD (2005) The Arabidopsis R2R3 MYB proteins FOUR LIPS and MYB88 restrict divisions late in the stomatal cell lineage. Plant Cell 17:2754-2767

Lake JA, Woodward FI, Quick WP (2002) Long-distance $\mathrm{CO}_{2}$ signalling in plants. J Exp Bot 53:183-193

Lucas JR, Nadeau JA, Sack FD (2006) Microtubule arrays and Arabidopsis stomatal development. J Exp Bot 57:71-79

Lukowitz W, Mayer U, Jurgens G (1996) Cytokinesis in the Arabidopsis embryo involves the syntaxin-related KNOLLE gene product. Cell 84:61-71

Lukowitz W, Roeder A, Parmenter D, Somerville C (2004) A MAPKK kinase gene regulates extra-embryonic cell fate in Arabidopsis. Cell 116:109-119

MacAlister CA, Ohashi-Ito K, Bergmann DC (2007) Transcription factor control of asymmetric cell divisions that establish the stomatal lineage. Nature 445:537-540

Mizukami Y, Fischer RL (2000) Plant organ size control: AINTEGUMENTA regulates growth and cell numbers during organogenesis. Proc Natl Acad Sci USA 97:942-947

Nadeau JA, Sack FD (2002) Control of stomatal distribution on the Arabidopsis leaf surface. Science 296:1697-1700

Nadeau JA, Sack FD (2002) Stomatal Development in Arabidopsis. In: Somervile CR, Meyerowitz EM (eds) Arabidopsis Book. ASPB, Rockville, MD 
Ohashi-Ito K, Bergmann DC (2006) Arabidopsis FAMA controls the final proliferation/differentiation switch during stomatal development. Plant Cell 18:2493-2505

Pillitteri LJ, Sloan DB, Bogenschutz NL, Torii KU (2007) Termination of asymmetric cell division and differentiation of stomata. Nature 445:501-505

Schiefelbein J (2003) Cell-fate specification in the epidermis: a common patterning mechanism in the root and shoot. Curr Opin Plant Biol 6:74-78

Schiefelbein JW (2000) Constructing a plant cell. The genetic control of root hair development. Plant Physiol 124:1525-1531

Schroeder JI, Allen G, Hugouvieux V, Kwak JM, Wagner D (2001) Guard cell signal transduction. Annu Rev Plant Physiol Plant Mol Biol 52:627-658

Schroeder JI, Kwak JM, Allen GJ (2001) Guard cell abscisic acid signalling and engineering drought hardiness in plants. Nature 410:327-330

Serger R, Krebs E (1995) The MAPK signaling cascade. FASEB J 9:726-735

Serna L, Fenoll C (2000) Stomatal development and patterning in Arabidopsis leaves. Physiol Plant 109:351-358

Serna L, Torres-Contreras J, Fenoll C (2002) Clonal analysis of stomatal development and patterning in Arabidopsis leaves. Dev Biol 241:24-33

Shpak ED, Berthiaume CT, Hill EJ, Torii KU (2004) Synergistic interaction of three ERECTA-family receptor-like kinases controls Arabidopsis organ growth and flower development by promoting cell proliferation. Development 131:1491-1501

Shpak ED, McAbee JM, Pillitteri LJ, Torii KU (2005) Stmatal patterning and differentiation by synergistic interactions of receptor kinases. Science 309:290-293

Sollner R, Glasser G, Wanner G, Somerville CR, Jurgens G, Assaad FF (2002) Cytokinesisdefective mutants of Arabidopsis. Plant Physiol 129:678-690

Torii KU (2004) Leucine-rich repeat receptor kinases: structure, function, and signal transduction pathways. Int Rev Cytol 234:1-46

Torii KU (2006) Stomatal patterning and differentiation: emerging role of cell-cell signaling (in Japanese). Tanpakushitsu Kakusan Koso 51:145-154

Torii KU, Mitsukawa N, Oosumi T, Matsuura Y, Yokoyama R, Whittier RF, Komeda Y (1996) The Arabidopsis ERECTA gene encodes a putative receptor protein kinase with extracellular leucine-rich repeats. Plant Cell 8:735-746

von Groll U, Berger D, Altmann T (2002) The subtilisin-like serine protease SDD1 mediates cell-to-cell signaling during Arabidopsis stomatal development. Plant Cell 14:1527-1539

Wang H, Nugwenyama N, Liu Y, Walker JC, Zhang S (2007) Stomatal development and patterning are regulated by environmentally responseive mitogen activated protein kinases in Arabidopsis. Plant Cell doi10.1105/tpc106.048298

Waizenegger I, Lukowitz W, Assaad F, Schwarz H, Jurgens G, Mayer U (2000) The Arabidopsis KNOLLE and KEULE genes interact to promote vesicle fusion during cytokinesis. Curr Biol 10:1371-1374

Yang M, Nadeau JA, Zhao L, Sack FD (1999) Characterization of a cytokinesis defective (cyd1) mutant of Arabidopsis. J Exp Bot 50:1437-1446

Yang M, Sack FD (1995) The too many mouths and four lips mutations affect stomatal production in Arabidopsis. Plant Cell 7:2227-2239

Zhao L, Sack FD (1999) Ultrastructure of stomatal development in Arabidopsis (Brassicaceae) leaves. Am J Bot 86:929-939 


\title{
Ethylene-induced hyponastic growth in Arabidopsis thaliana is controlled by ERECTA
}

\author{
Martijn van Zanten ${ }^{1, *}$, L. Basten Snoek ${ }^{1, \dagger}{ }^{\dagger}$ Evelien van Eck-Stouten ${ }^{2}$, Marcel C.G. Proveniers ${ }^{2}$, Keiko U. Torii ${ }^{3}$, \\ Laurentius A.C.J. Voesenek ${ }^{1}$, Anton J.M. Peeters ${ }^{1}$ and Frank F. Millenaar ${ }^{1, \neq}$ \\ ${ }^{1}$ Plant Ecophysiology, Institute of Environmental Biology, Utrecht University, Padualaan 8, $3584 \mathrm{CH}$ Utrecht, The Netherlands, \\ ${ }^{2}$ Molecular Plant Physiology, Institute of Environmental Biology, Utrecht University, Padualaan 8, $3584 \mathrm{CH}$ Utrecht, \\ The Netherlands, and \\ ${ }^{3}$ Department of Biology, University of Washington, Seattle, WA 98195, USA
}

Received 21 July 2009; revised 11 September 2009; accepted 18 September 2009; published online 27 October 2009.

*For correspondence (fax +3130 2518366; e-mail m.vanzanten@uu.nl).

${ }^{\dagger}$ Present address: Department of Nematology, Wageningen University and Research Center, Droevendaalsesteeg 1, 6708 PB, Wageningen, The Netherlands.

${ }^{\ddagger}$ Present address: De Ruiter Seeds, Leeuwenhoekweg 52, 2660 BB Bergschenhoek, The Netherlands.

\begin{abstract}
SUMMARY
Plants can respond quickly and profoundly to detrimental changes in their environment. For example, Arabidopsis thaliana can induce an upward leaf movement response through differential petiole growth (hyponastic growth) to outgrow complete submergence. This response is induced by accumulation of the phytohormone ethylene in the plant. Currently, only limited information is available on how this response is molecularly controlled. In this study, we utilized quantitative trait loci (QTL) analysis of natural genetic variation among Arabidopsis accessions to isolate novel factors controlling constitutive petiole angles and ethylene-induced hyponastic growth. Analysis of mutants in various backgrounds and complementation analysis of naturally occurring mutant accessions provided evidence that the leucin-rich repeat receptor-like Ser/Thr kinase gene, ERECTA, controls ethylene-induced hyponastic growth. Moreover, ERECTA controls leaf positioning in the absence of ethylene treatment. Our data demonstrate that this is not due to altered ethylene production or sensitivity.
\end{abstract}

Keywords: ethylene, petiole, hyponasty, ERECTA, QTL, Vancouver-0.

\section{INTRODUCTION}

Plants have to adjust to their environment for optimal growth and survival. For instance, several species, including Arabidopsis thaliana, induce upward leaf movement driven by differential petiole elongation (hyponastic growth) to optimize performance under conditions of diminished environmental resource availability. This response is utilized to restore contact with the atmosphere under complete submergence (Millenaar et al., 2005), to outgrow low light intensities (Hangarter, 1997; Millenaar et al., 2005; Mullen et al., 2006) and canopy shade (Ballaré, 1999; Pierik et al., 2004, 2005), and to avoid high temperatures (Koini et al., 2009; Van Zanten et al., 2009a).

The phytohormonal interactions controlling hyponastic growth are increasingly well understood. When plants are submerged, ethylene becomes trapped inside the plant due to a diffusion barrier caused by the surrounding water This ethylene accumulation is the key trigger for hyponastic growth in several species including Rumex palustris, rice (Oryza sativa) and $A$. thaliana (Voesenek and Blom, 1989; Hoffmann-Benning and Kende, 1992; Cox et al., 2003 Millenaar et al., 2005). Additionally, ethylene is also associated with shade-induced hyponastic growth in tobacco (Nicotiana tabacum) (Pierik et al., 2003, 2004), but is dispensable for low light intensity-induced hyponastic growth and is antagonistic to heat-induced hyponastic growth in Arabidopsis (Millenaar et al., 2009; Van Zanten et al., 2009a). Abscisic acid (ABA) antagonizes ethyleneinduced hyponastic growth in Arabidopsis (Benschop et al., 2007), and the hyponastic growth response to ethylene is independent of auxin action (Millenaar et al. 
2009; Van Zanten et al., 2009b). Nevertheless, little is known of the molecular mechanisms and signaling routes operating to integrate environmental stimuli to induce a functional hyponastic growth response.

Together with the study of induced genetic variation (mutants), assessment of natural genetic variation using quantitative genetic tools can be utilized to isolate novel genetic components controlling a trait of interest (Koornneef et al., 2004; Nordborg et al., 2005; Weigel and Nordborg, 2005; Shindo et al., 2007). Extensive natural phenotypic variation among $A$. thaliana accessions has been demonstrated previously for constitutive petiole angles and ethylene-induced hyponastic growth (Millenaar et al., 2005; Hopkins et al., 2008). We exploited this natural variation to isolate quantitative trait loci (QTL) for ethylene-induced hyponastic growth. Subsequent analysis of near-isogenic lines (NILs), induced mutants in various genetic backgrounds and complementation of naturally occurring mutant accessions indicated that the leucine-rich repeat receptor-like Ser/Thr kinase gene ERECTA $(E R)$ explains one of the identified major-effect QTLs. In addition to ethylene-induced hyponastic growth, ER mediates leaf positioning in the absence of ethylene treatment. We demonstrate that these responses are not a consequence of ER-mediated effects on ethylene production or sensitivity.

\section{RESULTS}

\section{QTL analysis reveals several loci controlling ethylene- induced hyponastic petiole growth}

To identify functional genetic components controlling leaf positioning and ethylene-induced hyponastic growth, we performed QTL analysis. For this purpose, petiole angles were measured in a population of recombinant inbred lines (RILs) derived from a cross between the accessions Cape Verde Islands-0 (Cvi-0) and Landsberg erecta (Ler) (AlonsoBlanco et al., 1998) before (initial angle; IA) and after $6 \mathrm{~h}$ of ethylene treatment $(6 \mathrm{hE})$. Calculation of the broad-sense heritability $\left(\mathrm{BSH} ; \mathrm{H}^{2}\right)$ indicated that $43 \%(\mathrm{IA}), 45 \%(6 \mathrm{hE})$ and $50 \%$ ETRA (ethylene response angle, which is the subtracted angle $6 \mathrm{hE}-\mathrm{IA}$ ) of the total variation is explained by genetic variation in the RILs. The mapped OTLs above the permutation-calculated likelihood-of-odds (LOD) threshold (Van Ooijen, 1999) for each trait are shown in Figure 1 and described in Table 1.

For all traits, QTLs with both negative and positive allelic effects were identified, implicating the existence of both positive and negative molecular regulators contributing to the overall petiole angle phenotype. The QTLs IA3-1 and $6 h E 3$ were co-located at the top of chromosome 3. Both have a positive Ler effect (Figure 1 and Table 1). IA3-3 and ETRA3 were co-located at the bottom of chromosome 3 .

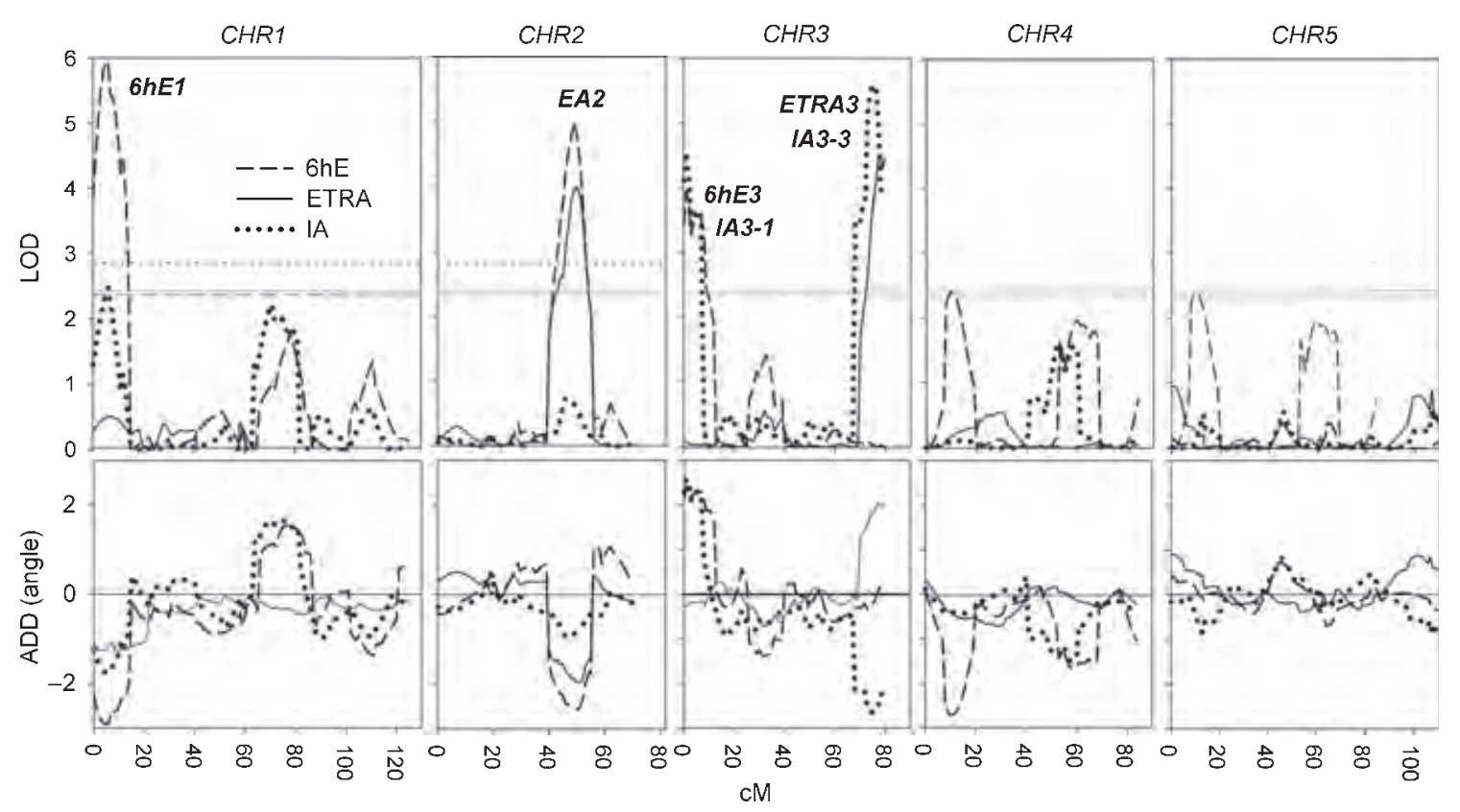

Figure 1. OTL profile for initial petiole angle and ethylene-induced hyponastic growth traits.

Position of QTLs mapped for petiole angle traits in the Ler $\times$ Cvi-0 RIL population (in cM). Upper panels show the LOD profile for each trait per chromosome (CHR). QTL names are indicated near each QTL peak. The values mapped are for initial petiole angles (IA; dotted lines) measured prior to treatment at $t=0 \mathrm{~h}$, absolute petiole angles after $6 \mathrm{~h}$ of ethylene treatment $\left(5 \mu \mathrm{L} \mathrm{L} \mathrm{L}^{-1}\right)(6 \mathrm{hE}$; dashed lines), and the difference between initial angle and the absolute angle after $6 \mathrm{~h}$ of treatment (ethylene response angle, ETRA; closed lines). The horizontal dotted, solid and dashed lines indicate the $95 \%$ confidence thresholds at LOD 2.92 (IA), 2.42 (ETRA) and $2.41(6 \mathrm{hE})$, respectively. The EA2 OTL indicate the position where OTLs for both ethylene-induced angle traits co-locates. The lower panels show the additive effect (angle) for the Ler allele compared to the population mean. 
Table 1 Characteristics of the OTLs described in this study

\begin{tabular}{llllllr}
\hline Trait & OTL name & Chr & LOD & top & ADD & \multicolumn{1}{c}{ R2 } \\
\hline IA & IA3-1 & 3 & 4.5 & 1 & 2.58 & 4.55 \\
& IA3-3 & 3 & 5.6 & 75 & -2.64 & 5.57 \\
& Total & & & & -0.06 & 10.12 \\
$6 \mathrm{hE}$ & 6hE1 & 1 & 6.0 & 5 & -2.92 & 13.39 \\
& EA2 & 2 & 5.0 & 49 & -2.56 & 9.76 \\
& 6hE3 & 3 & 3.9 & 1 & 2.429 & 8.37 \\
& Total & & & & -3.05 & 31.52 \\
ETRA & EA2 & 2 & 4.0 & 50 & -1.95 & 10.80 \\
& ETRA3 & 3 & 4.5 & 80 & 2.025 & 11.45 \\
& Total & & & & 0.079 & 22.25 \\
& & & & & & \\
\hline
\end{tabular}

QTLs mapped in the Ler $\times$ Cvi-O RIL population for initial petiole angles (IA) measured prior to treatment at $t=0 \mathrm{~h}$, absolute petiole angles after $6 \mathrm{~h}$ of ethylene treatment $\left(5 \mu \mathrm{L}^{-1}\right)(6 \mathrm{hE})$, and the difference between initial angle and the absolute angle after $6 \mathrm{~h}$ of treatment (ethylene response angle, ETRA).

$\mathrm{Chr}$, the chromosome to which the QTL was mapped; LOD, likelihood of difference score; top, position of maximum QTL LOD value (in $\mathrm{CM}$ ); $A D D$, additive effect (Ler allele effect difference from mean, in degrees); R2, explained variance (percentage). The sums of R2 and the additive effects are given in the last row for each trait (total).

These QTLs had opposite effects to each other. Furthermore, co-locating QTLs for $6 \mathrm{hE}$ and ETRA mapped to a region approximately $50 \mathrm{cM}$ on chromosome 2 . These ethylenespecific OTL were together re-named ethylene-angle 2 (EA2). Last, a trait-specific QTL for $6 \mathrm{hE}$ mapped to the top of chromosome 1 (Figure 1 and Table 1).

The initial petiole angle and absolute angle after $6 \mathrm{~h}$ of ethylene treatment for a heterozygous $F_{1}$ line derived from a cross between Ler and Cvi-0 were intermediate between the parental Ler and Cvi-0 angles (Figure 2a,b). This suggests that both accessions lack dominant loci controlling absolute petiole angles. However, the hyponastic growth response angle (RA) of the $F_{1}$ line equaled that of Cvi-0 after ethylene treatment (Figure 2c). Thus, Cvi-0 probably contains a dominant positive locus involved in control of the response to ethylene.

\section{Evaluation of the complex EA2 QTL}

We focused on characterization of the QTLEA2, because this locus appears to control ethylene-induced hyponastic growth specifically (e.g. no IA OTLs mapped to this location), and both OTLs contributing to EA2 (6hE and ETRA) had a consistent negative Ler effect.

To confirm EA2, we measured petiole angles in a nearisogenic line (NIL LCN2-7; Keurentjes et al., 2007) containing a single Cvi-0 introgression spanning the complete EA2 OTL region (30.3-71.7 cM; outer Ler markers on chromosome 2). Unexpectedly, the initial angle of LCN2-7 was significantly lower than that for either parental line $(P<0.001$; Figure 2a). The absolute angles after $6 \mathrm{~h}$ ethylene treatment were statistically indistinguishable between Ler and LCN2-7 (Fig- ure 2b). However, the response of LCN2-7 to ethylene was identical to that of Cvi-0 and the heterozygous Ler $\times \mathrm{Cvi}^{-0} \mathrm{~F}_{1}$ line, and thus was significantly stronger than $\operatorname{Ler}(P<0.001$; Figure 2c). These data were confirmed by analysis of pairwise subtracted response kinetics (Figure 2d), measured using a time-lapse camera set-up (Millenaar et al., 2005) which corrects for circadian and diurnal influences on leaf movement as well as for the initial angles. These data show that the Cvi-0 introgression strongly enhances the response to ethylene (Figure 2d).

Together, these results indicate that the LCN2-7 introgression contains a Cvi-0 locus that was not detected by our QTL analysis that negatively affects initial petiole angles. However, introgression of LCN2-7 confirmed the negative Ler effect QTL for ethylene response.

\section{ERECTA receptor-like kinase controls initial petiole angle and induced hyponastic growth}

The EA2 QTL mapped to the ERECTA (ER) locus. One of the obvious candidate genes underlying this QTL is the ER gene. $E R$ encodes a leucine-rich repeat receptor-like Ser/Thr kinase, and Ler carries a missense mutation within the conserved region of the kinase domain (Torii et al., 1996). Therefore, ER segregates between Landsberg erecta and Cvi-0 (Van Zanten et al., 2009c; and references therein). To test whether ER controls hyponastic growth, we measured initial petiole angles and induced hyponastic growth in er mutant alleles in various genetic backgrounds (Torii et al., 1996), and in the original Landsberg wild-type line (Lan-0) from which Ler was isolated (Redei, 1962).

Lan-0 exhibited a significantly $(P<0.001)$ lower initial petiole angle than Ler (Figure 2e). To test whether this was caused by er, we introduced the Col-0 ER locus, including the promoter sequences, into Ler by transformation (ERLer). This transgenic line complemented all characteristic plant architectural phenotypes typically attributed to er, including the compact rosette appearance (Figure 3a-k), reduced plant height, increased inflorescence thickness and decreased silique length (Figure $31-n)$. Initial petiole angles in $E R$-Ler were significantly $(P<0.01)$ lower than in Ler, and were indistinguishable from those in Lan-0 (Figure 2e), suggesting that ER controls petiole positioning. Interestingly, no such difference in initial petiole angle could be found between the er-105 null mutant and its parental line Columbia-0 (Col-0) or between the er-104 null mutant and Wassilewskija (Ws), although the typical architecture traits (Figure 2) attributed to er were observed in these lines. Several other er alleles isolated in the Col-0 background (Lease et al., 2001) also showed the significantly reduced initial angle (Figure 4a), suggesting that ERmediated control of initial petiole angles is not specific for the Landsberg background, but is allele-dependent.

No significant differences in absolute angles after $6 \mathrm{~h}$ ethylene treatment (Figure 2f) were found between er 


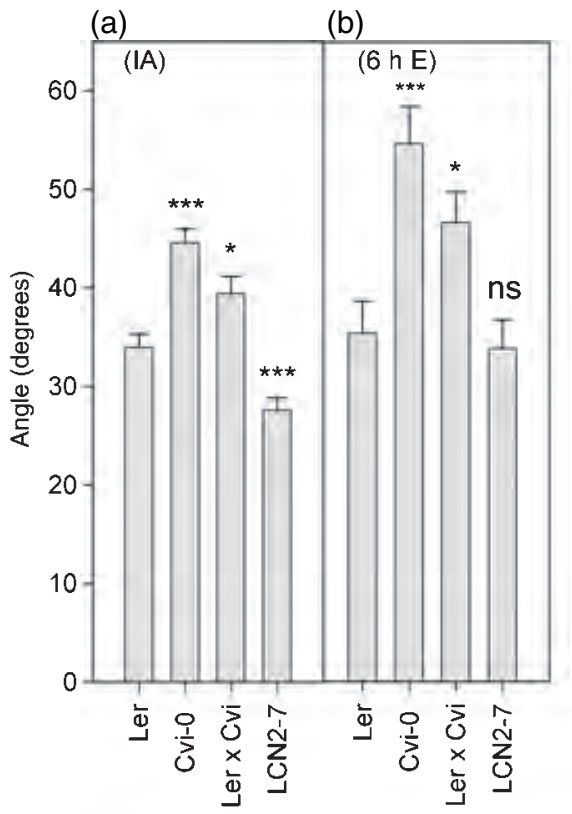

(e)

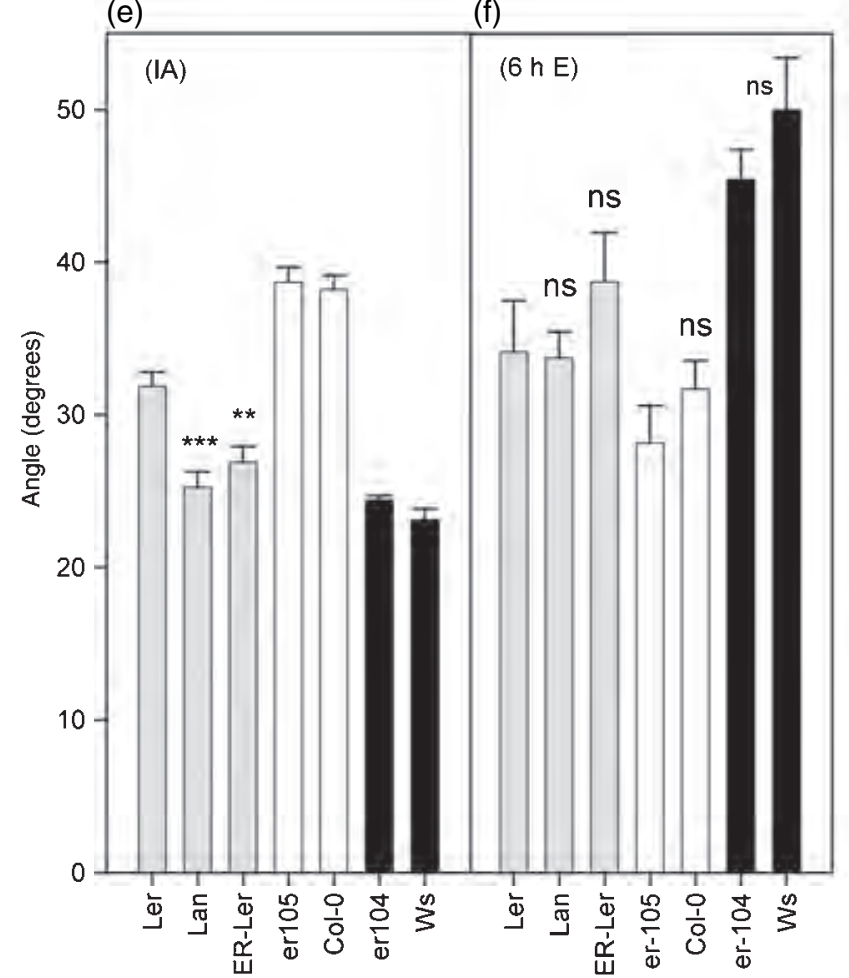

(c)

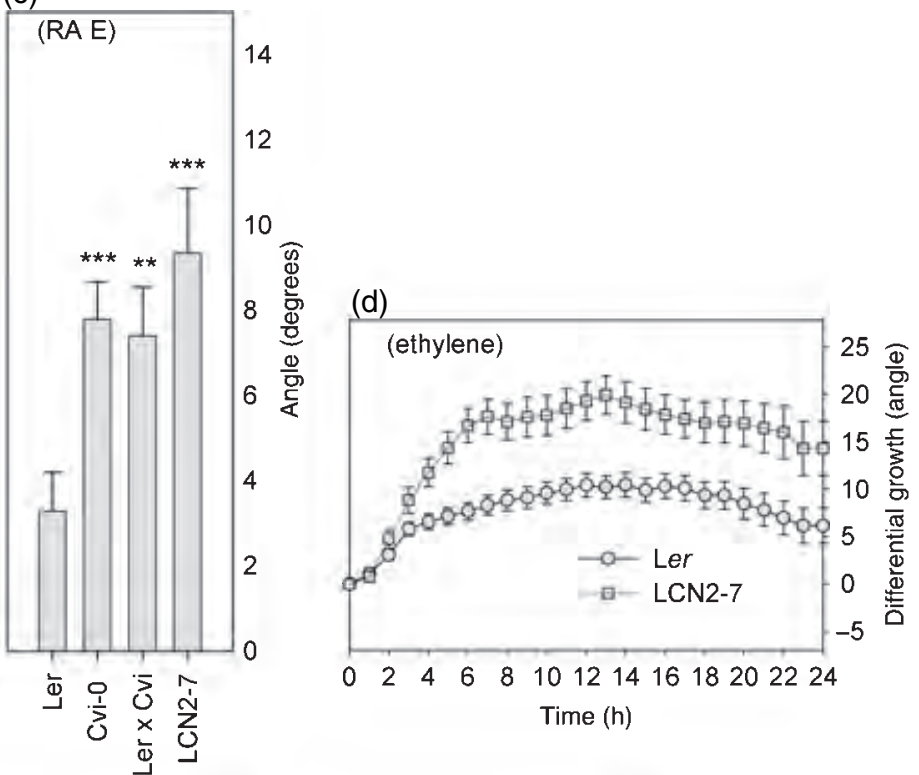

(g)

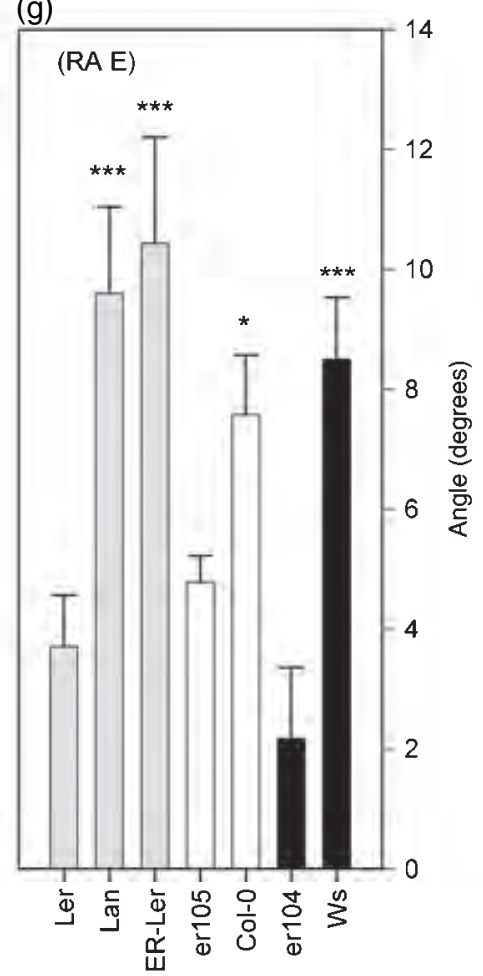

Figure 2. Genetic dissection of the EA2 QTL.

$(\mathrm{a}-\mathrm{c})$ Absolute petiole angles (a) before (initial angle; $\mathrm{IA} ; t=0 \mathrm{~h}$ ) and (b) after 6 h ethylene treatment $\left(6 \mathrm{~h} \mathrm{E} ; 5 \mu \mathrm{L} \mathrm{L}^{-1}\right)$; (c) response angle (RA E, which is the difference between the angles at 0 and $6 \mathrm{~h}$ ) for the RIL parents, heterozygous $\mathrm{F}_{1}$ and LCN2-7.

(d) Effect of exposure to ethylene on petiole angles of LCN2-7 (squares) and Ler (circles). Values are pairwise-subtracted ( $n \geq 12$ ).

$(\mathrm{e}-\mathrm{g})$ Petiole angles (e) before (initial angle; $I \mathrm{~A} ; t=0 \mathrm{~h}$ ) and (f) after $6 \mathrm{~h}$ ethylene treatment $\left(6 \mathrm{~h} \mathrm{E;} 5 \mu \mathrm{L} \mathrm{L}^{-1}\right) ;(\mathrm{g})$ response angle (RA E, which is the difference between the angles at 0 and $6 \mathrm{~h}$ ) for the er mutants, their corresponding wild-types and the ER-Ler complemented transgenic line.

Significance levels: ${ }^{*} P<0.05,{ }^{*} P<0.01,{ }^{* * *} P<0.001$ (by Student's $t$ test compared to Ler, $n \geq 12$ ). ns, not significant. Error bars represent SE.

alleles, ER-Ler and their respective wild-types, which is in accordance with the observations made on LCN2-7 (Figure $2 b$ ). However, several lines in the broader set of eralleles in the Col-0 background did show a significantly reduced absolute petiole angle after $6 \mathrm{~h}$ of ethylene treatment (Figure $4 b, c$ ). 

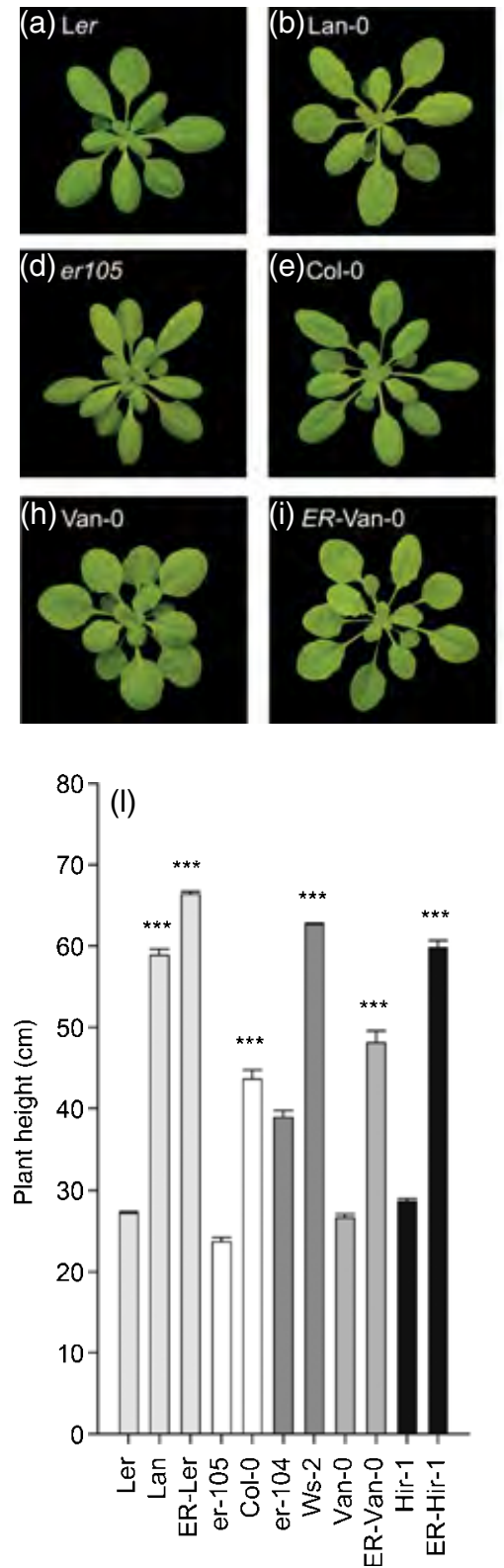
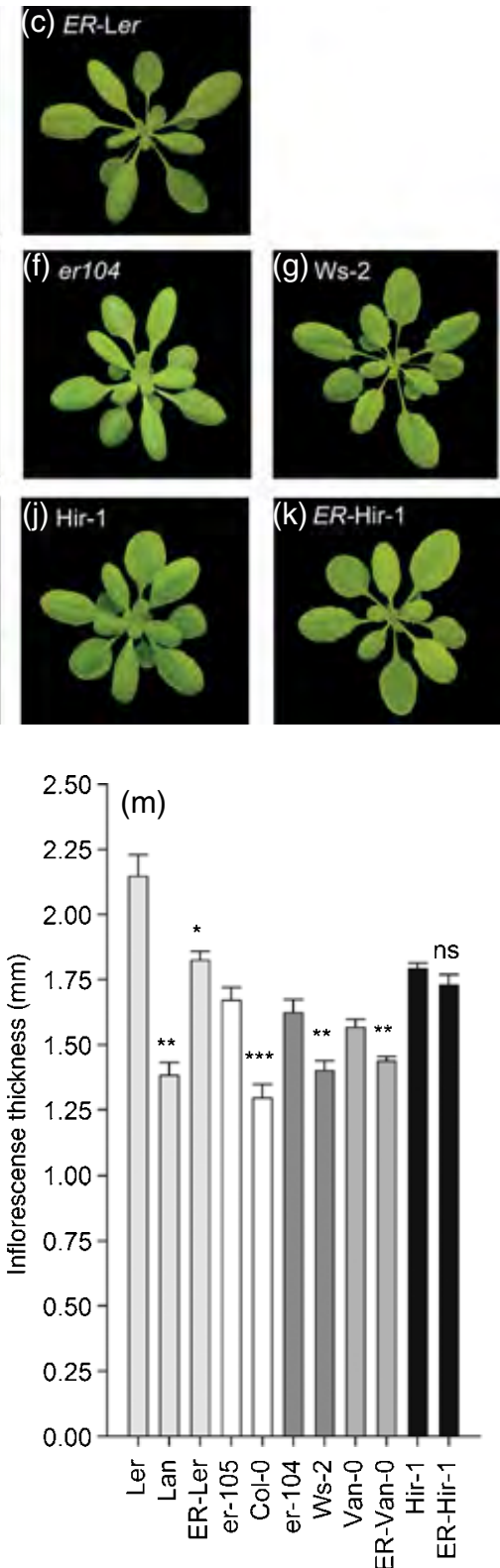

Figure 3. Effect of ER on plant architecture phenotypes in Arabidopsis thaliana.

$(a-g)$ Morphology of induced er mutants (a, d, f) and their respective wild-types (b, e, g); (c) ER-complemented Ler. $(\mathrm{h}-\mathrm{k})$ Naturally occurring er accessions $(\mathrm{h}, \mathrm{j})$ and $E R$-complemented lines of these accessions $(\mathrm{i}, \mathrm{k})$.

$(\mathrm{I}-\mathrm{n})$ Effects of ER on plant height (I), inflorescence stem thickness $(\mathrm{m})$ and silique length ( $\mathrm{n}$ ) for the lines shown in (a)-(k). Error bars represent SE ( $n>10)$. Significance levels: ${ }^{*} P<0.05,{ }^{*} P<0.01,{ }^{* * *} P<0.001$ by Student's $t$ test, ns, not significant.

The response angles after $6 \mathrm{~h}$ of ethylene treatment revealed, without exception, that lines carrying functional $E R$ are able to respond more strongly to ethylene (Figure $2 \mathrm{~g}$ ). This is in accordance with the results shown in Figure 2(c) for Cvi-0, the Ler $\times$ Cvi-0 $F_{1}$ and LCN2-7. These results were confirmed by analysis of the pairwise corrected response kinetics (Figure 5). Lan-0 exhibited a stronger response to ethylene than Ler throughout the experimental period (Figure $5 \mathrm{a}$ ), and the ER-Ler response was similar to that of Lan-0 (Figure 5a,b). In agreement, er-105 had an attenuated response to ethylene compared to Col-0 (Figure $5 \mathrm{c}$ ). However, kinetic analysis did not reveal differences between er-104 and Ws-2 (Figure 5d).

In addition to these induced mutations in er, at least two naturally occurring accessions carry a malfunctioning er allele, as shown by allelism tests (Koornneef et al., 2004; Van Zanten et al., 2009c). We complemented these lines, Vancouver-0 (Van-0) and Hiroshima-1 (Hir-1), with the Col-0 ER allele. This led to an increase in plant height, inflorescence length and silique length (Figure 3), and led to a stronger 


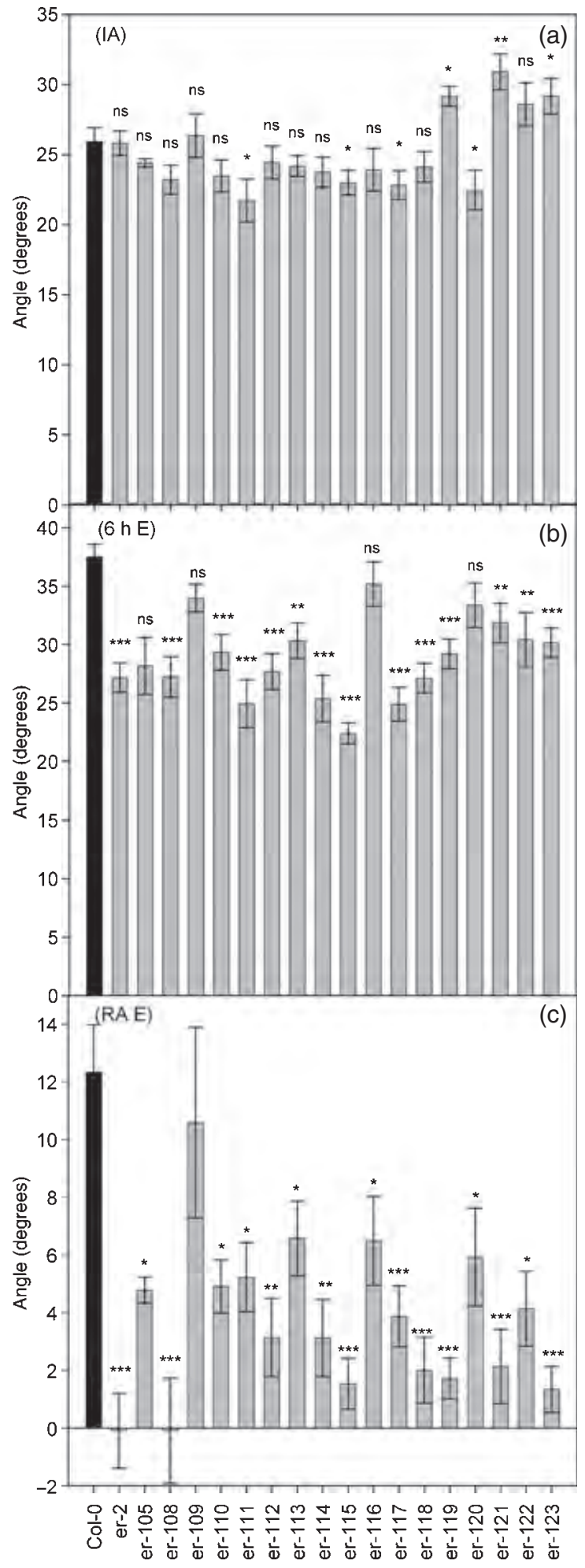

Figure 4. Initial petiole angles and induced hyponastic growth of Col-0 er alleles.

(a) Initial angles (IA) before treatment. (b) Absolute angles (6hE) and (c)

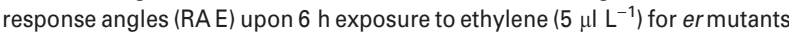
in the Col-0 genetic background. The values for Col-0 wild-type are shown as a black bar. Error bars represent SE $(n \geq 4)$. Significance levels: ${ }^{*} P<0.05$; ${ }^{*} P<0.01 ;{ }^{* *} P<0.001$ by Student's $t$ test. ns, not significant. induction of hyponastic growth upon ethylene treatment (Figure 5e,f). Features, that are all typically associated with functional ER. Together, these data provide evidence that ER positively controls ethylene-induced hyponastic growth and negatively controls leaf positioning in the absence of ethylene treatment. However, these effects are allele-dependent.

\section{Molecular nature of the naturally occurring er allele}

To unravel the molecular nature of the naturally occurring er allele, the corresponding genomic region of the er locus in Van-0 was sequenced. The Van-0 er possesses numerous deletions and insertions, as well as four amino acid substitutions, including a methionine-to-isoleucine substitution at the initiation codon (Figure $5 \mathrm{~g}$ ). Because Van-0 er does not possess the proper initiation codon, it is most likely a null mutant. Our results identify the unique molecular lesions in this naturally occurring er allele, which may have implications on growth and development in natural populations of this accession.

\section{ERECTA does not influence petiole angles via modulation of ethylene production or sensitivity}

The effects of er on initial petiole angle and on hyponastic growth might be explained by er effects on ethylene production or sensitivity. Transcription of several genes encoding early ethylene signal transduction components, as well as catalytic enzymes in the ethylene biosynthesis route, are up-regulated in the presence of ethylene (Wilkinson et al., 1995; Hua et al., 1998; Millenaar et al., 2009). As a default measurement, we compared transcriptome data of several ethylene signaling and biosynthesis genes, obtained using microarrays that were hybridized with cDNA from the aerial parts of 7-day-old Ler and Lan-0 plants (Snoek, 2009), with data for Col-0 petioles treated for $3 \mathrm{~h}$ in air (control) or with ethylene (Millenaar et al., 2006). Indeed, several ethylene signaling and biosynthesis genes were significantly up-regulated in ethylene-treated petioles as compared to controls (Figure 6a,b) (Millenaar et al., 2006). The same perception genes were not significantly differentially expressed between Lan-0 and Ler. The only exception was ERS1 $(P=0.036)$ (Figure 6c), which showed a slightly higher expression in Lan-0 compared to Ler.

In contrast, several ethylene-inducible ACC-oxidase ethylene biosynthesis genes were significantly induced in Ler compared to Lan-0 (Figure 6d). Accordingly, ethylene release from Ler was significantly $(P<0.001)$ higher than from Lan-0 (Figure 6e). However, the ER-complemented line (ER-Ler) released ethylene to a similar extent as Ler. This suggests that ER does not control ethylene production, but that an $E R$-independent polymorphism, segregating between Ler and Lan-0, controls the enhanced ethylene production. In agreement, ethylene release from er-104 and er-105 was similar to their respective wild-types (Figure 6e). 

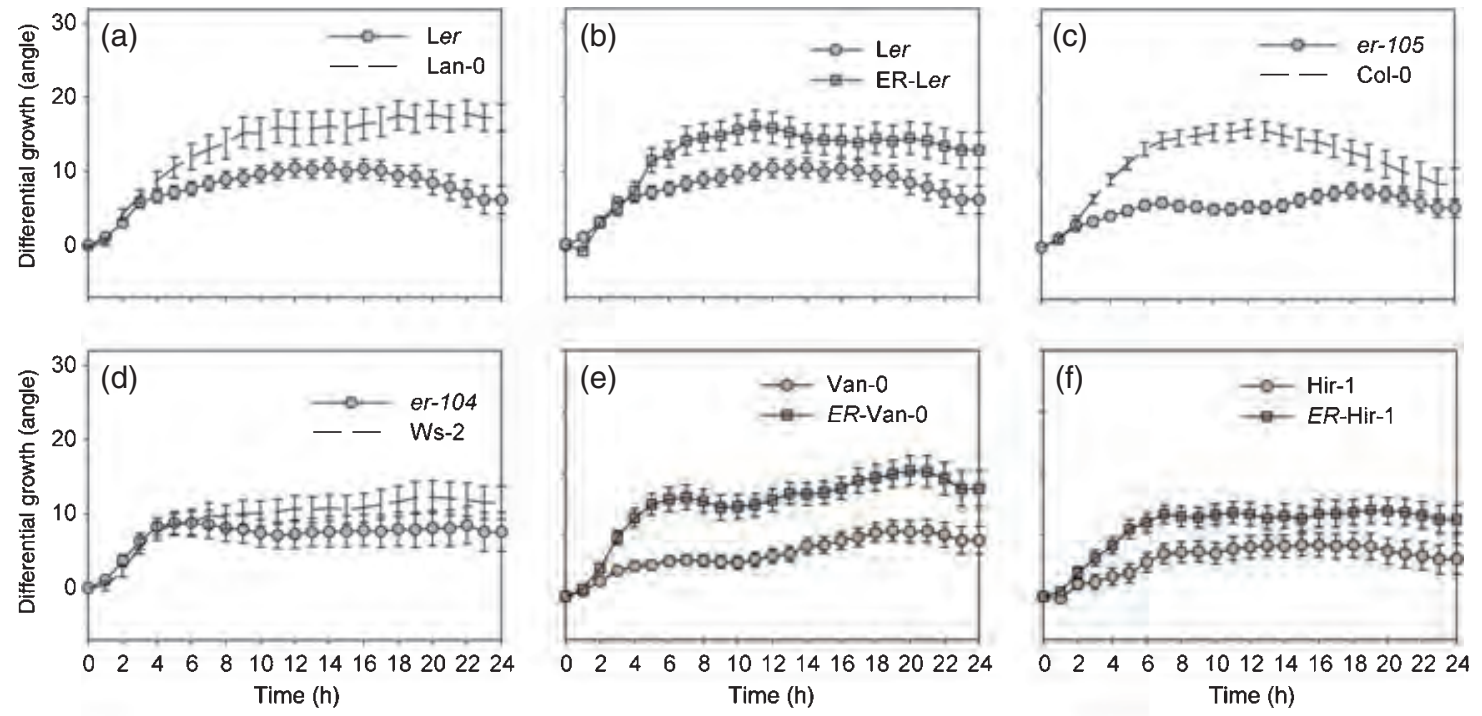

(g)

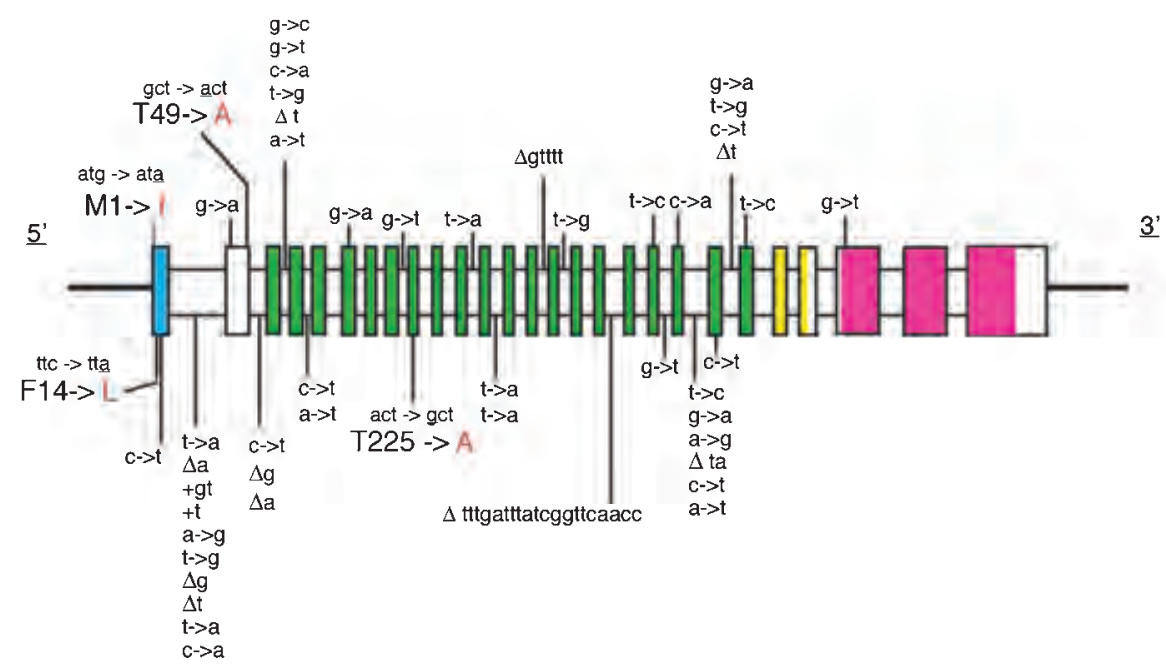

Figure 5. Effects of $E R$ on kinetics of ethylene-hyponastic growth.

(a-f) Effect of exposure to ethylene $(5 \mu \mathrm{L} \mathrm{L}$ ) on petiole angles of er mutants (circles) in the Ler background (a, b), Col-0 background (c), Ws-2 background (d) and the natural erecta mutant accessions Van-0 (e) and Hir-1 (f). Values for corresponding wild-types are shown as dashed lines, and those for the ER-complemented transgenic lines of Ler (b), Van-0 (e), and Hir-1 (f) are indciated by squares. Values are pairwise-subtracted. Error bars represent SE $(n \geq 12)$.

(g) DNA sequence of the naturally occurring Van-0 erecta mutant allele. The locations and consequences of polymorphisms are shown. Tall boxes represent exons and short boxes represent introns. Blue, green, yellow and pink correspond to the locations of the signal sequence, the leucine-rich repeat (LRR) domain, the transmembrane domain, and the protein kinase domain, respectively. Letters in red indicate amino acid substitutions.

Moderate and high ethylene concentrations inhibit cell elongation of hypocotyls in etiolated seedlings, whereas low and ambient concentrations of ethylene stimulate hypocotyl elongation (Guzman and Ecker, 1990; Hua and Meyerowitz, 1998; Pierik et al., 2006). We generated ethylene dose-response curves (triple response assay) to assess ethylene sensitivity in the various lines used here. No differences in ethylene sensitivity were observed between Lan-0 and Ler or between Col-0 and er-105 (Figure 6f,g). In contrast, er-104 showed increased hypocotyl length at low as well as at higher ethylene concentrations compared to Ws-2 wild-type (Figure 6h). This suggests that er-104 may be more sensitive at low ethylene concentrations (stronger growth induction) and less sensitive at high ethylene concentrations (less growth reduction).

Together, these data indicate that ER does not control ethylene production and does not have a general effect on ethylene sensitivity. It is therefore unlikely that ER-mediated control of ethylene production or sensitivity can explain the $E R$ effects on initial petiole angles and hyponastic growth phenotypes.

\section{DISCUSSION}

In this study, QTLs for initial petiole angles and ethyleneinduced hyponastic growth were mapped with the aim of isolating novel factors controlling these important traits, 

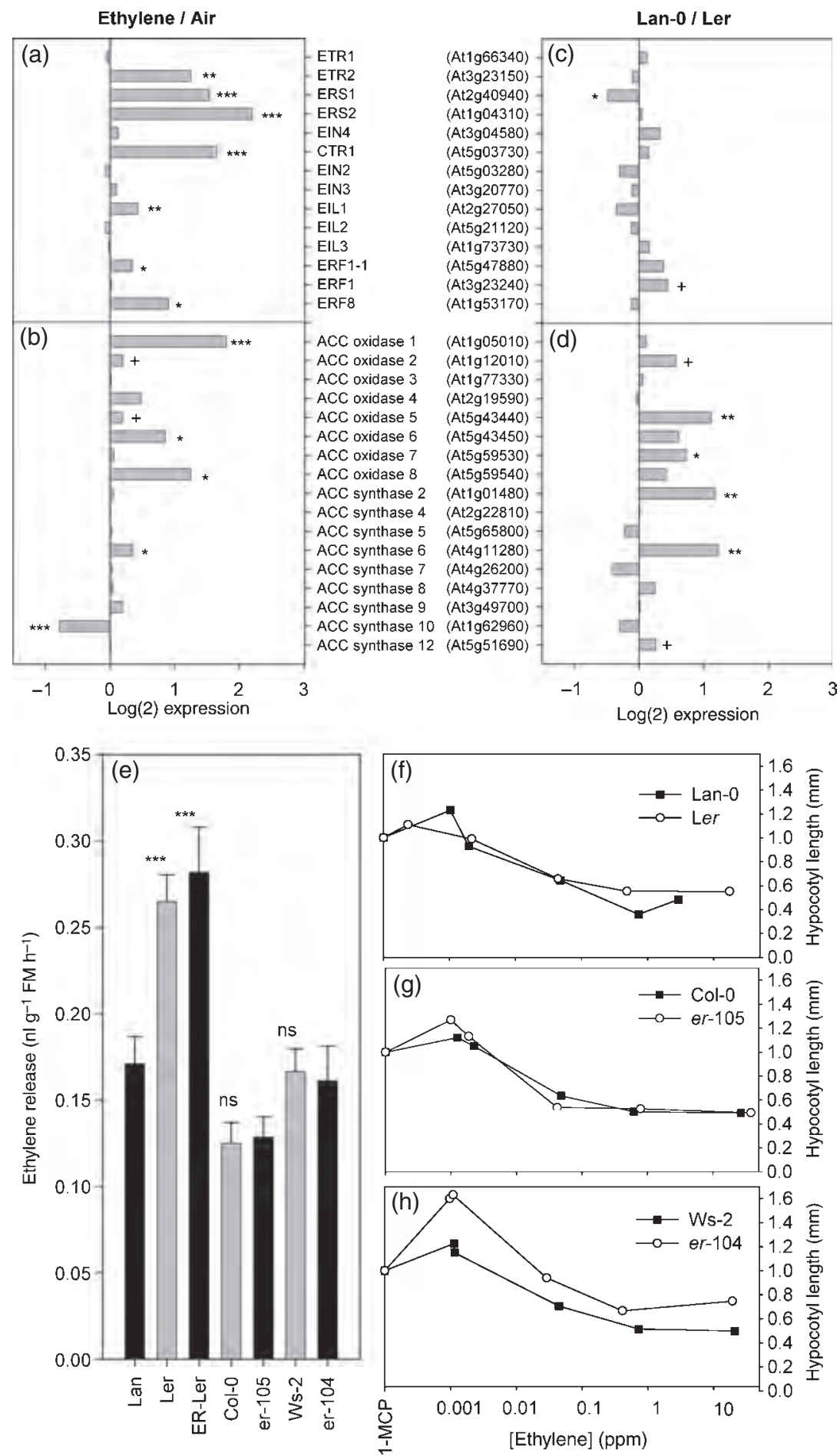

Figure 6. ER effects on transcription of ethylene-related genes, ethylene production and ethylene sensitivity.

(a-d) Expression ratios of $(a, c)$ ethylene signaling and (b, c) ethylene biosynthesis genes (AGI codes in parentheses) of plants treated with ethylene $\left(5 \mu l L^{-1}\right)$ relative to air controls (a, b) (ATH1 Affymetrix microarrays, data and procedures are described by Millenaar et al., 2006) and of Lan-0 plants compared to Ler (c, d) (CATMA spotted arrays, data and procedures are described by Snoek, 2009). The values shown are $\log (2)$ values of the ratio obtained by dividing raw arbitrary expression data of the respective microarrays.

(e) Ethylene release $\left(\mathrm{nl} \mathrm{g}^{-1}\right.$ fresh mass $\mathrm{h}^{-1}$ ) of er mutants, ER-complemented Ler and corresponding wild-types.

(f-h) Ethylene dose-response curves for hypocotyl growth in etiolated er mutant (white circles) and wild-type (black squares) seedlings. 1-MCP indicates plants in which ethylene perception was pharmacologically blocked.

Significance levels were determined by two-tailed Student's $t$ test $(\mathrm{a}, \mathrm{b}, \mathrm{e}-\mathrm{h})$ or are adjusted $P$-values $(\mathrm{c}, \mathrm{d}):{ }^{+} P<0.1,{ }^{*} P<0.05,{ }^{*} P<0.01,{ }^{*} * * P<0.001$. ns, not significant. (e) $n \geq 14$ samples of three pooled plants each; (f) $n \geq 8$. Error bars represent SE. 
associated with escape from diminished growth conditions (Millenaar et al., 2005, 2009).

Until now, few genes controlling leaf movements have been described in $A$. thaliana. Probably best understood in this respect are circadian clock-controlled diurnal leaf movements (Millar and Kay, 1991). Extensive natural variation has been observed in circadian/diurnal leaf movements among Arabidopsis accessions (Swarup et al., 1999; Edwards et al., 2005), and some of the observed variation in initial petiole angles and induced hyponastic growth phenotypes among the RILs assessed in this study may be due to circadian effects on petiole angles. Accordingly, some QTLs for circadian leaf movement co-located with QTLs identified in this study (Swarup et al., 1999; Edwards et al., 2005).

Detailed analysis of the EA2 QTL using NILs, induced er mutants in various genetic backgrounds and natural er accessions complemented with ER provided direct evidence that ER controls ethylene-induced hyponastic growth. However, we did not observe differences in ethylene sensitivity or transcription levels of ethylene perception components between er mutants and wild-types, or between Lan-0 and Ler. Because transcription of ethylene receptor genes is known to be induced by ethylene (Wilkinson et al., 1995; Hua et al., 1998; Millenaar et al., 2009), we conclude that ER functions downstream of ethylene signaling in the control of hyponastic growth.

Correction for circadian leaf movements by calculation of pairwise subtracted leaf angles (Benschop et al., 2007) showed a clear remaining effect of ethylene on petiole angles in the tested lines. Hence we can rule out the possibility that ER effects on circadian clock-associated leaf movement account for the EA2 QTL. Despite this, ER does most likely also control circadian leaf movement (Swarup et al., 1999).

In contrast to ethylene receptor genes, several ethylene biosynthesis genes were more highly expressed in Ler than in Lan-0. Accordingly, we observed significantly enhanced ethylene release in Ler. However, this is not associated with segregating $E R$ alleles, because the complemented $E R$-Ler line also showed enhanced ethylene release. In addition, er mutants in other genetic backgrounds did not differ from their wild-type counterparts with respect to the levels of ethylene release. Consequently, there must be a second, as yet unknown, segregating polymorphism between Lan-0 and Ler that controls ethylene production. Given this, we can also exclude the possibility that the observed differences in IA between Lan- 0 and Ler can be explained by the observed differences in ethylene release between these lines, because complementation of Ler with $E R$ resulted in reduction of the initial angles to values similar to those for Lan-0 despite the fact that this line maintained enhanced ethylene production. Therefore, we conclude that, in addition to a direct positive ER effect on ethylene-dependent leaf movement, ER plays a negative role in ethylene-independent leaf positioning. This supports the observation that several er mutants in the Col background showed increased initial angles, and that the NIL LCN2-7 (carrying the Cvi-0 ER allele in a Ler isogenic background) had a significantly lower IA than Ler.

However, no QTL for IA mapped to the ER locus, and the parental line Cvi-0 (which has a functional ER) had a higher initial angle than Ler. Perhaps, closely linked segregating loci with contrasting effects on initial petiole angles are to be found in the vicinity of the ER locus, hampering their identification by QTL analysis. Alternatively, other Cvi-0 loci may have an effect on initial angle that is dominant over the repressing effects of the functional ER in this parent. Notably, Cvi-0 has a less active phytochrome $B$ (phyB) allele (Filiault et al., 2008). Loss-of-function mutations in phy $B$ result in a constitutive shade avoidance phenotype, a syndrome that typically includes enhanced leaf angles (Fankhauser and Casal, 2004; Mullen et al., 2006). Because the phyB gene is only approximately $15 \mathrm{cM}$ upstream of $E R$, it is likely that the Cvi-0 phyB allele prevented detection of an IA QTL on the ER locus.

The $E R$ locus has been isolated previously in diverse quantitative genetic studies for, among others, growthrelated traits (Van Zanten et al., 2009c) including plant height and leaf morphology (Alonso-Blanco et al., 1999; PérezPérez et al., 2002; Ungerer et al., 2002; El-Lithy et al., 2004), hormone and light responses (Borevitz et al., 2002), resistance to pathogen attack (Godiard et al., 2003; Llorente et al., 2005), transpiration efficiency (Masle et al., 2005) and epidermal leaf cell size (Tisné et al., 2008). Our data show that the penetration of ER effects on ethylene-induced petiole angles and ethylene-independent effects on initial angles depends on the genetic background. Such background dependency has been shown previously, as effects of er on plant development are weaker in Col-0 er mutants compared to Ler (Llorente et al., 2005). In addition, enhanced disease susceptibility against Plectosphaerella cucumerina, typically attributed to er in Ler, was not detected in the natural er accession Van-0 (Llorente et al., 2005). Similarly, we observed no er effects on ethyleneinduced hyponastic growth for the strong er-104 allele (Torii et al., 1996), which is derived from the Ws accesssion. This is in sharp contrast to the clear er effect on ethylene-induced hyponastic growth in Col-0, Ler, Van-0 and Hir-1. The traitspecific variation in the penetration level of er phenotypes between genetic backgrounds may be explained by ERmediated effects on genetic canalization/buffering (Hall et al., 2007), a process that renders developmental pathways insensitive for environmental 'noise'. As a result, organisms have stereotyped phenotypes in different environments (Waddington, 1942; Hornstein and Shomron, 2006). In particular, ER affected canalization of rosette leaf number (flowering time) under long-day conditions, without affecting the mean (Hall et al., 2007). One of the consequences of canalization of a developmental pathway is that polymorphisms do not necessarily lead to phenotypic variation 
(Waddington, 1942; Hornstein and Shomron, 2006; Hall et al., 2007). Canalization may thus explain why the strong er-104 allele did not result in altered responses to ethylene compared to wild-type and whyER-mediated control of initial angles was observed between Ler and Lan-0/ER-Ler but not between er104 and Ws, and was allele-specific in several Col-0 er alleles. Canalization of other typical ER-mediated developmental traits, including plant height, root length and hypocotyl length, were not affected by ER (Hall et al., 2007; Sangster et al., 2008), suggesting that the canalization potential is trait-specific rather than general. Accordingly, in our study, all lines, including the complemented natural eralleles, carrying a functional ER showed increased plant height.

Only limited data have been published on the mechanism of direct ER action. Recently, two putative secreted ligands, epidermal patterning factors 1 and 2 (EPF1 and EPF2), have been identified as a regulators of stomatal patterning and differentiation that act upstream of ER (Hara et al., 2007, 2009; Hunt and Gray, 2009). These molecules might represent ligands for ER in stomatal development, one of the many developmental traits regulated by ER (Shpak et al., 2005). We recently re-analyzed data on trans regulation of gene transcription at the ER locus (Van Zanten et al., 2009c) obtained from a genetical genomics experiment by Keurentjes et al. (2007). Gene ontology (GO) analysis of these genes revealed over-representation of several phytohormone and abiotic-environment associated classes (including response to abscisic acid stimulus, auxin stimulus and ethylene stimulus, wounding and heat), indicating that those GO classes are potentially mediated by ER. ER is also required for reducing plant sensitivity to heat stress during adaxial-abaxial polarity formation in leaves and promotes leaf adaxial cell-fate specification (Xu et al., 2003; Qi et al., 2004). Hyponastic growth is driven by differential cellelongation responses of cells at the abaxial as opposed to the adaxial side of the petiole (Cox et al., 2003). It may not be just coincidence that ER is indirectly associated with such factors, which are also known to control hyponastic growth (Millenaar et al., 2005, 2009; Benschop et al., 2007; Van Zanten et al., 2009b). However, whether and how ER exerts control on hyponastic growth by modulating one or more of these pathways remains to be studied.

\section{EXPERIMENTAL PROCEDURES}

\section{Plant material and growth conditions}

Col-0 (N1092), Ler (N1624), Ws (N915), er-2 (N3401) and er-108-er123 (N3912-N3927) were obtained from the Nottingham Arabidopsis Stock Center (NASC IDs in parentheses). The Ler $\times$ Cvi-0 RIL population (N22000; Alonso-Blanco et al., 1998), the NIL LCN2-7 (Keurentjes et al., 2007), and Van-0, Hir-1 and Lan-0 (N1298) were kind gifts from M. Koornneef (Laboratory of Genetics, Wageningen University and Research Centre, The Netherlands). er-104 (Ws) and er-105 (Col) have been described by Torii et al. (1996), and er-108er-123 by Lease et al. (2001). Correctness of the generated $F_{1}$ crosses between Ler and Cvi-O was checked using the SSLP markers
NGA128 and NGA162, which are polymorphic between Ler and Cvi0 (Bell and Ecker, 1994), using a standard PCR procedure.

Plants were grown on a mixture of fertilized potting soil and perlite (1:2) as described by Millenaar et al. (2005) under the following conditions: $20^{\circ} \mathrm{C}, 70 \% \mathrm{v} / \mathrm{v}$ relative humidity, a $9 \mathrm{~h}$ shortday photoperiod with $200 \mu \mathrm{mol} \mathrm{m}{ }^{-2} \mathrm{sec}^{-1}$ photosynthetic active radiation. Pots were automatically saturated daily with tap water at the start of the photoperiod. Before potting, seeds were kept for 4 days in the dark at $4^{\circ} \mathrm{C}$ to synchronize germination.

All plants, except the RILs, were used at developmental stage 3.83.9 according to Boyes et al. (2001). RILs were measured 25 days after germination, which was just before the earliest-flowering RILs started to bolt. Although the developmental stages of the RILs were variable due to the inherent mixed genetic make-up of RILs, the extent of the hyponastic growth response was robust until a few days after bolting (data not shown), and therefore did not interfere with the measurements.

In all cases, plants were transferred to the experimental set-up, under growth-room conditions, 1 day prior to the treatment to allow acclimatization. All experiments started $1.5 \mathrm{~h}$ after the start of the light period to minimize variation that may be induced by circadian and diurnal rhythms (Salter et al., 2003).

\section{Experimental design and data analysis}

For QTL analysis, 156 individual RILs were used, which were randomly divided into four groups containing approximately 50 RILs each, with 10 RILs overlapping between experiments. All experiments were performed twice on separate batches. Data from 3 to 12 individuals per RIL per treatment were obtained.

The frequency distribution of trait values for QTL analysis was close to a Gaussian distribution, and trait values were used in the analyses without mathematical transformation. The genetic map for the Ler $\times$ Cvi RIL population was obtained from Alonso-Blanco et al., 1998. The core maps were used for initial QTL mapping. For fine mapping, available markers were added (up to 1 per cM). QTLs were calculated by composite interval mapping (CIM) using the free program QTL Cartographer (http://statgen.ncsu.edu/qtlcart/ WQTLCart.htm) with a forward and backward regression method and a window size of $7 \mathrm{cM}$. Genome-wide likelihood of odds thresholds (LOD; Van Ooijen, 1999) for each trait were estimated in OTL Cartographer using 1000 permutations. Broad-sense heritability (BSH) was calculated as part of the between-line variance attributed to the total variance, using variance components of the general linear model procedure type III ANOVA using SPSS software (12.0.1, http://www.spss.com) and Excel 2003 (Microsoft, http:// www.microsoft.com). The bi-variate linear regression correlation coefficients are Pearson two-tailed, and were calculated using a general linear model in SPSS. Statistical comparisons between two lines or treatments were performed using a two-tailed Student's $t$ test (Excel).

Plants used for fixed time-point measurements were manually photographed from the side. Angles were measured using the freeware algorithm IMAGEJ (http://rsb.info.nih.gov/ij). For all replicate plants, two petioles were measured and the mean value was used for further analysis. Values outside the $95 \%$ confidence interval for each line in each treatment were removed prior to further analysis. Absolute petiole angles are defined as the angle between the horizontal and the petiole/lamina junction through the base of the petiole in the rosette center. Response petiole angles are the calculated difference between the initial absolute angle and absolute angle reached after $6 \mathrm{~h}$ treatment on the same plants.

To measure petiole angle kinetics, a custom-built computerized digital camera system was used (Cox et al., 2003; Millenaar et al., 2005). To enable continuous photography, no dark period was 
included in the $24 \mathrm{~h}$ experimental period. Plants were placed singly in glass cuvettes with the petioles to be studied positioned perpendicular to the axis of the camera. Cuvettes were continuously flushed with air (or ethylene if indicated) at a flow rate of $10 \mathrm{~L} \mathrm{~min}^{-1}$. To facilitate the measurements, any leaf that was obscuring the petiole being photographed was removed. Additionally, the petiole was marked at the petiole/lamina junction with orange paint (http:// www.productpilot.com/en/suppliers/royal-talens-bv/). These preparations did not influence the response of the petiole (Millenaar et al., 2005). Digital photographs were taken every $10 \mathrm{~min}$. The angles of the petioles were measured on these digital images using the PC-based KS400 (version 3.0) image analysis system (http:// www.vision.zeiss.com/) with a custom-made macro. To correct for diurnal and circadian effects on leaf movement (Millar and Kay, 1991; Salter et al., 2003), values of response angles were pairwisesubtracted in all cases (Benschop et al., 2007). This was done by calculating the differences between the angles of treated and control plants for each time point. New standard errors for the differential responses were calculated by taking the square root of the sum of the two squared standard errors.

Plant height, stem thickness and silique lengths were measured when the last flower on the plant developed a silique. Plant height was measured from the root/shoot junction to the inflorescence top. Stem thickness was measured approximately $1 \mathrm{~cm}$ above the root/ shoot junction using a caliper, and silique lengths were measured from representative pedicles in the topmost part (approximately $10 \mathrm{~cm}$ ) of the main inflorescence stem.

\section{Ethylene treatments, release measurements and triple response assays}

Unless stated otherwise, ethylene application was performed as described by Millenaar et al. (2005). Pure ethylene (Hoek Loos BV; http://www.lindegasbenelux.com) and air (70\% relative humidity) were mixed using flow meters and controllers (Brooks Instruments BV, http://www.brooksinstrument.com//) to generate a concentration of $5 \mu \mathrm{L} \mathrm{L}^{-1}$ ethylene. This was flushed continuously through glass cuvettes containing the plants at a flow rate of $10 \mathrm{~L} \mathrm{~min}^{-1}$ and then vented away. Ethylene concentrations were checked regularly using a gas chromatograph (GC955; Synspec, http://www.synspec.nl/), and remained constant for the duration of the experiment.

Ethylene release from rosettes was measured $1.5 \pm 1 \mathrm{~h}$ after the start of the photoperiod. Measurements were performed as described by Millenaar et al. (2005). Whole rosettes of approximately $300 \mathrm{mg}$ were placed in a syringe with a volume of $1.5 \mathrm{ml}$. Ethylene was allowed to accumulate in the syringe for $15-20 \mathrm{~min}$, after which the air was analyzed using a gas chromatograph (GC955; Synspec). This short time frame prevented wound-induced ethylene production, which started after $20 \mathrm{~min}$.

For the triple response assay, sterile seeds were placed on halfstrength MS/agar plates containing $4 \mathrm{~g} \mathrm{~L}^{-1}$ plant agar (Duchefa, http://www.duchefa.com/) and $0.22 \mathrm{~g} \mathrm{~L}^{-1}$ Murashige \& Skoog (Duchefa), placed in air-tight jars, and kept for 4 days at $4^{\circ} \mathrm{C}$ in the dark. Thereafter, the plates were transferred to $200 \mu \mathrm{mol} \mathrm{m}{ }^{-2} \mathrm{sec}^{-1}$ light for $6 \mathrm{~h}$. Ethylene was administered in a dilution series starting with pure ethylene (Hoek Loos BV), and injected using a syringe, and then the jars were packed in aluminum foil and left in the dark for 5 days at $20^{\circ} \mathrm{C}$. After 5 days, ethylene concentrations were measured using a gas chromatograph (GC955; Synspec) before the plants were photographed. Hypocotyl lengths were measured using ImageJ software. To allow comparison, values were normalized relative to plants in which ethylene perception was blocked by injecting $1 \mu \mathrm{L} \mathrm{L} \mathrm{L}^{-1} 1$-methylcyclopropene ('Ethylbloc'; Floralife, http:// www.floralife-europe.de).

\section{Plant transformation}

The pKUT196 plasmid vector (Torii et al., 1996) containing the functional Col-0 ER locus, including the promoter and terminator, was used for plant transformation. This vector was transformed by electroporation into Agrobacterium tumefaciens strain C58C1 (pMP90). Recombinant bacteria were selected using $50 \mu \mathrm{g} \mathrm{ml}^{-1}$ spectinomycin (Duchefa), and the presence of the vector was checked by PCR (data not shown). Subsequently, plants were transformed by floral dipping (Clough and Bent, 1998). Selection of $\mathrm{T}_{1}$ plants was performed on selective agar plates containing $4 \mathrm{~g} \mathrm{~L}^{-1}$ plant agar (Duchefa), $0.22 \mathrm{~g} \mathrm{~L}^{-1}$ Murashige \& Skoog (Duchefa) and $70 \mu \mathrm{g} \mathrm{ml}^{-1}$ gentamycin (Duchefa). Gentamycin-resistant $T_{1}$ plants were transferred to standard soil, and all exhibited the typical ERassociated tall-plant phenotype. Single seed descendants of the $T_{1}$ plants were re-selected in the $T_{2}$ and $T_{3}$ generation on gentamycin/ agar plates to obtain homozygous $T_{3}$ lines.

\section{DNA sequencing}

Two-week-old Arabidopsis Van-0 seedlings were subjected to genomic DNA preparation. Sequencing and analysis of the Van-0 ER locus was performed as described by Lease et al. (2001). The Genbank accession number for the genomic DNA sequence of Van-0 er is G0368569.

\section{ACKNOWLEDGEMENTS}

We thank Rob Schuster and Nelson Lau for their technical assistance in sequencing the Van-0 er allele, and Kylee Peterson for editing. Part of this work was supported by the Netherlands Organization for Scientific Research (NWO), Program Genomics Grant 050-10-029 (to L.B.S. and A.J.M.P.) and by US Department of Energy grant DE-FG02-03ER15448 (to K.U.T.).

\section{REFERENCES}

Alonso-Blanco, C., Peeters, A.J.M., Koornneef, M., Lister, C., Dean, C., van den Bosch, N., Pot, J. and Kuiper, M.T.R. (1998) Development of an AFLP based linkage map of Ler, Col and Cvi Arabidopsis thaliana ecotypes and construction of a Ler/Cvi recombinant inbred line population. Plant J. 14, 259271.

Alonso-Blanco, C., Blankestijn-de Vries, H., Hanhart, C.J. and Koornneef, M. (1999) Natural allelic variation at seed size loci in relation to other life history traits of Arabidopsis thaliana. Proc. Natl Acad. Sci. USA, 96, 4710-4717.

Ballaré, C.L. (1999) Keeping up with the neighbours: phytochrome sensing and other signalling mechanisms. Trends Plant Sci. 4, 97-102.

Bell, C.J. and Ecker, J.R. (1994) Assignment of 30 microsatellite loci to the linkage map of Arabidopsis. Genomics, 19, 137-144.

Benschop, J.J., Millenaar, F.F., Smeets, M.E., Van Zanten, M., Voesenek, L.A.C.J. and Peeters, A.J.M. (2007) ABA antagonizes ethylene-induced hyponastic growth in Arabidopsis. Plant Physiol. 143, 1013-1023.

Borevitz, J.O., Maloof, J.N., Lutes, J. et al. (2002) Quantitative trait loci controlling light and hormone response in two accessions of Arabidopsis thaliana. Genetics, 160, 683-696.

Boyes, D.C., Zayed, A.M., Ascenzi, R., McCaskill, A.J., Hoffman, N.E., Davis, K.R. and Gorlach, J. (2001) Growth stage-based phenotypic analysis of Arabidopsis: a model for high throughput functional genomics in plants. Plant Cell, 13, 1499-1510.

Clough, S.J. and Bent, A.F. (1998) Floral dip: a simplified method for Agrobacterium-mediated transformation of Arabidopsis thaliana. Plant J. 16, 735-743.

Cox, M.C.H., Millenaar, F.F., de Jong van Berkel, Y.E.M., Peeters, A.J.M. and Voesenek, L.A.C.J. (2003) Plant movement; submergence-induced petiole elongation in Rumex palustris depends on hyponastic growth. Plant Physiol. 132, 282-291.

Edwards, K.D., Lynn, J.R., Gyula, P., Nagy, F. and Millar, A.J. (2005) Natural allelic variation in the temperature-compensation mechanisms of the Arabidopsis thaliana circadian clock. Genetics, 170, 387-400. 
El-Lithy, M.E., Clerkx, E.J., Ruys, G.J., Koornneef, M. and Vreugdenhil, D. (2004) Quantitative trait locus analysis of growth-related traits in a new Arabidopsis recombinant inbred population. Proc. Natl Acad. Sci. USA $135,444-458$.

Fankhauser, C. and Casal, J.J. (2004) Phenotypic characterization of a photomorphogenic mutant. Plant J. 39, 747-760.

Filiault, D.L., Wessinger, C.A., Dinneny, J.R., Lutes, J., Borevitz, J.O., Weigel, D., Chory, J. and Maloof, J.N. (2008) Amino acid polymorphisms in Arabidopsis phytochrome B cause differential responses to light. Proc. Natl Acad. Sci. USA, 105, 3157-3162.

Godiard, L., Sauviac, L., Torii, K.U., Grenon, O., Mangin, B., Grimsley, N.H. and Marco, Y. (2003) ERECTA, an LRR receptor-like kinase protein controlling development pleiotropically affects resistance to bacterial wilt. Plant J. 36, 353-365.

Guzman, P. and Ecker, J.R. (1990) Exploiting the triple response of Arabidopsis to identify ethylene-related mutants. Plant Cell, 2, 513-523.

Hall, M.C., Dworkin, l., Ungerer, M.C. and Purugganan, M. (2007) Genetics of microenvironmental canalization in Arabidopsis thaliana. Proc. Natl Acad. Sci. USA, 104, 13717-13722.

Hangarter, R.P. (1997) Gravity, light and plant form. Plant Cell Environ. 20, 796-800.

Hara, K., Kajita, R., Torii, K.U., Bergmann, D.C. and Kakimoto, T. (2007) The secretory peptide gene EPF1 enforces the stomatal one-cell-spacing rule. Genes Dev. 21, 1720-1725.

Hara, K., Yokoo, T., Kajita, R., Onishi, T., Yahata, S., Peterson, K.M., Torii, K.U. and Kakimoto, T. (2009) Epidermal cell density is auto-regulated via a secretory peptide, EPIDERMAL PATTERNING FACTOR2 in Arabidopsis leaves. Plant Cell Physiol. 50, 1019-1031.

Hoffmann-Benning, S. and Kende, H. (1992) On the role of abscisic acid and gibberellin in the regulation of growth in rice. Plant Physiol. 99, 1156-1161.

Hopkins, R., Schmitt, J. and Stinchcombe, J.R. (2008) A latitudinal cline and response to vernalization in leaf angle and morphology in Arabidopsis thaliana (Brassicaceae). New Phytol. 179, 155-164.

Hornstein, E. and Shomron, N. (2006) Canalization of development by microRNAs. Nature Genet. 38, S20-S24.

Hua, J. and Meyerowitz, E.M. (1998) Ethylene responses are negatively regulated by a receptor gene family in Arabidopsis thaliana. Cell, 94, 261271.

Hua, J., Sakai, H., Nourizadeh, S., Chen, Q.G., Bleecker, A.B., Ecker, J.R. and Meyerowitz, E.M. (1998) EIN4 and ERS2 are members of the putative ethylene receptor gene family in Arabidopsis. Plant Cell, 10, 1321-1332.

Hunt, L. and Gray, J.E. (2009) The signaling peptide EPF2 controls asymmetric cell divisions during stomatal development. Curr. Biol. 19, 864-869.

Keurentjes, J.J., Bentsink, L., Alonso-Blanco, C., Hanhart, C.J., Blankestijn-De

Vries, H., Effgen, S., Vreugdenhil, D. and Koornneef, M. (2007) Development of a near-isogenic line population of Arabidopsis thaliana and comparison of mapping power with a recombinant inbred line population. Genetics, 175, 891-905.

Koini, M.A., Alvey, L., Allen, T., Tilley, C.A., Harberd, N.P., Whitelam, G.C. and Franklin, K.A. (2009) High temperature-mediated adaptations in plant architecture require the bHLH transcription factor PIF4. Curr. Biol. 19, 408413.

Koornneef, M., Alonso-Blanco, C. and Vreugdenhil, D. (2004) Naturally occurring genetic variation in Arabidopsis thaliana. Annu. Rev. Plant Biol. $55,141-172$.

Lease, K.A., Lau, N.Y., Schuster, R.A., Torii, K.U. and Walker, J.C. (2001) Receptor serine/threonine protein kinases in signalling: analysis of the erecta receptor-like kinase of Arabidopsis thaliana. New Phytol. 151, 133143.

Llorente, F., Alonso-Blanco, C., Sánchez-Rodriguez, C., Jorda, L. and Molina, A. (2005) ERECTA receptor-like kinase and heterotrimeric $G$ protein from Arabidopsis are required for resistance to the necrotrophic fungus Plectosphaerella cucumerina. Plant J. 43, 165-180.

Masle, J., Gilmore, S.R. and Farquhar, G.D. (2005) The ERECTA gene regulates plant transpiration efficiency in Arabidopsis. Nature, 436, 866-870.

Millar, A.J. and Kay, S.A. (1991) Circadian control of cab gene transcription and mRNA accumulation in Arabidopsis. Plant Cell, 3, 541-550.

Millenaar, F.F., Cox, M.C., van Berkel, Y.E., Welschen, R.A., Pierik, R., Voesenek, L.A.C.J. and Peeters, A.J.M. (2005) Ethylene-induced differential growth of petioles in Arabidopsis. Analyzing natural variation, response kinetics, and regulation. Plant Physiol. 137, 998-1008.
Millenaar, F.F., Okyere, J., May, S.T., van Zanten, M., Voesenek, L.A.C.J. and Peeters, A.J.M. (2006) How to decide? Different methods of calculating gene expression from short oligonucleotide array data will give different results. BMC Bioinformatics, 7, 137.

Millenaar, F.F., van Zanten, M., Cox, M.C.H., Pierik, R., Voesenek, L.A.C.J. and Peeters, A.J.M. (2009) Differential petiole growth in Arabidopsis thaliana: photocontrol and hormonal regulation. New Phytol. 184, 141-152.

Mullen, J.L., Weinig, C. and Hangarter, R.P. (2006) Shade avoidance and the regulation of leaf inclination in Arabidopsis. Plant Cell Environ. 29, 10991106.

Nordborg, M., Hu, T.T., Ishino, Y. et al. (2005) The pattern of polymorphism in Arabidopsis thaliana. PLoS Biol. 3, e196.

Pérez-Pérez, J.M., Serrano-Cartagena, J. and Micol, J.L. (2002) Genetic analysis of natural variations in the architecture of Arabidopsis thaliana vegetative leaves. Genetics, 162, 893-915.

Pierik, R., Visser, E.J.W., de Kroon, H. and Voesenek, L.A.C.J. (2003) Ethylene is required in tobacco to successfully compete with proximate neighbours. Plant Cell Environ. 26, 1229-1234.

Pierik, R., Cuppens, M.L., Voesenek, L.A. and Visser, E.J. (2004) Interactions between ethylene and gibberellins in phytochrome-mediated shade avoidance responses in tobacco. Plant Physiol. 136, 2928-2936.

Pierik, R., Millenaar, F.F., Peeters, A.J.M. and Voesenek, L.A.C.J. (2005) New perspectives in flooding research: the use of shade avoidance and Arabidopsis thaliana. Ann. Bot. 96, 533-540.

Pierik, R., Tholen, D., Poorter, H., Visser, E.J. and Voesenek, L.A. (2006) The Janus face of ethylene: growth inhibition and stimulation. Trends Plant Sci. 11, 176-183.

Qi, Y., Sun, Y., Xu, L., Xu, Y. and Huang, H. (2004) ERECTA is required for protection against heat-stress in the AS1/AS2 pathway to regulate adaxialabaxial leaf polarity in Arabidopsis. Planta, 219, 270-276.

Redei, G.P. (1962) Supervital mutants in Arabidopsis. Genetics, 47, 443-460.

Salter, M.G., Franklin, K.A. and Whitelam, G.C. (2003) Gating of the rapid shade avoidance response by the circadian clock in plants. Nature, $\mathbf{4 2 6}$, 680-683.

Sangster, T.A., Salathia, N., Undurraga, S., Milo, R., Schellenberg, K., Lindquist, S. and Queitsch, C. (2008) HSP90 affects the expression of genetic variation and developmental stability in quantitative traits. Proc. Natl Acad. Sci. USA, 105, 2963-2968.

Shindo, C., Bernasconi, G. and Hardtke, C.S. (2007) Natural genetic variation in Arabidopsis: tools, traits and prospects for evolutionary ecology. Ann Bot. 99, 1043-1054.

Shpak, E.D., McAbee, J.M., Pillitteri, L.J. and Torii, K.U. (2005) Stomatal patterning and differentiation by synergistic interactions of receptor kinases. Science, 309, 290-293.

Snoek, L.B. (2009) Natural variation in shade and ethylene-induced differentia growth and transcription in Arabidopsis. PhD Thesis, Utrecht University The Netherlands.

Swarup, K., Alonso-Blanco, C., Lynn, J.R., Michaels, S.D., Amasino, R.M., Koornneef, M. and Millar, A.J. (1999) Natural allelic variation identifies new genes in the Arabidopsis circadian system. Plant J. 20, 67-77.

Tisné, S., Reymond, M., Vile, D., Fabre, J., Dauzat, M., Koornneef, M. and Granier, C. (2008) Combined genetic and modelling approaches reveal that epidermal cell area and number in leaves are controlled by leaf and plant developmental processes in Arabidopsis. Plant Physiol. 148, 1117-1127.

Torii, K.U., Mitsukawa, N., Oosumi, T., Matsuura, Y., Yokoyama, R., Whittier, R.F. and Komeda, Y. (1996) The Arabidopsis ERECTA gene encodes a putative receptor protein kinase with extracellular leucine-rich repeats. Plant Cell, 8, 735-746.

Ungerer, M.C., Halldorsdottir, S.S., Modliszewski, J.L., Mackay, T.F. and Purugganan, M.D. (2002) Quantitative trait loci for inflorescence development in Arabidopsis thaliana. Genetics, 160, 1133-1151.

Van Ooijen, J.W. (1999) LOD significance thresholds for OTL analysis in experimental populations of diploid species. Heredity, 83, 613-624.

Van Zanten, M., Voesenek, L.A.C.J., Peeters, A.J.M. and Millenaar, F.F. (2009a) Hormone- and light-mediated regulation of heat-induced differential petiole growth in Arabidopsis thaliana. Plant Physiol. doi: 10.1104/ pp. 109.144386

Van Zanten, M., Millenaar, F.F., Cox, M.C.H., Pierik, R., Voesenek, L.A.C.J. and Peeters, A.J.M. (2009b) Auxin perception and polar auxin transport are not always a prerequisite for differential growth. Plant Signal. Behav. 4, in press. 
Van Zanten, M., Snoek, L.B., Proveniers, M.C.G. and Peeters, A.J.M. (2009c) The many functions of ERECTA. Trends Plant Sci. 14, 214-218.

Voesenek, L.A.C.J. and Blom, C.W.P.M. (1989) Growth responses of Rumex species in relation to submergence and ethylene. Plant Cell Environ. 12, 433-439.

Waddington, C.H. (1942) Canalization of development and the inheritance of acquired characters. Nature, 150, 563-565.

Weigel, D. and Nordborg, M. (2005) Natural variation in Arabidopsis. How do we find the causal genes?. Plant Physiol. 138, 567-568.

The Genbank accession number for the Van-0 er sequence is G0368569.
Wilkinson, J.Q., Lanahan, M.B., Yen, H.-C., Giovannoni, J.J. and Klee, H.J. (1995) An ethylene-inducible component of signal transduction encoded by Never-ripe. Science, 270, 1807-1809.

Xu, L., Xu, Y., Dong, A., Sun, Y., Pi, L., Xu, Y. and Huang, H. (2003) Novel as 1 and as2 defects in leaf adaxial-abaxial polarity reveal the requirement for ASYMMETRIC LEAVES 1 and 2 and ERECTA functions in specifying leaf adaxial identity. Development, 130, 4097-4107. 


\title{
Dysregulation of cell-to-cell connectivity and stomatal patterning by loss-of-function mutation in Arabidopsis CHORUS (GLUCAN SYNTHASE-LIKE 8)
}

\author{
Jessica M. Guseman ${ }^{1}$, Jin Suk Lee ${ }^{1}$, Naomi L. Bogenschutz ${ }^{1, *}$, Kylee M. Peterson ${ }^{1}$, Rebecca E. Virata ${ }^{1}$, Bo Xie ${ }^{2}$, \\ Masahiro M. Kanaoka ${ }^{3}$, Zonglie Hong ${ }^{2}$ and Keiko U. Torii ${ }^{1,4, t}$
}

\begin{abstract}
Summary
Patterning of stomata, valves on the plant epidermis, requires the orchestrated actions of signaling components and cell-fate determinants. To understand the regulation of stomatal patterning, we performed a genetic screen using a background that partially lacks stomatal signaling receptors. Here, we report the isolation and characterization of chorus (chor), which confers excessive proliferation of stomatal-lineage cells mediated by SPEECHLESS (SPCH). chor breaks redundancy among three ERECTA family genes and strongly enhances stomatal patterning defects caused by loss-of-function in TOO MANY MOUTHS. chor seedlings also exhibit incomplete cytokinesis and growth defects, including disruptions in root tissue patterning and root hair cell morphogenesis. CHOR encodes a putative callose synthase, GLUCAN SYNTHASE-LIKE 8 (GSL8), that is required for callose deposition at the cell plate, cell wall and plasmodesmata. Consistently, symplastic macromolecular diffusion between epidermal cells is significantly increased in chor, and proteins that do not normally move cell-to-cell, including a fluorescent protein-tagged SPCH, diffuse to neighboring cells. Such a phenotype is not a general trait caused by cytokinesis defects. Our findings suggest that the restriction of symplastic movement might be an essential step for the proper segregation of cell-fate determinants during stomatal development.
\end{abstract}

KEY WORDS: Arabidopsis, Stomatal patterning, Callose synthase, Plasmodesmata, Cytokinesis

\section{INTRODUCTION}

The generation of tissue patterns in multicellular organisms requires coordinated cell proliferation and differentiation. Encapsulated by cell walls, pattern formation in plants occurs in the absence of cell migration. The last step of plant cell division, cytokinesis, is marked by a cell plate separating the two daughter cells. It is thought that positional information instructs the newly divided plant cells to acquire specific cell fate. One mechanism for transmitting and receiving such information is receptor kinase-mediated signaling, in which secreted ligands diffusing through the apoplast are received by receptors in neighboring cells. Plants possess large numbers of receptor-like kinases (RLKs) to regulate diverse aspects of development (Dievart and Clark, 2004; Shiu and Bleecker, 2001; Torii and Clark, 2000). The second mechanism is direct cell-to-cell movement of regulatory molecules, such as transcription factors and RNAs, through the symplastic continuum via plasmodesmata (Lucas and Lee, 2004; Oparka and Roberts, 2001).

Stomatal patterning has emerged as a model in which to study how positional cues influence functional tissue patterning. In Arabidopsis, stomata differentiate from a subset of protodermal cells called meristemoid mother cells (MMCs). An MMC undergoes an

\footnotetext{
1Department of Biology, University of Washington, Seattle, WA 98195, USA. ${ }^{2}$ Department of Microbiology, Molecular Biology and Biochemistry, University of Idaho, Moscow, ID 83844, USA. ${ }^{3}$ Division of Biological Science, Graduate School of Science, Nagoya University, Nagoya, Aichi 464-8602, Japan. ${ }^{4}$ PREST, Japan Science and Technology Agency, Tokyo, Japan.

*Present address: Molecular and Cellular Biology Graduate Program, University of Washington, Seattle, WA 98195, USA

†Author for correspondence (ktorii@u.washington.edu)
}

asymmetric entry division that creates a meristemoid and a larger sister cell called a stomatal lineage ground cell (SLGC). The meristemoid reiterates a few rounds of asymmetric cell divisions that amplify surrounding SLGCs. The meristemoid eventually differentiates into a guard mother cell (GMC) that divides symmetrically to form two guard cells constituting a stoma (Bergmann and Sack, 2007; Nadeau and Sack, 2002b). The SLGCs might differentiate into pavement cells or become MMCs, which initiate additional stomatal cell lineages.

Molecular genetic studies have revealed both positive and negative regulators of stomatal development. Three closely-related basic helix-loop-helix (bHLH) proteins, SPEECHLESS (SPCH), MUTE and FAMA, specify the initiation of the stomatal lineage, differentiation of meristemoids to GMCs, and the terminal differentiation of guard cells, respectively, together with SCREAM (SCRM; also known as ICE1) and SCRM2 bHLH proteins (Kanaoka et al., 2008; MacAlister et al., 2007; Ohashi-Ito and Bergmann, 2006; Pillitteri et al., 2008; Pillitteri et al., 2007). Two Myb proteins restrict the division of GMCs (Lai et al., 2005). A ligand-receptor signaling pathway inhibits entry into the stomatal lineage and orients asymmetric division. The components of the pathway include putative ligands, EPIDERMAL PATTERNING FACTOR 1 (EPF1) and EPF2, a putative receptor, TOO MANY MOUTHS (TMM), three redundant receptor-like kinases, ERECTA (ER), ERECTA-LIKE 1 (ERL1) and ERL2, and mitogen-activated protein kinase cascades mediated via YODA, MKK4/5 and MPK3/6 (Bergmann et al., 2004; Hara et al., 2007; Hara et al., 2009; Hunt and Gray, 2009; Nadeau and Sack, 2002a; Shpak et al., 2005; Wang et al., 2007). It remains unclear how the actions of these positive and negative regulators are spatially coordinated during epidermal development. 
To better understand the regulation of stomatal patterning, we performed a sensitized genetic screen using er erll as a starting background. We report a new stomatal patterning mutant, chorus (chor). CHOR represents a weak allele of the putative callose synthase gene GSL8 (also known as CALLOSE SYNTHASE 10), which was recently shown to be required for cytokinesis (Chen et al., 2009; Thiele et al., 2009). Although Chen et al. also observed that gsl 8 mutants show stomatal patterning defects, both the exact nature of the defects and the mechanism by which loss-of-function mutations in a callose synthase gene leads to stomatal clustering remain unclear. On the basis of histochemical callose deposition and cell-to-cell diffusion assays, we suggest a potential role of plasmodesmatal macromolecular trafficking as a mechanism for proper stomatal patterning.

\section{MATERIALS AND METHODS \\ Plant materials}

Arabidopsis thaliana Columbia $(\mathrm{Col})$ accession was used as a wild type. Mutants and lines used in this study were in the Col background unless otherwise specified. The chor mutant was derived from an EMS (ethyl methanesulfonic acid)-mutagenized er-105 erll-2 population and was outcrossed three times to wild type before analysis. The er-105 erll-2 background possesses an additional gll mutation, which confers trichomeless leaves. A T-DNA insertion allele of GSL8 ( $g s l 8-2$; GK 851 C04) was obtained from the Nottingham Arabidopsis Stock Centre (NASC). The following transgenic lines and mutants have been described previously: proERL1::GUS, proTMM::GUS, er-105, erl1-2, er-105 erl1-2 and er-105 erl1-2 erl2-1 (Shpak et al., 2004; Shpak et al., 2005); spch-3 (Pillitteri et al., 2007); tmm-1 and proTMM:GUS-GFP (Nadeau and Sack, 2002a); proMUTE::GFP (Pillitteri et al., 2008); proCaMV35S:YFP-PDCB1 (Simpson et al., 2009); atnack1-1 (Nishihama et al., 2002); and scd1-2 (Falbel et al., 2003). The transgenes and known mutant alleles were introduced into chor/ + via genetic crosses. Seedlings were grown in modified Murashige-Skoog media with $1 \times$ Gamborg B5 vitamins and $1 \%$ w/v sucrose at $21^{\circ} \mathrm{C}$ under long-day conditions (18/6 light-dark cycle). Plates (90 mm diameter, $25 \mathrm{~mm}$ thickness) were sealed with surgical tape (Micropore, $3 \mathrm{M}$ ) to minimize condensation.

\section{Genetic screen}

er-105 erl1-2 seeds were treated with $0.3 \%$ (v/v) EMS overnight. Seeds were harvested from individual M1 plants, and $528 \mathrm{M} 2$ seed populations were visually screened for seedling epidermal phenotypes.

\section{Genotyping}

Mutant genotypes were verified by PCR-based analyses. The chor mutant allele was detected by derived Cleavage Amplified Polymorphic Sequence (dCAPS) (Neff et al., 1998), using the primer pair chorus dCAPS F-NlaIV and chorus dCAPS R-NlaIV, and subsequent digestion with NlaIV. The TDNA insertion site of $g s l 8-2$ was detected by the primer pair At2g36850 M18F and GABI-K 08409. See Table S1 in the supplementary material for primer sequences. Genotyping of er-105, erl1-2, erl2-1, tmm-1 and spch-3 has been described previously (Lukowitz et al., 2004; Pillitteri et al., 2007; Shpak et al., 2004).

\section{Map-based cloning of CHORUS}

A mapping population of chor was generated by crossing B1F1 chor $/+$ er erll to Ler. Initially, a bulked segregant analysis (Lukowitz et al., 2000) was performed using a combined pool of 33 chor seedlings from the mapping population, which revealed the strong linkage of chor to the mid-bottom of chromosome 2 at SSLP markers MSAT2.36 and MSAT2.9 (Loudet et al., 2002). Further fine mapping was performed within this interval using two SSLP markers (KTL T20F21 and KTL F3G5) and a total of 1831 F2 chor $(-/-)$ seedlings, which located $C H O R$ within the $\sim 145-\mathrm{kb}$ interval encompassing the BACs F13K3 and T1J8. All 36 predicted open-reading frames within this interval were sequenced, and this led to the identification of a single $\mathrm{G} \rightarrow \mathrm{A}$ substitution at position 13447 of At $2 \mathrm{~g} 36850$, which encodes GSL8 (CalS10). The SSLP, CAPS, and dCAPS markers for map- based cloning of $C H O R$ were designed based on the information available in Monsanto Arabidopsis Polymorphism and Ler Sequence Collection (http://www.Arabidopsis.org/Cereon/index.jsp). For primer sequences and amplified fragment sizes in Col and Ler, see Table S1 in the supplementary material. For the list of sequencing primers, see Table S2 in the supplementary material.

\section{RNA extraction and RT-PCR}

Total RNA was isolated from 14-day-old wild-type and chor seedlings. cDNA synthesis and reverse-transcription (RT)-PCR were performed as described previously (Bemis and Torii, 2007), using the following primer pairs. For CHOR and chor, chorus cDNA F (5'-CAGCTTCATTACAATGCAATTCC-3') and chorus cDNA R (5'-TCAAGTTACCAAAATATAGTCAACC-3'); for Actin control, ACT 2-1 (5'-GCCCATCCAAGCTGTTCTCTC-3') and ACT 2-2 (5'-GCTCGTAGTCAACAGCAACAA- $3^{\prime}$ ); for sequencing RT-PCR products, chorus cDNA Seq F (5'GCTCTACTGTTGATCATTTACA-3').

\section{Histochemical analysis and microscopy}

Differential interference contrast (DIC) microscopy was performed according to Bemis and Torii (Bemis and Torii, 2007). For confocal microscopy, seedlings were treated with either propidium iodide (PI) or FM4-64 (Molecular Probes, Invitrogen). Fluorescence of GFP, YFP and PI/FM4-64 were simultaneously captured using a Zeiss LSM510 Meta or a Zeiss LSM700 (excitation, $488 \mathrm{~nm}$ and $543 \mathrm{~nm}$; emission, 505-530 nm, 545$585 \mathrm{~nm}$ and $570-610 \mathrm{~nm}$, respectively) with a $20 \times$ objective lens. For histochemical callose staining, seedlings were infiltrated with $0.1 \mathrm{mg} / \mathrm{ml}$ Aniline Blue fluorochrome (Biosupplies Australia) in 0.1\% Silwett L77 (Lehle Seeds, Round Rock, TX) for 60 minutes and, prior to mounting, wild type and chor were counterstained briefly with FM4-64 to highlight the cell periphery. The abaxial side of rosette leaves was observed with a Zeiss LSM700 (excitation, $405 \mathrm{~nm}$; emission, 475-500 nm). Aniline Blue-stained root hairs were observed with an Olympus BX51 epifluorescence microscope with a UV/B filter.

\section{Quantitative analysis of stomatal phenotype}

Twelve-day-old seedlings of wild type, chor, er erll and er erll chor were subjected to DIC microscopy. Four non-overlapping frames of cotyledons, excluding the mid-vein region, and numbers of stomata and their cluster size were quantified using ImageJ.

\section{Transient transformation of seedling leaf epidermis and cell-to-} cell diffusion assay

Arabidopsis 10-day-old seedling rosette leaves were transiently transformed as previously described (Kanaoka et al., 2008). Briefly, microcarriers were prepared with $100 \mathrm{ng}$ of plasmid containing proCaMV35S::eCFP (von Arnim et al., 1998), a combination of proCaMV35S::eCFP and proCaMV35S::3xGFP (J.S.L. and K.U.T., unpublished), or a combination of proCaMV35S::eCFP and proCaMV35S::SPCH-YFP (Lynn Pillitteri and K.U.T., unpublished). These plasmids were coated on $1.0-\mu \mathrm{m}$ gold particles and bombarded using the PDS-1000/He particle delivery system (Bio-Rad, Hercules, CA, USA). Specimens were observed under a Zeiss LSM 510 Meta or an LSM700 confocal microscope. CFP, GFP or YFP fluorescence was monitored 6 hours after the bombardment.

\section{Accession numbers}

The GenBank Accession number for the GSL8 (CalS10) mRNA sequence is GQ373182.

\section{RESULTS \\ chor, a stomatal patterning mutant obtained via a sensitized screen}

We conducted a genetic screen using a sensitized er erll background, in which two of the three ER-family genes are absent. er erll plants develop an epidermis with an elevated stomatal density but are otherwise normal (Fig. 1) (Shpak et al., 2005). On rare occasions, paired stomata were observed both in wild-type and er erl 1 cotyledons $(0.11 \%$ in wild type; $0.57 \%$ in er 

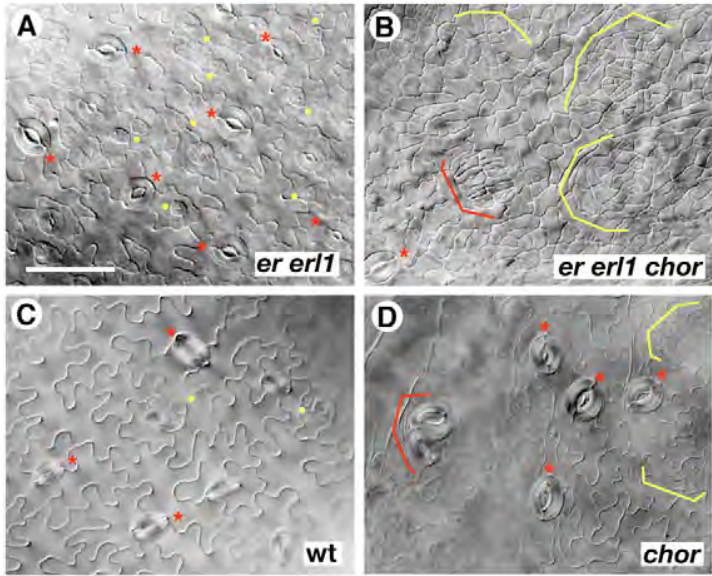

E
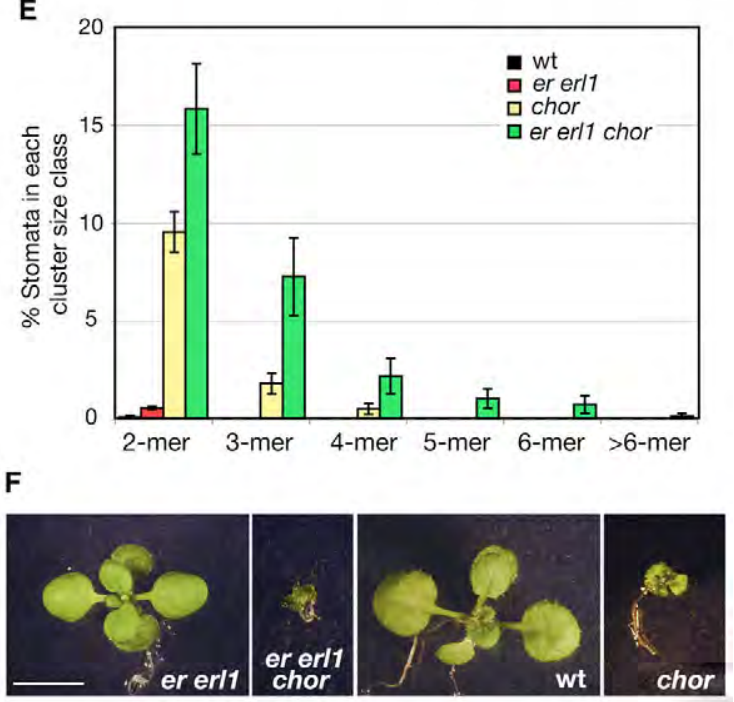

Fig. 1. chor mutant exhibits growth and stomatal patterning

defects. (A-D) Abaxial rosette leaf epidermis from 2-week-old seedlings of er erl1 (A), er erl1 chor (B), wild type (wt; C) and chor (D). er erl1 produces a higher density of stomata (red asterisks) and stomatal precursors (yellow dots) than wild type, but it does not develop two stomata in contact (A,C). In er erl1 chor, aggregates of stomata (red bracket) and small, highly divided epidermal cells (yellow brackets) are visible (B). This phenotype is less prominent in chor (D). Images are taken under the same magnification. Scale bar: $50 \mu \mathrm{m}$. (E) Percentage of stomata in each cluster size class. Abaxial cotyledons from 12-dayold seedlings of wt, er erl1, chor and er erl1 chor were subjected to quantitative analysis. Average values are shown. Error bars indicate s.e.m. Number of seedlings analyzed: $n=8$, wt and chor; $n=7$, er erl1 and er erl1 chor. Total number of stomata counted: wt, $n=1771$; chor, $n=715$; er erl1, $n=1921$; er erl1 chor, $n=2721$. (F) Representative 12day-old plants of (from left to right) er erl1, er erl1 chor, wt, and chor in a wild-type background. Images are taken under the same magnification. Scale bar: $5 \mathrm{~mm}$.

erl1; Fig. 1E). Neither genotype developed stomatal clusters with three or more adjacent stomata $(n=0 / 1771$ and $0 / 1921$ for wild type and er erl1, respectively; Fig. 1E). By contrast, er erl1 erl2 triple loss-of-function mutants develop excessive numbers of clustered stomata (Shpak et al., 2005). We reasoned that in the absence of two of the three receptors, plants would be more sensitive to the loss of any additional regulators of stomatal patterning.
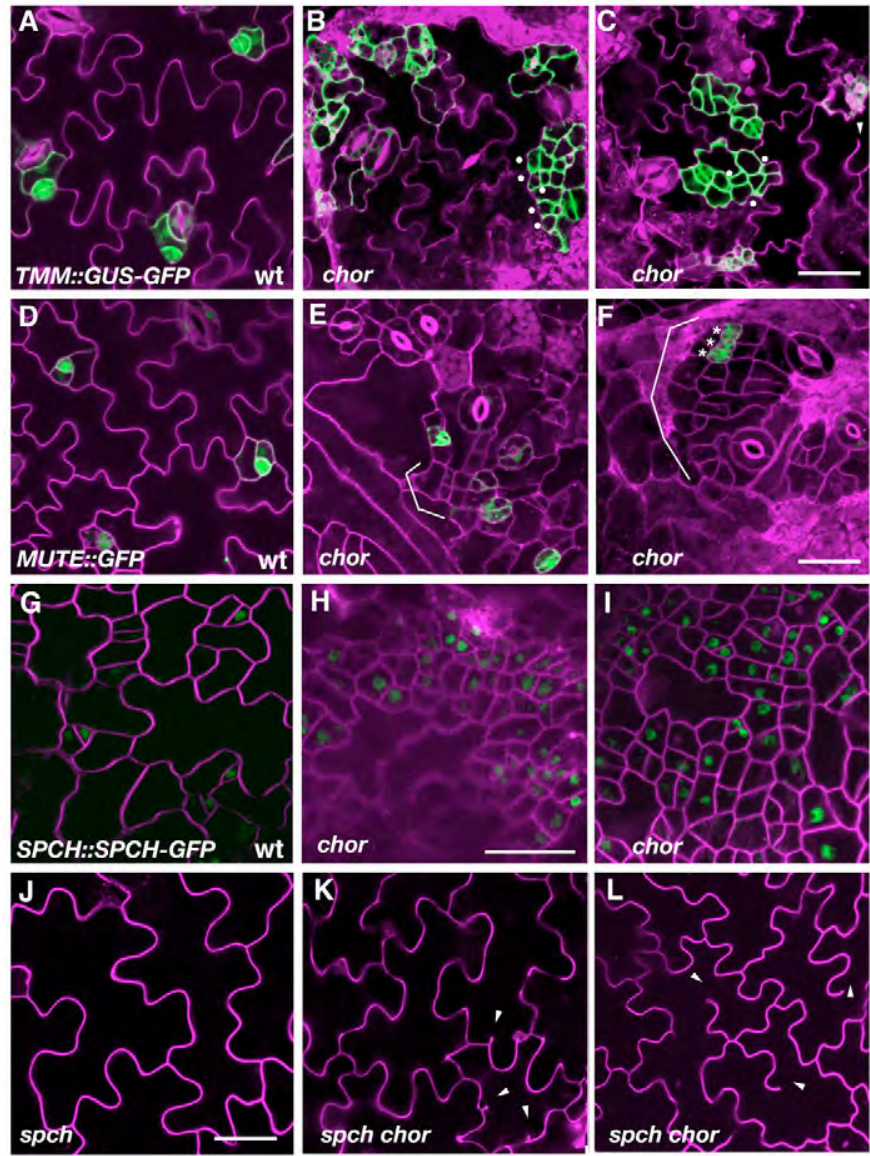

Fig. 2. CHOR is required to restrict stomatal cell-lineage divisions.

(A-C) Promoter activity of TMM (proTMM::GUS-GFP) in 6-day-old cotyledon epidermis of wild type $(A)$ and chor $(B, C)$. In chor, clusters of small cells (dots) show high GFP signals. Pavement cells in chor occasionally show incomplete cytokinesis (arrowhead). (D-F) Expression of MUTE (proMUTE::MUTE-GFP) in 6-day-old cotyledon epidermis of wild type (D) and chor (E, F). Clusters of small stomatal-lineage cells are in brackets. MUTE expression in adjacent meristemoids (asterisks) predicts the eventual formation of clustered stomata. (G-I) Expression of SPCH (proSPCH::SPCH-GFP) in 3-day-old cotyledon epidermis of wild type $(\mathrm{G})$ and chor $(\mathrm{H}, \mathrm{I})$. More cells express GFP signals in chor than in wild type. (J-L) Effects of spch on the excessive stomatal-lineage divisions in chor. Shown is 10-day-old cotyledon epidermis of spch (J) and spch chor $(\mathrm{K}, \mathrm{L})$. Small, highly divided cells are no longer produced in spch chor $(\mathrm{K}, \mathrm{L})$, whereas incomplete cytokinesis of pavement cells is still evident (K,L, arrowheads). Cell peripheries were highlighted by propidium iodide (PI; A-F,J-L) or FM4-64 (G-I). A-F, G-I, and J-L were taken under the same magnification. Scale bars: $20 \mu \mathrm{m}$.

We isolated a line (\#67). In the mutant leaves, multiple stomata are often formed in a compact aggregate with stomatal pairs $(15.8 \%)$, trios (7.26\%), quartets $(2.19 \%)$, and 5-10 adjacent stomata (1.95\%; Fig. 1B,E). In addition, aggregates of small epidermal cells, many resembling meristemoids, are formed in sectors across the epidermis (Fig. 1B). Segregation analysis showed that the mutation is recessive and monogenic $\left(207: 602, \chi^{2}=0.149, P=0.700\right)$. The mutant was named chorus (chor) owing to its aggregated stomatal phenotype. 

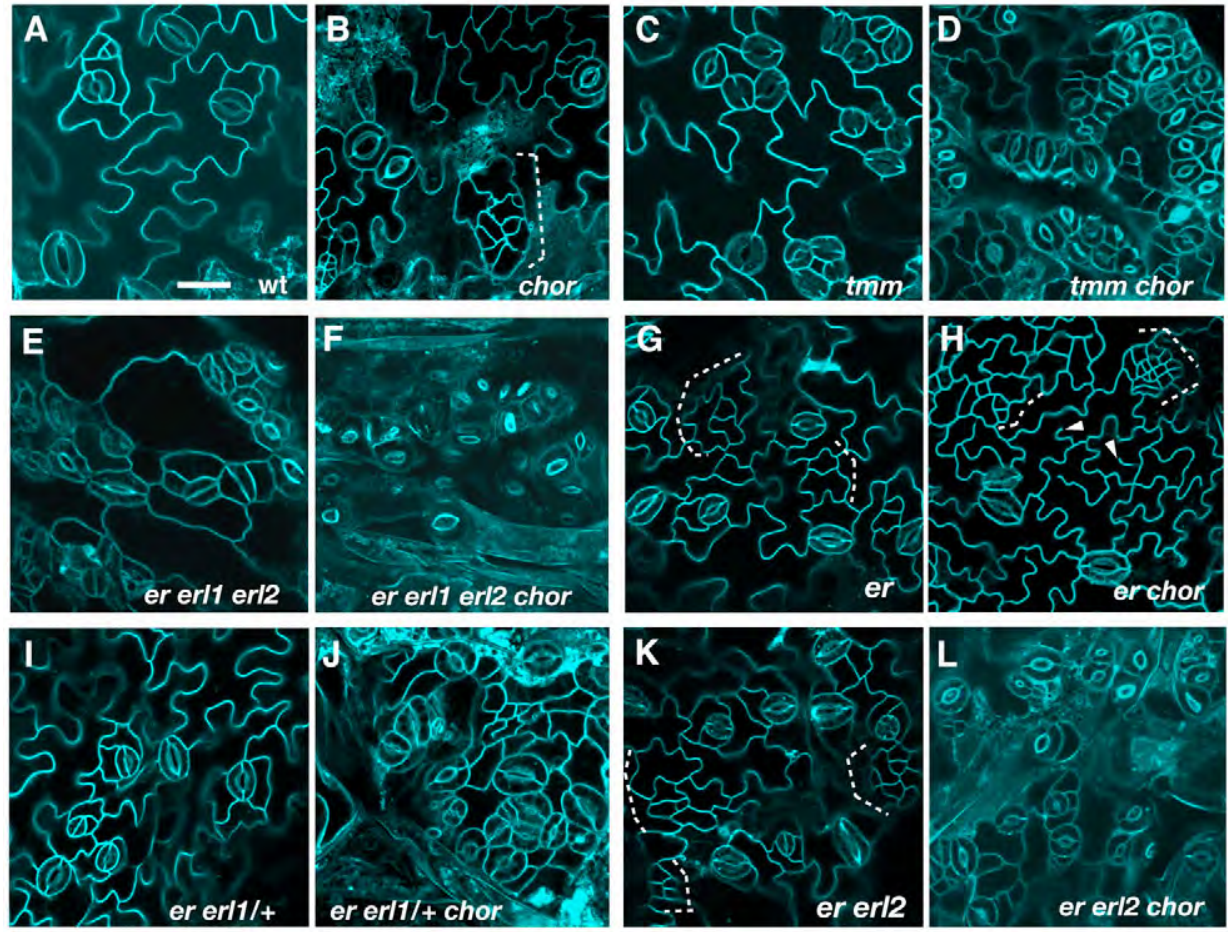

Fig. 3. Genetic interactions of chor with stomatal patterning mutants. (A-L) Abaxial epidermis of rosette leaves (first pairs) from 12-day-old seedlings of the following genotypes: wild type (wt, A), chor (B), tmm (C), tmm chor (D), er erl1 erl2 (E), er erl1 erl2 chor (F), er (G), er chor $(\mathrm{H})$, er erl1/+ (I), er erl1/+ chor (J), er erl2 (K) and er erl2 chor (L). chor in a wild-type background exhibits weak stomatal patterning defects and islands of small cells (dotted bracket; B), but the chor mutation severely enhances stomatal clustering in tmm (C,D) and in the er er/1 erl2 triple mutant (E,F). er er/1/+ and er er/2 seedling leaves do not exhibit stomatal patterning defects $(\mathrm{I}, \mathrm{K})$; however, introduction of chor confers severe stomatal clustering defects $(\mathrm{J}, \mathrm{L})$. The er chor double-mutant phenotype appeared additive, with increased small cells (dotted bracket) and incomplete cytokinesis (arrowheads, G,H). Images are taken under the same magnification. Scale bar: $20 \mu \mathrm{m}$
We introduced chor into the wild-type background to test whether the observed phenotype requires er erll. The epidermal phenotype of chor leaves in the wild-type background was much milder than that of chor er erl1. Numbers of stomatal pairs (9.4\%), trios $(1.93 \%)$ and quartets $(0.44 \%)$ were diminished. No stomatal clusters larger than quartets were found in chor $(n=0 / 715$; Fig. 1D,E). Similarly, fewer small epidermal cells were observed (Fig. 1D). Thus, chor phenotypes are enhanced by the loss of function in ER and ERL1. chor seedlings showed pleiotropic phenotypes, including severe dwarfism, short roots with disrupted cell files, short and branched root hairs, and seedling lethality (Fig. 1F; see also Figs S1 and S2 in the supplementary material). Furthermore, incomplete cytokinesis was frequently observed in chor (see Fig. S3 in the supplementary material). These findings suggest that $C H O R$ is required for proper cell division and tissue patterning throughout plant organs, including stomatal patterning.

\section{CHOR is required for restricting initiation and divisions of stomatal-lineage cells, as well as cytokinesis of epidermal cells}

The chor leaf epidermis exhibited incomplete cytokinesis and excessive epidermal cell proliferation (Fig. 1). We hypothesized that the latter defect is due to excessive entry into the stomatal lineage. To test this, we examined the expression patterns of stomatal-lineage markers in chor. TMM marks early stomatallineage cells, including MMCs, meristemoids and SLGCs (Fig. 2A). Clusters of small, highly divided epidermal cells in chor seedlings exhibited high reporter activity of proTMM::GUS-GFP (Fig. 2B,C), suggesting that the chor mutation causes a vast increase in stomatal lineage divisions in a disorganized manner. MUTE is specifically expressed in late meristemoids that are differentiating into GMC (Fig. 2D) (Pillitteri et al., 2008; Pillitteri et al., 2007). Occasionally, multiple adjacent cells showed high MUTE promoter activity (Fig. 2F, asterisks), which is likely to predict the eventual formation of stomatal clusters.
The excessive formation of small stomatal-lineage cells is a hallmark of SPCH overexpression (MacAlister et al., 2007; Pillitteri et al., 2007). We therefore tested whether $S P C H$ is ectopically expressed in chor, and whether $S P C H$ is required for the chor epidermal phenotype. $S P C H$ was expressed in early stomatallineage cells (Fig. 2G), and the spch mutant was devoid of stomatal cell lineages (Fig. 2J). Numerous SPCH-positive cells were observed in the young cotyledon epidermis of chor (Fig. 2H,I). Introduction of spch into chor eliminated all small, stomatal-lineage cells (Fig. 2K,L). By contrast, incomplete cytokinesis of pavement cells still occurred in the chor spch double mutant (Fig. 2K,L, arrowheads). These findings point to two independent roles of $C H O R$ in regulating leaf epidermal development: $S P C H$-mediated initiation and division of stomatal cell lineage; and $\mathrm{SPCH}$ independent cytokinesis. In support of this idea, the root epidermis of spch chor exhibited disrupted cell files with incomplete cytokinesis (see Fig. S1 in the supplementary material).

\section{chor mutation exaggerates stomatal patterning defects and confers a breakdown of redundancy among cell-cell signaling components}

The stomatal clustering phenotype of chor in the wild-type background remained relatively mild (Fig. 1). Therefore, the TMM/ER-family signaling pathway might still be able to largely enforce stomatal spacing in chor. To test this, we investigated the genetic interactions of $C H O R$ with $T M M$ and $E R$-family genes (Fig. 3). Quantitative analysis of stomatal cluster size was performed for key genotypes (see Fig. S4 in the supplementary material). In tmm, adjacent stomata are formed (Fig. 3C) (Nadeau and Sack, 2002a). The tmm chor double mutant developed massive clusters of stomata far more severe than those in either the tmm or the chor single mutant (Fig. 3B-D; see also Fig. S4 in the supplementary material). Similarly, the chor mutation intensified the stomatal cluster phenotype of the er erll erl2 triple mutant (Fig. 3E,F). 
We sought to understand whether $C H O R$ acts in an independent pathway from (i.e. additive interaction) or an overlapping pathway with (i.e. synergistic interaction) the TMM/ER family. For this purpose, we genetically dissected the contribution of each $E R$-family member. The three $E R$-family genes exhibit unequal redundancy, and a stomatal cluster phenotype is manifested only when the entire family is lost (Figs 1, 3) (Shpak et al., 2005). er single mutants occasionally produce SLGCs that do not accompany differentiated stomata (Fig. 3G, dotted lines) (Shpak et al., 2005). The er chor epidermis formed severely divided patches of small cells (Fig. $3 \mathrm{H}$, dotted brackets), but otherwise appeared similar to that of chor (Fig. $3 \mathrm{H}$, arrowheads), thereby suggesting additive effects.

In contrast to er chor, er erll chor and er erl 2 chor triple mutants exhibited severe stomatal clustering phenotypes (Fig. 1B; Fig. 3H,L; see also Fig. S4 in the supplementary material). We further tested whether such synergistic effects can be observed even when only a single copy of ERL1 is eliminated. Indeed, er erll/t chor also showed a strong stomatal clustering phenotype (Fig. 3J). We conclude that chor intensifies stomatal cell-cell signaling defects and breaks down the functional redundancies among $E R$-family genes, with sensitivity to the dosage of ERLs.

\section{Identification of CHORUS as GLUCAN SYNTHASE LIKE 8}

To understand the molecular nature of $C H O R$, we performed mapbased cloning. Our fine mapping delineated the $C H O R$ locus within a $145-\mathrm{kb}$ interval on chromosome 2 (Fig. 4A). Sequencing the interval led to the identification of a single G-to-A substitution at position 13447 of At2g36850, which encodes a putative callose synthase, GSL8 (CALS10) (Fig. 4B). Because the original chor mapping population contained a mixed genome of Col/WS and Ler, wild-type GSL8 alleles from WS and Ler were also sequenced. Both alleles possess $\mathrm{G}$ at this position, confirming that $C H O R$ is not a naturally occurring polymorphism.

Sequence analysis of the wild-type GSL 8 transcripts revealed misannotation of GSL8 in the existing database (see Materials and methods). Based on the experimental data, GSL8 is a large gene consisting of 50 exons with a coding region of 5712 bp (GenBank Accession number GQ373182). In chor, the G-to-A substitution disrupted the splice acceptor site at intron 46 (Fig. 4B), which resulted in three major splicing variants (Fig. 4C). Variant 1 adopted the $A G$ at +1 created by the chor G-to-A substitution and was one base pair shorter than the CHOR mRNA (see Fig. S5 in the supplementary material). Variants 2 and 3 were 60 and 130 bp longer than the $C H O R$ mRNA, respectively. Variant 2 was produced by a cryptic splice acceptor site at $-60 \mathrm{bp}$ from the correct splicing acceptor site, whereas variant 3 was unspliced (see Fig. S5 in the supplementary material). All splicing variants caused frameshifts, each of which truncated the coding sequence starting from exon 47 and added instead a short stretch of 19, 10 and 16 out-of-frame amino acids, respectively (see Fig. S5 in the supplementary material).

GSL8 encodes a large integral membrane protein of 1905 amino acids with sixteen predicted transmembrane helices. The three splicing variants all result in truncation at the sixth extracytoplasmic hydrophilic loop and removal of five C-terminal transmembrane helices (Fig. 4D). To confirm that CHOR is GSL8, we characterized the phenotype of the available GSL8 T-DNA insertion allele, gsl8-2 (Toller et al., 2008; Chen et al., 2009). It was originally reported that gsl8 confers male gametophytic lethality (Toller et al., 2008). However, data obtained by us and recently reported by other groups (Chen et al., 2009; Thiele et al., 2009) show that gsl8 mutations result in seedling lethality with severe dwarfism (see Fig. S6 in the
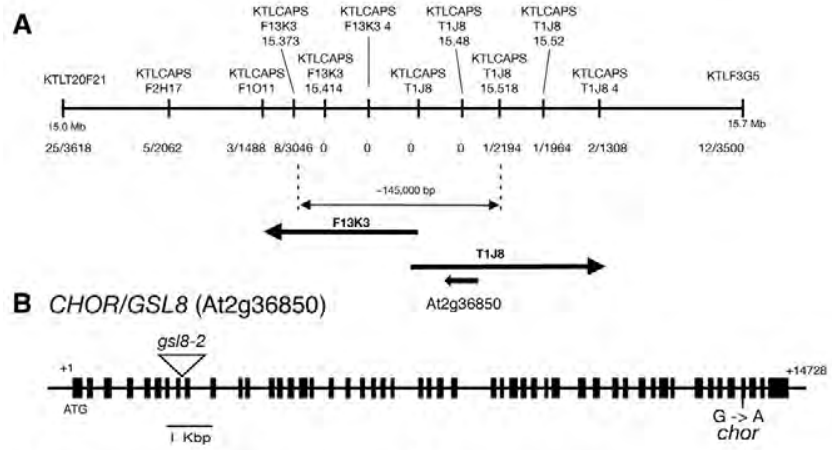

C

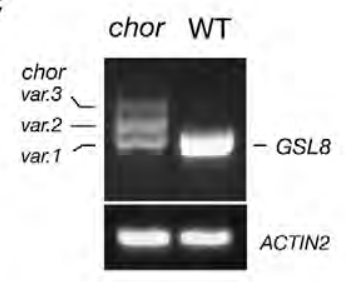

D

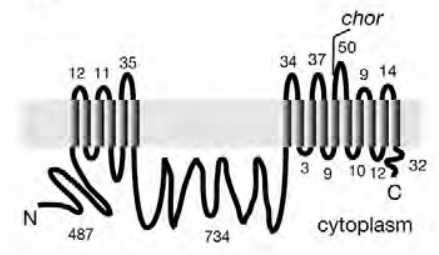

E
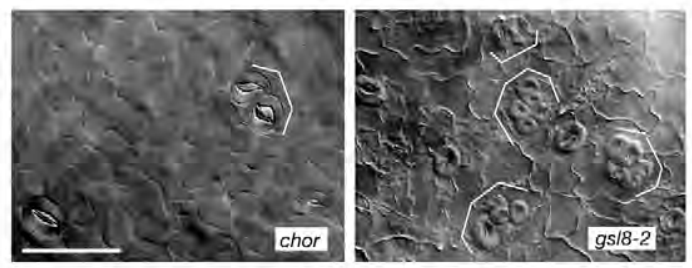

Fig. 4. Map-based cloning of CHOR as GSL8. (A) Physical mapping around the $C H O R$ locus. The locations of molecular markers, the number of recombinants, and the corresponding BAC clone are indicated. The 145-kb interval and the position of the CHOR locus (At2g36850) are indicated as a double-headed arrow and a broad arrow, respectively. (B) CHOR (GSL8) gene structure. Boxes indicate exons; lines, introns. In chor, the G-to-A substitution occurred at the splice acceptor site on intron 46. (C) RT-PCR analysis of chor reveals the presence of three splicing variants $(1363,1293$ and 1232 bp; see Materials and methods), none of which corresponds to the size of wild type (1233 bp). (D) Topology of the GSL8 protein. The GSL8 protein possesses 16 transmembrane helices. The chor mutation results in a truncation of the last three transmembrane helices and the C-terminal cytoplasmic tail. (E) Stomatal patterning defects of $g s / 8-2$. Shown is adaxial cotyledon epidermis of 12-day-old chor (left) and gs/8-2 (right). gs/8-2 has aggregations of many more stomata than those seen in chor (brackets). Scale bar: $50 \mu \mathrm{m}$.

supplementary material). Cotyledons and leaves of gsl8-2 plants exhibited similar stomatal patterning defects to those in chor, indicating that the chor phenotypes are due to loss of function in GSL8 (Fig. 4E; data not shown). The fact that the degree of stomatal clustering was less severe in chor than in gsl8-2 (Fig. 4E) suggests that chor might represent a partial loss-of-function allele. GSL8 is expressed broadly throughout plant organs/tissues, based on the available expression data (Winter et al., 2007).

\section{Both cell-plate and plasmodesmatal callose depositions are diminished in the developing chor leaf epidermis}

Callose, a linear 1,3- $\beta$-glucan polymer, accumulates in various tissues/cells/organelles during normal development (Stone and Clarke, 1992). The incomplete cytokinesis observed in chor pavement 

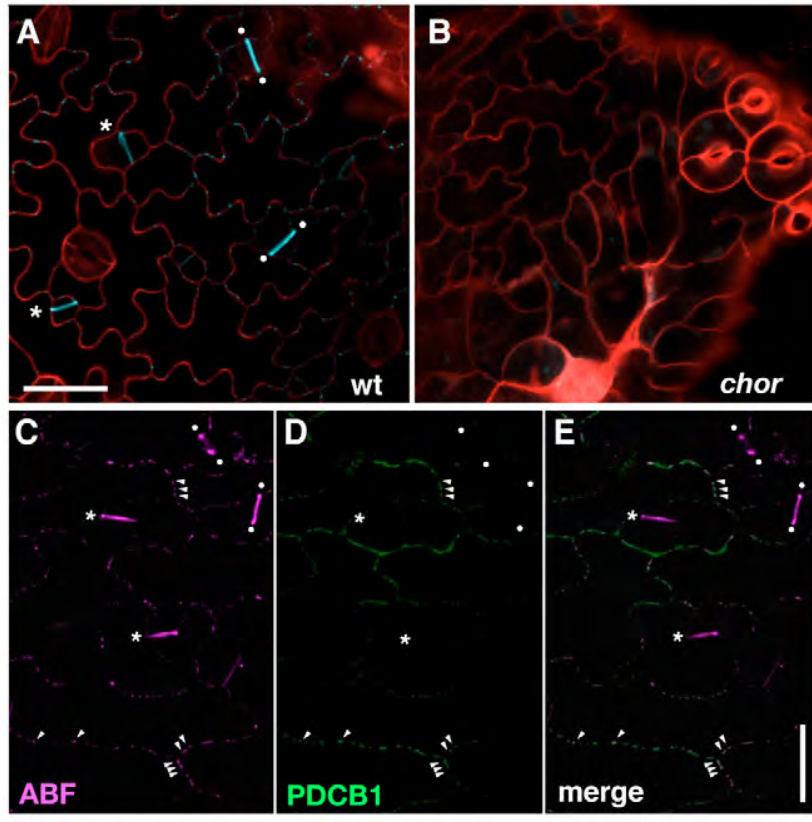

Fig. 5. Reduction in cell-plate, cell-wall and plasmodesmatal callose deposition in chor seedling epidermis. (A) Abaxial rosette leaf epidermis of a 2-week-old wild-type seedling. Callose deposition is notable at the cell plates of newly divided meristemoids (asterisks) and GMC (dots). Strong punctate signals, characteristic of plasmodesmatal callose deposition, are detected at the cell periphery. (B) Abaxial rosette leaf epidermis of a 12-day-old chor seedling under the same callose staining treatment as the seedling in A. FM4-64 was used to highlight the cell periphery. (C-E) Colocalization (e.g. arrowheads) of a plasmodesmata marker YFP-PDCB1 (D, green) and callose staining (C, magenta) supporting the identity of the fluorescent punctae. Merged images are shown in E. Note that callose depositions at the cell plates (asterisks) and ventral cell walls of stomata (dots) do not co-localize with YFP-PDCB1. Images are taken under the same magnification. Scale bars: $20 \mu \mathrm{m}$.

cells (see Fig. S3 in the supplementary material) is consistent with the recent finding that GSL8 acts as a cell-plate callose synthase (Thiele et al., 2009). In addition to its role in the cell plate, callose deposition imposes physical constraints to the symplastic channel (Levy et al., 2007b; Radford et al., 1998). To address the role of GSL8 during stomatal development, we examined callose deposition in wild-type and chor seedlings using a callose-specific dye, Aniline Blue fluorochrome (Fig. 5) (Stone et al., 1984). In the wild-type rosette leaf epidermis, bright, linear signals marked cell plates in dividing cells (Fig. 5A, asterisks and dots). In addition, strong, punctate fluorescent signals were detected on the walls of epidermal cells, most notably in pavement cells and SLGCs (Fig. 5A), which is characteristic of plasmodesmatal callose deposition (Levy et al., 2007a; Simpson et al., 2009). Consistently, these punctate signals co-localized with the plasmodesmatal marker YFP-PDCB1 (Fig. 5E) (Simpson et al., 2009).

In contrast to the wild type, almost no Aniline Blue signals were detected in the chor leaf epidermis (Fig. 5B). We also noted the absence of callose deposition in chor root hairs and abnormal root hair morphology (see Fig. S2 in the supplementary material). On the basis of these findings, we conclude that GSL 8 is required for the proper accumulation of callose at cell plates, cell walls and plasmodesmata.

\section{Loss of CHOR (GSL8) causes relaxation of cell-to- cell connection in the epidermis}

Because GSL8 is required for both cell-plate and plasmodesmatal callose deposition (Fig. 5), we postulated that the overproliferation of stomatal-lineage cells might be due to the reduction in plasmodesmatal callose deposition. As a first step towards gaining mechanistic insight, we investigated whether symplastic cell-tocell connections are altered in the chor epidermis. Monomeric soluble CFP was used as a reporter to monitor passive diffusion from cell to cell. Specifically, CaMV35S::CFP was bombarded to the leaf epidermis, and diffusion of CFP signals from the point of initial CFP expression was assayed. A similar method has been recently used to assess cell-to-cell trafficking (Levy et al., 2007a; Simpson et al., 2009). In nearly all cases in wild type $(n=19 / 21)$, a single cell at the site of transformation showed a bright CFP signal (Fig. 6; see also Fig. S7 in the supplementary material). A few surrounding cells showed a dim CFP signal, indicating diffusion (Fig. 6; see Fig. S7 in the supplementary material). chor epidermal cells without obvious cytokinesis defects were examined in diffusion assays. An average of four to five adjacent cells in the chor epidermis showed strong CFP fluorescence with many more surrounding cells showing dim CFP signals (Fig. 6; see Fig. S7 in the supplementary material). Total numbers of CFP-expressing cells per site in the chor epidermis $(16.8 \pm 2.68$, mean \pm s.e.m. $)$ were significantly higher than those in the wild-type epidermis $(5.7 \pm 1.24 ; P<0.0006)$.

To address whether the size exclusion limit (SEL) was increased in chor, we simultaneously introduced trimeric GFP $(3 \times \mathrm{GFP})$ and control CFP via co-bombardment and monitored their diffusion (Fig. 6). In wild type, all cells expressing $3 \times$ GFP $(n=22 / 22)$ were solitary (Fig. 6A,C). Thus, unlike with CFP (Fig. $6 \mathrm{~A}, \mathrm{~B})$, there was no diffusion of $3 \times \mathrm{GFP}$, indicating that $3 \times \mathrm{GFP}$ is beyond the SEL in the wild-type epidermis. By contrast, in the chor epidermis, a cluster of epidermal cells expressed $3 \times \mathrm{GFP}$ (Fig. 6A,C). In some cases, a cell adjacent to the site of transformation exhibited a dim GFP signal (Fig. 6A, asterisk), indicating that $3 \times \mathrm{GFP}$ was able to spread into neighboring cells in the chor epidermis. These results suggest that the reduced plasmodesmatal callose deposition caused by the chor mutation is associated with an increase in symplastic connectivity between leaf epidermal cells.

\section{chor mutation allows cell-to-cell movement of SPCH-YFP in the leaf epidermis}

In chor, SPCH-mediated stomatal cell-lineage divisions become excessive and dysregulated (Fig. 2). To explore the mechanistic link between increased cell-to-cell connections and stomatal patterning, we tested the diffusion of functional SPCH-YFP in wild-type and chor epidermis. For this purpose, SPCH-YFP and control CFP constructs were co-bombarded (Fig. 7). In wild type, YFP signal was detected solely in the nucleus of a single cell at the site of transformation ( $n=20 / 20$; Fig. 7A,C), indicating that SPCH-YFP does not diffuse from cell to cell. By contrast, in chor epidermis multiple adjacent cells expressed SPCH-YFP (Fig. 7A,C). In some cases, neighboring cells exhibited a dim YFP signal in the nucleus (Fig. 7A, asterisk). The presence of a continuous cell periphery indicates that these YFP-positive nuclei are not likely from a single multinucleate cell. The results demonstrate that SPCH-YFP diffuses from cell-to-cell in the chor epidermis and suggests that the failure to properly contain cell-fate determinants in chor causes the stomatal patterning defects. 


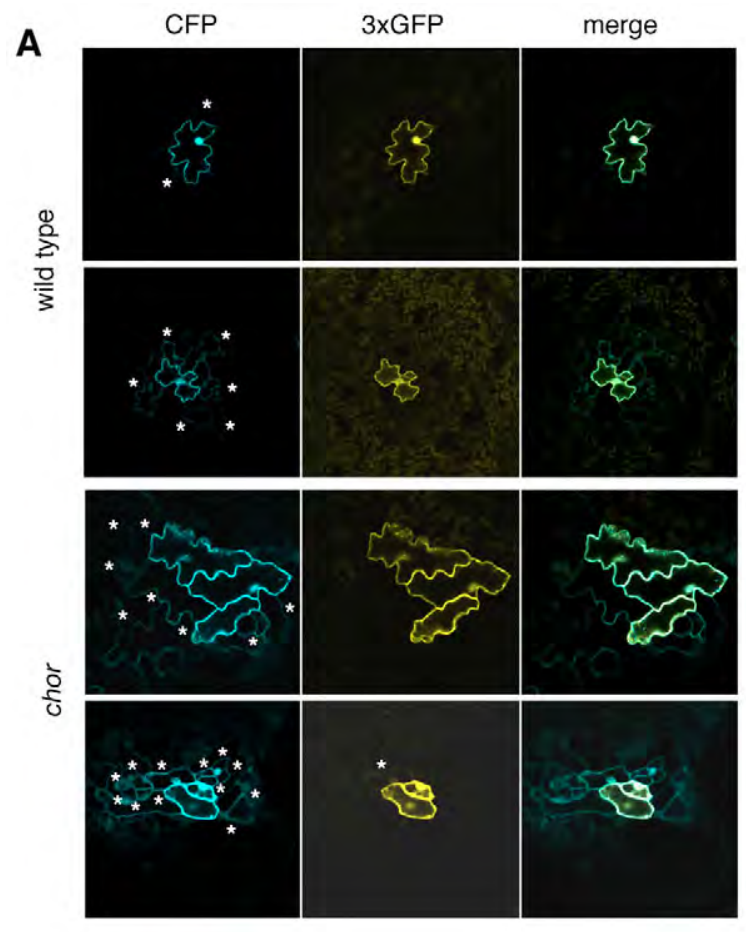

B

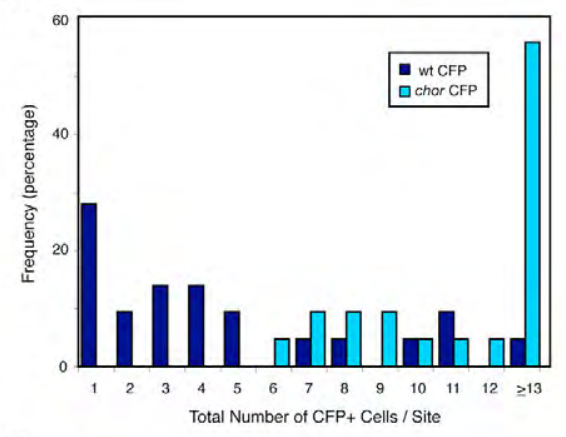

C

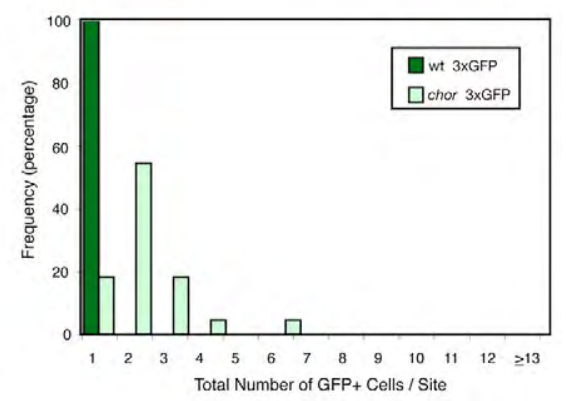

Fig. 6. Increased cell-to-cell macromolecular diffusion in chor. (A) Representative images of 10-day-old wild-type (top) and chor (bottom) epidermis expressing co-bombarded monomeric CFP (cyan, left) and trimeric GFP (yellow, middle). Merged images are shown on the right. Bright signals are detected at the site of transformation. Dim signals in surrounding neighbors (asterisks) indicate diffusion. Images are taken under the same magnification. (B,C) Quantitative distribution analysis of the number of cells expressing CFP (B) or $3 \times G F P(C)$ per site. Only continuous clusters of cells surrounding a cell (or cells) co-expressing strong CFP/GFP signals, which imply a single transformation event, were considered as a site ( $n=22$, wild type and chor).

\section{Epidermal phenotypes and symplastic connectivity in cytokinesis-defective mutants}

Because GSL8 is required for cytokinesis, incomplete cell division within the epidermis might also contribute to the dysregulated partitioning of regulatory macromolecules. To clarify this, we examined the epidermal phenotype and cell-to-cell connectivity in two well-described cytokinesis mutants, atnack1 (hinkel) (Nishihama et al., 2002; Strompen et al., 2002) and scd1 (stomatal cytokinesis-defective 1) (Falbel et al., 2003). Like chor, atnackl and $s c d 1$ seedlings exhibit dwarfism but produce rosette leaves and survive for 2-3 weeks after germination (data not shown).

Consistent with the previous descriptions in tobacco leaves (Nishihama et al., 2002), cotyledon and rosette leaf epidermis of atnack 1 showed occasional incomplete cytokinesis of pavement cells (Fig. 8A, arrowheads) and GMCs (Fig. 8A, asterisks), the latter of which resulted in abnormal stomata with incomplete separation of the guard cells. As reported previously (Falbel et al., 2003), incomplete cytokinesis and reduced lobing of pavement cells are also observed in the $s c d 1$ epidermis (Fig. 8A, plus signs). $s c d 1$ GMCs failed to divide and expand (Fig. 8A). In spite of these defects, neither atnackl nor $s c d 1$ epidermis showed excessive production of stomatal-lineage cells or severe stomatal clusters (Fig. $8 \mathrm{~A}$ ), indicating that stomatal patterning phenotypes in chor are not simply due to defective cytokinesis.

Next we investigated cell-to-cell diffusion of SPCH-YFP and soluble CFP in atnack1 and scd1 leaf epidermis (Fig. 8B-D), and compared the results with wild type and chor (Fig. 7). Among the four genotypes, only chor exhibited a significant difference in CFP diffusion (Tukey's HSD test with ANOVA, $P<0.01$ ). As in wild type, SPCH-YFP was detected solely in the nucleus of a single cell at the site of transformation in atnackl $(n=16 / 16)$ and $s c d 1$ $(n=20 / 20$; Fig. 8B,D). The results are inconsistent with the hypothesis that leakiness of cells is a common consequence of incomplete cytokinesis.

\section{DISCUSSION}

From a sensitized genetic screen, we have identified $C H O R$, which is required for organized initiation of the stomatal cell lineage and proper stomatal patterns. The molecular identity of CHOR as a putative callose synthase, GSL8, and seedling callose staining data demonstrate that GSL8 is required for cell-plate and plasmodesmatal callose deposition. A series of cell-to-cell diffusion assays illuminates the link between altered symplastic connectivity and stomatal fate specification.

Among the twelve putative callose synthase genes [GSLs (CalSs)] (Hong et al., 2001), GSL1 (CalS11), GSL5 (CalS12), GSL2 (CalS5), GSL8 (CalS10) and GSL10 (CalS9) have been shown to be required for microgametogenesis and pollen development (Dong et al., 2005; Enns et al., 2005; Nishikawa et al., 2005; Toller et al., 2008). Recent reverse-genetic studies by Chen et al. showed that most gsl mutants exhibited a nearly normal seedling growth phenotype (Chen et al., 2009), suggesting that many callose synthase genes act redundantly during sporophytic development. Our findings extend the recent reports (Chen et al., 2009; Thiele et al., 2009), and reveal roles of GSL8 in addition to its role in cytokinesis. Swollen, branched root hairs in chor and gsl8-2 (see Fig. S2 in the supplementary material) 


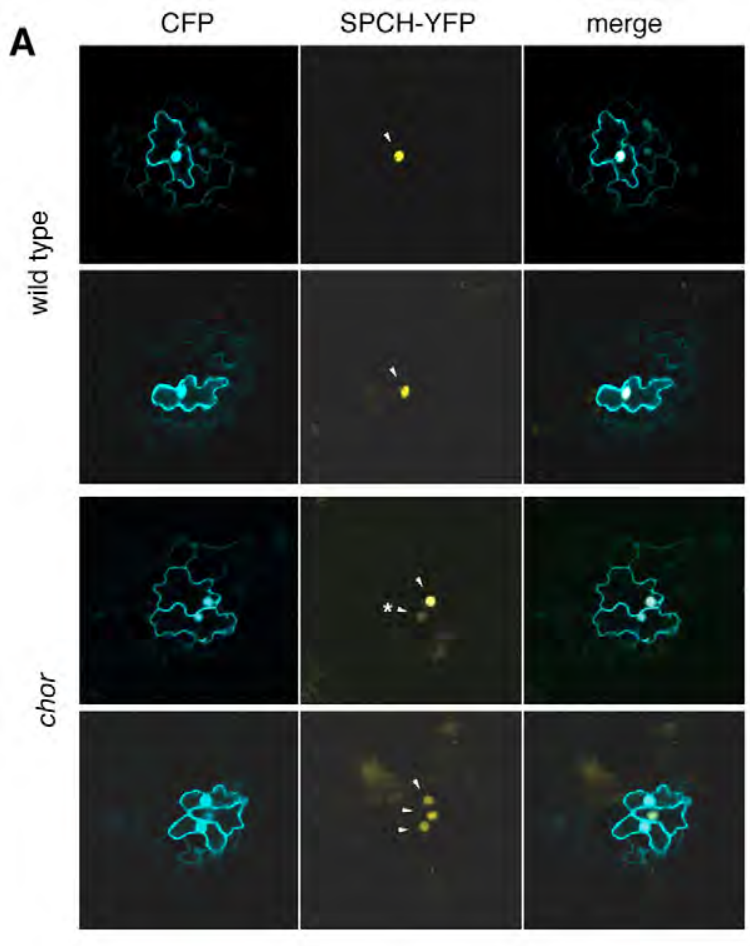

B

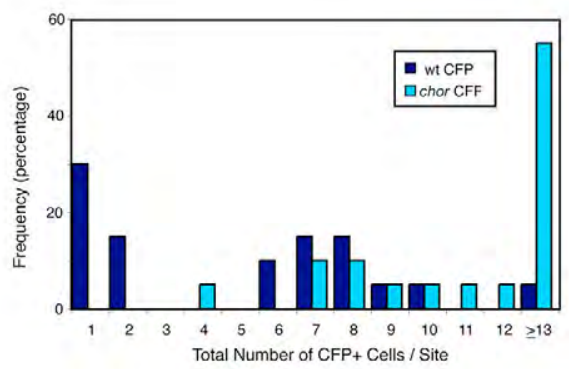

C

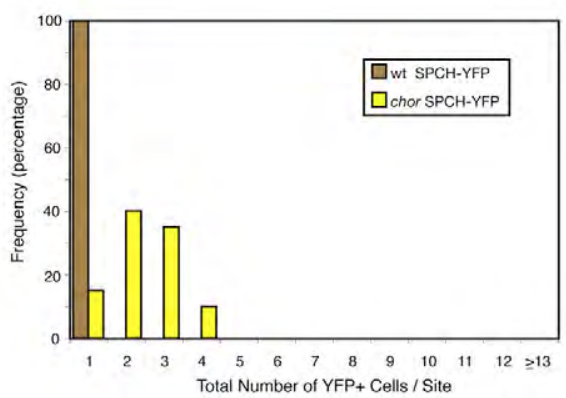

Fig. 7. Cell-to-cell diffusion of SPCH-YFP in chor. (A) Representative images of 10-day-old wild-type (top) and chor (bottom) epidermis expressing co-bombarded monomeric CFP (cyan, left) and SPCH-YFP (yellow, middle). Merged images are shown on the right. SPCH-YFP was detected solely in the nuclei (arrowheads). Dim YFP signals in neighboring nuclei (asterisks) indicate diffusion. Images are taken under the same magnification. (B,C) Quantitative distribution analysis of the number of cells expressing CFP (B) or SPCH-YFP (C) per site. In wild type, SPCH-YFP was only detected in one cell/site ( $n=20$, wild type and chor).

indicate that root-hair callose is necessary for proper root-hair-cell polarity and morphogenesis. Thiele et al. (Thiele et al., 2009) reported that seedlings of massue, which is allelic to chor, exhibit normal root-hair morphology. The difference between our observations and those of Thiele et al. (Thiele et al., 2009) might be due to specific growth conditions, such as a difference in mineral ion concentrations in the growth media, which affect root hair morphology and patterning (Muller and Schmidt, 2004; Yang et al., 2008).

Callose turnover at the plasmodesmata is likely to be determined by an intricate balance of callose synthase- and $\beta-1,3$ glucanase activities. Recent proteomic analyses of plasmodesmatal proteins led to the identification of a $\beta-1,3$ glucanase, AtBG_ppap (Levy et al., 2007a), as well as of GPI-anchored plasmodesmatal neck proteins that anchor callose via direct binding (Simpson et al., 2009). A loss-of-function mutation in AtBG_ppap conferred increased plasmodesmatal callose deposition and reduced cell-to-cell diffusion of free GFP (Levy et al., 2007a), a phenotype that is opposite to that of chor.

The fact that GSL8 is required for both cell-plate and plasmodesmatal callose deposition complicates the interpretation of chor epidermal phenotypes, as incomplete cytokinesis could also contribute to cell leakiness. Our cell-to-cell diffusion assays using atnackl and $s c d 1$ demonstrate that this is not likely to be the case. AtNACK1 encodes a kinesin-related protein that is required for cellplate expansion (Nishihama et al., 2002; Strompen et al., 2002). $S C D 1$ encodes a protein with a DENN domain and WD-40 repeats, which is implicated in the trafficking of secretory vesicles during cell-plate formation and polar cell growth (Falbel et al., 2003).
Although atnackl and scdl produced aberrant stomata with incomplete GMC divisions, neither mutant showed overproliferation of stomatal-lineage cells or severe stomatal clusters. Thus, the overproduction of stomatal-lineage cells in chor is likely to be a consequence of reduced plasmodesmatal callose deposition.

chor conferred massive stomatal cluster formation in plants lacking TMM or partially lacking members of the ER family (Figs 1,3 ), suggesting that $C H O R$ is required for full manifestation of inhibitory signaling via TMM and the ER family. How do cell leakiness and loss/reduction in a transmembrane signaling system together result in severe stomatal cluster formation? We propose the following model based on our findings (Fig. 9). In wild type, stomatal cell-fate determinants are tightly contained in stomatal initials (i.e. MMC) and precursors (i.e. meristemoids). Inhibitory signals, such as EPF peptides, further prevent the neighboring cells form adopting a stomatal-lineage fate (Fig. 9A). In the absence of CHOR, cell-fate determinants leak from the stomatal initials/ precursors to their neighbors, causing excessive entry divisions. However, functional TMM/ER-family signaling can target these cell-fate determinants and enforce stomatal patterning to some extent (Fig. 9B). The loss of CHOR in combination with the loss of TMM/ER-family signaling (or with even a slight reduction in signaling, such as a heterozygous loss of ERL1 in er chor) results in catastrophic and excessive stomatal differentiation (Fig. 9C).

Several lines of evidence support the hypothesis that SPCH might be such a cell-fate determinant. First, excessive entry into stomatal cell lineages, as seen in chor, is characteristic of $\mathrm{SPCH}$ overexpression. Second, spch loss-of-function mutation eliminates 


\section{A}

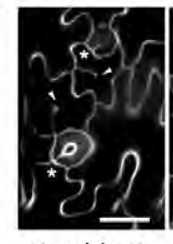

atnack1 cot
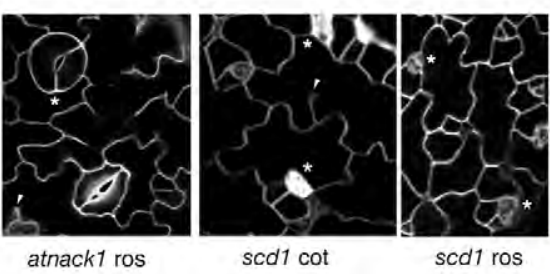

B

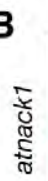

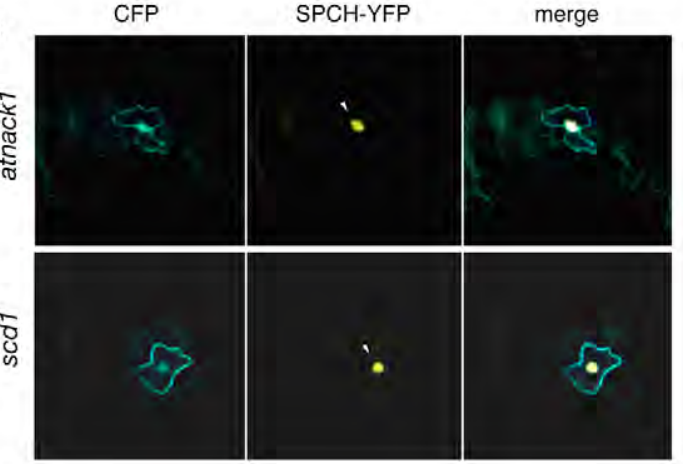

C

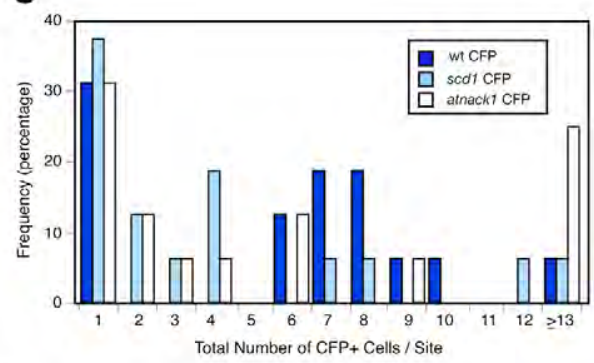

D

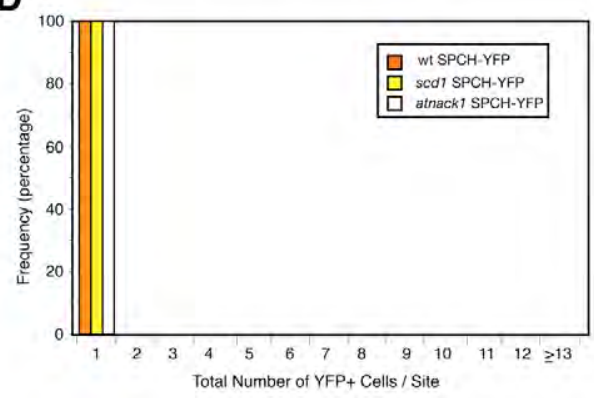

Fig. 8. Epidermal phenotypes and cell-to-cell diffusion assays in two cytokinesis mutants, atnack1 and scd1. (A) Shown are cotyledon and rosette leaf epidermis of 10-day-old atnack1 (left) and scd1 (right) seedlings. Both mutants exhibit occasional cytokinesis defects in pavement cells (arrowheads) and stomata (asterisks), but otherwise do not show the stomatal patterning phenotype seen in chor. (B) atnack1 (top) and scd1 (bottom) epidermis expressing co-bombarded monomeric CFP (cyan, left) and SPCH-YFP (yellow, middle). Merged images are shown on the right. SPCH-YFP was detected solely in the nuclei (arrowheads) of solitary cells. Images are taken under the same magnification. (C,D) Quantitative distribution analysis of the number of cells expressing CFP (C) or SPCH-YFP (D) per site. SPCH-YFP was detected in only one cell/site ( $n=20$, wild type and scd1; $n=16$, atnack1).

all of the small, highly divided cells in chor cotyledons and leaves, whereas incomplete cytokinesis defects in pavement cells remain unaffected. Third, more cells in the chor protoderm than in wild type express SPCH-GFP protein driven by its own promoter. Lastly, SPCH-YFP spreads from cell to cell in the chor epidermis. It has been proposed that the inhibitory cell-cell signal mediated via TMM/ER-family receptors targets and inhibits SPCH activity via YODA-dependent phosphorylation (Lampard et al., 2008). In this regard, it is worth mentioning that the expression of a phosphorylation-resistant version of SPCH also conferred a breakdown of redundancy among ER-family genes (Lampard et al., 2008).

ERLs were more sensitive than ER to the effects of chor (see Fig. 3; see Fig. S4 in the supplementary material). This further supports our hypothesis that $S P C H$ activity becomes excessive and dysregulated in chor. ER and ERLs have redundant yet unique roles during stomatal development. $E R$ is expressed throughout protodermal tissues, while ERLs are specifically expressed in stomatal-lineage cells (meristemoids and SLGCs) (Shpak et al., 2005). Consistently, ERLs inhibit the differentiation of meristemoids, while ER only has an earlier role (Shpak et al., 2005). It is possible that while the TMM/ER-family-mediated signaling pathway targets $\mathrm{SPCH}, \mathrm{SPCH}$ in turn promotes the expression and hence the function of ERLs.

Our studies reveal an exciting link between the regulation of intracellular macromolecule trafficking and stomatal patterning. Selective trafficking of regulatory molecules, such as transcription factors and small RNAs, influences cell-fate specification in plants, such as root tissue patterning and coordinated growth within the shoot apical meristem (Lucas and Lee, 2004; Oparka and Roberts,
2001). Thus far, intercellular movements of key transcription factors have not been observed during stomatal development. Rather, all five key stomatal bHLH proteins are expressed solely in specific

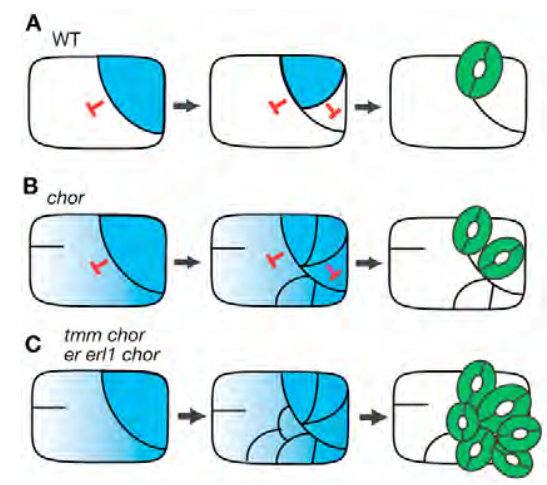

Fig. 9. Model of phenotypical consequences of the loss of $\mathrm{CHOR}$ (GSL8) and signaling receptors. (A) In wild type, stomatal cell-fate determinants (cyan) are contained in a precursor cell, and inhibitory signals (red T bar) prevent its neighbors from adopting a stomatallineage fate. (B) In chor, cell-fate determinants (cyan) may leak through plasmodesmata, which results in excessive stomatal entry divisions. The functional inhibitory signals, however, are able to prevent the stomatal differentiation of neighbors at some level. This results in modest stomatal clusters. Incomplete cytokinesis also occurs in chor. (C) In the absence of both CHOR (GSL8) and signaling receptors (such as TMM), the loss (or reduction at the threshold level in er erl1) of inhibitory signals together with the leakage of cell-fate determinants leads to catastrophic stomatal patterning defects. 
stomatal precursors (SPCH, MUTE and FAMA), or in stomatal precursors throughout stomatal development (SCRM and SCRM2), but not in their neighbors, consistent with their intrinsic, cellautonomous actions (MacAlister et al., 2007; Ohashi-Ito and Bergmann, 2006; Pillitteri et al., 2008; Pillitteri et al., 2007).

Owing to their nature as turgor-driven valves, mature guard cells constituting stomata are symplastically isolated and are consistently devoid of functional plasmodesmata (Willmer and Saxton, 1979). However, ultrastructural studies in some plant species have shown that developing stomatal precursors and their neighbors are connected via plasmodesmata (Kaufman et al., 1970; Willmer and Saxton, 1979). Imposing restrictions in cell-to-cell trafficking by callose deposition therefore could be a crucial means of segregating key cell-fate determinants while maintaining integrity between neighboring cells. In addition to regulatory proteins, movement of small RNAs might influence stomatal patterning. It has been reported that the microRNA miR824 and its target AGL16 are localized in different cell types within stomatal cell lineages, and this observation led to the hypothesis that they might act non-cell autonomously via plasmodesmata to regulate satellite meristemoid divisions (Kutter et al., 2007). Future studies to determine the distribution and cell-to-cell trafficking of these regulators in chor, and a precise delineation of cytokinesis and the plasmodesmatal functions of CHOR (GSL8), might reveal the exact mechanism by which cell-to-cell trafficking enforces proper stomatal patterning.

\section{Acknowledgements}

We thank Lynn Pillitteri and Jennifer Nemhauser for comments; Lynn Pillitteri, Michiko Sasabe, Yasunori Machida, Sebastian Bednarek, Andrew Maule, and NASC for plasmids and mutants/transgenic lines; Bruce Godfrey for assisting particle bombardment; Greg Martin and Amanda Rychel for assisting confocal microsopy; Tetsuya Higashiyama and Narie Sasaki for providing facilities to M.M.K. This work was supported by NSF (IOB0744892), DOE (DE-FG0203ER15448) and JST-PREST awards to K.U.T., NSF (MCB0548525 and IOB0543923) awards to Z.H., and Japan NEXT Grant-in-Aid for Young Scientists (Start-up:20870020 and B:21770041) to M.M.K. K.M.P. is an NSF graduate research fellow.

\section{Competing interests statement}

The authors declare no competing financial interests.

\section{Supplementary material}

Supplementary material for this article is available at http://dev.biologists.org/lookup/suppl/doi:10.1242/dev.049197/-/DC1

\section{References}

Bemis, S. M. and Torii, K. U. (2007). Autonomy of cell proliferation and developmental programs during Arabidopsis aboveground organ morphogenesis. Dev. Biol. 304, 367-381.

Bergmann, D. C. and Sack, F. D. (2007). Stomatal development. Annu. Rev. Plant. Biol. 58, 163-181.

Bergmann, D. C., Lukowitz, W. and Somerville, C. R. (2004). Stomatal development and pattern controlled by a MAPKK kinase. Science 304, 1494 1497.

Chen, X. Y., Liu, L., Lee, E., Han, X., Rim, Y., Chu, H., Kim, S. W., Sack, F. and Kim, J. Y. (2009). The Arabidopsis callose synthase gene GSL8 is required for cytokinesis and cell patterning. Plant Physiol. 150, 105-113.

Dievart, A. and Clark, S. E. (2004). LRR-containing receptors regulating plant development and defense. Development 131, 251-261.

Dong, X., Hong, Z., Sivaramakrishnan, M., Mahfouz, M. and Verma, D. P. (2005). Callose synthase (CalS5) is required for exine formation during microgametogenesis and for pollen viability in Arabidopsis. Plant J. 42, 315-328.

Enns, L. C., Kanaoka, M. M., Torii, K. U., Comai, L., Okada, K. and Cleland, R. E. (2005). Two callose synthases, GSL1 and GSL5, play an essential and redundant role in plant and pollen development and in fertility. Plant Mol. Biol. 58, 333-349.

Falbel, T. G., Koch, L. M., Nadeau, J. A., Segui-Simarro, J. M., Sack, F. D. and Bednarek, S. Y. (2003). SCD1 is required for cytokinesis and polarized cell expansion in Arabidopsis thaliana. Development 130, 4011-4024.
Hara, K., Kajita, R., Torii, K. U., Bergmann, D. C. and Kakimoto, T. (2007). The secretory peptide gene EPF1 enforces the stomatal one-cell-spacing rule. Genes Dev. 21, 1720-1725

Hara, K., Yokoo, T., Kajita, R., Onishi, T., Yahata, S., Peterson, K. M., Torii, K. U. and Kakimoto, T. (2009). Epidermal cell density is auto-regulated via a secretory peptide, EPIDERMAL PATTERNING FACTOR2 in Arabidopsis leaves. Plant Cell Physiol. 50, 1019-1031.

Hong, Z., Delauney, A. J. and Verma, D. P. (2001). A cell plate-specific callose synthase and its interaction with phragmoplastin. Plant Cell 13, 755-768.

Hunt, L. and Gray, J. E. (2009). The signaling peptide EPF2 controls asymmetric cell divisions during stomatal development. Curr. Biol. 19, 864-869.

Kanaoka, M. M., Pillitteri, L. J., Fujii, H., Yoshida, Y., Bogenschutz, N. L., Takabayashi, J., Zhu, J. K. and Torii, K. U. (2008). SCREAM/ICE1 and SCREAM2 specify three cell-state transitional steps leading to Arabidopsis stomatal differentiation. Plant Cell 20, 1775-1785.

Kaufman, P. B., Petering, L. B., Yocum, C. S. and Baic, D. (1970). Ultrastructural studies on stomata development in internodes of Avena sativa. Amer. J. Bot. 57, 33-49.

Kutter, C., Schob, H., Stadler, M., Meins, F., Jr and Si-Ammour, A. (2007) MicroRNA-mediated regulation of stomatal development in Arabidopsis. Plant Cell 19, 2417-2429.

Lai, L. B., Nadeau, J. A., Lucas, J., Lee, E. K., Nakagawa, T., Zhao, L., Geisler, M. and Sack, F. D. (2005). The Arabidopsis R2R3 MYB proteins FOUR LIPS and MYB88 restrict divisions late in the stomatal cell lineage. Plant Cell 17, 27542767.

Lampard, G. R., Macalister, C. A. and Bergmann, D. C. (2008). Arabidopsis stomatal initiation is controlled by MAPK-mediated regulation of the bHLH SPEECHLESS. Science 322, 1113-1116.

Levy, A., Erlanger, M., Rosenthal, M. and Epel, B. L. (2007a). A plasmodesmata-associated beta-1,3-glucanase in Arabidopsis. Plant J. 49, 669682.

Levy, A., Guenoune-Gelbart, D. and Epel, B. (2007b). 及-1,3 GlucanasesPlasmodesmatal gate keepers for intercellular communication. Plant Signal. Behav. 2, 404-407

Loudet, O., Chaillou, S., Camilleri, C., Bouchez, D. and Daniel-Vedele, F. (2002). Bay-0 x Shahdara recombinant inbred line population: a powerful tool for the genetic dissection of complex traits in Arabidopsis. Theor. Appl. Genet. 104, 1173-1184.

Lucas, W. J. and Lee, J. Y. (2004). Plasmodesmata as a supracellular control network in plants. Nat. Rev. Mol. Cell Biol. 5, 712-726.

Lukowitz, W., Gillmor, C. S. and Scheible, W. R. (2000). Positional cloning in Arabidopsis. Why it feels good to have a genome initiative working for you. Plant Physiol. 123, 795-805.

Lukowitz, W., Roeder, A., Parmenter, D. and Somerville, C. (2004). A MAPKK kinase gene regulates extra-embryonic cell fate in Arabidopsis. Cell 116, 109 119.

MacAlister, C. A., Ohashi-Ito, K. and Bergmann, D. C. (2007). Transcription factor control of asymmetric cell divisions that establish the stomatal lineage. Nature 445, 537-540.

Muller, M. and Schmidt, W. (2004). Environmentally induced plasticity of roo hair development in Arabidopsis. Plant Physiol. 134, 409-419.

Nadeau, J. A. and Sack, F. D. (2002a). Control of stomatal distribution on the Arabidopsis leaf surface. Science 296, 1697-1700.

Nadeau, J. A. and Sack, F. D. (2002b). Stomatal Development in Arabidopsis. Rockville, MD: American Society of Plant Biologists. doi: 10.1199/tab.0066 http://www.aspb.org/publications/arabidopsis/.

Neff, M. M., Neff, J. D., Chory, J. and Pepper, A. E. (1998). dCAPS, a simple technique for the genetic analysis of single nucleotide polymorphisms: experimental applications in Arabidopsis thaliana genetics. Plant J. 14, 387-392.

Nishihama, R., Soyano, T., Ishikawa, M., Araki, S., Tanaka, H., Asada, T., Irie, K., Ito, M., Terada, M., Banno, H. et al. (2002). Expansion of the cell plate in plant cytokinesis requires a kinesin-like protein/MAPKKK complex. Cell 109, 8799.

Nishikawa, S., Zinkl, G. M., Swanson, R. J., Maruyama, D. and Preuss, D. (2005). Callose (beta-1,3 glucan) is essential for Arabidopsis pollen wall patterning, but not tube growth. BMC Plant Biol. 5, 22.

Ohashi-Ito, K. and Bergmann, D. C. (2006). Arabidopsis FAMA controls the final proliferation/differentiation switch during stomatal development. Plant Cell 18, 2493-2505

Oparka, K. J. and Roberts, A. G. (2001). Plasmodesmata. A not so open-andshut case. Plant Physiol. 125, 123-126.

Pillitteri, L. J., Sloan, D. B., Bogenschutz, N. L. and Torii, K. U. (2007) Termination of asymmetric cell division and differentiation of stomata. Nature 445, 501-505

Pillitteri, L. J., Bogenschutz, N. L. and Torii, K. U. (2008). The bHLH protein, MUTE, controls differentiation of stomata and the hydathode pore in Arabidopsis. Plant Cell Physiol. 49, 934-943.

Radford, J. E., Vesk, M. and Overall, R. L. (1998). Callose deposition at plasmodesmata. Protoplasma 201, 30-37. 
Shiu, S. H. and Bleecker, A. B. (2001). Plant receptor-like kinase gene family: diversity, function, and signaling. SCi. STKE 2001, RE22.

Shpak, E. D., Berthiaume, C. T., Hill, E. J. and Torii, K. U. (2004). Synergistic interaction of three ERECTA-family receptor-like kinases controls Arabidopsis organ growth and flower development by promoting cell proliferation. Development 131, 1491-1501.

Shpak, E. D., McAbee, J. M., Pillitteri, L. J. and Torii, K. U. (2005). Stomata patterning and differentiation by synergistic interactions of receptor kinases. Science 309, 290-293.

Simpson, C., Thomas, C., Findlay, K., Bayer, E. and Maule, A. J. (2009). An Arabidopsis GPI-anchor plasmodesmal neck protein with callose binding activity and potential to regulate cell-to-cell trafficking. Plant Cell 21, 581-594.

Stone, B. A. and Clarke, A. E. (1992). Chemistry and physiology of higher plant $1,3-\beta$-glucans (callose). In Chemistry and Biology of $(1,3) \beta$-glucans (ed. B. A. Stone and A. E. Clarke), pp. 365-429. Bundoora, Australia: La Trobe University Press.

Stone, B. A., Evans, N. A., Bonig, I. and Clarke, A. E. (1984). The application of Sirofluor, a chemically defined fluorochrome from aniline blue for the histochemical detection of callose. Protoplasma 122, 191-195.

Strompen, G., El Kasmi, F., Richter, S., Lukowitz, W., Assaad, F. F., Jurgens, G. and Mayer, U. (2002). The Arabidopsis HINKEL gene encodes a kinesin-related protein involved in cytokinesis and is expressed in a cell cycle-dependent manner. Curr. Biol. 12, 153-158.
Thiele, K., Wanner, G., Kindzierski, V., Jurgens, G., Mayer, U., Pachl, F. and Assaad, F. F. (2009). The timely deposition of callose is essential for cytokinesis in Arabidopsis. Plant J. 58, 13-26.

Toller, A., Brownfield, L., Neu, C., Twell, D. and Schulze-Lefert, P. (2008). Dual function of Arabidopsis glucan synthase-like genes GSL8 and GSL10 in male gametophyte development and plant growth. Plant J. 54, 911-923.

Torii, K. U. and Clark, S. E. (2000). Receptor-like kinases in plant development. Adv. Bot. Res. Adv. Plant. Path. 32, 226-268.

von Arnim, A. G., Deng, X. W. and Stacey, M. G. (1998). Cloning vectors for the expression of green fluorescent protein fusion proteins in transgenic plants. Gene 221, 35-43.

Wang, H., Ngwenyama, N., Liu, Y., Walker, J. and Zhang, S. (2007). Stomatal development and patterning are regulated by environmentally responsive mitogen-activated protein kinases in Arabidopsis. Plant Cell 19, 6373.

Willmer, C. M. and Saxton, R. (1979). Stomata and plasmodesmata. Protoplasma 100, 113-124

Winter, D., Vinegar, B., Nahal, H., Ammar, R., Wilson, G. V. and Provart, N. J. (2007). An "electronic fluorescent pictograph" browser for exploring and analyzing large-scale biological data sets. PLoS One 2, e718.

Yang, T. J., Perry, P. J., Ciani, S., Pandian, S. and Schmidt, W. (2008). Manganese deficiency alters the patterning and development of root hairs in Arabidopsis. J. Exp. Bot. 59, 3453-3464. 


\title{
Out of the Mouths of Plants: The Molecular Basis of the Evolution and Diversity of Stomatal Development ${ }^{\mathbb{W}}$
}

\author{
Kylee M. Peterson, ${ }^{\mathrm{a}, 1}$ Amanda L. Rychel, ${ }^{\mathrm{a}, 1}$ and Keiko U. Torii ${ }^{\mathrm{a}, \mathrm{b}, 2}$ \\ a Department of Biology, University of Washington, Seattle, Washington 98195 \\ b PREST, Japan Science and Technology Agency, Tokyo 102-0075, Japan
}

\begin{abstract}
Stomata are microscopic valves on the plant epidermis that played a critical role in the evolution of land plants. Studies in the model dicot Arabidopsis thaliana have identified key transcription factors and signaling pathways controlling stomatal patterning and differentiation. Three paralogous Arabidopsis basic helix-loop-helix proteins, SPEECHLESS (SPCH), MUTE, and FAMA, mediate sequential steps of cell-state transitions together with their heterodimeric partners SCREAM (SCRM) and SCRM2. Cell-cell signaling components, including putative ligands, putative receptors, and mitogen-activated protein kinase cascades, orient asymmetric cell divisions and prevent overproduction and clustering of stomata. The recent availability of genome sequence and reverse genetics tools for model monocots and basal land plants allows for the examination of the conservation of genes important in stomatal patterning and differentiation. Studies in grasses have revealed that divergence of SPCH-MUTE-FAMA predates the evolutionary split of monocots and dicots and that these proteins show conserved and novel roles in stomatal differentiation. By contrast, specific asymmetric cell divisions in Arabidopsis and grasses require unique molecular components. Molecular phylogenetic analysis implies potential conservation of signaling pathways and prototypical functions of the transcription factors specifying stomatal differentiation.
\end{abstract}

\section{INTRODUCTION}

The evolution of stomata (Greek for mouths; singular, stoma) was a crucial adaptation occurring some 400 million years ago that made it possible for plants to thrive on land, which they began to colonize some 450 million years ago. To survive in the dry atmosphere, plants must protect against desiccation yet allow for the gas exchange necessary for photosynthesis and respiration. A watertight epidermis with closable valves, the stomata, was the solution. Stomata are composed of paired, symmetric guard cells that operate by changing turgor pressure, a system that forms an effective fail-safe against dehydration, since low turgor closes stomata. Transpiration, or water loss through stomata, promotes upward and outward water movement in plants and cools the plant surface via thermal dissipation. The distribution of stomata is far from random; they are evenly distributed, an adaptation allowing gases to reach interior tissues effectively, and they adhere closely to the one-cell spacing rule (Sachs, 1991), which means that stomata are always found with at least one nonstomatal epidermal cell separating them. This one-cell spacing rule is important for proper opening and closing of the stomatal aperture, which requires efficient exchange of water and ions with neighboring nonstomatal cells. Stomatal development must be tightly controlled to accomplish these goals.

The fundamental physiological importance of stomata is underscored by the observation that their evolution predates that of

\footnotetext{
1 These authors contributed equally to this work.

${ }^{2}$ Address correspondence to ktorii@u.washington.edu.

WOnline version contains Web-only data.

www.plantcell.org/cgi/doi/10.1105/tpc.109.072777
}

flowers, leaves, roots, and even vascular systems (Freeman, 2008) (Figure 1). The fossil record indicates that the earliest nonvascular and vascular plants, such as Cooksonia, Rhynia, and Aglaophyton, possessed a simple architecture of bifurcated stems with apical spore capsules (Edwards et al., 1998), very different from modern land plants. Yet these ancient plants possessed stomata that were strikingly similar to those of extant land plants, such as Arabidopsis thaliana (Edwards et al., 1998; Taylor et al., 2005). In Figure 1, mature stomata and their neighboring epidermal cells over a large phylogenetic distance are presented to demonstrate the evolutionarily ancient derivation of the stoma.

If the evolution of stomata was indeed a single vital event in land plant evolution, what are the underlying genes and molecular mechanisms that gave rise to stomata? What are the molecular bases that explain the diversity of stomatal patterns seen today? Recent discoveries of key genes controlling stomatal development in the model plant Arabidopsis now provide molecular tools with which to tackle these questions. In this review, we first introduce Arabidopsis genes controlling stomatal development. We further describe the evolutionary conservation and uniqueness of stomatal development as well as the functions of orthologous genes in other plant species as an attempt to promote interest and discussion among the diverse disciplines of botany, paleobotany, and evolutionary biology.

\section{STOMATAL DEVELOPMENT IN ARABIDOPSIS}

\section{Cell-State Transitions}

Stomata in Arabidopsis develop through a process of asymmetric cell division (Figure 2) that produces the anisocytic stomatal 


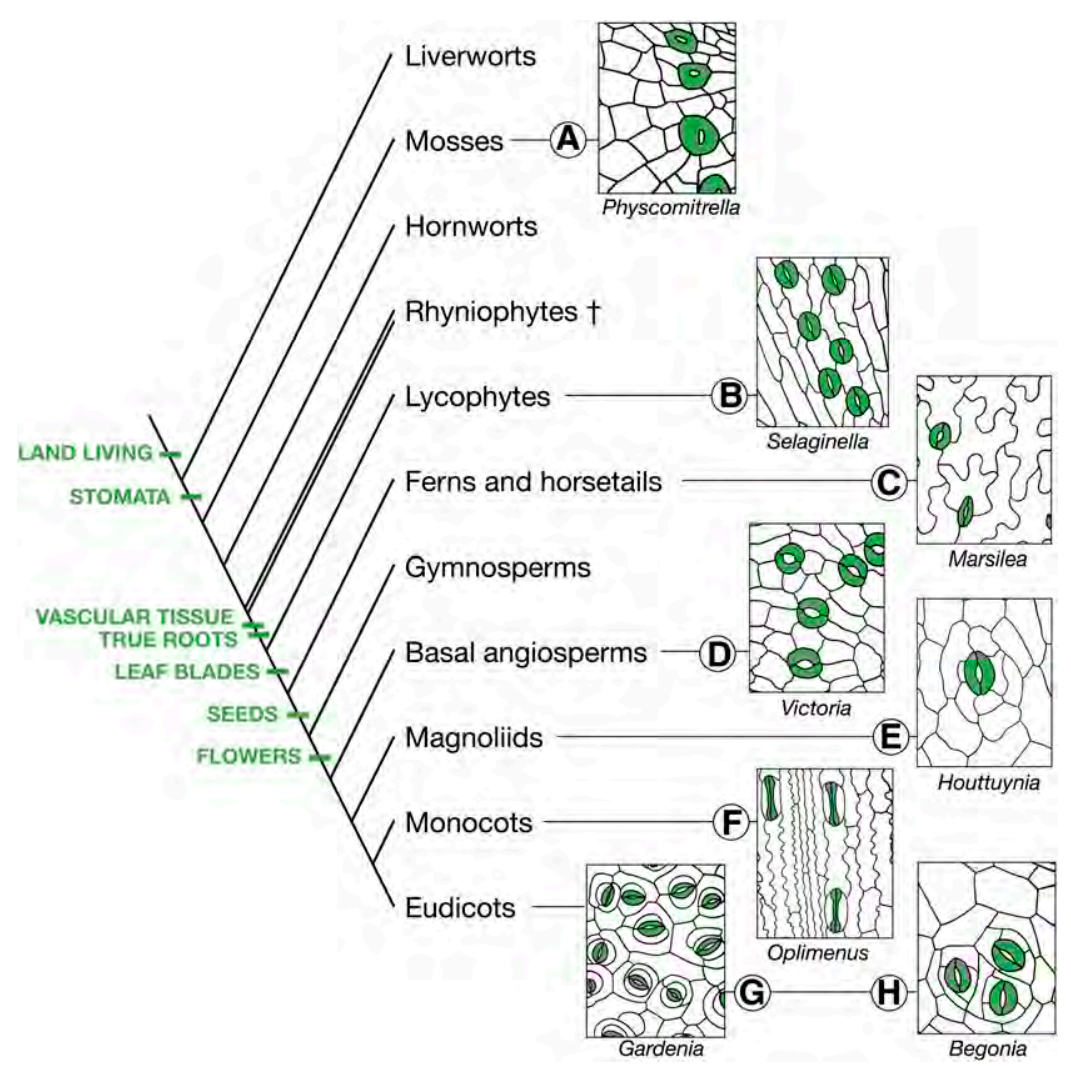

Figure 1. Diversity of Stomata across Land Plant Taxa.

A phylogenetic tree of extant and extinct $(\dagger)$ land plants includes evolutionary traits supporting success on land. The wide diversity of stomatal complexes among these groups is represented by epidermal tracings of $P$. patens ([A]; nonvascular), Selaginella kraussiana ([B]; lycophyte, vascular), Marsilea macropoda ([C]; fern, vascular), Victoria amazonica ([D]; Nymphaeaceae, basal angiosperm), Houttuynia cordata ([E]; Piperales, magnoliid), Oplimenus hirtellus ([F]; Poales, monocot grass), Gardenia taitensis ([G]; Gentianales, eudicot angiosperm), and Begonia rex-cultorum 'Roberta' ([H]; Cucurbitales, eudicot angiosperm). Stomata are colored green. Note that the stomata of Physcomitrella have a single GC, while the GCs of Oplimenus, a grass, have a dumbbell shape. Only Houttuynia, Gardenia, and Begonia show evidence of asymmetric amplifying divisions within the stomatal lineage. Physcomitrella is traced from a scanning electron microscopy image by L. Pillitteri.

complexes commonly seen in Brassicaceae, in which each stoma has one smaller and two larger neighboring cells arising from asymmetric divisions within the stomatal lineage (Esau, 1977). First, in the protoderm, a population of cells initiates the stomatal lineage and divides asymmetrically to form a stomatal precursor cell called a meristemoid. The meristemoid reiterates asymmetric divisions several times, usually from one to three, creating sister cells called stomatal lineage ground cells (SLGCs) of decreasing size while renewing the meristemoid state (Figure 2). The meristemoid then differentiates into a guard mother cell (GMC). The GMC divides once symmetrically to form a pair of cells that develop the thickened walls and mature chloroplasts characteristic of stomatal guard cells (GCs). SLGCs eventually become pavement cells but may divide asymmetrically as well, producing a meristemoid away from the original stoma (Figure 2). The stomatal complex may be formed exclusively through multiple asymmetric divisions, in which case it is clonal, or if fewer asymmetric divisions occur the stoma may be adjacent to the lineage of another protodermal cell (Serna et al., 2002).
Two groups of basic helix-loop-helix (bHLH) transcription factors regulate the major cell-state transitions through stomatal differentiation (Figure 2). The first group is encoded by the paralogs (closely related genes arising from duplication within a genome) SPEECHLESS (SPCH), MUTE, and FAMA, which operate sequentially to regulate the cellular identity of each step in the developmental process (Ohashi-Ito and Bergmann, 2006; MacAlister et al., 2007; Pillitteri et al., 2007b). SPCH controls the initial asymmetric division with which protodermal cells enter the stomatal lineage; spch mutants are entirely without stomatal lineage cells, having only puzzle piece-shaped pavement cells interlocking over the entire shoot surface (MacAlister et al., 2007; Pillitteri et al., 2007b). Next to act is MUTE, which governs the meristemoid's cessation of asymmetric divisions and its transition to GMC identity. Plants without functional MUTE also develop no stomata, but rather than lacking the stomatal lineage entirely they show rosette-like patterns of inwardly spiraling meristemoid cell divisions with aborted meristemoids in the center (MacAlister et al., 2007; Pillitteri et al., 2007b, 2008). Lastly, FAMA appears to control the switch from the GMC to GC 
A

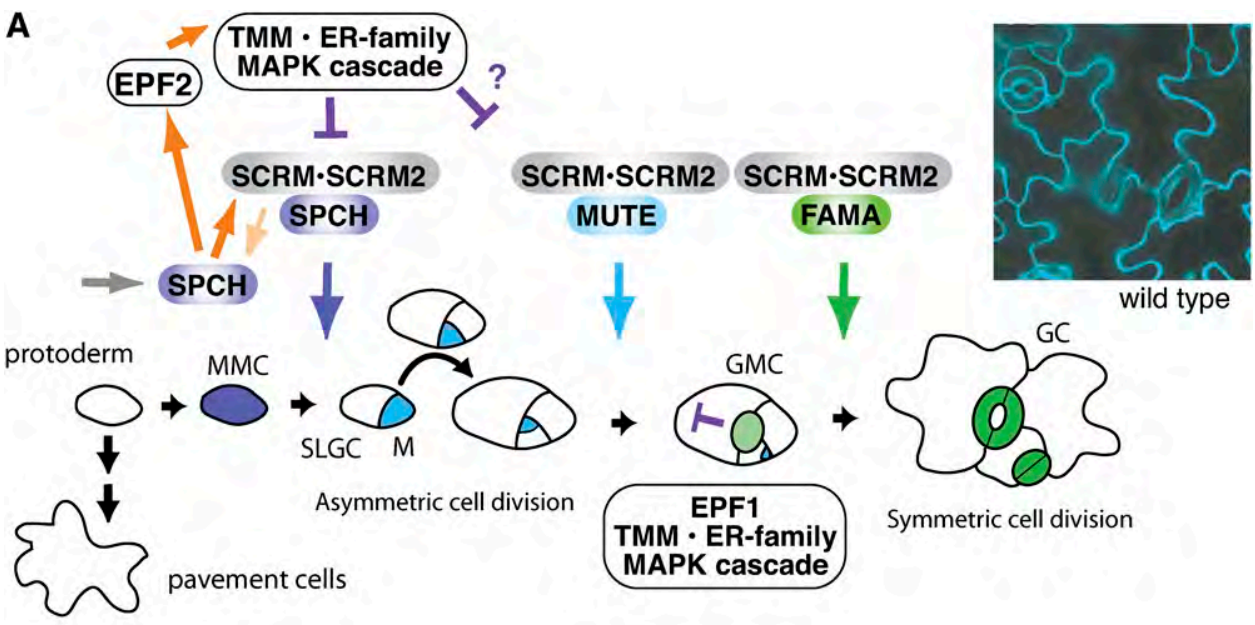

B (gof cell-cell signaling)

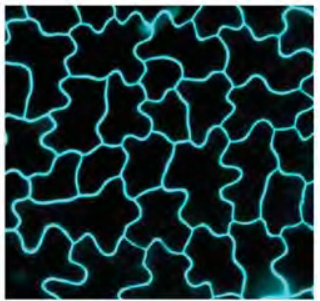

scrm scrm2

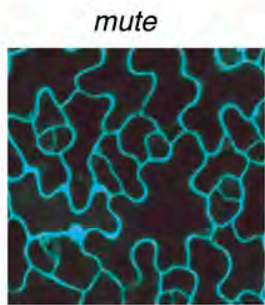

(scrm scrm2/+)

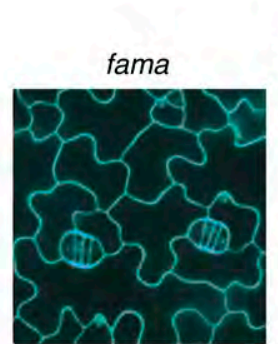

(scrm) (lof cell-cell signaling)

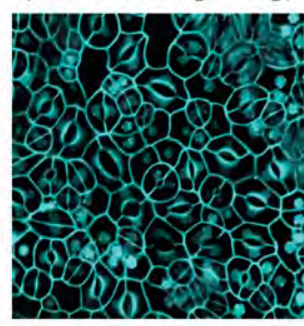

scrm- $D$

Figure 2. Stomatal Development in Arabidopsis.

(A) Schematic diagram of stomatal development. The cell states of stomatal precursors are driven by three paralogous bHLH transcription factors, which likely dimerize with SCRM and SCRM2 as a mechanism for coordinated action. Initial specification of the stomatal cell lineage, in which a protodermal cell becomes a meristemoid mother cell (MMC), is controlled by SPCH. Protodermal cells not entering the stomatal lineage differentiate into pavement cells. The MMC divides asymmetrically to form a meristemoid (M) and SLGC and may reiterate similar divisions several times. MUTE controls the cell-state transition from M to GMC, and FAMA is required for correct division of the GMC into GCs forming a functional stoma. It is proposed that a MAP kinase signaling cascade following putative ligands EPF1 and EPF2 (EPF1 expressed in GMC, light green, and EPF2 expressed in MMC, blue, and M, cyan) perceived by TMM and the ER family of RLKs acts to suppress stomatal identity in cells adjacent to developing stomata; new meristemoids can differentiate at least one cell away, as shown near GMC. An image of wild-type epidermis is shown at the top right.

(B) Epidermal phenotypes of stomatal differentiation mutants. Shown are the rosette leaf epidermis of (from left) scrm scrm2, mute, fama, and scrm-D. scrm scrm2 produces epidermis solely composed of pavement cells, a phenotype identical to that of spch as well as gain-of-function mutants in stomatal cell-cell signaling genes. mute and fama produce epidermis with arrested stomatal precursor cells similar to scrm scrm2/+ and scrm, respectively. scrm-D produces epidermis solely composed of stomata, a phenotype similar to loss of function in stomatal signaling genes. Images are reproduced from Kanaoka et al. (2008).

identity; in fama mutants, the stomatal precursors halt at the GMC state, resulting in "caterpillars" of excessively symmetrically divided cells instead of mature GCs from a single symmetric division (the many "false mouths" of the Roman goddess of rumor, Fama) (Ohashi-Ito and Bergmann, 2006).

Regulating the sequential action of the master regulators are the second group of bHLH proteins, encoded by the broadly expressed paralogs SCRM and SCRM2, which have partially overlapping functions. The originally discovered gain-of-function mutation, scrm- $D$, causes all epidermal cells to take on stomatal fate, while successive loss of SCRM and SCRM2 creates phenotypes recapitulating spch, mute, and fama mutants (Kanaoka et al., 2008). The bHLH proteins function through dimerization
(Massari and Murre, 2000), and SCRM and SCRM2 are capable of interacting with SPCH, MUTE, and FAMA in vivo (Figure 2) (Kanaoka et al., 2008). This suggests a possible mode of regulation in which transiently expressed stomatal lineage-specific bHLH proteins heterodimerize with widely expressed bHLH partner proteins to control a developmental progression (Pillitteri and Torii, 2007).

Other factors also act to promote stomatal development. Formation of satellite (secondary) meristemoids is controlled by microRNA miR824, which targets AGAMOUS-LIKE16 (AGL16) mRNA for degradation (Kutter et al., 2007). An AGL16 mRNA that no longer matches miR824 due to silent mutations causes additional reentry of SLGC into the stomatal lineage, resulting 
in more satellite meristemoids, a phenotype opposite to that of the ag/16-1 dosage reduction mutant (Kutter et al., 2007).

Loss of function in a MYB transcription factor, FOUR LIPS $(F L P)$, causes parallel pairs of stomata (Yang and Sack, 1995). Its paralog, At $M Y B 88$, is partially redundant with $F L P$ : in flp myb88 double mutants, massive fama-like stacks of cells are frequently formed, although some are able to differentiate terminally into GCs (Lai et al., 2005). It is therefore likely that FLP and MYB88 together restrict the symmetric division of GMC, though unlike FAMA, they do not necessarily specify terminal differentiation into the GC state. Consistent with their redundant roles in GC differentiation, FLP and MYB88 share an amino acid substitution that is unique among known plant MYBs (Lai et al., 2005). The roles of FLP and MYB88 echo the ongoing pattern in which a gene and its paralog have redundant or related functions, which is consistent with evolution through gene duplication.

\section{Patterning and Signaling}

A repressive cell-cell signaling pathway in Arabidopsis maintains proper stomatal patterning as defined by the one-cell spacing rule (Figure $2 \mathrm{~A}$ ). Interactions among the partially redundant ERECTA (ER) family of leucine-rich repeat receptor-like kinases (LRR-RLKs) and the LRR receptor-like protein TOO MANY MOUTHS (TMM) affect stomatal spacing and density (Shpak et al., 2005). The function of TMM is organ dependent and complex: in tmm mutants, leaves show clusters of stomata, but stems develop no stomata at all despite the entry of some cells into the stomatal lineage (Bhave et al., 2009). Similarly, the three ER family RLKs exhibit combination-specific interactions with TMM in specific organ types, implying that different combinations of receptor complexes inhibit particular steps of stomatal differentiation. Consistent with the receptor model, two putative ligands of TMM and ER family RLKs, EPIDERMAL PATTERNING FACTOR1 (EPF1) (Hara et al., 2007) and EPF2 (Hara et al., 2009; Hunt and Gray, 2009) restrict the later and the earlier steps of stomatal cell fate specifications, respectively (Figure 2A). The cell-cell signals in turn appear to activate a mitogen-activated protein (MAP) kinase signaling cascade including the MAPKKK YODA (Bergmann et al., 2004), MPKK4/5, and MAPK MPK3/6 (Wang et al., 2007), to suppress stomatal development in neighboring cells. In general, MAP kinase cascades target transcription factors and regulate their activities via phosphorylation. Recently, SPCH was shown to be a MAP kinase target, thus providing a mechanism by which stomatal suppression signaling might affect stomatal cell state identity directly (Lampard et al., 2008). Recently, additional signaling ligands have been discovered: STOMAGEN/EPF-LIKE9 (EPFL9), which promotes stomatal differentiation through TMM, and CHALLAH/EPFL6, which plays a role in tissue-specific stomatal patterning (Abrash and Bergmann, 2010; Kondo et al., 2010; Sugano et al., 2010). These additional players highlight the roles of the EPF family of signaling ligands in various aspects of stomatal development and may clarify the complex actions of TMM/ER family RLKs.

STOMATAL DENSITY AND DISTRIBUTION1 (SDD1) also acts as a negative regulator of stomatal formation (Berger and Altmann, 2000), but although it has been shown to require
TMM for function (von Groll et al., 2002), it seems to be independent of EPF1 (Hara et al., 2007), EPF2 (Hara et al., 2009; Hunt and Gray, 2009), STOMAGEN/EPFL9 (Kondo et al., 2010; Sugano et al., 2010), and CHALLAH/EPFL6 (Abrash and Bergmann, 2010). Although SDD1 encodes a membraneassociated subtilisin-type protease (Berger and Altmann, 2000), it appears unlikely that it directly processes any known member of the EPF family of stomatal ligands.

\section{Innate Polarity}

Asymmetric cell division within the stomatal lineage is highly polar: The meristemoid divides at angles of exactly $60^{\circ}$ from each previous division, forming an orderly inward spiral of SLGCs with a triangular meristemoid inside (Serna et al., 2002; Pillitteri et al., 2007b). When spacing divisions occur, the satellite meristemoids always divide away from any neighboring stoma to maintain the one-cell spacing rule. While cell-cell signaling and bHLH transcription factors determining the state of asymmetrically dividing stomatal precursors are well characterized, factors directing the innate polarity that allows spacing to emerge from the asymmetric cell division process have remained elusive, until the recent discovery of a unique protein, BREAKING OF ASYMMETRY IN THE STOMATAL LINEAGE (BASL) (Dong et al., 2009). The BASL protein fused with green fluorescent protein (GFP) is localized to the nuclei of dividing stomatal lineage cells and also to the cell cortex opposite the site of the most recent asymmetric division, which appears to be BASL's main site of activity (Dong et al., 2009) (Figure 3). The ectopic overexpression of BASL confers abnormal cell polarization. Interestingly, the loss of asymmetry in a bas/ mutant resulted in adoption of the stomatal fate, as depicted by the accumulation of a MUTE:nuclear GFP reporter fusion protein in both daughter cells (Dong et al., 2009). This observation implies that the asymmetric divisions in stomatal lineage cells are necessary for specifying the fate of the SLGC, but not the meristemoid, and a rapid cell polarization via a BASL-mediated mechanism is required to do so. This bas/ phenotype is the opposite of tmm er, where both daughter cells of a meristemoid adopt the SLGC fate (Shpak et al., 2005). While genetic studies suggest that $B A S L$ operates in a separate pathway from the TMM/ER family (Dong et al., 2009), the latter is required for proper positioning of GFP-BASL. Understanding the mechanism that achieves a peripheral localization of BASL may illuminate the intersection of signaling and innate asymmetry.

\section{Stomatal Patterning and Differentiation in Grasses}

In grasses, stomatal complexes develop from nonclonal cells through a polarized division process (Figure 4). The cells are organized in cell files throughout the plant, which are visible on the macro scale as the typical parallel venation of the monocot (Figure 1F). Spacing is maintained by the specification of nonadjacent cell files to develop stomata, which begin with an asymmetric division toward the leaf apex for all cells in the file. One of the cells produced by the asymmetric division differentiates directly into a GMC, which before dividing again signals the 


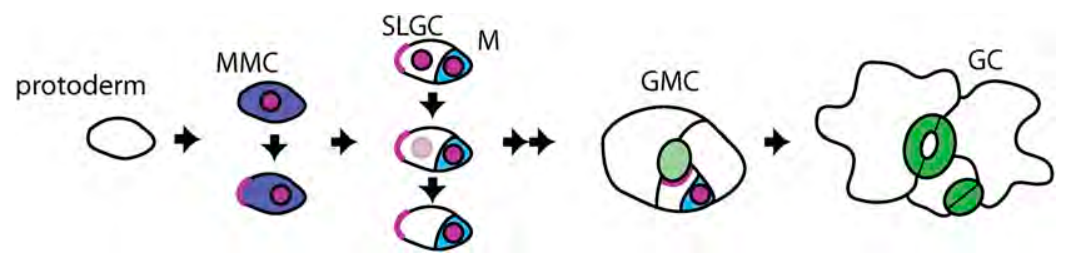

Figure 3. Localization of BASL.

BASL acts as a molecular signal instructing stomatal lineage cells to divide away from it. BASL protein appears initially in the nuclei of meristemoid mother cells (MMC), which differentiate from protodermal cells. The protein then localizes in a second location at the cell periphery opposite the site of the future asymmetric division. Following that division, BASL remains at the cell periphery but fades away from the nucleus of the larger daughter cell (SLGC), which loses stomatal lineage identity; it remains in the nucleus of the meristemoid (M), which may further asymmetrically divide. BASL is not found in later stomatal lineage cells, such as (GMCs or GCs. However, when satellite meristemoids are formed by SLGCs that resume stomatal lineage fate, BASL appears at the SLGC periphery next to the stomatal lineage cell, providing a mechanism for maintenance of the one-cell spacing rule. (Based on data presented in Dong et al. [2009].)

neighboring cells in adjacent cell files to divide asymmetrically toward the GMC. These divisions produce subsidiary cells (SCs) important for stomatal action (Figure 4). Subsequently, the GMC makes a single symmetric division and differentiates two GCs in a distinctive dumbbell shape (Gallagher and Smith, 2000). GCs with this shape require less water to open than those with a kidney shape (Raschke, 1979), so they may be an adaptation to drought conditions.

The recent availability of genome sequences and reversegenetics tools for model monocot plants, rice (Oryza sativa) and maize (Zea mays), allows for the examination of the conservation of genes important in stomatal patterning and differentiation. Grasses contain orthologs (genes in different species that evolved from a common ancestor) of the master regulatory genes of Arabidopsis stomatal differentiation, SPCH, MUTE, and FAMA (Liu et al., 2009). Although the transcripts of the two Os SPCH genes were detected only in coleoptiles, one of them showed a decrease in entry into the stomatal lineage when mutated (Liu et al., 2009). This suggests that in rice, at least one of the SPCH genes controls initial cell lineage divisions; it may have additional functions that are redundant with the second Os $\mathrm{SPCH}$, in which a mutation has not yet been found. Os MUTE and maize Zm MUTE were expressed earlier in development than their Arabidopsis ortholog, at the time of cell file specification. Ectopic overexpression of Os MUTE and Zm MUTE in Arabidopsis caused conversion of epidermal cells into stomata to varying degrees, a phenotype similar to that of At MUTE overexpression (Liu et al., 2009), and they were able to partially rescue the Arabidopsis mute mutant when expressed under the native Arabidopsis promoter (Liu et al., 2009). In mutants of Os FAMA, GCs fail to differentiate into the dumbbell shape of wild-type GCs, implying that Os FAMA controls GC fate, though there are none of the extra cell divisions seen in Arabidopsis fama plants (Liu et al., 2009).

This series of studies provides interesting implications. First, the SPCH-MUTE-FAMA bHLH genes diverged before the evolutionary split between monocots and dicots. Second, they have maintained their overall functions as regulators of stomatal differentiation across these widely separated taxa. Third, the orthologs have differences in their specific functions that reflect the differences in the specifics of stomatal ontogeny between dicots (Arabidopsis) and monocots (grasses). For instance, while both At fama and Os fama mutants fail to differentiate mature GCs, only the At fama mutant forms characteristic tumors of supernumerary GMC-like cells (Ohashi-Ito and Bergmann, 2006; Liu et al., 2009). These differences could be reconciled if we assume that, in Arabidopsis, a transition from a proliferative state to terminal differentiation of stomata requires a strong brake to halt cell division machinery. There is no actively dividing meristemoid state during stomatal differentiation in grasses; consequently, Os FAMA may not have acquired (or retained) the specific function to restrict cell division. The role of Os MUTE earlier in development, relative to At MUTE, may also suggest that these bHLH proteins acquired specific functions reflecting the uniqueness of stomatal development in grasses.

Formation of stomatal complexes in grasses (Figure 4) involves asymmetric cell divisions in subsidiary mother cells (SMCs), members of GMC-neighboring cell files that give rise to SCs, which have a highly specialized function as ion reserves for GC movement. Such an asymmetric cell division outside the stomatal lineage does not exist in Arabidopsis. Therefore, it is of great interest to unravel the molecular identities of the genes controlling subsidiary cell division and polarity in grasses. Loss-offunction mutations in PANGLOSS1 (PAN1) lead to aberrant asymmetric cell division and SC patterning (Gallagher and Smith, 2000). Recently, PAN1 was cloned and shown to encode an atypical group III LRR-RLK without kinase activity (Cartwright et al., 2009). It follows that PAN1 might associate with other RLKs with functional kinase domain to transmit signals. In Arabidopsis, an atypical LRR-RLK without kinase activity is known to regulate developmental processes (Chevalier et al., 2005). Interestingly, PAN1 protein is localized specifically at the plasma membrane of the SMC at the sites of contact with GMC. This is consistent with the hypothesis that PAN1 receives GMC signals to regulate the site of cytokinesis. Such polar localization has not been observed for the Arabidopsis stomatal LRR receptor TMM (Nadeau and Sack, 2002), and no member of LRR group III in Arabidopsis has been found to affect stomatal development. Thus, PAN1's function may highlight the unique regulation of stomatal patterning in grasses. Maize PAN2 is required for proper subcellular localization of PAN1 (Cartwright et al., 2009). The molecular identity of 


\section{A}

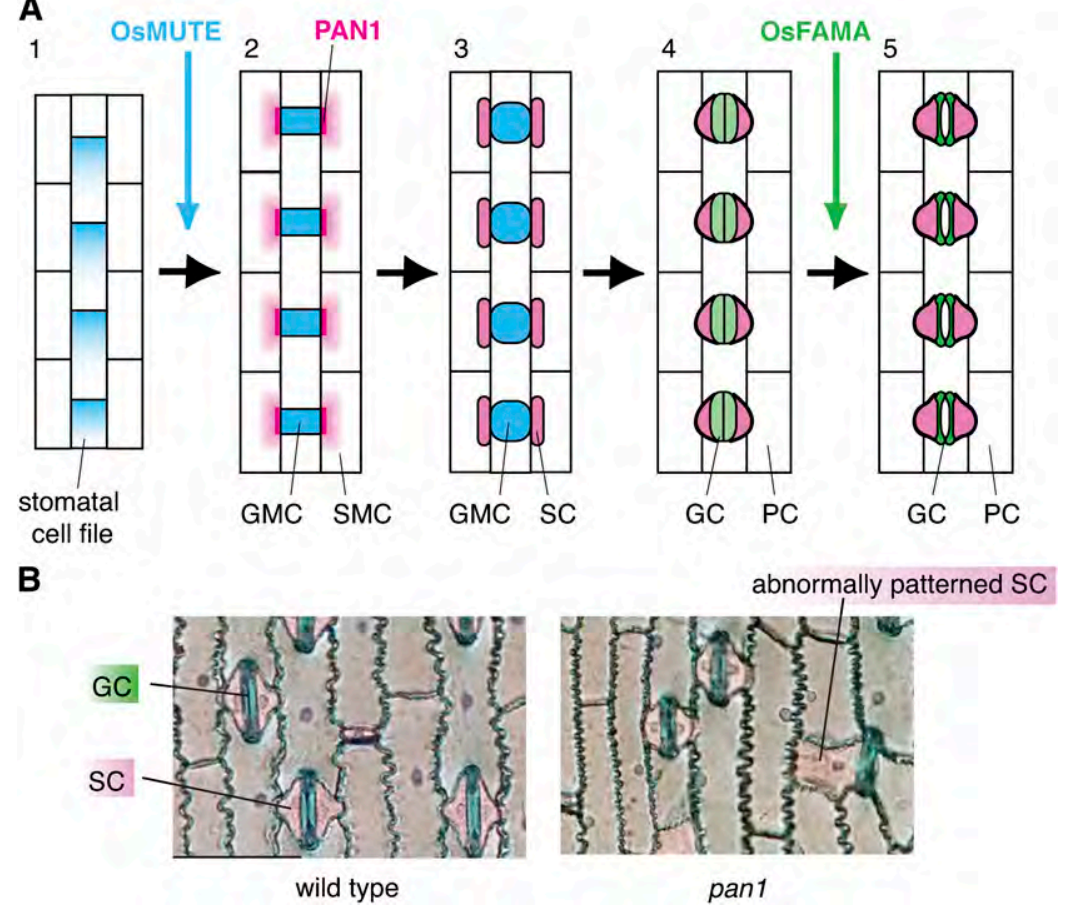

Figure 4. Stomatal Development in Grasses.

(A) Schematic diagram of stomatal development in grasses. (1) During early epidermal development in grasses, stomatal and nonstomatal cell files are specified, and cell division polarity is established in the stomatal cell file. This polarity will ensure that the one-cell spacing rule is maintained. (2) Os MUTE controls a single asymmetric division toward the leaf apex in the stomatal cell file, which creates GMCs (blue). Neighboring cell files (SMC; pink gradient) receive a signal via putative receptor PAN1 (magenta), which localizes at the area of GMC contact and polarize in preparation for division. (3) SMCs divide asymmetrically toward PAN1 to form SCs (pink), which will act as ion reservoirs for the operation of mature stomata. (4) GMCs divide once symmetrically to form GCs (light green). (5) Finally, GC and SC terminally differentiate, forming mature dumbbell-shaped stomata (dark green). Os FAMA is required for the differentiation of GCs, though not their symmetric division.

(B) Leaf epidermal peel from the wild type (left) and pan1 mutant maize (right). GC and SC are stained in blue and pink, respectively. Unlike the wild type, the pan 1 mutant occasionally fails to produce proper asymmetric divisions that give rise to SC, resulting in abnormal SC patterning. Images kindly provided by Laurie Smith (University of California, San Diego).

PAN2 is not known, so we cannot speculate yet on whether this protein and its function may be unique to grasses.

\section{Diversity of Stomata and Stomatal Development}

In extant land plant species, stomata appear on the sporophyte shoot epidermis of nearly every lineage. For most plants, stomata are evenly distributed in the shoot areas where they appear, and they adhere closely to the one-cell spacing rule (Sachs, 1991), allowing them to open and close effectively. Therefore, the diversity of stomata consists of variations on a theme: stomata are spaced at least one cell apart, but the specifics differ. Classic anatomical studies suggest that differences in stomatal patterning reflect the types, positions, and numbers of cell divisions that give rise to the stomatal complex (Esau, 1977; Payne, 1979). In this section, we describe stomatal development in other plant taxa and speculate regarding their possible alterations of regulatory mechanisms known in Arabidopsis.

In mosses and lycophytes (Figures $1 \mathrm{~A}$ and 1B), stomata develop through a simple process of a single asymmetric division followed by differentiation of a GMC, then GCs (Payne, 1979). We could speculate that the ancient function of SPCH-MUTE-FAMA family proteins may be to initiate the stomatal cell lineage and differentiate into GMC directly. In ferns, MMC in a cell file undergo one, or sometimes two, asymmetric division toward the leaf apex, then differentiate into GMC and GC (Apostolakos et al., 1997) (Figure 5A). This implies cell polarity mechanisms different from Arabidopsis.

In basal angiosperms, such as Victoria (Figure 1D), stomata appear to arise without amplifying divisions. (For terminology and evolution of stomatal complexes among basal angiosperms, see Carpenter [2005].) By contrast, the magnoliid Houttuynia shows a clear and extended inward spiral of cells around each stoma, which we hypothesize arise through an extended process of asymmetric division resulting from delayed MUTE activity (Figure 5B).

Gardenia and Begonia (Figures $1 \mathrm{G}$ and $1 \mathrm{H}$ ) show some of the diversity found within eudicots, a category that also includes Arabidopsis (Figure 2). In Gardenia, it appears that most, if not all, protodermal cells enter the stomatal lineage, implying that the unknown mechanism by which MMCs arise operates at higher 
A

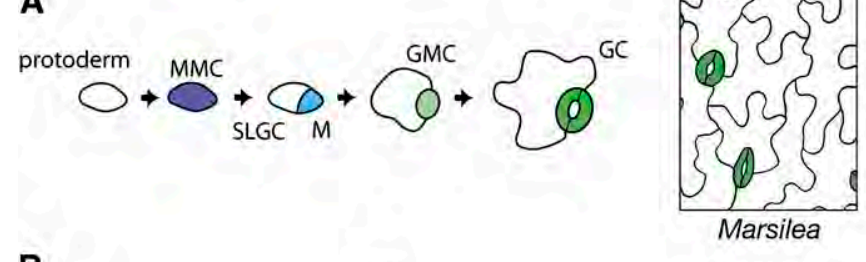

B
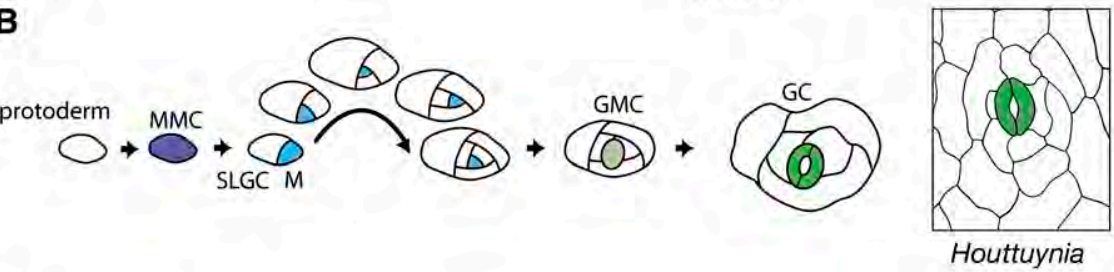

C
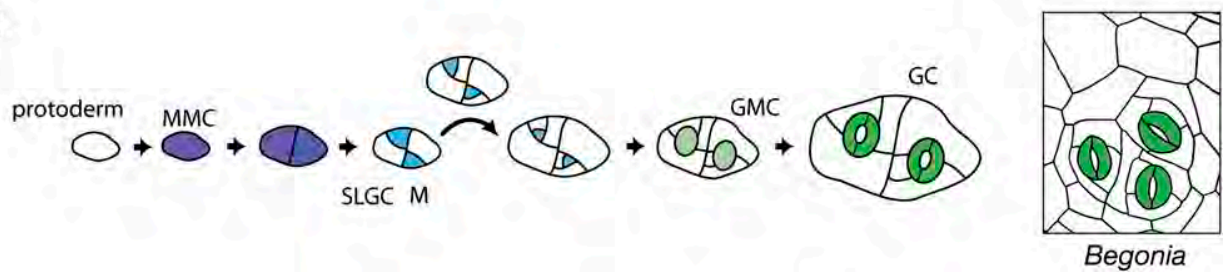

Figure 5. Developmental Hypotheses for Stomatal Complex Diversity.

(A) Stomata in the fern Marsilea appear to develop through a process lacking amplifying divisions of a meristemoid (cf. Arabidopsis in Figure 2A).

(B) Houttuynia (magnoliid) stomata are surrounded by a spiral arrangement of cells that suggests a large number of amplifying divisions.

(C) In Begonia, a eudicot, stomata arise in groups that can be explained by early division of a stomatal precursor, such as an MMC, and retention of MMC identity by the daughter cells.

density than in Arabidopsis. In Begonia, small groups of stomata are separated by pavement cells. Figure $5 \mathrm{C}$ shows a hypothetical developmental process giving rise to such a stomatal complex: Instead of dividing asymmetrically, the MMC divides symmetrically one or more times and all daughter cells retain MMC identity. Asymmetric division then proceeds as in Arabidopsis, allowing the one-cell spacing rule to be maintained, and the mature Begonia epidermis has groups of up to four stomata (Figure 5C).

\section{Phylogenetic Perspective on Stomata Development}

The recent availability of diverse land plant genomes, such as the moss Physcomitrella patens and model crop monocot plants rice and maize, allows for examination of the origin and conservation of genes important in stomatal patterning and spacing. Since stomata evolved only once in the ancestor of the mosses (Raven, 2002), it is expected that plants that arose from different basal land plant lineages, such as $P$. patens, would also possess some if not all of the genes found to be essential for stomatal patterning and differentiation in Arabidopsis. Stomata in moss form through a single asymmetric cell division to produce a GMC, which will divide partially or completely to produce a single GC or a pair of GCs surrounding a pore (Payne, 1979). Congruent with this relatively simplified developmental sequence to mature GC formation, the moss $P$. patens does not have genes encoding SPCH or MUTE. $P$. patens, however, has two FAMA-like genes (Figure 6A; see Supplemental Data Set 1 online), which likely function in moss as they do in Arabidopsis, rice, and maize: to control the final differentiation step to GCs. Based on our SPCHMUTE-FAMA gene tree and taxonomic sample, it is not clear whether there was only a single stomatal master regulatory bHLH gene in the ancestor of land plants or if SPCH and MUTE have been subsequently lost in $P$. patens. It is fascinating, however, to speculate that a single stomatal bHLH protein in early land plants may have had a function to create stomatal-lineage cells and immediately trigger GC differentiation. Later, as this gene duplicated, its roles may have subfunctionalized and even acquired new functions to mediate amplifying and spacing asymmetric cell divisions. The evolution of the leaf blade (Figure 1) in vascular plants perhaps benefited from additional mechanisms to space stomata correctly during leaf laminar expansion, such as amplifying divisions of meristemoids and recruiting cell-cell signaling components to impinge directly on the stomatal differentiation process. In this regard, it is interesting that At SPCH possesses a specific MAP kinase target domain not found in At FAMA (Lampard et al., 2008).

Land plants also share common signaling components for the spacing of stomata. TMM, a member of the LRR-RLP family, functions as part of a signaling complex that regulates the spacing of stomata in Arabidopsis (Nadeau and Sack, 2002). TMM is present in a single copy in poplar, grass, and moss genomes, which may reflect its integral role in enforcing correct stomatal spacing (Figure 6B; see Supplemental Data Set 2 online). In Arabidopsis, TMM is proposed to associate with ER family receptors, which are Ser/Thr kinases (Shpak et al., 2005). TMM is specific to the stomatal lineage, whereas ER family members play 
A

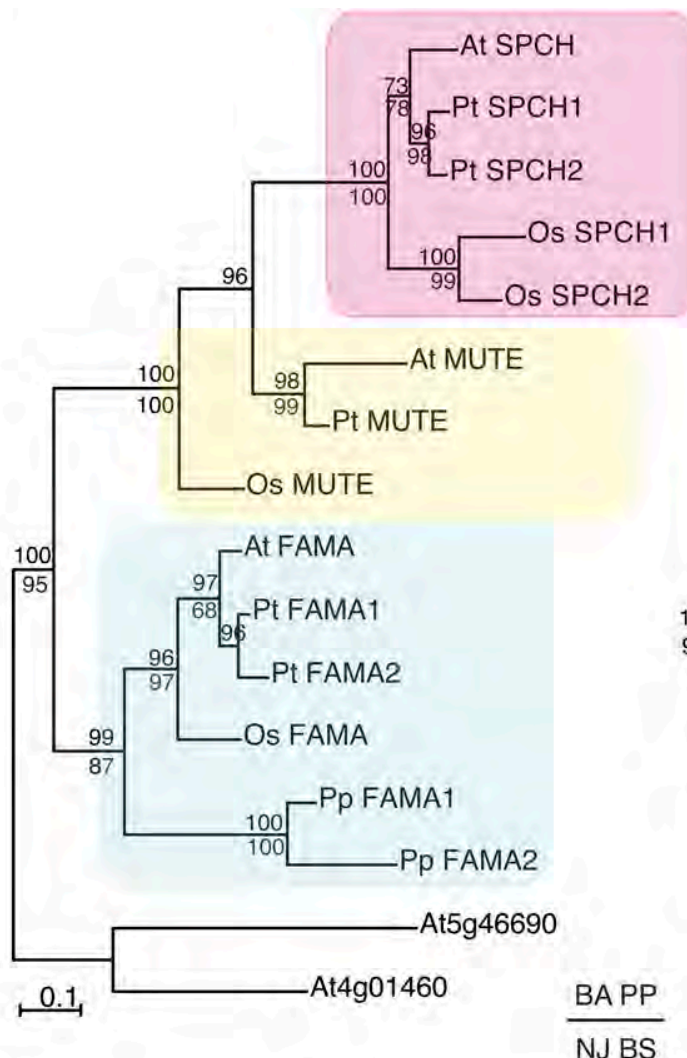

B
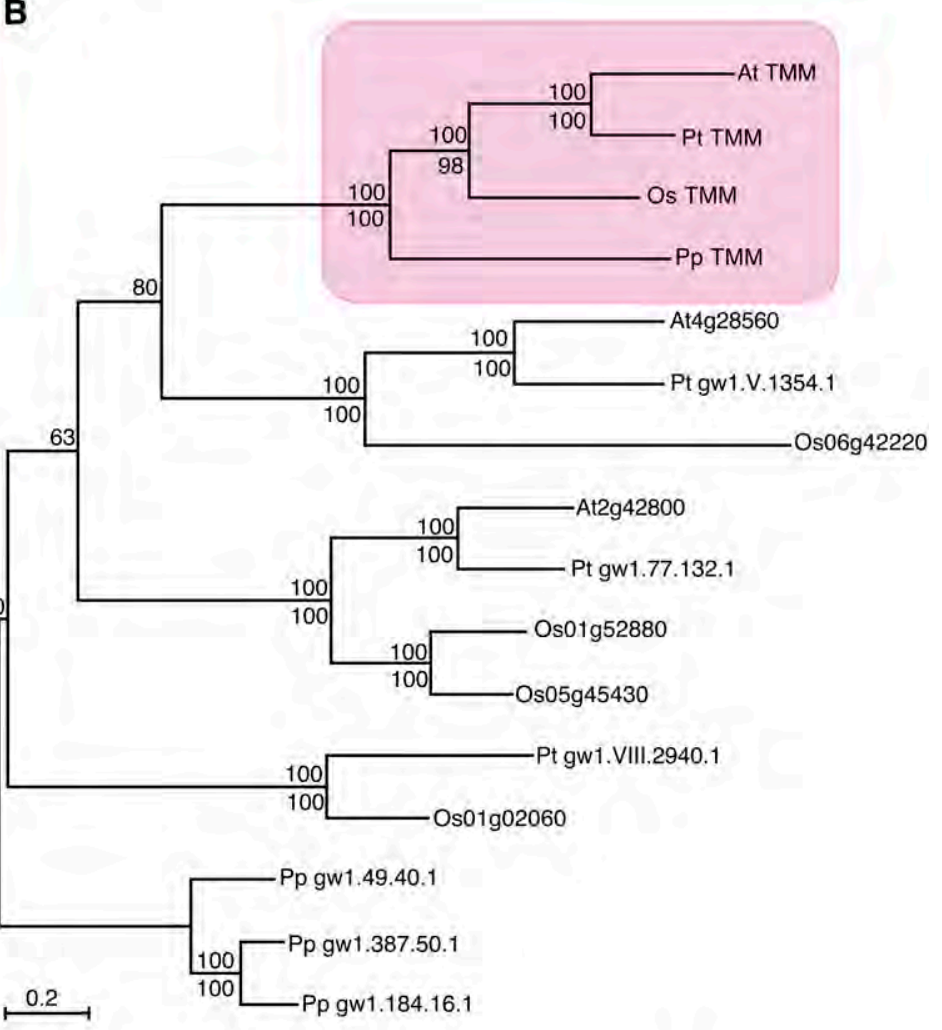
C At_YDA $P t^{-}$YDA 1 PE YDA2 $P t^{-}$YDA3 OS YDAI OS YDA OS YDA consensus

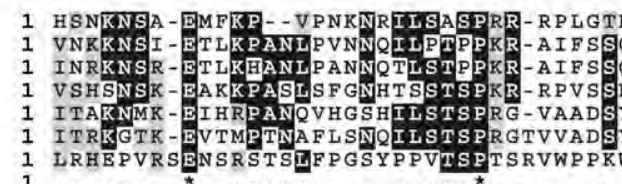
GTHVKNLQIPQRDLVL CSAPDSLLSSPSRSPMRS F I PDQVSNH GLL I 1 INRKNSR-ETLKANLPANNQTLSTPRKR-AIFSSQVQNLQIPHR-VAFFSAPDSSMSSPSRSPMRAFGTEQVINNGFW

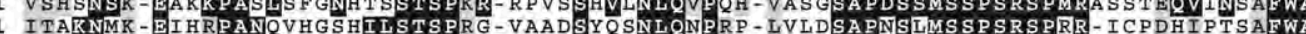
VIMRT

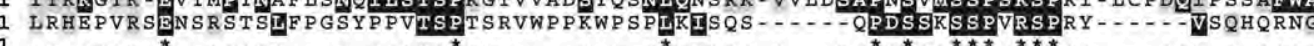

At YDA PE-YDA1 PE YDA2 Pt YDA3 OS YDA 1 OS YDA2

PP YDA

consensus

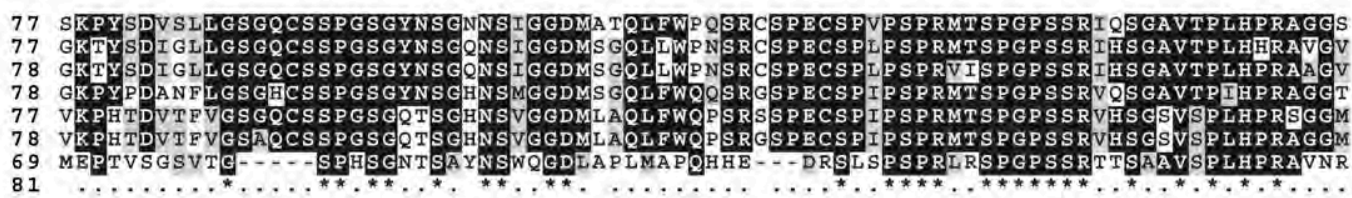

At_YDA Pt YDA1 Pt YDA2 $P t^{-}$YDA 3 Os YDA1 OS_YDA PP_YDA coñsensus

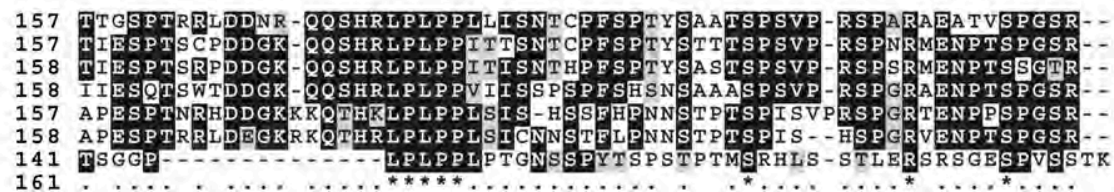

Figure 6. Stomatal Patterning and Spacing Genes Are Conserved among Embryophytes.

(A) A gene tree of master regulatory bHLH transcription factors (SPCH, MUTE, and FAMA) in stomatal development. Amino acid sequences from bHLH and ACT domains were aligned and the tree constructed using Bayesian and neighbor-joining methods. The Bayesian phylogram is shown. The pink, yellow, and cyan rectangles highlight the SPCH, MUTE, and FAMA clades, respectively. Posterior probability values are indicated above the nodes, and bootstrap values over 50 (100,000 replicates) are indicated below the nodes.

(B) A gene tree of TMM, which encodes an LRR receptor-like protein necessary for proper stomatal spacing in Arabidopsis. The entire amino acid sequence was aligned and the tree constructed as in (A). The Bayesian phylogram is shown. The pink rectangle highlights the single copy TMM clade. Posterior probability values are indicated above the nodes, and bootstrap values over 50 (100,000 replicates) are indicated below the nodes.

(C) Amino acid alignment of the N-terminal portion of YODA (YDA), a MAPKKK, which is essential to regulate the activity of the protein in Arabidopsis. YDA is also required for appropriate stomatal spacing. At, Arabidopsis thaliana; Pt, Populus trichocarpa; Os, Oryza sativa; Pp, Physcomitrella patens. See Supplemental Methods and Supplemental Table 1 online for detailed bioinformatic and phylogenetic methods and gene ID numbers. 
additional roles in various developmental processes in Arabidopsis (Shpak et al., 2004; Pillitteri et al., 2007a; Hord et al., 2008; van Zanten et al., 2009, 2010). Thus, TMM may be necessary for recognition of ligands or receptor complex activation specific to the stomatal lineage in all land plants.

YODA is a MAPKKK thought to act downstream of TMM and is present in multiple copies in rice and poplar and in single copy in Arabidopsis and $P$. patens (Figure 6C). Although MAPKKKs comprise a large gene family, YODA can be identified by its unique $\mathrm{N}$-terminal Ser-rich regulatory region (see Supplemental Data Set 3 online). Closely related MKKKs in Arabidopsis appear to be lacking this region (see Supplemental Data Set 3 online), and if it is deleted in YODA, the protein becomes constitutively active, resulting in a stomata-less phenotype (Lukowitz et al., 2004). Given the high level of sequence conservation in the region of this protein in $P$. patens relative to Arabidopsis, we predict that the $\mathrm{N}$-terminal region regulates its YODA activity as well. Like ER family RLKs, YODA regulates multiple developmental processes in Arabidopsis in addition to stomatal patterning, such as embryo polarity/patterning, inflorescence elongation, and flower development (Lukowitz et al., 2004). Therefore, it would be interesting to see whether YODA might also be an essential component in the regulation of stomatal spacing in moss.

Although close anatomical descriptions of stomatal development exist for many taxa, little is known of the molecular and evolutionary basis of stomatal formation across land plants. Valuable insights into ancestral and conserved mechanisms of stomatal development will no doubt be gained from further studies on basal land plants. In the moss sporophyte, stomatal development is relatively more similar to that of grasses than that of eudicots, in that one asymmetric division gives rise to a GMC directly, which then divides symmetrically once to form paired GCs (Ziegler, 1987). However, unlike grasses, there are no subsidiary cells formed from outside the stomatal lineage. To reach a deeper understanding of novel mechanisms responsible for generating stomatal diversity (Figure 1), comparative studies of closely related, yet morphologically divergent, stomatal phenotypes are needed.

\section{Future Perspectives}

With recent advances in understanding the molecular processes controlling stomatal development in the model eudicot Arabidopsis, alongside the completion of several genome projects in monocot crop plants and in basal land plants, we are beginning to find that many of the molecular mechanisms that control stomatal development in Arabidopsis are part of an ancient machinery. As shown by PAN1 in maize and BASL in Arabidopsis, some of the unique aspects of stomatal development relate to the type and number of asymmetric cell divisions associated with the formation of the stomatal complex. Further identification of orthologs of stomatal regulator genes in phylogenetically and evolutionarily important species and analysis of their developmental functions may unravel the molecular basis of the evolution of stomatal development.

Several exciting questions remain: For instance, how is the stomatal lineage first established? What initiates $\mathrm{SPCH}$ expression, and what are the cellular dynamics that set up the entry into asymmetric cell divisions of the stomatal lineage? When did amplifying cell divisions come to play a key role in stomatal development, and is that innovation correlated with specific features of MUTE?

A second line of questions revolves around the intersection of environmental signaling and stomatal development. Some recent work is beginning to explain how light signals integrate with the stomatal development program (Boccalandro et al., 2009; Casson et al., 2009; Kang et al., 2009), and other work is examining how $\mathrm{CO}_{2}$ regulates stomatal density (Gray et al., 2000). In addition, while a direct role between temperature and stomatal patterning has not been determined, SCRM, which is also known as ICE1, is an important transcriptional activator of cold response genes (Chinnusamy et al., 2003). During the evolution of land plants, when and how did such network connections of environmental signaling pathways to stomatal development emerge? Clearly, we now have the moleculargenetic tools in hand to explore the evolution of this landmark developmental innovation.

\section{Supplemental Data}

The following materials are available in the online version of this article.

Supplemental Table 1. Gene Names and IDs Used for the Phylogenetic Analysis.

Supplemental Data Set 1. Text File of Amino Acid Alignment of the bHLH Domain and the C-Terminal ACT-Like Domain of the SPCH, MUTE, and FAMA Orthologs Used for the Phylogenetic Analysis Presented in Figure 6A.

Supplemental Data Set 2. Text File of Amino Acid Alignment of the Full-Length At TMM and Its Orthologs Used for the Phylogenetic Analysis Presented in Figure 6B.

Supplemental Data Set 3. Text File of Amino Acid Alignment of the N-Terminal End of YODA and Related Genes Presented in Figure 6C.

Supplemental Methods.

Supplemental References.

\section{ACKNOWLEDGMENTS}

We thank Laurie Smith (University of California, San Diego) for the images of maize wild-type and pan1 epidermis (Figure 4B) and Lynn Pillitteri for the scanning electron microscopy image of $P$. patens stomata (Figure 1A). Our research is supported by grants from the National Science Foundation (IOB-0744892 and MCB-0855659), by the U.S. Department of Energy (DE-FG02-03ER15448), and by PREST, Japan Science and Technology Agency, to K.U.T. K.M.P. is a National Science Foundation Graduate Research Fellow.

Received November 13, 2009; revised January 22, 2010; accepted February 1, 2010; published February 23, 2010.

\section{REFERENCES}

Abrash, E.B., and Bergmann, D.C. (2010). Regional specification of stomatal production by the putative ligand CHALLAH. Development 137: 447-455. 
Apostolakos, P., Panteris, E., and Galatis, B. (1997). Microtubule and actin filament organization during stomatal morphogenesis in the fern Asplenium nidus. 1. Guard cell mother cell. Protoplasma 198: 93-106.

Berger, D., and Altmann, T. (2000). A subtilisin-like serine protease involved in the regulation of stomatal density and distribution in Arabidopsis thaliana. Genes Dev. 14: 1119-1131.

Bergmann, D.C., Lukowitz, W., and Somerville, C.R. (2004). Stomatal development and pattern controlled by a MAPKK kinase. Science 304: 1494-1497.

Bhave, N.S., Veley, K.M., Nadeau, J.A., Lucas, J.R., Bhave, S.L., and Sack, F.D. (2009). TOO MANY MOUTHS promotes cell fate progression in stomatal development of Arabidopsis stems. Planta 229: 357-367.

Boccalandro, H.E., Rugnone, M.L., Moreno, J.E., Ploschuk, E.L., Serna, L., Yanovsky, M.J., and Casal, J.J. (2009). Phytochrome B enhances photosynthesis at the expense of water-use efficiency in Arabidopsis. Plant Physiol. 150: 1083-1092.

Carpenter, K.J. (2005). Stomatal architecture and evolution in basal angiosperms. Am. J. Bot. 92: 1595-1615.

Cartwright, H.N., Humphries, J.A., and Smith, L.G. (2009). PAN1: A receptor-like protein that promotes polarization of an asymmetric cell division in maize. Science 323: 649-651.

Casson, S.A., Franklin, K.A., Gray, J.E., Grierson, C.S., Whitelam, G.C., and Hetherington, A.M. (2009). Phytochrome B and PIF4 regulate stomatal development in response to light quantity. Curr. Biol. 19: 229-234.

Chevalier, D., Batoux, M., Fulton, L., Pfister, K., Yadav, R.K., Schellenberg, M., and Schneitz, K. (2005). STRUBBELIG defines a receptor kinase-mediated signaling pathway regulating organ development in Arabidopsis. Proc. Natl. Acad. Sci. USA 102: 9074-9079.

Chinnusamy, V., Ohta, M., Kanrar, S., Lee, B.H., Hong, X., Agarwal, M., and Zhu, J.K. (2003). ICE1: A regulator of cold-induced transcriptome and freezing tolerance in Arabidopsis. Genes Dev. 17: 1043-1054.

Dong, J., MacAlister, C.A., and Bergmann, D.C. (2009). BASL controls asymmetric cell division in Arabidopsis. Cell 137: 1320-1330.

Edwards, D., Kerp, H., and Haas, H. (1998). Stomata in early land plants: An anatomical and ecophysiological approach. J. Exp. Bot. 49: 255-278.

Esau, K. (1977). Stomata. In Anatomy of Seed Plants eds (New York: John Wiley \& Sons), pp. 88-99.

Freeman, S. (2008). Green plants. In Biological Sciences, 3rd ed (San Francisco, CA: Pearson Education), pp. 626-663.

Gallagher, K., and Smith, L.G. (2000). Roles for polarity and nuclear determinants in specifying daughter cell fates after an asymmetric cell division in the maize leaf. Curr. Biol. 10: 1229-1232.

Gray, J.E., Holroyd, G.H., van der Lee, F.M., Bahrami, A.R., Sijmons, P.C., Woodward, F.I., Schuch, W., and Hetherington, A.M. (2000). The HIC signalling pathway links $\mathrm{CO} 2$ perception to stomatal development. Nature 408: 713-716.

Hara, K., Kajita, R., Torii, K.U., Bergmann, D.C., and Kakimoto, T. (2007). The secretory peptide gene EPF1 enforces the stomatal onecell-spacing rule. Genes Dev. 21: 1720-1725.

Hara, K., Yokoo, T., Kajita, R., Onishi, T., Yahata, S., Peterson, K.M., Torii, K.U., and Kakimoto, T. (2009). Epidermal cell density is autoregulated via a secretory peptide, EPIDERMAL PATTERNING FACTOR2 in Arabidopsis leaves. Plant Cell Physiol. 50: 1019-1031.

Hord, C.L.H., Sun, Y.J., Pillitteri, L.J., Torii, K.U., Wang, H., Zhang, S., and Ma, H. (2008). Regulation of Arabidopsis early anther development by the mitogen-activated protein kinases, MPK3 and MPK6, and the ERECTA and related receptor-like kinases. Mol. Plant 1: 645-658.

Hunt, L., and Gray, J.E. (2009). The signaling peptide EPF2 controls asymmetric cell divisions during stomatal development. Curr. Biol. 19: 864-869.
Kanaoka, M.M., Pillitteri, L.J., Fujii, H., Yoshida, Y., Bogenschutz, N.L., Takabayashi, J., Zhu, J.K., and Torii, K.U. (2008). SCREAM/ ICE1 and SCREAM2 specify three cell-state transitional steps leading to Arabidopsis stomatal differentiation. Plant Cell 20: 1775-1785.

Kang, C.Y., Lian, H.L., Wang, F.F., Huang, J.R., and Yagn, H.Q. (2009). Cryptochromes, phytochromes, and COP1 regulate light-controlled stomatal development in Arabidopsis. Plant Cell 21: 2624-2641.

Kondo, T., Kajita, R., Miyazaki, A., Hokoyama, M., Nakamura-Miura, T., Mizuno, S., Masuda, Y., Irie, K., Tanaka, Y., Takada, S., Kakimoto, T., and Sakagami, Y. (2010). Stomatal density is controlled by a mesophyll-derived signaling molecule. Plant Cell Physiol. 51: 1-8.

Kutter, C., Schob, H., Stadler, M., Meins, F., Jr., and Si-Ammour, A. (2007). MicroRNA-mediated regulation of stomatal development in Arabidopsis. Plant Cell 19: 2417-2429.

Lai, L.B., Nadeau, J.A., Lucas, J., Lee, E.K., Nakagawa, T., Zhao, L., Geisler, M., and Sack, F.D. (2005). The Arabidopsis R2R3 MYB proteins FOUR LIPS and MYB88 restrict divisions late in the stomatal cell lineage. Plant Cell 17: 2754-2767.

Lampard, G.R., Macalister, C.A., and Bergmann, D.C. (2008). Arabidopsis stomatal initiation is controlled by MAPK-mediated regulation of the bHLH SPEECHLESS. Science 322: 1113-1116.

Liu, T., Ohashi-Ito, K., and Bergmann, D.C. (2009). Orthologs of Arabidopsis thaliana stomatal bHLH genes and regulation of stomatal development in grasses. Development 136: 2265-2276.

Lukowitz, W., Roeder, A., Parmenter, D., and Somerville, C. (2004). A MAPKK kinase gene regulates extra-embryonic cell fate in Arabidopsis. Cell 116: 109-119.

MacAlister, C.A., Ohashi-Ito, K., and Bergmann, D.C. (2007). Transcription factor control of asymmetric cell divisions that establish the stomatal lineage. Nature 445: 537-540.

Massari, M.E., and Murre, C. (2000). Helix-loop-helix proteins: Regulators of transcription in eucaryotic organisms. Mol. Cell. Biol. 20: 429-440.

Nadeau, J.A., and Sack, F.D. (2002). Control of stomatal distribution on the Arabidopsis leaf surface. Science 296: 1697-1700.

Ohashi-Ito, K., and Bergmann, D.C. (2006). Arabidopsis FAMA controls the final proliferation/differentiation switch during stomatal development. Plant Cell 18: 2493-2505.

Payne, W.W. (1979). Stomatal patterns in embryophytes - Their evolution, ontogeny and interpretation. Taxon 28: 117-132.

Pillitteri, L.J., Bemis, S.M., Shpak, E.D., and Torii, K.U. (2007a). Haploinsufficiency after successive loss of signaling reveals a role for ERECTAfamily genes in ovule development. Development 134: 3099-3109.

Pillitteri, L.J., Bogenschutz, N.L., and Torii, K.U. (2008). The bHLH protein, MUTE, controls differentiation of stomata and the hydathode pore in Arabidopsis. Plant Cell Physiol. 49: 934-943.

Pillitteri, L.J., Sloan, D.B., Bogenschutz, N.L., and Torii, K.U. (2007b). Termination of asymmetric cell division and differentiation of stomata. Nature 445: 501-505.

Pillitteri, L.J., and Torii, K.U. (2007). Breaking the silence: Three bHLH proteins direct cell-fate decisions during stomatal development. Bioessays 29: 861-870.

Raschke, K. (1979). Movements of stomata. In Physiology of Movements, Encyclopedia of Plant Physiology, Vol. 7, W. Haupt and M.E. Feinleib, eds (Berlin: Springer-Verlag), pp. 383-441.

Raven, J. (2002). Selection pressures on stomatal evolution. New Phytol. 153: 371-386.

Sachs, T. (1991). Stomata as an example of meristmoid development. In Pattern Formation in Plant Tissues (Cambridge, UK: Cambridge University Press), pp. 101-117.

Serna, L., Torres-Contreras, J., and Fenoll, C. (2002). Clonal analysis of stomatal development and patterning in Arabidopsis leaves. Dev. Biol. 241: 24-33.

Shpak, E.D., Berthiaume, C.T., Hill, E.J., and Torii, K.U. (2004). 
Synergistic interaction of three ERECTA-family receptor-like kinases controls Arabidopsis organ growth and flower development by promoting cell proliferation. Development 131: 1491-1501.

Shpak, E.D., McAbee, J.M., Pillitteri, L.J., and Torii, K.U. (2005). Stomatal patterning and differentiation by synergistic interactions of receptor kinases. Science 309: 290-293.

Sugano, S.S., Shimada, T., Imai, Y., Okawa, K., Tamai, A., Mori, M., and Hara-Nishimura, I. (2010). Stomagen positively regulates stomatal density in Arabidopsis. Nature 463: 241-244.

Taylor, T.N., Kerp, H., and Hass, H. (2005). Life history biology of early land plants: Deciphering the gametophyte phase. Proc. Natl. Acad. Sci. USA 102: 5892-5897.

van Zanten, M., Basten Snoek, L., van Eck-Stouten, E., Proveniers, M.C., Torii, K.U., Voesenek, L.A., Peeters, A.J., and Millenaar, F.F. (2010). Ethylene-induced hyponastic growth in Arabidopsis thaliana is controlled by ERECTA. Plant J. 61: 83-95. van Zanten, M., Snoek, L.B., Proveniers, M.C., and Peeters, A.J. (2009). The many functions of ERECTA. Trends Plant Sci. 14: 214-218.

von Groll, U., Berger, D., and Altmann, T. (2002). The subtilisin-like serine protease SDD1 mediates cell-to-cell signaling during Arabidopsis stomatal development. Plant Cell 14: 1527-1539.

Wang, H., Ngwenyama, N., Liu, Y., Walker, J., and Zhang, S. (2007). Stomatal development and patterning are regulated by environmentally responsive mitogen-activated protein kinases in Arabidopsis. Plant Cell 19: $63-73$.

Yang, M., and Sack, F.D. (1995). The too many mouths and four lips mutations affect stomatal production in Arabidopsis. Plant Cell 7: 2227-2239.

Ziegler, H. (1987). The evolution of stomata. In Stomatal Function, E. Zeiger, G.D. Farquhar, and I.R. Cowan, eds (Stanford, CA: Stanford University Press), pp. 29-57. 


\title{
Arabidopsis ERECTA-Family Receptor Kinases Mediate Morphological Alterations Stimulated by Activation of NB-LRR-Type UNI Proteins
}

\author{
Naoyuki Uchida ${ }^{1}$, Kadunari Igari ${ }^{1}$, Naomi L. Bogenschutz ${ }^{2}$, Keiko U. Torii ${ }^{2}$ and Masao Tasaka ${ }^{1, *}$ \\ ${ }^{1}$ Graduate School of Biological Sciences, Nara Institute of Science and Technology, 8916-5 Takayama, Ikoma, 630-0192 Japan \\ ${ }^{2}$ Department of Biology, University of Washington, Seattle, WA 98195, USA \\ *Corresponding author: E-mail, m-tasaka@bs.naist.jp; Fax, +81-743-72-5489 \\ (Received December 15, 2010; Accepted March 14, 2011)
}

Shoot apical meristems (SAMs), which maintain stem cells at the tips of stems, and axillary meristems (AMs), which arise at leaf axils for branch formation, play significant roles in the establishment of plant architecture. Previously, we showed that, in Arabidopsis thaliana, activation of NB-LRR (nucleotide-binding site-leucine-rich repeat)-type UNI proteins affects plant morphology through modulation of the regulation of meristems. However, information about genes involved in the processes was still lacking. Here, we report that ERECTA (ER) receptor kinase family members cooperatively mediate the morphological alterations that are stimulated by activation of UNI proteins. uni-1D is a gain-offunction mutation in the UNI gene and uni-1D mutants exhibit early termination of inflorescence stem growth and also formation of extra AMs at leaf axils. The former defect involves modulation of the SAM activity and is suppressed by er mutation. Though the AM phenotype is not affected by a single er mutation, it is suppressed by simultaneous mutations of $E R$-family members. It was previously shown that trans-zeatin ( $\mathrm{tZ}$ )-type cytokinins were involved in the morphological phenotypes of uni-1D mutants and that expression of CYP735A2, which is essential for biosynthesis of tZ-type cytokinins, was modulated in uni-1D mutants. We show that this modulation of CYP735A2 expression requires activities of ER-family members. Moreover, the ER activity in UNI-expressing cells contributes to all morphological phenotypes of uni-1D mutants, suggesting that a cross-talk between ER-family-dependent and UNI-triggered signaling pathways plays a significant role in the morphological alterations observed in uni-1D mutants.

Keywords: Arabidopsis thaliana - Axillary meristem • ERECTA • NB-LRR • Shoot apical meristem • UNI.

Abbreviations: $A M$, axillary meristem; eQTL, expression quantitative trait locus; ER, ERECTA; GUS, $\beta$-glucuronidase; NB-LLL, nucleotide-binding site-leucine-rich repeat; QTL, quantitative trait locus; SA, salicylic acid; SAM, shoot apical meristem; $\mathrm{tZ}$, trans-zeatin.

\section{Introduction}

Shoot apical meristems (SAMs) and axillary meristems (AMs) play significant roles in the establishment of plant architecture. The SAMs, which are maintained at the tips of stems, are indeterminate structures and are the source of stem cells from which all post-embryonic aerial organs are derived (Brand et al. 2000, Schoof et al. 2000, Clark 2001, Lenhard and Laux 2003, Kondo et al. 2006, Muller et al. 2006, Tucker and Laux 2007, Miwa et al. 2008, Muller et al. 2008). On the other hand, AMs, which are required for branch formation, arise at leaf axils, which are boundary tissues between stems and leaves (Schmitz and Theres 2005, Aida and Tasaka 2006). Changes in activities of these above-ground meristems affect plant morphology.

The uni-1D mutant of Arabidopsis thaliana harbors a gain-of-function mutation in the UNI gene, which encodes a member of the CC-NB-LRR (coiled-coil nucleotide-binding site-leucine-rich repeat) family (Igari et al. 2008). The heterozygous uni-1D/+ plants show interesting morphological phenotypes, i.e. early termination of inflorescence stem growth after formation of several fruits and formation of extra AMs at leaf axils (Igari et al. 2008), while the homozygous uni-1D plants exhibit severe growth defects soon after germination and stop growing at a vegetative stage. Although it was shown that trans-zeatin (tZ)-type cytokinins were involved in morphological alterations of uni-1D/+ plants (Igari et al. 2008), further aspects of these processes are still not clear. Because NB-LRR-type proteins function as molecular sensors to trigger intracellular signaling pathways upon stimulation by ligands (Jones and Dangl 2006, Bent and Mackey 2007, Padmanabhan et al. 2009), the uni-1D mutation probably converts UNI proteins into an active state without an activation event, which triggers downstream signaling pathways (Igari et al. 2008); however, such a ligand for activation of UNI proteins has not yet been identified. Many NB-LRR-type proteins have been reported to function in plant immunity as sensors for pathogen-derived factors to activate defense responses (Jones and Dangl 2006, Bent and Mackey 2007, Padmanabhan et al. 2009) and uni-1D plants

Plant Cell Physiol. 52(5): 804-814 (2011) doi:10.1093/pcp/pcr032, available online at www.pcp.oxfordjournals.org

(C) The Author 2011. Published by Oxford University Press on behalf of Japanese Society of Plant Physiologists.

All rights reserved. For permissions, please email: journals.permissions@oup.com 
also show some aspects of pathogenesis-related responses such as the up-regulation of PATHOGENESIS-RELATED GENE 1 (PR1) and PR5 through the salicylic acid (SA) pathway (Igari et al. 2008). However, it has been shown that inactivation of the SA pathway did not affect the morphological phenotypes of uni-1D/+ mutants (Igari et al. 2008) and so far there has been no evidence that $U N I$ is involved in plant immunity.

The ERECTA (ER) family consists of ER, ER-LIKE 1 (ERL1) and $E R L 2$ (Shpak et al. 2004) and encodes leucine-rich repeat receptorlike kinases in a subfamily of transmembrane-type signaling receptors in plants (Torii et al. 1996, Shpak et al. 2004). The ER-family members are broadly expressed in various tissues (Yokoyama et al. 1998, Shpak et al. 2004) and play roles in diverse aspects of plant development by mediating cell-cell signals that sense and coordinate organ development (Torii et al. 1996, Shpak et al. 2004, Tisne et al. 2008). Though a loss-of-function mutation in the $E R$ gene affect inflorescence architecture but do not cause severe growth defects (Torii et al. 1996), simultaneous loss of activities of more than one ERfamily member confers extreme dwarfism (Shpak et al. 2004), abnormal flower development (Shpak et al. 2004, Pillitteri et al. 2007, Hord et al. 2008) and defects of stomatal patterning and differentiation (Shpak et al. 2005). Furthermore, ER has been identified as a major trait in various quantitative trait locus $(\mathrm{QTL}) /$ expression quantitative trait locus (eQTL) analyses of environmental stresses and developmental processes, suggesting that ER could be a modulator of signaling pathways in response to changes in external and/or internal conditions (Keurentjes et al. 2007, Tisne et al. 2008, Ghandilyan et al. 2009, El-Lithy et al. 2010, Terpstra et al. 2010, van Zanten et al. 2010a, van Zanten et al. 2010b).

In this study we report that ER-family members cooperatively mediate morphological alterations that are stimulated by activation of UNI proteins. Early termination of inflorescence stem growth observed in uni-1D/+ mutants is suppressed by er mutation. On the other hand, though the AM phenotype is not affected by single er mutations, it is suppressed by simultaneous mutations of more than one ER-family member. Cytokinin biosynthesis pathways and responses to cytokinins are modulated in uni-1D mutants, and these modulations require activities of ER-family members. Furthermore, ER activities in UNI-expressing cells contribute to all morphological phenotypes of uni-1D mutants, suggesting that a signaling cross-talk between ER-family-dependent and UNI-triggered signaling pathways plays a significant role in the morphological alterations observed in uni-1D mutants.

\section{Results}

\section{Early termination of inflorescence stem growth caused by the uni-1D mutation is restored in the Ler background.}

The Arabidopsis uni-1D mutant was originally isolated in the Wassilewskija (Ws) accession (Igari et al. 2008) and the original
uni-1D/+ heterozygous plants $[$ uni-1D/+ (Ws)] showed two morphological phenotypes: early termination of inflorescence stem growth after formation of several fruits, which resulted in short inflorescences, and formation of extra AMs at leaf axils, which contributed to a bushy shape (Igari et al. 2008). The combination of these two phenotypes resulted in the unique architecture of uni-1D/+ plants (Fig. 1A) (Igari et al. 2008). When the uni-1D allele was introgressed into the Columbia (Col) background [uni-1D/+ (Col)], no obvious difference in plant architecture was observed between uni-1D/+ (Ws) and uni-1D/+ (Col) (Fig. 1A, B). On the other hand, when the uni-1D allele was introgressed into the Landsberg erecta (Ler) background [uni-1D/+ (Ler)], the early cessation of stem growth was largely rescued (Fig. 1C).

When Arabidopsis wild-type plants were grown under normal conditions, no axillary bud was formed at the axils of cotyledons (Fig. 1D), while the formation of axillary buds was observed even at cotyledon axils in the original uni-1D/+ (Ws) plants (Fig. 1E) (Igari et al. 2008). Both uni-1D/+ (Col) and uni-1D/+ (Ler) plants also showed formation of axillary buds at axils of cotyledons (Fig. 1F, G).

Thus, among the morphological phenotypes of the original uni-1D/+ mutants in the Ws background, the early cessation of inflorescence growth is restored specifically in the Ler background.

\section{$E R$ is required for early termination of inflorescence stem growth in uni-1D/+ plants}

Because the Ler accession is known to harbor a loss-of-function mutation in the ER gene (Torii et al. 1996), which functions in various developmental aspects of plants (Torii et al. 1996, Shpak et al. 2003, Shpak et al. 2004, Shpak et al. 2005, Woodward et al. 2005, van Zanten et al. 2009), we examined whether the er mutation in Ler plants is responsible for the rescue of rapid termination of inflorescence growth in uni-1D/+ (Ler) plants. To this end, a functional $E R$ genomic fragment was introduced into uni-1D/+ (Ler) plants. The resulting uni-1D/+ $E R^{+}$(Ler) plants showed early cessation of stem growth (Fig. 2A) and the architecture of the uni-1D/+ER (Ler) plants was equivalent to that of the the original uni-1D/+ (Ws) plants (Figs. 1A, 2A). These observations strongly suggest that the presence of ER activities is associated with early termination of inflorescence stem growth in uni-1D/+ plants. To examine this possibility further, er loss-of-function mutants in Ws and Col backgrounds were crossed with uni-1D/+ (Ws) plants and uni-1D/+ (Col) plants, respectively. Inflorescence stems of er uni-1D/+ plants elongated well in both backgrounds (Fig. 2B, C) and, accordingly, inflorescence SAMs of er uni-1D/+ plants continuously produce flowers as in wild-type plants (Fig. 2D, F), while in uni-1D/+ plants only a few flowers were formed and then flower formation stopped (Fig. 2E) (Igari et al. 2008). When we examined expression patterns of WUS, a central player in stem cell maintenance in SAMs, WUS expression was detected in central domains of inflorescence SAMs of 

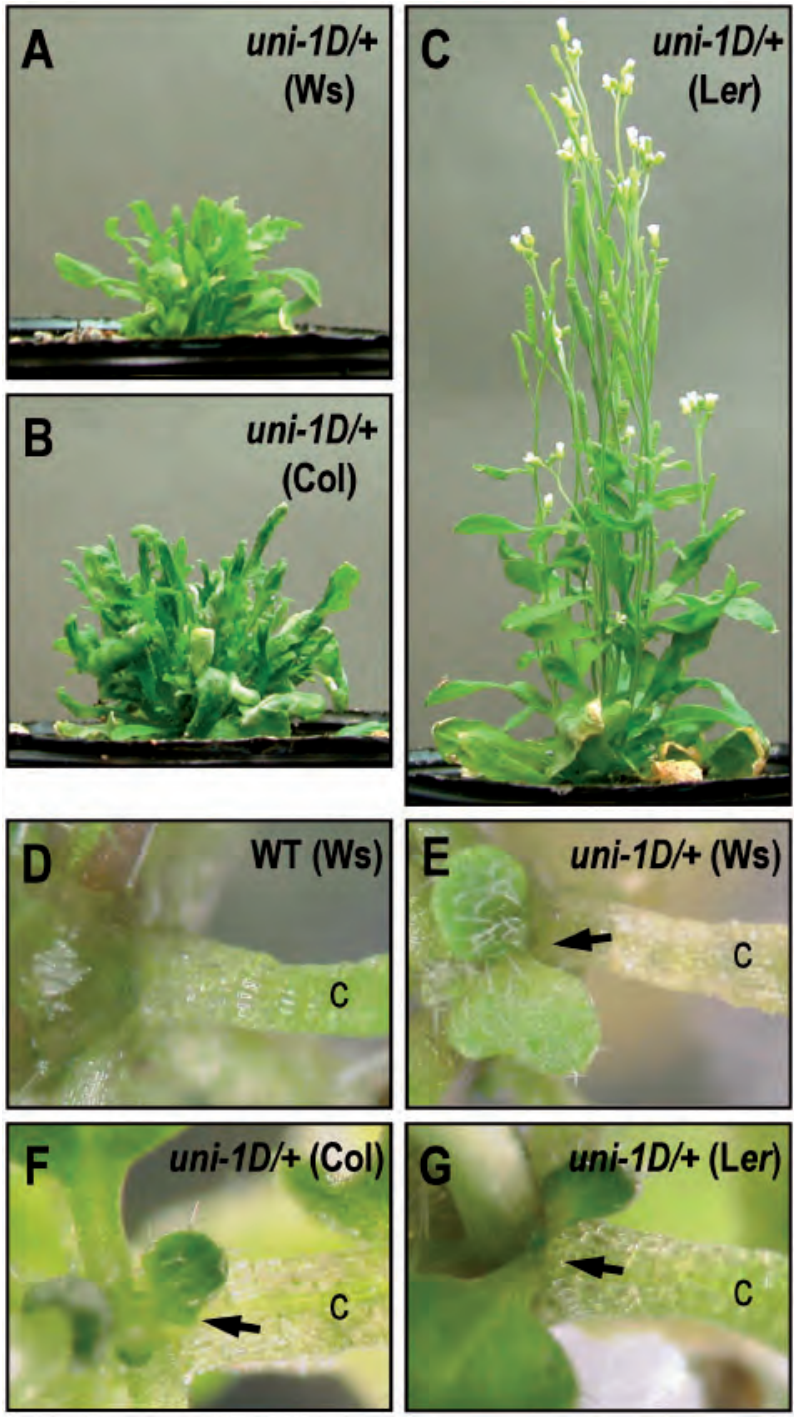

Fig. 1 Morphological characteristics of uni-1D/+ plants in various accession backgrounds. (A-C) Forty-five-day-old uni-1D/+ plants in Ws (A), Col (B) and Ler (C) backgrounds. (D-G) Observation of the axils of cotyledons (indicated by C) of 19-day-old wild-type (WT, Ws) (D), uni-1D/+ plants in Ws (E), Col (F) and Ler (G) backgrounds. C indicates cotyledons and arrows indicate formation of axillary buds.

wild-type and er uni-1D/+ plants by in situ RNA hybridization (Fig. 2G, I, arrowheads). In contrast, in uni-1D/+ mutants, inflorescence SAMs were apparently small and the expression level of WUS was below the detection limit (Fig. $2 \mathrm{H}$, arrowhead). WUS is also expressed in floral meristems during flower development (Fig. 2G, arrow) (Laux et al. 1996, Lenhard et al. 2001, Lohmann et al. 2001) and this expression in floral meristems was not affected in uni-1D/+ plants (Fig. $2 \mathrm{H}$, arrow). These observations were in accordance with the fact that uni-1D/+ plants were able to produce normal flowers once flower formation started (Fig. 2E) (Igari et al. 2008). Thus, ER is required for early termination of inflorescence stem growth in uni-1D/+ plants.
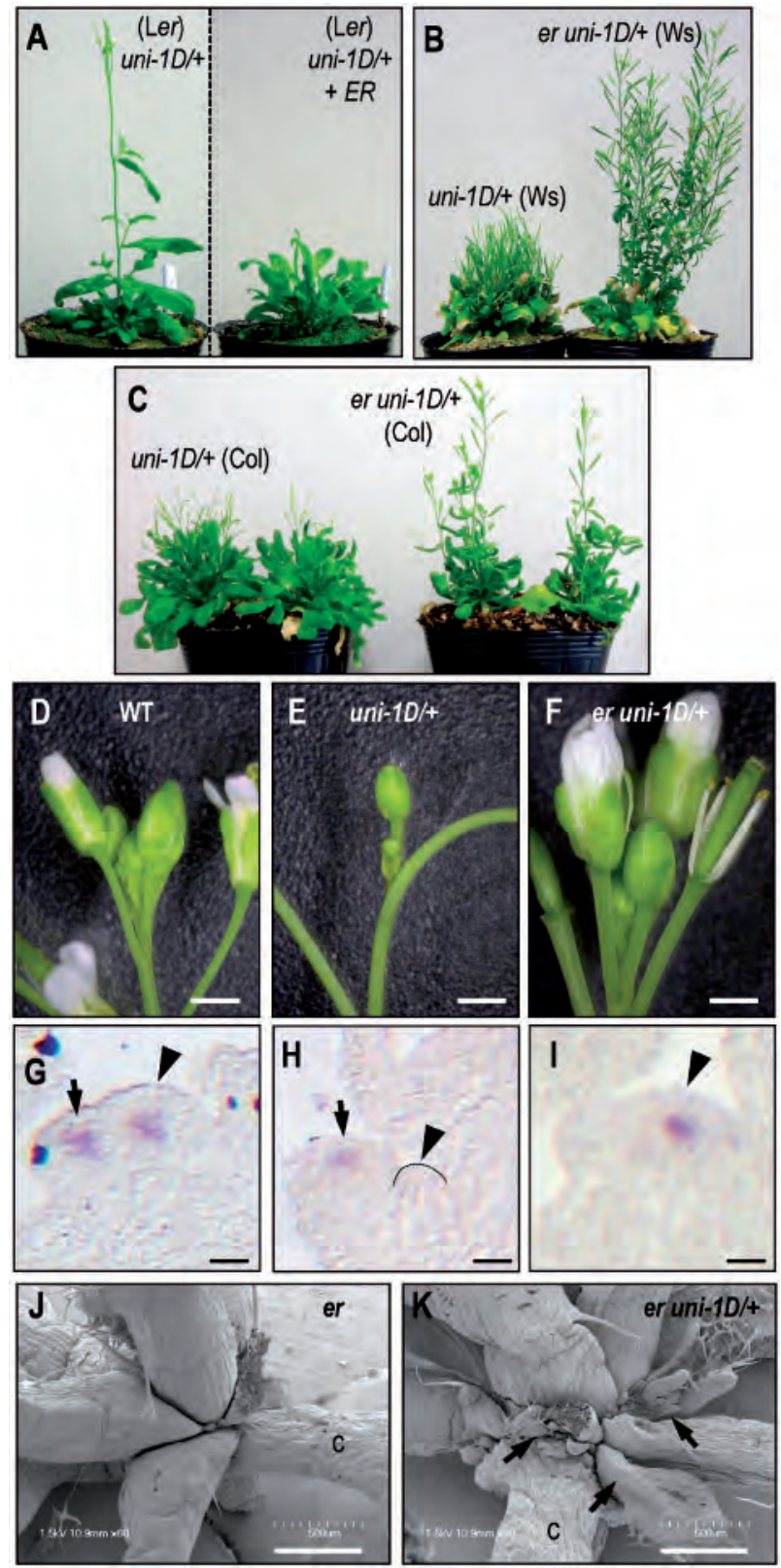

Fig. 2 ER is required for the early termination of stem growth in uni-1D/+ plants. (A) A 38-day-old uni-1D/+ (Ler) plant (left) and a uni-1D/+ plant harboring the $E R$ genomic transgene (right). (B) A 76-day-old uni-1D/+ (Ws) plant (left) and an er uni-1D/+ plant (right). (C) A 56-day-old uni-1D/+ (Col) plant (left) and an er uni-1D/+ plant (right). (D-F) Inflorescence tips of wild-type (WT) $(D)$, uni-1D/+ (E) and er uni-1D/+ (F) plants. Bars $=1 \mathrm{~mm}$. (G-I) Expression patterns of WUS mRNA in inflorescence tips of wild-type $(G)$, uni-1D/+ $(H)$ and er uni-1D/+ (I) plants. Arrowheads indicate inflorescence SAMs and arrows indicates floral meristems. Bars $=50 \mu \mathrm{m}$. $(\mathrm{J})$ and $(\mathrm{K})$ Scanning electron microscopy images of 20-day-old er (J) and er uni-1D/+ (K) seedlings. c indicates cotyledons and arrows indicate formation of axillary buds. Bars $=500 \mu \mathrm{m}$. 
As shown in Fig. 1G, the Ler background did not affect the formation of extra axillary buds at axils of cotyledons that was observed in the original uni-1D/+ plants in the Ws background, suggesting that the ER function is not involved in this phenotype. When we compared er uni-1D/+ plants with er mutants in the original Ws background, er mutants formed no axillary buds at the axils of cotyledons and the first pair of leaves (Fig. 2J). On the other hand, in er uni-1D/+ plants, formation of axillary buds was observed even at both the axils of cotyledons and the first pair of leaves (Fig. 2K). These results indicate that the er mutation does not affect formation of extra axillary buds at leaf axils in uni-1D/+ plants.

\section{$E R$ is not involved in up-regulation of $P R$ genes in uni-1D/+ plants}

uni-1D/+ mutants exhibit a high level of expression of the pathogenesis-related genes $P R 1$ and PR5 (Igari et al. 2008). We next investigated whether ER is required for up-regulation of these $P R$ genes. As previously reported (Igari et al. 2008), PR1 and $P R 5$ expression was increased in uni-1D/+ mutants compared with wild-type plants (Fig. 3). The same degree of $P R 1 / 5$ upregulation was also detected in uni-1D/+ plants harboring the er mutation (Fig. 3). These results indicate that the ER activity does not influence PR1/5 expression in uni-1D/+ mutants. Thus, ER function is specifically involved in early cessation of inflorescence growth of uni-1D/+ plants but not in either formation of extra axillary buds at leaf axils or up-regulation of $P R$ genes.

\section{The ER activity in the UNI promoter active region contributes to the SAM defect of uni-1D/+ plants}

$E R$ is expressed in a broad range of tissues during diverse developmental processes (Yokoyama et al. 1998, Shpak et al. 2004,

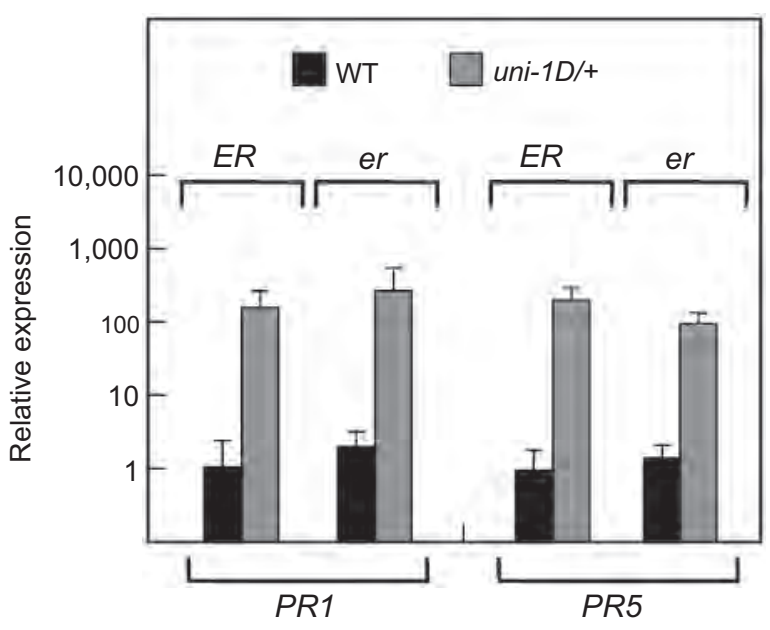

Fig. 3 ER function is not involved in up-regulation of PR genes in uni-1D/+ plants. Expression levels of PR1 and PR5 in the aerial parts of 7 -day-old seedlings were analyzed by quantitative real-time PCR. The expression levels were normalized with respect to that of $\beta$-TUBULIN. Data shown are means of three independent samples, with error bars representing the SD.
Shpak et al. 2005). Because both ER and UNI have protein structures that can act as starting points to trigger intracellular signaling pathways, we hypothesized that the signaling pathways activated by them could cooperate in the same cells for the SAM defect of uni-1D/+ plants. To examine this possibility, we made constructs in which the ER coding sequence is translationally fused to a FLAG-tag at its C-terminus and is driven by the ER promoter (ProER:ER-FLAG) or by the UNI promoter (ProUNI:ER-FLAG). er mutant plants developed short inflorescence stems (Fig. 4A) and clustered flower buds at the top of the inflorescence (Fig. 4D), as previously described (Torii et al. 1996). Introduction of the ProER:ER-FLAG transgene into the er mutant rescued all er phenotypes (Fig. 4B, E) with flower buds folded on to each other to cover the SAM (Fig. 4E). When the expression pattern of ER-FLAG proteins was examined by immunohistochemistry using anti-FLAG antibodies, the signals were broadly detected in the inflorescence tip (Fig. 4H), in agreement with previous reports (Yokoyama et al. 1998, Shpak et al. 2004). The ProER:ER-FLAG transgene was then introduced into er uni-1D/+ plants. The suppression of early termination of inflorescence growth of uni-1D/+ plants by er mutation was complemented by the ProER:ER-FLAG as expected (Fig. 4J-M).

Next, the ProUNI:ER-FLAG transgene was introduced into the er mutant. The promoter region used in this experiment was previously shown to be sufficient to induce all uni-1D/+ phenotypes when it was fused to UNI cDNA harboring the uni-1D mutation (Igari et al. 2008). The ProUNI:GUS transgenic plants showed strong $\beta$-glucuronidase (GUS) activity in regions beneath the SAM and also in the vasculature (Supplementary Fig. S1A-C). In the ProUNI:ER-FLAG transgenic plants, the ER-FLAG proteins were also detected in regions beneath the SAM by anti-FLAG immunohistochemistry (Fig. 4I), which is in accordance with the result using the ProUNI:GUS transgenic plants (Supplementary Fig. S1B, C). The short inflorescence stems and clustered flower buds at the inflorescence tip with the er mutation were rescued by ProUNI:ER-FLAG (Fig. 4C, F) as well as ProER:ER-FLAG (Fig. 4B, E). The ProUNI:ER-FLAG transgene was then introduced into er uni-1D/+ plants. er uni-1D/+ plants harboring the ProUNI:ER-FLAG exhibited uni-1D/+ morphology (Fig. 4J, K, N), showing that the ER activity in the region where the UNI promoter is active contributes to the SAM defect observed in uni-1D/+ mutants.

\section{ER-family members cooperatively mediate the AM phenotype of uni-1D/+ plants}

$E R$ has two family genes, ERL1 and ERL2, and it has been reported that the simultaneous loss of activities of ER-family members affects various events in plant development (Shpak et al. 2004, Shpak et al. 2005, Pillitteri et al. 2007, Hord et al. 2008). Therefore, we next examined whether activities of the other ER-family members are also involved in uni-1D/+ morphological phenotypes. We first checked the effects of simultaneous loss of ERL1 and ERL2 functions. As shown in 
ProER:ER-FLAG ProUNI:ER-FLAG
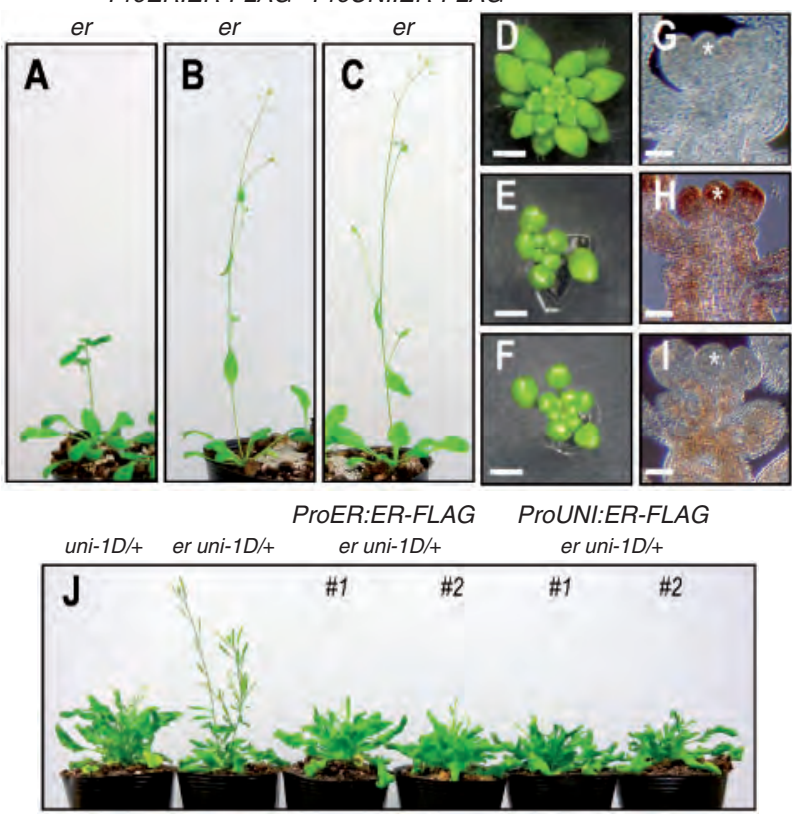

ProER:ER-FLAG ProUNI:ER-FLAG

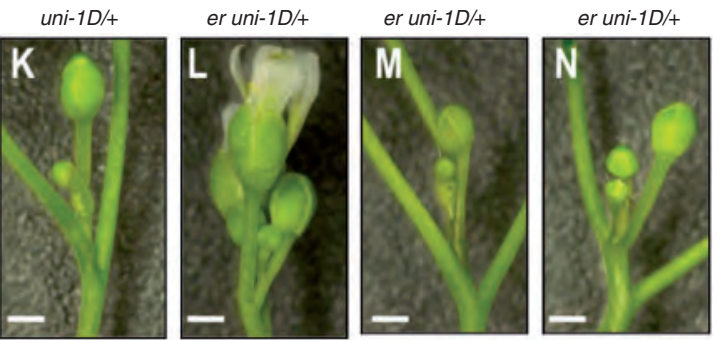

Fig. 4 The ER activity in the UNI-expressing cells is sufficient for the uni-1D/+ SAM defect. (A-C) Twenty-eight-day-old er (A), er ProER:ERFLAG (B) and er ProUNI:ER-FLAG (C) plants. (D-F) Inflorescence tips at the age of $28 \mathrm{~d}$ of er (D), er ProER:ER-FLAG (E) and er ProUNI:ERFLAG (F) plants. Bars $=1 \mathrm{~mm}$. (G-I) Immunohistochemistry using anti-FLAG antibody. ER-FLAG expression patterns in inflorescence SAMs of er (G), er ProER:ER-FLAG (H) and er ProUNI:ER-FLAG (I) plants. Asterisks indicate the SAMs. Bars $=50 \mu \mathrm{m}$. (J) Plants of the indicated genotypes at $59 \mathrm{~d}$ old. Two independent transgenic lines were used to generate er uni-1D/+ ProER:ER-FLAG and er uni-1D/+ ProUNI:ER-FLAG plants, respectively. (K-N) Inflorescence tips of uni-1D/+ (K), er uni-1D/+ (L), er uni-1D/+ ProER:ER-FLAG (M) and er uni-1D/+ ProUNI:ER-FLAG (N) plants. Bars $=1 \mathrm{~mm}$.

Supplementary Fig. S2, erl1 erl2 uni-1D/+ plant still showed the original uni-1D/+ morphology. Accordingly, the formation of extra axillary buds, which was not seen in wild-type plants (Fig. 5A), was observed in erl1 erl2 uni-1D/+ plants as well as in uni-1D/+ and er uni-1D/+ plants (Fig. 5B-D). We next examined the effects of other combinations of mutations among $E R$ family members. Most er erl1 uni-1D/+ plants still produced extra axillary buds (Fig. 5E), while er erl2 uni-1D/+ plants did not show this phenotype (Fig. 5F), demonstrating significant roles for $E R$ and $E R L 2$ in the AM phenotype of uni-1D/+ plants. To examine clearly the contribution of ERL1 to the AM

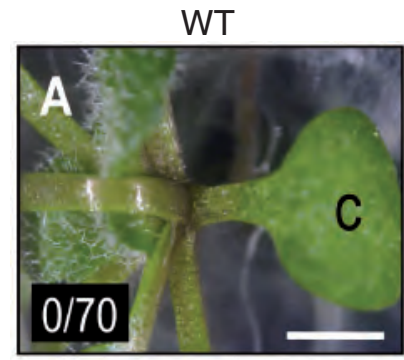

er uni-1D/+

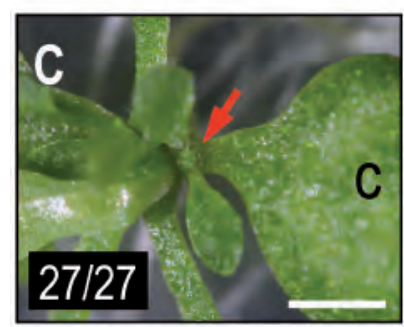

er erl1 uni-1D/+

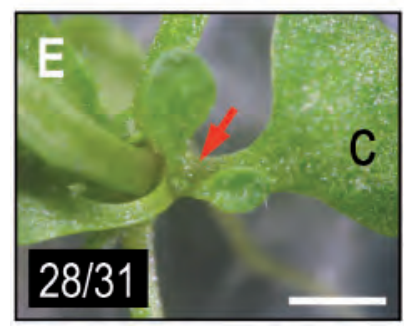

er erl1/+ erl2/+ uni-1D/+

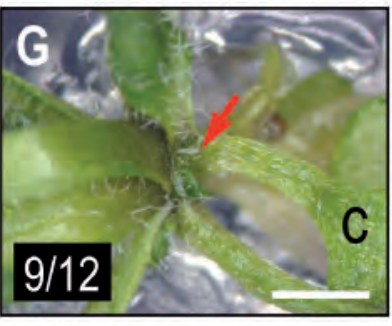

er erl1 erl2/+ uni-1D/+

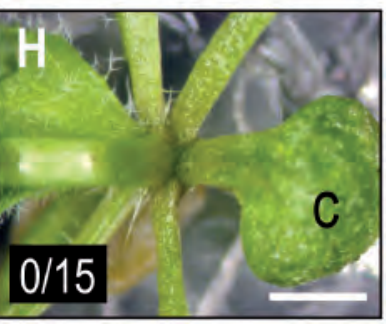

Fig. 5 ER-family members cooperatively mediate the AM phenotype in uni-1D/+ plants. The axils of cotyledons (indicated as C) of 16-day-old wild-type (WT) (A), uni-1D/+ (B), er uni-1D/+ (C), erl1 erl2 uni-1D/+ (D), er erl1 uni-1D/+ (E), er erl2 uni-1D/+ (F), er erl1/+ erl2/+ uni-1D/+ (G) and er erl1 erl2/+ uni-1D/+ (H) plants. Numerals at the lower left indicate the number of plants that produced extra axillary buds during the vegetative stage per total number of plants examined. er erl1/+ erl2/+ uni-1D/+ (G) and er erl1 erl2/+ uni-1D/+ $(\mathrm{H})$ plants were selected by genotyping among progeny of er erl1/+ erl2/+ uni-1D/+ plants because plants harboring the er erl 1 erl2/+ genotype are sterile (Pillitteri et al. 2007). c indicates cotyledons and arrows indicate formation of extra axillary buds. Bars $=3 \mathrm{~mm}$.

phenotype in addition to $E R$ and $E R L 2$, we next compared er erl1/+ erl2/+ uni-1D/+ plants with er erl1 erl2/+ uni-1D/+ plants. Most er erl1/+ erl2/+ uni-1D/+ plants still form extra axillary buds (Fig. 5G), while er erl1 erl2/+ uni-1D/+ plants did not exhibit this phenotype (Fig. $5 \mathrm{H}$ ), indicating that ERL1 also 
contributes to the AM phenotype of uni-1D/+ plants together with $E R$ and ERL2.

\section{Cytokinin response and CYP735A2 expression are up-regulated in uni-1D/+ mutants but are suppressed by attenuation of activities of ER-family members}

Among combinations of mutations of ER-family genes, we found the er erl1/+ erl2 uni-1D/+ plants showed a similar morphology to er erl1/+ erl2 plants without any sign of the morphological alterations observed in uni-1D/+ plants (Fig. 6A, Supplementary Fig. 3). It has been reported that tZ-type cytokinins accumulated in uni-1D/+ plants and, correspondingly, expression of the cytokinin-responsive ARR5 gene was upregulated in uni-1D/+ plants (Igari et al. 2008). When cytokinins were artificially reduced in uni-1D/+ seedlings, ARR5 up-regulation and also morphological alterations were suppressed, suggesting the importance of cytokinins for the phenotype (Igari et al. 2008). Therefore, we first checked the effect of attenuation of $E R$-family activities on the cytokinin response by determining the expression level of ARR5. As shown in Fig. 6B, $A R R 5$ expression was up-regulated in uni-1D/+ plants compared with wild-type plants, but this ARR5 up-regulation was suppressed in er erl1/+ erl2 uni-1D/+ plants. Among active cytokinins, only tZ-type cytokinins are specifically accumulated in uni-1D/+ plants (Igari et al. 2008) and the production of the tZ-type cytokinins requires the CYP735A enzyme in their biosynthesis pathway (Takei et al. 2004). CYP735A2 expression was up-regulated in uni-1D/+ plants compared with wild-type plants (Fig. 6C) (Igari et al. 2008), but this CYP735A2 up-regulation was not observed in er erl1/+ er/2 uni-1D/+ plants (Fig. 6C), indicating that activities of ER-family members contribute to the modulation of CYP735A expression in uni-1D/+ plants.

\section{Activities of ER-family members in the UNI promoter active region contribute to all morphological phenotypes of uni-1D/+ plants}

Because the ER activity in the region where the UNI promoter is active contributed to the SAM phenotype of uni-1D/+ plants (Fig. 4), we next examined whether a similar scenario could be occurring in the case of the suppression of all uni-1D/+ morphological phenotypes by attenuation of activities of ER-family members. The suppression of the uni-1D/+ phenotypes by er erl1/+ erl2 was complemented by the introduction of the ProER:ER-FLAG transgene as expected (Fig. 7). When the ProUNI:ER-FLAG transgenes were introduced into er erl1/+ erl2 uni-1D/+ plants, the ProUNI:ER-FLAG transgene had the same effect as the ProER:ER-FLAG transgene (Fig. 7). These observations show that the ER activity in the region where the UNI promoter is active is sufficient for uni-1D/+ plants to exhibit morphological alterations. Together, these data indicate that ER-family members cooperatively contribute to all
A
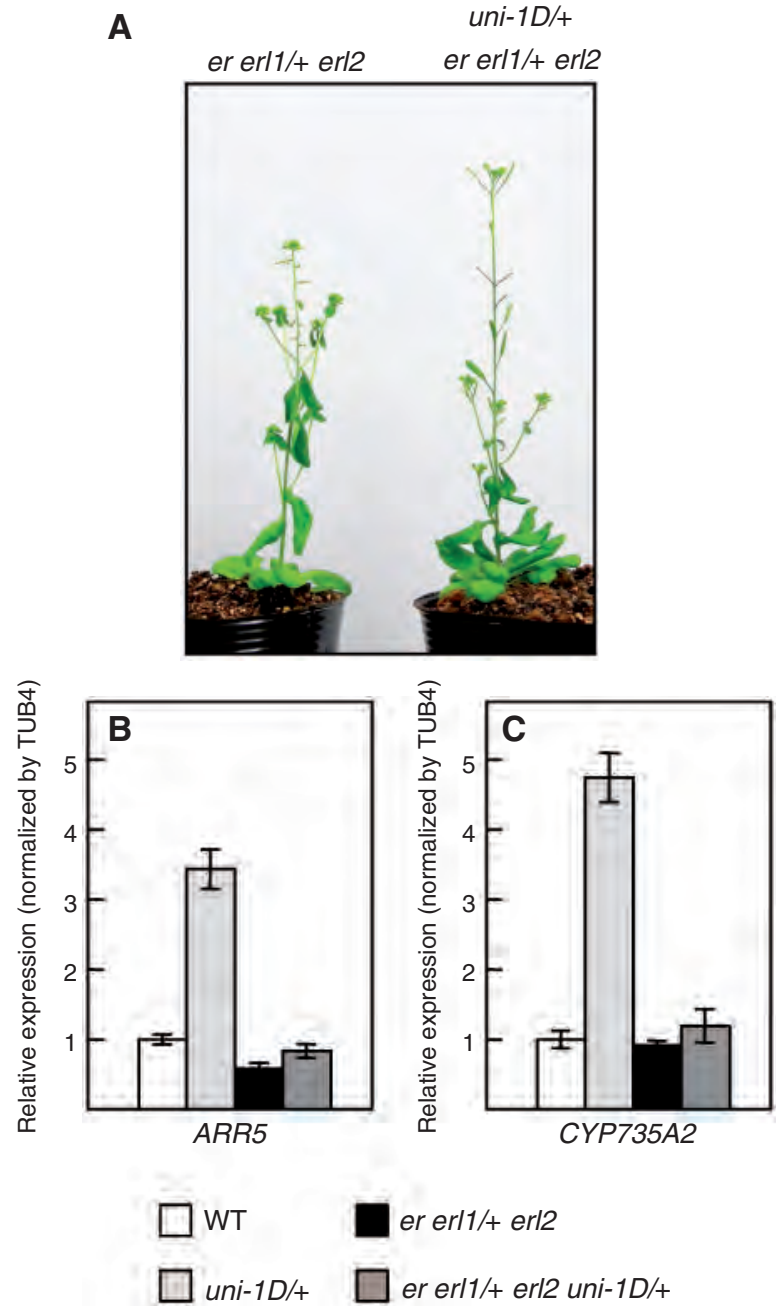

er erl1/+ erl2

er erl1/+ erl2 uni-1D/+

Fig. 6 ER-family members cooperatively mediate up-regulation of ARR5 and CYP735A2 in uni-1D/+ plants. (A) er erl1/+ erl2 (left) and er erl1/+ erl2 uni-1D/+ (right) plants at $34 \mathrm{~d}$ old. (B and $C$ ) Expression levels of ARR5 (B) and CYP735A2 (C) in the aerial parts of 10-day-old seedlings were analyzed by quantitative real-time PCR. The expression levels were normalized with respect to that of $\beta$-TUBULIN. Data shown are means of three independent samples, with error bars representing the SD.

morphological phenotypes observed in uni-1D/+ plants by exerting their functions in the UNI promoter active regions.

\section{Discussion}

\section{Relationships between signaling pathways} triggered by UNI and those triggered by ER-family members

The structure of UNI is related to the NB-LRR family. Most well-studied NB-LRRs serve as intracellular immune receptors (Jones and Dangl 2006, Caplan et al. 2008). These NB-LRRs are activated by specific pathogen effectors and they then trigger 


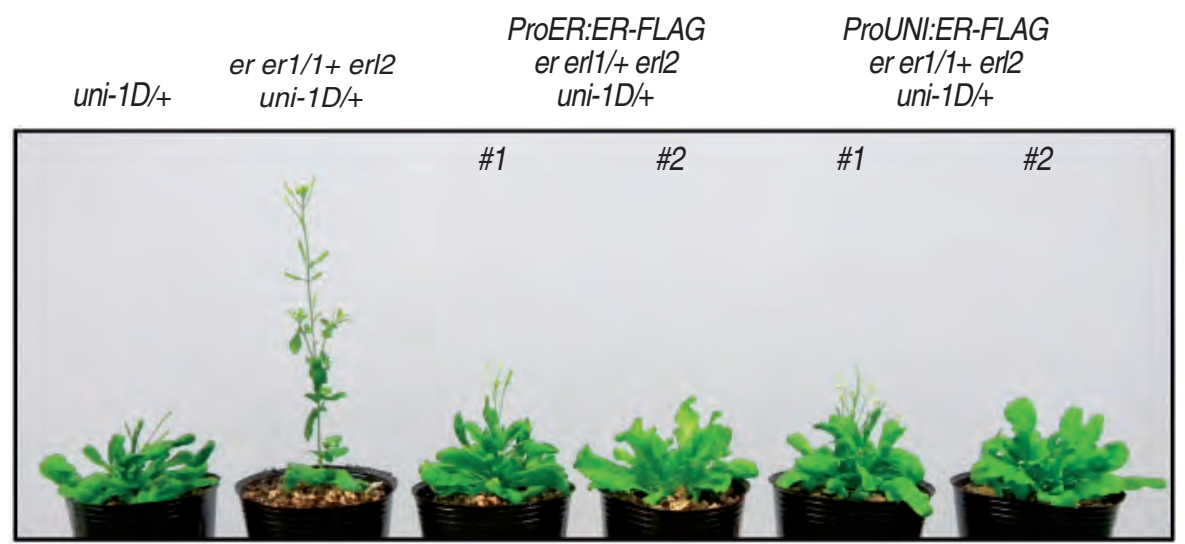

Fig. 7 The ER activity in UNI-expressing cells contributes to all morphological phenotypes of uni-1D/+ mutants. Plants at $48 \mathrm{~d}$ old with the indicated genotypes. Two independent transgenic lines were used to generate ProER:ER-FLAG er erl1/+ erl2 uni-1D/+ and ProUNI:ER-FLAG er erl1/+ erl2 uni-1D/+ plants, respectively.

defense responses. Interestingly, uni-1D plants have morphological phenotypes that can be genetically separated from the pathogenesis responses (Igari et al. 2008). Some NB-LRR-related factors also play roles in the biological processes that are distinct from pathogenesis-related regulations (Faigon-Soverna et al. 2006, Hewezi et al. 2006, Bomblies et al. 2007, Alcazar et al. 2009) and therefore UNI could be classified as belonging to this type of NB-LRR-related factor. As UNI has a protein structure that is potentially able to serve as a molecular switch in response to a ligand that can activate UNI proteins, it would be possible for UNI proteins to sense changes in the internal or external environment, via reception of an as yet unidentified ligand, and then function as a molecular switch to trigger the signaling for morphological regulation. The unidentified ligands to activate UNI proteins could be missing in wild-type Arabidopsis plants under normal growth conditions. This speculation is in accordance with the fact that the loss of UNI function by a T-DNA insertion induced no obvious phenotype (Igari et al. 2008).

Because both ER-family proteins and UNI proteins have structures that can act as starting points to trigger intracellular signaling pathways, and also because they play roles in the UNIexpressing cells for morphological phenotypes of uni-1D/+ plants (Figs. 4, 7), it would be interesting to investigate intracellular signaling cross-talk between signaling pathways triggered by ER-family proteins and those triggered by UNI proteins. Furthermore, it would be interesting to analyze how these signaling pathways are connected to regulation of CYP735A2 expression (Fig. 6).

\section{Different thresholds of activities of ER-family proteins for each uni-1D/+ phenotype}

uni-1D/+ mutants exhibit two morphological phenotypes: early termination of inflorescence stem growth and formation of extra AMs at leaf axils (Fig. 1) (Igari et al. 2008). When only $E R$ was mutated in uni-1D/+ plants, the former defect was suppressed (Fig. 2). On the other hand, attenuation of ER-family activities in uni-1D/+ plants resulted in suppression of not only the inflorescence defect but also the AM defect (Fig. 5). This difference in threshold of ER-family activities required for each phenotype of uni-1D/+ plants could be explained by the fact that proper development of each tissue needs different levels of ER-family activities. Loss-of-function mutations just in the ER gene can induce phenotypes in inflorescence architecture (Torii et al. 1996) though ER-family members are broadly expressed in various tissues, including inflorescences (Yokoyama et al. 1998, Shpak et al. 2004). Developmental processes of other tissues, such as flowers (Shpak et al. 2004, Pillitteri et al. 2007, Hord et al. 2008) and stomata (Shpak et al. 2005), are affected only when activities of more than one ER-family member are simultaneously lost. Thus, the development of inflorescences could be more sensitive to a reduction in total activities of ER-family proteins compared with developmental processes in other tissues. This could be a reason why the inflorescence defect of uni-1D/+ mutants was suppressed by single er mutations (Fig. 2) and the AM phenotype was more tolerant (Fig. 5).

\section{Relationships between cytokinins and uni-1D/+ phenotypes}

Though cytokinin is known to be an important hormone, playing significant roles in various developmental processes in plants (To and Kieber 2008, Werner and Schmulling 2009, Perilli et al. 2010), reports on its involvement in initiation processes of AM formation are limited. Interestingly, it was reported that infection of Arabidopsis with Rhodococcus fascians provokes ectopic formation of AMs, resulting in an overall bushy appearance (Vereecke et al. 2000, Manes et al. 2004), and that R. fascians produces cytokinin derivatives during its pathology, which are poor substrates of cytokinin degradation enzymes of host plants (Pertry et al. 2009). The R. fascians cytokinins modulate plant morphology through 
recognition by plant cytokinin receptors (Pertry et al. 2009), triggering fast activation of ARR5 expression (Depuydt et al. 2008). In uni-1D/+ mutants, formation of extra AMs was associated with modulation of cytokinin biosynthesis pathways and up-regulation of ARR5 expression (Fig. 6) (Igari et al. 2008), resembling the events provoked by $R$. fascians infection, except for the source of the cytokinins. These observations suggest the importance of cytokinin regulation to alter plant morphology through activation of AM formation. Because ER-family members were involved in the formation of extra AMs in uni-1D/+ plants (Fig. 6), it is an attractive hypothesis that ER-family members play an important role in the morphological alterations observed in $R$. fascians infection.

It has been shown that a feedback relationship between WUS and cytokinin signaling controls meristem homeostasis (Leibfried et al. 2005, Gordon et al. 2009). WUS creates a domain of high cytokinin responsiveness by negatively regulating ARR genes that can function as inhibitors of the cytokinin response (Leibfried et al. 2005). In turn, the localized high responsiveness to cytokinin establishes a spatial domain in which stem cell fate is specified through induction of WUS (Gordon et al. 2009). In uni-1D/+ plants, expression of $A R R 5$, an inhibitor of the cytokinin response, was up-regulated (Fig. 6) and WUS expression was altered (Fig. 2), suggesting that the homeostasis between WUS and cytokinin signaling could be disrupted and that this disruption could be involved in morphological alterations of uni-1D/+ plants. Because ER-family members participate in this event (Fig. 6), it would be of interest to investigate whether ER-family members play a role in the feedback loop between WUS and cytokinin signaling for meristem homeostasis.

Although the levels of ARR5 and CYP735A2 transcripts in uni-1D/+ plants were reduced to the wild-type level by er erl1/ + erl2, those in wild-type plants were not significantly affected by er erl1/+ erl2 (Fig. 6). This implies that ER-family members do not play important roles in cytokinin-related regulation under normal conditions but exert their functions to affect expression of CYP735A2 and ARR5 in response to changes in growth conditions via stimuli such as activation of UNI proteins. Alternatively, the remaining activity of ERL1 in er erl1/+ er/2 plants could be enough to maintain proper regulation of the cytokinin-related genes in the wild-type background.

\section{Possibility of coordination between environmental changes and morphological regulation via NB-LRR-related proteins and the ER-family}

The functional UNI promoter (Igari et al. 2008) has activities mainly outside meristems (Supplementary Fig. S1), although uni-1D/+ mutants exhibit meristem-related morphological phenotypes. This could suggest that activation of UNI proteins outside meristems affects the regulation of meristems. Furthermore, ER has been identified as a major trait in various QTL/eQTL analyses regarding environmental stresses and developmental processes, suggesting that ER could be a modulator of signaling pathways in response to changes in external and/or internal conditions (Keurentjes et al. 2007, Tisne et al. 2008, Ghandilyan et al. 2009, El-Lithy et al. 2010, Terpstra et al. 2010, van Zanten et al. 2010a, van Zanten et al. 2010b). Taken together, it would be attractive to hypothesize the existence of a system that connects environmental changes to morphological regulation via UNI proteins and ER-family receptor kinases. Because there are about 150 NB-LRR-related genes in the sequenced genome of A. thaliana (Meyers et al. 2003) and the biological roles of most of them have not been investigated, the possibility that some of them could function in such systems for the coordination between environmental changes and morphological regulation cannot be excluded. Future reports on this type of system involving NB-LRR-related genes should open up further aspects of the relationships between morphological regulation and environmental responses and it would be interesting to examine the involvement of ER-family receptor kinases in such systems.

\section{Materials and Methods}

\section{Plant materials}

The uni-1D allele originally identified in the Ws accession was introgressed six times into Col and Ler. pKUT196 (Ler) harboring the ER genomic fragment was described previously (Shpak et al. 2003). The er-2 mutant in the Col background (CS3401) and the er-201 mutant in the Ws background (FLAG_395D02) were obtained from ABRC and INRA, respectively. er-105, erl1-2 and erl2-1 mutants in the Col background were reported previously (Torii et al. 1996, Shpak et al. 2004).

\section{Plasmids and plant transformation}

For ProUNI:GUS construction, the $2.2 \mathrm{kbp}$ promoter region upstream from the translation start codon of the UNI gene was inserted into the $\mathrm{pBI} 101$ vector.

To construct ProER:ER-FLAG:ERterm, PCR was performed using the primer pair ERg4359 (CAACAATGATCTGGAAGG AC) and ERECTAnostopBamHI.rc (CGCGGATCCGCGCTCAC TGTTCTGAGAAATAAC), and the amplified fragment was inserted into the Sacl/BamHI site of pKUT195 to generate pNBL111, which harbors the ER genomic fragment from the 1,678 base promoter region to the unique $\mathrm{Xbal}$ site in the coding region, the ER cDNA fragment from the Xbal site to the end of the open reading frame without the stop codon, and the 1,964 bp ER terminator region. The $3 \times$ FLAG cassette with a stop codon (pNLB112) was generated by PCR using pF3PZPY122 [a gift from Dr. Xing-Wang Deng (Feng et al. 2003)] as a template with the primer pair: 3XFlag5 (GAAGTT CATTTCATTTGGAGAG) and 3XFlag3 Bglll.rc (GAAGATCTTC GACTTTATCGTCATCGTC) and cloned into pCR2.1 (Invitrogen). Subsequently, the BamHI/Bglll fragment of the $3 \times$ FLAG cassette was inserted into the BamHI site of pNLB111 to generate ProER:ER-FLAG:ERterm (pNLB115). This construct was introduced into the er-105 mutant and rescued 
the er phenotypes. For construction of ProER:ER-FLAG and ProUNI:ER-FLAG used in experiments described in this study, the fragment containing the ER coding region, $3 \times$ FLAG-tag and the ER $3^{\prime}$-untranslated region was amplified by PCR using the genome prepared from er-105 mutants harboring the original ProER:ER-FLAG transgene. The PCR products were then fused to the $2 \mathrm{~kb} E R$ promoter and the $2.2 \mathrm{~kb} U N I$ promoter, respectively, in the $\mathrm{PBIN} 30$ vector.

MP90 Agro strains harboring each plasmid were used for plant transformation. Ws wild-type plants and er-2 mutants in the Col accession were parent lines for transformation of ProUNI:GUS and ER-FLAG series, respectively.

\section{Quantitative real-time PCR}

RNA isolation, synthesis of first-strand CDNA and real-time PCR for $A R R 5$ were performed as previously described. Primers used for CYP735A2 were GCTCTTCCATCCACCACAACA and CGGA TTGTGCTTCGTTAGCA.

\section{GUS histology}

The procedures for GUS staining were described in Uchida et al. (2007). The stained material was cleared with chloral hydrate and then observed.

\section{In situ RNA hybridization}

In situ hybridization was performed according to Takada et al. (2001). Templates for transcription of a WUS antisense probe were described in Hamada et al. (2000).

\section{Immunohistochemistry}

Immunohistochemistry was performed using mouse anti-FLAG M2 antibody (Sigma, F1804) and ImmPRESS reagent anti-mouse Ig (Vector Laboratories, MP-7402) according to the manufacturer's instructions with the additional treatment of sectioned tissues with proteinase $K$ before the blocking stage. ImmPACT DAB (Vector Laboratories, SK-4105) was used as a peroxidase substrate.

\section{Supplementary data}

Supplementary data are available at PCP online.

\section{Funding}

This work was supported by the Ministry of Education, Culture, Sports, Science, and Technology (MEXT) [Grant-in-Aid for Scientific Research on Priority Areas (19060007), Grant-in-Aid for Scientific Research (A) (18207003) and Grant-in-Aid for Scientific Research (B) (22370019) to M.T., Grant-in-Aid for Young Scientists (B) (20770035 and 22770038) to N.U.]; US Department of Energy [DE-FG02-03ER15448 to K.U.T.]; US National Science Foundation [IOS0744892 to K.U.T.]; Sumitomo Foundation [funding to N.U.].

\section{Acknowledgments}

We thank Dr. Xing-Wang Deng (Yale) for the gift of pF3PZPY122. We also thank Ms. Eriko Tanaka for technical assistance.

\section{References}

Aida, M. and Tasaka, M. (2006) Morphogenesis and patterning at the organ boundaries in the higher plant shoot apex. Plant Mol. Biol. 60: 915-928.

Alcazar, R., Garcia, A.V., Parker, J.E. and Reymond, M. (2009) Incremental steps toward incompatibility revealed by Arabidopsis epistatic interactions modulating salicylic acid pathway activation. Proc. Natl Acad. Sci. USA 106: 334-339.

Bent, A.F. and Mackey, D. (2007) Elicitors, effectors, and R genes: the new paradigm and a lifetime supply of questions. Annu. Rev. Phytopathol. 45: 399-436.

Bomblies, K., Lempe, J., Epple, P., Warthmann, N., Lanz, C., Dangl, J.L. et al. (2007) Autoimmune response as a mechanism for a Dobzhansky-Muller-type incompatibility syndrome in plants. PLoS Biol. 5: e236.

Brand, U., Fletcher, J.C., Hobe, M., Meyerowitz, E.M. and Simon, R. (2000) Dependence of stem cell fate in Arabidopsis on a feedback loop regulated by CLV3 activity. Science 289: 617-619.

Caplan, J., Padmanabhan, M. and Dinesh-Kumar, S.P. (2008) Plant NB-LRR immune receptors: from recognition to transcriptional reprogramming. Cell Host Microbe 3: 126-135.

Clark, S.E. (2001) Cell signalling at the shoot meristem. Nat. Rev. Mol. Cell Biol. 2: 276-284.

Depuydt, S., Dolezal, K., Van Lijsebettens, M., Moritz, T., Holsters, M. and Vereecke, D. (2008) Modulation of the hormone setting by Rhodococcus fascians results in ectopic KNOX activation in Arabidopsis. Plant Physiol. 146: 1267-1281.

El-Lithy, M.E., Reymond, M., Stich, B., Koornneef, M. and Vreugdenhil, D. (2010) Relation among plant growth, carbohydrates and flowering time in the Arabidopsis Landsberg erecta $\times$ Kondara recombinant inbred line population. Plant Cell Environ. 33: 1369-1382.

Faigon-Soverna, A., Harmon, F.G., Storani, L., Karayekov, E., Staneloni, R.J., Gassmann, W. et al. (2006) A constitutive shade-avoidance mutant implicates TIR-NBS-LRR proteins in Arabidopsis photomorphogenic development. Plant Cell 18: 2919-2928.

Feng, S., Ma, L., Wang, X., Xie, D., Dinesh-Kumar, S.P., Wei, N. et al. (2003) The COP9 signalosome interacts physically with SCF COI1 and modulates jasmonate responses. Plant Cell 15: 1083-1094.

Ghandilyan, A., Ilk, N., Hanhart, C., Mbengue, M., Barboza, L., Schat, H. et al. (2009) A strong effect of growth medium and organ type on the identification of QTLs for phytate and mineral concentrations in three Arabidopsis thaliana RIL populations. J. Exp. Bot. 60: 1409-1425.

Gordon, S.P., Chickarmane, V.S., Ohno, C. and Meyerowitz, E.M. (2009) Multiple feedback loops through cytokinin signaling control stem cell number within the Arabidopsis shoot meristem. Proc. Natl Acad. Sci. USA 106: 16529-16534.

Hamada, S., Onouchi, H., Tanaka, H., Kudo, M., Liu, Y.G., Shibata, D. et al. (2000) Mutations in the WUSCHEL gene of Arabidopsis thaliana result in the development of shoots without juvenile leaves. Plant J. 24: 91-101. 
Hewezi, T., Mouzeyar, S., Thion, L., Rickauer, M., Alibert, G., Nicolas, P. et al. (2006) Antisense expression of a NBS-LRR sequence in sunflower (Helianthus annuus L.) and tobacco (Nicotiana tabacum L.): evidence for a dual role in plant development and fungal resistance. Transgenic Res. 15: 165-180.

Hord, C.L., Sun, Y.J., Pillitteri, L.J., Torii, K.U., Wang, H., Zhang, S. et al. (2008) Regulation of Arabidopsis early anther development by the mitogen-activated protein kinases, MPK3 and MPK6, and the ERECTA and related receptor-like kinases. Mol. Plant 1: 645-658.

Igari, K., Endo, S., Hibara, K., Aida, M., Sakakibara, H., Kawasaki, T. et al. (2008) Constitutive activation of a CC-NB-LRR protein alters morphogenesis through the cytokinin pathway in Arabidopsis. Plant J. 55: 14-27.

Jones, J.D. and Dangl, J.L. (2006) The plant immune system. Nature 444: 323-329.

Keurentjes, J.J., Fu, J., Terpstra, I.R., Garcia, J.M., van den Ackerveken, G., Snoek, L.B. et al. (2007) Regulatory network construction in Arabidopsis by using genome-wide gene expression quantitative trait loci. Proc. Natl Acad. Sci. USA 104: 1708-1713.

Kondo, T., Sawa, S., Kinoshita, A., Mizuno, S., Kakimoto, T., Fukuda, H. et al. (2006) A plant peptide encoded by CLV3 identified by in situ MALDI-TOF MS analysis. Science 313: 845-848.

Laux, T., Mayer, K.F., Berger, J. and Jurgens, G. (1996) The WUSCHEL gene is required for shoot and floral meristem integrity in Arabidopsis. Development 122: 87-96.

Leibfried, A., To, J.P., Busch, W., Stehling, S., Kehle, A., Demar, M. et al. (2005) WUSCHEL controls meristem function by direct regulation of cytokinin-inducible response regulators. Nature 438: 1172-1175.

Lenhard, M., Bohnert, A., Jurgens, G. and Laux, T. (2001) Termination of stem cell maintenance in Arabidopsis floral meristems by interactions between WUSCHEL and AGAMOUS. Cell 105: 805-814.

Lenhard, M. and Laux, T. (2003) Stem cell homeostasis in the Arabidopsis shoot meristem is regulated by intercellular movement of CLAVATA3 and its sequestration by CLAVATA1. Development 130: 3163-3173.

Lohmann, J.U., Hong, R.L., Hobe, M., Busch, M.A., Parcy, F., Simon, R. et al. (2001) A molecular link between stem cell regulation and floral patterning in Arabidopsis. Cell 105: 793-803.

Manes, C.L.D., Beeckman, T., Ritsema, T., Van Montagu, M., Goethals, K. and Holsters, M. (2004) Phenotypic alterations in Arabidopsis thaliana plants caused by Rhodococcus fascians infection. J. Plant Res. 117: 139-145.

Meyers, B.C., Kozik, A., Griego, A., Kuang, H. and Michelmore, R.W. (2003) Genome-wide analysis of NBS-LRR-encoding genes in Arabidopsis. Plant Cell 15: 809-834.

Miwa, H., Betsuyaku, S., Iwamoto, K., Kinoshita, A., Fukuda, H. and Sawa, S. (2008) The receptor-like kinase SOL2 mediates CLE signaling in Arabidopsis. Plant Cell Physiol. 49: 1752-1757.

Muller, R., Bleckmann, A. and Simon, R. (2008) The receptor kinase CORYNE of Arabidopsis transmits the stem cell-limiting signal CLAVATA3 independently of CLAVATA1. Plant Cell 20: 934-946.

Muller, R., Borghi, L., Kwiatkowska, D., Laufs, P. and Simon, R. (2006) Dynamic and compensatory responses of Arabidopsis shoot and floral meristems to CLV3 signaling. Plant Cell 18: 1188-1198.

Padmanabhan, M., Cournoyer, P. and Dinesh-Kumar, S.P. (2009) The leucine-rich repeat domain in plant innate immunity: a wealth of possibilities. Cell. Microbiol. 11: 191-198.

Perilli, S., Moubayidin, L. and Sabatini, S. (2010) The molecular basis of cytokinin function. Curr. Opin. Plant Biol. 13: 21-26.
Pertry, I., Vaclavikova, K., Depuydt, S., Galuszka, P., Spichal, L., Temmerman, W. et al. (2009) Identification of Rhodococcus fascians cytokinins and their modus operandi to reshape the plant. Proc. Natl Acad. Sci. USA 106: 929-934.

Pillitteri, L.J., Bemis, S.M., Shpak, E.D. and Torii, K.U. (2007) Haploinsufficiency after successive loss of signaling reveals a role for ERECTA-family genes in Arabidopsis ovule development. Development 134: 3099-3109.

Schmitz, G. and Theres, K. (2005) Shoot and inflorescence branching. Curr. Opin. Plant Biol. 8: 506-511.

Schoof, H., Lenhard, M., Haecker, A., Mayer, K.F., Jurgens, G. and Laux, T. (2000) The stem cell population of Arabidopsis shoot meristems in maintained by a regulatory loop between the CLAVATA and WUSCHEL genes. Cell 100: 635-644.

Shpak, E.D., Berthiaume, C.T., Hill, E.J. and Torii, K.U. (2004) Synergistic interaction of three ERECTA-family receptor-like kinases controls Arabidopsis organ growth and flower development by promoting cell proliferation. Development 131: 1491-1501.

Shpak, E.D., Lakeman, M.B. and Torii, K.U. (2003) Dominant-negative receptor uncovers redundancy in the Arabidopsis ERECTA leucine-rich repeat receptor-like kinase signaling pathway that regulates organ shape. Plant Cell 15: 1095-1110.

Shpak, E.D., McAbee, J.M., Pillitteri, L.J. and Torii, K.U. (2005) Stomatal patterning and differentiation by synergistic interactions of receptor kinases. Science 309: 290-293.

Takada, S., Hibara, K., Ishida, T. and Tasaka, M. (2001) The CUP-SHAPED COTYLEDON1 gene of Arabidopsis regulates shoot apical meristem formation. Development 128: 1127-1135.

Takei, K., Yamaya, T. and Sakakibara, H. (2004) Arabidopsis CYP735A1 and CYP735A2 encode cytokinin hydroxylases that catalyze the biosynthesis of trans-Zeatin. J. Biol. Chem. 279: 41866-41872.

Terpstra, I.R., Snoek, L.B., Keurentjes, J.J., Peeters, A.J. and van den Ackerveken, G. (2010) Regulatory network identification by genetical genomics: signaling downstream of the Arabidopsis receptor-like kinase ERECTA. Plant Physiol. 154: 1067-1078.

Tisne, S., Reymond, M., Vile, D., Fabre, J., Dauzat, M., Koornneef, M. et al. (2008) Combined genetic and modeling approaches reveal that epidermal cell area and number in leaves are controlled by leaf and plant developmental processes in Arabidopsis. Plant Physiol. 148: 1117-1127.

To, J.P. and Kieber, J.J. (2008) Cytokinin signaling: two-components and more. Trends Plant Sci. 13: 85-92.

Torii, K.U., Mitsukawa, N., Oosumi, T., Matsuura, Y., Yokoyama, R., Whittier, R.F. et al. (1996) The Arabidopsis ERECTA gene encodes a putative receptor protein kinase with extracellular leucine-rich repeats. Plant Cell 8: 735-746.

Tucker, M.R. and Laux, T. (2007) Connecting the paths in plant stem cell regulation. Trends Cell Biol. 17: 403-410.

Uchida, N., Townsley, B., Chung, K.H. and Sinha, N. (2007) Regulation of SHOOT MERISTEMLESS genes via an upstream-conserved noncoding sequence coordinates leaf development. Proc. Natl Acad. Sci. USA 104: 15953-15958.

van Zanten, M., Basten Snoek, L., van Eck-Stouten, E., Proveniers, M.C., Torii, K.U., Voesenek, L.A. et al. (2010a) Ethylene-induced hyponastic growth in Arabidopsis thaliana is controlled by ERECTA. Plant J. 61: 83-95.

van Zanten, M., Snoek, L.B., Proveniers, M.C. and Peeters, A.J. (2009) The many functions of ERECTA. Trends Plant Sci. 14: 214-218.

van Zanten, M., Snoek, L.B., van Eck-Stouten, E., Proveniers, M.C., Torii, K.U., Voesenek, L.A. et al. (2010b) ERECTA controls low 
light intensity-induced differential petiole growth independent of phytochrome B and cryptochrome 2 action in Arabidopsis thaliana. Plant Signal. Behav. 5: 284-286.

Vereecke, D., Burssens, S., Simon-Mateo, C., Inze, D., Van Montagu, M., Goethals, K. et al. (2000) The Rhodococcus fascians-plant interaction: morphological traits and biotechnological applications. Planta 210: 241-251.

Werner, T. and Schmulling, T. (2009) Cytokinin action in plant development. Curr. Opin. Plant Biol. 12: 527-538.
Woodward, C., Bemis, S.M., Hill, E.J., Sawa, S., Koshiba, T. and Torii, K.U. (2005) Interaction of auxin and ERECTA in elaborating Arabidopsis inflorescence architecture revealed by the activation tagging of a new member of the YUCCA family putative flavin monooxygenases. Plant Physiol. 139: 192-203.

Yokoyama, R., Takahashi, T., Kato, A., Torii, K.U. and Komeda, Y. (1998) The Arabidopsis ERECTA gene is expressed in the shoot apical meristem and organ primordia. Plant J. 15: 301-310. 


\title{
The presence of multiple introns is essential for ERECTA expression in Arabidopsis
}

\author{
RUCHA KARVE, ${ }^{1}$ WUSHENG LIU, ${ }^{1}$ SPENCER G. WILLET, ${ }^{1}$ KEIKO U. TORII, ${ }^{2}$ and ELENA D. SHPAK ${ }^{1,3}$ \\ ${ }^{1}$ Department of Biochemistry, Cellular and Molecular Biology, University of Tennessee, Knoxville, Tennessee 37996, USA \\ ${ }^{2}$ Department of Biology, University of Washington, Seattle, Washington 98195, USA
}

\begin{abstract}
Gene expression in eukaryotes is often enhanced by the presence of introns. Depending on the specific gene, this enhancement can be minor or very large and occurs at both the transcriptional and post-transcriptional levels. The Arabidopsis ERECTA gene contains 27 exons encoding a receptor-like kinase that promotes cell proliferation and inhibits cell differentiation in above-ground plant organs. The expression of ERECTA very strongly depends on the presence of introns. The intronless ERECTA gene does not rescue the phenotype of erecta mutant plants and produces about 500-900 times less protein compared with the identical construct containing introns. This result is somewhat surprising as the region upstream of the ERECTA coding sequence effectively promotes the expression of extraneous genes. Here, we demonstrate that introns are essential for ERECTA mRNA accumulation and, to a lesser extent, for mRNA utilization in translation. Since mRNA produced by intronless ERECTA is degraded at the $3^{\prime}$ end, we speculate that introns increase mRNA accumulation through increasing its stability at least in part. No individual intron is absolutely necessary for ERECTA expression, but rather multiple introns in specific locations increase ERECTA expression in an additive manner. The ability of introns to promote ERECTA expression might be linked to the process of splicing and not to a particular intron sequence.
\end{abstract}

Keywords: gene expression; intron-mediated enhancement; splicing; intron; ERECTA

\section{INTRODUCTION}

Most genes of higher eukaryotes contain introns, noncoding DNA sequences that are transcribed and then removed from pre-mRNA molecules through a process called splicing. Frequently introns are critical for gene function as they might be necessary for alternative splicing or could contain vital noncoding RNAs and ORFs. In addition, introns have the ability to promote gene expression. For example, maize alcohol dehydrogenase-1 expression in a transient assay is increased 50-100 times when introns are included (Callis et al. 1987). Introns are critical for rabbit $\beta$-globin expression in animal cell cultures (Buchman and Berg 1988), for expression of the mouse thymidylate synthase gene (Deng et al. 1989), and for human purine nucleoside phosphorylase (Jonsson et al. 1992). While endogenous introns can enhance the expression of many genes, in most cases the effect is rather modest, and only in a limited number of cases, such as the

\footnotetext{
${ }^{3}$ Corresponding author.

E-mail eshpak@utk.edu.

Article published online ahead of print. Article and publication date are at http://www.rnajournal.org/cgi/doi/10.1261/rna.2825811.
}

rabbit $\beta$-globin gene or maize alcohol dehydrogenase- 1 , it is dramatic (Lu and Cullen 2003).

The most well understood mechanism of gene expression regulation by introns is through the presence of cis-regulatory elements in intronic sequences. For example, the second intron of the Arabidopsis gene AGAMOUS contains binding sites for several transcription factors, including LEAFY and WUSCHEL (Sieburth and Meyerowitz 1997; Hong et al. 2003). Alternatively, introns can also promote gene expression through the recruitment of splicing factors. During the past decade, it has become clear that the different steps of mRNA biogenesis such as transcription, $5^{\prime}$ end capping, splicing, and the cleavage and polyadenylation of the $3^{\prime}$ end are mutually dependant (Moore and Proudfoot 2009; Hocine et al. 2010). Thus, introns can promote transcription by recruiting spliceosomal proteins that directly interact with the RNA polymerase II complex and stimulate transcriptional elongation (Fong and Zhou 2001; Lin et al. 2008). There are multiple reports suggesting that splicing and polyadenylation can be linked: Several splicing factors interact directly with the $3^{\prime}$ end processing machinery (Millevoi et al. 2002; Kyburz et al. 2006), specific intronic sequences can positively regulate polyadenylation (Lou 
et al. 1996), the $3^{\prime}$ splice site of the last intron is able to enhance poly(A) site cleavage (Rigo and Martinson 2008), and spliced mRNAs are often more efficiently polyadenylated than are identical intronless mRNAs (Huang and Gorman 1990; Niwa et al. 1990; Lu and Cullen 2003). As the 3' end influences mRNA stability, transport, and translation, the splicing-induced changes to its structure should alter the level of gene expression.

Finally, during splicing a complex of proteins is deposited on mRNA 20-24 nucleotides (nt) upstream of an exon-exon junction (Le Hir et al. 2000). An exon junction complex (EJC) consists of four core proteins (eIF4A-III, MLN51, Y14, and Magoh) and a variable number of proteins associated with it in a transient manner (Tange et al. 2005). The EJC is well known for its role in nonsense-mediated mRNA decay (Tange et al. 2004). For some intron-containing genes, the EJC is required for efficient mRNA export to the cytoplasm as it is able to recruit mRNA export factors (Zhou et al. 2000; Le Hir et al. 2001). In other cases, the EJC is believed to increase the translation of spliced transcripts, possibly through interaction with the small ribosomal subunit. The human PYM protein interacts with both the EJC and the small ribosomal subunit, and its knockout results in reduced translational efficiency of spliced mRNAs but does not change the translation of intronless mRNAs (Diem et al. 2007).

Why is the presence of introns extremely significant for expression of some genes but not others? In mammals only a small proportion of genes are naturally intronless $(\sim 5 \%$ in Homo sapiens and $\sim 8 \%$ in Mus musculus). It is speculated that for most mammalian genes, splicing is essential for efficient $3^{\prime}$ end formation of mRNA and its export from the nucleus. Naturally intronless genes and some introncontaining genes have cis-elements that promote these processes, and thus, their expression becomes independent of splicing. Such elements have been identified in human c-Jun gene (Guang and Mertz 2005), mouse H2a gene (Huang and Carmichael 1997), and several viral genes (Malim et al. 1989; Ernst et al. 1997). In plants the proportion of intronless genes is higher than in mammals: $21.7 \%$ in Arabidopsis and $19.9 \%$ in rice (Jain et al. 2008). The removal of introns from many plant genes is not detrimental to their function, based on effective complementation of numerous Arabidopsis mutants by cDNA sequences. Thus, for most plant genes, the $3^{\prime}$ end formation of mRNA and its export from the nucleus is probably not dependent upon splicing.

At the same time, there are still quite a few examples when introns are essential for plant gene expression (Callis et al. 1987; Dean et al. 1989; Rose and Last 1997; Maas et al. 1991; Rose 2008). The mechanism by which they function is not clear except in cases when cis-regulatory elements are found in intronic sequences. Historically, stimulation of plant gene expression by introns has been called intron-mediated enhancement (IME) (Mascarenhas et al. 1990). In described cases of IME, introns often increase mRNA accumulation (Callis et al. 1987; Dean et al. 1989; Rose and Last 1997; Wang et al. 2002), but sometimes they also have an effect on translation (Bourdon et al. 2001; Curi et al. 2005; Lu et al. 2008). In several cases, nuclear run-on transcription assays suggested that the increase in mRNA accumulation was not due to changes in transcription initiation or elongation but instead occurred at a later step (Dean et al. 1989; Rose and Last 1997; Rose and Beliakoff 2000). For efficient IME, introns have to be within the transcribed sequences and in their natural orientation (Callis et al. 1987; Mascarenhas et al. 1990; Clancy et al. 1994; Curi et al. 2005). The position of introns in a gene is significant, and often IME is stronger when introns are located close to the 5' UTR (Rose 2004). While IME requires at least a partial assembly of the spliceosome, the completion of splicing is not always essential (Rose and Beliakoff 2000; Rose 2002). Recently it was suggested that promoter proximal introns are enriched in sequences that elevate gene expression, and an algorithm for finding such sequences called IMEter was proposed and tested in Arabidopsis (Rose et al. 2008). However, in a later study in rice, the IMEter predictions were not supported (Morello et al. 2011). While one can expect that recruitment of splicing factors and the EJC proteins have the same effect on gene expression in plants as it does in mammals, and therefore should be involved in IME, this effect has not been demonstrated experimentally.

To gain more knowledge of gene expression regulation in plants, we have investigated the Arabidopsis gene ERECTA. The ERECTA gene encodes a receptor-like kinase that is involved in regulation of plant size and epidermis development (Torii et al. 1996; Shpak et al. 2004; Shpak et al. 2005). Orthologs of ERECTA can be found in many different plant species, including Bryophytes, and in most plants, the family is represented by several paralogs. In Arabidopsis, ERECTA belongs to a family of three genes (the others are ERL1 and ERL2) that have overlapping functions and are critical for plant growth. Mutation of all three genes leads to severe dwarfism and dramatically increases the proportion of stomata in the epidermis (Shpak et al. 2004, 2005). The phenotype of single erecta mutants is not so striking but is still very distinct. Erecta plants have compact inflorescence with flowers clustered on the top; short and thick stems; small, round flowers; and short siliques (Torii et al. 1996).

Here we demonstrate that the intronless ERECTA gene cannot rescue this phenotype, while intron-containing ERECTA can. The presence of introns is required for mRNA accumulation, and in this way, IME of ERECTA is similar to IME of most other plant genes. Based on our data, we speculate that IME of ERECTA expression does not require specific intron sequences but is related to some aspect of splicing and operates at least in part through promotion of $3^{\prime}$ end mRNA formation. 


\section{RESULTS}

\section{The intronless ERECTA gene produces a dramatically reduced amount of $\mathrm{mRNA}$}

The 9.4-kb genomic fragment of ERECTA, including the intron-containing coding sequence (here will be referred to as gERECTA), and the $1.7-\mathrm{kb} 5^{\prime}$ and $1.9-\mathrm{kb} 3^{\prime}$ regions rescued the erecta-105 knockout mutant in all 14 independent transgenic T1 Arabidopsis plants we examined (Fig. 1A). However, when the intronless sequence of ERECTA (here will be referred to as $C E R E C T A$ ) was placed between the same promoter and terminator regions, the construct did not complement erecta-105 even partially in all 43 transgenic lines that we obtained (Fig. 1A). We confirmed the presence of the ER::cERECTA construct by PCR analysis in 11 of the transgenic lines.

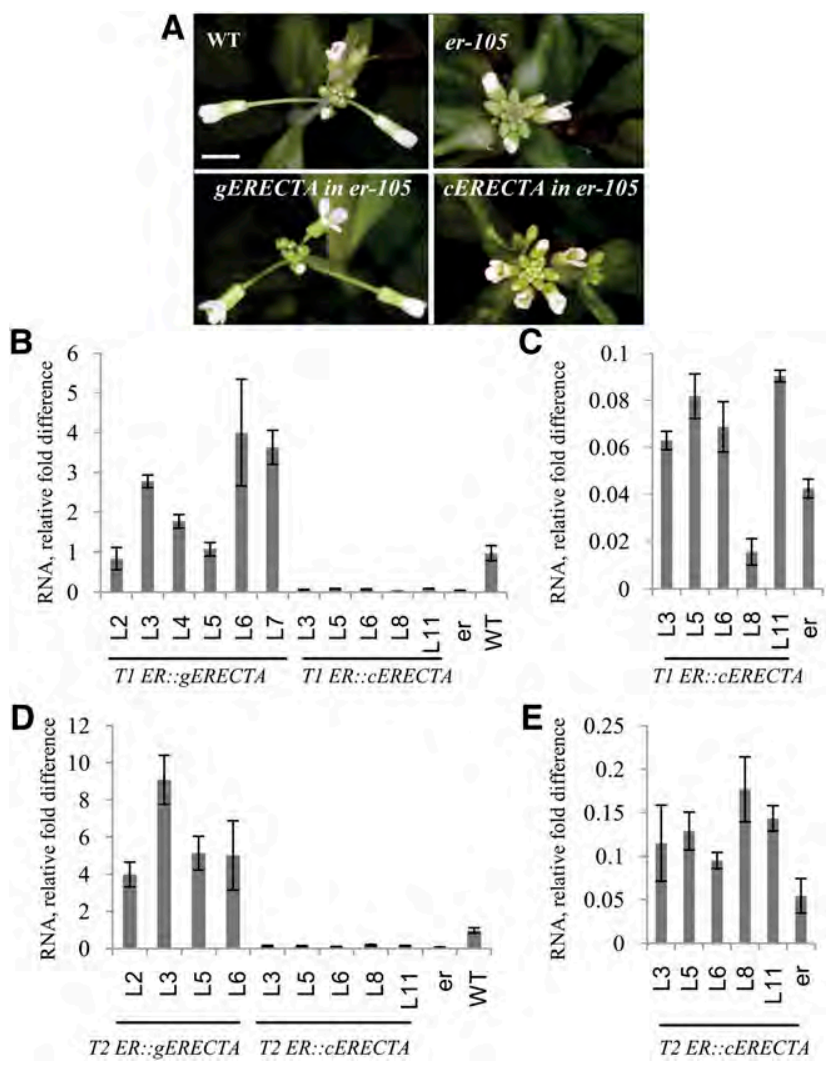

FIGURE 1. The intronless ERECTA gene does not rescue the erecta knockout mutant and produces dramatically reduced amount of mRNA. (A) Complementation of erecta-105 with ER::gERECTA and ER::cERECTA. Bar, $3 \mathrm{~mm}$. (B-E) The amount of ERECTA mRNA in $E R:: c E R E C T A$ plants is lower and in ER::gERECTA plants is equal or higher compared with the wild type as determined by qRT-PCR with $A C T I N-2$ as an internal control. The total RNA was isolated from T1 inflorescences $(B, C)$ and 15-d-old gentamicin selected T2 seedlings $(D, E)$ of multiple independent transgenic lines. Graphs $C$ and $E$ demonstrate that ERECTA mRNA is expressed at a higher level in ER::cERECTA plants compared with the erecta-105 mutant. The mean of three technical replicas is plotted; error bars, $1 \mathrm{SD}$.
In a previous study, ERECTA RNA gel blot analysis detected only one RNA species (Torii et al. 1996). All 97 expression sequenced tags (ESTs) available for the ERECTA gene correspond to the CDNA structure used in our experiments. An extensive reverse transcription PCR (RT-PCR) analysis of the ERECTA gene segments detected that introns 6,20 , and 21 are less efficiently spliced than are others (data not shown). However, they contain multiple stop codons and will most likely lead to nonsense-mediated decay of mRNA. Thus, we were not able to detect any alternatively spliced ERECTA mRNA.

To determine whether introns are necessary for transcription and/or mRNA stability or impact the later stages of expression, we compared ERECTA levels in transgenic lines to those in the wild type and in erecta-105 using quantitative RT-PCR (qRT-PCR). T1 cERECTA inflorescences of the five analyzed transgenic lines had at least 10 times less ERECTA than did the wild type (Fig. 1B). Subsequent T2 generation transgenic seedlings had at least 5.5 times less (Fig. 1D). We detected higher levels of ERECTA in CERECTA lines (two to three times) compared with the erecta-105 mutant, suggesting that some ERECTA is expressed from this construct (Fig. 1C,E). T1 gERECTA inflorescences had up to four times more ERECTA compared with the wild type, and T2 gentamicin selected seedlings had from 2.8-9.5 times more (Fig. 1B,D). All gERECTA lines in which we measured ERECTA mRNA levels contained only one T-DNA insert based on their 3:1 segregation ratio on selection plates. During cloning we made sure to place both gERECTA and cERECTA in the vector oriented such that the terminator, but not the promoter of, ERECTA was adjacent to the $235 \mathrm{~S}$ promoter regulating the aacC1 gentamicin resistance gene. Therefore it is not likely that the $d 35 S$ promoter is able to influence ERECTA expression in our constructs. Higher ERECTA expression in the gERECTA lines could be due to negative regulation of expression in its endogenous location by elements outside of the $9.4-\mathrm{kb}$ region or by the chromatin structure. While ERECTA $m R N A$ is expressed at five to nine times higher levels in gERECTA lines 3 and 5, the phenotype of plants was indistinguishable from the wild type (Supplemental Fig. S1). We were not able to determine whether higher expression at the mRNA level is translated into a larger amount of protein produced. ERECTA mRNA consistently accumulated at considerably lower levels in the cERECTA lines and at considerably higher levels in the gERECTA lines, strongly suggesting that the ERECTA gene experiences IME of gene expression occurring at the stage of mRNA accumulation.

\section{Importance of introns in the ERECTA gene for protein production}

As the $c E R E C T A$ lines did produce some mRNA, we wanted to investigate whether introns are necessary for any later stages of mRNA metabolism. It was not possible to directly 
compare the accumulation of ERECTA protein in our transgenic plants as the endogenous ERECTA protein is produced at a very low level (Shpak et al. 2003), and reliable measurements of its quantity by Western blotting analysis are extremely challenging. To overcome this problem, we transformed erecta-105 with the ER::gERECTA-RLUC and $E R: \because c E R E C T A-R L U C$ constructs where the ERECTA sequences were fused to the Renilla luciferase (RLUC) coding region and expressed under control of the endogenous ERECTA promoter and the $35 S$ terminator. ER ::gERECTARLUC complemented the mutation, while ER::cERECTA$R L U C$ did not. Complementation by ER ::gERECTA-RLUC was partial in more than half of the T1 plants, presumably due to inhibition of ERECTA function by the addition of luciferase to its $\mathrm{C}$ terminus.

We characterized two independent transgenic lines for each construct with qRT-PCR analysis and Renilla luciferase assay using homozygous transgenic seedlings carrying a single T-DNA insert. In $c E R E C T A$ seedlings we detected around 8.9 and 14.1 times less and in gERECTA 3.1 and 1.2 times more ERECTA compared to the wild type (Fig. 2A). Similar to the cERECTA lines, the cERECTA-RLUC lines produced ERECTA mRNA above the erecta-105 level (Fig. 2B). A RT-PCR analysis with primers annealing upstream of the first ATG and downstream from the stop codon demonstrated that the length of the ERECTA-RLUC coding region was of the same size in $C E R E C T A-R L U C$ and gERECTA-RLUC transgenic plants and only one species of mRNA was present (Supplemental Fig. S2). The sequencing results confirmed that the sequence of $c E R E C T A-R L U C$ and gERECTA-RLUC mRNA is identical and the presence of introns does not result in alternative splicing.

By measuring luciferase activity, we detected a high level of ERECTA-RLUC protein in the gERECTA-RLUC lines (Fig. 2C). Two cERECTA-RLUC lines exhibited statistically significantly higher luciferase activity compared with that of the wild type, suggesting that they also produced some ERECTA-RLUC protein (Fig. 2C). However, there was 486941 times less protein in the $c E R E C T A-R L U C$ lines compared with the gERECTA-RLUC plants (Fig. 2B), which is considerably larger than the difference in mRNA levels (11-43 times), suggesting that the small amount of $c E R E C T A-R L U C$ mRNA produced in transgenic plants is not translated efficiently.

\section{The mRNA produced by the intronless ERECTA gene is less stable at the $3^{\prime}$ end}

The difference in mRNA accumulation could be due to a different rate of transcription initiation/elongation or unequal mRNA stability. As mRNA degradation often begins with deadenylation at the $3^{\prime}$ end followed by degradation in a $3^{\prime} \rightarrow 5^{\prime}$ direction by the cytoplasmic exosome (Houseley and Tollervey 2009), we checked whether there is any difference in the stability of the $3^{\prime}$ end of $C E R E C T A$ and gERECTA mRNA.
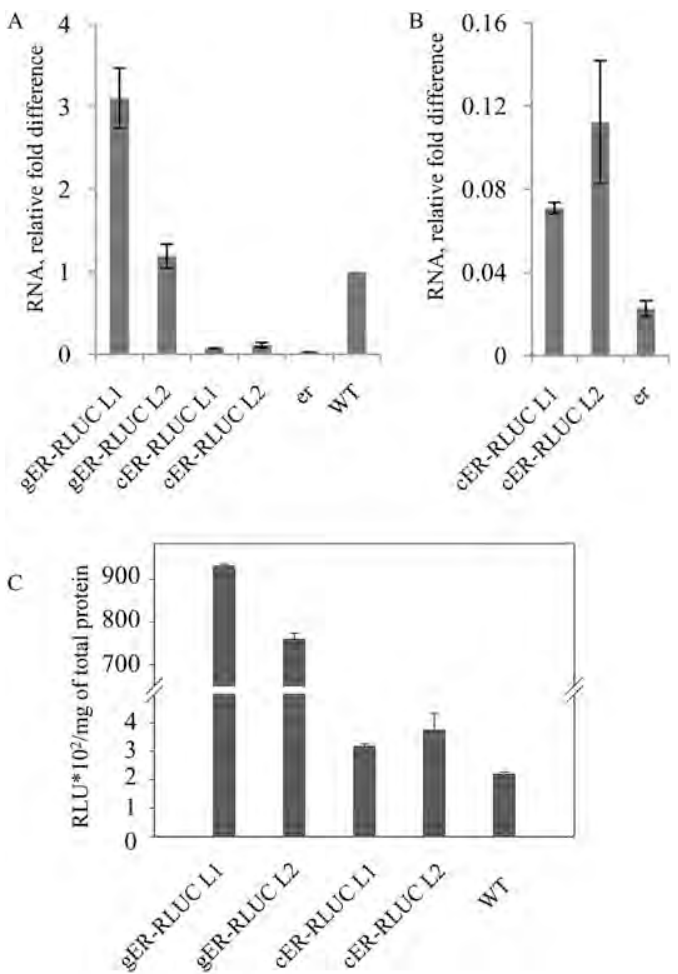

FIGURE 2. The cERECTA-RLUC gene produces 11-43 times less mRNA and 486-941 times less protein compared with the gERECTARLUC gene. $(A, B)$ The amount of ERECTA mRNA in ER::cERECTA$R L U C$ plants is dramatically lower compared with the wild type and ER::gERECTA as determined by qRT-PCR with ACTIN-2 as an internal control. The total RNA was isolated from T5 seedlings of two independent transgenic lines. Graph $B$ demonstrates that ERECTA is expressed at a higher level in ER::cERECTA-RLUC plants compared with the erecta105 mutant. The mean of two biological replicas is plotted; error bars, 1 SD. (C) Measurements of luciferase activity per milligram of total protein isolated from seedlings. RLU indicates relative light units. The mean of three biological replicates is plotted; error bars, $1 \mathrm{SD}$.

Semiquantitative RT-PCR analysis with primers annealing at different regions along the length of ERECTA or ERECTA$R L U C$ cDNA suggested that the $3^{\prime}$ end is less stable in both cERECTA and cERECTA-RLUC compared with gERECTA and $g E R E C T A-R L U C$ (Fig. 3). When we used primers annealing closer to the $3^{\prime}$ end of ERECTA, there was a higher discrepancy in mRNA accumulation in the $C E R E C T A$ versus gERECTA lines compared with when we used primers that annealed closer to the $5^{\prime}$ end (Fig. 3B). We observed the same phenomena in the $c E R E C T A-R L U C$ versus gERECTARLUC transgenic lines (Fig. 3C). The decreased stability of the $3^{\prime}$ end was not dependant on a particular 3' UTR sequence as two different UTRs were used in the ERECTA and ERECTA-RLUC constructs (Fig. 3A).

The ligation-mediated poly(A) test (LM-PAT) (Salles and Strickland 1999) and RT-PCR analysis of 5' to $3^{\prime}$ end-ligated mRNAs (Kuhn and Binder 2002) were used to examine the presence and length of the poly(A) tail. LM-PAT detected a poly(A) tail in the gERECTA-RLUC lines but not in 

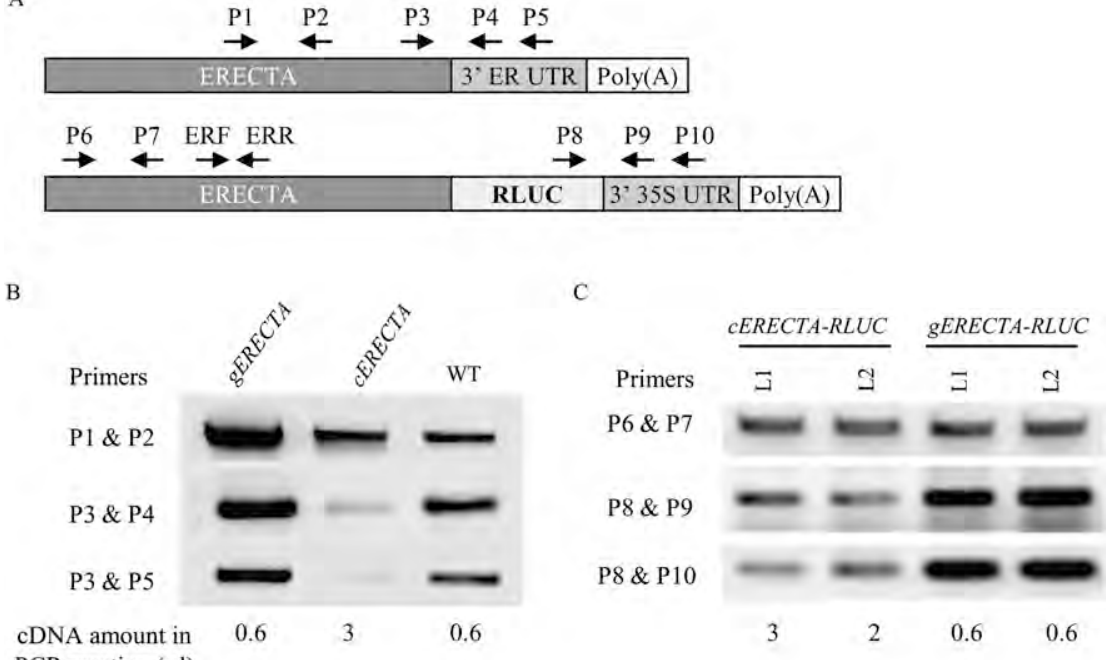

The binding of specific mRNAs to the oligo-dT primer depends on the polyadenylation status of an individual RNA, where mRNAs with a longer poly $(\mathrm{A})$ tail bind more efficiently than do those having a shorter tail. To determine whether mRNA produced by the intronless ERECTA-RLUC gene is efficiently polyadenylated or not, we measured the mRNA level with qRT-PCR before and after selection for polyadenylated mRNAs (Fig. 4B). The relative fold difference of cERECTA-RLUC to ACTIN-2 did not change significantly after selection for polyadenylated mRNAs, suggesting similar levels of polyadenylation for these two genes. In contrast, the ratio of gERECTA$R L U C$ to $A C T I N-2$ increased five to seven times after selection, indicating a higher level of gERECTA-RLUC polyadenylation compared with both $c E R E C T A-R L U C$ and $A C T I N-2$. These data imply that cERECTA-RLUChas a poly(A) tail similar to ACTIN-2 but that gERECTA-RLUC is polyadenylated more efficiently, which can mean both a longer $\operatorname{poly}(\mathrm{A})$ tail or a higher percentage of mRNA molecules that have a $\operatorname{poly}(\mathrm{A})$ tail. To our knowledge, the length of the poly(A) tail in Arabidopsis ACTIN-2 mRNA is not known. Our attempts to determine it by LM-PAT were not successful. Therefore, introns

cERECTA-RLUC (Fig. 4A). The measured length of the poly(A) tail in $g E R E C T A-R L U C$ was up to $120 \mathrm{nt}$ based on the size of the amplified fragment. Sequencing confirmed that the poly $(\mathrm{A})$ addition site is $13 \mathrm{nt}$ downstream from the AATAAA sequence of the $35 S$ terminator as reported earlier (Sanfaçon et al. 1991), and we concurringly detected 120-130 adenines. LM-PAT assays were not successful in determining the length of the poly(A) tail in gERECTA, possibly due to the shortness of the tail. But sequencing of a fragment obtained in a RT-PCR reaction with the ERg7044 and PAT.rc primers indicated a tail of 17-25 adenines located $184 \mathrm{nt}$ downstream from the stop codon after the sequence GTTTTTCT. RT-PCR analysis of 5' to $3^{\prime}$ end-ligated mRNAs identified the same site of polyadenylation in endogenous ERECTA and the same length of a poly(A) tail. Multiple ERECTA ESTs possess the same poly(A) addition site; however, there are also two additional poly $(\mathrm{A})$ addition sites 155 and 169 nt downstream from the stop codon that we did not observe. We were unable to detect any poly(A) tail in cERECTA or $c E R E C T A-R L U C$ by LM-PAT. In addition, RT-PCR analysis of $5^{\prime}$ to $3^{\prime}$ end-ligated mRNAs was not successful in determining the $3^{\prime}$ end sequence of those mRNAs, suggesting a heterogeneous nature of the $3^{\prime}$ end. in the ERECTA gene might increase mRNA accumulation through increasing its stability at the $3^{\prime}$ end, and in this model, their function would resemble the role introns play in mammalian $\beta$-globin gene expression, where mRNA expression is enhanced by splicing and splicing is necessary for efficient $3^{\prime}$ end processing ( $\mathrm{Lu}$ and Cullen 2003).

\section{The length of the poly(A) tail is important for ERECTA translation in vitro}

Since our analysis demonstrated that endogenous ERECTA has a fairly short $\operatorname{poly}(\mathrm{A})$ tail, we investigated whether the extent of ERECTA polyadenylation is critical for its translation and whether a short poly $(\mathrm{A})$ tail might be one of the reasons for low protein expression. To verify whether the poly (A) tail is necessary for ERECTA translation, an $\mathrm{RNaseH}$ treatment in the presence of the oligo-dT primer was used to remove it from gERECTA-RLUC mRNA and other mRNAs in the total RNA sample. RNaseH specifically degrades the RNA in RNA:DNA hybrids and does not degrade unhybridized RNA. Removal of the poly(A) tail completely abolished gERECTA-RLUC translation in vitro in competitive conditions (Fig. 5A). ERECTA-RLUC RNA produced by in vitro 


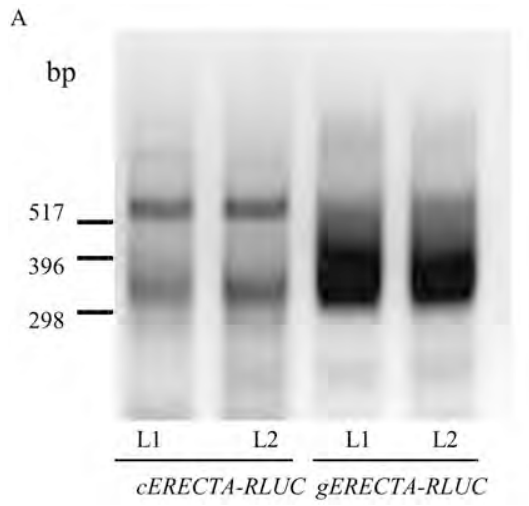

B

Total RNA

RNA eluted from oligo(T) beads
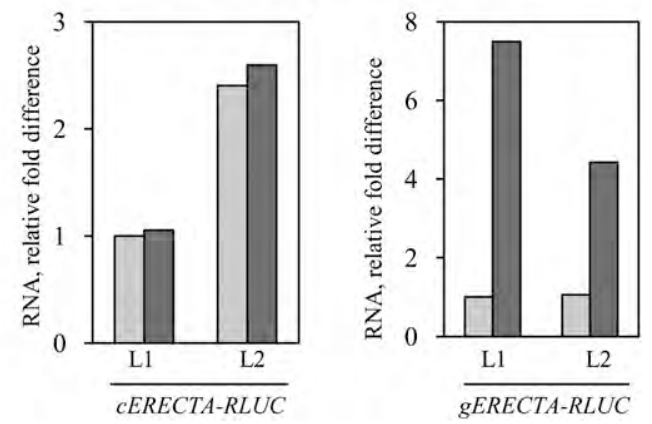

FIGURE 4. cERECTA-RLUC mRNA has a significantly lower level of polyadenylation compared to gERECTA-RLUC. (A) Poly(A) tail-length assay of total RNA detected about 120 adenines (the amplified fragment is $\sim 400$ bp long, of which 280 bp encodes a segment of RLUC, the 3' UTR sequence, and the oligo-dT anchor primer) at the end of gERECTA-RLUC but none in cERECTA-RLUC. The sequencing determined that the two weak bands in $c E R E C T A-R L U C$ are unspecific products of amplification. The identity of the major band and the poly(A) length in the gERECTA-RLUC sample was confirmed by sequencing. (B) ERECTA-RLUC to ACTIN-2 ratio before and after elution from oligo(dT) beads as determined by qRT-PCR. The selection for polyadenylated mRNAs does not change $c E R E C T A-R L U C / A C T I N-2$ ratio but significantly increases the gERECTA-RLUC/ ACTIN-2 ratio. RNA was isolated from T3 homozygous seedlings. The mean of three technical replicas is plotted.

transcription does not have a poly(A) tail, but it is efficiently translated in our reaction, most likely because it does not have to compete for ribosomes with other mRNAs. The fact that its translation is not changed by oligo-dT/RNAseH treatment indicated that this treatment did not lead to internal degradation of ERECTA-RLUC mRNA (Fig. 5B).

Next, we examined whether lengthening the poly $(A)$ tail can increase cERECTA-RLUC and gERECTA-RLUC in vitro translation. For this analysis we used mRNA that was eluted from an oligo-dT column and already had some poly(A) tail. After treatment with poly(A) polymerase, $c E R E C T A-R L U C$ and $g E R E C T A-R L U C$ were translated 7.5 and 2.3 times better, respectively, compared with untreated samples (Fig. 5C). Thus, the length of a poly(A) tail is critical for ERECTA translation in competitive conditions in vitro, and the decrease in $c E R E C T A-R L U C$ translation in transgenic plants is likely the result of changes in the $3^{\prime}$ end mRNA structure.

\section{The sequence in front of the ERECTA gene functions as a strong promoter}

The first intron of ERECTA is larger in size, at $346 \mathrm{nt}$, than the other introns at 69-164 nt. Introns located near the translational start site are known to alter transcription (Callis et al. 1987; Gidekel et al. 1996). However, inclusion of the first intron in the ERECTA cDNA (the construct pESH212) did not result in complementation of erecta-105 based on phenotypic analysis of multiple T1 plants (Table 1; Fig. 6A). Moreover, analysis of ERECTA expression by a promoter GUS construct (Shpak et al. 2004) was consistent with in situ hybridization (Yokoyama et al. 1998) and microarray data (analysis of available data by Genevestigator and eFP browser) suggesting that elements upstream of the translational start site are sufficient for proper ERECTA expression. ERECTA promoter was successfully used for expres- sion of CLAVATA1 and LhG4 in Arabidopsis (Dievart et al. 2003; Song et al. 2006) as it is considered to be a relatively strong promoter for shoot meristematic tissues. In addition, replacement of the ERECTA promoter with the $35 \mathrm{~S}$ promoter in front of the $c E R E C T A$ sequence does not result in a functional construct (data not shown). All these data suggest that introns might be important for transcription elongation, $3^{\prime}$ end formation, or mRNA stability but not for transcription initiation.

\section{While the presence of introns is important, no individual intron is absolutely necessary for ERECTA expression}

Of the 26 introns in the ERECTA gene, 19 separate exons encoding individual leucine-rich repeats (LRRs) (Torii et al. 1996). To determine which specific introns are essential for ERECTA function, we expressed several constructs with varying sets of introns in erecta-105 and analyzed the phenotype of T1 transgenic plants (Table 1; Fig. 6A). The last three introns separate exons that encode the kinase domain of ERECTA. Their removal does not decrease the functionality of the construct (pKUT 199), nor does their inclusion into cERECTA (pDLH 101) restore the construct's complementation capability. However, the presence of introns between exons encoding LRRs was significant for the functionality of the ERECTA gene. Two constructs, one with introns 1-14 (pESH210) and the other with introns 15-23 (pESH217), were able to rescue the erecta-105 mutant (Fig. 6A,B), although the first construct was slightly less effective (Fig. 6D). These data show that none of the individual ERECTA introns are absolutely obligatory for the functionality of the gene.

Further removal of introns from both groups reduced or abolished the ability of constructs to complement the 

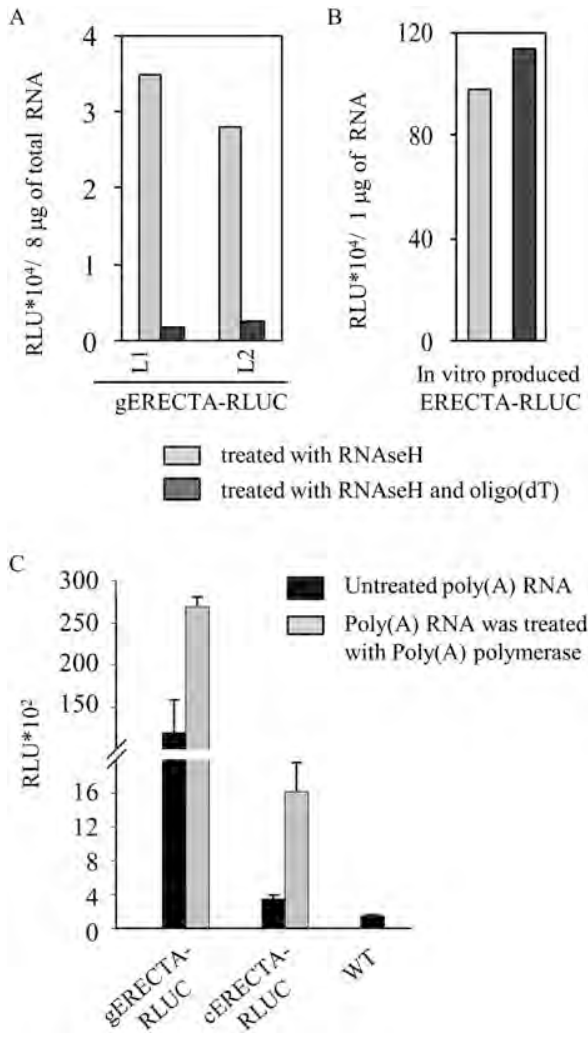

FIGURE 5. The length of the poly(A) tail is important for ERECTA translation in vitro. $(A, B)$ Removal of the poly(A) tail decreased ERECTA translation in vitro in competitive conditions. $(A)$ When the poly(A) tail is removed by oligo(dT)/RNAseH treatment, gERECTARLUC mRNA is not translated in vitro. (B) RNAseH does not change translation of ERECTA-RLUC RNA produced in vitro without a poly(A) tail in noncompetitive conditions. $(C)$ Elongation of the poly(A) tail increases in vitro translation of both $c E R E C T A-R L U C$ and gERECTA-RLUC. Plant produced mRNA eluted from oligo(dT) beads was used as a starting material.

mutation. For example, while a construct with the first 14 introns (pESH 210) was able to complement the mutant, constructs with introns $1-10$ (pESH 222) or with 11-14 (pESH 221) were not able to do so (Table 1). Our data also suggest that a functional ERECTA gene is not characterized by the presence of a minimal number of introns but by the presence of introns in the correct locations. For example, ERECTA with five introns (introns 19-23; pESH 225) rescued the mutant, while ERECTA with 10 introns (introns 1-10; pESH222) did not (Fig. 6A).

We chose two constructs, pESH 210 and pDHL 101, for further analysis in T3 generation. In T1and T2 generations, the first one partially complemented erecta and the second did not. Analysis of mRNA expression in T3 transgenic seedlings established that the ability of constructs to complement the erecta mutant (Fig. 6B,D) is correlated with mRNA abundance (Fig. 6C), just as in the case with the $c E R E C T A$ and gERECTA constructs (Fig. 1B,D), thus again confirming the importance of introns for mRNA accumulation. These data suggest that ERECTA expression depends on the presence of introns in multiple specific locations and that introns function in an additive manner to ensure a high level of mRNA accumulation.

\section{Expression of individual ERECTA exons is dependent on the presence of an intron}

A search for introns that are required for ERECTA expression through generation of transgenic plants proved to be very time consuming. So a different approach was used to dissect the individual introns that can affect ERECTA expression. We created constructs in which different segments of the ERECTA gene with or without introns were fused with Renilla luciferase and placed under control of $35 S$ promoter and terminator. These constructs, together with a construct encoding firefly luciferase (FLUC; as an internal control), were transiently expressed in Arabidopsis seedlings. By measuring luciferase activity in protein extracts, the expression of constructs with introns was compared to expression of corresponding constructs without introns.

This assay demonstrated that small segments of the ERECTA gene can change their expression in an introndependent manner (Fig. 7A). While the expression level of one construct (exons 23-27) was not affected by intron presence, the expression of a majority of the constructs was. For example, the expression of exons 17-21 and exons 2-7 increased 11 times when they were separated by introns. In other cases, the presence of introns moderately increased the expression of constructs: Introns increased expression of exons 8-13 1.6 times and of exons 14-19 2.6 times. The results of this experiment were similar to the data we obtained from the analysis of transgenic plants. Thus, introns 23-26 did not confer functionality to ERECTA (pKUT199) (Table 1) and were not able to increase expression of exons 23-27 (Fig. 7A). A construct with introns 19-23 (pESH225) (Table 1) was able to complement the erecta-105 mutant, and introns increased expression of constructs with exons 17-21 and exons 20-23 (Fig. 7A).

More constructs were created with the intent of finding the smallest segment with expression dependent on the presence of an intron; some of them are presented in Figure 7B. In general, decreasing the size of constructs reduced the difference in expression with and without introns. For example, the presence of introns in the construct with exons 20-23 increased its expression 5.5 times (Fig. 7A). Breaking down this segment of ERECTA into smaller pieces containing only two exons (constructs with exons 20 and 21, 21 and 22, or 22 and 23) reduced the effect to only a 2-2.8 times boost of expression (Fig. 7B). In no cases have we observed that the inclusion of an intron in a construct leads to decreased expression. Further analysis of the construct with exons 22-23 demonstrated that the increased expression imparted by an intron occurs at both the protein (Fig. 7D) and mRNA levels (Fig. 7E) and, in both cases, leads to roughly doubling the expression. 
TABLE 1. Ability of an ERECTA gene with varying number of introns to complement the erecta-105 mutant

\begin{tabular}{|c|c|c|c|c|c|}
\hline $\begin{array}{c}\text { ERECTA gene structure in the } \\
\text { construct }\end{array}$ & $\begin{array}{c}\text { Introns in } \\
\text { the } \\
\text { construct }\end{array}$ & $\begin{array}{c}\text { Intron } \\
\text { count }\end{array}$ & $\begin{array}{l}\text { Ability to } \\
\text { rescue } \\
\text { the } \text { erecta } \\
\text { mutation }\end{array}$ & $\begin{array}{c}\text { \# of fully or } \\
\text { partially } \\
\text { complemented } \\
\text { plants/total T1 } \\
\text { plants analyzed }\end{array}$ & $\begin{array}{c}\text { Name of the } \\
\text { construct }\end{array}$ \\
\hline 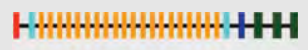 & $1-26$ & 26 & +++ & $14 / 14$ & pKUT 196 \\
\hline I & 0 & 0 & - & $0 / 43$ & pKUT 524 \\
\hline H & 1 & 1 & - & $0 / 30$ & pESH 212 \\
\hline rwhwhen & $1-23$ & 23 & +++ & $20 / 20$ & pKUT 199 \\
\hline 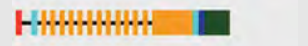 & $1-14$ & 14 & ++ & $27 / 29$ & pESH 210 \\
\hline lwhum & $1-10$ & 10 & - & $0 / 38$ & pESH 222 \\
\hline whe & $11-14$ & 5 & - & $0 / 36$ & pESH 221 \\
\hline Whuth & $11-23$ & 13 & +++ & $32 / 36$ & pESH 218 \\
\hline whwh & $15-23$ & 9 & +++ & $29 / 36$ & pESH 217 \\
\hline W & $15-18$ & 4 & - & $0 / 38$ & pESH 224 \\
\hline $\mathrm{HW}$ & $19-23$ & 5 & ++ & $54 / 54$ & pESH 225 \\
\hline 배배 & $24-26$ & 3 & - & $0 / 27$ & pDLH 101 \\
\hline
\end{tabular}

Exons are represented as boxes and introns as lines in the structure of created ERECTA constructs. Red, blue, orange, dark blue, and green boxes represent exons encoding a signal sequence, segments with paired cysteines, LRR repeats, a transmembrane domain, and a kinase domain, respectively. The size of exons and introns boxes is scaled. The ability to rescue the erecta mutation is as follows: +++ , complete complementation; ++ , partial complementation (final plant height and pedicel length are intermediate between those of wt and erecta-105); and -, no complementation.

present. We examined exons 22 and 23 to gain some insight into the mechanism by which introns increase gene expression. First, we hypothesized that some aspect of splicing is important for ERECTA expression, but not the nature of the intron. Consistent with this hypothesis, intron 13 , which does not change expression of exons 13 and 14, increased the expression of exons 22 and 23 to the same degree as did intron 22 (Fig. 7C). We then searched for an expression inhibiting sequence that requires the presence of an intron. Replacement of exon 22 with exon 13 abolished dependence of construct expression on the presence of an intron $(13+23)$ (Fig. 7C), while replacement of exon 23 with exon 14 did not $(22+14)$ (Fig. 7C), suggesting that an inhibiting sequence might be located in exon 22. Furthermore, placing the intron in front of exon 22 did not increase expression of the construct $(23+$ 22) (Fig. 7C), suggesting that a splice site needs to be located behind a putative inhibiting sequence. We hypothesize that exon 22 has a sequence that is able to inhibit some process that is important for mRNA accumulation, such as transcription elongation, $3^{\prime}$ end formation, or post-transcriptional mRNA stability. The assembly of spliceosome or deposi-

Based on our data, we were able to analyze whether the ability of ERECTA introns to promote gene expression correlates with their IMEter score (Rose et al. 2008). Several ERECTA introns gave a positive IMEter score: intron 1 (score 24), intron 16 (6.4), intron 2 (6), intron 19 (4), intron 26 (3.5), intron 15 (1.3), and intron 4 (0.3). All other introns had a negative score. These scores did not correspond to the introns' ability to enhance gene expression. While introns 20 , 21 , and 22 had a high ability to enhance expression in our experimental system, they had negative IMEter scores.

Our data show that multiple introns increase ERECTA expression in an additive manner and that the presence of introns is significant for mRNA accumulation. However, not all introns are able to influence ERECTA expression. The placement of introns between exons 23-26, 4-5, or 13-14 does not alter expression of the corresponding constructs.

\section{The ability of ERECTA introns to increase expression is not sequence specific}

As most Arabidopsis genes do not require introns to be expressed, we reasoned that there may be sequences in the ERECTA exons that inhibit expression unless introns are tion of EJC proteins might somehow obstruct the function of such a sequence.

\section{DISCUSSION}

The reduced ability of cDNA constructs to complement mutants, especially when expressed under their native promoters, is a widespread, but unfortunately not well documented, phenomenon due to the negative result nature of these experiments. In pursuit of increased transgene expression, an intron is routinely inserted into commercially available mammalian expression vectors, but it is not known whether its presence in these vectors increases expression of the majority of cDNA inserts or improves expression only under selected circumstances. Deeper understanding of intron involvement in regulation of gene expression is critical both for the basic knowledge of gene function and, at a practical level, for increased protein production in research and industry.

Here, we examined the function of introns in the regulation of ERECTA gene expression. The vital role introns play in ERECTA expression makes this gene an excellent model system. The intronless ERECTA gene is not able to rescue the 

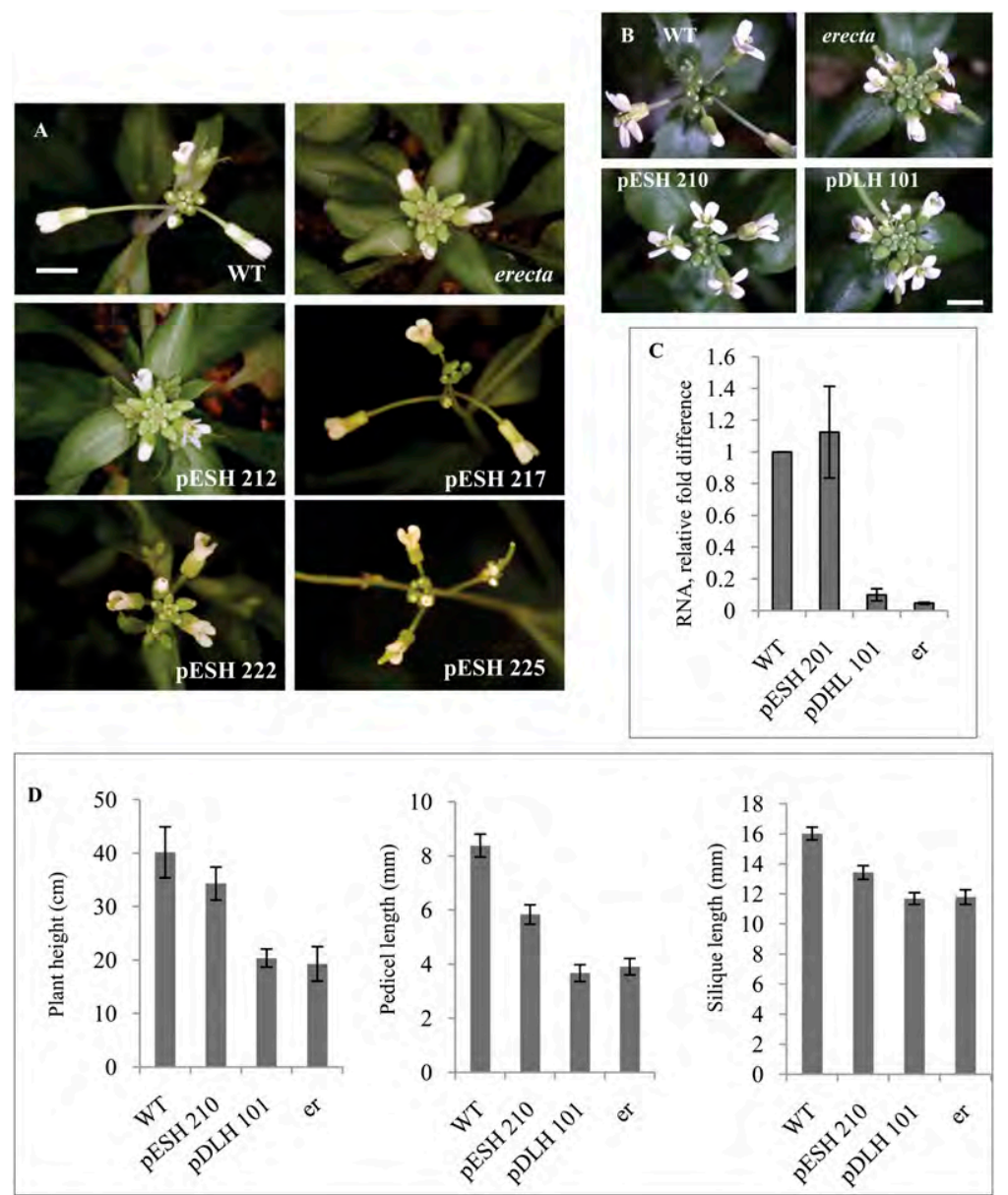

FIGURE 6. The presence of multiple introns is critical for ERECTA gene function and mRNA accumulation, but no particular intron is essential. $(A, B)$ Complementation of the erecta-105 mutant with ERECTA constructs containing selected introns; bar, $3 \mathrm{~mm}$. The structure of constructs is described in Table 1 . (C) The ability of a construct to complement erecta-105 correlates with the amount of mRNA produced. qRT-PCR analysis demonstrates that pDLH101 plants produce dramatically less ERECTA mRNA and pESH201 plants produce an equal amount compared with the wild type. The total RNA was isolated from T3 gentamicin selected seedlings. $(D)$ Morphometric analysis of fully grown 8 -wk-old plants of the wild type, erecta-105, and of T3 transgenic plants expressing pDLH101 and pESH210 in an erecta-105 background. Twenty-one plants were analyzed for plant (inflorescence) height. Lengths of 40 mature pedicels and siliques on the main inflorescence stem (eight measurements per stem) were analyzed. Bars, average values $\pm 1 \mathrm{SD}$.

erecta mutant, and it produces at least 10 times less mRNA compared with the endogenous gene. The endogenous ERECTA protein is produced in very small amounts that are difficult to detect, but by fusing ERECTA with Renilla luciferase, we were able to study protein accumulation. We observed that the intronless ERECTA gene generates 500-900 times less protein compared with an identical construct containing introns. Therefore, the presence of introns in the ERECTA gene is required both for mRNA accumulation and for its efficient translation. Inefficient translation of mRNA produced by the intronless gene is likely due to its degradation at the $3^{\prime}$ end, as we were unable to detect the poly(A) tail and found decreased stability of the $3^{\prime}$ UTR. This instability is not related to the structure of the $3^{\prime}$ UTR as we detected it using both the endogenous ERECTA 3' UTR and the $35 S$ terminator.

Having observed defects of the poly(A) tail, we investigated the importance of poly(A) for ERECTA translation. Normally, the Poly(A)-Binding Protein complex (PABP) binds the poly(A) tail and has a stimulatory effect on translation due to its interaction with eIF4G (Le et al. 1997; Imataka et al. 1998), a part of the cap-binding complex that mediates the attachment of the $43 \mathrm{~S}$ pre-initiation complex. PABP-eIF4G interactions confer an advantage under competitive conditions, ensuring a stronger association of the cap binding complex with mRNA (Proweller and Butler 1997; Borman et al. 2000). Removal of the poly(A) tail from ERECTA mRNA demonstrated that its presence was critical for ERECTA translation in competitive conditions in vitro. In accordance, elongation of the poly $(\mathrm{A})$ tail enhanced translation of ERECTA, and this effect was stronger for mRNA produced by the intronless gene, suggesting an altered nature of its tail.

The decreased accumulation of ERECTA mRNA produced by the intronless gene theoretically can be due to impairment in transcription initiation or elongation, inefficient formation of the $3^{\prime}$ end, or formation of defective mature ribonucleoparticles (mRNPs) that are less stable. The high activity of the ERECTA promoter suggests that it is able to successfully initiate transcription on its own. While hypothetically it is possible that the ERECTA exons carry multiple elements that negatively regulate transcription initiation and that the presence of introns can counterbalance them, this possibility seems to be far-fetched. Thus, we presume that inefficiency in the later steps of biogenesis of mRNPs is responsible for reduced expression of the intronless ERECTA gene. Not only does the intronless ERECTA gene produce less mRNA, but the mRNA produced has a deficient $3^{\prime}$ end. As polyadenylation of mRNA is known to be important for mRNA stability by protecting mRNA from degradation by $3^{\prime}$ exonuclease (Houseley and Tollervey 2009), its decline could be one of the reasons for decreased ERECTA accumulation. However, other scenarios involving abortive transcription or changed composition of mRNPs leading to mRNA degradation are possible as well.

While multiple introns promote ERECTA expression in an additive manner, none of the introns is essential. Introns 

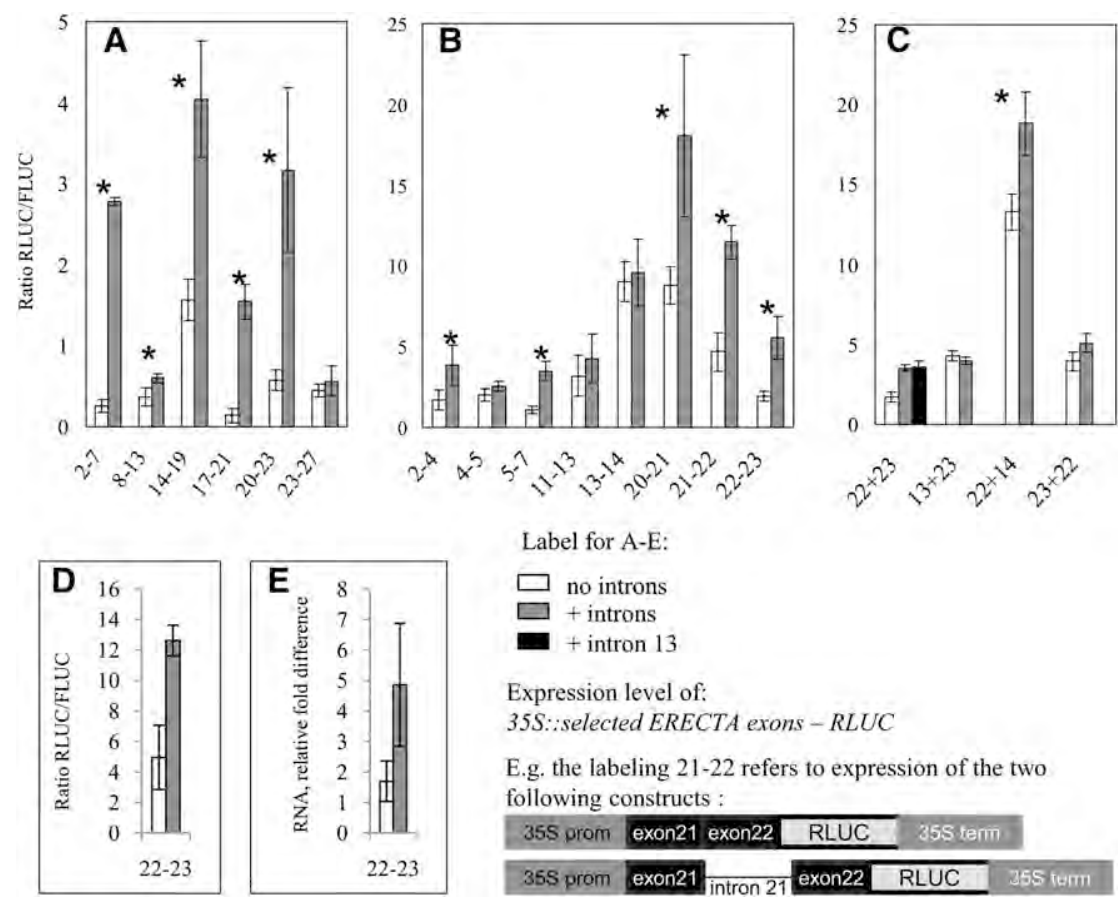

Label for A-E:

$$
\begin{aligned}
& \square \text { no introns } \\
& \text { + introns } \\
& + \text { + intron } 13
\end{aligned}
$$

Expression level of:

35S: :selected ERECTA exons - RLUC

E.g. the labeling 21-22 refers to expression of the two following constructs :

355 prom exon21 exon22 RLUC 355 term

355 prom exon21 intron 21 exon22 $\quad$ RLUC $35 S$ form

FIGURE 7. The level of expression for some ERECTA exons depends on the presence of introns. $(A-C)$ RLUC/FLUC luminescence ratio measured in protein extracts from transiently transformed Arabidopsis seedlings expressing selected ERECTA exons fused to RLUC (35S::ERECTA exons-RLUC) and FLUC as an internal control. The level of protein expression is compared for constructs encoding identical proteins but distinguished by the presence or absence of introns. ${ }^{\star} P<0.05$ based on Student $t$-test. $(D, E)$ The higher expression of introncontaining exons $22+23$-RLUC construct versus the identical construct but without intron is evident on both protein $(D)$ and mRNA $(E)$ levels. The amount of mRNA was determined by qRT-PCR with FLUC as an internal control and amount of protein by measurements of RLUC/FLUC luminescence ratio. The mean of three biological replicates is plotted; error bars, 1 SD for $A$ though $E$.

retain the ability to promote gene expression even when small segments of the ERECTA gene are expressed under control of the $35 S$ promoter. Our data suggest that it is not the sequence of introns but their specific location that is essential for efficient gene expression. It has been proposed that in plants, promoter proximal introns are enriched in signals that enhance gene expression (Rose et al. 2008). However, many ERECTA introns that contribute to a high level of expression are not promoter proximal and do not have a high IMEter score.

Here, we propose an additional mechanism for IME in plants. Our data suggest that the ability of introns to promote ERECTA gene expression might be independent of their sequence and instead be related to recruitment of spliceosomal or EJC proteins. These proteins are capable of influencing multiple stages of mRNA biogenesis. For example, the assembly of a spliceosome might promote transcription elongation or efficient cleavage and polyadenylation of the ERECTA 3' end. Alternatively, due to their association with EJC proteins, spliced mRNAs might have a different rate of cytoplasmic polyadenylation/deadenylation and, as a result, experience increased stability.
Recently it has been reported that ERECTA expression is decreased in an emu mutant (Furumizu et al. 2010). EMU encodes a protein homologous to yeast HPR1, a component of the THO complex. This complex plays a role in mRNA biogenesis and is needed for efficient transcriptional elongation, $3^{\prime}$ end processing, and mRNA export (Rondon et al. 2010). It is recruited to genes during transcription and is involved in formation of optimal mRNPs. In yeast, THO mutant phenotypes are suppressed by slowing down transcription (Jimeno et al. 2008), and THO might be critical for highly expressed genes and genes that require a fast assembly of mRNPs. Whether THO functions in an analogous manner in plants and what its role is in ERECTA expression are interesting questions for future research. One possibility is that both splicing and THO contribute to efficient assembly of ERECTA mRNPs that are critical for this gene's expression.

Why does ERECTA expression depend so strongly on the presence of introns? While it is hypothesized that for most mammalian genes efficient $3^{\prime}$ end formation and export from the nucleus relies on recruitment of spliceosome (Guang and Mertz 2005), this is obviously not the case for plants. Plants have a much higher proportion of intronless genes $(\sim 20 \%$ in Arabidopsis versus $\sim 5 \%$ in $H$. sapiens), and the removal of introns alters gene expression only in selected cases. Thus, the formation of the $3^{\prime}$ end and mRNA export from nucleus should be less dependent on splicing for the majority of plant genes. We speculate that exon sequences of some plant genes, including ERECTA, have cis-elements preventing efficient $\mathrm{mRNP}$ biogenesis. For example, exons might have sequences that inhibit transcription elongation, titrate out polyadenylation factors, or lead to mRNA instability. During splicing these sequences could be concealed by spliceosomal proteins or by an EJC. The degree to which gene expression depends on the presence of introns could hinge on the number and strength of those sequences. Based on the facts that the ERECTA gene functions best when multiple introns are present and that the degree of complementation of the erecta mutant increases gradually with intron number, we expect to find multiple sequences in exons inhibiting mRNPs biogenesis. So far we have not identified such elements by exon sequences analysis. On the one hand, at this point we do not have complete information about which exons might carry such sequences, and on the other hand, the predicted 
RNA cis-acting elements might be determined partially by secondary structure.

The ERECTA gene has a rather distinctive structure that is shared by many other Arabidopsis LRR receptor-like kinases. Of the 27 exons in the ERECTA gene, 20 encode tandem LRRs of 24 amino acids each. Every LRR is encoded by a separate exon, and introns of similar sizes are present at the exact same "phase 2" position, between the second and third nucleotides of the codon for leucine at position 13 (underlined) in the consensus $P^{\star \star} L G^{\star} L^{\star \star} L^{\star \star} L^{\star} L^{\star \star} N^{\star} L^{\star} G^{\star} I$ (asterisks represent nonconserved amino acids) (Torii et al. 1996). The identical gene structure is found in the Arabidopsis ERL1 and ERL2 paralogs of ERECTA, as well as ERECTA genes in other species as evolutionarily distant as Physcomitrella patens (data not shown). Analysis of the published gene structure of 220 Arabidopsis LRR receptor-like kinases (Shiu and Bleecker 2003) revealed that about half of them (111) did not possess a single intron in the gene segment encoding LRRs. The other half (93) had most LRRs encoded by separate exons with introns placed in exactly the same position as in ERECTA, between the second and the third nucleotides of the codon for leucine that is analogous to Leu13 in ERECTA. The consistent and repeated location of introns in LRR-containing receptors suggests that they may have evolved by exon duplication. In some genes, introns might have later become functionally important and involved in regulation of gene expression. An analysis of IME occurrence in ERECTA family genes in Arabidopsis and other species and in other LRR receptors could help determine how common IME is in these gene families.

It is also possible that the importance of introns for ERECTA expression is gene specific and related to ERECTA function in the regulation of cell proliferation (Shpak et al. 2003, 2004; Pillitteri et al. 2007). Endogenous ERECTA mRNA has a poly(A) tail of 17-25 adenines. The shorter length of the poly(A) tail in mRNAs that regulate the cell cycle and a specific control of its deadenylation has been proposed to be responsible for delimiting their expression during the cell cycle (Beilharz and Preiss 2007). In addition to the $3^{\prime}$ UTR, the presence of introns in ERECTA might influence the length of poly(A) and thus contribute to tight regulation of ERECTA expression.

The ability of ERECTA to regulate plant morphology and transpirational efficiency (Masle et al. 2005) makes it an attractive target of genetic manipulation in crop plants. Expression of ERECTA in erecta-105 or the wild type using the $35 S$ promoter and the endogenous ERECTA terminator rescues the mutant phenotype but does not result in bigger or faster growing plants compared to the wild type (data not shown). In this article, we described several transgenic lines where expression of ERECTA under control of its own promoter and terminator resulted in a three to nine times increase of RNA levels, but plants were still phenotypically identical to the wild type. One possibility is that ERECTA expression is tightly regulated at the post-transcriptional level and that to manipulate plant size one has to address it. For example, expression of ERECTA under control of terminators that are able to promote formation of a longer poly(A) tail might be advantageous for increased protein accumulation. Knowledge of ERECTA mRNP biogenesis will further contribute to our understanding of this gene's expression and might indicate novel means to manipulate its expression and, therefore, plant size.

\section{MATERIALS AND METHODS}

\section{Generation of transgenic plants}

The plasmid carrying gERECTA (pKUT 196) has been described previously (Godiard et al. 2003). To construct a plasmid carrying cERECTA (pKUT 524), the cDNA sequence of ERECTA was cloned as a SpeI fragment into a pZP222 vector carrying the ERECTA promoter and terminator sequences (pKUT 522). For cloning purposes, an NcoI restriction site was introduced in front of the first ATG in pKUT 524. To construct plasmids carrying gERECTARLUC (pESH 427) and cERECTA-RLUC (pWSL123), the Renilla luciferase $(R L U C)$ sequence and the $35 S$ terminator from pAVA 696 (Subramanian et al. 2006) were cloned behind ERECTA into pKUT 196 or pKUT 524, replacing the endogenous ERECTA terminator. The stop codon at the end of the ERECTA sequence was changed to the coding sequence of Ala in pESH427 and to Gly-Ser-Ala in pWSL123.

The structures of the ERECTA gene in the following constructs (pESH 212, pKUT 199, pESH 210, pESH 222, pESH 221, pESH 218, pESH 217, pESH 224, pESH 225, and pDLH 101) are described in Table 1. The backbone of all these plasmids was the vector pZP222, and all constructs carried the endogenous ERECTA promoter and terminator in the same orientation as in pKUT 522. These plasmids were constructed by combining different ERECTA segments from pKUT196 ( $g$ ERECTA) and pKUT524 (cERECTA). We did not introduce any novel restriction sites inside of the ERECTA sequence but, instead, used the endogenous ERECTA restriction sites during cloning.

The described plasmids were introduced into an Agrobacterium tumefaciens strain GV3101/pMP90 by electroporation and into erecta-105 plants by the floral dip method (Clough and Bent 1998). The transgenic plants were selected based on gentamicin resistance. The Arabidopsis erecta-105 knockout mutant (Colombia ecotype) has been described previously (Torii et al. 1996). The conditions for plant growth were as described earlier (Shpak et al. 2003).

\section{RNA isolation and cDNA synthesis}

Total RNA was isolated from 7-d-old seedlings grown on MS plates or from floral buds of plants grown in soil using the Spectrum Plant RNA Isolation Kit (Sigma-Aldrich). The RNA quantification was done by both spectrophotometry and nondenaturing electrophoresis.

Poly(A) mRNA was isolated using the PolyATtract mRNA Isolation System III (Promega) as follows: Total RNA $(\sim 35 \mu \mathrm{g})$ was heat denatured, incubated with a biotinylated oligo-dT probe in the presence of $0.5 \times$ SSC, and then incubated for $15 \mathrm{~min}$ with prewashed MagneSphere streptavidin beads. The beads were 
washed three times with $0.1 \times$ SSC solution before elution of poly(A) mRNA with $35 \mu \mathrm{L}$ of water.

The total RNA was treated with RNAse-free RQ1 DNAse (Promega). First-strand cDNA was synthesized from 1 or $2 \mu \mathrm{g}$ of total RNA or $10 \mu \mathrm{L}$ of eluted poly(A) mRNA with random hexamer primers using a ProtoScript M-MuLV Taq RT-PCR kit (NEB). Use of total RNA and random hexamer primers for cDNA synthesis was significant as it allowed measuring the level of ERECTA mRNA independently of its polyadenylation status. As a control for genomic DNA contamination, we used primers flanking intron-containing regions and analyzed products by gel electrophoresis or used RNA directly for PCR.

\section{qRT-PCR and semiquantative RT-PCR}

qPCR was performed with a MyiQ Real-Time PCR Detection System (BioRad) using Sso Fast EvaGreen Supermix (Bio-Rad). Each experiment contained three technical replicates and was performed in a total volume of $20 \mu \mathrm{L}$ with $2 \mu \mathrm{L}$ of the five times diluted first-strand reaction. Cycling conditions were $1 \mathrm{~min}$ at $95^{\circ} \mathrm{C}$ and then 50 repeats of $30 \mathrm{sec}$ at $95^{\circ} \mathrm{C}, 30 \mathrm{sec}$ at $61.6^{\circ} \mathrm{C}$ for the ERECTA or $59.3^{\circ} \mathrm{C}$ for the ACTIN-2, and $30 \mathrm{sec}$ at $72^{\circ} \mathrm{C}$ followed by the melt curve analysis. The qPCR ERF and qPCR ERR primers were used to amplify ERECTA and ERECTA-RLUC; the sbrActin2F and sbrActin2R primers were used to amplify ACTIN-2 (At3g18780); the Rluc891 and 35Sterm.rc primers were used to amplify ERECTA exons 22-23-RLUC; and the Fluc qPCR F and Fluc qPCR R primers were used to amplify FLUC. The Ct values were calculated using the iQ5 software (Bio-Rad). The fold difference in the gene expression was calculated using relative quantification or the $2^{-\Delta \Delta \mathrm{Ct}}$ method (Livak and Schmittgen 2001).

Semiquantative RT-PCR was used to assay the stability along the length of ERECTA mRNA. PCR was performed for $2 \mathrm{~min}$ at $93^{\circ} \mathrm{C}$ and then with varying numbers of cycles for $30 \mathrm{sec}$ at $93^{\circ} \mathrm{C}$, for $30 \mathrm{sec}$ at $58^{\circ} \mathrm{C}$, for $60 \mathrm{sec}$ at $72^{\circ} \mathrm{C}$, and then for $3 \mathrm{~min}$ at $72^{\circ} \mathrm{C}$. The amount of cDNA used for reaction was as in the figure legend. Sets of primers used are described in the figure legend, and sequences are given in Supplemental Table S1.

\section{Renilla luciferase activity assay}

ERECTA-RLUC protein expression was measured by monitoring Renilla luciferase activity with a 20/20n single tube luminometer in transgenic seedlings using the Renilla Luciferase Reporter Assay (Promega). The protein concentration in each sample was determined using the Bradford reagent (Sigma).

\section{Poly(A) tail length assays}

The LM-PAT assay was performed according the method described by Salles and Strickland (1999). One microgram of total RNA was heat denatured for $7 \mathrm{~min}$ at $65^{\circ} \mathrm{C}$ in the presence of 20 ng of the phosphorylated oligo-dT primer (NEB), and then the ligation reaction was performed for $30 \mathrm{~min}$ at $42^{\circ} \mathrm{C}$ using the following conditions: $1 \times$ Reverse Transcriptase Reaction Buffer (NEB), $1 \mathrm{mM}$ DDT, $2 \mathrm{mM}$ dNTPs, $0.1 \mathrm{mM}$ ATP, and $10 \mathrm{U} \mathrm{T} 4$ DNA ligase (NEB). The ligation was continued for $2 \mathrm{~h}$ at $12^{\circ} \mathrm{C}$ in the presence of $200 \mathrm{ng}$ of the oligo-dT anchor primer (Invitorgen). The first-strand cDNA was synthesized for $1 \mathrm{~h}$ at $42^{\circ} \mathrm{C}$ with $10 \mathrm{U}$ of MuLV-RT (NEB). The amplification reaction was done with the Rluc891 and PAT.rc primers.
The RT-PCR analysis of $5^{\prime}$ to $3^{\prime}$ end-ligated mRNAs was performed according the method described by Kuhn and Binder (2002) with slight modifications. Ten microliters of total RNA was de-capped with $5 \mathrm{U}$ of tobacco acid phosphatase (TAP; Epicentre) in supplied buffer for $2 \mathrm{~h}$ at $37^{\circ} \mathrm{C}$ and then phenol-chloroform extracted, ethanol precipitated, and dissolved in $10 \mu \mathrm{L}$ of water. Five microliters of decapped RNA was $5^{\prime}$ to $3^{\prime}$ end-ligated with $40 \mathrm{U}$ of T4 RNA Ligase 1 (NEB) in supplied buffer supplemented with $1 \mathrm{mM}$ ATP for $24 \mathrm{~h}$ at $4^{\circ} \mathrm{C}$ and then again phenolchloroform extracted, ethanol precipitated, and dissolved in 10 $\mu \mathrm{L}$ of water. The cDNA synthesis reaction was performed with 1-3 $\mu \mathrm{L}$ of the circularized RNA and ERECTA-specific primer ERg2550.rc. The $5^{\prime}$ and $3^{\prime}$ end sequences of mRNA were established by sequencing the amplified fragment obtained in a PCR reaction with the produced CDNA and the ERg7201 and ERg1790.rc primers. To verify that cDNA synthesis was successful, a control PCR reaction with the ERg1761 and ERg2339.rc primers was performed.

\section{In vitro transcription}

The ERECTA gene was amplified by Phusion high-fidelity polymerase (NEB) using the ER-T7 and 35Sterm.rc primers and pWSL123 as a template. The ER-T7 primer contained a T7 promoter in front of the ERECTA 5' UTR sequence. The 35-Sterm.rc primer annealed to ERECTA downstream from the stop codon but upstream of the putative polyadenylation site. PCR product was used for in vitro transcription reactions with T7 RNA Polymerase (NEB) and rNTPs (NEB).

\section{Poly(A) tail removal by RNase $H$ and poly(A) tail elongation}

Total RNA $(8 \mu \mathrm{g})$ from T3 gERECTA-RLUC transgenic plants was heat denatured for $5 \mathrm{~min}$ at $70^{\circ} \mathrm{C}$ and then incubated for 10 min at $42^{\circ} \mathrm{C}$. Poly(A) tail digestion was carried out for $1 \mathrm{~h}$ at $37^{\circ} \mathrm{C}$ in the presence of oligo-dT (NEB) and $5 \mathrm{U}$ of RNAseH (NEB). Two control reactions were performed: the first without addition of oligo-dT and the second with $1 \mu \mathrm{g}$ of in vitro transcribed ERECTA RNA (a poly(A) tail is absent) instead of total RNA. After the digestion, RNA was extracted with phenol-chloroform-isoamyl alcohol (25:24:1), precipitated with ethanol, and then resuspended in $10 \mu \mathrm{L}$ of water. The RNA $(3.25 \mu \mathrm{L})$ was subsequently used for in vitro translation and luciferase activity assay.

Seventeen microliters of poly(A) eluted mRNA (as described above) from T3 gERECTA-RLUC and $c E R E C T A-R L U C$ transgenic plants was incubated with $10 \mathrm{U}$ of poly(A) polymerase (NEB) in the presence of $1 \times$ poly $(\mathrm{A})$ polymerase buffer, $1 \mathrm{mM} \mathrm{ATP}$, and 20-40 $\mathrm{U}$ of RNAse inhibitor in the $25 \mu \mathrm{L}$ volume for $20 \mathrm{~min}$ at $37^{\circ} \mathrm{C}$. Control reactions were performed without the poly(A) polymerase. Then RNA was extracted with phenol-chloroformisoamyl alcohol (25:24:1), precipitated with ethanol in the presence of polyacryl carrier (1:400 dilution; MRC, Inc.), and then resuspended in $10 \mu \mathrm{L}$ of water. The RNA $(3.25 \mu \mathrm{L})$ was subsequently used for in vitro translation and luciferase activity assay.

Prior to the in vitro translation reaction, the RNA was denatured for $3 \mathrm{~min}$ at $65^{\circ} \mathrm{C}$. In vitro translation was done for $90 \mathrm{~min}$ at $30^{\circ} \mathrm{C}$ using a rabbit reticulocyte lysate system (Promega) with total volume of $12.5 \mu \mathrm{L}$. To measure in vitro ERECTA-RLUC 
production, $2 \mu \mathrm{L}$ of translation mixture were used in a Renilla luciferase assay system (Promega).

\section{Transient transformation of Arabidopsis seedlings with plasmids carrying short ERECTA segments fused to Renilla luciferase (RLUC)}

To create plasmids for the transient expression experiment, we amplified short segments of the ERECTA gene using as templates plasmids carrying gERECTA (such as pKUT 196) or cERECTA (such as pKUT524) and cloned these segments in a frame with C-terminal RLUC into NcoI digested pAVA 696 (Subramanian et al. 2006). This cloning placed the fusion proteins under control of the $35 \mathrm{~S}$ promoter and the $35 \mathrm{~S}$ terminator. To create plasmids with novel arrangements of ERECTA introns and exons (such as exon22-intron13-exon23; exon13-intron13-exon23; exon13-exon23; exon22-intron13-exon14; exon22-exon14; exon23-intron22-exon22), we used overlap extension PCR: We generated PCR products encoding individual exons and introns and then placed them together in the PCR reaction, where they annealed and primed off each other to give a full-length product. The sequence of all amplified fragments and their orientation in pAVA 696 were confirmed.

Arabidopsis 10-d-old seedlings were transiently transformed with created vectors and pAVA $315(35 S:: F L U C)$ as an internal control. As we compared expression of fusion proteins from genes that are identical except for the presence or absence of introns, we took special care to use the same amount of plasmids encoding such genes $(1 \mu \mathrm{g}$, the concentration determined by both spectrophotometric measurements and confirmed by gel electrophoresis). For transient transformation, we used $1.1 \mu \mathrm{m}$ tungsten microcarriers (BioRad) and a PSD-1000/He particle bombardment system (Bio Rad). Three independent transformation experiments were performed for each plasmid. Arabidopsis seedlings were harvested $24 \mathrm{~h}$ after bombardment and assayed by the Dual Luciferase Reporter Assay System (Promega) or $16 \mathrm{~h}$ after bombardment and used for RNA extraction. The RLUC activity was divided by the FLUC activity to normalize for transfection efficiencies.

\section{Oligo DNA sequences}

Primer sequences are available as supplementary data (Table S1).

\section{SUPPLEMENTAL MATERIAL}

Supplemental material is available for this article.

\section{ACKNOWLEDGMENTS}

We thank Albrecht von Arnim for comments on the manuscript and DanYu Kong for confirming the sequence of ERECTA-RLUC mRNAs. We also thank Albrecht von Arnim for plasmid constructs (pAVA 696 and pAVA 315). This work was supported in part by a start-up fund from University of Tennessee and in part by the National Science Foundation (IOS-0843340 to E.S.). The initial part of the work was supported by Department of Energy (grant no. DE-FG02-03ER15448 to K.U.T.)

Received May 23, 2011; accepted July 30, 2011.

\section{REFERENCES}

Beilharz TH, Preiss T. 2007. Widespread use of poly(A) tail length control to accentuate expression of the yeast transcriptome. RNA 13: 982-997.

Borman AM, Michel YM, Kean KM. 2000. Biochemical characterization of cap-poly(A) synergy in rabbit reticulocyte lysates: the eIF4G-PABP interaction increases the functional affinity of eIF4E for the capped mRNA 5'-end. Nucleic Acids Res 28: 4068-4075.

Bourdon V, Harvey A, Lonsdale DM. 2001. Introns and their positions affect the translational activity of mRNA in plant cells. EMBO Rep 2: 394-398.

Buchman AR, Berg P. 1988. Comparison of intron-dependent and intron-independent gene expression. Mol Cell Biol 8: 4395-4405.

Callis J, Fromm M, Walbot V. 1987. Introns increase gene expression in cultured maize cells. Genes Dev 1: 1183-1200.

Clancy M, Vasil V, Curtis HL, Vasil IK. 1994. Maize Shrunken-1 intron and exon regions increase gene expression in maize protoplasts. Plant Sci 98: 151-161.

Clough SJ, Bent AF. 1998. Floral dip: a simplified method for Agrobacterium-mediated transformation of Arabidopsis thaliana. Plant J 16: 735-743.

Curi GC, Chan RL, Gonzalez DH. 2005. The leader intron of Arabidopsis thaliana genes encoding cytochrome $c$ oxidase subunit $5 \mathrm{c}$ promotes high-level expression by increasing transcript abundance and translation efficiency. J Exp Bot 56: 2563-2571.

Dean C, Favreau M, Bond-Nutter D, Bedbrook J, Dunsmuir P. 1989. Sequences downstream of translation start regulate quantitative expression of two petunia $r b c S$ genes. Plant Cell 1: 201-208.

Deng T, Li Y, Johnson LF. 1989. Thymidylate synthase gene expression is stimulated by some (but not all) introns. Nucleic Acids Res 17: 645-658.

Diem MD, Chan CC, Younis I, Dreyfuss G. 2007. PYM binds the cytoplasmic exon-junction complex and ribosomes to enhance translation of spliced mRNAs. Nat Struct Mol Biol 14: 1173-1179.

Diévart A, Dalal M, Tax FE, Lacey AD, Huttly A, Li J, Clark SE. 2003. CLAVATA1 dominant-negative alleles reveal functional overlap between multiple receptor kinases that regulate meristem and organ development. Plant Cell 15: 1198-1211.

Ernst RK, Bray M, Rekosh D, Hammarskjold ML. 1997. A structured retroviral RNA element that mediates nucleocytoplasmic export of intron-containing RNA. Mol Cell Biol 17: 135-144.

Fong YW, Zhou Q. 2001. Stimulatory effect of splicing factors on transcriptional elongation. Nature 414: 929-933.

Furumizu C, Tsukaya H, Komeda Y. 2010. Characterization of EMU, the Arabidopsis homolog of the yeast THO complex member HPR1. RNA 16: 1809-1817.

Gidekel M, Jimenez B, Herrera-Estrella L. 1996. The first intron of the Arabidopsis thaliana gene coding for elongation factor $1 \beta$ contains an enhancer-like element. Gene 170: 201-206.

Godiard L, Sauviac L, Torii KU, Grenon O, Mangin B, Grimsley NH, Marco Y. 2003. ERECTA, an LRR receptor-like kinase protein controlling development pleiotropically affects resistance to bacterial wilt. Plant J 36: 353-365.

Guang S, Mertz JE. 2005. Pre-mRNA processing enhancer (PPE) elements from intronless genes play additional roles in mRNA biogenesis than do ones from intron-containing genes. Nucleic Acids Res 33: 2215-2226.

Hocine S, Singer RH, Grünwald D. 2010. RNA processing and export. Cold Spring Harb Perspect Biol 2: a000752. doi: 10.1101/cshperspect. a000752.

Hong RL, Hamaguchi L, Busch MA, Weigel D. 2003. Regulatory elements of the floral homeotic gene AGAMOUS identified by phylogenetic footprinting and shadowing. Plant Cell 15: 12961309.

Houseley J, Tollervey D. 2009. The many pathways of RNA degradation. Cell 136: 763-776.

Huang Y, Carmichael GG. 1997. The mouse histone H2a gene contains a small element that facilitates cytoplasmic accumulation 
of intronless gene transcripts and of unspliced HIV-1-related mRNAs. Proc Natl Acad Sci 94: 10104-10109.

Huang MTF, Gorman CM. 1990. Intervening sequences increase efficiency of RNA 3' processing and accumulation of cytoplasmic RNA. Nucleic Acids Res 18: 937-947.

Imataka H, Gradi A, Sonenberg N. 1998. A newly identified N-terminal amino acid sequence of human eIF4G binds poly(A)binding protein and functions in poly(A)-dependent translation. EMBO J 17: 7480-7489.

Jain M, Khurana P, Tyagi A, Khurana J. 2008. Genome-wide analysis of intronless genes in rice and Arabidopsis. Funct Integr Genomics 8: $69-78$.

Jimeno S, Garcia-Rubio M, Luna R, Aguilera A. 2008. A reduction in RNA polymerase II initiation rate suppresses hyper-recombination and transcription-elongation impairment of THO mutants. Mol Genet Genomics 280: 327-336.

Jonsson JJ, Foresman MD, Wilson N, McIvor RS. 1992. Intron requirement for expression of the human purine nucleoside phosphorylase gene. Nucleic Acids Res 20: 3191-3198.

Kuhn J, Binder S. 2002. RT-PCR analysis of $5^{\prime}$ to $3^{\prime}$-end-ligated mRNAs identifies the extremities of cox2 transcripts in pea mitochondria. Nucleic Acids Res 30: 439-446.

Kyburz A, Friedlein A, Langen H, Keller W. 2006. Direct interactions between subunits of CPSF and the U2 snRNP contribute to the coupling of pre-mRNA $3^{\prime}$ end processing and splicing. Mol Cell 23: $195-205$.

Le H, Tanguay RL, Balasta ML, Wei CC, Browning KS, Metz AM, Goss DJ, Gallie DR. 1997. Translation initiation factors eIF-iso4G and eIF-4B interact with the poly(A)-binding protein and increase its RNA binding activity. J Biol Chem 272: 16247-16255.

Le Hir H, Izaurralde E, Maquat L, Moore M. 2000. The spliceosome deposits multiple proteins 20-24 nucleotides upstream of mRNA exon-exon junctions. EMBO J 19: 6860-6869.

Le Hir H, Gatfield D, Izaurralde E, Moore M. 2001. The exon-exon junction complex provides a binding platform for factors involved in mRNA export and nonsense-mediated mRNA decay. EMBO $J$ 20: 4987-4997.

Lin S, Coutinho-Mansfield G, Wang D, Pandit S, Fu XD. 2008. The splicing factor SC35 has an active role in transcriptional elongation. Nat Struct Mol Biol 15: 819-826.

Livak KJ, Schmittgen TD. 2001. Analysis of relative gene expression data using real-time quantitative PCR and the $2^{-\Delta \Delta C(\mathrm{~T})}$ method. Methods 25: 402-408.

Lou H, Gagel RF, Berget SM. 1996. An intron enhancer recognized by splicing factors activates polyadenylation. Genes Dev 10: 208-219.

Lu S, Cullen BR. 2003. Analysis of the stimulatory effect of splicing on mRNA production and utilization in mammalian cells. RNA 9: 618-630.

Lu J, Sivamani E, Azhakanandam K, Samadder P, Li X, Qu R. 2008. Gene expression enhancement mediated by the $5^{\prime}$ UTR intron of the rice rubi3 gene varied remarkably among tissues in transgenic rice plants. Mol Genet Genomics 279: 563-572.

Maas C, Laufs J, Grant S, Korfhage C, Werr W. 1991. The combination of a novel stimulatory element in the first exon of the maize Shrunken-1 gene with the following intron 1 enhances reporter gene expression up to 1000-fold. Plant Mol Biol 16: 199207.

Malim MH, Hauber J, Le SY, Maizel JV, Cullen BR. 1989. The HIV-1 rev trans-activator acts through a structured target sequence to activate nuclear export of unspliced viral mRNA. Nature 338: 254257.

Mascarenhas D, Mettler IJ, Pierce DA, Lowe HW. 1990. Intronmediated enhancement of heterologous gene expression in maize. Plant Mol Biol 15: 913-920.

Masle J, Gilmore SR, Farquhar GD. 2005. The ERECTA gene regulates plant transpiration efficiency in Arabidopsis. Nature 436: 866-870.

Millevoi S, Geraghty F, Idowu B, Tam J, Antoniou M, Vagner S. 2002. A novel function for the U2AF 65 splicing factor in promoting pre-mRNA $3{ }^{\prime}$-end processing. EMBO J 3: 869-874.
Moore MJ, Proudfoot NJ. 2009. Pre-mRNA processing reaches back to transcription and ahead to translation. Cell 136: 688-700.

Morello L, Giani S, Troina F, Breviario D. 2011. Testing the IMEter on rice introns and other aspects of intron-mediated enhancement of gene expression. J Exp Bot 62: 533-544.

Niwa M, Rose SD, Berget SM. 1990. In vitro polyadenylation is stimulated by the presence of an upstream intron. Genes Dev 4: 1552-1559.

Pillitteri LJ, Bemis SM, Shpak ED, Torii KU. 2007. Haploinsufficiency after successive loss of signaling reveals a role for ERECTA-family genes in Arabidopsis ovule development. Development 134: 30993109.

Proweller A, Butler JS. 1997. Ribosome concentration contributes to discrimination against poly(A) ${ }^{-}$mRNA during translation initiation in Saccharomyces cerevisiae. J Biol Chem 272: 6004-6010.

Rigo F, Martinson HG. 2008. Functional coupling of last-intron splicing and $3^{\prime}$-end processing to transcription in vitro: the poly(A) signal couples to splicing before committing to cleavage. Mol Cell Biol 28: 849-862.

Rondon AG, Jimeno S, Aguilera A. 2010. The interface between transcription and mRNP export: From THO to THSC/TREX-2. Biochim Biophys Acta 1799: 533-538.

Rose AB. 2002. Requirements for intron-mediated enhancement of gene expression in Arabidopsis. RNA 8: 1444-1453.

Rose AB. 2004. The effect of intron location on intron-mediated enhancement of gene expression in Arabidopsis. Plant J 40: 744751.

Rose AB. 2008. Intron-mediated regulation of gene expression. In Nuclear pre-mRNA processing in plants (ed. ASN Reddy, M Golovkin), pp. 277-290. Springer, Berlin-Heidelberg.

Rose AB, Beliakoff JA. 2000. Intron-mediated enhancement of gene expression independent of unique intron sequences and splicing. Plant Physiol 122: 535-542.

Rose AB, Last RL. 1997. Introns act post-transcriptionally to increase expression of the Arabidopsis thaliana tryptophan pathway gene PAT1. Plant J 11: 455-464.

Rose AB, Elfersi T, Parra G, Korf I. 2008. Promoter-proximal introns in Arabidopsis thaliana are enriched in dispersed signals that elevate gene expression. Plant Cell 20: 543-551.

Salles FJ, Strickland S. 1999. Analysis of poly(A) tail lengths by PCR: the PAT assay. Methods Mol Biol 118: 441-448.

Sanfaçon H, Brodmann P, Hohn T. 1991. A dissection of the cauliflower mosaic virus polyadenylation signal. Genes Dev 5: 141-149.

Shiu SH, Bleecker AB. 2003. Expansion of the receptor-like kinase/ Pelle gene family and receptor-like proteins in Arabidopsis. Plant Physiol 132: 530-543.

Shpak ED, Lakeman MB, Torii KU. 2003. Dominant-negative receptor uncovers redundancy in the Arabidopsis ERECTA Leucinerich repeat receptor-like kinase signaling pathway that regulates organ shape. Plant Cell 15: 1095-1110.

Shpak ED, Berthiaume CT, Hill EJ, Torii KU. 2004. Synergistic interaction of three ERECTA-family receptor-like kinases controls Arabidopsis organ growth and flower development by promoting cell proliferation. Development 131: 1491-1501.

Shpak ED, McAbee JM, Pillitteri LJ, Torii KU. 2005. Stomatal patterning and differentiation by synergistic interactions of receptor kinases. Science 309: 290-293.

Sieburth LE, Meyerowitz EM. 1997. Molecular dissection of the AGAMOUS control region shows that cis elements for spatial regulation are located intragenically. Plant Cell 9: 355-365.

Song SK, Lee MM, Clark SE. 2006. POL and PLL1 phosphatases are CLAVATA1 signaling intermediates required for Arabidopsis shoot and floral stem cells. Development 133: 4691-4698.

Subramanian C, Woo J, Cai X, Xu X, Servick S, Johnson $\mathrm{CH}$, Nebenfuhr A, von Arnim AG. 2006. A suite of tools and application notes for in vivo protein interaction assays using bioluminescence resonance energy transfer (BRET). Plant J 48: $138-152$. 
Tange TO, Nott A, Moore MJ. 2004. The ever-increasing complexities of the exon junction complex. Curr Opin Cell Biol 16: 279-284.

Tange TO, Shibuya T, Jurica MS, Moore MJ. 2005. Biochemical analysis of the EJC reveals two new factors and a stable tetrameric protein core. RNA 11: 1869-1883.

Torii KU, Mitsukawa N, Oosumi T, Matsuura Y, Yokoyama R, Whittier RF, Komeda Y. 1996. The Arabidopsis ERECTA gene encodes a putative receptor protein kinase with extracellular Leucine-rich repeats. Plant Cell 8: 735-746.
Wang H, Lee MM, Schiefelbein JW. 2002. Regulation of the Cell Expansion Gene RHD3 during Arabidopsis Development. Plant Physiol 129: 638-649.

Yokoyama R, Takahashi T, Kato A, Torii KU, Komeda Y. 1998. The Arabidopsis ERECTA gene is expressed in the shoot apical meristem and organ primordia. Plant J 15: 301-310.

Zhou Z, Luo MJ, Straesser K, Katahira J, Hurt E, Reed R. 2000. The protein Aly links pre-messenger-RNA splicing to nuclear export in metazoans. Nature 407: 401-405. 